3019101010

If

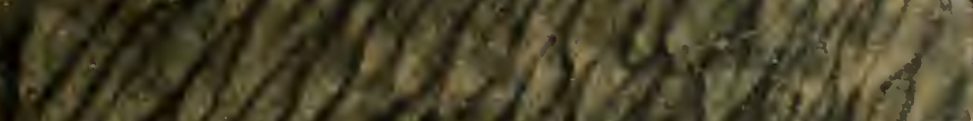

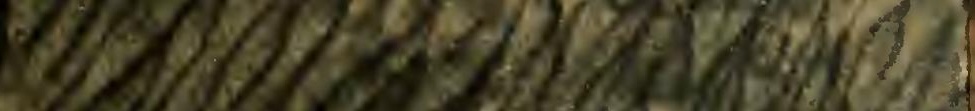
1.5.

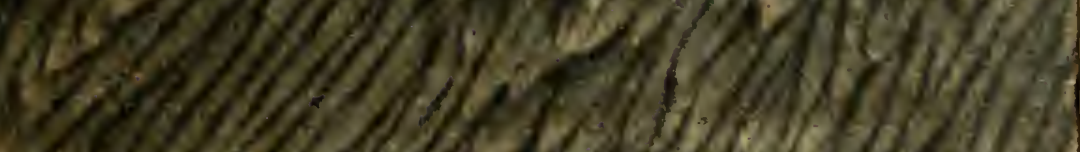

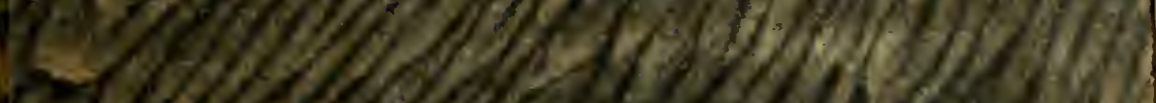

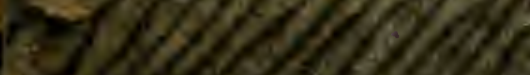

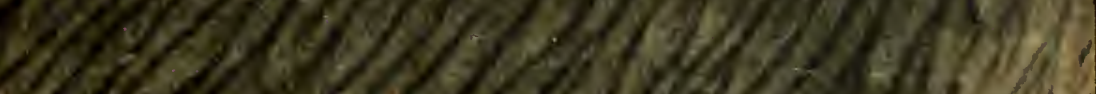

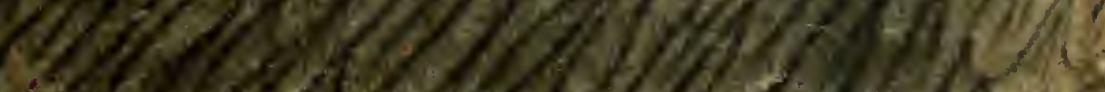

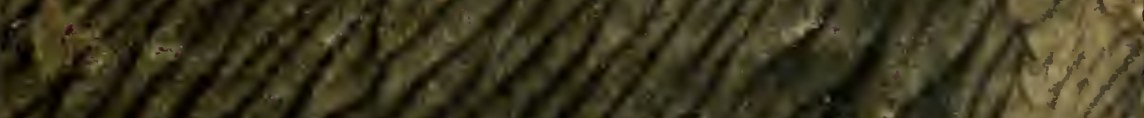

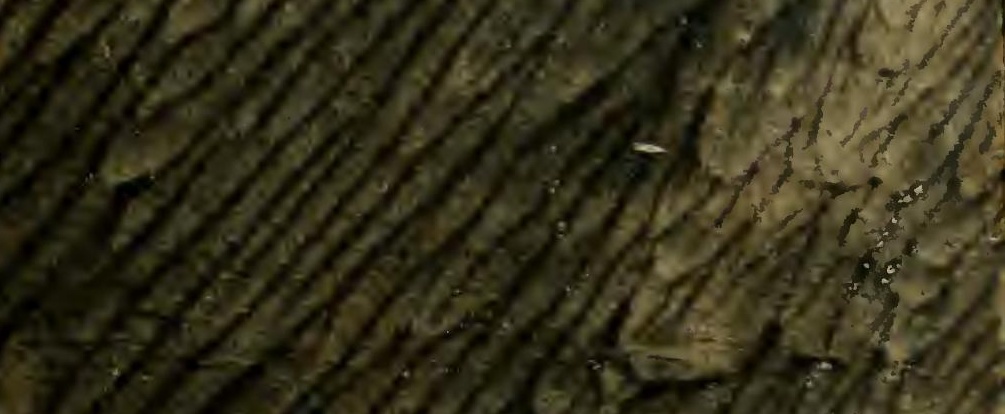

i.

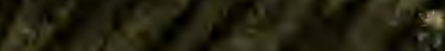

1. 18

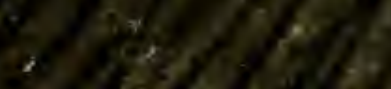

B. 22 



$$
\frac{b}{630}
$$


+ wi andoy
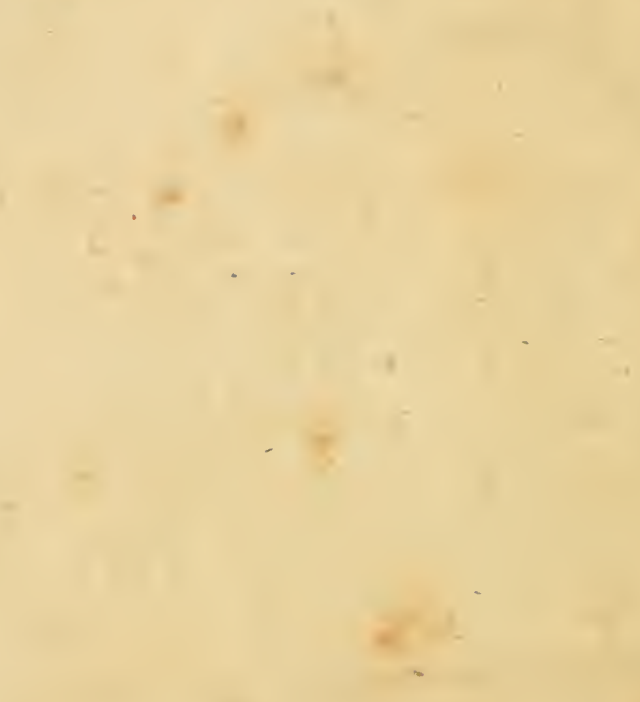


\section{ENTOZOORUM}

SYNOPSIS

C.LAllacot

CUI ACCEDUNT

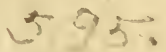

IIATISSA DUPLEX ET INDICES

LO C U P LET ISSI M I.

\section{A UCTORE}

\section{CAROLO ASIMUND RUDOLPHI}

PIILOS. ETT MED. DOCT, HUJUS PROF. P. O. MUSEI ANATOMI, DÍ́ECTORE, REGI A CONSIL. MED. INTINIS, COLLEGII MEDICO. TLCIINICI PRIMARII MEMBRO, ACAD. SCIFNT. BEROLIMENSIS, HOLNIIES. NEAPOLIT. ET PETROPOLIT. ACAD. NAT. CURIOS, LEOPOLDIN. GEORGOPIILORUII FLORENTIN. SOC. MIED IFOLMILNS, MIONSPELIENS. PARISIENS MEDICO-PIYYS. ERLANGCNS. VETERIN, HAFNIINS. PIIYSICAR. GOTTING. HALENS. JENENS. MLARUUR. ET ROSTOCHIENS NAT. SCRUT. BEROLIN, ET MOSOUENS. LINNAEAN. LIPS. SYDENIIATT. IIALIAS. TIINIRALOG.

JENEXS. SODALI.

C U. TI A B. III A E NEIS.

BEROLINI,

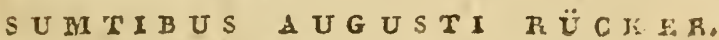
I 8 I. 
C.". Siltrietlerere

(5itroli (5)motant of atrus

i) $\pi .2$. 11. 


\title{
VIRO GELEBERRIMO
}

\section{JO. GODOFR. BREMSER}

IMPERIALIS MUSEI VIENNENSIS CUSTODI

\section{HEI. IT N THOLO G O SUM MTO}

\author{
AMICO INTEGERRIMIO
}

\section{U J U S J U S S U S G R I P S I T}

C U J U S O FE S C R I BER E P O T U I T

OPUS HOCCE QUALEUNQUE

C RATISSIMA MEITE

D. D. D.

A U C $\quad \mathrm{T} \quad \mathrm{O} \quad \mathrm{R}$. 



\section{P R A E F A T I O.}

Animalium Ordines, si Luvvaer et nostra tempora comparamus, ad nuum omines maximo quidem specierum numero locupletatos, horum tamen nulium qram Entozoorum illum aeque adauctum videbinus. JiNTAEUS undecim tantum vermes intestinales (Gordıuñ médinensem; Ascaridem vermicularem, lumbricoidem; 'Fasciolam hepaticám, intestinalem, barbatam; Hydram Hydatulam; Taeniam Solium, rulgarem, latam, caninam.) in Systematis Naturae Editione Duodecima (T. 1. P. II. Holm. 1767.) admisit G GELINUS in decima tertia (T.I. P. VI. 1790.) Redit, Palladis, O. Fr. MÜlleri, Goezin, Biociin, Wenneri, aliorumque laboribus utens, Entozoorum species ducentas nonaginta et rovem; ZEDERus vero (Anleitung zur Naturgeschichte der Eingeveide- 
würmer. Bamberg. '1803.) tercentas nonaginta et unam enumeraverunt. Quod ipse ante decem et quod excurrit annos edidi opus (Entozoorum Historia naturalis. Amstelaed. Yoll, II. 1808-1810.) sexcentas et tres; quod nunc edo ultra mille et centum species continet. Quarum si multas dubias invenires, hoc nihil interest, multo plures enim inter Gmelinianas etc. dubiae fuerunt, quo eadem. saltem ratio maneat.

Entozoologia edita domicilium mutavi et Berolinum petii, ubi Museum Regium Anatomicum directioni meae commissum, olin anatomiae humanae fere suli, nunc autem comparatae quoque dicatum, ideoque curam assidnam poscens, aliaq̣ue negotia bene multa ne ab Entozoorum studio penitus forsan avocassent, nisi Bremserus accessisset, et Musei Viennensis divitias exponendo, acta laudando, agenda demonstrando, inconstantiam meam vituperando, in viam relictam non sine vi me reduxisset,

Quo facto, me de studio nostro melius non merere posse mihi visus sum, quam si Italiam adirem, quo Entozoa Redio visa, plurimam partem dubia, recognıscerem, novàque indagarem. N'eque spes fefellit, variisque Italiae locis, praesertim autem Arimini 
et Neapoli multa Rediana, sed multo plura reperi nova, passim egregia, tam generalem Entozonrum indolem illustrantia, quam syștematis lacunas explenia. Micruscopio vero et simplici et composito instructus iter feci, omnia viva aut saltem recentia sedulo investigavi, observata mox litteris mandavi; redux autem Berolini onnia iterum de novo examinari et cum collectione mea comparavi.

Dum ipse vero in Italia Entozois indagandis occupatus fui, cel, AB Olfens, cujus dissertationem egregriam pag. 607. recensui, Brasiliam adiit, et quam plurima a se detecta, partim iconibus illustrata transmisit.

$A b$ altera parte Viri ill, et cel. Acbers, Bummenbacit, Bojanus, Braun, Cuvier, DoezIiNgli, Gaede, Klug, Lichitenstein, Nesti, Nitzcen, Otto, Recrlebex, Spedalieri, Tiedemane, Treutlen multa eademque passim maximi momenti benevole mecum communicaverunt, uti suis locis grata mente notavi.

Tanta tamen, ac nunc datur, nunquam offerre potuissem, nisi IMuseum Imperrale Viennense thesauros suos, opera fidem fere superante paratos, de quibus p. 602. fuse locutus sum, benignissime mihi aperuisset, 
quid? quod novissima, "quae optimus NATTE $\mathrm{TI}$ in Brasilia detexit plurina, describenda et, huic operi adjicienda permisisset, quod quidem beneficium laudibus meis majus est.

Singulis ceterum hujus libri sectionibus, quas Elenchus refert, harmmque singulis capitibus, nec non ordinibus et generitus in Mantissa enumeratis necessaria semper pracmisi, quae lectores benevoli conferant.

Dabam Berolini Calendis Maji 1819. 


\section{E l e ńch us.}

Synopsis, p. 1-200.

Nematoidea p. 1.

Acanthocepliala p. 65 .

Tremaioda p. 32.

Cestoidea p. 127.

Cystica p. 177.

Entozoa dubia p. 184.

Mantissa, p. $201-629$.

Sectio I. Systematica p. $203-569$;

Nematoidea p. 205.

Acanthocephala p. 309.

Trematoda p. 337.

Cestoidea p. 440.

Cystica p. 536.

Dubia p. 553 .

Sectio II. Anatomico-Physiologica p. 570-601.

Cap. 1. Generalia. p. 571.

Cap. 2. De nervis Entozoorum p: 574.

Cap. 3. De organis reproductionis p. 5\%9.

Cap. 4. De organis generationis, ovis, horumque nidis. p. 585 .

Cap. 5. De Entozoorum vita, varietatibus, monstris et morbis. p. 595. 


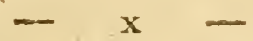

Sec:io III. Bibliographica p. 602-629. Appendix, sive Mantissa Altera (praeserim Brasiliana continens). p. $653-714$.

Nematoideir p. 634 .

Aranthocephala $\mathrm{p}, \sigma_{3}$.

Trematoda p. 67. .

Cestoidea p. 68 .

Cystica P. 709.

Dubia p. 710.

Index systematicus animalium, in quibus Entozoa hactenus reperta sunt, locis qua occurrunt simul inslicatis. p. 715 .

Index alphabeticus (generum) animalium in quibus

Entozoa reperta sunt. p. 789 .

Index Entozoorum alphabeticus, p. 793.

Explicatio Tabularum p. 809.

Corrigenda p. 811. - 


\section{Pars 1.}

\section{Entozoorum Synopsis.}


Entozoa signo crucis notata mihi visa non sunt, 1 


\section{Ordo I. \\ $\mathrm{N}$ e mat o id e a.}

Corpus teres elasticum. Tractus intestinalis hinc ore, illine ano terminatus. Alia individua mascula, alia feminea.

$$
\text { Genus I. Filaria. }
$$

Corpus teres, elasticum, subaequale, elongatum. Os orbiculare. Genitale masculum: spiculunt sim. plex.

$$
\text { A. Ore simplici. }
$$

1. Filaria medinensis Gmex.

F. longissima, capite attenuato, cauda maris sub: ulata, inflexa, feminae semiterti, acutiuscula incurva.

Ent. I. p. 55. n. 1. Mantiss. n. I. F. medinensis. Hab. in Hominis.tela cellulosa subcutanea, praesertim pedum, in regionibus tropicis.

2. Filaria gracilis R.

F. longissima, cauda attenuata, maris spiraliter devoluta obtusiuscula, feminae inflexa, асu. tiuscula.

Ent. 1. p. 57. n. 2. Mant. n. 2. F. gracilis. Hab. In savo abdominis Simiac Capucinae Be- 


\section{- 6}

rolini Januàro reperi. In illo S. dpollas, FGs nisci, Sphingis. Cat. Ent, Viein.

3. Filaria attenuata $\mathrm{F}_{2}$.

F. longissima, utrinque obrusa, postỉce attenua. ta, caudae maris apice complanato inflexo.

Ent. I. p. 58. 1. 3. F. attenuata, p. 70. 1. 17. F。 aquilae. n. 18. F. Falconum. p. 71. 11. 19. Fo, Strigis. Mant. n. 3. F. attenuata.

Hab, in abdomine aliisque partibus Falconum: praesertim $F$. peregrini; Strigium et corvorums de quibus conf. Mantissa.

4. Filaria obtusa $\mathbf{R}$.

F. crassiuscula, aequalis, capite aeutirusculo, cau. da obtusa,

Ent. I. P. 59. n. 4. F. obtusa,

Hab. In cavo abdominis Hirundinis rusticas Gryphiae Majo reperi, Ibid, in Hirundino urbica et riparia. Cat. Ent. Vienzi.

5. Filaria unguiculata $\mathbf{R}$.

F, crassiuscula, utrinque attenuata, capite obruso, cauda feminae reflexa unguiculata.

Ent. I. p. 72, n. 25. F. Alaudae, Mant, D.5. F. un, guiculátz.

Hab. In abdomine Alawda arvensis repertam KurG dedit.

6. Filaria aftinis $\mathrm{R}$.

F. crassiuscula, subaequalis, capite truncato y cauda feminae obtusa.

Mant. n. 6. F. affinis.

Hab. in Fringillae nova specie hispanica, Mus: Vienn.

7. Filaria abbreviata $\mathrm{R}$.

F. crassiuscula, aequalis, capite tenuiore obru: so, cauda feminae rotundata. 


\section{$=-5=$}

Mant. n. 7, F, abbreviata.

Hab, circa oculum Mgtacillae stajazinac. Mus, Vienn.

8. Filaria fusca $\mathbf{R}$.

F. crassiuscula, fusca, utrinque obtusa, cauda feminae tenuiore.

Mant. n. 8. F, fusca.

Hab. In abdomine Pleuronectis manci Neapoli Junio reperi.

9. Filaria sanguinea $\mathrm{R}$.

F. crassiuscula, sanguinea, utrinque obtusa, cals. da feminae tenuiore,

Mant. n. 9. H. sanguinea.

Hab. Sub Cyprini Gibelionis cute pinnae cau. dalis Berolini Martio reperi

o. Filaria rubella $\mathrm{F}$.

F. elongata, rubella, antrorsum tenuior, capito acutiusculo, cauda obtusa.

Mant. n. 10. F. rubella,

Hab. In ventriculo Ranae temporariae KIUG Berolini reperit. Ipse ibidem Octobri in $R$. csa culentae vesiculis ventriculi et intestinorung; sed minorem offendi. As huc 2, 5. I. ?

1. Filaria truncata $\mathbf{P}$.

F. elongata, capite truncato, caudae crassioris acumine brevissimo obtusiusculo;

Ent. 1. p. 59. n. 5. F, truncata.

Hab. In larva Tineae Padellae Nrxzsczi Augu-

sto reperit.

12. Filaria ovata ZED.

F. corpore antrorsum attenuato, 'capite ovato; cauda rotundata.

Ent. 1. p.60. n.6. Mant, n. 12. F, ovata. $\mathrm{Hab}_{\mathrm{b}}$ in abdomine Cyprini Gobionis et Phoxini. 


\section{$=6 .-$}

13. Filaria capsularia $\mathrm{R}$.

$F_{t}$ corpore antrorsum attenuato, ore orbiculari marginato cauda obtusa cum acumine.

Ent. r.p.67.n. 7. F. capsularia,

Hab. In Clupeae Harengi abdomine intra vis. cerum praesertim hepatis tunicam externam, complicatam, saepe gregariam, rarissime in illius tubo cibario, Gryphiae reperi.

\section{B. Ore papilloso vel labiato.}

14. Filaria papillosa $R$.

F. ore orbiculari colloque papillosis, corpore suba€quali, postice attenuato, cauda incurvata.

Ent. 1. p. 62, n. 8. Mant, n, 14. F. papillosa.

Hab. in Equi pectoris, abdominis aliisque cavis 15. Filaria cormata $\mathbf{R}$.

F. capitis nodulis tribus obsoletis, corpore subaequali, caudae acumine brevi obtuso.

Ent. I. p.65. n, 9. F. coronata.

Hab, sub Coraciae Garrulae cute jugulari, versus aures, vel inter colli musculos.

16. Filaria acuminata R. $\downarrow$

F. capite quadrinodi, corpore aequali, caudae obtusae acumine tenui recto.

Ent, I, p, 66, n. Io, F, acuminata',

Hab. in larva Noctuae Nuptae. Goez.

17. Filaria truncatula $\mathrm{R}$.

F. capitis truncati ore papillis sex cincto, corpore tenuissimo retrorsum increscente, caurla....

Ent. I. p. 78, n. 39, F. Phalangii. Mant. n. 17.

F. truncatula.

Hab. in abdomine Phalangii cornuti et Opilioniser 


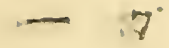

is. Filaria plicata R.

F. capite attenuato, labio oris plicato, cauda.obtusa.

Ent, I. p. 67, n. Ir. Mant, n. Is. F. plicata.

Hab. in Erucis ZEDER, Bombycis Salicis, Mantiss.

19. Filaria globiceps $\mathbf{R}$.

F. capitis, rotundati papillis obsoletis, corpore aequali, cauda depressa obtusissima.

Mantiss, n. 19: F. globiceps.

Hab. In Uranoscopi scabri abdomine et genita. libus, inque Blennii Phycis ovariis Junio et Julio Neapoli reperi.

$$
\text { species dubiae. }
$$

20. Filaria Hominis bronchialis. I

Ent. I. p. \&2. n. I. Hamularia subcompressa.

Mant. n. 20. F, bronchialis.

Hab. ad Hominis bronchos. Treutrer.

21. Filaria Vespertilionis. +

Hab. in abdomine $V$ espertilionis discoloris, Cat. Ent. Vienn. mspt.'

22. Filaria Vulpis. +

Ent. I. p. 68. n. 13. F. vulpis.

Hab, in Vulpis abdomine. P. Camper. Num potius Ascaris intestinum transgressa? In Vulpis rene, Richir. An petius Strongylus Gigas?

23. Filaria Leonis, +

Ent, I+ p. 68, n. I4. F. leonis.

Hab, sub Leonis pelle. Reor.

24. Filaria Mustelarum subcutanea. I

Ent, I, p. 69, n. I5. Mant, n. 24. F. Mustelarim.

Hab.'sub cute Mustelarum Foinae, Martis it Putorii. 


\section{- $8-$}

25. Filaria Mustelarum pulmonalis.

Ent. 2: p 263. n. 3. Vermis gen, dub, Mantiss, n. 25. F. Mustelarum pulmonalis.

Hab. in pulmonibus Mustelarnm Foinae, Martis et Putorii.

26. Filaria Erinacei. \&

Hab. in pulmonibus Erinacei vulgaris Catal. Entoz. Vientu.

27. Filaria Leporis subcutanea. \&

Ent. 1. p. 69. n. 16. F. leporis.

$\mathrm{Hab}$, in Rossiae australis Lepore timido circa regionem lumbarem et coxam. Paltas,

28. Filaria Leporis pulmonalis. $f$

Mantiss, n. 28. F. leporis pulmonalis,

Habo in bronchiis Leporis timidi. Froedict, An ad Strongylum 11. 17.?

29. Filaria Musculi.

Hab. in abdomine Mluris Musculi circa ventriculum et hepar. Cat, Ent. Vienll. mseppt,

30. Filaria cervi. I

Hab. in abdomine Cervi Elaphi, Cat.Ent.Viennn. 31. Filaria Bubali \&

Hab, in abdomine Bovis Bubali, Cat, Ent. Vientu,

32. Filaria Collurionis subcutanea. $f$

Ent. 1. p. 71, n. 20. F. collurionis. Mant, n. 32. F, Collurionis subcutanea,

Hab. sub pelle Lanii Collurtonis, pomerani et minoris. Cat. Ent. Viẹnn. mscpt.

33. Filaria Collurionis pulmonalis. $\downarrow$

Ent, I. p. 83, n. 2. Hamularia cylindrica. Mant. $\mathrm{n}, 33, \mathrm{~F}$, collurionis pulmonalis.

Hab, in Lanii Collurionis pleura, pulmonibus. 
34. Filaria Meropis. +

Hab. in mesenterio INeropis dpiasiri. Cat, Ent. Vienln.

35. Filaria Sturni.

Ent. r. p. 73, n, 26. F. Sturni.

Hab. in Starni cavo pectoris et pulmonibus, PaILAS.

36. Filaria Turdorum. +

Hab, in abdomine Turdi pilaris et viscivori, eandemque esse speciem ac n, 33. F, Motacillarum, Cat, Ent. Vientr.

37. Filaria Carduelis.

Ent, I. p. 73. n, 27, F, carduelis.

Hab. in Fringillae carduelis coxa., Sprgenuus,

38. Filaria motacillarum. $t$

$\mathrm{Hab}$, in abdomine Motacillae Rubeculae et $\mathrm{O}$ nanthes, eandemque esse specicm ac illam Turdorum n, 36. Cat. Ent. Vienn, In Catalogo manuscripto enumeratur exiam Filaria cirea aures, genas et collum. MIotacillae P7ilomelas reperta.

39. Filaria Ciconiae. $f$

Ent, 1. p. 7r. n. 23. F. Ciconiae.

Hab. in abdomine et sub pelle Ardeate cico. niae.

40. Filaria Ardeae nigrae. $t$

Mantiss. n. 3y. F. Ardeae nigrae.

Hab. in abdomine Ardeae nigrae. Rosa.

41. Filaria Ardeae cinereae.

Ent. 1. p. 72. n. 24. F. Ardeae cinereae.

Hab. in Ardea cinerea prope pedis tendinem. braun, Sub pelle femoris, Cat. Enti Vienr. mucptiss, 
42. Filaria Tringae. †

Hab, in Tringa alpina, sub pelle aurium. Cat. Enit. Vienn.

43. Filaria Charadrii.

$\mathrm{Hab}$, in Charadrio minore sub pelle narium et aurium. Cat. Ent. Vienn.

44. Filaria Sternae. t

Hab. in Sterna Leucopareia, intestinis extus adhaerens. Cat. Eut. Vienn.

45. Filaria Colymbi. †

Hab. in abdomine Colymbi cristati. Cat, Ent. Vienn, mscpt.

46. Filaria Lari.

Mantiss. n. 46. F. Lari.

Hab. in Laro minuto sub cute colli, IIus, Vienn 47. Filaria Cygni. †

Ent. I. p. 7r. n. 21, F. cygni.

Hab. in Cygni abdomine et intestinis, Redr. 48. Filaria Anatis.

Ent. I. p. $71,-n, 22, \mathrm{~F}$, anatis.

Hab, in Anate, cordi adhaerens. Paullinus.

49. Filaria Colubri austriaci. I

Hab, in oesophago Colubri austriaci. Cat. Ent. Vienn.

50. Filaria Colubri, americani. I

Ent. 1. p. 73, n, 28. Fo colubri.

Hab, in intestinis 'speciei Colubri americanae. liosc.

51. Filaria Ranae esculentae. I

Hab. sub cute Ranae esculentae. Cat. Ent. Vienn. An ad n, 10, ?

52. Filaris piscium.

Ent, x, p. 7\%, 1, 29, Mant, n, 52, F, piscium. 
Hab. In variorum piscium marinorum carne musculari, cavo abdominis, hepate etc. occurrere dicitur species ista hybrida et excludenda, de qua mantissa conferatur.

53. Filaria Zenis Fabri. †

$\mathrm{Hab}$, in Zenis Fabri hepate et intestinis. Cat. Ent. Vien!n.

54. Filaria Spari Auratae.

Hab, in ovariis Spari Auratae. Catal. Enttoz. Vicnn!.

55. Filaria Coleopterorum. †

Hab. in Coleopteris, quorum forsan Filariae cum insequentibus conjungendae sunt, quemadn:o. dum Filaria Carabi blapoidis in Cat, Ent. Vienn. huc trahitur.

56. Filaria Silphae.

Ent. I. p. 76, n. 3r. F. Silphae.

Hab. in Silpha obscura, Goeze.

57. Filaria Chrysomelae Tanaceti. f

Ent. I. p. 76. n. 32. F. Chrys. Tanaceti,

Hab. in Chrysomela Tanaceti. Froelicir.

58. Filaria Chrysomelae Alni. †

Ent. 1. p. 77, n. 33. F. Clirys. Alni.

Hab, in Chrysomela Alni. Holten.

59. Filaria Buprestis. †

Ent. I. p. 77. n, 34. Filaria Buprestis.

Hab, in Buprestis specie non dicta. Bovcher d'Abвeville.

60. Filaria Forficulae.

Ent, 1. p. 77. n. 35. Mant, n, 6o, F. forficulae. Hab. in Forficula Auricularia.

61. Filaria Locustae. I

Ent, r. p. 77, n, 36, F, locustae, 


\section{$-82-$}

Synonymis addatur: ZnNANNi sopra le cavallette. p. 8, 9. Tab, I, n. 3.

Hab. in Locusta viridissima. Frisch, In $\mathrm{L}_{\text {r }}$ verrucivora. Roeser. In L. Heluitogia. Cat. Ent. Vienn.

62. Filaria Cercopidis t

Ent, I. p. 78. n. 37. F. Cercopidis,

Hab. in Cercopide spunzaria. RoEser. 65. Filaria Araneae. $t$

Ent. I. p. 78, n. 38. F, àraneae.

Hab. in Araneae specie non dicta, Latremiz. 64. Filaria Monoculi. \$

Hab. in Monoculo Apode Dux Scriwarzbergo Rudolstamiensts.

65. Filaria Erucarum.

Ent. I. p. 79. n, 4I. Mant, n. 65. F. erucarum.

Hab, in larvis Papilionum Antiopes, Betulae, Polychlori, Quercus, Urticae; Sphingis Euphorbiae; Bombycum Alni, Cajae, Ouercus, Sálicis, Trifoliz, Ziczac; Pyralidis Pomanae; erucis mortuis etiam in pyris et ponis reperilur. Plures certe species, Conf, $n_{0}, \mathbf{I} \mathbf{r}_{\text {, I6, I8, }}$

66. Filaria Phryganeae. I

Ent. I. P. 81. 1. 43. F. Phryganeae.

Hab. in larvis Phryganeorun, DEGEER,

67. Filaria Tenthredinis. I

Ent, I. p. 82. n. 43. Fo tenthredinis.

Hab, in larvis Tenthredinum, Bergaraxis; 
Genus II, Trichosoma.

(Capilläria ZEDERL.)

Corpits teres elasticum tenuissimum retrorsum in. sensibili modo incresceins. Os punctiforme. Gimitale masculum: filum simplex vaginatun.

1. Trichosoma brevicolle $\boldsymbol{R}$.

T. parte capillari corpore curvato breviori, cau. da feminae obtusa.

Ent, I. p. 86. n. I. Trichocephalus capillaris. Habitat in intestinis coecis Anseris. Schrank, Nitzscir, Anatis Querquedulae, potissimum Junio et Julio, ZEDER.

2. Trichosoma obtusiusculum $\boldsymbol{R}$.

T. parte capillari corpore incurvo subaequali, cauda feminae obtusiuscula.

Mant. n. 2. T. obtusiusculum.

Hab, inter Arcleae Gruis tunicas ventriculi, Mlus, Vienn.

3. Trichosoma obtusum $R$.

T. parte capillari corpori subaequali, cauda ob. tusa, vagina penis duplo longioris recta.

Ent. 1. p. 87. n, 2. Trichocephalus tenuissimus, Mant, n. 3. T, obtusum.

Hab. in intestinis coecis Strigis Aluconis, $B u$. bonis, Dasypodis, Oti, Passerinae. An huc no. 10.?

4. Trichosoma inflexum $R$.

T. parte capillari corpori subaequali, cauda in. flexa et vagina penis brevi obtusis. Mant, n. 4. T inflexum.

Hab, in intestinis Turdi cyanei, Mus, 'Ficnn, 
5. Trichosoma longicolle $R$.

T. parte capillari corpore longipri, cauda femi. nae obtusa emarginata.

Ent. I. p. 84. n. 3. Hamularia nodulosa. Mantiss. n. 5. T. longicolle.

Hab, in intestinis praesertim crassis Phasiani Galli et colchici; in coecis Tetraonis Urogalli, Perdicis et Tetricis.

6. Trichosoma Plica R.

T. parte capillari corpore incurvo longiori, cauda feminae obtusa.

Mant. n, 6. T. Plica.

Hab, In Canis Lupi vesica urinaria Berolini Januario reperi.

\section{species dubiac.}

Omnes fere generis hujus species dubias vocare et intactas relinquere potuissem, verum enim discrimen plurimarum nos fugit; paucarum tamen differentias specificas exempli quidem caussa ponere tentavi, a succéssoribus emendandas, rem - maribus rarius occurrentibus - non adeo facilem. Optimas e contra differentias in genere Trichocephali insequente, cujus individua mascula cognita sunt, invenies.

7. Trichosoma Vespertilionis. $f$

Hab, in intestinis Vespertilionis Lasiopteri, Cat.

Ent, Vienn, nuscptus.

8. Trichosoma Putorii. \&

Hab, in duodeno Mustelae $\boldsymbol{P}_{\text {utorii, Cat, Ent. }}$ Vienn. mscpt.

9. Trichosoma Erinacei. $t$.

Hab. in intestinis tenuibus Erinacei europaei. Cat, Ent, Vienn, 


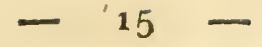

10. Trichosoma Falconum. ' f

Mantiss. n. Io. T, falconum.

Hab, in intestinis crassis Fálconis Buteonis et Milvi. An ad n. 3.?

11. Trichosoma Corvorum. †

Mantiss. n. I1. T. corvorum,

Hab. in intestinis Corvorum Caryocatactis, Mo nedulae, Picae.

12. Trichosoma Picorum. I

Hab, in intestinis Picorum cani, majoris, viridis. Cat. Ent. V. mscpt.

13. Trichosoma Alaudae. I

$\mathrm{Hab}$. in intestinis crassis Alaudae arvensis, Cat, Ent. V. mscpt.

14. Trichosoma Turdi. +

Hab, in intestinis Turdi Viscivori, Cat, Ent, V.: an ad n. 4. ?

15. Trichosoma Fringillae. I

Hab. in intestinis Fringillae caelebis. Cat. Ent. Vienn.

16. Trichosoma Hirundinis, +

Hab. in intestinis Hirundinis rusticae. Cat, Ent. Vienn.

17. Trichosoma Caprimulgi. †

Hab. in intestinis Caprimulgi europaei, Catal. Ent. V. mscpt.

18. Trichosoma Columbae. †

Hab. in intestinis crassis Columbae domesticae, Cat. Ent. V. mscpt.

19. Trichosoma Vanelli. I

Hab, in intestinis Tringae Fanelli, Cat, Entoz, Vionp mscpt. 


\section{- $16-$}

20. Trichosoma Cha adrii. $f$

Hab inter Charadrii minoris et Himantopodis

tunicas ventriculi. Càt. Ent. $V$. mscpt.

21. Trichosoma Carbonis. I

PHab. in intestínis Pelecani Carbonis. Cat. Ent. Viein.

22. Trichosoma Crotali.

Hab. in intestinis Crotali Durissi, Cat, Ent. $V$.

\section{Genus III. Trichocephalus.}

Corpus teres elasticum, parte, antica capillari su. bito in crassiorem transeunte. Os orbiculare. Genitale masculum simplex viginatum.

\section{A. Incomes.}

*. Trichocephalus dispar $\mathbf{R}$.

T. parte capillari longissima, capite acuto, corpore maris spiraliter involuto, feminae sub. recto, vaigina penis obovata.

Ent. I. p. 88. n. 3. T. dispar.

Hab. in Hominis intestinis crassis, praesertim coeco, vulgatissimus.

2. Trichocephalus palaeformis. $R$.

- T, parte capillari longissima, capite acutiuscu. 1o, corpore maris spiraliter involuto, feminae subrecto, vagina penis basi cylindrica, apice lato, truncato.

Mant. n. 2. Tr. palaeformis.

Frab. in intestinis crassis, praesertim coeco-Simiae Sphingis, Ursinae, Sylvani, Patae, Sabaeae

3. Trichocephalus affinis $\mathbf{P}$. T. parte capillarí longissima, capite acuto, cor. 


\section{- $17-$}

pore maris subspirali, feminae rectiusculo; vagina penis brevi, cylindrica, obtusa.

Ent. I. p. 92. n. 4. Mant, n, 3. Trich. affinis.

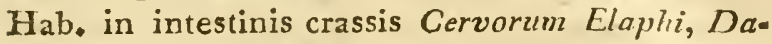
mae, Capreoli; Antiloparun Dorcadis, Rupicaprae; Bovis Tauri; Ovis Ammonis et Arietis. 4. Trichccephalus unguiculatus. $\mathrm{R}$.

Tr. parte capillari longissima, capite unguiculato, corpore maris spirali, feminae rectius. culo, vagina penis longissima tenuissima truncata.

Ent. I. p. 93, n. 5. Mant, n, 4. Trich, unguicu. latus.

$\mathrm{Hab}$, in intestinis crassis, praesertim coeco Leporis timidi, variabilis, Cuniculi feri, Arctomydis Citilli.

5. Trichocephalus depressiusculus $\mathbf{R}$.

Tr. parte cápillari longissima, capite attenuato, corpore maris spirali, feminae rectiusculo, vagina penis tenui, cylindricä.

Ent. 1.p.94. n.6. Mant.n.5. Tr, depressiusculus, Hab, in coeco Canis familiaris et Vulpis. 6. Trichocephalus crenatus $\mathbf{R}$.

Tr. parte capillari longissima, capite acutiuscu1o, corpore maris spirali, feminae rectiusculo, vagina penis cylindrica truncata brevi cincta vesicula biloba.

Ent. I. p. 95. n.7. Mant. K. 6. Tr. crenatus،

Hab, in intestinis crassis Suis ferae et domesticae.

7. Trichocephalus nodosus $\mathrm{R}$.

Tr. parte capillari longiore corpore maris spirali, feminae rectiusculo, capite acuto, vagina penis infundibuliformi truncata cincta vesicu. cula rotundatas 
Ent. I. p.96. n. 8. Mant. n. 7. Trich. nodosus. Hab. in intestino coeco Murium amphibii, arvalis, Musculi, Ratti, sylvatici.

\section{B. Armati.}

8. Trichocephalus echinatus $\mathbf{R}$.

Tr. capite echinato, parte capillari corpore spirali breviore.

Ent, I. p. 98. n. 9. Tr, echinatus,

Hab, in ventriculo Lacertae Apodis LINN. (Chamaescurae SchNeId, Bipedis LAcíptide; CuVLER.)

$$
\text { Species dubiae. }
$$

9. Trichocephalus Lemuris.

Mant. n. 9. Trich. Lemuris.

Hab, in intestino coeco Lemuris Mongoz, Mus. Vienn.

10. Trichocephalus Castoris.

Hab, in intestinis crassis Castoris Fibri. Cat, Ent. Vienn.

11. Trichocephalus Cameli. I

$\mathrm{Hab}$, in intestinis crassis Cameli Bactriani et

Dromedarii, Cat, Ent. Vienn. mscpt.

$$
\text { Genus IV. Oxyuris. }
$$

Corpus teres; elasticum, parte postica (feminae) subulata. Os orbiculare, Penis vaginatus.

1. Oxyuris cuirvula $\mathrm{R}$.

O. capite nudo, parte subulata ovifera, (Mas ignoratur.)

Ent. 1. p. 100. n. 1. Oxyuris curvula. $\mathrm{Hab}$, in Equi coeco, toto anno. 


\section{$-19-$}

2. Oxyuris alata $R$.

O. capite alato, parte subulata ovifera,

Mant, n. 2. Ox, alata.

Hab. in intestinis crassis Melis Taxi, Mus, Vienn.

3. Oxyuris ambigua $\mathrm{R}$.

O. parte crassa ovifera, Maris cauda incurvz ante apicem penem raginatum emittente,

Mant, 11. 3. Ox, ambigua.

Hab. in intestinis crassis Leporis Cuniculi et

"timidi. Mus. Vienn.

Species dubiae.

Ipsemet nonnisi species dictas veras Oxyurides agnoscere possum, amicissimo vero $\mathbf{B} R E M$. SERo, Helminthologo summo, cujusque auctoritas alioquin apud me summa est, adhuc enume. rantur:

Oxyuris vermicularis Hominis et Simiae $P a$. nisci: Ascaris nobis ejusdem nominis n, 3r. nec non

Oxyuris Muris amphibii, arvalis, Musculi, Arctomydis Citilli: Ascaris obvelata nobis $\mathbf{n}, 32$.

\section{Genus V. Cucullanus.}

Corpus teres elasticum, postice attenuatum. Capitis ore orbiculari, cucullo striato. Genitale masculum: spiculüm duplex.

1. Cucullanus elegans ZED.

C. capite obtuso, cucullo globoso, cauda maris acutiuscula inflexa, utrinque alata, feminae recta, obtusa.

Ent. r. p. I02. n. I. C. elegans ZEn. p. 107. n. 3 .

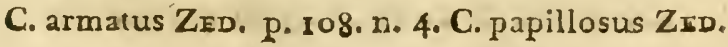


-p. Ir3. n. 7. C. coronatus ZED. Mantiss, n. I.

C. elegans.

Hab. in intestinis et abdomine Muraenae Anguillae, Gadi Lotae, Percae Luciopercae, fluviatilis, cernuae, Zingel, Esocis Lucii, Cy: prini Aspii.

2. Cucullanus truncatus $\mathbf{R}$.

C. capite obtuso, cucullo ovali; cauda maris acuta bialata, feminae attenuata, acumine bre. vi truncato.

Mant. n. 2. C. truncatus.

Hab. In Siluri Glanidis intestinis Gryphiae Octor bri reperi.

3. Cucullanus alatus $\mathrm{R}$.

C. capite obtuso, cucullo globoso; caudae maris inflexae acutae ala solitaria.

Ent. 1. p. 106. n. 2. C. alatus.

Hab- In intestinis Pleuronectis maximi Gryphiae Junio et Augusto reperi. Num alam recte vidi? Num species a prima vere differt?-

4. Cucullanus globosus ZED.

C. capite globoso, oris margine tumido, collo gracili, cauda acuta, maris breviori inflexa, feminae longiori recta.

Ent. 1. p. II I. n. 6. C. globosus.

Hab. in intestinis Salmonis Truttae (Martio)

ZeDer; Farionis, Cat. Ent. Vienn.

5. Cucullanus melanocephalus $\mathbf{R}$.

C- capite obtuso, cucullo globoso, cauda mas. cula incurva obtusa bialata.

Mant. n. 5. C. melanocephalus.

Hab. In intestinis Scombri Coliae, Rochei, Sardae Junio et Julio Neapoli repexi, 


\section{$-21=$}

6. Cucullanus foveolatus $R$.

C. capite obtuso, cucullo globoso, cauda acuta nuda, maris incúrva, feminae recta.

Ent. I. p. Iog. n. 5. Mant. 11.6. C. foveolatus.

Hab. in Gadorum intestinis. O. Fr. Mürter.

- Ipse in intestinis Blennii Phycis (Junio), MTle raenae Cassini (Julio), Helenae (Augusto) Neapoli reperi.

7. Cucullanus abbreviatus $R$.

C. capite obtuso, cucullo subgloboso, apice cau. dali brevi acuto.

Mant. n. 7. C. abbreviatus.

Hab. In intestinis Percce cirrosae Majo Romae reperi.

8. Cucullanus minutus $\mathbf{R}$.

C. capite et cucullo globosis, cauda acuta, ma ris inflexa, feminae recta depressa.

Mant. n. 8. C. minutus.

Hab. in Pleuronecte Passere. Mus, Vienn.

9. Cucullanus heterochrous $\mathbf{R}$.

C. capite cuneiformi papilloso, cucullo elliptico, cauda utriusque sexus rectiuscula.

Ent. I. p. II . n. 8. C. heterochrous.

Hab. In intestinis Pleuronectum Flesorum saepissime a me dissectorum semel (Gryphiae Majo 1800) et quidem copiose reperi.

$$
\text { species dubiae. }
$$

10. Cucullanus Testudinis. $t$

Hab. In intestinis Testudinis orbieularis marem et feminam repertos esse. Catal, Ent. $V, m$.

11. Cucullanus Hydri. \pm

Hab. In intestinis Hydri caspii feminam inventam esse, Cat. Ent. Vienn, mscptus. 
12. Cucullanus Accipenseris. $f$

Ent. I. p. 11 7. n. Ir. C. Accipenseris.

Hab. in Accipensere (certe Sturione) Amitdgaard.

In Acc. Husonis intestinis marem et feninam repertos esse. C. E. V.m. An Ophiostoma sphaerocephalum mihi n. 3. dictum?

13. Cucullanus Platessae.

Ent. J. p. I 16, n. 9. C. platessàe.

Hab, in intestinis Pleuronectis Platessae. RetNH.

Treviranus. An ad speciem octavam?

14. Cucullanus Soleae. $t$

Hab. In intestinis Pleuronectis Soleae marem et

feminam repertos esse, C. E. V. m.

15. Cucullanus Percae. $t$

Ent. I. p. 117. n. 10. C. Percae.

Hab. in Perca Norvegican Abildgaard.

16. Cucullanus Spari + .

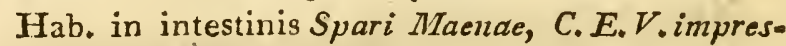
sus; sed in catalogo manuscripto signum in. terrogationis adjectum est.

17. Cucullanus Tincae. $\downarrow$

Hab, in intestinis Cyprini Tincae. C. E. V. An ad speciem primam, quam ipse saltem in $C_{y}$ prino Aspio reperi.

\section{Genus VI. Spiroptera.}

Corpus teres elasticun utringue attenuatum. Os orbiculare. Penis inter alas caudae spiraliter de. volutae laterales emergens.

A. Ore nudo.

1. Spiroptera megastoma $\mathrm{R}$. $\mathrm{S}_{\mathrm{P}}$. capitis discreti ore magno nudo, cauda femi- 


\section{- $23-$}

nae rectiuscula acuta, maris simphiciter spirali, corpusculis retundis ad basin penis styliformis. Mant, n. I. Sp. megastoma.

Hab, in Equi tuberculis ventriculi morbosis. Reckieben.

2. Spiroptera sterëura $\mathbf{R}$.

Sp. ore orbiculari nudo, caudae femineae rectae apice rigido styliformi, rotundato, masculae simpliciter spiralis vagina penis monophylla. Mant. n. 2. Sp. sterëurá.

$\mathrm{Hab}$, in Falcone naevio, inter membranam nictitantem et oculi bulbum, nec non in meátu auditorio, Mus. Vienn.

3. Spiroptera strongylina $\mathbf{R}$.

Sp. ore orbiculari nudo, caudae femineze apice depresso acutiusculo, masculae simpliciter spiralis alis latis radiatis, pene longissimo.

Mant. n. 3. Sp. strongylina.

Hab, in Suis ferae ventriculo, ITus, Vienn.

4. Spiroptera nasuta $\mathbf{R}$.

Sp. capitis acuti ore nudo, caudae femineae apice acuto inflexo, masculae spira subtriplici. Mant. n. 4. Sp. nasuta.

$\mathrm{Hab}$, in ventriculo Fringillae domesticae, M. V. 5. Spiroptera laticeps $\mathbf{R}$.

Sp. capite alis subsagittato, cre nudo, caudae femineae apice depresso obtusiusculo, masculae spira subtriplici.

Mant. n. 5. Sp. laticeps.

$\mathrm{Hab}$, in Falconis Lagopodis oesophago, $M_{0} V_{\text {. }}$

\section{B. Ore papilloso.}

6. Spiroptera alata $\mathbf{R}$.

Sp, ore papilloso, capite caudaque utriusque 


\section{$-24-$}

sexus alatis, feminea apice brovi depresso, mascula in spiram triplicem deflexa.

Ent. 1. p. 189. n. 51. Ascaris sagittata. Mant. n.6. Sp. alata.

Hab. in ventriculo et intestinis Ardeae nigrae. Braun, Froelich.

7. Spiroptera laticaudata $R$.

Sp. capite papilloso, colli dente utrinque breviusculo, caudae femineae apice depresso acutiusculo, masculae rotundato-alatae spira sim. plici.

Mant. n. 7. Sp. laticaudata.

Hab. inter Otidis Tetracis tunicas ventriculi. Mus, Vienu.

8. Spiroptera bidens. $\mathbf{R}$.

Sp. capite papilloso discreto, colli denticulo utrinque laterali, caudiae femineae apice bre. vi incurvo, masculae spira duplici.

Mant. n. 8. Spiroptera bidens.

Hab. inter Meropis Apiastri tunicas ventriculi. Mlus. Vienn.

9. Spiroptera bicuspis $\mathbf{R}$.

Sp. capite papilloso, colli cuspide utrinque latérali, . caudae femineae apice recurva, masculae spira duplici.

Mant. n. 9. Sp. bicuspis.

Hab, inter Tringae helveticae tunicas ventriculi. Mus. Vienn.

10. Spiroptera strumosa $\mathbf{R}$.

- Sp. capite papilloso, tuberculo sub basi colli, caudae femineae apice depresso obtuso, masculae spira duplicì.

Ent. ̌. p. 193. n. 55. Ascaris strumosa. Mant, n. 10. Sp. strumosa. 


\section{$-\quad 25$}

Hab. In ventriculo Talpae europaeae Gryphiae Aprili reperi.

11. Spiroptera quadriloba $\mathrm{R}$.

Sp. capitis utrinque bilobi ore papilloso, caudae femineae apice acuto. (Mas ignotus.)

Mant. n. 1I. Sp. quadriloba. $H_{a b}$, in oesophago Pici viridis. IM. V.

12. Spiroptera contorta $R$.

Sp. capite papilloso, feminae corpore spiraliter contorto, cauda depressa, inflexa, apice acu. - tiusculo. (Mas ignotus.)

Ent. r. p. 198. n. 67. Ascaris testudinis, Mant, n. 12. Sp. contorta.

Hab. In Testudinis orbicularis ventriculo hujusque tuberculis morbosis Arimini Majo reperi.

13. Spiroptera Anthuris $R$.

Sp. ore papilloso, caudae femineae apice depresso incurvo, obtusiusculo, masculae spira. simplici, vagina penis longi tetraphylla.

Mant. n. 13. Sp. Anthuris.

Hab. inter Corvorum, Coraciae Garrulae et Orioli Galbulae tunicas ventriculi. $M . V$.

14. Spiroptera attenuata $R$.

Sp. capite papilloso, caudae femineae ápice atte. nuato excavato, masculae spira simplici, va* gina penis composita.

Mant. n. 14. Sp. attenuata.

Hab. inter Hirundinis urbicae, rusticae et ripa. riae tunicas ventriculi. $M . V$.

15. Spiroptera Cystidicola $\mathrm{R}$.

Sp. capite papilloso, caudae femineae apice inflexo obtusiusculo, masculae spira duplici, pene longiuscule: 
Ent. r. p. I22. n. 4. Ophiostoma Cystidicola. Mant. n. 15. Sp. Cystidicola.

Hab. in vesica natatoria Salmonis Farionis et Thymalli lati, hujusque oesophago, ubi No. vembri mense Berolini reperi.

16. Spiroptera uncinata $R$.

Sp. capite papilloso, caudae femineae apice deflexo unciformi, masculae spira simplici, pene breviusculo.

Mant. n. 16. Sp. uncinata.

Hab. In Anseris domestici tuberculis oesophageis Kuvg Berolini Septembri mense reperit.

17. Spiroptera elongata $R$.

Sp. capite papilloso, feminae longissimae cauda apice deflexo uncinato. (Mas ignotus.)

Mant. n. 17. Sp. elongata.

Hab. inter Sternae nigrae tunicas ventriculi Mus. Vienn».

18. Spiroptera revoluta $R$.

Sp. 'capite papilloso, caudae femineae apice depresso divaricato, masculae revoluto, spira duplici.

Mant. n. I8. Sp. revoluta.

Hab. inter Charadrii Himantopodis tunicas ventriculi. $M . V$.

19. Spiroptera leptoptera R.

Sp. capite papilloso, caudae femineac apice acuto, depresso, masculae tenuissime alatae spira duplici.

Mant. n. 19. Sp. leptoptera.

Hab. in vertriculo Falconis Buteonis, cineracei, Nisi, Tinnuncinii. M. V.

20. Spiroptera euryoptera $\mathbf{R}$.

Sp. capite papillose subdiscreto, caudae femi- 


\section{- $27-$}

neae apice obtusiusculo, masculae latissime alatae spira triplici.

Mant. n. 20. Sp. euryoptera.

Hab. inter Lanii Collurionis, Excubitoris et minoris tunicas ventriculi. $M . V$.

21. Spiroptera sanguinolenta $\mathbf{R}$.

Sp. capite papilloso, caudae femineae apice depresso obtusiusculo, masculae spira subduplici, vagina penis filiformis monophylla.

Ent. I. p. 242.n. 26. Strongylus lupi. Mant. n. 2, Sp. sanguinolenta.

Hab. In' Canis Lupi tuberculis ventriculi morbosis, rarius in hoc vel duodeno liberam Berolini Januario reperi.

22. Spiroptera obtusa $\mathbf{R}$.

Sp. capite papilloso, caudae femineae apice brev̀i conico, masculae spira duplici, pene inflexo.

Ent. I. p. 170. n. 36. Ascaris obtusa. Mant. n. 22. Sp. obtusa.

Hab. In ventriculo Muris Musculi Gxyphiae Junio reperi.

$$
\text { Species dubiac. }
$$

23. 'Spiroptera Hominis.

Mant. n. 23. Sp. hominis.

Hab. in Hominis vesica urinaria. Barnetr.

24. Spiroptera Simiae.

Ent. I. p. 67. n. 12. Filaria alata. Mant. n. 24. Sp. Simiae.

Hab. inter Simiae Maimonis tunicas ventriculi。 Albers.

25. Spiroptera Leonis.

Ent. I. p. 242. n. 28. Strongylus Leonis.

Hab. in Leonum tuberculis oesophageis. REDr. 


\section{$-28$}

26. Spiroptera Tigridis. +

Ent. I. p. 243.n.29. Strongylus Tigridis.

Hab. in Tigridis gula et ventriculo. Du.Hardr.

27. Spiroptera Ursi Arcti.

Mant. n. 27. Sp. Ursi. An huc Ent. 1. p. 244. n. 27. Sírongylus Ursi.

Hab. in Ursi Arcti oesophago. $M . V$.

28. Spiroptera Hystricis. †

Ent. I.p. 245. 1. 3I. Stronigylus Eystricis.

Hab. in Iystricis cristatae tuberculis oesopha. geis. Redr.

29. Spiroptera Citilli.

Mant. n. 29. Sp. Citilli.

Hab. in ventriculo Arctonydis Citilli.- $M . V$. 30. Spiroptera Falconis.

Mant. n. 30. Sp. Falconis.

Hab. in pede Falconis rufipedis. M. $V$.

31." Spiroptera Strigis. I

Hab. inter Strigis Scopis tunicas ventriculi. Cat E. $V$.

32. Spiroptera Upupae. †

Hab. inter Upupae Epopis tunicas ventriculi. Cat. $E, V$.

33. Spiroptera Sturni. †

Hab. inter Sturni Cincli tunicas ventriculi. Cat. E. V.

34. Spiroptera Falcinelli. +

Hab. inter Tantali Falcinelli tunicas ventriculi. Cat. E. V.

35. Spiroptera Gallinulae.

Ent. 1.p. 237.n. 20. Strongylus horridus.

Hab. In oesophago Scolopacis Gallinulae Julio

Gryphiae reperi. 
36: Spiroptera Vanelli. †

Ent. I. p. 239. n. 23. Strongylus Vanelli.

Hab. in intestinis Tringae Vanelli. Schraxk. In.

ter illius tunicas ventriculi. C, E. $V$.

37. Spiroptera Fulicae. $f$

- Hab. inter Fulicae atrae tunicas ventriculi, C.E.V.

38. Spiroptera Sternae.

Ent. 1. p. 239. n. 22. Strongylus ambiguus.

Hab. Inter Sternae Hirundinis tunicas oesophagi Julio Gryphiae reperi.

39. Spiroptera Colymbi.

Ent. I: p. 238. n. 21. Strongylus, crenulatus.

Hab. In Colymbi septentrionalis bulbo ventriculi Julio Gryphiae reperi.

40. Spiroptera Anatis. I

Hab. inter Anatis glacialis tunicas ventriculi. C. E. $V^{*}$. mscpt.

Genus VII. Physaloptera.

Corpus teres elasticum utrinque attenuatum. Os orbiculare. Cauda maris deflexa utrinque alata, vesicam inferam sistens. Penis tuberculo emissus.

1. Physaloptera clausa $\mathrm{R}$.

Ph. Ore nudo, caudae fémineáe depressae apice incurvo papillato, masculae vesicula infera utrinque clausa serifera.

Mant. n, I. Ph. clausa.

Hab. in ventriculo Erinacei europaei. Mus. $r$.

2. Physaloptera alata $R$.

Ph. capitis alati ore papilloso, caudae masculae vesica aperta apicem haud attingente, fernineae rectae apice brevi depresso. 
Ent. 2. p. 375. Vermis dubius Falconis Nisi. Mant. n. 2. Ph. alata.

Hab. in Falconis Nlsi, pennati ventriculo, in intestinis F. Gallici. M. $Y$.

3. Physaloptera abbreviata $R$.

-Ph. ore subpapilloso, caudae femineae obtusae acumine brevissimo conico, masculae vesicula aperta apicem haud attingente.

Mànt. n. 3. Ph. abbreviata.

Hab. in ventriculo et intestinis Lacertae margaritaceae. $M . V$.

4. Physaloptera retusa R.

Ph. ore papilloso, caudae femineae apice bre. vissimo conico, masculae vesicula patentissima retusa.

Mant. n. 4. Ph, retusa.

Habitaculo non indicato Olferś maxima copia ex Brasilia misit.

$$
\text { species a } u \text { bia. }
$$

5. Physaloptera tenuicollis $\mathrm{R}$.

Mant. n. 5. Ph. tenuicollis.

Hab. in intestinis Falconis Haliaëti. M.V.

\section{Genus VIII. Strongylus.}

Corpus teres elasticum utrinque attentzatum. Os orbiculáre vel angulatum. Apex caudae masculac cerminatus bursa penem emittente.

A. Ore orbiculari, aculeato. Sclerosto. sn $a t a$.

1. Strongylus armatus $\mathbf{R}$. Str. cápite globoso truncato, oris aculeis rectis densis, bursa maris triloba, cauda feminae obtusiuscula. 


\section{- $31-$}

Ent. I. p. 204. n. I. Mant. n. r. Str. armatus. Hab. Major in intestinis crassis, praesertim coeco Equi, Asini et Muli; Minor in aneurysmati. bus arteriarum Equi mesentericarum, uterque vulgaris.

2. Strongylus dentatus $\mathbf{R}$.

Str. capitis obtusi dentibus anticis recurvis, bur. sa maris triloba, cauda feminae subu!ata.

Ent. I. p. 209.n.2. Mant.n. 2. Str. dentatus.

Hab. In intestinis pratsertim crassis Suis et ferae et domesticae Octobri, Novembri et Februa. rio Gryphiae reperi.

B. Ore orbiculari noduloso seu papil. loso.

3. Strongylus Gigas $\mathrm{R}$.

Str. capitis obtusi ore papillis sex planiusculis cincto, bursa maris integra truncata, cauda feminae rotundata.

Ent. I. p. 2 Io. n. 3. Mant. n. 3. Str. Gigas.

Hab. in renibus Hominis, Canis, Lupi, Vulpis, Vulpis americanae, Martis, Equi et Tauri; in omento Gulonis; in intestinis Lutrae; in Phocae vitulinae pulmonibus, hepate, intestinis, de quibus Mantissa conferatur.

4. Strongylus papillosus $\mathbf{R}$.

Str. capitis obtusi ore papillis sex conicis cincto, corpore crenato, bursa maris integra ob. longa patente, cauda feminae obtusá.

Ent. I. p. 214. n.4. Mant. n. 4. Str. papillosus.

Hab. In oesophago Corvi Caryocatactis Junio mense Gryphiae reperi.

5. Strongylus tubifex Nitzscir.

Str. capitis obtusi ore papillis sex conicis cin- 


\section{- $3^{2}$}

cto, corporis crenati parte media inflata, bursa maris integra obliqua contracta, cauda feminae obtusa.

Ent. I. p. 21/4. 11. 4. sub Strongylo praecedente. p. 240. n. 24. Str. mergorum. n. 25. Str. anatis. Mant. n. 5. Str. tubifex.

Iab. in oesophago Colymbi septentrionalis, arctici, cristati, minoris; Mergi Merganseris, albclli, serratoris; Anatis domesticae, Creccae; Pelecani Carbonis et pygmaei.

6. Strongylus contcrtus $\mathbf{R}$.

Str. capitis obtusi ore subtrinodi, bursa maris compressa quadriloba, cauda feminae acuta recurva.

Ent. I. p. 2:6. n. 5. Str. contortus.

Hab: Fabricus in Ovis Ariei is intestinis No. vembri reperit; ipse eodem mense Gryphiae in Agni ventriculo quarto copiosissimum in. veni.

7. Strongylus filicollis $\mathbf{R}$.

Str. capitis alati ore subtrinodi, collo capillari longissimo, bursa maris integra, hinc produ. cta, cauda feminae obtusiuscuia.

Ert. r. p. 217.n.6. Mant. n. 7. Str. filicollis.

Hab. In Ovis Arietis intestinis tenuibus saepius aestate et auctumno Gryphiae offendi; in Cervo Capreolo hyeme Nixzsch reperit.

\section{Ore undo.}

8. Strongylus Filaria R.

Str. capite obtuso, corpore subaequali, bursa ma. ris integra obliqua, cauda feminae acuta. Ent. 1. p. 219. n. 7. Str. Filaria. Hab. in-Ovis Aristis arteria aspera et bronchis, 


\section{$-\quad 33$}

saepe copiosissimus et letiferus. Srck, Froraran. Iisdem locis in Ove Anmone. C.E.V. 9. Strongylus hypostomus $\mathrm{R}$.

Str, capitis subglobosi gibbi ore seminfero, corpore subacquali, bursa maris integra truncata, caudae fernineae apice brevi acuto.

Mart. n. 9. Str. hypöstomus.

Hah. in coeco Antilopes Rupicapras. Arus. V. 10. Strongylus radiatus $\hat{h}$.

Str. capite obtuso, bursa maris biloba, lobis inaequalibus, tottindatis, cauda feininae subulata.

Ent. I. p. 220. n. 8. Str, radiatus,

Hab. In intestinis Bovis Tauri tenuibus Octo. bri tt Novembri Gryphiae reperi.

i I. Strongylus venulosus $R$.

Str. capite obtuso, bursa maris subbiloba trun. cata, cauda feminae obtusiuscula.

Ent, 1.p.221. n. 9. Str. venulosus.

Hab. In intestinis praesertim tenuibus Caprae Hirci Augusto Gryphiae reṕeri.

12. Strongylus ventricosus $R$.

Str. capite attenuato alato, maris corpore postice ińcrassato, bursa obtusa, feminae antrorsum auctae cauda subulata.

Ent. I. p. 222. n. Io. Str. ventricosus.

Hab. In intestinis tenuibus Cervi Elaphi Februario reperi, Ibidem in Cervo Dama. IIIus, Vienti.

13. Strongylus auricularis $\mathrm{ZED}_{\mathrm{ED}}$

Str. capite obtuso alato, bursa maris biloba, feminae cauda subulata.

Ent. 1. p. 223. 1. 11. Str. auricularis.

$\mathrm{Hab}$, in intestinis Lacertae caerulescentis, viri- 


\section{$-34-$}

dis, agilis; Anguis fragilis; Hylae arboreae, Rance temporariae, esculentae; Bufonis vulgaris, viridis, ignei, fusci; Salanzandrae lacu. stris, atrae.

14. Strongylus denudatus $\mathbf{R}$.

Str. capite nudo, oris orbicularis margine tumidulo, cauda feminae subulata.

Marat. n. r4. Str. denudatus.

Hab. in Colubri tesselati pulmonibus, Mus. V.

15. Strongylus striatus $\mathrm{Z} E \mathrm{ED}$.

Str. maris collo, feminae toto corpore transversim striatis, margine denticulatis, bursa illius henisphaerica, hujus cauda acuta.

Ent. I. p+225, n. 12. Str. striatus.

$\mathrm{Hab}$, in bronchiis Erinacei europaei, a ZEDERo Januario, a me Julio Gryphiae repertus,

16. Strongylus inflexus $\boldsymbol{R}$.

Str. capite obtuso, corpore retrorsum attenuato, bursa maris inflexa, cauda feminae unguicuIata.

Ent. I. p. 227. n. I3. Str, inflexus,

Hab, in bronchiis et cavo tympani Delphini Phocaenae, KLEIN, P. Gamper, Arbers,

17. Strongylus retortaeformiș ZED. Str, capillaris, capite obtuso, ore orbiculari, bur. sa maris biloba, cauda feminae subulata.

Ent, I, p. 229. n. 14. Mant, n', 17. Str. retortaeformis.

Hab. minor in Leporis Cuniculi et timidi intestinis, in hoc Zedero auctumno, mihi Au. gusto, in illo Bralnio visus; major a Treutxero Januario in Lep. cimidi brónchuis repertus. 


\section{- 35}

18. Strongylus nodularis $\mathrm{R}$.

Str. capite truncato discreto, bursa maris obliqua biloba, cauda feminae subulata,

Ent. I. p. 230, n. 15. Mant. n, I8. Str, nodularis.

Hab. in Anseris domestici oesophago, ventriculo et duodeno, mili Novembri Gryphiae obvius; eodem mense a Nitzschio inter Ana* tis segetum tunicas ventriculi repertus.

19. Strongylus capitellatus $R$.

Str. capite globoso discreto, caudae femineae apice obtuso incurvo.

Mant. n. ig。 Str. capitellatuś.

Hab. In intestinis Caprimulgi europaei tenuibus

Treutler Augusto reperit.

20. Strongylus trigonocephalus $\mathbf{R}$.

Str. labio capitis trigono, bursa maris biloba, cauda feminae obtusiuscula.

Ent. I. p.231, n. 16. Mant. n, 20, Str. trigonoce. phalus.

Hab. in Canis familiaris ventriculo, in hujus et intestinorum tuberculis, in corde.

21. Strongylus tetragonocephilus $R$.

Str.' labio capitis tetragono, bursa maris biloba, cauda feminae acuta inflexa.

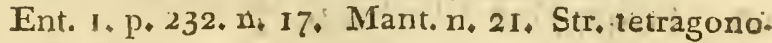
cephalus,

$\mathrm{Hab}$, in intestinis Canis $\bar{\nu}_{\text {ulpis, a Froenrchro }}$ Octobri, Novembri a Zedero et Treutuero repertus.

22. Strongylus criniformis $R$.

Str. labio capitis tetragono, bursa maris bilobz, inaequali, cauda feminae obtusiusccula.

Ent, I. p. 234. D. 18. Str. crinifarmis, 


\section{- $3^{6}-$}

Hab. In intestinis Melis Taxi tenuibus Octobri

Gryphiae copiosissimum reperi.

23. Strongylus tubaeformis ZED.

Str. labio capitis tetragono,' bursa maris obco. nica, cauda feminae acuta, ..

Ent. I: p, 236, n, 19. Str, tubaeformis.

Hab. in Felis Cati duodeno Zederus Aṕrili reperit.

\section{species, dubiae.}

24. Strongylus Lupi.

Hab. in intestinis Canis Lupi, C.M. V., mspt. 25. Strongylus Vulpis glandularis.

Mant. n. 25. Sr. Vulpis gland,

$\mathrm{Hab}_{\text {, in }}$ Julpis glandulis mesenterii, Mus, $\boldsymbol{V}_{*}$ 26. Strongylus Vulpis oesophageus.

Mant. n, 26. Str. Vulpis oesoph.

Hab. in Vulpis oesophago. Mlus. $V$.

27. Strongylus Myoxi: ま

Hab, in intestinis Myoxi Gliris, C, E. W. 28. Strongylus Suis.

Eut. 1.p. 246.n. 34. Mant. n. 28. Str. Suis.

Hab, in bronchis Apri. EBEx. Suis domesticae MODEER,"MUU. $Y$.

-29. Strongylus Elephanti. †

' Hab. in Elephanti indici hepate. C.E. $\boldsymbol{V}$. 30. - Strongylus Cameli. I

Hab. in Cameli Dromedarii et Bactriani pulmonibus. C.E.V.nust.

51. Strongylus Capreoli. †

Ent. 1.p. 244.11. 32 Str. Capreoli.

Hab, in Cervi Capreoli tuberculis renum, RED.

An potius Spiroptera? 


\section{$=37-$}

32. Strongylus Dorcadis, $t$

- Hab, in pulmonibus Antilopes Dorcadis, C. E. V. miscptus,

35. Strongylus Ammonis.

Hab, in intestinis Ovis Ammonis, $C, E, V$.

34. Strongylus Vitulorum. t

'Ent, í, p. $245+$ D. 33 .

Hab. in Vitulormm trachea et bronchis, accumulatus saepe perniciósus.

35. Strongylus Tardae.

Ent. r. p. 24r. n. 25. Str. Tardae.

Hab. In Otide Tarda Septembri Gryphiae femineum reperi.

36. Strongylus Ardeae stellaris. $\dot{T}$

$\mathrm{Hab}$, in Ardcae stellaris ventriculo. C.E. V. $\mathrm{m}_{\mathrm{e}}$ 37. Strongylus Viperae. †

$\mathrm{Hab}$, in intestinis Viperae Reäi, C.E. $\boldsymbol{L}^{*}$ 38. Strongylus Colubri. t

Hab, in intestinis Colubri austriaci, C.E.Y.

\section{Genus IX. Ascaris.}

Corpus teres elasticum utrinqué attenuratum. 'Cáput trivalve. Genitale masculum: spiculuni cinplex. A. Corpore utrinque aequaliter atitenut at o.

$$
\text { a. Capite nudo: }
$$

1. Ascaris lumbricoides LiNX. Asc, capite nudo, corpore utrinque sulcato, cauda obtusiuscula.

Ent. I. p. I24. n. I. Mánt, n. I. Asc.lumbricoides. Hab. in intestinis tenuibus Hominis, Shis, $\mathbf{B o}$. vis, Equi et Asini. 


\section{$-38$}

8. Ascaris distans $\mathbf{R}$.

Asc. Capite nudo, corpore aequabili, cauda maris inflexa acuta, feminae recta subulata.

Ent. I. p. I28. n. 2. Asc. distans.

Hab. In intestinis Simiae Sabaeae crassis Bero.

lini Martio reperi.

3. Ascaris vesicularis Froelich.

Asc. capite nudo, corpore utrinque aequaliter attenuato, cauda subulata rectiuscula, maris utrinque alata.

Ent. I. p. I29. n. 3. Asc. vesicularis. p. 198. n. 66. Asc. Urogalli. Mant. n, 3. Asc, vesicularis. Hab. in intestinis crassis, praesertim coecis Otidis Tardae; Phasiani Galli, colchici, picti, nyethemeri; Pavonis cristati; Numidae Mlelea. gris; Tetraonis Urogalli, Bonasias, Coturnieis, Perdicis, saxatilis,

4. Ascaris inflexa Zed.

Asc. capite nudo, corpore utrinque aequaliter attenuato, caudae femineae acumine brevi inflexo:

Ent. 1. p. 129. n, 3, sub praecedente, ib. p, 132, et n. 4. Mant. n, 4, Asc, inflexa,

Hab, in intestinis tenuibus Phasiani Galli; Ana. tis Boschadis, domesticae, acutae, moschatae. An huc n. 99.?

5. Ascaris subulata $R$.

Asc. capite nudo, corpore utrinque aequaliter attenuato, cauda subulata, feminae recta de. pressa, maris inflexa, utrinque alata.

Mant. n. 5. Asc. subulata.

Hab. in intestinis Caprimulgi ruficollis, Mus, $V$. An huc n. 90.? 


\section{$=39$.}

6. Ascaris adunca R.

Asc. capite caudaque utriusque sexus incurva acuta nudis, 'corpore utrinque aequaliter attenuato,

Ent. I. p* 133, n. 5. Mant. n. 6. Asc, adunca, Hab. In ventriculo et intestinis Clupeae Alosae Gryphiae Majo, Arimini Aprili reperi.

7. Ascaris constricta $\mathrm{R}$.

Asc. capite nudo, truncato, corpore utrinque aequaliter attenuato, cauda rectiuscula obtusa. Ent, 1. p. 134. n. 6. Mant. n. 7. Assc. constricta.

Hab. In Trachini Draconis tunica intestinorum peritoneali Parisiis Majo, Neapoli Junio reperi,

8. Ascaris rotundata $\mathrm{R}$.

Asc. capite rotundato nudo, corpore utrinque aequaliter attenuato, apice caudae brevi acuto inflexo,

Mant. n. 8. Asc, rotundata,

Hab. In Squali Galei ventriculo Arimini Majo reperi. In ventriculo Squali glauci, IMus, V.

9. Ascaris osculata $\mathrm{R}$.

Asc. capitis nudi valvulis orbicularibus marginatis, corpore utrinque aequaliter attenuato, utriusque sexus apice caudae brevissimo acuto.

Ent. I. p. 135. n. 7. Asc, osculata.

Hab. In ventriculo Phocae vituliuse Gryphiae Novembri reperi,

10. Ascaris pellucida $\mathbf{R}$.

Asc. capitis nudi valvulis magnis obtusis, corpore utrinque aequaliter attenuato, apice cau. dae obtuso.

Mant. n. Io. Asc. pellucida.

Hab. Sul Upupae Epopis tunica hepatis TrEvT.

LER reperit. 


\section{$\sim 40$}

11. Ascaris gulosa $\mathrm{R}$.

Asc. "cápitis' nusti valvulís élongatis, corpore utrinque aequaliter attenuato, cauda feminae recta subulata, maris acumine brevi divaricato. Mant. n. '1. Asc. gulósa.

Hab. In Festrictinis tabulatae coeco et anteriore coli parte Novembri Berolini reperi.

12. Ascaris dactyluris R."

Asc. capite nudo, corpore utrinque aequaliter attenuato, cauda feminae longa subulata, maris arice brevi obtuso depresso.

Ent, 2* p. 266, n. 9* Vermis dubius Testudinis terrestris." Mant, n. I2. A

Hab. In intestinis crassis Testudinis graecae Romae Majo reperi.

13. Ascaris spinicainda OEFERs.

Asc. capite nudo, corpore utrinque subaequalitêr attenuato, cauda feminae recta subulata, marisinicurva, alata, cuspidata.

Mant. n. 15. Asc, spinicauda.

Hab. In Lacerta Teguixin repertam OleEns ex Brasilia misit.

14. Ascaris acuminata Scurrants.

Asc. capite nudo, corpore utrinque aequaliter atteruato, cauda obtusa mucronata, mariş recta, feminae inflexa.

Ent. I. p. 136. n. 8. Asc acuminata.

Hab. in intestino tenui Hylae arboreae, Ranae esculentae et lemporariae, in qua saepius reperi. b. Capite alato.

15. Ascaris transfuga $R$.

Asc. capitis ala utrinque lineári longa, caudae obtuso-acutae nulla, corpore sulcato aequa. liter attenuato. 
Mant. n. 15. Asc, transfuga

Hab', in intestinis, Ursi arcti et maritimi, MIus. Vienn.

16. Ascaris leptoptera $\mathbf{R}$.

Asc. capitis ala utrinque Ionga lineari vel latiuscula, caudae acutiusculae nulla, corpore aequaliter attenuato.

Ent, I. p. 137+n. 9. Mant. n. 16. Asc, lept.

$\mathrm{Hab}$. In Leonis oesophago et ventriculo Decembri Berolini reperi.

17. Ascaris microptera $\mathrm{F}$.

Asc. capitis ala utrinque lineari brevi, caudae obtusae nulla, corpore aequaliter attenuato. Mant. n. 17. Asc, microptera.

Hab. In oesophago et intestinis tenuibus Canis Lupi Berolini Januario reperi.

18. Ascaris marginata $R$.

Asc. capitis ala utrinque semilanceolata, caudae obtusiusculae vix conspicua, corpore aequaliter attenuato.

Ent. r. p. 138. n. ro. Asc. marginata. Hab, in intestinis Canis familiaris tenuibus vulgaris.

19. Ascaris triquetra Scrmanr.

Asc. capitis ala utrinque semielliptica, caudac acutae latiuscula, corpore aequaliter attenuato. Ent. I. p. 139. n. II. Asc. márginata.

Hab, in intestinis Canis $\mathbb{F} u l p i s$ tenuibus vulgaris.

20. Ascaris brachyoptera $\mathrm{R}$.

Asc. ala capitis utrinque lato-elliptica, caudac papillatae nulla, corpore aequaliter attenuato. Mant. n. 20. Asc: brachyoptera.

Hab. in intestinis Viverrae Genettae. Mus, $V_{*}$ 


\section{$-42-$}

21. Ascaris mystax ZED.

Asc, ala capitis utrinque semiovata, caudae acutiusculae lineari, corpore aequaliter attenuato. Ent. I. p. 140. n, r2. Asc. mystax.

Hab. frequentissima in intestinis tenuibus Felis Cati-clomestici; etiam feri et Lyncis. Mus $V$.

22. Ascaris Perspicillum h.

Asc. ala capitis urinque lineari, valvulis marginatis, corpore aequaliter attenuato, cauda acuta Ent. I. P, I4I. H. I3. Asc. perspicillum.

Hab. in intestinis tenuibus Meleagridis Gallo. pavonis Decembri Gryphiae reperi.

23. Ascaris semiteres ZED.

Asc. ala capitis utrinque lineari, corpore aequa. liter attenuato subtus planiusculo, cauda obtusiuscula.

Ent. I. P, I 43. n. I4. Mant. n. 23. Asc, semiteres. $\mathrm{Hab}$, in intestinis Tringae Vanelli. Froelichro Septembri, Julio ZeDero, mihi Junio Gryphiae obvia,

24. Ascaris depressa ZED.

Asc, ala capitis discreti depressi, caudaeque obtuso-acutae lineari, corpore aequaliter vel antrorsum magis attenuato.

Ent, I. p. 143. n. 15. Asc. depressa. p. 189. n. 32. Asc spiralis, Mant, n, 24. Asc, depressa.

Hab. in intestinis Vulturtum, Falconum et Strigium, de quibus Mantissa conferatur.

25. Ascaris ensicaudata ZeD.

Asc, ala capitis caudaeque ensiformis latiori, corporis aequaliter vel antrorsum magis attenuati vix conspicua.

Ent, I.p. I45, n, 16. Asc. ensicaudata. p. 191. n.54. Asc, lancea. Mant, n, 25. Asc, ensicaudáta. 


\section{$-43$}

Hab. in Turdorum intestinis, de quibus Man. tissa conferenda.

26. Ascaris crenata ZED.

Asc, ala capitis depressi corporisque utrinque aequaliter attenuati crenata, caudae obtusae acuta, spiculis foliaceis.

Ent. I. p. 146. n. I7. Asc. crenata.

Hab. in intestinis Sturni vulgaris Comes De Borke, Zeder Aprili; Braun dedit.

27. Ascaris fallax $R$.

Ascaris ala capitis oblitterata, valvulis obtusis, corpore utrinque aequaliter attenuato, cauda obtusa maris marginata deflexa, feminae rectiuscula.

Mant. n. 27. Asc. fallax.

Hab. in ventriculo et intestinis Lacertae viridis. Mus. Tienn.

28. Ascaris nigrovenosa ZED.

Asc. ala capitis caudaeque maris reflexae, feminae inflexae latiore, corporis utrinque aequaliter attenuati tenuissima.

Ent. I.p.147.n. 18. Mant. n.28..Asc.nigro.venosa. Hab. In Anguis fragilis, Thanae temporariae, esculentae, Bufonis cinerei pulmonibus reperi; in Bufonis ignei, viridis, fusci pulmonibus. Cat, Ent. $V$.

29. Ascaris Acus Brocir.

Asc. ala capitis tenuissima, corpore utringue aequaliter attenuato, calida inaris ácuta, feminae obtusa.

Ent. 1. p. 150. n. rg. Asc. Acus.

Hab. in intestinis Esocis Lucii frequentissima, rarior in Esocis Belones, abdonine, ubi ipse Majo Gryphiae reperi. An huc n. 135.? 
50. Ascaris angulata $\mathrm{f}$.

Asc. capitis truncati ala utrincue exstante; cox: pore aequaliter attenuato, cauda maris recta obtusiuscula, feminae tenuiore subreflexa.

Ent. r. p. 152. n. 20. Asc. angulata:

Hab. In Cotti Scorpii mesentèrio Gryphiae Decembri reperi.

B. Parte antica crassiore.

a. Capite alato.

31. Ascaris vermicularis Livn:

Asc. capitis obtrsi membrana utrinque vesicu:lari, corpore antice crassiore, cauda máris in. flexa obtusa, feminae recta subulata.

Ent. r. p. 152. n. 2r. Mant. n. 3\%. Asc, vermi$\therefore$ cularis.

Hab. in Hominis intestinis crassis, praesertim recto. An quae in. Simiae Panisci craósis occurrit, eadem?

32. Ascaris ohvelata $R$.

Asc. capitis obtusi membrana utrinque vesicu. lari, corpore antice crassiore, acumine caudae masculae incurvae, femincae rectae brevi obtusiusculo.

Ent. 1.p. 155. n. 22, Mant, n, 32. Asc; obvelata. - Hab. In intestinis Muris musculi crassis Novem. bri Gryphiae reperi. Ibid. in'Ihire amphibia, arvali, Arctomyde Citillo. C.E.V.

53. Ascaris acutissima ZED t.

Asc. capitis acuti rnembràna laterali lineari, cauda subulata longissima.

Ent. I. p. 156.11.23. Asc. acutissima.

Hiab. In coeco Sciuri vulgaris ZeDER Augusto reperit, 


\section{- $45-$}

34. Ascaris dispar Schrank. †

- Asc. capitis acuti membrana laterali latiore, re. liqui corporis tenuiore, cauda subulata.

Ent. I. p. 257. n. 24. Asce dicpar.

Hab. in coecis Aisserum saginatorum, ZFDER (in quibus ipse nunquara reperi.) X́n Ansere canadensi." C. E. $V$.

35. Ascaris maculnsa $\mathrm{F}$.

Asc. ala capitis utrinque semielliptica, "corpore antice crassiore, cauda obtusa cum acumine; feminae recla, maris oblique truncata.

Ent. 1. p. 58 . n. 25. Asc. maculosa.

$\mathrm{Hab}$. In intestinis tenuibus Columbae domesti= cae (gutturosae) Gryphiae Junio reperi. Ibid. in $C$. risoria. C. $E, V$.

36. Ascaris truncata $\mathrm{ZED}$.

Asc. ala capilis utrinque lineari, corpore antice crassiore, cauda obtusa cum acumine, mascula incurva, feminea recta.

Ent. x. p. 195. n. 60. Mant. n. 36. Asc, truncata. Hab. in intestinis Psittaci aestivi. Froexict. Leucocephati tt domingensis. Mus, $V$.

\section{b. Capite nudo.}

37. Ascaris dentata ZED.

Asc. capitis nudi valvulis exiguis, corpore antice crassiore, cauda maris spiraliter involuta, feminae brevi inflexa.

Ent. 1. p. 160. n: 26. Mant. n. 27. Asc. dentata. Hab. in ventriculo et intestinis Cyprini Barb: 38. Ascaris gracilescens $R$.

Ascaris capitis nudi valvulis latiusculis, corpore antice crassiore, apice caudali depresso acuto.

Mant, n, 38. Asc. gracileścens. 


\section{- $46-$}

Hab. In peritoneo intestinorum Clupeae Spratti et Encrasicoli Arimini Aprili et Majo, Neapoli Junio reperi. Ex intestinis Spratti, $M, V$.

39. Ascaris compar Schrank.

Asc. capitis nudi valvulis latiusculis, corpore antice crassiore, cauda maris oblique truncata, alata, feminae recta, obtusiuscula.

Ent. r. p. 16r.n.27. Mant. n.39. Asc. compar. Hab. in intestinis crassis Tetraonis Lagopodis. 40. Ascaris leptocephala $\mathrm{R}$.

Asc. capitis discreti nudi valvulis magnis, corpore antrorsum crassiore, cauda maris obtusa, ferninae acuta.

Mant. n. 40. Asc. leptocepliala.

Hab. In Bufonis crucirti hydatide lienis GaEde Berolini Junio reperit, In ventriculo Sala. mandrae palustris Oтто Vratislaviae. In intestinis Bufonis ignei, Mus, Vienn.

41. Ascaris incisa $R$.

Asc. capite obtuso nudo, corpore antice crassiore, acumine caudae brevi conico.

Ent. I. p. 163. n. 28. Asc: incisa.

Hab. In vesiculis Talpae europaeae peritoneali-

bus Gorzıus Majo, ipse Aprili Gryphiae reperimus.

\section{Parte postica crassiore.}

a. Capite nudo.

42. Ascaris pusilla $R$.

Ascaris capite acutiusculo nudo, caudae 'cras. sioris acumine obtuso,

Ent, 1. p. 164. n.29. Asc. pusilla.

Hab. In Erinacei europaei vesiculis peritonei Julio et Septembri Gryphia reperi, 


\section{- $47-$}

43. Ascaris brevicaudata ZED.

Asc. capite nudo tenuiore, caudae crassioris acumine masculae incurvo, feminae subreflexo.

Ent. 1. p. 165. n. 30. Asc. brevicaudata. p. 199. n. 68. Asc. Salamandrae terrestris. Mant, n. 43. Asc. brevicaudata.

Hab. in intestinis Colubri Nutricis, Anguis fra. gilis, Salamandrae atrae, maculosae, Bufonis cinerei, viridis, ignei, fusci et in Bufonis viridis hydatidibus variarum partium.

44. Ascaris echinata $R$.

Asc. capitis nudi valvulis magnis acutiusculis, corpore retrorsum crassiore aculeis reflexis armato, cauda obtusa mucronata.

Mant. n: 44. Asc. echinata.

Hab, in Geckone Algesirae reperta. Mus. V.

45. Ascaris megatyphlon $\mathrm{h}$.

Asc. capitis nudi valvulis acutiusculis, corpore retrorsum crassiore, cauda maris truncata, feminae subulata.

Mant. n. 45. Ascaris megatyphlon.

Hab. in coeco Lacertae Iguanae' spiritu vini servatae magna copia reperi.

45. b. Ascaris tenuicollis $\mathrm{P}$.

Asc. capitis nudi discreti valvulis obtusis longiusculis, corpore retrorsum crassiore, cauda maris oblique truncata, cuspidata incurva, fe. minae recta subulata.

Mant. n. 45. b. Asc. tenuicollis.

Hab. In ventriculo Crocodili Lucii Heidelbergae Martio 1818 mortui Tiedemans reperit. 46. Ascaris extenuata $R$.

Asc, capitis nudi valvulis exiguis, corpore rem 


\section{- 48}

trorsum crassiore, caudae obtusae mucrone longo, tenuissimo. Mánt. n. 46. Asc. extenúata.

Hab. In recto Lacertae margaritaceae Algesira reperta....Mus. $V$.

\section{Ascaris leptura R:}

$\therefore$ Asc. capitis nudi valyulis obtusis, corpore re. trorsum crassiore, cauda feminae subulata recta, maris inflexa, acumine longo reflexo,

Mant. n. 47. Asc. leptura.

Hab: in intestinis Testudinis Mydae, Mus. $V$. 48. Ascaris microcephala $\mathrm{r}$.

Asc. capite nuclo constricto, cauda crassioris apice brevissimo incurvo.

Ent. I. p. 167. n. 3I. Mant. n. 48. Asc. microcephala.

Hab. In Ardeae comatae abdomine Nitzs CiI Majo reperit. Ipse in A. Nycticoracis oesophago et ventriculo Arimini Aprili offendi: 49. "Ascaris gibbosa R." !

Asc. capite tenui, colio strumoso, caudae cras. sioris apice subtus plano.

Ent. I, p. 167. n. 32. Asc. gibbosa.

Hab. in intestinis Phasiani Galli rarissima, ZE. DERo soli visa.

50. Ascaris sulcata $\mathrm{R}$ :

Asc. capitis discreti válvulis majusculis, corpore - retrorsum crassiore utrinque sulcato, caudae femineae apice brevissino conico.

Mant. n. 50. Asc. sulcata.

Hab. in intestinis Testudinis Mydae. Mus.V. Vienn.

51. Ascaris spiculigera $\mathrm{R}$.

Asc, capite nudo, corpore retrorsum crassiore, 


\section{- $49-$}

caudae incurvac apice brevissimo, maris acúto, feminae obtusiusculo.

Ent. 1. p. 168. n. 33. Mant. n. 51. Asc. spicu. ligera:

Hab. in oesophaga et ventriculo Peleccri; Cno. crotali ANDr. JuRiNe; ihidem in P. Carbone ipse Majo Berolini reperi; in $P$. cristato et pygmaeo: Mus. $V$.

52. A.caris variegata $R$.

Asc. copite nudo, corpore retrorsum crassiore, cauda feminae recta obtusinscula cum acu. mine brevi.

Ent. I. p. 169. n. 34. Asc. variegata.

Hab. In Colymbi septentrionalis oesophago Grypliae Augusto solitariam reperi. An huc n. 97? 53. Ascaris simplex $\mathrm{R}$.

Asc. capite nudo, corpore retrorsum crassiore, cauda obtusa.

Ent. 1. p. 170. 11. 35. Asc. simplex.

Hab. in ventriculo primo Delphini Phocaenae. AleERS An huc n. 82.?

54. Ascaris labiata $R$.

Asc. capitis nudi valvulis maximis, corpore retrorsum crassiore, cauda acuta.

Fnt. 1. p. 172.n. 37. Mant. n. 54́. Asc. labiata.

Hab. In intestinis Muraence Anguillae Gryphiae Augusto, M. Congri et IIyri Neapoli Junio et Julio reperi.

55. Ascaris truncatula F.

Asc. capite nudo truncato, corpore retrorsum crassiore, caudae acumine obtuso.

Ent. r. p. 173. n. 38. Asc. truncatula.

Hai. Iercae fluviatilis in intestinis Decembri, in liepatis hydatide Julio et Octobri; hepatis 


\section{- 50 -}

tunica involutam Aprili, inter dorși muscu. los Junio; in Percae Luciopercae peritoneo Majo Gryphiae reperi.

56. Ascaris crassicauda $\mathrm{F}$.

Asc. capitis nudi valvulis magnis truncatis, corpore retrorsum crassiore, cauda obtusiuscula. Mant. n. 50. Asc, crassicauda.

Hab, in intestinis Labri Tincae. Mus, $V_{\text {. }}$ 57. Ascaris aucta $\mathrm{R}$.

Asc. capite acutiusculo nudo, corpore retrorsura crassiore, cauda obtusiuscula inflexa alata.

Ent. 1. p. 175. n. 39. Asc. aucta.

Hab.' In Blcniti vivipari tubo intestinali saepius copiosam, interdum in peritoneo, semel inter branchias solitariam Gryphiae reperi.

58. Ascaris cuneiformis ZED. †

Asc. capite tenuissimo, corpore retrorsum cras. siore, caluda alata mucronata.

Ent. I. p. 177. n. 40. Asc. cuneiformis.

Hab. in variorum Cyprinorum intestinis rarior. Zener. In int. Cyprini cultrati et Idi. Cat. Ent. Vienn.

59. Ascaris obtusocaudata ZED.

Asc. capite nudo, corpore retrorsum crassiore, cauda mucronata, membrana laterali antice dorso, postice abdomini approximata.,

Ent. 1. p. 177. n. 41. Asc. obtusocaudata, p. 202. n. 74. Asc. Truttae. Mant. n. 59. Asc. obtusocaudata.

Hab. in ventriculo et intestinis Salmonis Fariouis et Truttae.

6o. Ascaris Capsularia R.

Asc. capite caudaque acutiuscula nudis, corpore retrorsum crassiore. 


\section{- $51-$}

Ent, r: p. 179. n. 42. Asc. Capsularia.

Hab. In Salmonis Salaris intestinis liberam, et

cavo abdominis, peritoneo involutam, saepius

Gryphiae verno tempore reperi.

61. Ascaris rigida $R$.

Asc. capite discreto nudo, corpore retrorsum crassiore, apice postico papillari, cauda maris incurva, feminae recta.

Ent. I. p. IS1. n. 43. Mant. n.61. Asc. rigida. Hab. in Lophii piscatorii tubo cibario. MüsLER. Muls. $Y$.

62. Ascaris acuta MülL. †

Ascaris capitis discreti valvulis rotundatis, corpore retrorsum crassiore, cauda obtusa mu. cronata.

Ent. I. p. 182. n. 44. Asc. acuta.

Hab. in Pleuronecte Rhombo, Mürier.

63. Ascaris incurva $R$.

Asc. capitis nudi valvulis exiguis, corpore re. trorsum crassiore, apice caudali longiusculo semitereti, acuto, incurvo.

Ent. 2. p. 276. n. 86 . Vermis dubius Xiphiae. Mant. n. 63. Asc. incurva.

Hab. in Xiplitae Gladii tuberculis intestinorum morbosis. SPEDALIERI.

$$
\text { b. 'Capite alazo. }
$$

64. Ascaris clavata $R$.

Asc. ala capitis utrinque lineari, corpore retrorsum crassiore, cauda obtusiuscula.

Ent. I.p. 183. n. 45. Mant. n. 64. Asc. clavata. Hab. in ventriculo Gadi barbati, Fabricius; in abdomine Merlangi, GAEDE; in faucibus Gadi Acolefini ipse verno tempore reperi. 


\section{$-52-$}

65. Ascaris collaris $\mathrm{R}$.

Asc. ala capitis utrinque lineari, corpore retrorsum crassiore, cauda obtusa mucronata.

Ent. T. p. 184. n. 46. Mant. n. 65. Asc. collaris.

Hab. In intestinis. Pleuronectis Flesi et maximi Gryphiae, Neapoli Julio in abdomine $P$. manci reperi.

66. Ascaris tenuissima $\mathrm{ZED}$.

Asc. ala capitis tenui, corpore retrorsum crassiore, cauda subulata.

Ent. 1. p. 185. 1. 47. Asc. tenuissima.

Hab. in intestinis Gadi Lotae. ZfDER, C.E.V.

67. Ascaris mucronata ScIrnaNK. $t$

Asc. ala capitis utrinque latiuscula, corpore retrorsum crassiore, cauda mucronata.

Ent. I: p. 186. n. 48. Asc, mucronata.

Hab. in ventriculo Gádi L_otae. Zener. C.E.V. 68. Ascaris cuspidata $R$.

Asc. ala capitis utrinque tenuissima, corpore retrorsum crassiore, cauda obtusa cuspidata.

Mant. n. 6s. Asc. cuspidata,

Hab. In colo duarum Larvarum Geotrupis Nas sicornis Berolini Majo reperi. An huc n. 140,? 69. Ascaris succisa $\mathrm{R}$.

Asc. ala capitis utrinque lineari, corpore retror. sum crassiore, cauda truncata.

Ent. I. P 187. n. 49. Asc. succisa.

Hab. in intestinis Rajae clavatae. ReInH, TrEviranus, 'R. Miraleti. C. E. $V$.

70. Ascaris céphalopera $\mathrm{R}$.

Asc. ala capitis utrinque semilanceolata brevi, corporis retrorsurn crassioris xulla, apice caudali obtusiusculo.

Nant, 1. 70, Asc, ceplaloptera. 
$\mathrm{Hab}$, in intestinis Viperae Redi, a qua dejectam

NESTr Florentiae dedit.

\section{Ascaris holoptera R.}

Asc. ala capitis corporisque retrorsum crassio.

ris tenui, caudae inflexae apice acuto.

Mant. n. 7. Asc. holoptera.

Hab. in intestinis Testudinis Mydae. Mus, V. 72. Ascaris serpentulus $\mathrm{R}$.

Asc. ala capitis corporisque retrorsum crassioris lineari, cauda inflexa obtusa mucronata.

Ent. I. p. 19I. n. 53, Mant. n. 72. Asc, serpentulus. .

Hab. in intestinis Ardeae cinereae, Braun; FroeIICu Julio,

$$
\text { Species dubiae. }
$$

73. Ascaris Canis Lagopodis. I

Ent. r.p. 194. n. 56. Asc. Canis Lagopodis.

Hab. in Cane Lagopode. Forsan ad n. Ig. amandanda.

74. Ascaris Canis aurei. $t$

Hab. in intestinis Canis aurei. C. E. V.mspt. 75. Ascaris Tigridis.

Ent. 1.p. 194.11.57. Mant. 1. 75. Asc. Tigridis.

Hab, in intestinis Felis Tigridi,s. MTus, V.

76. Ascaris Mustelarum. †

Ent. 1. p. 194. n. 58. Asc. martis.

Hab. in intestinis Mustelae Martis, Mystaci n. $2 \mathbf{r}$. proxina. Gozze. In intestinis Toinae, C, E. . . 77. Ascaris Gulonis, $t$

Ent. 2. B. 374. n. 58.b. Asc. Gulonis.

Hab. in Gulonis jejuno. Palias.

78. Ascaris Didelphidis. $†$

$\mathrm{Hab}$, in intestinis Didelphidis nutriacae. C,E.V. 
79. Ascaris Castoris, $t$

Ent. 1. p. 195. n. 59. Ascaris Castoris.

Hab. in intestinis Castoris Fibri. Perrault. 80. Ascaris Dipodis. $\downarrow$

Hab. in intestinis Dipodis Sagittae. C.E.V. 81. Ascaris Ovis.

Hab. in intestinis Ovis domesticae. C.E.V.m. 82. Ascaris Delphini. t

Mant. n. 82. Asc. Delphini.

Hab. in ventriculo Delphini gangetici. LeBber. 83. Ascaris Laniorum. +

Mant. n. S3. Asc. Laniorum.

Hab. in intestinis Lanii Collurionis et minoris. Froflich, C. E. $V$.

84. Ascaris Cornicis.

Ent. I. p. I9ז. n.6ı. Asc, Cornicis.

Hab. In Corvi Cornicis ventriculo. SoemmerRrsG. Ipse in iliins tenuibus Gryphiae Junio reperi.

85. Ascaris Corvi glandarii.

Ent. 1. p. 197. n. 62. Mant. n. 85. Asc. Corvi glandarii.

Hab. in intestinis Corvi slandarii. Oтто. 86. Ascaris Corvi frugilegi. †

Ent. I. p. 197. n. 63. Asc. Corvi frugilegi.

Hab, in intestinis Corvi frugilegi. SCHRANK.

Cat. Ent. $V$.

87. Ascaris Picae.

Mant. n. 87. Asc. Picae.

Hab. In intestinis tenuibus Curvi Picae Novembri Gryphiae repcri.

88. Ascaris Alaudae.

Hab. in intestinis Alaudae trivialis, C, E. $V, m$. 


\section{$-55-$}

89. Ascaris Emberizae. I

Hab. in intestinis Emberizae Hortulanae, Cat. Ent. V. miscpt.

90. Ascaris Caprimulgi. +

Hab. intestinis Caprimnlgi europaei. Cat. E. V. mispt. an ad 5.?.

91. Ascaris Ciconiae. +

Mant. n. gr. Asc. Ciconiae.

Hab. in intestinis Ardeae Ciconiae. Cat. E. $V$.

In ventriculo Ardeae nigrae. RosA.

92. Ascaris Ardearum. †'

Hab. in intestinis Ardeae comalae et purpureae. An ad n, 48 vel iz.?

33. Ascaris Charadriorum. I

Hab. in intestinis Charadrii Himantopodis, Morinelli, Oedicnemi, pluvialis, C.E.V.m. 94. Ascaris Ralli. t

Hab. in intestinis Ralli Crecis, Cat. Ent.Vienn. manuscriptus.

95. Ascaris Glareolae.

Mant. n. 95. Asc. Glareolne.

Hab. In intestinis coecis Glareolne anstriacae Arimini Aprili reperi,

96. Asc. Sternae.

Mant, n. 9ó. Asc. Sternae.

Hab. In duarum, Sternarum nigrantum thydatidibus intestinorum Arimini Aprili reperi.

97. Ascaris Colymborum. I

Hab. in intestinis Colymbi auriti, cormuti, cristati, minoris. C. E. V.m. An ad n. 52.? 98. Ascaris Lari. +

Ent. I. p. 198. 1. 65. Asc. Lari.

Hab. in intestinis Lari cinerarii, Bıocis. 


\section{- $56-$}

99. Ascaris Fulignlae.

Ent. x. P. 197. n. 64. Asc. Fuligulae.

Hab. in intestinis Anatis Fuligulae. Brocr. An ad n. 4.?

100. Ascaris Mergorum.

Hab. in intestinis Mlergi DMerganseris et Serra. toris. Cat. E. V. mllscpt.

101. Ascaris Ammodytis. $t$

Hab. in intestinis Vipera Ammodytis. Cat. mspt. An ad n. 70.?

102. Ascaris Torpedinis.

Mant. n. ro2. Asc. Torpedinis.

Hab. in ventriculo Torpedinis marmoratae, M. $V$.

103. Ascaris Pastinacae. $t$

Hab, in intestinis Rajae Pastinacae. C.E.V.m.

104. Ascaris Squali. $t$

Ent. I. p z00. n. 7r. Ascaris lineata.

Hab. in intestinis Squali. Bosc. An excluso synonymo Gordium spectante ad n. 8.?

105. Ascaris Helopis. $t$

Paltas Zoographia Rosso-Asiatica. T. III. (Petrop. I813. 4.) p. 102. Asc̈aris fusco-rubroque annulata.

Hab. saepe copiose in recto Accipenseris Helo. pis. Pallas.

106. Ascaris Orthragorisci.

Mant. n. Io6. Asc. Orthragorisci.'

Hab. In intestinis Orthragorisci Molae Neapoli. Júlio reperi.

107. Ascaris Hippocampi.'

Mant. n. 107. Asc. Hippocampi.

Hab. In intestinis Syngnathi Hippocampi Arimini Aprili reperi. 


\section{- $57-$}

108. Ascaris Centrisci.

Mant. 11. I08. Asc. Centrisci.

Iiab. In peritoneo Centrisci Scolopacis Neapoli

Julio reperi.

109. Ascaris Ophidii barbati.

Mant. n. rog. Asc. Ophidii barbati.

Hab. In mesenterio Ophidii barbati Neapoli

Junio reperi.

130. Ascaris Ophidii imberbis.

Mant. n. 110. Asc. Oph. ímberbis.

Hab. In mesenterio Ophidii imberbis Neapoli

Julio reperi.

11. Ascaris Uranoscopi.

Mant. n. III. Asc. Uranoscopi.

Hab. In peritoneo Uranoscopi scabri Neapoli Junio reperi.

112. Asćaris Gadi minuti.

Mant. n. II2, Asc. Gadi.

Hab. In abdomine Gadi minuti Arimini Majo reperi.

113. Ascaris Phycis.

Mant. n. II3. Asc. Phycis.

Hab. Inter Blennii Phycis appendices pyloricas Arimini Majo reperi.

114. Ascaris Gobii. I

Hab. in intestinis Gobii, Jozo. C. E. V. mspt.

115. Ascaris Scorpaenae.

Mant. n. I15. Asc. Scorpaenae.

Hab. In intestinis Scorpaenae Scrofae Arimini Aprili reperi.

116. Ascaris Fabri.

Mant. n. Ir6. Asc. Fabri.

Hab. in ventriculo Zenis Fabri Aximini Aprili reperi. 


\section{$-58-$}

117. Ascaris Soleae.

Hab. in intestinis Pleuroinectis Soleae. Cat. Ent. Vienn. mscpt.

118. Ascaris Linguatulae.

Mant. n. Irs. Asc. Linguatulae.

Hab. In Pleuronectis Linguatulae mesenterio Neapoli Julio reperi.

119. Ascaris Maenae.

Mant. 11. I1.9. Ásc. Maenae.

Hab. In intestinis Spari Maenae Arimini Apri-

li reperi.

120. Ascaris Boopis.

Hab. In peritoneo Spari Boopis Arimini Majo reperi.

121. Ascaris Spari Spicre.

Mant. n. I2I. Asc. Spari Spicre.

Hab. In Spari Spicre peritoneo Neapoli Junio reperi.

122. Ascaris Smaris. I

Hab. in intestinis Spari Snaris. C. E. V.mspt.

123. Ascaris Cynaerli.

Mant. n. 123. Asc. Cynaedi.

Hab. In peritoneo Labri Cynaedi Neapoli Ju. nio reperi.

124. Ascaris Labri lusci.

Mant. n. 124. Asc, Labri lusci.

Hab. In abdomine Labri lusci Neapoli Junio reperi.

125. Ascaris Novaculae.

Mant. n. 125. Asc. Novaculae.

Hab. In Labri Novaculae (Coryphaenae Linn.) peritoneo Junio et Julio Neapoli reperi,

126. Ascaris Sciaenae.

Mant. n. 226. Asc. Sciaenae. 


\section{- 59}

Hab. In peritoneo Sciaenae Umbrae Augusto Speziae reperi.

127. Ascaris Gasterostei.

Ent. I. p. 201. n. 72. Asc. Gasterostei.

Hab. In intestinis Gasterostei aculeati, Farr.

crus. Ipse Gryphiae Junio reperi.

128. Ascaris Mulli.

Mant, n, 128. Asc. Mulli.

Hab. In intestinis Mulli rubescentis Arimini Aprili reperi.

129. Ascaris Lyrae.

Mant. n. 129. Asc. Lyrae.

Hab. In mesenterio Triglae Lyrae Junio Neapoli reperi.

150. Ascaris Barbatulae. †

IIab. in intestinis Cobitidis Barbatulae. C. E.V.

131. Ascaris Siluri.

- Ent. r. p. 20 r. n. 73. Asc. Siluri.

Hab. intestinis Siluri Glanidis. Bцogrr. Ipse Gryphiae Majo reperi.

132. Ascaris Albulae. I

Ent. I. p. 202. 11. 75. Asc. Albulae.

Hab. in Salmonis Albulac tuberculis morbosis ventriculi et branchiarum. Koetreuter.

133. Ascaris Salmonis Omul. I

Pallas Zoographia Asiatico-Rossica T. III. (Petrop. ISI3. 4.) p. 409.

Hab. Ascarides et Taeniolae in Salmonis Omul ventriculo frequentes, coecis inhaerentes ea. que saepe perfodientes.

134. Ascaris Sauri.

Hab. In Osmeri Sauri mesenterio Neapoli Ju. nio reperi, 
135. Ascaris adiposa Schrank. I

Ent. I. p. 202. n. 76. Asc. adiposa.

Hab, in abdomine Esocis Lucii. Schrank. An ad n. 29. ?

136. Ascaris Argentinae.

Mant. n. 1.36: Asc. Argentinae.

Hab. In abdomine Argentinae Sphyraenae Romae Majo reperi.

137. Ascaris Atherinae.

Mant. n, 137. Asc. Atherinae.

Hab. In intestinis et peritoneo Atherinae Hepscti Neapoli Junio reperi.

158. Ascaris Clupearum. I

Ent, 1. p.203. 1. 77. Mant. n, 138. Asc, Clupearum.

Hab. in abdomine Clupeae Harengi, O. FABRI. Cius.

139. Ascaris Cyprini erythrophthalmi.

Ent. 2. p. 375. n. 78 .

Hab. in intestinis Cyprini erythrophthalmt. B R $\Lambda$ UN,

140. Ascaris Lucani. I

Mant. n. I40. Asc. Rucani.

Hab. in intestinis Lucani Capreoli, Froesrch. An ad n. 68.?

$$
\text { Genus X. Ophiostoma. }
$$

Corpus teres elasticun utringue attenuatum. Caput bilabiatum, labio superiore et inferiore.

1. Ophiostoma cristatum R. $t$

Oph. capitis rotundati labio superiore galeato, inferiore depresso, truncato, collo denticulato, cauda feminae obtusa mucronata. 


\section{$-6 i$}

Rictularia cristata Froelick, Mant, n, I, Oph. cristatum.

Hab, in intestinis tenuihus Myoxi Muscardini, Froelich, Myoxi Gliris el Dryadis, C.E.V.

2. Ophíostoma mucronatum $R$.

Oph. capitis obtusi labiis depressis aequalibus, cauda feminae obtusa inucronata.

Ent. T. p. I:7.n. 1. Mant. n.2. Oph. mucronatum. Hab. In intestinis $V$ espertilionis auriti Gryphiae Decembri 1803 et 1804 reperi. In int, Vesp. lasiopteri et murini. MHus. $V$.

3. Ophiostoma sphaerocephalum $R$.

Oph. capitis rotundi labio superiore tumido, inferiore minore, utroque sed loc ninus fisso, cauda maris incurva alata, feminae rectiuscu. la depressa.

Ent, I. p. Iss. n* 50. Asc. sphaerocephala, Mant, n. 3. Oph. sphaerocephalum.

Hab. In intestinis crassis Accipenseris Sturionis Gryphiae et Berolini Junio mense reperi.

4. Ophiostoma dispar R. I

Oph. capitis obtusi labio superiore longiore, cauda maris mucronata, feminae obtusiuscula. Ent. I.p. Irg. n. 2. Oph. dispar.

Hab. in intestinis Phocae foetidae et groenlandicae. O. Fabricius.

5. Ophiostoma lepturum R. I

Oph. capitis angustati labio inferiore longiore, cauda tenuissima.

Ent. I. p. 12r. n. 3. Oph. lepturum.

Hal, in intestinis Coryphacuae Mippuridis, Tr. LESIUS.

(Ophiostoma Cystidicola Ent, I, p. I22.'n. 4. ad Spiropteras relatum est, conf. harum n, 15.) 


\section{$-62-$ \\ Genus XI. Liorhynchus.}

Corpus elasticum seres, Caput evalve, oris tubulo eniessili, laevi.

$\therefore$ Liorhynchus truncatus R.

Liorh. capitis truncati tuhulo elabiato, corpore utrinque atenuato, cauda acutissima.

Ent. r. p. 247. n. I. L. truncatus.

Hab. In intestinis Melis Taxi praesertim tenui-

bus copiosissimos Gryphiae Octobri mense I 792 (neque postea) reperi.

2.) Liorhynchus gracilescens $\mathbf{R}$.

Liorh. capitis truncati tubulo elabiato, corpore retrorsum altenuato, cauda acuta.

Ent. I. p. 348. n. 2. Liorh. gracilescens,

Hab. in ventriculo Phocae barbatae, O, FABRI. crus.

3. Liorhynchus denticulatus $R$.

Liorh, capitis tubulo labiato, corpore denticulorum antrorsum majorum seriebus plurimis armato, caucla maris involuta, alata, feminae recta.

Ent. I. p, 249, n, 3, Mant. n, 3. Liorh, denticulatus.

Hab. in ventriculo ITuracnae Anguillae. Zeder (Julio) et Hürser, a quo specimina mea ha. beo. An Spiroptera? 


\section{Ordo II.}

\section{A c a n thoceph a 1 a.}

Corpus teretiusculum, utriculare, elasticum. Proboscis seriatim uncinata-retractilis. Individurs alia mascula, alia feminea.

Genus XII. Echinorhynchus.

(Character Ordinis etiam ille Generis anici.)

A. Collo corporeque inermibus. a. Collo brevissimo vel nullo.

a. Boboscide subglobosa.

1. Echinorhynchus Gigas, GozzE.

Ech. Proboscide subglobosa, collo vaginațo, corpore longissimo, cylindrico, retrorsum decrescente.

Ent. I. p. 25I.n. I. Mant. n. I. Ent. Gigas,

$\mathrm{Hab}$. in intestinis tenuibus Suis domesticas et ferae, vulgaris.

2. Echinorhynchus Spirula. Orfers,

Ech. Proboscide subglobosa, collo subnullo, corpore utrinque attenuato, postice crassiori incurvo.

Mant, n. 2, Ech, Spirula, 


\section{- 6r子'-}

Hab. In intestinis crassis Simiae Frosaliae reper. tum Oxfers ex Brasilia misit.

3. Lchinorhynchus ricinoides $\mathbf{R}$.

Ech. Proboscide subglobosa, collo brevissimo vaginato, corpore utrinque attenuato, antice crassiore.

Ent. 1. P. 253, n, 2. Ech, ricinoides,

Hab. In Upupae Epopis mesenterio specimina duo Gryphiae Julio mense reperi, In illius intestinis. Cat, Ent. Vienzı.

4. Echinorhynchus napaeformis $\boldsymbol{R}$.

Ech. proboscide subglobosa, collo brevissimo - vaginato, cotpore antrorsum crassissimo,'retrorsum attenuato.

Ent. I. p. 254. n. 3. Ech, napaeformis.

Hab. In colo Erinacei ezropaei Septembri Gry. phiae reperi.

5. Echinorhynchus compressus $\mathrm{R}$.

Ech. Proboscide subglobosa, collo brevissimo vaginato, corpore compresso, antrorsum latiore.

Ent. 1. p. 255. n. 4. Ech. compressus.

Hab. In Corvi Monedulae recto Gryphiae Majo reperi.

6. Echinorhynchus oligacanthus $\mathrm{R}$.

Ech. Proboscide subglobosa, uncinorum validissimorum seriebus tribus, collo brevissimo, corpore retrorsum subattenuato.

Mant. n. 6. Echi oligacanthus.

Hab. Intestino inferiori Colubri quadrilineati. cxtus adhaerentem Florentiae Augusto mense reperi.

7. Echinorhynchus oligacanthoides $\mathbf{R}$

Ech. Proboscide subquadrata, uncinorum vali- 


\section{$-65$}

dissimorum seriebus quatuor, collo subnullo; corpore maris cylindrico, feminae antrorsum crassiore.

Mant. n. 7. Ech, oligacanthoides.

Hab. In Colubri olivacei tunica intestini peritoneali repertum Oxfers ex Brasilia misit. 8. Echinorhynchus tuberosus ZED. +

Ech. Proboscide subglobosa, uncinorum tenuium serie unica, collo vaginato brevissimo, corpore oblongo.

Ent. 1. p. 257- n. 5. Mant. n. 8. Ech. tuberosus. Hab. Intestinis Cyprini rutili adhaerentem $O$. Fr. Mücter semel copiosum reperit. Aa huc n, 99, ?

9. Echinorhynchus clavaeceps ZED. I

Ech. Proboscide subglobosa, uncinorum tenui. un seriebus paucis, collo subnullo, corpore cylindrico, antrorsum decrescente.

Ent. 1. p. 258. n. 6. Ech, clavaeceps. p. 3 i 3. n. 57 . Ech. rutili.

Hab. in intestinis Salmonis Hluchonis; Cobitidis

Barbatulae, Taeniac; Cyprini Alburni, aurati, Barbi, Bramae, Carpionis, erythrophthalmi, Gobionis, Nasi, Phoxini, rutili, Tineas.

\section{B. Probuscide ovali.}

10. Echinorhynchus globulosus $\mathrm{R}$.

Ech. Proboscide ovali, uncinorum seriebus sex vel octo, collo brevissimo, corpore oblongo. Ent.1. p.259. n.7. Mant. n. 10. Ech. globulosus Hab. In intestinis Nuraenae Anguillae Gryphiae Maio, Junio et Novembri; Gobii nigri Arimini Aprili et Junio Neapoli; Spari Denticis Arimini Majo et Julio Neapoli; Pleuronectis Linguatulae Neapoli Julio; Sciaenae 
Umbràe Speziae Augusto; Sphyraeinae $S_{p e t}$ Neapoli Junio reperi. In Gobio Aphya. Mus. Nienn. In Gobio Gozo. Cat. Ent. I.

\%. Proboscide oblonga medio incrassata.

11. Echinoshynchus Pumilio R.

Ech. Proboscide medio incrassata, uncinorum brevissimorum seriebus quatuor vel sex, collo nullo, corpore cylindrico.

Mant. n. I . Ech. Pumilio.

Hab. in intestinis Lophii piscatorii, Mrzs, V.

12. Echinorhynchus inaequalis $R$.

Ech. Proboscide medio incrassata, uncinorum

- minutissimorum seriebus viginti quatuor, collo brevi, corpore postice attenuato.

Ent. 1. p. 261. n. 8. Ech. inaequalis.

Hab. in ventriculo Falconis Buteonis. ANDr. JuRINe.

13. Echinorhynchus globocaudatus $Z_{\text {ED }}$.

Ech. Proboscide inedio incrassata, uncinorum seriebus vigintiquatuor, collo nullo, corpore longissimo, postice rotundato.

Ent. I. 1, 264, n. I I, Mant. n, 13, Ech, globo. candatus.

Hab. in intestinis Strigis passerinae, $\mathrm{Z} \times D E R$. Aluconis, Nitzscr.

14. Echinorhynchus cinctus R.

Ech. Proboscide longa medio incrassata, unci, norum minutissimorum seriebus quadraginta, corpore oblongo, antrorsum attenuato, postice lineari.

Mant. n. 14. Ech. cinctus.

Hab. In mesenterio Colubri atrovirentis (Arimini, Majo) et Viperae Redi (Bononiae Sep- 


\section{$-67$}

tembri 1817 acceptae, Majo 18rs Berolini mortuae) reperi.

j. Proboscide apice incrassata s. clavata.

15. Echinorhynchus bacillaris $R$.

Ech. Proboscide cylindrica apice aucta, uncinorum seriebus triginta, corpore lengissimo, apice caudali inflexo.

Ent. 1.p. 301. n. 38. Nant. n. 15. Ech, bacillaris.

Hab, in intestinis Mergi minuti, BLосн.

- 16. Echinorhynchus agilis $\mathrm{R}$.

Ech.-Proboscide clavata, uncinorum seriebus tribus, collo brevissimo, corpore utrinque attenuato, densissime transversim striato.

Mant, n. 16, Ech. agilis.

Hab. In intestinis Mugilis Ceplaali Augusto Speziae reperi.

17. Echinorhynchus fusiformis ZED.

Ech. Proboscide subclavata, uncinorum seriebus octo ad duodecim, collo nullo, corpore utrinque attenuato.

Ent. I. p. 26I. n. 9. Ech. fusiformis. p. 270. n. I6. Ech. inflatus, Mant. n. I7. Ech. fusiformis. Háb. In intestinis Salmonis Salaris Majo Gryphiae reperi. Salm. Thymalli, Farioniis, -Truttac. Gat. Ent. V.

8. Proboscide basi incrassata sive conica.

18. Echinorhynchus Haeruca R.

Ech. Proboscide conica, uncinorum seriebus

sex vel octo, collo subaequali, corpore longo tereti, antrorsum crăssiore.

Ent, 1. p. 265. n. 12. Mant. n. 18. Ech. Hảeruca. Hab. In intestinis Ranae telhporariae et esculentae vere et aestate Gryphiae, Bufonis ignei Berolini Martio reperi, Búfonis cinerei, C, $E_{*} V$. 


\section{$-68$}

3. Proboscide cylindrica sel lineari.

19. Echinorhynchus angustatus $\mathbf{~}$.

Ech. Proboscide cylindrica, uncinorum seriebus octo ad viginti, collo brevissimo, corpore utrinque atteruato.

Ent, I. p. 266. n, I3. Ech, angustatus, p. 268. n* If. Ech. affinis. Mant. n. 19. Ech. angustatus. Hab. In intestinis Gadi Lotae (Gryphiae Septernbri); Pleuronectis Flesi (ib. Junio); Soleae (Neapoli Julio); Percae fluviatilis (Gryphiae saepissime, vario anni tempore); Gasterostei aculati (ib. Junio); Siluri Glanidis (ib. Majo); iEsocis Lucii (ib. et Drerolini saepius); Relo. nes (Gryphiae, Majo) reperi. In Cotto Gobio, Perca Lucioperca et cernua, Cat. E. $K_{\text {. }}$

\section{Echinorhynchus simplex R.}

Ech. Proboscide cylindrica, uncinorum minu torum seriebus multis, collo nullo, corpore tereti subaequali.

Ent. 1. p. 270. n. 15. Ech, simplex.

Hab. in Trigla Gurnaidi. Ratuke, In intestinis Iriglae adriaticae. Cat. E. V.

21. Echinorhynchus falcatus Frozicr. $t$

Ech. Proboscide cylindrica, uncinorum tenuissimorum seriebus sex vel octo, collo nullo, corpore tereti aequali incurvo.

Ent. 1. p.' 27 r. n. 17. Eich. falcatus.

Hab. in intestinis Salamandrae atrae. Froenica, Cat. Ent. $V$.

2\%. Echinorhynchus gracilis $\mathrm{R}$.

Ech. Proboscide cylindrica, uncinorum minuto. rum seriebus decem vel duodecim, collo nul1o, corpore cylindrico retrorsum attenuato. Mant. 2. 22, Ech. gracilis, 


\section{$-69$}

Hab. In intestinis Coraciae garrulae Treutuer plurimos Augusto reperit.

23. Echinorhynchus areolatus $\mathrm{h}$.

Ech. Proboscide cylindrica, uncinorum minuto. rurn seriebus viginti, collo subnullo, corpore utrinque praesertim retrorsum attenuato.

Mant. n, 23. Ech, areolatus.

$\mathrm{Hab}$. in intestinis Motacillae Atricapillae, M. $V$. 24. Echinorhynchus cylindraceus Scrranx. I

Ech. Proboscide lineari, uncinorum (? serrato. rum) seriebus undecim, collo subnullo, cor. pore longissimo antrorsum angustato.

Ent. r, p. 272. 12, 18. Mant, n. 14. Ech. cylindraceus.

Hab, in intestinis Pici majoris et viridis. GoezE, Octobri.

25. Echinorhynchus tumidulus $\mathbf{R}$.

Ech. Proboscide lineari, uncinorum tenuissimo. rum seriebus triginta, collo nullo, cospore cylindrico retrorsum attenuato.

Mant, n, 25. Ech, tumidulus.

Habitaculo non indicato Orfers 'ex Brasilia misit.

26. Echinorhynchus transversus $R$.

Ech. Proboscide lineari, uncinorum mediocrium seriebus vigintiquatuor ad triginta, collo nullo, corpore utrinque, praeserim antrorsum attenuato.

Mant. n, 26. Ech. transversus,

Hab, in intestinis Turdi cyanei, Turdi n. sp., Sturmi vulgaris, Motacillae stapazinae. Mus. Vienn Turdi Merulao et saxatilis. Cat, E. $V$.

27. Echinorhynchus micracanthus $R$.

Ech, Proboscide subcylindrica, uncinorum zai- 


\section{$=70$}

nutissimorum seriebus plurimis, collo nullo, curpore cylindrico antrorsum crassiore. Mant, n. 27. Ech. micracanthus.

Hah. In intestinis Matacillae (perperam Ficedulae dictae) Florentiae Augusto reperi. In int. Loxiae Coccothraustis, Fringillae caelebis, Mis. Vienn. Motacillae atricapillae, nisoriae, Oenanthes, Alandae arvensis. Cat, E.V.

28. Echimoshynchus spiralis $R$.

Ech. Prroboccide lineari, uncinorum minutorim seriebus triginta, collo nullo, corpore tereti, aequali, longissimo.

Ent. 1.p 273.n. 19. Mant. n.28. Ech. spiralis.

Hab. ili intestinis Ardeae minutae, Nrтzscr et ego (Arimini, Aprili) reperimus,

29. Echinorhynchus caudatus Zeder. +

Ech Probocide cylindrica medio constricta, un. cinorum seriebus viginti septem, collo nullo, corpore tereti longo,

Ent. 1. p. 274 n. 20. Mant. n. 2G. Ech. caudatus. Hab. in intestinis Falconis rasvii, lagopodis, Mitvi, Bnteonis, rufi, pennati, cyanei, Tinnunculi, cineracei. Cat. E. $V$.

3o. Echinorhynchus Tuba R.

Ech. Proboscidis linearis plurimis uncinorum seriebus armatae tuba apicis incrassata, collo nullo, corpore tereti longo.

Fnt. 1. p. 25. n. 21. Mant, n. 30. Ech. tuba. Hab in intestinis Strigis Bubonis, Cat. Ent. Vicull. Aluconis, Mülzer, Froelich, stridulae (Jannario), Goeze; flammeae, ego Gryp'liae $\therefore$ artio.

31. Echinorhynchus aequalis R. $f$ Ech. proboscide cylindrica, uncinorum seriebus 


\section{- 71}

plurimis, crassitie corporis longi aequalis, collo nullo.

Ent. I. p. 2277. n. 22. Ech. aequalis.

Hab. in intestinis crassis Strigis Oti, Februario, Martio, Goeze, Comes de Borke.

32. Echinorhynchus Acus R.

Ech. Proboscide lineari, uncinorum seriebus viginti, collo nullo, corpore longissimo, retrorsum subattenuato.

Ent. 1. p. 278. n. 23. Ech. Acus. p. 28r. n. 24. Ech. lineolatus, p. 317, n, 6r, Ecli, Lophii, Mant. n. 32. Ech, Acus.

Hab. in intestinis Gadi Aeglefini, barbati, Callariae (in quo Gryphiae Majo reperi), lusci, Merlangi, Merluccii, Molvae, virentis, Cotti Scorpii, Lophii piscatorii.

33. Echinorhynchus moniliformis BREMSER. Ech. Proboscide cylindrica, uncinorum tenuis. simorum seriebus duodecim, collo nullo, corpore longissimo moniliformi, cauda tereti.

Mant. n. 33. Ech. moniliformis.

Hab, in intestinis Muris arvalis, Mus. V. Crivceti. Cat. E. V.

\section{Longicolles,}

34. Echinorhynchus porrigens $\boldsymbol{R}$.

Ech. Proboscidis cylindricae receptaculo maximo obconico, collo filiformi, corpore cylin. drico antrorsum attenuato.

Ent. 1. p.304. n. 40, Ech. Balaenae, Mant, n. 34 . Ech, porrigens.

Hab. in intestinis tenuibus Balaenae rostratae. WALTER.

35. Echinorhynchus filicollis $\mathrm{r}$.

Ech.'Proboscidis (semper latentis) receptaculo 


\section{$-7^{2}$}

magno sphaerico, collo filiformi, corpore ob. longo utrinque obtusissimo.

Ent. 1. p. 283. n. 25. Ech. filicollis. p. 304. n. 4. Ec Ech. Anatis mollissimae. Mant. ñ. 35. - Ech. filicollis.

Hab. in intestinis sinatis Boschadis ferae (in qua Gryphiae Septembri), Fuligulae, Sponsae, mollissimae; Fulicae atrae (in qua Julio Gryphiae reperi).

36. Echinorhynchus tereticollis $\mathbf{R}$.

Ech. Proboscidis cylindricae uncinorum seriebus sedecim ad viginti armatae, colloque tenuioris receptaculo globoso, corpore postice attenuato,

Ent. 1. p. 284. n. 26. Mant. 1. 36. Ech. tereticollis. Hab. In intestinis Accipenseris Sturionis (Arimini Majo), Mhuraenae Anguillae (Gryphiae Augusto), Gadi Lotae (ib. Septembri), Blennii vivipari (ib. Aprili et Octobri), Cotti Scorpii (ib. Septembri), Percae cernuae (ib. Aprili) Jluviatilis (ib. Decembri), Siluri Glanidis (Berolini Julio), in ventriculo Pleuronectis Flesi (Gryphiae vario anni tempore) reperi. In Gado Mlerlango Kilonii repertum GaEDE dedit, In Accipensere IIusone, Cotto Gobio Cat. Ent. Vienuı.

37. Echinorhynchus nodulosus Scurank.

Ech. Proboscidis subclavatae uncinorum seriebus duodecim ad sedecim armatae receptaculo subgloboso, collo conico, corpore postice attenuato:

Ent. I. p. 287. n.27. Ech.nodulosus, p.314.n. 56. Ech. barbi. p. 317. n. 59, Ech. bramae, Mant. n. 37. Ech. nodulosuz。 


\section{- 75}

Hab. In intestinis Cyprini Jesis (Gryphiae; A prili), Timbac, (ib. Junio) reperi. Dobulac, Zener. Barbi, Idli, Phoxini, Gobionis. Cat.E. Jicnn. Gadi Callariae, Braun. Lotae, Nitzsch.

38. Echinorhynchus ovatus ZEDER. T

Ech. Proboscide subclavata, uncinorum seriebus novendecim armata, angustiore collo cylindrico longissimo, corpore obovato.

Ent. I. p. 290.n. 28. Ecl. obovatus.

Hab. rarins in intestinis $C_{y}$ rinorum, plerumque in eorundem, nec non Gadi Lotae, Salmonis Truttae, et Esocis Luacii mesenterio. ZeDER.

39. Echinorhynchus sphaericus R.

Ech. Proboscide lineari, uncinorum seriebus duodecim, collo conico, corpore subsphaerico. Ent. r. p. 29z. n. 29. Ech. sphaericus.

Hab. In Cottorzm Scorpiorum complurium peritoneo satis multos Gryphiae Septembri mense reperi,

B. Collo vel corpore'armata.

40. Echinorhynchus gibbosus R.

Ech. Proboscide cylindrica transversa, collo nullo, corporis undique aculeati parte antica subglobosa, postica tereti.

Ent. I. p. 292.n. 30. Ech gibbosus.

Hab. In peritoneo Cyclopteri Lumpi, Gryphiae,

Februario, Trachini Draconis, Parisiis Majo reperi.

41. Echinorhynchus strumosus R:

Ech. Proboscide cylindrica transversa, collo nullo, corporis parte antica subglobosa aculeata, postica tereti inermi.

Ent, I, p. 293.n. 31. Ech. strumosus, 
Hab. In intestinis tenuibus Phocae vitulinae Gryphiae Novembri reperi.

42. Echinos hynchus ventricosus $R$.

Ech. Proboscide cylindrica recta, collo conico, corporis parte antica subglobosa, reliqua tereti, illis undique hac postice, aculeatis.

Ent, I. p. :04. 11. 32. Ech. ventricosus.

Hab. In intestinis tenuibus Mustclae Putorib Gryphiae Decembri reperi.

43. Echinorhynchus striatus Goeze.

Ech. Proboscide subclavata, collo conico inermi, corporis parte antica aculeata, postica tenuiore sulcata.

Ent. r. p. 26 . n. 10. Mant. n. 43. Ech. striatus.

Hab. in intestinis Ardeae cinereae. Goeze. MTus. Vienn. Anatis Oloris, Falconis Albicillae, Cat. Ent. Vienn.

44. Echinorhynchus versicolor $R$.

Ech. Proboscide subovali, collo inermi, corporis oblongi constricti parte antica, aculeatissima. Ent. I. p. 295. 11. 33. Ech. minutus. p. 296. n. 34 . Ech. contrictus. p. 298. 11, 35. Ech. collaris. Mant. n. 44. Ech. versicolor.'

Hab. In intestinis Anatis Fuligulae (Gryphiae Novembri), Fulicae atrae (Aprili, Berolini) reperi, In Anate fusca, Januario, Gozze. Fulica Chloropode, Zener. In Anate Boschade domestica, Gozze Septembri, fera et Ansere (Junio) Eroelich.

45. Echinorhynchus pyriformis Bremsen. Ech. Proinscide lineari, collo nullo, corporis obovati parte antica aculeatissima.'

Mant. n, 45. Ech, pyriformis.

Hab. in intestinis Turdi Merulae. Mus. Vienn. 


\section{- $75-$}

46. Echinorhynchus Hystrix Brens.

Ech. Proboscidis cylindricae parte antica angustata, collo brevi, corpore antrorsum crassissimo aculeato, apice caudali tenui subnudo.

Mant. n, 46. Ech. Hystrix.

Hab. in intestinis crassis Pelecani Carbonis, Junio. Mus. Vienn.

47. Echinorhynchus Pristis R.

Ech. Proboscide cylindrica, uncinorum seriebus triginta ad quadraginta, collo nullo, corpore longissimo filiformi postice obtuso, antice aculeato.

Ent, I. p. 299. n. 36. Ech, Pristis, p. 312. n. 5 I.

Ech. Scombri. Mant, n, 47. Ech. Pristis.

Hab. In infestinis Esocis Belones, Gryphize, Majo; Scombri Scombri, Arimini Aprili; Sc.Coliae Neapoli Junio reperi.

48. Echinorlynchus subulatus ZED. $\downarrow$

Ech. Proboscide oblongo-ovali, uncinorum seriebus octo, collo brevissimo parteque corpo. ris antica cylindrica aculeatis, postica subulatà inermi.

- Ent. 1. p. 300. n. 37. Mant. n. 48. Ech. subulatus. Hab. in intestinis Clupeae alosae. Hermann. 49. Echinorhynchus vasculosus $\mathrm{R}$.

Ech. Proboscide ovali, uncinorum seriebus decem, corporis parte antica subconica aculeata, reliqua cylindrica colloque conico nudis. Mant. n. 49. Ech. vasculosus.

Hab. In abdomine tt intestinis Spari (Bramae) Raji Neapoli Junio et Julio reperi.

$$
\text { species dubiae. }
$$

50. Echinorhynchus Mustelae.

Mant. n. 50. Ech. Mustelae. 


\section{$-76$}

$\mathrm{Hab}$, in mesenterio Mustelae vulgaris, Mus. Tienn.

51. Echinorhynchus Soricis. †

Hab. in intestinis Soricis Aranei. C.E.V.mspt. 52. Echinorhynchus Erinacei abdominalis. I Hab. in Erinacei eurspaei mesenterio, diversus et a napaeformi (n. 4. Erinacei intestinali) et ab insequente. Cat. Ent. I. mspt.

53. Echinorhynchus Erinacei subcutaneus. $\downarrow$ Hab. sub pelle Erinacci europaei et ab antecedente et a napiformi (n. 4.) diversus. Cat, E. V. miscptus.

54. Echinorhynchus Citilli. I

Hab. in intestinis Arctomydis Citilli, C. E, V.

55. Echinorhynchus Muris. †

Pseudoechinorhynclius Goezrr. Haeruca Muris ZED. Ent. I. 1.302. n. 39. Ech, muris.

Hab, in ventriculo Muris musculi a Comite Dr Borke repertus. An hujus generis?

56. Echinorhynchus Falconis cyanei. †

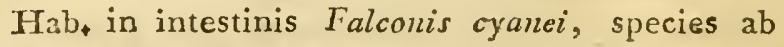
Ech, caudato n. 2g, diversa. Cat, E. $V_{*}$

57. Echinorhynchus Haliaëti. †

Hab. in intestinis Falconis Haliaëti. C.E.V.m.

58. Echinorhynchus Collurionis. $†$

Hab. in intestinis Lanii Collurionis. C.E.V.m. 59. Echinorhynchus Cornicis. I

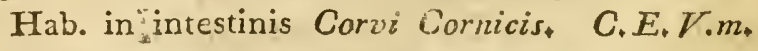
6o. Echinorhynchus Picae. †

Hab, in intestinis Corvi Picae, species ab Ech. compresso n. 5. diversa, Cat, t. $V_{\text {. }}$ 
161. Echinorhynchus Coraciae. $t$

Hab. in omento Coraciae garrulae, species ab

Ech. gracili n, 22. diversa. O, E, V.

62. Echinorhynchus Orioli.

Hab. in intestinis Orioli Galbulae. C. E.V.

63. Echinorhynchus Alandae. †

Hab, in intestinis Alsudae nemorosae et trivia.

lis. C.E. V.mrpt. An ad n. 27.

64. Echinorbynchus Minscicapae †

Hab. in intestinis Muscicupae collaris. Cut. E.

V. mscptus.

65. Echinorhynchus Iotacillae atricapillae f

Hab. in omento Mot, atricapillae, species ab

Ech, areolato n. 23. diversa, C, E. $V . m$. An ad sequ.?

66. Echinorhynchus Sylviarum. †

Hab. in intestinis Sylviae Fitis, Lusciniae, Phï-

lomelae, Phoehicuri, rubeculäe, rubicolae, Troglodytis. C.E.V.

67. Echinorhynchus Ruhetrae. $t$

- Hab. in intestinis Motacillae rubetrae. C. $E . V$. mspt. An ad pratcedentem?

68.. Echinorhynchus Pari. $\downarrow$

Hab. in intestinis Pari majoris. C. E. $V$.

69. Echïnorhynchus Hirundinum. †

Hab. in intestinis Hirundinis rusticae et Apodis.

C. E. $V . m s p t$.

70. Echinothýnchus Tardae.

Ent. I. p. 308. n. 45. Ech. Tardae.

Hab. In intestinis Otidis Tardae Septembri Gryphiae reperi, ob proboscidem retractam con dignoscendum. 


\section{- $78 \div$}

71. Echinorhynchus Gruis. It

Hab. in intestinis Ardeae Gruis. C, E.V.m. 72. Echinorhynchus Ardeae purpureae. $\ddagger$

Hab, in intestinis-Ardcae purpureae Cat. Ent.

Vienn. mscptus.

73. Echinorhynchus Ardeae albae. $t$

Eint. I. p. 307. 11. 43. Ech. Ardeae albae.

Hab. in Ardeae albae intestinis et bursa FABRIcro dicta. PeDi.

74. Echinorhynchus Vanelli. t

Ent. I. p. 30s. Ech. Vanelli.

Hab. in intestinis Tringae Vanelli, GozzE, Sp̃ecies peculiaris. C. E.V.

75. Echinorhynchus Morinelli, †

Hab. in intestinis Charadrii Morinelli Cat. E. V. mscptus.

76. Echinorhynchus Oedicnemi. $†$

Hab. in intestinis Charadrii Oedicnemi. Cat. E. Vienn.

77. Echinorhynchus Charadrii pluvialis. $t$

Hab. in intestinis Charadrii pluvialis, C.E.V.

78. Echinorhynchus Anatum. †

Hab. in intestinis Anatis Clangulaé, Creccae, Ma. rilae, niracae, Penelopes, rufinae. Cat. E.V. Sirgúli vel ad $n$. 35 . vel ad n. 44. pertinebunt, a Brevsero conjunctos, quo commotus huc retuli.

79. Echinorhynchus Sternae.

Hab. in intestinis Sternae Cantiacae. Cat. Ent. Vienn. mscptus.

80. Echinorhynchus Husonis. $\downarrow$.

- Hab. in intestinis Accipenseris Husonis, species ab Ech. tereticolli n. 36. diversa. C, E. $V$. 


\section{$-99$}

81. Echinorhynchus Accipenseris rutheni $\mathrm{Hab}$. in intestinis Accipenseris rutheni. Cat.E. V. mseptus.

82. Echinorhynchus Sturionis.

Hab. in intestinis Accipenseris Sturionis. Gozz:. Species ab Ech. tereticolli n, 36. diversa, forsan ad Ech. angustatum n. 19. pertinet.

83. Echinorhynchus Wachniae.

Mant. n. 83. Ech. Wachniae.

Hab. in intestinis Gadi Wachniae. Turesius. Ad

- Ech. Acum n. 32. pertinebit. 34. Echinorhynchus Gobii. +:

Ent. I. p. 309. n. 47. Ech. Gobii.

Hab. in intestinis Cotti Gobii, species ab Ech. tereticolli n. 36. qui pariter in hoc pisce oc. currit, diversa. Cat. E. $V$.

85. Echinorhynchus Scorpaènae. +

Hab. in intestinis Scorpaenae Scrofae. Cat, $E$. Vienn.

86. Echinorhynchus Fabri, i

Hab. in intestinis Zenis Fabri. C, E. V.

87. Echinorhynchus Pleuronectis maximi. to Ent. I. p. 31 o, n. 4\%. Ech. Pleur. maximi.

Hab, in intestinis pleuronectis maximi, O. Fr. MürLfer. IPse in pisce vulgatišsimo nunquatm Echinorhynchum vidi, neque in Scorpacna, neque in Zene Fabro, neque in Labris, Tri. glis, Eperlanis, Argentinis, aliisque postèa enu. merandis, sed ormia ounibus non contin. gunt.

88. Echinorhynchus Platessae. $†$

Ent, 1. p. 3io. n. 49. Ech, Platessat. 


\section{$-80$}

Hab. in intestinis Pleuronectis Platessae. O. Fr. MüLLER.

89. Echinorhynchus Platessoidae. †

Ent. r. 1. 310. n. 49. Ech. Platessoidae.

$\mathrm{I} a \mathrm{~b}$, in ventriculo Pleuronectis Platessoidae, $\mathrm{O}$.

Fabiicrus. An hujus generis?

90. Echinorhynchus Labri. \$

Hab. in intestinis Labri Tincae., C. E. V.m.

91. Echinorhynchus Sciaenae.

Mant, n. 9r. Ech. Sciaenae.

Hab. In mesenterio Sciaenae Aquilae Neapoli Junio reperi.

92. Echinorhynchus Triglae. . t.

Hab. in intestinis Triglae adrialicae. Cat. $\boldsymbol{E}$.

Vienn. inscptus.

93. Echinorhynchus Salmonum. $t$

Ent. I. p. 312. n. 52. Ech. sublobatus. p. 313. Ir, 53. Ech. Lavareti.

Hab, in intestinis Salmonis Farionis, Salvelini, Thymalli. Cat. Ent. V. Vel ad tereticollem vel ad nodulosim (n. 36 et 37. ) quos BREMSER conjungit, In intest. Salmoris Salaris, Hermane. Lavareti, Koelreuter. Huchonis, Cat. E. V. miscptus.

94. Echinorhynchus Eperlani. +

Fnt. I. P. 313. n. 54. Ech. Eperlani.

Hab, in abdomine et intestinis Salmonis Eperlani. Martin.

95. Echinorhynchus Argentinae. $t$

Ent. 1. p. 3:4. n. 55. Ech. Sphyraenae.

Hals. in abdomine Argentinae Sphyraenae. Red 96. Echinorhynchus Atherinae.

Mant, n. 96. Ech, Atherinae. 


\section{$-81$}

Hab. In intestinis Atherinae Hepseti Neapoli - Junio reperi.

97. Echinorhynchus Idhari. t

Ent, 1. p. 3r6. n. 53. Eih. Idbari

Hab. in intestinis Cyprini Idbari, O. Fr. Mür. LER. Ad Ech, nodulosum n, $3 \%$ pertinebit, 98. Echinorhynchus Carassii. +

Ent. I. p. 317. n. tio. Ech. Carassii。

Hab.' in intestinis Cyprini Carassii. Braun. In Catalogo Vienrensi a reliqutis Gyprinorum Echinorhynchis pariter sejungitur. 


\section{Ordo 11 I.}

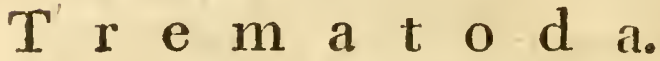

Corpus depressum vel teretiusculum, molle. Pori suctorii. Omnia individua androgyna.

\section{Genus XIII. Monostoma.}

Corpus molle, teretiusculuni vel depressum. Porus anticus solitarius.

A. Pari apertura infera. Hypostoma.

8. Monostoma caryophyllinum $\mathbf{R}$.

M. capite obtuso, ore amplissimo rhomboidali infero, corporis depressi apice postico acutiusculo.

Ent. I. p. 325. n. I. M. caryophyllinum.

Hab. In intestinis Gasterostei aculeati Gryphiae Junio mense rarius reperi.

2. Monostoma gracile $R$. $t$

M. capite obtusiusculo, ore ovali infero, corporis depressi apice postico acuto.

Ent. 1. p. 326. n. 2. M. gracile.

Hab. in abdomine Sulmonis Eperlani. Acharivs.

3. Monostoma cochleariforme R. t

M. capite obtuso discreto, ore ovali infero, cor. pore teretiuscula. 


\section{$-83$}

Ent, I. p. 326. n. 3. M. cochleariforme.

Hab. in intestinis Cyprini Barbi. Schrank.

B. Pori apertura äntica. Monostoma.

4. MIonostomá foliaceum $\mathbf{R}$.

M. poro minimo rotundo, rarius papillaeformi, corporis ovalis utrinque obtusi ventre plano, dorso convexiusculo.

Mant. n. 4. M. foliaceum.

Hab. In abdomine Accipenseris Sturionis Arimini Majo reperi.

5. Monostoma orbiculare $\mathbf{R}$.

M. Poro oblongo.ovato, corpore orbiculari hine convexo illinc concavo.

Mant. n. 5. M. orbiculare.

Hab. In intestinis Spari Salpae' Neapoli Junio et Julio reperi.

6. Monostoma Crucibulum R.'

M. Pori crucibuliformis profundi margine exstante, corpore subelliptico antrorsum atte. nuato, ventre planiusculo, dorso convexo.

Mant. n. 6. M. Crucibulum.

Hab. In intestinis Muraenarum Congri et Cassi$u$ Neapoli Junio et Julio reperi,

7. Monostoma capitellatum $\mathrm{R}$.

M. Poro subglobuso, corpore teretiusculo lineari, apice postico obtuso.

Mant. n. 7. M. capitellatum.

Hab. In intestinis praesertim inferioribus Spari Salpae Neapoli Junio et Julio reperi.

8. Monostoma lineare $\mathbf{R}$.

M. Poro orbiculari exiguo, corpore depresso lineari, postice obtusissimo.

Mant. n. 8. M. lineare. 


\section{- $84-$}

Hab. in intestinis Tringae Vauelli, Mus. Vienn. An huc n. 27:?

9. Monostoma crenulatum R.

M. Poro crenulato, corpore teretiusculo, antrorsum gracilescente, postice obtuso.

Ent. 1. p. 328: n. 4. M. crenulatum.

Hab. It intestinis Motacillac Phocnicuri Gry. phiae Majo reperi.

10. Monostoma attenuatum $\mathrm{f}$.

M. Poro orbiculari, corpore depressiusculo, án. trorsum attenuato, postice rotundato. Ent. I. p. 328. n. 5. M. attenuatum.

- Hab.. In intestinis coecis Scolopacis Gallinagimis Gryphiae Julio reperi. In illis Anatis clypeatae. Braun.

11. Monostona ocreatum ZED. .

M. Poro orbiculari, corpore teretiusculo lon: gissimo, apice postico divaricato.

Ent. I. p. 329: n. 6. M. ocreatum.

Hab. 'in intestinis Talpae curoppaeae. Goeze, $\mathrm{Z}_{\mathrm{r}}$. Der, Nitzscr (a quo accepi), Cat. E. $V_{*}$

12. Monostoma rerrucosum ZED.

M. Poro orbiculari, corpore oblongo-ovato, depressiusculo, papillarum ventralium serie triplici.

Ent. I. p. 331. n. 7. M. verrucosum.

Hab. in intéstinis crassis Anatis Anseris et Puerquedulac. ZnDER. Boschatis domesticae et sem getum. Mus. Vienn.

13. Monostoma ellipticum R.

M. Poro orbiculari, corpore depressiusculo re. trorsum incrassato.

Ent. 1. p. 333. n, 8, Mant. n. 13. M, elliptis cLuس. 


\section{$-85$}

Fab. In pulmonibus Bufonis ignei (Berolini Junio G.aede réperit), B. cinerei. Mus. $V$.

14. Monostoma Cornu $R$.

M. Poro oblongo subintegerrimo, corpore tereti incurvo, retrorsum decrescente.

Ent. 1. p. 346. n. 7. Amphistoma Cornu. Mant, n. Iq. M. Cornu.

Hab, in intestinis Ardeas, cinereae (Novembri), Gozze; Nycticoracis. Mus. $V$.

15. Monostoma prismaticum ZED,

MI. Poro orbiculari exiguo, corpore subtriquetro utrinque obtuso.

Ent. I. p. 335. n. 10, M. prismaticum.

Hab. Iu abdomine Corvi frugilegi ZEDER solitarium reperit.

16. Monostoma mutabile Zisn. t.

M. Poro urbiculari, collo conico, corpore oblongo, hinc plano, illinc convexo, postice rotundato.

Ent. 1. p. 333. n. 9. M. mutabile.

Hab. In ajdomine Fulicae Chloropodis ZEner Junio solitarium offendit; in intestinis avis dictae. Cat. E. $\boldsymbol{V}$.

17. Monostoma temuicolle $R$.

M. Poro orbiculari, collo tereti, retrorsum 2t. tenuato breviore! còrpore maximo supra convexo, subtus planiusculo:

Mant. n. 17. M. ienuicolle?

Hab. inter carnes Lampridis guttáti, BAzKer. 18. Munostoma filicolle $R$.

M. Poro orbiculari, collo, longissimo filiformi, corpore crasso sipra convexo, subtus planiusculo.

Mant. n. 18. M. filicolle. 


\section{$\therefore 86$}

Hab. Inter Bramae Raji precessus spinosos vertebrarum dorsi spurios Neapoli Julio reperi. An huc 11, 30.?

19. Monostoma ventricosum $\mathrm{R}$.

M. Capite discreto subconico tenui, corpore antice crassissimo subgloboso, postice cylindrico.

Ent. I. p. 335. n. Ir. M. ventricosum.

Hab. In abdomine Motacillae Lusciniae Majo

Grypliae reperi. An Amphistoma?

20. Monostoma trigonocephalum R:

M. Capite discreto trigono, corpore tenuiore supra convexo, subtus concavo.

Ent. I. p. 336. n, 12. Mant. n+ 20. M, trigonocephalum.

Hab. In intestino crasso Testudinis Mydae Majo Arimini reperi.

21. Monostoma galeatum R.

M. capitis discreti subtetragoni margine superiore sexdentato, corpore tereti, retrorsum attenuato.

Mant. n. 2I. M. galeatum:

Hab. In intestinis Centronoti glalci' Neapoli Augusto reperi.

22. IMonostoma sulcatum R.

M. capite discreto ovali, corpore angustiori lineari depresso, hinc sulcato.

Ent. I. p. 337. n. I3. M. sulcatum.

Hab. In intestinis Ranae Pipáe-spiritu vini ser. vatae specimina complura reperi.

23. Monostoma macrostomum $\mathrm{R}$.

M. capite ovali discreto, corpore tenuiore hinc convexo, illinc concavo.

Ent. I. p. 337. n. 14. M. macrostomum.

Hab. In intestinis Lari cinerarii specimina plu. rima Gryphiae Julio reperi. An Amphistoma? 


\section{$=87$}

species dubiae.

24. Monostoma (Hypostoma) Maraenulae. $f$

Fnt. 1. p. 339. n. 16. M. Maraenulae.

Hab. In vesicula ventriculo Salmonis Maraenulae adnexa. Braus. Speciei primae affine.

25. Monostoma (? Hypostoma) Idi $f$

Hab. in intestinis Cyprini ldi. Cat, E. V. 26. Monostoma Vespertilionis. I

Hab. in intestinis Kespertilionis lasiopteri. Cat. E. V. mseptus.

27. Monostoma Vanelli.

Mant. n. 27. M. Vanelli.

Hab. in thorace Tringae Vanelli pulmonibus adhatrens. Mus. $\boldsymbol{V}$.

28. Monostoma Himantopodis. $f$

Hab. in intestinis Charaärii Himantopodis. Cat. E. V.mescpt.

29. Monostoma Marilae $f$

Hab, in intestinis Anatis Marilae, C, F.V.

30. Monostoma Molae.

Mant. n. 3o. M. Molae.

Hab. Inter musculos Orthragorisci Molae dorsales Neapoli Julio reperi, an ad n. I8, amandandum?

Genus, XIV. Amphistoma.

Corpus molle teretiusculum. Porus anticus et poo sticus solitarii.

A. Capite discreto.

1. Amphistoma longicolle R.

Amph. Capite subcordato trilobo, collo tenui longiore: corpore teretiusculo obtuso; poro antico inaequali majore, postico orbiculari. 
Mant. n. I. Amph.' longicolle.

Hab. in intestinis Ardeae albae, HüBner; Aro deae stellaris, Lari ridibundi et Atricillae. Mus. Viènntr.

2. Amphiostoma Serpens Nitzscri:

Amph. capite, subovato, collo crassiusculo, cor: pore teretiusculo truncato, poro antico magno obliquo, postico exiguo prominulo. Manto n. 2. Amph, serpens.

Hab. in intestinis Ealconis Haliaëti. NIтzscr.

5. Amphistoma macrocephalum $R$.

Amph. Poro capitis subglohosi magno lobato, caudali exiguo crenato, corpore teretiusculo. incurvo,

Ent. I. P. 370. n. I. A. macrocephalum. p. 343. n. 3. A. striatum. p. 352, n. 10. A. Falconts Palumbarii. Mant. n. 3. A. macrocephalum. Hab. in intestinis Falconum et Strigium vulgatisssimum. Ipse in Bubone (Gryphiae Julio), flammea (Novembri ib.), Oto (ib. Febr., Junio Berol), Falcone apivoro (Berol. Majo) repéri. Abildgaard in Ulula. In F. Timunuculo Froe. Ircr. In F. Albicilla, Chrysaëto, naevio, galtico, lägopode, cineraceo, rufo, Milvo, Haliaëto, Buteone, Palumbario, peregrino, lana. rio, pennato, cyaneo, Niso, subbuteone, Lithofalconc, rufipede, Leucosomate; in Str. Brachyoto, Alusone, passerinc, dasypode. C.E.I.

4. Amphistoma microstomum R.

Amph. Porò capitis subconici parvo orbiculari, postico lobato, corpore teretiusculo.

Ent. I. p. 342, 1. 2. Amph. microcephalum. Hab. In duodeno Corvi Caryocatactis Gryphiae Octobri reperi. 


\section{$-89$}

5. Amphistoma isostomum R. $f$

Amph Poris cdpitis subconici, corporisque obconici integerrimis subaequalihus.

Ent. I. p. 352: n. 11. Ainph. Tadornae. Mant.

n. 5. A. isostomum.

Hab, in intestinis Anatis Tadornae. AmindGaAd.

6. Amphistoma gracile $\mathrm{R}$.

Amph. Poro-capitis elongati lobato, caudali exiguo prominente, corpore utrinque attenuato.

Mant. n. 6. Amph. gracile.

Hab. in intestinis Mergi Merganseris et Albelli. Nins, Vienn.

7. Amphistoma erraticum $R$.

Amph. Poro capitis niaximi campanulati sublobato, caudali immerso, corpore utrinque attenuato incurvo.

Ent, 1. p. 344.n. 5. Mant. 11. 7. Amph. erraticum. Hab. In intestinis et abdomine Colymbi septenttrionalis Augusto, in int. Scolopacis Gallinaginis Julio Gryphiae reperi, In int, Col, arctici, Scol. Rusteicolas. MHus. V.

8. Amphistoma urnigerum $\mathrm{R}$.

Amph. Poro capitis subcampanulati magnno, cre. nato, corpore tereti subaequiali. Mant. n. 8. Amph. urnigerum:

Hab. in Ranae escrlentae hydatidibus viscerum et intestino recto, $\mathbf{I}$ Tus. $V$.

9. Amphistoma Cornu Nitzscr.

Amph. Capite maximo subbilobo, corpore re. trorsum increscente incurvato, poro postico - exiguo integerrimo. Miant. n, 9. Amph. Cornu.

Hà, in intestinis Ardeae cinereae. Nitzscr. Gar 
zettae, Mus. Vienu. Non confundendum cum Monostomate Cornu n. 14. olim mihi perperam Amphistomate dicto.

10. Amphistoma cornutum $\mathbf{H}$.

Amph. Poro capiris hemisphaerici multilobato, corpore crenato, hinc convexo, illinc concavo, postice truncato.

Ent. 1. p. 34 in 4. Amph. cornutum.

Hab. In intestinis Charadrii, pluvialis Gryphiae Octobri reperi.

11. Amphistoma Sphaerula $R$.

Amph. Poro capitis subglobosi tumido multilobato, postico integerrimo, corpore oblongo. Ent. I. p. 345. n, 6. Mant. n. I1. Amph. Sphaerula.

Hab. In intestinis Corvi Cornicis Gryphiae Novembri reperi.

12. Amphistoma pileatum $\mathrm{R}$.

Amph. capite orbiculari depresso, corpore angustiore, tereti, poro postico exiguo.

Ent. I. p. 338. n. 15. Monostoma pileatum. Mant. n. 12. Amph. pileatum.

Hab. In intestinis Sternae Hirundinis Gryphiae Julio reperi. St. Cantiacae. Mus. $V$.

13. Amphistoma denticulatum $\mathrm{R}$.

Amph. Poro capitis lanceolato-ovati immerso, caudali orbiculari exiguo, corpore tereti po. stice rotundato, subdenticulato.

Mant. n. I3. Amph. denticulatum.

Hab, in intestinis Alcedinis 1spidae. Mus, $V$.

$$
\text { B. Capite continuó. }
$$

14. Amphistoma subclavatum $\mathbf{R}$.

Amph. Corpore conico, postice obtusissimo, poro antico exiguo, caudali amplissimo. 


\section{$-9^{1}$}

Ent. I. p. 348. n, 8. Mant. n. 14. Amph. subcla. vatum.

Hab. In intestino recto Ranae temporariae saepius, semel in intestinis $R_{0}$. esculentae Berolini Octobri, semel ib, Julio in Bitfonis ' $\mathrm{g}_{\text {ruei }}$ vesica urinaria reperi. In intest. Hylae arboreae, Bufonis cinerei, Gowze.

15. Amphistoma truncatum $\mathrm{R}$.

Amph. corpore depressiuscnlo, antrorsum attenuato, postice truncato, poris orbicularibus, antico exiguo, caudali majore marginato.

Mant. n. 5. Amph. truncatum.

Hab. In Phocae vitulinae junioris ventriculo et intestinis Berolini Junio maxima copia reperi. Eadem copia in hepate Phocae vituilnae, Отто, Vratislaviae.

16. Amphistoma nnguiculatum $R$.

Amph. corpore oblongo depresso, poro antico exiguo, caudali 'emarginato majore:

Mant. n. 16. Amph. unguiculatum.

Hab. In intestinis Tritonis palustris Berolini Martio reperi.

17. Amphistoma conicum R.

Amph. Corpore tereti retrorsum increscente, obtuso, poris integerrimis, antico minirno, caudali magno.

Ent. I. p. 349. n. 9. Mant, n, 17. Amphistoma conicum.

Hab. In Bovis ventriculo primo saepe copiose, parcius in secundo, Gryphiae, reperi. In Cervo Elapho, Wredow. Dama, Mus. V. In Ovis arietis ingluvie. TreuteEk.

18. Amphistoma subtriquetrum $\mathrm{f}$. Amph. Corpore depressiusculo retrorsum in. 
crescente rotundato, poris orbicularibus, an. tico exiguo, caulali magno, infero.

Mant, n. I8. Amphistoma subtriquetrum.

Hab. In Castoris Fibri coeco et colo Berolini

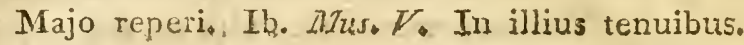
WaLter.

$$
\text { Species dubiae. }
$$

19. Amphistoma Falconis peregrini.

Mant. 17. is Amph. F. peregrini.

Hab. In intestinis tenuibus Falconis peregrini Berolini Noũembri reperi.

20. Amphistoma Lari glauci, t

Hab. in intestinis Lari glanci, diversum ab Amphistomate longicolli Lari ridibundi, n. I. Cat. Eut. Viennu.

21. Amphistoma Anatis querquedulae. $t$. $H_{a b}$, in intestinis Anatis Querquedulae. $C, E, V$.

\section{Genus, $X V$. Distoma}

Corpus molle, depressum aut teretiusculum. Pori solitarii, anticus et ventralis.

$$
\begin{aligned}
& \text { A. Iuermia. } \\
& \text { a. Plana vel depressa. } \\
& \text { a. Poro ventrali majore. }
\end{aligned}
$$

1. Distoma hepaticum Abirdgand.

D. obovatum, planum, collo subconico, brevis. șimo, poris orbicularibus, ventrali majore.

Ent. 1. P. 352. n. 1. Mant n. 1. D. hepaticum.

Hab. in Hominis vesica fellea; in Halmaturi siggntei; Leporis timidi, Guniculi; Sciuri entropaei; Canneli bactriani; Corvi Llaphi, Capreali, Damae; Alutilopes Kevellac, Corinnae; 
Bovis tauri ct domestici; Caprac; Ovis; Equi, Asini; Suis hepate, inde passim in intestina delatum. In ove vulgatissimum.

2. Distoma ovatum $\mathrm{R}$.

D. ovatum, planum, poris orbicularibus, ventrali majore.

Ent. I. p. 357 . n. 2. 0. ovatum.

Hab. in avium bursa Fabricro dicta. Ipse in Corvo Pica Junio, Anate clypeata et Fulica jigra (cmmibus pullis) Julio Gryphiae ri ri. In Corvo frugilean, I. C. H. Mixxer. In Cornice, Cat. Ent. $V$,

\section{Distoma cuneatum R.}

D. depressum, cuneatum, poris orbicularibus; ventrali majore.

Ent. 1. p. 358. n. 3. D. cuneatum.

Hab. In intestinis Otidis Tardae Gryphiae Septembri reperi.

4. Distoma dendriticum $R$.

D. planúm lanceolato - ovatum, poris liemisphae. ricis $v \in$ ntrali majore.

- Mant. n. 4. D. dendriticum.

Hab. In intestinis Xiphiae Gladii. Spedaiterr.

5. Distoma tenuicolle $\mathrm{R}$.

D. oblongum planum, collo angustato, poris hemisphaericis, ventrali majore.

Mant. n':5. D. tenuicolle.

Hab. In hepate Phocae barbatrae TREUTIER Aprili reperit.

6. Distoma carnosum R.

D. depressum ovatum, collo cónico, poris glo. busis, ventrali maximo.

Mant. in. 6 D. carnosum.

Hab. In intestinis Spari Dentiacis Arimini Majo, Julio Neapoli reper̦i. 


\section{- $94-$}

\section{Distoma hians $\mathbf{R}$.}

D. planum subellipticum, antrorsum magis at. tenuatum, poris orbicularibus distantibus, ventrali majore.

Ent. I. p. 359. n. 4* Mant, n. 7. D. hians, .

Hab. In oesophago Ardeae nigrac Gryphiae Sep. tembri reperi.

8. Distoma cucumerinum $R$.

D. planum ellipticum, poris orbicularibus approximatis, ventrali majore.

Ent. 1. 1. 360. n. 5. D. cucumerinum.

Hab. In Avis ripariae trachea Rousseau Parisiis reperit.

9. Distoma lucipetum $\mathrm{F}$.

D. depressum oblongum, collo elongato angustato, poris orbicularibus distantibus inferis, ventrali majore.

Mant. $n+9 . D$. lucipetum.

Hab. sub Lari glauci et fusci membrana oculi nictitante. Mus. $V$.

10. Distoma pulchellum R.

D. depressum, subellipticum, antrorsum magis decrescens, poris globosis inferis distantibus, ventrali majore.

Mant. n. 10. D. pulchellum.

Hab. In intestinis Labri cynaedi Neapoli Junio reperi.

13. Distoma incisum R. t

D. planum, ovale, poris subglobosis, ventralis majoris margine postico bifido.

Ent. 1. 1. 36r. n. 6. D. incisum.

ILab. in ventriculo Anarrhichae Lupi, RathxE.

12. Disıma holostomun R.

D. depressum, oblongum, retrorsum decrescens, 


\section{- $95-$}

antice truncatum, poris amplissimis, terminali orbiculari, ventralis nıajoris apertura transversa.

Mant. n. I2. D. holostomum.

Hab. in intestinis Ralli aquatici. Mus. $V$.

13. Distoma trànsversale $R$.

D. depressum, oblongum, collo angustato, poro antico orbiculari - exiguo, ventralis maximi apertura transversa.

Ent. r.p.36r.n. 7. Mant. n. 13. D. transversale. Hab. In ventriculo Cobitidis fossilis Gryphiae Julio, Aprili Berolini reperi. In C. Taeniae intestinis. Cat. $E V$.

14. Jistoma tumidulum $\mathrm{R}$.

D. depressum, lineare, utrinque obtusúm. poris remotis, antico globoso, ventralis majoris apertura transversa.

Mant. n. 14. D. tumidulum.

Hab. in intestinis Syngnathi Ilippocampi Mus. Vienn. S. Acus. Cat. E. V.

15. Distoma Atomon $\mathrm{R}$.

D. oblongum planum, collo angustato, poris orbicularibus, antico exiguo, ventrali majore.

Ent. I. p. 362 . n. 8. D. Atomon.

Hab. in ventriculo Pleuronectis Flesi Gryphiae Majo reperi.

16. Distoma polymorphum $\mathbf{R}$.

D. depressiusculum subovatum, crenatum, postice emarginatum, pori antici margine tumido, ventralis majoris recto.

Ent. 1. p. 363. n. 9. Mant. n. 16. D. polymorphum.

Hab. in intestinis Muraenae Anguillae Gryphiae Majó et Augusto reperi. 


\section{$-9^{6}$}

17. Distoma glohiporum $\mathrm{R}$.

D. depressiusculum oblongum, collo subtus excavate, poris globosis, ventrali majore.

Ent. 1. p. 364 , n. 10. D. globiporum.

Hab. In intestinis Cyprini Bramae et erythiro-

- phthalmi Gryphiae aliquoties reperi. In Carpione. Froeltch, Zeder; Tinca, Moneer; $\mathrm{Na}$ so, Cat. E. V. (In Perca fuvialili, Zentr; an parasiticum?)

18. Distoma caudiporum $\mathrm{fr}$.

D. depressinsculum, corpore subelliptico, caudat $r \in t r a c t i l i s$ angustioris apice excavato, poris globosis, ventrali majore.

Mant. n. Is. D. caudiporum.

Hab. In intestinis Zenis Fabri Arimini Aprili reperi.

19. Distoma cygnoides ZED.

D. depressiusculum oblongum, collo angustàto teretiusculo, poris orbicularibus, ventrali majore.

Ent. I. p.367. n. Ir. Mànt. n. 19. D. cygnoides.

Hab. In vesica urinaria Ranac esculentae Gryp!iae; in illa Bufonis ignci GaEde Berolini Junio reperinus.

20. Distoma cymbiforme $\mathrm{h}$.

D. depressum, ovatum, collo crena discreto an. guctato, poris globosis remotis, ventrali majore.

Mant. 11. 20. D. cymbiforme.

Hab. In vesica urinaria Testudinis mydae Majo Arimini reperi.

21. Disloma folum Olfers.

D. depressum, corpore ovato, collo angustato, poris orbicularibus, ventrali majore. 


\section{$-97$}

Mant. n. 2r. D. folium.

Hab, 'In vesica urinaria Esocis Lutii Oreers Julio Berolini reperit,

\section{Distoma seriale $\mathrm{R}$.}

D. corpore plano subquadrato, collo angustato, pori antici apertura oblongiuscula, ventralis majoris orbiculari.

Ent, 1. p. 368. n. 12. D. seriale。

Hab. in sanguine dorsali Salmonis alpini, O. FA BRICIus. (Iste autem sanguis dorsalis nil nisi Renes apud varios auctores olim male pro sanguinis coagulo habitos significat.)

23. Distoma simplex $\mathrm{R}_{\text {。 }}$

D. depressiusculum lineare, collo conico continuo, poris orbicularibus, ventrali majore.

En:. 1. p. 370, n, 13. D. simplex.

$\mathrm{Hab}_{\text {, in intestinis Gadi Aeglefui }}$ O, Fr. Mür. LER।

24. Distoma divergens $\mathrm{f}$.

D. oblongum planum, collo conico divergente, poris globosis, ventrali majore.

Ent. 1. p. 37 I. n.' 14. Mant, n. 24, D. 'divergens.

Hab, in intestinis Blenii vivipari, Zoega, MüLLER, Ipse in illis Bleniii Gattorugine, cornuti ct tentaculati Arimini Aprili et Majo reperi:

25. Distoma fascialuin $\mathrm{R}_{\text {}}$

D. depressum ellipticum, collo attenuato, paris globosis, ventrali majore:

Mant. $16,25 . \mathrm{D}$. fasciatum:

Hab. In intestinis Labri Tincaè Junio; irgelopis

et Percae marinae Julio-Neapoli reperi.

26. Distomà sinuatum $R$.

D. depressum oblongum, 'margine sinuatum; 
collo conico, poris distantibus orbicularibus, ventrali majore.

Mant. n. 20. 10. sinuatum.

Hab. In intestinis Ophidii imberbis Neapoli Julio reperi.

37. Distoma fulvum $\mathrm{R}$.

D. depressum, corpore ovali, collo angustato, poris subglobosis marginatis distantibus, 'ven." trali majore.

Mant. n. 27, D. fulvum.

Hab. In intestinis Gadi Molvae Neapoli Junio reperi. In Gado mediterraneo. Mus, $V$.

28. Distomą oxycephalum $\mathrm{h}$.

- D. planum lineare poștice obtusiusculum, collo antrorsum angustato, poris orbicularibus in. feris, antico exiguo, ventrali magno.

Mant. n. 28. D. oxycephalum.

Hab, in intestinis Anatis Boschadis, Froerich,

Nitzscri; $A_{*}$ clypeatae, Mus. $V$.

29. Distoma complanatum $\mathbf{R}$.

D. oblongum planum, collo angustato, poris magnis, antico orbiculari, ventralis majoris apertura I:ngitudinali.

Mant. n. 29. D. complanatum.

Hab. In oecoplago Ardeae cinereae Rosenthax Berolini Aprili reperit.

\section{Distoma macrourum $\mathrm{R}$.}

D. depressum oblongurn, postice attenúatum, collo tenui, pori antici apertura orbiculari, ventralis majuris longitudinali.

Ent. 1. p. 370, n. 15. D. longicauda.

Hab. in Corvi Cornicis liepate et vesicula fel. lea. Andr. Jurine.

31. Distoma albiculle $\mathrm{R}$.

D, depressum oblougum utrinque attenuatum, 


\section{- 99}

poris semiglobosis approximatis, ventrali ma. jore.

Mant. n. 31. D. albicolle.

$\mathrm{Hab}$, in Falconis pennati hepate et cystide fel. lea. Mhus. $V$.

32. Distoma Naja R.

D. depressum, corpore elongato angusto, collo dilatato ovali, poris globosis approximatis, ventrali majore:

Ent. I. p. 434 n. 7. D. Colubri Natricis pulmona. le. Mạnt. n. 32, D. Naja.

Hab. Ir Colubri Natricis pulmonibus Berolini aestate reperi.

B. Poro antico majure.

33. Distoma variegatum $\mathrm{R}$.

D. depressim, elongatum, antrorsum attenuatum, poris orbicularibus remotis, antico ma. jore.

Mant. n. 33. D. variegatum.

Hab. In pulmonibus Ranae esculentae Berolini Octobri reperi.

34. Distoma capitellatum $\mathrm{R}$.

D. depressum, parte corporis anteriore subel. liptica, posteriore lineari, yoris globosis, antico majore discreto.

Mant. n. 34. D. capitellatum.

Hab. In vesicula fellea Uranoscopi scabri Aprili, Majo et Junio, Arimini et Neapoli reperi.

35. Distoma delicatulum $R$.

D. planum ellipticum, collo subtereti, poris orbicularibus, antico majore.

Ent. I. p. 373. n. 16. D. delicatulum,

Hahitaculum in Anatis sponsae vesicula fellea. Bralx. 
36. Distoma crystallinum $\mathrm{R}$.

D. depressiusculum subellipticum, poris globosis remotis, antico majore,

Niant. n, 36. D. crystaliinum.

Hab. In vesicula fellea Ranae esculentaie et temsporariae, inque utritusque et Bufomis viridis êt ignei hydatidibus mesenterii et hepatis GAEDE Majo et Junio Berolini reperit. Ipse ibidem Augusto in Viperae Beri hydatidibus cordis offendi.

37. Distoma maculosum $\mathrm{R}$.

D. depresstim oblongo-ovatum, poris orticu. laribus, antici majoris margine tumidulo.

Ent. 1. p. 374. 1. 17. Mant, n. 37. D. maculosum. $\mathrm{Hab}$, In intestinis Caprimulgi europaei Septembri, Hirundinis rusticae Majo, Gryphiae reperi. In illis H. Apodis Froencrer Junio; H. urbicae ZeDER Augusto; $I$. ripariae Cat, $E, V$.

38. Distoma Linguatula $\mathbf{R}$.

D. depressoplanum ellipticum, poris orbicula. ribus, antico majore.

Mant. n. 38: D. Linguatula.

Habitac. non indicato Olfers ex Brasilia misit 39. Distoma elegans $P_{\mathbf{r}}$

D. depressiusculum ovále, apertura pori antici majoris oblenga, ventralis orbiculari.

Ent. 1. p. 375. n. 18. D. elegans.

Hab. In intestinis Fringillae domesticae (pullo. rum) Gryphiae reperi; in illis Fr, caelebis et montanae. Cat. E., $V_{\text {s }}$

40. Distoma cirratum $R$.

D. depressiusculum ovale, antice punctatum, apertura pori terminalis majoris oblonga, ven. tralis orbiculari, 
Ent. r, p. 376. n, I9. D. cirratum.

Hab. In intestinis crassis Corvi MIonedulae et Picae Gryphiae Junio reperi.

41. Distoma nanum $\mathrm{R}$.

D. planum ovale, medio contractum, apertura pori antici duṕlo majoris oblonga, ventralis orbiculari.

Ent. r. p. 376. n. 20. D. nanum,

Hab. In intestinis crassis Scolopacis. Gallinulae Julio Gryphiae reperi.

42. Distoma micrococcum $\mathbf{R}$.

D. depressiusculum subellipticum, apertira pori - antici majoris oblonga, ventralis orbiculari.

Mant, n, 42. D. micrococcum.

Hab. In intestiuis Glareolae austriacae Arimini Aprili reperi.

43. Distoma fuscatum $R$.

D. depressiusculum oblongum, apertura pori antici maximi longitudinali, ventralis orbiculari,

Mant. 2.43. D. fuscatum.

Hab. In intestinis tenuibus Tetraonis Cotzarnicis Anconae Majo reperi.

44. Distoma ringens $R$.

D. depressiusculum oblongum, poris orbicula. ribus remotis, antico majore tumido.

Mant. $11.44 . \mathrm{D}$. ringens.

Hab, in intestinis Pici tridactyli. Mus. $\boldsymbol{V}$.

45. Distoma involutum R. \&

D. planum, obovatum, margine inflexo, poris orbicularibus, antico majore.

Ent. I. p. 377. n. 21. D, involutum.

Hab. In intestinis Upupae Epopis ZeDsí Julio reperit. 
46. Distoma crassicolle $\mathrm{R}$.

D. planum, corpore retrorsum decrescente, pqris globosis distantibus, antico majore.

Ent. x. p. 378. n, 22. Mant. n. 46. D. crassicolle.

Hab. In intestinis Salamandrae maculatae (hercynicae) Berolini auctumno repéri. In recto S. atrae. Froelich. Cat. E. $V$.

47. Distoma tereticolle $R$.

D. depressum lineare, subcrenatum, collo tereti, poris globosis, antico majore.

Ent. I. p. 379. n. 23. Mant, n. 47. D. tereticolle.

Hab. In ventriculo Esocis Lucii majoris fere nullo non tempore, in illa Percae Luciopercae semel Gryphiae reperi. In Salnone Trutta Axor. Jurine; Farione, Huchone, Cat. E. V.

48. Distoma relatinosum $\mathrm{R}$.

D. depressiusculum elongstum, utrinque obtusum, antrorsum attenuatum, poris globosis remotis, antico majore.

Mant. n. 48. D. gelatinosum.

Hab. In intestinis Testudinis Mydae Arimini Majo reperi.

49. Distoma megastomum $\mathbf{l}$.

D. depressum, oblongum, utrinque obtusissimum, collo parum angustiore, poris globosis remotis, antico majore.

Mant. n. 49. D, megastomum.

Hab. In ventriculo Squali Galei Arimini Majo reperi.

50. Distoma heterostomum $\mathrm{R}$.

D. depressum oblongum transversim striatum, collo angustiore, pori antici majoris apertura triangulari, ventralis oblonga.

Ent, I. p.381. n.24. Mant, n.50, D. heterostomum. 


\section{- $103-$}

Hab. in-Ardeae purpureac oesophago, ANDR, Jurive; sub illius lingua, Rosa,

51. Distoma mentulatum $R$.

D. depressum elongatum, pori antici majoris apertura oblonga, ventralis orbiculari, cirro tenui.

Ent. I. p. 433. n. 70, D. Colubri Natricis intestinale. Mant. n. 5I, D. mentulatum.

Hab. In intestinis Colubri Natricis Julio Bero. lini, Lacertae maculatae Aprili Arimini re. peri; in illis Lacertae agilis Gaede Julio Berolini offendit,

52. Distoma clavigerum $\mathbf{R}$.

D. depressum, ovato-ellipticum, antrorsum an. gustatum, poris orbicularibus, antico majore,

- cirro clavato.

Mant, n, 52, D. clavigerum.

$\mathrm{Hab}$. In Bufonis viridis intestinis tenuibus $\mathrm{Or}_{\mathrm{r}}$ FERS b'erolini Julio copiosum reperit. In illis $B u f$. cinerci, Ranae temporariae, esculentae, Hylae arboreae, Cat. E. V.

\%. Poris aequalibns.

5j. Distoma Squamula R.

D. depressum sublunatum, poris orbicularibus exiguis remotis aequalibus,

Mant. 11. 53. D. squamula.

Hab. in intestinis Mustelae Putorii. Mus. V. 54. Distoma caudale $\mathrm{R}$.

D. depressum, subellipticum, postice acuminatum, poris subaequalibus, antico obliquo, ven. trali angulato.

Ent. I. p. 382. n. 25. D. caudale.

$\mathrm{Hab}$, in intestino recto Corvi Caryocatactis, $\mathrm{ZE-}$ DER; glandarii, Pyrrhicoracis, Cat.E. V. 
55. Distoma soleaeforme R.

D. planum subellipticum, antrorsum increscens, poris subaequalibus globosis.

Ent. 1. p. 384. n. 26. D. soleaeforme.

Hab. in ventriculo Triglae Gurnardï. Rathry.

56. Distoma pusillum ZED.

D. pianum ellipticum, utrinque obtusissimum, poris distantibus orbicularibus, aequalibus,

Ent. 1. p. 384* n. 27. D. pusillum.

Hab. Sub cute Erinacei europaei Braun Majo reperit. Conf. n. 130.

57. Distoma macrostomum $\mathbf{R}$.

D. planum oblongum, antrorsum increscens, cauda appendiculata, poris orbicularibus anıplissimis subaequalibus.

Ent. T. p. 386 n. 28. D. macrostomum.

Hab. In intestino recto Motacillae Lusciniae Gryphiae Junio reperi. In intestinis Motac, Ravae, cinercae, Misoriae, Ruviatilis, C.E.V.

58. Distoma mesostomum $\mathrm{R}$.

D. planum oblorgum, antrorsum increscens, postice acutiusculum, poris orbicularibus ąmplis. simis subaequalibus.

Ent. x. p. 387. 17. 29. D. mesostomum. .

Hab. In intestino recto Turdi iliaci Novembri Gryphiae reperi.

59. Distoma microstomum R.

D. depressum sublineare, collo subelliptico, poris exiguis subglobosis aequalibus.

Ent. 1. P 388. n. 30. D. microstomurn.

Hab. In intestinis Pleuronectis Soleae Majo Pa. riciis reperi.

6o. Distoma trilobum $\mathbf{R}$.

D. depressum, antice trilobum, retrorsum de- 


\section{$-105$}

crescens, postice obtusum, poris orbiculari.

bus exiguis aequalibus.

Mant. n. 6o: D. trilobum.

Hab. in intestinis Pelecani Carbonis. Mus. V.

61. Distoma hyalinum $\mathrm{h}$.

D. depressum oblongum utrinque obtusum, po. ris globosis mediocribus aequalibus.

Ent. I. p. 389. n. 3r. D. hynlinum.

Hab, in intestinis Salmonis Eriocis. O, FrLED, MüLLER,

62. Distoma isostomum $R$.

D. depressum oblongum utrinque obtusum, po. ris globosis remotis aequalibus.

Mant. n. 62. D. isostomum.

$\mathrm{Hab}$, in Astaci fuviatilis cluctibus biliferis, Orтo; ad illius nervorum ganglia, Carus.

63. Distoma irroratum $\mathrm{h}$.

D. depressum, sublineare, utrinque obtusum; poris globosis aequalibus.

Mant. n. 63. D. irroratum,

Hab. In ventriculo Testudinis Mydae Arimini Majo reperi.

64. Distoma flexuosum $\mathrm{R}$.

D. depressiusculum elongatum, utrinque attenuatum, poris minutis subaequalibus.

Ent. 1.p.389. n. 32. D. flexuosum.

Hab. In ventriculo et intestinis Talpae europaeae Hildebrandt, auditor quondam noster pracmatura morte scientiis iereptus et ego Gry. phiae aestate reperimus. 


\section{$-106=$ \\ b. Tereituscula. \\ a. Poro ventrali majore.}

65. Distoma clavatum R. $\downarrow$

D. tercs transversim rugosum, postice incrassatum, poris subglobosis, ventrali majore.

Ent. 1. p. 391. 11. 33. Mant. n. 65. D. clavatum.

Hab. in ventriculo Scombri. Pelamidis.

66. Distuma cylindraceum ZED.

D. teres, collo conico crassiore, poris orbicularibus, ventrali majore.

Ent. 1. p. 393. n. 34. D. cylindraceum:-

Hab. In-Ranae temporariae pulmonibus Aprili, Majo et Junio Gryphiae saepe et copiose reperi. In illis Ranae esculentae, ZEDEK et BRAUN.

67. Distoma Granulum R. †

D. teres utrinque decrescens, poris globosis, ventrali majore.

Ent. 1. p. 394. n. 35. D. granulum.

Hab. in intestinis Cotti Scorpii, ZoeaA, MüLIER.

68. Distoma inflexum $\boldsymbol{R}$.

D. teres, collo tenuiore inflexo, poris globo: is. ventrali majore.

Ent. 1. p. 395.n. 36. D. inflexum.

Hab. In intestinis Cyprini Jesis Gryphiae Aprili reperi.

69. Distoma varicum $\mathrm{ZED}$.

D. teres, collo corpori aequali divergente, po. ris globosis inferis, ventrali majore.

Ent. 1. p. 396. n. 37. D. varicum.

Hab. In ventriculo Salmonis Salaris Gryphiae Majo reperi, In intestinis S. Thymalli. Cat. Ent. Vicnn, 
70. Distoma ocreatum f.

D. teres, collo antrorsum tenuiore divergente, cauda retractili, poris globosis, ventrali majore.

Ent. I. p. 397. n. 38. D. ocreatum.

Hab. In intestinis Clupeae Harengi Gryphiae Majo reperi.

71. Distoma gibbosum $R$.

D. teretiusculum, abdomine gibboso, poris subglobosis, ventrali pedunculato majore.

Entoz. 1. p. 399. n. 39. Mantiss, n, 71, D. gibbosum.

Hab. In ventriculo Esocis Belones Majo et Junio Gryphiae reperi. An parasiticum in Colymbo cristato? Mus, $V_{\text {. }}$

72. Distoma furcatum Brens.

D. teres filiforme, pori ventralis submajoris pe. dunculo divaricato collum aequante.

Mant, n. 72. I). furcatum,

$\mathrm{Hab}$, in intestinis Illulli surmuleti. Mrus. $V$. Ipse in illis Mulli rubescentis Arimini Aprili, Gadi ITolvae Junio Neapoli reperi.

73. Distoma fractum $\mathrm{R}$.

D. teres, retrorsum attenuatum, poris remotis, antico oblongo, ventrali glcboso majore sui. pedunculato.

Mant. n. 73. D. fractum.

Hab. In intestinis Spari Salpae Junio et Julio Neapoli reperi.

74. Distoma genu R.

D. teres oblongum abdomine prominente, poris remotis inferis, ventrali majore globoso, antico hemisplacrico:

Mant. n, 74, D, genu. 


\section{- 108}

Hah. In intestinis Labri lusci Neapolì Junio reperi.

75. Distoma ventricosum $\mathrm{R}$.

D. teres oblongum abdomine prominente, poris globosis, ventrali majore.

Mant. n. 75. D. ventricosum.

Hab. In intestinis Clupsace Alosae Arimini Aprili offendi.

76. Distorna haccigerum $R$.

D. teres postice increscens rotundatum, poris orbicularibus, ventrali majore.

Mant. n. 76. D. baccigerum.

Hab. In intestinis Atherince Hepseti Junio Neapoli reperi.

77. Distoma Ascidia R.

D. teres antrorsum attenuatum, postice obtu. sum, ventre prominente, poris hemisphaericis approximatis, ventrali majore.

Mant. n. 77. D. Ascidia.

Hab. In intestinis Spari Boopis (Majo et Junio, Arimini et Neapoli) et Pagri (ibid, Julio) reperi.

78. Distoma labiatum $R_{\text {. }}$

D. teres antrorsum attenuatum, postice obtusum poris inferis subglobosis, ventrali majore.

Mant. n. 78. D. labiatum.

Hab. In hepate Synguashi pelagici Neapoli Ju. lio reperi.

79. Distoma apertum $\mathrm{R}$.

D. teres antrorsum attenuatum, apice postico obtuso hiante, poris subglobosis, ventrali majore.

Mant. n. 79. D. apertum.

Hab. In intestinis Apogonis (Mulli imberbis Lrsv.) Junio et Julio Neapoli reperi. , 


\section{$-109$}

80. Distoma microsoma $\boldsymbol{R}$.

D. teres antrorsuin attenuatum, postice obtu. sum, poris globosis remotissimis, ventrali majore.

Mant. n. 8o. D. microsoma.

Hab. In intestinis Percae marinat Junio Nea. poli reperi.

81. Distoma globulus $\mathrm{R}$.

D. subglobosum, abdomine tumido, poris orbicularibus, ventrali majore.

Mant, n. Sr. D. globulus.

Hab. In intestinis Anatis Fuligulae Gryphize

Novembri reperi.

82. Distoma excavatum $R$.

D.' collo supra convexó subtus concavo, antror. sum attenuato, longiore: corpore tereti; po. ris orbicularibus; ventrali majore.

Ent. I. p. 339. n. 40. Mant. n. 82, D. excavatum.

Hab, In intestinis Ardeas Ciconiae tenuibus

Gryphiae Julio reperi. In illis A. Nyctico. racis. - Cat. E. V.

83. Distoma spathaceum $\mathbf{R}$.

D. collo planiusculo subovato, breviore: corpore tereti; poris orbicularibus, ventrali ma. jore.

Mant. n. 83. D. spathaceum.

Hah, in intestinis Lari glauci, Mus, $V_{*}$

84. Distoma spatulatum $\mathbf{R}$.

D. colli plani antrorsum increscentis marginibus lateralibus inflexis, antico truncato, corpore tereti longissimo, poris orbicularibus, ventrali majore.

Mant. n. 84. D, spatulatum.

Hab. in intestinis Ardeae minutae. Mius. F. 


\section{Distoma appendiculatum $R$.}

D. teres, crenatum, cauda brevi attenuata retra. ctili, poris globosis, ventrali majore.

Ent. I. p. 400. 1. 4 I. D. appendiculatum. p. 404. n. 44. D. crenatum, p. 437, n. 77. D. Clupeae rhenanae. Mant. n. 85. D. appendiculatum.

Hab. In ventriculo Torpedinis marmoratae Arimini Aprili; Accipenseris Sturionis ib. Majo; in inteśtinis Ophidii barbati, ib. eod.; $O p h$. Vassalli, Neapoli Junio; Zenis Apri, ib. Julio; Pleuronectis maximi, Gryphiae Junio, Aprili Arimini; Pl: Linguatulae, Julio Neapoli; $G a$ sterostei aculeati, Gryphiae Junio; Triglae Hirnndinis et adriaticae Arimini Majo; Sal-

- monis Salaris, Gryphiae eod. mense; Osmeri Sauri, Junio Neapoli; Clupeae Alosae, Arimini Aprili reperi, In Pleur. Passere et So. lea. Mus, V.

86. Distoma affine R.

D. teres, corpore ovato, cauda elongata obtusa, poris globosis, ventrali majore.

Mant, n. 86. D. affine.

Hab. In intestinis Percac cirrosac Arimini Majo reperi.

87. Distoma rufoviride R.

D. teres, retrorsum attenuatum, cauda retractili, poris hemisphaericis, ventrali majore.

Mlant. n. 37. D. rufoviridé.

Hab. In ventriculo Muraenae Congri Julio Nea. poli reperi.

88. Distoma grandiporum $\mathbf{R}$.

D. teres, retrorsum attenuatum, cauda retractili, poris hemisphaericis, antico exiguo, ventrali maximo. 
Mant. n. 88. D. grandiporum.

Hab. In ventriculo Muraenae Helenae Neapoli Junio reperi.

89. Distoma pallens $\mathrm{f}$.

D. teretiusculum, elliptico.oblongum, pororum subglobosorum apertura antici longitudinali, ventralis majoris transversa.

Mant. n. 89. D. pallens.

Hab. In intestinis Spari Auratae Neapoli Augusto reperi.

B. P̈oro antico majore.

go. Distoma areolatum R.

D. teretiusculum, utrinque obtusum, poris globosis, antico majore.

Ent. 1. p. 401. n. 42 . Mant. n. 90. D. areolatum.

Hab. in intestinis pleuronectis Platessue O. Fr. Müller; ipse in illis Pl. mançi Neapoli Junio reperi.

91. Distona gracilescens $\mathrm{R}$.

D. teres, corpore oblongo, cauda attenuata, po. ris globosis, antico majore.

Mant, n. 9r. D. gracilescens.

Hab. In intestinis Lophii piscatorii Tergesti Aprili reperi.

92. Distoma torulosum R.

D. teres, antice obtusum sensim retrorsum decrescens, poris globosis, ántico majore.

Mant. n. y2. D. torulosum.

Hab. In intestinis Siluri Glanidis Gryphiae Octobri reperi.

93. Distoma tubarium $R$.

D. terts utrinque attenuatum, poris hemispliaericis, antico majore.

Mant. n. 93. D, tubarium. 
Hab. In intestinis Sciaenae Umbrae Augusto Speziae reperi.

94. Distoma filiforme R.'

D. teres, filiforme, utrinque obtusum, pori antici majoris apertura oblonga, ventralis orbiculari.

Mant. n. 94. D. filiforme.

Hab. In intestinis Cepolae Taeniae Arimini Aprili reperi.

95. Distoma excisum $\mathbf{R}$.

D. teres, inaequale, collo subtus concavo, poris globosis, antico majore emarginato.

Mant. n. 95. D. excisum.

Hab. In ventriculo Scombri Scombri Arimini Majo, in intestinis Scombri Coliae Junio et Julio Neapoli reperi,

\%. Poris aequalibus.

96. Distoma alatum Z Z

D. parte anteriore depressiuscula expansa, pos steriore teretiuscula, poris orbicularibus sub. aequalibus.

Ent. I. p. 402. n. 43. Mant. n. 96. D. alatum.

Hab. In Canis Vulpis duodeno Martio Gryphiae, in ventriculo et duodeno Canis Lupi Bero. lini Januario, copiosissimum reperi.

97. Distoma crassiusculum R: $t$

D. teres, subovatum, poris semiglobosis aequa. libus approximatis.

Ent. I. p. 408. n. 46. D. crassiusculum.

Hab. In vesicula fellea dưrum Falconum Haliaë-

torum Bravn Augusto mense copiose reperit.

98. Distoma punctum ZED.

D. teré ovatum, poris semiglobosis distantibus aequalibus: 


\section{$-113$}

Ent. r. p. 409. n. 47. D. punctum.

Hab. ir intestinis Cyprini Barbi. Mus, Vienn.

Zenerus in illius recto Julio reperit.

99. Distonna fuscescens $R$.

D. corpore terti obtusiusculo, poris globosis distantibus aequalibus.

Mant. n. 99. D. fuscescens.

Hab. - In intestinis Spari Denticis Arimini Majo reperi.

$$
\begin{gathered}
\text { B. Ar mata. } \\
\text { a. Nodulosa seu papillosa. }
\end{gathered}
$$

100. Distoma nodulósuñ ZED.

D. teres, ovaturn, collo tenuiore, poro antico - nodulis sex cincto.

Ent. I. 4ío. n. 48. D. nodulosum.

Hab. in intestinis Percarum. Ipse in Perca Lu* cioperca, et fluviatili Decembri, Januario, Februario t Martio saepe maxime copiosum Gryphiae reperi. In P. cernua ZoegA et Zeder. In P. Aspero Schrank. In P. Zinlgel. Cat. Ent, Vienn.

101. Distoma laureatum ZED.

D. depressiusculum oblongum, poro antico no. dulis sex cincto.

Ent. I. p. 413, 12.49. Mant. n, IoI. D. Iau. reatum.

Hab. in intestinis 'Salmonum. Ipse in Farione saepe et copiose in insula Rugia Augusto teperi. In Tructa, Froelich auctumno, ZEDER Majo. In S. alpino et Thymallo, Cat. Ent. Viemn.

I02. Distoma lineare $\mathbf{R}$.

D. planum lintare, collo ténuiore, poro antico nodulis sex-cincto. 


\section{$-114-$}

Ent. r. p. 414. n. 50. Mánt, n. 102. D. lineare. Hab. In intestinis crassis Phasiani Galli. (luorum pullorum) Gryphiae Octohri reperi. Huc pertinebit etiam D. trachea Georgir.Montagis in trachea pullorum Phasiani Galli, colchici Tetraonis Perdicis, Pavonis et Anatis renert'n!n.

\section{b. Echinata. (Echinostuma.)}

103. Distoma trigonocephalum $R$.

D. depressiusculum, oblongum, collo antrorsum attenuato, capite trigono, echinis cincto posticeque, vage obsito.

Fnt. 1. p. 415. n. 51, D. trigonocephalum.

Hab. In intestinis ommibus Melis Taxi copiosissimum auctumno; in illis trium Erinaceorun europaeornm eodem tempore sparsum; Mustelarm Pntorionm plurimorun Octobri, Novembri et Decembri; in Mustelae vulga. ris tenuibus Julio, Gryphiae reperi. In illis Foinaé repertum Treutur dedit.

104. Distonia acanthoides $R$.

D. dępressum, sublineare, postice attenuatum, capite conico; echinis cincto posticeque cumulato.

Mant. ni. I04. D. acanthoidless.

Hab- In intestinis Phocae vitulinae tenuibus Junio Eerolini reperi.

105. Distoma bilobum $R$.

D. planum lineare, postice'oltusiusculum, capite bilobo, lobis semilunaribus disco et margine spinosis.

Mant. n. 105. 1). bilobum.

Hab. in intestinis Tantali Falcinelli. Mus, Vienn. 
106. Distona echinatum ZED.

D. planum, elongatum, capite reniformi, cincto echinis alternis̀.

Ent. I. p. 4.32. n. 67. D. gruis, p. 4rs. n. 52.

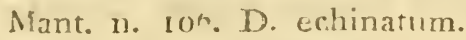

Hab. in intestinis Ardene comatce, Gruis: 'Colymbi minoris; Pelecani Carbonis, pygmaei; Anatis Boschadis domesticae et ferae, ferinae, clypeatae, streperae. Penelopes, Nyracae.

107. Distrima uncinatum ZED. †

D. planum, lineare, collo latiusculo, capite subreniformi cincto echinis, antice divérgen. tibus.

Ent. 1. p. 420. n. 53. Mant, n. 10\%. D. uncinatum.

Hab. In intestinis crassis Fulicae Chloropodis Zener Julio solitarum, complura Froelich Májo invenerunt.

108. Distoma militare $\mathbf{R}$.

D. planum lineare, collo corpori spimioso subaequali, capite subreniformi echinis cincto. Ent. 1. p. 421. 11. 54. Mant. 11. 103. D. nilitare.

Hab. In intestino recto Scolopacis Arquatae Gryphiae Januario reperi. In intestinis $S c$. Grllimiae, Hindebrandt; lialli Porzanae, Mius. Vienn.

iog. Distoma echinocephalum Ti.

D. planum sublineare, postice attenuatum, capite subrowando echinis cincto.

Ent. 1: p. 429. n. 62. D. Milvi. Mant, n. Iog. D. echinocephalum.

Hab. In intestino recto Falconis Milvi Treur. a. in Augusto reperit. 
110. Distoma cinctum $\mathrm{R}$.

D. depressiusculım oblongum, collo corpori subaequali, capite suborbiculari echinis cincto.

Ent. I. p. 422. n, 55. D. cinctum.

Hab. in intestinis Tringae Vanelli, WeIgex.

11. Distoma apiculatum $\mathrm{R}$.

D. depressiusculum oblongum, collo subaequali uncinato, capite subconico echinis cincto.

Ent. 1. p. 423. n. 56. D. apiculatum.

Hab. In intestino crasso Strigis Stridulae,

Reich; Rammeae ipse (Novembri, Gryphiae) reperimus.

12. Distoma denticulatum R.

D. teretiusculum, collo subaequali uncinato, capite subconico, basi tumido, echinis cincto. Ent. I. p. 424. n. 57. Mant. n. I12. D. denticulatum.

Hab. In intestinis Sternae Hirundinis Julio Gryphiae reperi. In illis St, cantiacae, Mus. Vienn. St. nigrae, Cat. Ent. Vienn.

13. Jistoma spinulosum $\mathrm{R}$.

D. teretiusculum, collo corpore tenuiore, capite subconiro, basi tumido, echinis cincto. Ent. I. p. 425. n. 58. Mant. n. 113. D. spinu. losum.

Hab. In intestinis Lari naevii Junio, Lari cinerarii, Julio, Colymbi. septentrionalis Augusto, Gryphiae reperi, In illis C. cristati, Mus. Vieinlı.

114. Distoma ferox $\mathrm{R}$.

D. planum lineare, collo subgloboso, capite subreniformi exiguo, echinis cincto.

Ent. 1. p. 426. n. 59. Mant. n. 115. D. ferox. Hab. In intestinis Ardeae Ciconiace Aprili et 
Julio, A. Jigrae Septembri, Gryphiae reperi. Huc etiam 11. 137. pertinere videtur.

\section{Distoma fallax $\mathrm{R}$.}

D. teres sibaequale, collo tenuiore, capite sub. globoso echinis cincto.

Miant. 17. 115. D. fallax.

Hab. In ventriculo Uranoscopi scabri Junio et Julio Neapoli reperi.

116. Distoma laticolle $\mathrm{R}$.

D. depressum, corpore sublineari, collo dilatato, utrinque aculeato, capite subgloboso echinis cincto.

Mant. n. II6. D. Jaticolle.

Hab. In intestinis Carancis trachuri Arimini et

Neapoli, Aprili et Julio reperi.

117. Distoma Lima $\mathrm{R}$.

D, depressiusculum elongatum, parte corporis anteriore aculeata, poro antíco oblongo, ven. - trali 'orbiculari.

Ent. I. p. $427 \cdot$ n. 60. D. Lima.

Hab. In intestinis Vespertilionis auriti Mrütcen, Goeze, Aprili, Werget Octobri; V. muriuz ipse saepius vario tempore (Gryphiae) reperimus. In horum at $\mathrm{V}$. discoloris, lasiopteri, Pipistrelli et-Ferri equini, Cat. Ent. Vienn. mscpt.

118. Distoma exasperatum $\mathrm{R}$.

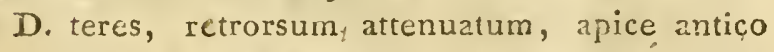
rotundato, postico-emarginato, undique aculeatum, utriusque pori apertura transversa.

Mant. n. II8. D. exasperatum.

Hab. in intestinis Soricis Eremitae. Mus, Vienu.

119. Distoma eristatum R.

D. teres, torulosum, aculeatum, cauda attenu. 


\section{$-118-$}

ata nuda, poro antico oblongo, ventrali subglobóso.

Mant. 1. irq. D. cristatum.

Hab. In ventriculo Stromatis Fiatolae Majo Arimini reperi.

120. Diroma hispidum AnILDGARD.

D. teres, collo et anteriore corporis parte spinosissimis, poris globosis, ventrali majort. Ent. 1. p. 535. 12. 7.1. D. sturionis, Mant, I20.

D hispidum.

Hab. In intestinis Accipenseris Sturionis Majo Ariunini, Berolini Junio reperi.

421. Distoma scabrum ZED.

D. teres, collo hispido, corpore laevi, poris globosis, antico minore echinis cincto.

Ent. I. p- 400. 11. 43. Mant. 1, 121. D, scabrum

Hab. In Gadi burbati ventriculo MüLter No'vembri, in intestinis G. Molvae ego Junio Neapoli, reperimus,

122. Distoma contortum $R$.

D. teres longissimun duriusculum retrorsum atteuuatum, collo spinuloso elongato subtus concavo, poris subglobosis ventrali pedunculato.

Mant. n. I22. D. contortum.

Hab. In branchiis Orthragorisci Molae Julio Neapoli reperi.

123. Distoma nigroflavum $\mathbf{R}$.

D. teres longisissimum molle, collo brevi sninuloso cylindrico, poris subglobosis, ven-

- trali pedunculato.

Ent. 2. p. 257. Schisturus paradoxus, Mant. 123. D. nigroflavum. 


\section{- 119}

Hab. In ventricnlo Orthragorisci Molae Neapoli Julio reperi.

$$
\text { Species dubiae. }
$$

124. Distoma Noctulae. $t$

Hah. in intestinis Vespertilionis Noctulae, diversum a D. Lima n. 117. Cat. Ent. Vienn!. 125. Distoma Musculi. †

Hab. in intestinis Mluris MIusculi. Cat, Ent. Vienn.

126. Distoma Chrysaëti. †

Ent. 1. p. 429. n. 61. D. chrysaëti.

Hab. in Falconis Chrysaëti cystide fellea, ArrubGAARD.

127. Distoma Buteonis. I

Ent. r. p. 430. n. 63. D. buteonis.

Hab. In intestinis tenuibus Falcouis Ruteonis Goeze Decembri reperit.

128. Distoma Falconis rufi. t

Hab, in intestipis Falconis rufi. Cat. Ent. Vienn. mscpt.

129. Distoma Aluconis intestinale. $\ddagger$

Hab. in intestinis Strigis Alneonis. Cat. Ent. Vienn. msept. an ad n. III. D. apiculatum.

130. Distoma Aluconis thoracicum. I

Ent. '. p. 385. n. 27. sub D. pusillo.

Hab. In Strigis Aluconis thorace Braun Aprili reperit at minus bene cum Erinacei D. , usillo n. 56. conjunxit, mammalium enim aviumque entozoa nunquam specie conveniunt.

131. Distoma Collurionis. $\mp$

Ent. x. p. 430. n. 64. D. Cullurionis. Hab. in intestinis Lanii Collurionis. Schrank. 
132. Distoma Meropis. +

Hab. in intestinis Meropis Apiastri, Cat. Ent. Vienn.

133. Distoma Turdi. +

Hab. in intestinis Turdi sascatilis, Cat, Ent. Vient. manuscript. an ad n. $5 \mathrm{~s}$.

134. Distoma Loxiae. $f$

Hab, in intestinis Loxiae Coccothraustis, Pyrrhulae, Chloriais, Cat. Ent, Vienu.

135. Distoma erraticum. †

Hab. in intestinis Fringillae Linariae, Motacillae albae, Pari caerilei, majoris, palustris, pendulini. Cat. Eut. Vienn.

136. Distoma Philomelae. I

Hab. in intestinis Motacillae Prílomelae. Cat. Ent. Vienn, mscpt.

137. Distoma Ardeae stellaris. +

Ent. I. p. 432. n. 68. D. Ardeae stellaris.

Hab. In intestinis Ardeae stellaris Goeze No-

vembri reperit, AdD, ferox n. 114. pertinere videtur.

138. Distoma Calidris. †

Hab, in intestinis Scolupacis Calidris. Cat. Ent. Vienn. mescpt.

139. Distoma Tringae helveticae. $t$

Hab. in intestinis Tringae helveticae, Cat. Ent. Vienn. mscpt.

140. Distoma Ralli. I

Hab. in intestinis Ralli aquatici. Cat. Ent. Vienn. mspt.

141. Distoma Anatis fuscae. †

Ent. x. p. 43I. 11. 05. D. Anatis fuscae. 


\section{$-121$}

Hab. in Anate fusca ab Abirgannio reper-

tum forsan ad D. echinatum n. In6. pertinet.

142. Distoma Anatis domesticáe. +

Ent. I. p. 431. 11. 66, D. Anatis domesticae.

Hab. Specimen unicum intestino Anatis do.

mesticae extus adhaerens (an bursa elapsum)

a Mü̈tzro repertum ad D, ovatum n. 2 . amandandum videtur.

143. Distoma Miergi, $t$

Hab. in intestinis Mergi Albclli. Cat. Ent.Vienn. 144. Distoma Testudinis. †

$\mathrm{Hab}$, in intestinis Testudinis orbicularis, Cat. Ent. Vienn.

145. Distoma Lacertae.

Hab. in intestinis Lacertae casrulescentis. Cat. Ent. Vienn.

146. Distoma Colubri murorum. †

Hab, in intestinis Colubri Murorim. C. E. $V$.

147. Distoma Colubri tesselati

Hab. in intestinis Colubri tesselati, C. E. V. mscpt,

148. Distoma Colubri americani $†$

Ent. I. p. 434. n. 72, D. col. americani.

Hab. In faucibus speciei Colubri americanae

Bosc copiosissime reperit.

149. Distoma Hylae. $f$

Hab. in vesica urinaria Hylae arboreae. C.E.V.

150. Distoma Cyclopteri. †

Ent. r. p. 4.38. n. 78. D. Cyclopteri.

Hab. In intestinis Cyclopteri Luinpi Farricius Aprili reperit.

151. Distoma Anarrhichae. $t$

Ent, 1. p. 435, n. 75.,D. Anarrhichae. . 


\section{$-122$}

Hab. In intestinis znarrhichae Inpi Rathke copiosius reperit, quam 1$)$. incisim. n, II. Secundun descriptionem pbiter factam ad D. appendiculatum n. 85. perinere videtur.

152. Distoma Wachiae. f

Mant. n. $15 \%$ D. Wachniae.

Hab. In intestinis Gadi Wachniae Truesues reperit, forsan ad D. scabrum n. I2I. pertinens.

153. Distoma Coryphaenae. *

Ent. I, P. 4:6. 11. 76. D. Coryphaenae.

Hab. in branchiis, intestinis et visceribus $C o$. ryphacnae Mippuidis, Bosc, Treesrus. Distomati clavalo 11.65 . nimis affine videlur.

154. Distoma Scorpaenae. †

Hab. in intestinis Scorpaenae Scrofae. C, E. $V$. 155. Distoma Spari. †

Hab. in intestinis Spari erythrini et Smaridis. Cat. Ent. Vienn.

156. Distoma Labri. †

Hab. in intestinis Labri rupestris. C. E. V.mscpt. 157. Distomc Triglae. t.

Hab. in intestinis Triglae Cuculi, C.E.V.mscipt. 158. Distoma Lucii.

Ent. I. p. 433. n. 79. D. Lucii,

Hab. In intestinis Esocis Lucii Januario Gry. phiae solitarium reperi: album, ellipticum, magnis poris instructum, vix lineam dimidiam longum, forsan novum.

159 'Distoma truncatum AbildgaArd. + Mant. n. I5\%. D. truncatum.

Hab. In 'ven'riculo Percae Laciopercae ArildGAakn reperit, an $D$, tereticollis $n .47$, spccimen mutilum, 
160. Distoma binode ZixD. * Ent. 1. 1. 439. n. 80. D. binode. Hab. in piscizm intestinis. Zoera. 161. Distona distichum ZeD. $t$ Ent. I. p. 4 40 . n. 8I. D. distichum. Hab. in piscinm intestinis. ZOEGA,

Genus XVI, Tristoma.

Corpus depressum. Pori dno antici simplices, teltius posticus radiatus. Inter illos os, probus. cidem? gmititens,

1. Tristoma coccineum Cuvier. Mant. n. I. Tr, coccineum.

Hab. in branchiis Orthragorisci MIolae, Xiphise Gladii, aliorumque maris mediterranci piscium. Cuvier.

2. Tristoma maculatum R.

Mant. 1. 2. Tr. maculatum.

Hab, Diodontis corpori adfixum Is MANTNIERE reperit,

\section{Genus XVII. Pentastoma.}

Corpus teretiusculum vel depressum. Os inter po. ros, utrinque binos, hamulum emittentes, lunatim positos.

1. Pentastoma taenioides $\boldsymbol{R}$.

P. depressum oblongum, postice attenuatum, transverse plicatum, margine crenatum.

Ent. 1. P. 44!, n. I. Polystoma. Mant. n, I.

Pentastoma tacnioides.

Hab. In sinubus frontalibus Canis vulgaris Gry- 
phize reperi. In illis Canir Lippi, Mus, Vienn. Equi, Chanzist, Mml, Greve.

2. Pentastoma denticulatum $\mathrm{r}$.

P. depressum oblongum, retrorsum decrescens, scriatim transverse denticulatum.

Hah. in superficie hepatis Caprae Hirci, AnILdgaARd; in Capra americalla, Florman.

3. Pentastoma emarginatum $\mathrm{R}$." †

P. depressum, oblongum, antice emarginatum, retrorsum decrescens, seriatim transverse denticulatum.

Tetragulus Çaviae Bosc. Mant. ñ. 3. P. emargi- natum.

Hab. in pulmonibus Çaviac Cobayae. Le Galıors 4. Pentasioma serratum R. †.

P. planum, subellipticum, seriatim transverse denticulatum.

Ent. 1. p. 449. n. 3. Polystoma serratum. Hab. In Leporis timidi pulmonibus Froelicr Decembri reperit.

5. Pentastoma proboscideum $\mathbf{R}$. $\ddagger$

P." teres subclavatum, utrinque obtusum, transverse rugosum, ore fisso protactili.

Porocephalus Crotali Humbolnt, Ent. r.p. 433. n. 69. Distoma Crotali Durissi. Mant. n. 5. P. proboscideum.

Hab. in Crotali Durissi pulmonibus et cavo abdominis, Alex ab Humboldt. 


\section{- 125}

Genus XVIII. Polystoma.

Corpus teretiusculum vel depressum. Pori sex an. tici, ventralis et posticus solitarii.

1. Polystoma integerrimum $R$.

P. depressum oblongtum postice obtusiusculum, poris sex anticis, uncinis duobus intermediis.

Ent. 1. p. 45 i. n. 4. P. integerrimum.

Hab. In vesica urinaria Rallae temporariae Zeder Septembri, ipse Junio Gryphiae reperimus. In illa R. esculentac Braun; $\mathcal{B}_{u}$ fonis variabilis, Mus. Vicnin.

2. Polystoma ocellatum R.

P. depressum ovatum apice postico obtusiusculo, poris sex anticis aggregatis inermibus. Mant, n, 2. D. ocellatum.

Hab. In faucibus Testudinis orbicularis Arimini Majo reperi.

3. Polyitoma Pinguicola ZED. +

P. depressum oblongum antice truncatum, pos. tice acuminatum, poris sex anticis lunatim. positis.

Ent. 1. p. 455 . n. 5. Mant. n. 3. P. Pinguicola. Hab.In tuberculo Ovarii humani Treuteer reperit. 4. Polystoma duplicatum R. †

P. depressum oblongum antice rotundatum, cor. pore utrinque attenuato, poris sex biforibus anticis lunatim positis, papillis duabus inter. mediis.

Mant. n. 4. P. duplicatum.

Hab. in branchiis Scombri Thynni, Defarocus. 
Species dubia.

5. Polystoma venarum ZED. +

P. depressum, lanceolatum, poris anticis sex.

Ent. I. p. 456 . n. 6. P. venarum.

Hab. In vena tibiali antica Flominis in fluvio lavantis disrupta Treutrrir specimina duo reperit. Pori icone non exprinuntur et vermes nil nisi Planariae fuisse videntur. 


\section{Ordo IT.}

- C e s t o i d e a.

Corpus elongatum depressum 'molle continuum. vel articulatum. Caput paucissimorum simpliciter labiatum, reliquorum bothriis vel osculis suctoriis duobus aut quatuor instructum. Omnia individua androgyna.

Genus XIX. Caryophyllaeus.

Corpus depressum continume. Caput dilatatnn fimbriatum, bilabiatnm, labio superiore et inferiore.

\section{Caryophyllaeus mutabilis $\mathrm{In}$.}

Ent. 2. p. 9. Mant. n, I. Caryophyllaeus mutabilis.

Hab. in intestinis Cyprinorum, vulgaris. Ipse in C. Blicca, Brama, Carassio et Gibelione reperi. In C. Carpione, Jese, Tinca, Brocix observavit. Catalngus Eintoznornm Ríusei Vienneusis manuscriptus pratterea sequentes enumerat Cyprinos. Caryopliyllacum foventes, Alburnum, amarum; Barbum, erythrophthal- 


\section{- 128}

- mum, Gobionem, Leuciscum, Nasum, Phoxinum, rutilum; enumerat etiam Cobitidem Ziar. batulam et Taeniam.

\section{Genus XX. Scolex.}

Corpus depressum continuum. Caput bothriis quatuor instructum.

1. Scolex polymorphus $\mathbf{R}$.

Ent. 2. p. 3. n. I. Scolex quadrilobus. p. 7. n. 4. Scolex Lophii, p. 8. n. 5. Sc. Cyclopteri. n. 6. Sc. Percae. Mant, n. I. Sculex polymorphus.

Hab. in intestinis Piscium et Cephalopterorum, passim in illorum abdomine. Ip e reperi in Torpcdine marmorata; Squalo Acanthio; Lo. phio piscatorio; Ophidio barbato; Stromate Fiatola; Gado Merlnccio; Lepadogustro Gouani; Gobionigro, minuto; Cotto Gobio; Scorpaena Porco; Pleuronecte maximo; Sparo Boope; Sciandra; Labro lusco; Apogone (Mullo imberbi Linn.); Clupea Encrasicolo; Polypo vulgari (Sepia Octopodia Lrsx.)

O. Fr. Mulicer in Lophio piscatorio, Pleuro. neete Rhombo, Platcssa et Linguatula, inque Percis, quarum species now dixit; Отто FABricius in Cycloptero Lumpo et Pleuronecte Solea invenernint.

Catalogus Viennensis Manuscriptus sequentes enumerat pisces Scolecem foventes, in quibus ipse non observavi: Rajam Mirale. tum, Pastinacam; Syngnathm Acum; Uranoscopum scabrum; Blenuium ocellarem; Ciepolam rubessentem; Gobium Jozo; Zenem Fabrum; Esocem Belonem. 
Scolex bilobus sive Lavareti Ent. 2. p. 5. n. 2. et tetrastomus s, Eperlani ib. p 6 n. 3 . Bothriocephali tenelli et Taeniolae sunt, de quibus nec non de consinili Taeniola Scolecem mentiente, a me in Clupeae nova specie re. perta, dubii generis Entozoa consulantur.

Geñus XXI. Gymnorhynchus.

Corpus depressum contintum, longissimum, colli receptaculo subgloboso. Caput bothriis duobus bipartitis instructum, proboscides quatuor nudas retrastiles emittens.

1. Gýmnorhynchus reptans $\mathrm{R}$. Scolex Gigas, Cirvrsz. Mant. n. I. G. reptáns, Hab. Inter omnes Bramae (Spari) Rnji, carnes Junio et Julio Neapoli reperi, neque ullum hujusmodi piscem Gymnorhynchis liberum vidi,

Genus XXII. Tetrarhynchus.

Corpus depressum continuum. Caput bothriis duo. bus bipartitis instructum, proboscides quabuor unccinatas retractiles emittens.

1. Tetrarhynchus megacephalus $\mathbf{R}_{\text {。 }}$

T. capitis conici bothriis subovatis profundis marginatis, corpore depresso, retrorsum decrescente, apice truncato papillato.

Mant. n. I. T. megacephalus.

Hab. In Squali stellaris abdomine-parieti dor sali adhaerentem Julio Neapoli reperi.

2. Tetrarhynchus grossus $\mathrm{R}$.

'T. capitls ovalis discreti bothriis oblongis pro. 


\section{- $130-$}

fundis marginatis, corpore depresso recto apice papillari.

Mant, n. 2. T. grossus.

Hab. in pisce maris Iaponici an Squalo? Tile. sius misit, locum qua invenerit, oblitus.

3. Tetrarhynchus attenuatus $R$.

T. capitis obtusi bothriis obconicis, corpore longissimo tenui, postice acutiusculo.

Nant. n. 3. T. attenuatus.

Hab. In branchiis Xiphiae Gladii Fiscuer reperit.

4. Tetrarhynchus discophorus R.

T. capitis rotundati bothriis profundis prbicularibus, corpore abbreviato depresso obtuso. Mant. n. 4. T. discophorus.

Hab. In branchiis interque ventriculi tunicas Bramae (Spari) Raji Junio Neapoli repéri.

5. Tetrarhynchus tenuicollis $\mathbf{R}$.

T. capitis subcordati bothriis bilobis, collo tereti postice attenuato, corpore ovato.

Mant. n. 5. T. tenuicollis.

Hab. It: ventriculo Pleuronectis Pegosae Arimini, Romae in peritơneo Lophii piscatoriz, Majo reperi.

6. Tetrarhynchus megabothrius $\mathbf{R}$.

T. capitis corpore depresso postice papillato majoris bothriis latissimis costatis bilóbis, ex. stantibus.

Ent. 2. p. 284. n. 39. Vermis dubius Sepiae officinalis. p. 285. n. 40. Vermis dubius. Se. piae Loliginis. Fasciola barbata Linv, Mant. n. 6. T. megabothrius.

Hab. Ipse inter ventriculi tunicas Scombri Sardae, (Neapoli Junio) et Sepiae officina- 
lis (Arimini Aprili) reperi. In Loligine, MARtix; nam Fasciola barbata Linsaei huc uti. que pertinere videtur.

7. Tetrarhynchus macrobothrius $R_{*}$

T. caritis corpore teretiusculo majoris bothriis longissimis costatis appressis, appendice pos. tica biloba.

Ent. I. p. 320. n. 2, T. papillosus. Mant. n. 7. T. macrobothrius.

Hab. Lnter Testudinis Mydae tunicas ventriculi Berolini Octobri reperi: specimina simillima Olfers ex Brasilia misit, forsan oryphaenae Hiphpuridis, cujus càput simul dedit. In dicti picis plurimis partibus \$osc. Tulesrụs inter Scombri Pelanidis musculos invenit, qua de re Mantíssa conferatur.

8. Tetrarhynchus appendiculatus $\mathrm{R}$.

T. capitis corpore depresso postice appendicu. lato brevioris hothrive cblongis angustis.

Ent. 1, p. 3is, n, 1. Mant. n. 8. T. appendi. culatus.

Hab, in Salmonis Salaris hepàte WAGLERo, mus. culis ( Kajo) Goezro visus, cujus specimina in Mhuseo Viennensi conservata examinavi.

9. Tetrarhynchus scolecinus $\mathrm{R}$.

"T. scapite exiguo subrctundo, bothriis lato-ellip. ticis auriculato, corpore depressiuscula con stric to obtusu.

Mant. ĥ. g. T. scólecinus?.

Hab. In carnibus Squali, stellaris innumeross. 5: in illis Squali Centrinae duos, Julio; in pinna ain Rajae oxyrhynçhoe, pectorali solitarium:Au. gusto, Neapoli reperi. 


\section{- $13^{2}-$}

10. Tetrarhynchus gracilis $\mathbf{R}$.

T. capite exiguo bothriis lato-ellipticis auriculato, corpore depressiusculo lineari subcon. stricto, postice acutiusculo.

Mant. n. 10. T. gracilis.

Hab. In novem Ammodytum Cicerelortum intes. tinis Neapoli Julio reperi.

$$
\text { species-dubiat. }
$$

11. Tetrarhynchus Squali. $t$

Hepatoxylon Bosc. Mant. n. It. T. squali. Hab, in Squali hepate, La Martiniere; ventri. culo, Latitllardiere.

12. Tetrarhynchus Pleuronectis maximi. + Mant. n. I2. T. Pleuronectis maximi.

$\mathrm{Hab}$, in carne linguae Pleur. maximi aliorum. gue piscium, Cuvder.

\section{Genus XXIII. Ligula.}

x. Statu ante evolutionem: Corpus depressum, continuum, longissinum, sulcollongitudinali medio exaratum. Neque capite neque genita. libus conspicuis.

2. Statu evoluto: Corpns depressum continuum longissimum. Caput bothrio utringue simplicissimo. Ovaria serie simplici aut duplici cum bemniscis in linea mediana.

$$
\text { A. Ovaris, distinctis. }
$$

1. Ligula uniserialis $\mathbf{R}$.

I. parte autica rugosa crassiuscula, corpore reliquo retrórsumı attenuato, ovariorum serie solitaria regulari. 


\section{$-153-$}

Ent. 2. p. 12. Mant. n. 1. L. uniserialis.

Hab, in intectinis Falconis fulvi, Braus, ala bicillae, Mfus. Vienu.

2. Tirula aliemans $\mathrm{R}$.

L. parte antica rugosa crassiuscula, reliqua re. trorsum attenuata, ovariorum serie duplici alieruante.

Iist. 2. p. 3. n. 2. Mant. n. 2. I. alternans, Hab. In intestinis Lari tridac'yli, HürNer. Lari parasitici, ridibundi, Mus, Vienn, In Laro cano nondum evoluta, ibidem.

3. Ligula interrupta $R$.

L. antice crassiuscula, postice attenuata, utrinque laevis et obtusiuscula, ovariis oppositis interruptis.

Ent. 2. P. I5. n. 3. I. interrupta. p.25. n. II. L. Mergorum. Mant. n, 3. L- interrupta.

Hab, in intestinis Colymbi auriti, Bцосн, HüвNER Mergi serratoris, Albelli, Mus. Vienn. In M. Mergansere, Nitzsch, nondum evoluta. An huc $L$. Pclecani Carbonis et pygmaei, illa capite instructa, Mlus. Vielun.

4. Ligula sparsa $\mathbf{R}$.

L. parte antica compressa, crassiuscula, corpore depresso subacquali, laevi, caudae apice te. nuissimo, ovariorum serie irregulari, solitaria vel alternante.

Ent. 2. p. 16. n. 4. L. sparsa. p. 26. n. 12. I. Colymbi cristati, p. 27. n. 13. L. Colymbi Immeris. Mant. n. 4. I, sṕarsa.

Hab. in intestinis Arcieac Ciconiae, Hradebrandx; Ardeac Egrettac, Nycticoracis, Totani chloropodis, Sternae Ilirundinis, nigrae, Colymbi 
septentrionalis, arctici, cristati, subcristati, Anatir Boschadis ferae. Mus. Viennu.

\section{Ligula nodosa R. +}

L. linearis, linea totius corporis punctis exarata, appendicis caudalis apice nodoso.

Ent. 2. p. 17. n. 5. Mant. n. 5. L. nodosa.

Hab. in abdorninis cavo Salmonis Truttae,

Schrank。

\section{B. Ovariis latentibus+}

6. Ligula simplicissima $\mathbf{R}$.

Ligula corpore depresso, linea media longitudinali impressa.

Ent, 2. p. 18. n. 6. Ligula contortrix. p. 20. n. 7. L. cingulum, p. 2 I. n. 8. L. constringens. p. 24. n. 9. L. acuminata. p. 28. 11. 14. L. Cobitidis, n. 15. L. salvelini, p. 29." n. 16. S. Wartmanni. n. 17. Cyprini Carpionis. p. 30. 11. 18. C. Tincae. n. 19. C. Gobionis. p. 3I. n. 20. L. Alburni. n. 21, L. Leucisci. Mant. n. 6, Ligula simplicissima.

Hab. in abdomine piscium fluviatilium, potissimum Cyprinorim: Branae, Cárassiae, erythrophthalmi, Alburni; Siluri Glanidis; Esocis Lucii: Percac fluviatilis. Luciopercac. Mus. Vienn. Ipse in Cyprinis Gobione, Gibelione, Brama, et nova specie italica, Barbo affini, vidi. In C. Blicca, Goeze; Leucisco, Pallas; rutilo, hürner. In Petrontyzonte branchiali, Schrank. In Cobitide Saenia. Fruscir, Bruch. In Salmone Salvelino, Schraxi ; ITartmanni, Froesich.

7. Ligula crispa $\mathbf{R}$. Mant, n. ?. L. crispa. 


\section{$-135$}

Hab. In intestino tenui Phocae vitulinae junioris ab Amanuensi meo Berolini Junio . reperta, an piscina, ad praecedentern amandanda?

Entozoa Ligulis simillima vide inter illa dubii generis n, 8, 9, I9 et 20 .

Genus XXIV. Triaenophorus.

Corpus elongatum atcpressum subarticulatum. Os bulabiatun, utrinque aculeis binis tricuspida. tis armatumn.

1. Triaenophorus nodulosus $\mathbf{R}$.

Ent. 2. p. 32. Tricuspidaria nodulosa. p. 237. n. II. Cysticercus Gadi Lotae. n. 12. Cyst. Percae. p. 24r. n. I5. Gystic, Lucii. Mant. I. Triaenophorus nodulosus.

Hab. In intestinis Percae fluviatilis et Esocis Lucii (saepissime varioque anni tempore) Gasterostei aculeati (Berol. Januario), Syne gnathi Hippocampi (Arimini Majo) liberum; cystide inclusum autem in hepate et mesen. terio Percae fluviatilis, Gasterostei aculeati et Esocis Lucii reperi. In hepate Cotcti Go. bii, Mus. Vienn. In hepate vel appendici. bus Salmonis Farionis, Huchonis, Thymalli, Truttae, C. E. V. In Esoce Bolune, O. Fr. MLÜLLER. 


\section{$=136$ \\ Genus XXV. Bothriocephalus.}

Corpus elongatum, depressum, articulatum. Caput subtetragonum, לothriis duobus sel quatuor oppositis.

A. Inermes (Gymobothrii).

a. 'Dibothrii.

1. Bothriocephalus latus Brrms.

B. capite bo:hriisque marginalibus oblongis, collo subnullo, articulis anticis rugaeformibus, insequentibus plurimis subquadratis, ultimis longiusculis,

Ent. 2. p. 70. n. I. Taeniza lata Lrmatai.

Mant, n. I. Bothr. latus.

Hab. in Hominis intestinis. Helvetiae et Russiae indigena; in Gallia passim; in Germania, Hqllandia, Anglia rarissime et fere in advenisy tantum occlirrit. In cadavere, quod sciam, nunquam repertus est.

2. Bothiocephalus plicatus $\mathbb{R}$.

B. capite compresso sagittato, bothriis lateralibus oblongis, collo nullo, articulis anticis et posticis angustissimis, mediis latissimis et brevissimis.

Ent. I, p. 308, n. 46. Echinorh. Xiphize. Mant, n. 2. Bothr. plicatus.

Hab. In intestino recto Xiphiae Gladii Bero. lini offendi. Ibidem repertum Spedalierr $T$ icini dedit.

3. Bothriocephalus claviceps $\mathrm{R}$.

B. capite oblongo, bothriis marginalibus, collo nullo, articulis anterioribus brevissimis, mediis oblongis, reliquis subquadratis, margine postico tumido. 


\section{$-137-$}

Ent, 2, p. 37, n. I, Mant. n. I. B. claviceps.

Hab. In Muraenae Anguillae intestino versus pylorum Majo et Novembri Gryphize copiose reperi. Fragmenta huc pertinere visa in intestinis Muraenae Cassini Neapoli Julio seperi,

4. Bothriocephalus proboscideus $\mathbf{R}$.

B. capite bothriisque marginalibus oblongis, collo isullo, corpore depresso medio sulcato, articulis brevissinis, antrorsum attenuatis.

Ent 2. p. 39. n. 2. Mant. 1. 4. B. proboscideus. Hab. In Salmonis Salaris appendicibus pyloricis primo vere Gryphiae saepe frequentissime reperi. In illis Salnonis Fluchonis, Mus. Vienu. Palias.

5. Bothriocephalus infundibuliformis $R$.

B. capite bothriisque iateralibus oblongis, collo nullo, articulis variis, primis rugaeformibis, sequentibus subinfundibuliformibus, reliquis brevioribus.

Ent. 2, p. 46. n. 5. Mant. n, 5. 13. infundibuliformis.

Hab. in intestinis Salmonis Salvelini et alpini. Mus, Vienn.

6. Bothriocephalus rugosus $\pi$.

B. capite subsagittato, bothriis lateralibus ob. longis, collo nullo, corpore depresso medio sulcato, articulis brevissimis inaequalibus.

Ent. 2. p. 42. n. 3. B. rugosus.

Hab. in appendicibus pyloricis Gadi Lotae et Mustelae, in hac Decembri a IVAGLiro et Goezro, in illa Septembri et Decembri Gryphiae a me repertus. 
7. Bothriocephalus microcephalus $R$.

B. capite sagittato, hothriis lateralibus oblongis, collo mullo, ariculis primis rugaeformibus, tum subqualratis, insequentibus brevissimis plurimis, reliquis angustatis.

Ent. 2. p. 213. n, 115. Taenia Tetrodontis Molae.

Mant. n. 7. B. microcephalus.

Hab. In Orthragorisci Molae branchiis, ventriculo et intestino summo Neapoli Julio reperi.

8. Bothriocephalus fragilis $R$.

B. capite cuneiformi, bothriis lateralibus orbi"cularibus, collo tereti articulisque brevissimis, corpore depresso, medio sulcato.

Ent. 2. p. 45. n. 4. B. fragilis.

Hab. In appendicibus pyloricis Clupeae Alosae Gryphiae Majo et Junio reperi.

9. Bothriocephalus granularis $\mathbf{R}$.

B. capite cuneiformi, bothriis marginalibus, collo teretiusculo, articulis crassiusculis, subglobosis.

Ent. 2. p. 48. n. 6. Mant. n. 9. B. granularis.

Hab. in intestinis Cyprini Phoxini, Mus.Vieni?.

10. Bothriocephalus rectangulum $\mathrm{R}$.

B. capite sagittato, bothriis lateralibus profundis, articulis anticis brevissimis, insequentibus sensim majoribus, tandem subquadratis. Ent. 2. p. 49. n. 7. Mant, n. 10. B. rectanguluin.

Ulab, in intestinis Cyprini Barbi, Bцосн, Schrank, Zeder, Mins. Vienth.

11. Bothriocephalus punctatus $R$.

B. capite bothriisque marginalibus oblongis, 
collo nullo, articulis corporis plani anterioribus elongatis, reliquis subquadratis.

Ent. 2. p. 50. 11. 8. Mant. n. 11. B. punctatus. Hah. In ventriculo Torpedinis ocellatae (ex

Pleuronecte semidigesto residuum) Neapoli Junio, in intestinis Gadi minuti (ib. eod.); Cotti scorpii (Gryphiae, vere); Pleuronectis maximi (ibiderr, saepissime, vario anni tein; pore; Arimisi, Aprili), Pl. Rhomb̈i (Neapoli Julio), Pl. Boscii (ib. eod), Pl. Pegusae (Arimini, Aprili) reperi. In Pl.Solea, Mus. Vienn. Trigla adriatica C. E. $Y$.

12. Bothriocephalus angustatus $\mathrm{R}$.

A. capite longissimo, cum bothriis lateralibus et articulis anterioribus elongatis, angustissimis, horum reliquis brevioribus, tandem sub. yuadratis.

Mant. n. I2. B. angustatus.

Hab. in intestinis Scorpaenae Scrofae. Mlus. Vienn.

13. Bothriocephalus crassiceps $R$.

B. capite magno subgloboso, bothriis marginalibus profundis, corpore serrato, articulorum inaequalium margine postico ntrinque exstante.

Nant. n. 13, I. crassiceps.

Hab. In intestino duodeno Gadi Merluccii Nea. poli Julio reperi.

\section{Bothriocephalus solidus IR.}

B. capite triangulari depresso, bothriis lateralibus costa dirisis, corpore planiusculo obo. vatolanceolato, linea media impressa utrinque sulcato.

Ent, 2. p. 57, n, 10, Mant, 11. I4. B, solidus, 


\section{$-140$}

Hab. In abriomine Gasterostei aculeati Gryphize et Berolini saepissime, praesertim Junio reperi. Crasterosteis comestis superstes invenitur variis in animalibus; in Cotto Scorpio, ZOEGA; ipse in Salmonis Salaris ventriculo (Majo) et Phocae vitulinae recto, Gryphize, ofiendimus.

15. Bothriocephalus nodosus F.

B. capite triangulari depresso, bothriis laterali. bus costa divisis, corpore plano obovato-lan. ceolato, ovariis nodiosis.

Ent. 2. p. 54. n. 9. B. nodosus.

Hab. In intestinis Colymbi cristati Julio, C. septentrionalis et Sternae Hirundinis Au. gusto, Gryphiat reperi. In illis Ardeae cinereae repertum Braun dedit, In Mergo Al. bello et Mergansere Buocn; $M$. serrato, $C_{0}$. lymbis Immere et Troile, AbiLdgand.

\section{Tetrabothrii.}

16. Bothriocephalus macrocephalus $R$.

B. capite subtetragono magno, antice truncato, bothriis lateralibus subquadrangulis utrinque binis, collo brevissimo, articulis depressis, anticis angustissimis, reliquis campanulatls. Ent, 2. p, 61, n, 11, Mant, n, 16. B. macroce. phalus.

Hab. In Colymbi septentriontalis oesophago, ventriculo, et intestinis summa copia Gryphiae Augusto reperi. In intestinis $\mathrm{Col}$. Immeris. Amilngando; Col. aretici, C, E. V.

17. Lothriocephalus cylindraceus $R$.

13. capite subtetragono exigıso, antice truncato, bothruis lateralibus utrinque binis, collo lon. 
giusculo, articulis depressiusculis, primis bre vissinis, tum brevibus, dein subquadratis, tandem campanulatis.

Mant. n. 17. B. cylindraceus. Hab in intestinis Lari glauci et Atricillas, Mlus. Vienu.

18. Bothriocephalus auriculatus $R$.

B. borhinis utrinque duobus patentissimis, collo breviusculo, articulis primis bacillaribus, in. sequentibus plurimis subquadratis, ultmis elongatis.

Mant.'n. 18. A. auriculatus.

Hab. In intestino crasso Torpedinis marmoratas Arimini Aprili, Julio Neapoli, Squali Ga. lei (?) Romat Majo reperi. Ex iisdem, MIus, Vicin.

19. Bothriocephalus tumidulus $\mathbf{R}$.

B. bothriis utrinque duobus ovalibus crassius. culis linea divisis, transversim striatis, collo brevissimo, articulis primis angustissimis elon. gatis, insequentibus subquadratis.

Mant. 1. 19. B. tumidulus.

Hab. in intestinis Rajue Pastinacae, Mus, Dienn.

B. Armati (omnes tetrabothrii). a. Uncinati, Onchobothrii.

20. Bothriocephalus coronatus $\mathbf{R}$.

B. borhriorum singulorum uncinis anticis dichotomis, cullo longiusculo, articulis primis rugatiormibus,' inscquentibus subquadratis va. riis, ultimis tlongatis.

Ent. 2. p. 213. 12. 116. Taenia Rajae Batis.

Mant. "n. 20. 13. coronatus.

Hab. In intestinis crassis. Torpedinis marmora- 
tae (Arimini Aprili, Neapoli Julio), T. ocellatae (ib. Junio) et Squali stellaris (Arim. Majo, Julio Ncapoli) reperi. In illis Rajae Pastinacae, Squali Squatinae, Mus. Vienu. Rajae Batis, Braun.

21. Bothriocephalus uncinatus $R$.

B. bothriorum singulorum uncinis utrinque anticis bifidis, collo brevissimo, artićulis primum rugaeformibus, insequentibus subquadratis variis.

Mant. n. 2I. B. uncinatus.

Hab. In intestinis crassis Squali Galei Arimini Majo reperi.

22. Bothriocephalus verticillatus $R$.

B. bothriis utrinque binis antice uncinatis, collo nullo, articulorum margine postico fimbriato.

Mant, n. 22: B. verticillatus.

Hab, In intestinis crassis Squali Galei Arimini Majo reperi.

b. Proboscidei, Rhynchobothrito

23. Bothriocephalus corollatus $\mathbf{R}$.

B. capite depresso, proboscidibus uncinatis bothriisque utrinque duobus, collo brevis. sino, articulis oblongis, foraminibus marginalib́us alternis.

Ent. 2. pit63. n. 12. Mant. n. 23. B. corollatus. Hab. In, intestinis, crassis Rajae Latis Parisis, in ventriculo Rajae Rubi Arimini, in crassis Squali? Galei, Romae, Majo reperi, In illis Squali Spinacis, A BILDGaArd; Squatinae, Cat; Ent. Vienn.

24. Bribricephalus paleaceus R. † B. capite depresso,- proboscidibus (uncinatis) 


\section{- $143-$}

bothriisque utrinque duobus, collo brevissimo, articulis oblongis, foraminibus marginalibus unilateralibus.

Ent. 2. p. 65. n. 13. B. paleaceus.

Hab. In intestinis crassis Squali scanthiae FA. BRicius reperit.

Obs. Ob foramina unilateralia dicta hunc a praecedenté diṣtinxi, cun qua ceterum nimis congruit et forsan conjungendus érit.

$$
\text { Species dubiae. }
$$

25. Bothriocephalus Squali glanci. +

Hab. in interinis squali glauci. C.E $V: m s c p t$. 26. Brithriocephalus Lophii. †

Hab. in intestinis Lophii piscatorii. Nova spe. cies. C E. $V$.

27. Bothriocephalus Gadi Redianus. I

Ent. 2, p. $67^{\circ}$ n. 14. B. Gadi Merluccii.

Hab. in intestinis Gadi, Redi. Gadum illum Merluccium tuisse olim suspicatus sum. sed quos ipse in Merluccio reperi, Bothriocephali.Redianis saltem multo minores erant. Redianus vermis forsan ad. B. punctatum n. II. pertinet.

28. Bothriocephalus Gadi barbati. †

Ent. 2. p. 68. n. 15. Ab Othone Fabuicto in intestinis Gadi barbati repertus et jure forsan ad B. punctatum 11. I1. relatus, quem ipse saltem in Gado minuto reperi.

29. Bothrincephalus-Gadi Mrhuae. t 30. Bothrocephalus Gudi Callariae. †

Ent. 2. p. bu. n. 16. et 17. Utraque species ab ill. Pallas ad B, proboscidelum relata, de $\therefore$ quo dubitare licet. 
5x. Bothriocephalus Cepolae. $†$

IJab, in intestinis Cepolae rubescentis, Nova species. Cat. Ent, Vienn.

32. Bothriocephalus Barbatulae. †

Irab. in intestinis Cobitidis Barbatulae. Nova species, $C, E . V$.

35. Eothriocephalus Erincis. $\downarrow$

Ent, 2. p. 69, n. i 8 . Ab ill. Paxtas ad B.pro. boscideum olim relatus.

34. Bothriocephalus Carpionis. $†$

Ent. 2. p. 69. n. I9. Ab Otrone Fabricio, qui in Salmone Carpione reperit, dubie ad Bo. thriocephalum punctatum refertur.

Obs. Num Taenia anthocephala (Phocarum) 11. S. et Taenia octolobata (Percae norve. gicae) 1. 80. Bothriucephalis addendae sunt?

\section{Genus XXVI. Taenia.}

Corpus elongatum, depressum, articulatum, Osculle capitis quatuor suctoria.

$$
\text { A. I ner mes, }
$$

a. Capite simplici s. non rostellatos

1. Taenia expansa $\mathrm{R}_{\text {. }}$.

T. capite obtuco, collo nullo, articulis anterioribus brevissimis, reliquis subquạdratis, foraminibus marginalibus oppositis.

Ent. 2. p. 7. . .." 2. T. expansa. p. 200 . n. 83.

T. Caprooli. ant. ก. t. T expansa.

Hab. in intestinis tenuibus Ovis Arietis, prae.

sertum Aguorum, vulgatissima. In illis Anti- 


\section{- $145-$}

lopes Rupicaprae et Dorcadis, Mus. Vienn, Cervi Capreoli, Nrrzscri.

2. Taenia denticulata $R$.

T. capite tetragono, collo nullo, articulis brevissimis, foraminibus marginalibus oppositis, lemniscis dentiformibus.

Ent. 2. p. 79. n. 3. T. deniticulata.

Hab. in intestinis Bovis Tauri, Careper, Cha. bert, Havemann.

3. Taenia pectinata, Goeze.

T. capite obtuso, collo articulisque brevissimis, foraminibus marginalibus oppositis papillosis.

Ent, 2. p. 82. n. 4. Mant. n. 3: T. pectinata.'

$\mathrm{Hab}$. in intestinis tenuibus Leporis timidi, $\mathrm{Cu}_{\mathrm{u}}$ niculi, Arctomydis Marmotae.

4. Taenia lanceolata Goeze.

T. capite ovali, collo articulisque brevissimis, posticorum angulis obtusis, lemriscis margi. nalibus oppositis.

Ent. 2. p. 84. n. 5. Mant. n. 4. T. lanceolata. Hab. In intestinis Anatis Anseris frequentissimá, in illis, Anatis ferinae et Nyracae, Cat. Ent. Vienn. Reperi quoque in illis Colymbt subcristati, Aprili, Berolini,

5. Taenia plicata $\mathbf{R}$.

T. capite tetragono, corpori utrinque incum. bente, collo articulisque brevissimis; horum angulis lateralibus acutis.

Ent: 2. p. 87. n. 6. Mant. n. 5. T. plicata Hab. in intestinis tenuibus Equi Caballi, inse. quente rarior.

6. Taenia perfoliata GoEzE.

T. capite tetragono, postice utrinque bilobo, collo nullo, articulis perfoliatis. 
Iint. 2. p. 89- n. 7. T. perfoliata.

Hab. in coeco et colo Equi Caballi, vulgaris.

7. Taenia festiva $R$.

T. capite tetragono antrorsum incrassato, collo nullo, articulis anterioribus rugaeformibus, reliquis bacillaribus.

Mant. n. 7. T. festiva.

Hab. in ductibus hepaticis et vesica fellea Malmaturi gigantei. Minus. Vicnu.

8. Taenia anthocephala. R. if

T. capite subtetragono, lobis angularibus antrorsum eminentibus aucto, collo articulisque - brevissimis.

Ent. 2. p. 91. n, 8. T. anthocephala.

Hab, in intestino recto Phocae barbatae, O.FABricius. (Num species ista ad BothriocephaIos pertinet?)

9. Taenia omphalodes, Hermans.

T. capite subtetragono, postice contracto, collo nullo, articulis anticis brevissimis, reliquis subcuneatis, angulis acutis.

Ent. 2. p. 92. 9. Mant n. 9. T. omphalodes.

Hab. in intestinis Muris arvalis, HERMANN. In illo et MI. amphibio, Mrus. Vienn.

10. Taenia perlata, Goeze.

T. capite tetragono, collo longiusculo, articulis subcuneatis, posticis medio nodosis.

Ent. 2. 1. 95. 11. 12. T. perlata.

Hab. in intestinis tenuibus Falconis Buteonis. GoEze, Num ad Taeniam globiferam.n. 53. pertinet?

11. Taenia crenata Goeze.

T. capite hemisphaerico, antice nodulo aucte, collo longissimo, articulis transversis obtusis. 
Ent. 2. p. 97. n. 13. Mant. n. I I. T. crenata.

Hab. in intestinis Picorum. Conf. n. 91. Tae. niam crateriformem, ad quam crenata forsan amandanda erit.

32. Taenia nąsuta $\mathbf{R}$.

T. capite subliemisphaerico apiculato, collo brevi, articulis anterioribus brevissimis, reli. quis subinfundibuliformibus, variis. $\quad \therefore ' s$.

Ent. 2. p. 98. n. 14. T. nasuta.

Hab. In intestinis Pari majoris Zener Martio, ipse Octobri (Gryphiae) reperimus. In illis Pari caerulei, atri, palustris. C. E. Y. P crise tati. Cat. Viennz. mscpt.

13. Taenia tripunctatá. Brauñ.

T. capite obconico, collo brevi, articulis anterioribus capillaribus, reliquis latioribus ob. túsis.

Ent. 2. p. 99. n. I5. T. tripunctäta.

Hab. In intestinis Erinacei etirdpaed passib *." occurrit.

14. Taenia cucumeriûna. Btocri.

T. capite antrorsum attenuato obtuso, collo brevi continuo, articulorum ellipticorlin forznuinibus marginalibus oppositis.

Ent. 2. p. roo. n. I6. T. cucumerina."

Hab. in intestinis teriuibus Canis fámiliaris, vul: gatissima.

15. Taenia opuntioides $R$.

I. (capite colloque mihi non visis) articulis anterioribus subinfundibuliformibus, reliquis elliptico-ovatis, foraminibus marginalibus alternis.

Mant. n. I5. T. opuntioides. 


\section{$-148$}

Hab. In intestinis tenuibus Canis Lapi Berolini Januario reperi.

16. Taenia litteratá BAtsch.

T. capite obconico, truncato, osculis oblongis, collo brevi, articulorum subellipticorum foraminibús marginalibus alternis.

Ent. 2. p. 103. n. 17. T. litterata.

$\mathrm{Hab}$, in intestinis tenuibus Canis Tulpis, vulgaris.

17. Taenia dendritica, Goeze.

T. capite globoso, osculis hemisphaericis, collo longiusculo plano, articulorum oblongorum foraminibus marginalibus alternis.

Ent. 2. p. 104. n. 18. Mant. n. 17. T. dendritica.

Hab. in intestinis Sciuri vulgaris Nitzscir Decembri, Goeze Januario, Treutler Martio, ZEDER vere, invenerunt.

8. Taenia difformis $R$.

T. capite subgloboso, collo nullo, articulis anterioribus difformibus, insequentibus brevi. bus aequalibus, reliquis angustatis.

Mant. n. 18. T. difformis.

Hab, In intestinis tenuibus Cuculi canori Be. rolini Majo reperi.

19. Taenia angustata $\mathrm{R}$.

T. capite obcordato, collo elongato, articulis anterioribus brevissimis, insequentibus sub. quadratis, reliquis oblongis inaequalibus.

Mant. ni. 19. T. angustata.

Hab. in intestinis Melis Taxi, Mus. Vienn.

20. Taenia filicollis $\mathbf{R}$.

T. capite subgloboso discreto, collo longissimo. fuliformi, articulis ovariisque quadratis. 
Ent. 2. p. 106. n. 19. T. filicollis. Hab. In intestinis Gasterostei aculeati GoezD Augusto, ipse Junio (Gryphiae) iṇvenimus.

21. Taenia longicollis $\mathbf{R}$.

T. capite truncato, collo longissimo, articulis subquadratis, ovariis racemosis.

Ent. 2. p, 6. n. 3. Scolex tetrastomus. p. 107. n. 20. Taenia longicollis. p. 240 . n. 14. Cys. ticercus Salmonum. Mant. n. 2I. T. longicollis.

Hab. in intestinis, rarius in hepate Salmonum. Ipse in illis S. Lavareti Majo; Gryphiae; S. Maraenularum plurimarum Februario Berolini, ibidem variis annis Januario in Eperla. nis plurinis. In multis Salmonibus Wartmami $F_{\text {roelich }}$ Augusto; idem et $Z_{\text {EDer }}$ in S. Trutta. In S. Thymallo, Muss. Vienn. In hepate S. aipini et Farionis, Martiv.

22. Taenia ocellata $R$.

T. capite hemisphaerico, osculis profundis, collo longiusculo rugoso, articulis subqquadratis lineolatis.

Ent. 2. p. 108. n. 21. T. ocellata.

Hab. In Percae fluviatilis intestinis vario anni tempore copiose, rarissime in illius laepate, Gryphiae offendi. In intestinis Percae cermuae Palitas copiose; in illis Percae norve. gicae MürLfer rarius reperẹunt. (Iste quidem Percam in qua invenit marinam vocat, haec autem in maribus septentrionalibus non occurrit cf. Retzri Fauna Suecica p. 337.)

23. Taenia torulosa. BAtschị.

Taenia capite truncato, osculis orbicularibus 
marginatis, collo mediocri, articulis crassius. culis subrotundis.

Ent. 2. p. IrI. n. 22. T. torulosa.

Hab. In intestinis Cyprini Jesis Bzoch et ego Aprili (Gryphiae, ille Berolini) reperimus. In C. Orfo. Froetrar eodem, in C. Leucisco ZEDERus Majo mense invenerunt.

24. Taenia dispar, Goeze.

T. çapite optuso, osculis hemisphaericis, collo longissimo, articulis anterioribus suborbiculatis, posticis oblongis tenuioribus.

Ent. 2. p. I13. n. 23. Mant, n. 24. T. dispar. Hab. In intestinis tenuibus Geckonis vulgaris (Lacertae Stellionis Lins.) Speziae Septembri reperi, prorsus eandem, ac illas quas in Hyla arborea, in Bufone cinereo et viridi repertas Muscum Viennense obtulit. In Bufone fusco C. E. $V$. In Salamandrae atrae tenuibus Froextcr Augusto invenit.

25. Taenia tuberculata $\mathrm{I}$.

T. capite subgloboso, osculis orbicularibus, collo brevissimo, articulis planis subquadratis, foraminibus marginalibus papillaribus vage alternis.

Mant. n. 25. T. tuberculata.

Hab. in intestinis Lacertae novae speciei in Hispania (Algesirae) reperta. Mus. Vieinn.

8. Rostell a ta e (capitis rostello retractili, inermi).

26. Tacnia osculata, Goeze.

T. osculis hemisphacricis, rostelli apice excavato, collo nullo, articulis anterioribus brevissimis, reliquis subquadratis, foraminibus marginalibus vage alternis. 
Ent. 2, p. II5. n. 24. T. calycina, p. II6. n. 25. T. osculata. Mant. n. 26. T. osculata. Hab. In intestinis Siluri Glanidis Goeze Julio; ego Majo et Octobri Gryphiae, reperimus.

27. Taenia sphaerophora $R$.

T. capite obcordato, rostelli maximi apice sub. globoso, collo longo capillari, articulis anterioribus brevissimis, insequentihus subquadratis, reliquis elongatis.

Ent. 2, p. I19, n. 26. Mant, n, 27. T. sphae. rophora.

Hab. In intestinibus tenuibus Scolopacis Arquatae Januario Gryphiae reperi,

28. Taenia variabilis $R$.

T. capite subrotundo, rostello exiguo obtuso, collo brevissimo, articulis anterioribus angustissimis, insequentibus moniliformibus, infundibuliformibus, cyathiformibus, ultimis elongatis.

Ent. 2. p. 120, n. 27. Mant, n. 28. T. variabilis.

Hab. In intestinis tenuibus Tringae $\nabla$ anelli Junió et Julio Gryphiae reperi. In illis $T r$. hypoleucae, Glareolae; Scolopacis subarquatae, Gallinaginis et Calidris. Mus, Vienn.

29. Taenia laevigata $\mathbf{R}$.

T. capite subgloboso, rostello cylindrico obtuso, collo elongato latiusculo, articulis anteribus brevioribus, reliquis longioribus, ornnium angulis rotundatis.

Ent. 2. p. 208. n. I0I. Taenia Clraradrii Hiaticulae.

Mant, n. 29. T. laevigata.

Hab, in intestinis Charallrii Hiaticulae. Mus. 


\section{- $152-$}

Vienn. Eandem occurrẹe in Char, pluviai, cantiaco et minore. C. E. $V$.

3o. Taenia amphitricha $\mathbf{R}_{\text {, }}$

T. capite subrotundo, rostelli cylindrici apice nodoso, collo breviusculo. articulorum brevịorum longiorumque margine postico tumido, lemniscis alternis rectis.

Mant. n. 30. T. amphitricha.

Hab. in intestinis Tringae alpinae. Mus. Viera.

31. Taenia mutabilis $R$.

I. capite subglóboso, collo breviusculo, art:culis anterioribus brevissimis, insequentibu; angustioribus et longioribus, reliquis cam. panulatis, foraminibus marginalibus alternis.

Mant. n, 3r. T. mutabilis.

Hab. In intestinis Crotophagae minoris OtFrers in Brasilia reperit.

32. Taenia cyathiformis, Froęrch.

T. capite subcordato aequali rostello obtuso, collo articulisque anterioribus brevissimis, reliquis cyathiformibus.

Ent, 2. p. 122, n, 28. Mant. p. 32. T. cyathiformis,

Hab. in intestinis Hirundinis rusticae Majo Gryphiae, Arimini Aprili, H. ripariae ib. eod. reperi. In $\boldsymbol{H}$. urbica Goeze; H. IYelba, Muse Vięnz. H. Apode Froelich, Niтzsch. Augustio.

33. Taenia infundibuliformis, GoEze.

T. capite subrotundo, rostello cylindrico obtuso, collo brevissimo, articulis anterioribus brevissimịs, reliquis infundibuliformibus, foraminibus marginalibus vage al rnis.

Ent, 2. p. 123. n. 25. T. infundibuliformis. Hab. In intestinis Phașiani Galli vulgatissima, 


\section{$-153$}

vario anni tempore, frequens in Otide Tarda, Septembri, et Anate domestica, Decembri, Gryphiae a me reperta; rarior in Ansere etAnate Boschade fera, in quibus nunquam offendi. Catalogus Entozoorum Viennensis, ceteroquin ditissimus, solummodo Phasiamim Gallum Taeniae infundibuliformis babitaculum indicat.

34. Taenia villosa, Brocr.

T. capite subrotundo, rostello cylindrico, collo brevissimo, articulis anterioribus brevissimis, insequentibus longlusculis, reliquis infundibuliformibus, marginis posterioris angulo altero protracto, subulato.

Ent. 2. p. 126. n. 32.'T. villosa.

Hab. In Olidis Tardae intestinis Broch et ego (Septembri, Gryphiae) copiosissimam reperimus.

35. Taenia setigera, Froelich. †

T. capite obcordato, rostello pyriformi, collo articulisque anticis et posticis brevissimis, intermediis infundibuliformibus, marginis posterioris angulo altero protracto truncato.

Ent. 2. p. 128. n. 3I. T. setigera.

Hab. in intestinis Anserum pascentium tenuibus versus crassa, Froelich, Z Zeder.

36. Taenia vaginata $R$.

T. capite ovato, rostelloque exiguis, collo nullo, articulis anterioribus plurimis brevibus postice utrinque acutis, reliquis subquadratis, lemniscis alternis vaginatis.

Ent. 2. p. 208. n. 100. T. Charadii Himantopodis. Mant. n. 36. T. vaginata, 


\section{$-154-$}

Hab. in intestinis Charadii Himantopodis. Mus. Vienn.

37. Tạenia polymorpha $\mathrm{R}$.

- $\boldsymbol{T}$. capite angulato rostellato, collo brevissimo, articulis latiusculis brevibus acutis, lemniscis oppositis recurvis.

Mant. n. 37. T. polymorpha.

Hab. in intestinis Recurvirostrae Avocetiae. Mis. Vienn.

38. Taenia bacillaris, Goeze.

T. capite subrotundo, rostello pyriformi, collo elongato, articulis brevissimis, subcuneatis obtusis.

Ent, 2. p. I29. n. 32. T. filamentosa, p. I3I. n. 33. Mant. n. 38. T. bacillaris.

Hab. In intestinis tenuibus Talpac europaeae Majo, Julio et Septembri Gryphiae sed nunquam lemniscatam (flamentosam) reperi, qualis Gorzio Septembri occurrit.

39. Taenia sphenocephala $\mathbf{R}$.

T. capite subtriangulo, rostello cylindrico, collo longissimo, articulis brevissimis obtusis.

Ent. 2. p. 94. n. II. Mant, n. 39. T. spheno. ceplaala.

Hab. In intestinis Columbae Turturis Goeze et Zener Julio invenerunt. Exinde: Mus.Vienn. Ix illis C. Liviae, Mlus. Vienn, an huc per. tinet?

40. Taenia platycephala $R$.

T. capite disciformi tetragono, rostello obtuso, ariculis anterioribus brevissimis subcuneatis obtusis, ultimis elongatis infundibuliformibus.

Ent, 2. p. 94. n, 10. T. platycephala. p; 210. 


\section{- $155-$}

n. 107. T. Alaudae. Mant, n. 40, T. platycephala.

Hab. In intestinis MTotacillae Luscini

Gryphiae, Berolini Decembri reperi. In illis Motacillae stapazinae, nisoriae et Emberizae melanocephalae, Mus. Viein. Alaudae arvensis, Bravn. Alazdac camipestris, trivialis, cristatae, obscurae; Motacillac Currucae, hortenisis, Sylviae, flavae, Oenanthes, atricapillae, phoenicuri, rubeculae, C. E. $V$.

41. Taenia angulata $R$.

T. capite subgloboso, rostello crasso obtusissimo, collo subnullo, articulis brevissimis cu. neatis.

Ent. 1. p. 132. n. 34, 'T. maculata. p. 133. n. 35. T. angulata. p. 210 . n. I0з. T. Turdorum. Mant. n. 4I. T. angulata.

Hab. In intestinis Turdi pilaris Gryphiae Novembri, Turdi Merulae Berolini Octobri re. peri. In illis Turdi iliaci, Braun. Turdi n. sp. Muls. Vienn. T'. viscivori, musici, torquati, saxatilis. C. E. $V$.

42. Taenia laevis, BLocrr.

T. capite cylindrico, rostello pyriformi, collo longissimo, articulis brevissimis acutis.

Ent. 2. P. 135. n. 36. T. laevis,

Hab. In intestinis Anatis Clangulac et clypeatae

B сосн reperit. An ad $T$. sinuosam $n$. 80.?

43. Taenia aequabilis $\mathrm{P}$.

T. capite subrotundo, rostello obovato, collo articulisque brevissinis, horum plurimis acutis, posticis obtusiusculis.

Ent. 2. p. 135. n. 37. T. aequal,ilis。

$\mathrm{Hab}$. In intestinis tenuibus Anatis Cygăi No- 


\section{$-156$}

vembri Gryphiae reperi. In illis Anatis Olo. ris. C. E. V.

\section{Taenia tenuirostris $R$.}

T. capite subrotundo, rostello tenui subclavato, collo longiusculo, articulis anterioribus an. gustissimis brevissimis, insequentibus longioribus, reliquis brevibus utrinque acutis.

Mant. n. 44. T. tenuirostris.

Hab. In intestinis Mergi albelli Majo Berolini reperi. In illis Mergi Mlarganseris et serra. toris, Mus. Vienn.

45. Taenia inversa $\mathrm{F}$.

T. capite subrotundo, rostello minimo obtuso, collo articulisque anterioribus brevissimis, insequentibus angustatis, tum plurimis latis, rotundatis, ultimis elongatis.

Mant. n. 45. T. inversa.

Hab. in intestinis Sternac nigrae. MLus. Vionn. 46. Taenia capillaris $\mathbf{R}$.

T. capite subgloboso, rostello obovato, collo longissimo, articulis brevissimis, anterioribus inaequalibus.

Ent. 2. p. 138. n. 39. Mant. n.46. T. capillaris. Hab, in intestinis Colymbi auriti, Braun, in his et illis Col, subcristati. Mus. Vienn, An ad n. 84. T. multistriatam?

47. Taenia capitellata $R$.

T. capite subgloboso, restello filiformi capitato, collo brevi, articulis anterioribus angustis longiusculis, reliquis brevibus utrinque acutis, IEmniscis unilateralibus.

Ent. 2. p, 139. n, 40, Mant. In 47. T, capitellata. 


\section{- $157-$}

Hab, in intestinis Colymbi lmmeris Amindgaard. In illis C. arctici, Mus. Vienn.

48. Taenia fasciata $R$. $†$.

T. capite hemisphaerico compresso, rostello cylindrico acuto, collo longissimo, articulis brevissimis obtusis.

Ent. 2. p. I39. n. 4I. T. fasciata.

Hab. In intestinis Anatis Anseris Zeder Julio reperit. - An ad T. sinuosam n. S6. pertinet?

49. Taenia filum, Gozzz.

T. capite subgloboso, rostello cylindrico apice incrassato, collo longissimo, articulis subct. neatis, utrinque acutis.

Ent. 2. p. 140. n. 42. Mant. n. 49. T. filum.

Hab. In intestinis Scolopacis Rusticolae Aprili, Gallinulae Julio Gryphiae reperi. In illis Tringae ochropodis et pugnacis. Mus. Vienn.

50. Taenia microcephala $\mathbf{R}$.

T. capite exiguo continuo, rostello cylindrico, collo elongato, articulorum brevium angulis posticis acutis reflexis.

Mant. n. 50, T. micrucephala.

Hab, in intestinis Tantali Falcinelli, Mus. Vienn, 51. Taenia Linea, Goeż.

T. capite subgloboso, rostello obtusó, collo capillari, articulis anterioribus rugaeformibus, insequentibus plurimis infundibulifórmibus, ultimis campranulatis.

Ent. 2. p. 142. n. 43. Mant. n. 51. T. linea.

Hab. In intestinis Tetraonis Perdicis, Goeze, Zeder, Braun. Ipse in illis T. Coturuicutu complurium Anconae Majo reperi. Corif. Entozoon generis dubii n. 23. 
52. Taenia elliptica, Batscr.

'T. capite subgloboso, rostello pyriformi, collo arriculisque anterioribus brevissimis, mediis subquadratis, reliquis moniliformibus.

Ent. 2. p. 143. n. 44. T. cuneiceps. p. 195. n. 76. Mant. n. 52. T. elliptica.

Hab, in intestinis tenuibus Felis Cali domestici. 53. Taenia globifera Batscit.

- T. capite subgloboso, rostello obtuso, collo, sulunullo, articulis anterioribus elongatis margine postico tumido, insequentibus subcuneatis, reliquis obovatis aut orbicularibus.

Ent. 2. p. 145. n. 45. Mant. n. 4j. T, globifert.

Hab. In intestinis Falconis Tinnnnculi GaEDE, in illis F. Buteonis, lanarii, lagopodis, rufz repertam Mlus. Vienth. obtulerunt, In F. gal. lico, atro, cyanteo, Leucosomate, Subbuteone. C. E. .. F. Lithofalcone, pennato, peregrino. Ciat. mscpt.

54. Taenia Nymphaea Scrraxr. I

T. capite subgloboso, rostello cylindrico ob-' tuso, collo nullo, articulis anterioribus oblongis, reliquis brevissimis.

Ent. 2. p. 147. n. 46. T. Nymphaea.

Hab. in intestinis Scolo pacis Phacopodis, Schrank.

Forsan ad T.- variabilem 11. 28. pertinet,

55., Taenia gracilis R. t

T. capite subsloboso, rostello tenui, collo brevissimo, articulis anterioribus infundibuliformibus, reliquis subquadratis.

Ent. 2. p. 148. 11. 47. T. gracilis.

Hab. in intestinis Anatis Boschadis et Penelo. pes. Blocir. 


\section{$-159$}

56. Taenia pusilla, Gowze.

T. capite rostelloque rotundatis, collo brevi antrorsum angustato, articulis oblongis, posterioribus subparabolicis.

Ent. 2. p. 149. n. 4S. T. pusilla.

Hab. In intestinis tenuibus Muris Musculi aliquoties reperi. In illis $M$. Ratti, Goeze。

57. Taenia brevicollis $\mathbf{R}$.

T. capite subgloboso, rostello exiguo obtuso, collo brevi latiusculo, articulis anterioribus inaequalibus, insequentibus subquadratis, ultimis elongatis.

Mant. n. 57. T. brevicollis.

Hab. In intestinis Mustelae Ermineae Gaxde Junio Berolini reperit.

58. Taenia tenuicollis $\mathbf{R}$.

T. capite subrotundo, rostello truncato, collo elongato tenui, articulis anterioribus brevibu's obtusatis, insequentibus subcuneatis, reliquis angustis Iongioribus.

Ent. 2. p. 196. n. 77. Taenia Putorii p. 197. n. 78. T. Mustelae vulgaris. Mant. n. 58. $\mathrm{T}$, tenuicollis.

Hab. in intestinis Mustelae Putorii et vulga. ris, Mus. Vienll.

59. Taenia obtusata $\mathbf{R}$.

T. capite subrotundo, rostello tenui, articrlis obtusatis, anterioribus longioribus, reliquis latioribus.

Ent. 2. p. 19S. n. 80. T. vespertilionis, Mant. n. 59. T. obtusata.

Hab. in intestinis Vespertili mis murini, M.Tus. Viemll. 
6o. Taenia candelabaria Goeze.

T. capite artrorsum attenuato, rostello obtuso, collo longissimo, articulis anterioribus bre. vissimis obtusis, mediis campanulatis, reli. quis oblongis, medio contractis.

Ent. 2. p. 15r. n. 49. Mant. n. 60. T. candelabraria.

Hab. in intestinis Strigium, Aluconis, Comes de Borke; Brachyoti, Mus. Tienn. Bubonis, Oti, Scopis. C. E. V.

61. Taenia parallelipipeda $\mathrm{R}$.

T. capite subgloboso, rostello obtuso, coilo mediocri articulis anticis brevissimis, insequentibus subcampanulatis; reliquis parallelipedis, passim medio constrictis.

Ent. 2. p. 377. n. 15. b, Mant. n, 6r. T. pz. rallelipeda.

Hab. in Lanii Collurionis intestinis Aprili Gryphiae repertum Kreplin; in illis Lanii miroris Majo, Mus. Vienn. communicavit. In Lanio pomerano, C. E. V. An varietes prae. cedentis Taeniae?

62. Taenia farciminalis Batscr.

T. capite tetragono, rostello basi cylindrico, apice ovato, collo articulisque anticis brevissimis, insequentibus subcuneatis, tum ovali: bus, ultimis elongatis repandis.

Ent, 2. p. I53, n, bo. Mant, n. 62. T. farciminalis.

Hab. in intestinis Sturni vulgaris, Gozze, Bravn, C. E. $r$ :

63. Taenia stylosa $\dot{R}_{\text {. }}$

T. capite subgloboso, rostello cylindrico, collo nullo, articulis anterioribus brevissimis, inse. 


\section{$\therefore \quad 161$}

quentibus linearibus, posticis infundibuliformibus,

Ent. 2. p. 154. n. 5t. Mant. n. 63. T. stylosa. Hab. In intestinis Corvi glandarii Hildeerand - Februario Gryphiae recenter repertam dedit.

64. Taenia paradoxa $\mathbf{R}$.

T. capite subcorciato, rostello subclavato, basi vaginato, collo brevissimo, articulis anterio. ribus brevissimis, latiusculis, reliquis diffor. mibus,

Ent. 2. p. 155. n. 52. T. paradoxa.

Hab. In intestinis praesertim crassis Scolopacis Rusticolae copiosissimam Gryphiae Aprili reperi.

65. Taenia interrupta $\mathbf{f}$.

T. capite suligloboso, rostello subclavato, collo brevissimo, articulis anterioribus angustissimis insequentibus latioribus, ultimo rotundato maximo.

Ent. 2. p. 156. n. 53. T. interrupta.

Hab, In intestinis tenuibus Scolopacis Gallizulac Julio Gryphiae reperi.

66. Taenia oligntoma Nrxzscr.

T. capite rostelloque exiguo subglobosis, collo articulisque anterioribus brevissimis, reliquis majoribus, tandem subrotundis.

Mant. n. 66. T. oligotoma.

Hab: In intestinis Sternae fissipedis Niтzsck Ju. nio reperit.

67. Taenia flagellum Goeze.

T. capite subgloboso, rostello (non viso), collo longissimo, corpore antrorsum capillari, su- 
bito increscente, articulis anterioribus subcu. neatis, reliquis brevissinis.

Ent. 2. p. 157. 12. 54. Mant. n. 67. T. flagellum.

Hab, in intestinis Falconis Milvi. Gorze.

68. Taenia Malleus Guzze.

T. capite subgloboso, rostello cylindrico vaginató, collo articulisque obtusis brevissimis, corporis parte anteriore posteriori transversim imposita.

Ent, 2. p. 158. n. 55, Mant. n. 68. T. Malleus.

Hab. In intestinis Anatis domesticae Goeze Oc. tobri et Decembri; Zener in hac saepissime, rarius in Boschade fera et Puerquedula; rarissime in Ansere et Mergo Mergansere reperit. Ipse in Anate Penelope Junio, et consimilem in Pico medio Gryphiae offendi, et mons. tri quid subesse suspicor.

$$
\text { B. Armat a e. }
$$

69. Taenia Solium LinNaEt.

T. capite subhemisphaerico, discreto, rostello obtuso, collo ątrorsum increscente articulisque anterioribus brevissimis, insequentibus subquadratis, reliquis oblongis, omnibus obtusiusculis, foraminibus marginalibus vage alternis.

Ent. 2. p. 160. n, 56. Mant. n, 69. T, Solium.

Hab. in IIominis intestinis tenuibus. In Germania, Anglia, Hollandia, Oriente haec fere sola aut potissimum occurrit; in Gallia cum Bo. 


\section{$-163-$}

thriocephalo lato alternat. Eundem hominem utroque verme laborasse exemplum non est. In cadaveribus Taeniam passim Berolini re. perimus.

70. Taenia marginata BAтscri.

T. capite subrotundo, discreto, rostello obtiso, collo plano aequali, articulis anterioribus brevissinis, insequentibus subquadratis, posterioribus oblongis, angulis cbtusis, foraminibus marginalibus vage alternis.

Ent. 2. p. 165. n. 57. Mant. n.70. T. marginata. Hab. In intestinis tenuibus Canis Lupi Comes de Borke Februario, ipse Januario Berolini, reperimus.

71. Taenia intermedia $\mathbf{K}$.

T. capite sublemisphaerico, rostello crassissimo, collo plano aequali articulisque anterioribus brevissimis, mediis subcuneatis, postice acutis, reliquis oblongis, foraminibus marginalibus vage alternis.

Ent. 2. p. $16 \%$. n. 58 . T. intermedia.

Hab. In intestinis Mustelae Martis Novembri Berolini reperi.

72. Taenıa serrata Goeze.

T. capite subhemisphaerico, rostello obtuso, collo aequali plano árticulisque anterioribus brevissimis; reliquis subquadratis, postice utrinquae acutis, foraminibus marginalibus vase alternis.

Ent. 2. p. 169. n. 59. T. serrata.

Hab. in intestinis tenuibus Canis familiaris fere semper sed rarius copiosa occurrit.

73. Taenia crassiceps $\mathrm{R}$.

T. capite subcuneiformi, rostello obtuso, collo 


\section{- 164-}

subattenuato articulisque anterioribus brevis. simis, reliquis subquadratis obusis, foraninibus marginalibus vage alternis.

Ent. 2. P. 192, n, 60. T. crassiceps.

Hab. in intestinis tenuibus Canis Vulpis, vul. garis.

74. Taenia laticollis $\mathrm{i}$.

T. capite crasso discreto, rosteilo cylindrico; collo crassiusculo, articulis anterioribus brevissimis!, insequentibus subquadratis, ultimis oblongis, foraminibus marginalibus vage alternis, Mant. n. 74. 'T. Laticollis.

Hab, In intestinis Felis Lyncis, Mus. Vienn.

75. Taenia crassicollis R.

T. cupite crassiusculo colloque brevissimo continuis, rostello cylindrico, articulis anticis transversis, insequentibus cuneiformibus, postice acutis, reliquis oblongis; foraminibus marginalibus vage alternis.

Ent. 2. p. 173. n. 6r. T. crassicollis.

Hab. in intcstinis temuibus Felis Cati domes. tici vulgaris, In illis Cati feri, Revr, Comes

1 de Burk, C. E. $V$.

76. Taenia compacta R. i

T. capite colloque brevissimo continuis crassiusculis, rostello (non, visq),' corpore re. trorsum angusticre, articulis subcuneatis,

Ent. 2. p. 176. n. 62. T. compacta.

Frab. In intestinis Erinacei europaei Brocr hactenus solus reperit.

78. Taenia quadrata $R$.

T. rapite quadrato, rostello brevi, collo nullo, 


\section{$-165$}

articulis anticis brevissimis', reliquis longioribus, obtusis, foraminibus marginalibus vage altèrnis.

Mant, n. 78. T. quadrata.

Hal). in intestinis Muscicapac atricapillae et collaris, Ríus, Iicnt. M. Grisolae Cat, Ent. Vien!n. mscpt, an huc?

79. Taenia macror?yncha $\mathrm{h}$.

'T. capite iransverso, rostello cylindrico maximo, collo nullo, articulis brevissimis latissimisque, anzulis lateralibus elongatis reflexis

Ent. 2. p. 177. 11. 63. T. macrorhyncha,

$\mathrm{Hab}$, In intestinis Colynüi minoris Gryphiae

Octobri reperi.

8o. Taenia octolobata R. I

T. capite tetragono octclobato, rostello cylin. drico, articulis brevissimis, angulis lateralilibus papillaribus teflexis.

Ent, 2. p. I78. n, 64. T. octolobata.

Hab. In intestinis Percae norvegicae O. Fabricrus reperit. Bothrioceplaalis addidissem, nisi rostellum unicum in his nunquam visum du. bium me reddidisset.

81. Taenia straminea Goezt.

T. capite subgloboso, rostello pyriformi, collo longissimo, articulis brevissimis acutiusculis. Ent, 2. p. 18I, n. 65. T. straminea.

Hab. In intestinis Arctomydis, Criceti Gorze Septembri et Noverabri, Hindebrandt Sepa tembri invenerunt.

82. Taenia acuta $\mathrm{f}$.

T. capite subglohnso, rostello magno cylin:drico, collo longiusculo, articulis anterionhus brevissimis, inksequertibus sensim majo- 


\section{- $166-$}

ribus, tandem subcampanulatis, omnium angulis posticis acutis.

Mant. n. 82. T. acuta.

Hab. In intestinis Vespertilionis lasiopteri. Mus. Ficnul.

83. Taenia filifomis $\mathrm{R}$.

T. capite subrotundo, rostello (retracto viso), collo longissimo, corpore filiformi, articulis brevissinis.

Ent. 2. p. 182. n. 66. T. filiformis.

Hab. in intestinis tenuibus Psittaci erithaci

Goeze, C. E. V: Ipse illum aliquoties multosque alios Psittacos secui, sed nunquam Entozoa offendi.

84. Taenia multistriata $\mathrm{R}$.

T. capite pyriformi, rostello brevi, obtuso, collo longo, undulato, articulis brevişsimis obtusis, lemniscis unilateralibus brevibus subclayatis.

Ent. 2. p- 183. n. 67. Mant. n. 84. T. multistriata. Hab. In intestinis Colymbi minuti teuuibus Gryphiae Octobri reperi.

85. Taenia inflata $\mathrm{R}$.

T. capite obovato, rostello subgloboso, collo

- longo tenuissimo, passim inflato, articulis anterloribus brevissinis et angustissimis, reliquis subcuneatis acutis, lemniscis unilatéra. libus capillaribus longissimis.

Ent. 2. p. 208. n. 102, T. Fulicae, Mant. n. 85. T. inflata.

Hab, in intestinis Fulicae atrae, Mus. Vienn86. Taenia sinuosa $R$.

T. capite subrotundo, rostello obtuso colloque crenulato longiusculis, articulis brevissimis 


\section{- $167-$}

acutís, lemniscis unilateralibus brevissimis truncatis.

Ent. 2. p. 184. n. 68. Mant, n. 86. T. sinuosa. Hab. In Allatis domesticae intestinis Gryphiae Decembri reperi; in Anate Boschade fera Julio repertam Niтzsch misit. In Anate acuta Gorze Decembri offendit.

87. Taenia trilineata BArsci.

T. capite subgloboso, rostello cylindrico longo, collo articulisque brevissimis, horum angulis posterioribus reflexis.

Ent. 2. p. 136. n.38. Mant. n. 37. T. trilineata. Hab. In intestinis Anatis acutae et circiae Brocir, ego ịn illis A. clypeatae Novembri, Boschadis, Septembri Gryphiae; Nitzsch in lac Junio et Novembri, reperimus.

88. Taenia undulata $\mathrm{R}$.

T. capite antrorsum increscente, rostello obtuso, collo aullo, articulis brevissimis, angulis lateralibus obtusis, lemniscis unilateralibus setosis.

Ent. 2. p. 186, n, 69. Mant. n. 38. T. undulata,

Hah. In intestinis Corvi Corones Goeze et $\mathrm{ZE}$ DER; in illis C. Coracis Froelich, C. Cornicis Nrtzsch (Martio) invenerunt.

89. Taenia serpentulus, Schrank.

'T. capite antrorsum attenuato, rostello conico, collo longiusculo, articulis anticis brevissimis, mediis infundibuliformibus, posticis sub. campanula:is.

Ent. 2. p. 188. 17. 70. T. serpentulus, p. 202. n, 87. T. Corvi frugilegi. n. 8s. Taenia Cor- 


\section{- 168}

nicis. p, 203. $n+89, T$, Orioli, Mant, n. 89. T. serpentulus.

Hab. Goeze in variis Corvis reperit, sed a praecedente non distinxit: ZEDER in Corvo Corone reperit. Ipse in C. Cornice Novembri, in Pica Fetbrario, in Glandario Majo, in. Oriolo Galbula Julio Gryphiae offendi.

90. Taenia porosa $\mathbf{R}$.

T. capite subgloboso, rostello conico, colld articulisque ancrioribus brevissimis, his latis, reliquis longioribus et angustioribus, variis.

Ent. 2. p. 190. n. 71. Mant. n. 90. T. porosa.

Hab. In intestinis tenuibus Lari cinerarii Gryphiae Julio reperi, In illis Lari fusci, ridi. bundi, minuti. MIus. Tiezur.

91. Taenia crateriformis Goeze.

$T$. capite rotundo, rostollo cylindrico apice globoso, collo longiusculo, articulis anterioribus brevissimis, insequentibus sensim infunđibuliformibus, campanulatis, crateriformibus.

Ent. 2. P. Igr. n. 72. Nant. n. 9I. T. crateriformis.

Hab. In intestinis Pici majoris Goeze; ipse in illis Pici viricils Gryphiae Decembri, Junio Berolini; Yungis Torquillae Aprili Gryphiae; in abdomine Upupae Epopis Treutrer Majo, reperimus. In Pico Martio, viridi, cano, majore, medio, C. E. V.

92. Taenia longirostris $\mathrm{r}$.

T. capite subrotundo, rostello cylindrico longissimo, collo articulisque anterioribus brevissinis, insequentibus subcampanulatis, ul. timis elongatis.

Mant. 1. 92, T. longiostris. 
Hab. In intestinis tenuibus Glarealae àhstricease Arimini Aprili reperi.

93. Taenia scolecina $\mathbf{R}$.

T. capite subgloboso, rostello obtuso (parum excerto viso), collo brevi, articulis antcrioribus rugacformibus lemniscatis, posterioribus subquadratis ovigeris.

Mant. n. 93. T. scolecina.

Hab. in intestinis Pelccani Carbanis. MIus. Vienllt.

$$
\text { Species aubiae. }
$$

94. Taenia Vespertilionis auriti. †

Hab. in intestinis Vespertilionis auriti, C. $E, V$. $m s c p t$. vel ad Taeniam obtusatam n. 59, vel ad $T$. acutam 11. Sะ. pertinebit.

95. Taenia Canis Lagopodis.

Ent. 2. p. 193. n. 73. Hab. in intestinis Canis Lagopodis, Abídgand, $T$, litteratae n, $\mathbf{~}$. proxima videtur.

96. Taenia Felis Pardi.

Ent. 2. p. 193. n. 74, Hab. in Fele Pardo, Cuvier, a quo accepi, speciem T. marginatae n. 8o. affinem.

97. Taenia lineala Gorze. I

Ent. 2. p. 19i. n. 75. T. lineata.

Hab. in intestinis Cati feri ( Februario) Goezz. C. E. $V$. mscpt. ex quo speciem propriam et incrmem esse discitur.

98.' Taenia-Ursi maritimi.

Ent. 2. p. 197. n. 79. T. Ursi maritimi.

Hab. in intestinis Ursi maritimi, 'a quo dejec. tam Cuver mecum communicavit, speciem foraminibus vage alternis instructam, ad fa: 
miliam Taeniarum nobis n. $69-78$ dictarum pertinet.

99. Taenia Soricis.

Hab. in intestinis Soricis Aranei, fodientis, Eremitae C. E. $V$.

100. Trenia Didelphidis. +

Hab. in intestinis Didelphidis murinae. C.E.V.

101. Taenia Myoxi.

Mant. n. rol. T. Myoxi.

Hab. in intestinis Myyoxi Gliris, Mus, Vienn. Myoxi Dryadis, Cat. Ent. Vienn.

102. Taenia Musculi. †

IEnt. 2. p, 195. 11. 81. T. Musculi.

Hab. In Muris Mulsculi abdomine Pallas Sim-

birskii Decembri reperit. Forsan nova species.

103. Tacnia Ratti. +

Hab. in intestinis Muris Ratti et Decumani, propria species. $C_{*} E_{*}, \dot{H}$.

104. Taenia Muris sylvatici. †

Hab. in intestinis Muris sylvatici. C, E. V. miscpt.

105. Taenia Lemmi.

Ent. 2. p. 378. n. 8I, b. In intestinis Muris Lemmi O. Fr. MülLer reperit.

106. Taenia Muris capensis. †

Hab, in intestinis' Muris capensis, propria spe. cies. C, E. $V$.

107. Taenia Hyracis. t

Iint. 2. p. 199. n. 82, T. Hyracis.

Hial. In intestinis crassis Hyracis capcusis PAL-

I.As reperit,

108. Taenia Caprae.

Ent, 2. P. 200, n, 84. T. caprac. 


\section{$-171-$}

Hab. In intestino ileo Caprae Hirci Gryphiae Augusto reperi, proprian uti videtur speciem, inter $\boldsymbol{T}$. expansam $\mathbf{n}$. . et denticulatam n. 2. inserendam, cujus vero caput me fugit.

109. Taenia Zebrae. †

Ent. 2. $p$ 01. 11. 85. T. Zebrae.

Hah. in 'Equo Zebra, SANDER. 'T. plicatae n. 5. atinis.

110 Taenia Chrysaëii. †

Ent. 2. p. 201. 1. 86. In Falcone Chrysaëto

A BILDGAard detexit, nondum descriptam.

11. Pra nia Pici.

Ent. 2. p. 203. n. 90. T. Pici.

Hiab In Pici medii tubo intestinali olim Gry-

phiae sperimen reperiTaeniae Malleo n. 68. similinum, quod.casu perdidi, forsan cum omnibus Taeniis malleatis monstrositatem sistens, tumque ad $\mathbf{T}$, crateriformem $n .9 r_{0}$ pertinens,

112. Taenia Sittae.

Hal, in intestinis Sittae europaeae, nova spe. cies, C. E. $V$.

113 Taenia Meropis. I

Hab. in intestinis Meropis Apiastri, nova spe. cies, C. E. $V$.

114. Taenia Upupae.

Hab. in intestinis Uprupae Epopis, $C, E . V$. ubi inter novas species enumeratur, sed in ave dicta repertam, quam examinare miki contigit, ad T. crateriformem n. 9I. referre coactus sum.

115. Taenia Corvorum. $\ddagger$

Taeniae in intestinis Corvi Caryocatactis et 
"Moncdalac repertae in $C$. $E . V$. cum reliquis Corvorum Taenis pro min eademque specie. habentur, num illae itaque ad nostram $T$. undulatam $n-88$. vel ad serpentulum n. 89 . pertineant, nos fugit.

116. Taenia Curaciae. †

Hah. in intestinis Coraciae garmlae, nova species. C. E. $I^{2}$.

117. Tacnia Curvirostrae †

Int. 2. p. 2ri. n. rog. T. Loxiae curvirostrae. Hah. in intestinis foxiae Curvirostrae, nova species. Đuvamizich.

118. Taenia Coccothraustis. I

Hab. in intestinis Loxine Coccotirraustis, nova species. $C, E . V$.

119. Taenia Fringillarum.

'Ent. 2: p. 2rr. 11. II0. T. Fringillarum.

Hab, in intestinis Fringillae domesticae et cannabinae, Pallas. In illis $F$. caelebis, domesticae, Linariae, montanae, Spini. C. E. V.

120. Taenia Emberizarum. †

'Hab, in intestinis Embirizae Citrinellae, Oue. leae, Ilortulanae. C. E. V.

121. Taenia Urogalli. +

Ent. 2. p. 209. 21. 105. T. Urogalli.

Hă. in intestinis Tetraonis Urogalli, Moder. Propria videtar species.

122. Taenia Bonasiae.

Ent. 2. p. 210. n. 106. In Tetraone Bonasia

O. Fr. Müustrr reperit.

125. Taenia Phasiani colchici o t

Hab. in intestinis Phasiani colchici, nova species, $C, F, V$. 


\section{$-175$}

124. Taenial Struthionis, ?

Ent. 2. p. 20\%. n. 104. T. Struthiocamelí,

Hab, in Strutlitone Camielo. Hourrurs.

125. Trenta Lnguicula Braun.

Ent. 2. p. 20\%. n. 97. Mant, n. I2.5. T. Un* guicula.

Hab. in intestinis Ardens cinereae, Bunx; in illis Ciconiae, Mrs. Vienn. A.purpureac, "Nycti. coracis. C. H. F.

126. Taenia Gallinaginis. :

Ent. 2. p. 207. n. 98. T. Gallinaginis.

Hab, in intestinis Scolopacis Gallinaginis, Brauv,

127. Taenia Silicula Scurañx. 4

Ent. 2. p. 207. n. 99. T. Silicula.

Hab. in intestinis Scolopacis Tolmi Scrmanis.

128. Taenia Scolopacis. $\div$

Hab. in intestinis Scolopacis lapponicac, fus. cae, aegocephalae. C. E. V. mscpt. V'ti ad T. variabilem $n, 28 . \mathrm{vel}$ ad $\mathrm{T}$, filum $\mathrm{n}, 49$, pertineburit.

129. Taenia Tolani. I

Hab. In intestinis Totani chloropodis et stag. natilis. C. E. $V$. nesept. Forsan cum prioribus et insequente militant.

130. Taenia Tringae. +

Hab. In intestinis Tringae Interpretis. C, E, $I$. msćpt.

131. Taenia Ralli.

Ento, 2. p. 208 . 21. '103. T. Ralli.'

Hab. In intestinis Ralli Crecis, nòvae specie fragmenta Augusto Gryphiae reperi. 


\section{- 174}

132. Taenia Sternae. $\dot{\uparrow}$

Hab. in intestinis Sternae Ilimudinis C. E. $V$. Forsan ad T. oligotoman n. 66. pertinet, quàe in Sterna fissipede occurrit, cum hujus et Sternae Hirundinis Taenia in Catalıgo conjungatur.

133. Taenia Colymbi. Troiles. $t$

Ent. 2. p. 206. n. 94. ab Amigaarnto in Co. lymbo Troile reperta neque descripta.

134. Taenia Colymbi cristati. !

Ent. 2. p. 206. n. 9.5. I. 2.

Utraque a Pravaro in intestinis Colymbi cristati reperta, prior Taeniae multistriatae $\mathrm{n}_{\text {* }}$ 84., altera T. macrorhyncliae n. 79. affinis.

135. Taenia Colymbi cornuti. †

Hab, in intestinis Colymbi cornuti, C. E. $V$. mscpt.

136. Taenia Lari cani. †

Ent. 2. p. 206. 11. 96. T. Lari cani.

Hab. In intestinis Lari cani Braus reperit, a T. porosa fursan diversam, si articuli subcuneati vere posteriores dicendi sunt. Rostel. lum enim ab amico descriptum in $\mathbf{T}$. porosa nunquam penitus evolutum vidi.

137. Taenia Tadornae.

Ent. 2. p. 203. n. 91. Mant. n. 137. T. Tadómae.

Hab. In intestinis Anatis Tadornae Amildgard reperit, novam forsan speciem.

138. Taenia Alcae Picae. †

Ent. 2. p. 204. n. 92. T. Alcae Picae.

$\mathrm{Hab}$. Cum specie insequente in intestinis Alcac

Picae O. Fabrictus reperit, fuse sed incon. 


\section{$-175$}

grue descriptam; an Bothriocephalum nocosum?

139. Taenia armillaris $\mathrm{h} . \cdots$

Ent. 2 p. 205. n. 93. T. irmillaris.

Hab. Cum specie pratcedente diversissima in intestinis Alcae Picae O. Fabzrcius reperit, haud sufhicienter Gescriptarn.

140. Taeńia Iacertae.

Hab. in intestinis Lacertae orbicularis. Cat. Ent. Ticint.

141. Taenia Amphisbaenae.

Hab. in intestinis Aupllisbaene albae. Cat. Ent. Vienn.

142. Taenia Pollachii, †

Ent. 2. p. 212. 1. II. T. Gadi Pollachii.

Hab. In intestinis Gadi Polinchii se bellem ef raram Taeniae speciem invenisse Finfrx: refert, quam cum T. ocellata n. 22. coinparat, nonne tamen Bothriocephalum?

143. 'Taenia Belones. I

Ent, 2. p. 2t2. 11. I12. T. Belones.

Hab. O. Fr. MüLLER se in Esocis Belones intestinis Taenias duas reperisse, alteram articulatam, inarticulatam alteram refert; an hanc Triaęnophorum? an hujus varietatem illam?

144. Taenia Eperlani. I

Ent. 2, p. 2 I2. I1. II3. T. Fperlani.

Hab. Acmarils in cavo abdominis Salmonis Fiperlani reperit, quam ob collidefectum cuin T.longicolli 11. 21. conjungere non ausus sum.

145. Taenia Salmonis Omul. +

Palias (Zoographia Rosso-Asiatica. T. Ill. 
Petropoli 1853. 4. p. 409). Ascarides et Tae. niolas in ventriculo Salmonis Omul frequentes, coecis inhaerentes easque saepe perfodientes refert. Forsan Bothriocephalus, .

146. Taenia Idi. †

Eist. 2. p. 213. n. II4. Taenía Cyprini Idi.

What. Amtudgand in Cyprino Ido a se re. pertam non descripsit, forsan T. torulosam n. 23. varis in Cyprinis obviam. 


\section{Ordo V.}

\section{$C^{y}$ s $t$ i c a.}

Corpuis depressum iel teretiusculum, apice poste-

- riore in vesiculam abiens entozois singuilis solitariam rel pluribus communem. Cruput bothriis (2 vel 4) aut osculis suctoriis (4), uncinulorum. corona, vel proboscidibus quatuor uncinatis instructum. Organa sexus in nullis hactenus conspicua.

Genus XXVII. Anthocephalus.

(Vesica extoria dura elastica, continens alteram tenuiorem, in qua entozoon solitarium, cujus)

Corpus elongatum depressum, basi int vesicam abit caudalem ampliatam. Ciaput, (Tetrarhynchi).bothriis (2 vel, 4) et proboscidibus uncinatis (4) instructum.

\section{Anthocephalus elongatus $R$.} Anth. bothriis duobus ovalibus, collo teretius. culo, crassiore: corpore depresso; vesica caudali ovali, media subclavata, externa magua varia, 


\section{$=178=$}

Floriceps, Cuvien, Mant.' n. I. Anth. elongatus.

Hab. In mesenterio et hepate Orthragorisci Molae Juilo, in mesenterio Centronoti glauci Augusto, Neapoli reperi. Huc quóque referendus videtur, quem ib, in Sciaena Aquila offendi.

2. Anthocephalus gracilis $\mathrm{R}$.

Anth. bothriis duobus ovalibus, collo corporeque tenuiore teretiusculis filiformibus, vesica caudair oblonga, exiema ovato ielliptica. Mant. 1., 2. Anth. gracilis.

Hab. In peritoneo Scombri Rochéi Junio et Julio, in illo Spari Raji Juli Neapoli reperi.

3. Anthocephalus Granulum $\mathrm{R}$.

Anth. bothriis duobus divisis, collo tenui, corpore ovato, vesica externa fusca subglobosa minimas.

Mant. 11. 3. Anth. Granuluni.

Hab. In peritoneo Carancis trachuri Aprili, Spari Alcedinis Majo Arimini, in illo Scomb̈ri oliae Julio Neapoli reperi.

4. Anthocephalus macrourus $R$.

Anth. bothriis quatuor, collo tenui elongato insidente receptaculo ovali, terminato vesica longissima.

Mant. n. 4. Anth. macrourus.

Hab. In hydatibus visceralibus et hepate $S_{p a r i}$ cujusdam repertum Otrers ex Brasilia misit. 5. Anthotephialus interruptis $R$.

Anth. bothriis quatuor, antrorsum distantibus, corpore inatquali, interrupto, retrorsum depresso, vesica caudali . (non exhibita).

Mant, n, 5. Anth. interruptus. 
Hab, non indicato Orfers ex Brasilia misit.

Obs. Genus hoc Tetrarhyncho et Bothrio. cephalo corollato ui insequens Taeniae proximum, regionibus australioribus proprium, piscibus, quemadmodum insequentia mamrna. libus tantum infesta videntur.

\section{Genus XXVIII. Cysticercus.}

(Vesica exterua simplex, continens Entozoon so. litarium, cujus:).

Corpus teretiusculum vel depressum abiens in vesicain caudalem. Caput (Taeniae armatae) orculis suctoriis quatuor, rostelloque uncinato instructum.

- 1. Cysticercus fasciolaris R.

Cyst. capite subtetragono, collo nullo, corpore elongato depresso, vesica caudali exigua sub. globosa.

Ent. 2. p: 215. n. I. Mant. n, 2. Cyst, fasciolaris

Hab. in hepate. Ipse nonnisi in illo Muris Musculi et decumani, Pallas praeterea in arvalis et Ratti, Goeze in M. amphibii, Buocu in Vespertilionis non determinati hepate reperimus. In illo Vesp, auriti, Cat. Ent. Vienul. msciptus.

2. Cysticercus fistularis $R$,

Cyst. capite tetragono, corpore brevissimo tere. tiusculo, vesica cauda longissima.

Ent, 1. p. 218. n., 2. Mant, n, 2. Cyst. fistularis.

Hab. in Equi Caballi peritoneo. A. Chaberto 


\section{$\therefore \quad-180 \div$}

in Schola veterinaria Alfortensi detectus, passim quoque in nostra Bérolinensi reperitur.

3. Cysticercus tenuicollis $\mathrm{R}$.

Cyst. capite tetragono, collo brevi filiformi, corpore teretiusculo, resica caudali subglobosa, amplissima.

Ent. 2. p. 220. 11. 3. Cyst. tenuicollis. p. 234. n. 7. Cyst.: visceralis Simiae. Mant. n.3. Cyst. tenuicollis.

Hab. in mammalium herbivororum peritoneo et pleura. Ipse in Ove, Bove, Capra et Sue tam donestico quam fero reperi. In Ove Ammone, Cervo Elapho et Sciuro cinereo, "C. E. $V$. In Sciuro vulgari, Kuvg. In Capreolo ZeDer. In Autilope Dorcade, Tysox; Saiga, Pallas. Rupicapra et Fevella, C. E. $V_{\text {. }}$ mscpt. in Tarahldo. Nic. Stexonis, in Cervo Axi Amildgand, In Simia, Bloch, $S$, Sa. baea. C. E. V. mscpt.

4. Cysticercus cellulosae R.

Cyst. capite tetragono, collo brevissimo antrorsum increscente, corpore cylindrico longiore vesica caudali elliptica transversa.

Ent. 2. 'p. 226. n. 5. Mant, n. 4. Cyst. cellulosae.

Hab. In Hominis cerebro, corde rarius; in ejus. dem musculis quotannis aliquoties reperio. In Simia syluano et Pata, Treutler; S. Cejho, Mus. Vienn. In Suis domertici cerebro et omnibus partibus musculosis vulgatissi. mus occurrit, neque fero detst.

5. Cysticercus longicollis $\mathrm{R}$.

Cyst. capite tetragono, collo depresso longiore: corpore rugoso, vesica caudali subglobosa. 
Mant. n. 5. Cyst. longicollis.

Hab. in thorace Muris arvalis. Mus. Nienz. W 6. Cysticercus pisiformis ZED.

Cyst. capite globoso, collo tenui, corpore tereti antrorsum attenuato, aequali vesicae, cauciali subglobosae.

Ent. 2. p. 224. 11. 4. Mant. n. 6. Cyst, pisiformis.

Hab. in visceribus Leporis timidi et Cuniculi, rarius in illis Muris Mulsculi.

7. Cysticercus sphaerocephalus $R$.

Cyst. capite subgloboso, collo nullo, corpore hrevissimo, vesica caudali oblonga maxima.

Mant. n. 7. Cyst. sphaerocephalus.

Hab. in peritoneo Lemuris Mongoz, Mus. Vicm,

Conf. speciem insequentem.

$$
\text { Species dubiae. }
$$

8. Cysticercus crispus $\mathbf{R}_{\text {. }}$

Mant. n. 8. C. crispus.

Hab. in pleura Lemuris Mongoz. Mrus, Vienn. An speciti praecedentis varietas?

9. Cysticercus visceralis Hominis. $t$

Ent. 2. p. 233. n. 6. Cyst. visc. Homints,

Res valde ambigua, mihique in cadaverum hu. manorum aliquot millibus nunquam vísa. "

10. Cysticercus Canis.

Ent. 2, p. 234. n. 8. Gyst. Canis.

Hab. in Canis familiaris peritoneo. Chabert.

11. Cysticercus Putorii. †

Ent. 2. p. 235. n. 9. Cyst. Putorii.

Hab. in hepate Mustelae Puterii, Gorze.

12. Cysticercus Talpae. I

Hab, in hepate Talpae europaeae, C. E. $V$. 
13. Cysticercus Leporis variahilis.

Hab. in mesenterio Leporis variabilis. Mus. Vienn.

14. Cystercus Delphini. +

Ent. 2. p. 236. n. 10. Cyst. Delphini.

Hab. in Delphini Delphidis adipe, praesertim circa genitalia, bosc.

Obs. Species Cysticorum piscinae iu Entozoologiae P. 2. P. $237-24 \mathrm{I}$. n. II - I5. ZEDERI potissimum auctoritate, licet dubie, admissae, in hoc opere Cestoideis et praesertim 'Triacnophoro adjectae sunt, ubi de singulis singula conferantur.

Num autem entozoon dubii generis (n. 8.) in Erinacei hydatidibus thoracicis repertum huc pertfnet?

\section{Genus XXIX. Coenurus.}

Vesica simplex, in quam desinunt plurima Entozoa, gnornm:

Corpus elongatum depressiusculum, rugosum; Caput (Taeniae armatue) rostello uncinato guatuorque osculis suctoriis instructum.

1. Cocnurus cerebralis $\mathbf{R}$.

Ent. 2. p. 243. n. I. Coenurus cerebralis.

Hab, in Ovium vertiginosarum cerebro. Eadem quoque species in Bobus et Antilopis vertiginosis occurrere videtur. 
Genus XXX Echinococcus.

Vesica externa, simplex vel duplex, cujus superficici intcrnae insident entozoa plurima, arenulan mentientia, quorum:

Corpus obovatum; Caput (Tacniae armatae) untcinornm corona et osculis suctoriis instructum.

1. Echinococcus Hominis.

Ent. 2. p. 247. n. x. Mant. 11. x. Ech. hominis. Hab. in IIominis visceribus, praesertim hepate.

2. Echinococciis Simiae. †

Ent. 2. p. 250. ก. 2. Exh. Simiae.

Hab. In Simiae Cynomolgi visceribus thoracis et abdominis Blumenmach reperit. Speciem propriam in Simia lnuo repertam habet Cat. Ent. Vienn.

3. Echinococcus Veterinorum.

Ent. 2. p. 25r, n. 3. Ech. veterinornm.

$\mathrm{Hab}$. in thoracis et abdominis risceribus Ovis domesticae et Ammonis (Cat. Ent. Fienn.); Bovis Tauri feri et domestici; Cameli lBactriani et Dromedarii (Cat. Fint. Vienn.); Suis. 


\section{Entozoa vel Generis dubii, vel fictitia.}

1. Hominis.

Ent. 2. p. 258. Diceras rucle. Strzeri Ditra* chyceras rudis. Entozois genuinis vix adnumerandum, Nullius examini ab auctore subjectum.

2. Diacanthus polycephalus, Stienex.

Dyacanthus polycephalus STEBelio dictus (MECKEL's. Ar'chiv für Physiologie B. III. St, 2. Halle 1817. 8. p. $174-179$ 1'ab. 3. Fig. I - 5.) ab auctore pro novo entozoo. rum genere et quidem prolificante exhibitus, a puero dejectus, nil nisi ramentum vegetabile est, forsan Vitis viniferae. Illustris Blumenbachivs, cui auctor vermem pulatitium miserat, hunc mecūm communicare non recusavit, et ramento fusco adspecto, circa pedunculorum apices nequaquam tam regulari, qualem figurae citatae sistunt, vermem non esse in aprico erat; segmentis autem illius tenuibus microscopio subjectis, vasa spira. lia et scalaria, fibrae et cellulae naturam vege- 


\section{$-185-$}

tabilem visui obtulerunt, quo minus dubium ullum superesse possit.

3. Mecrelius, Academiae scientiarum Berolinensis olim decus, in illius historia anni 1766 . p. 34. vermes describit in pueri maniacofatui coeco a se repertos, qui procul dubio Muscarim larvae fuerunt. Error iste illo quidem tempore facile committi potuit, nos. tris autem diebus repeti non debuisset, ut Brrea, magnorum de entozois voluminum auctor, qui aliena misceat et fucata vendat, mox risum mox bilem noveat, qua de re in Mantissae sectione bibliographica fusius tractabitur.

4. Vespertilionis auriti.

Mantiss. Dub. n. 4 .

Hab. in intestinis Vespertilionis auriti, Mus. Kenzl. An Strongylus?

5. Vespertilionis discoloris.

Mantiss. dub. n, 5 .

Hab. in intestinis crassis Vespertilionis discoloris. Mus. Vienn. An Trichosoma?

6. Vespertilionis. I

Ent, 2. p. 262. n. I. Dub. Vespertilionis.

Hab.'sub pelle Vespertilionum, Redr, Entozoa pusilla nematoidea Ascaridem forsan sistentia. 7. Vulpis.

Mant, Dub, n. 7.

Hab. in Vulpis glandulis mesenterii. Ifus. Vienr. An Strongylus?

8. Erinacei europaei.

Mant: Dub. n. 8.

Hab. in Erinacei hydatidibus thoracis. Truesrvi. An Cysticercus vesica caudali oblitterata, 


\section{$-186-$}

utrum cum insequente et speciebus n. 19. et 20. novum entozoorum genus format, $\mathrm{Li}$. gulae et Gymnorhyncho affine?

9. Mygales moschatae. †

Ent. 2. 1) 24. n. 10. Ligula Soricis moschati.

Hab.sub cute MYgales moschatae, Gütdenstedt.

Cum praecedente et speciebus n. 19. et 20, novi forsan generis Cestoideorum.

10. Muris musculi vel amphibii. t

Ent. 2. p. 262. n. 2. Muris.

Hab. in Muris Musculi (vel amphibii) glandu-

lis inguinalibus. Renr. Nematoideum.

11. Myrmecophagae. +

Marcgravies (Brasil, p. 226.). se in Tamanduae s. Myrmecophagae didactylae intestinis multos teretes lumbricos reperisse refert.

12. Antilopes. †

Ent. 2. p. 265. n. 4.

Nematoideum a Soemmerrivgro in Antilope re. pertum et Gozzio missum.

13. Oris t

Ent. 2. p. 265. n. 5 .

Nematoideum in Ove repertum, habitaculo ul. terius non indicato.

14. Delphini. †

Ent. 2. p. 265. n. 6.

Hab. in visceribus et intestinis Delphini Del. phidis, Renr. Entozoa visceralia hydatidibus inclusa pro Cysticerco n. 14. haberi possent, sed ab ill, viro cum intestinalibus conjunguntur.

15. Falconis fulvi. t

Ent. 2. 1. 375. n. 7. 


\section{$-187$}

Nematoideum (forsan Physaloptera) a BrauNIo in Falconis fulvi thorace repertum.

16. Falconis Nisi. !

Ent. 2. p. 266 . n. 7 .

Hab. in Falconis Nisi tuberculis hepatis. Redr.

17. Falconis Buteonis. $t$

Fnt. 2. p. 350, n. I8.

Nematoidea a Goezio in intestinis Falconis $\mathbb{B} u$ -

tconis reperta.

18. Falconis naevii.

Mant. n. I8. Dub. Falconis naevii.

Hab. in naribus et orbita Falconis naevii. MIus.

Vienn. Nematoideum, nihilque erit nisi Filaria abbreviata, habitaculo an recte indicato?

19. Falconis.

Mant. Dub. n. rg.

Hab. In Falconis (Speciei sibi incertae) abdomine circa renes et ovaria. Tilisivs. Cum insequente et entozois n, 8. et 9. novum for. san Cestoideorum genus constituit.

20. Strigis accipitrinae.;

Mant. Dub. n. 20.

Hab. sub pelle hypochondriorum Strigis accis pitrinae, Nitzsch. Cum praecedente et speciebus n. 8. et $g$. proprii Cestoideorum generis videtur, Ligulae affinis.,

21. Lanii pomerani.

Mant. Dub. n. 2I,

$\mathrm{Hab}$, in abdomine Lanii pomerani. Mus, Tienn. Cestoideum.

22. Fringillae domesticae.

Mant. Dub. n. 22. 


\section{$-188$}

Hąh. in ventriculo Fringillae domesticae. Mus,

Tientr. Nematoideum paradoxum.

23 Perdicis saxatilis.

Nant, Dub. n. 23.

Hab. in abdomine Perdicis saratilis. Mus. Vienil. An Tacniolae? Confer Tacniam Lineam n. 5I.

24. Colymbi septentrionalis.

Ent. 2. p. 266. n. 8 .

Cestoideum maxime singulare in Colymbi sep. tentrionalis ventriculo' Gryphiae 'Augusto reperi.

25. Alcae Picae. I

Ent. I. p. 306. 1. 42. Echinorhynchus Alcae. Hab, in intestinis Alcae Picae, O. Fabutcivs, qui pro Ascaride habuit. Neque descriptio neque icones datae genus efficiunt.

26. Testudinis marinae. I

Fint 2."p. 267. n. 10.

Hab. in Tostudinis marinae (magnae, an $M y$. dae?) vesiculis renum. REDr.

27. Lacertae viridis.

Mant, Dub. n, 27.

Iah. in Lacertae viridis tuberculis hepatis. Mus. Viem. Novum forsan genus, Scoleci proximum. Conf, speciem insequentem.

28. Jacertae muralis.

Mant. Dub. n. 28 .

Hab. in Lacertae muralis tuberculis hepatis, praecedente ninus, ceterum idem. Mus. Vienn.

29. Lacertarum. †

Ent. 2. p. 269 . n:- 13 .

Nematoidea a REDIO in ventriculo et intestinis 


\section{- $189-$}

Lacertarum vulgarium (ergo viridis etc.) nec non in plurimis partibus Lacertae africanae reperit.

30. Serpentum. +

Ent. 2. P. 269 . n. I/s.

Pent similia Nematoidea ac in Lacertis (n. 2n.)

in serpentibus quoque reperit, in Viperis

scilicet (T'ipera Redi) et serpente bicipite

(Colubri Natricis, uti mihi videiur, monstro).

31. Colubri Natricis. t

Ent. 2. p. 270. n. 15.

Nematoideum in intestino Colubri Natricis a

Duce HorsteIx-BEck iepertum.

32. Colubri olivacei.

Mant. Dub. $12.3 \%$

Nematoideum in Colnbri olivacei ventriculo repertum Olfers ex Brasilia misit.

33. Ranae Pipae.

Ent. 2. p. 267 . n. II.

Nematoidea in Ranae Pipae spiritu vini servatae vesiculis intestinorum peritonealibus reperi.

34.' Ranarum, it

Ent. 2. p. 268 . ก. 12 .

Entozoa in Ranarum hydatidibus reperta CAIDAxI refert, ex descriptione nextiquam dijudicanda. 35. Protei anguini.

Mant. Dub. n. 35.

Nematoidea in trium Proteorum anguinorum: (Arimini, Aprili) recens mortuorum intes: tinis et hydatidibus viscerum reperi. Alind specimen in Protei ventriculo repertum, forsan ad Ascaridem leprocephalam n, 40, pertinens in Museo Fiennensi vidi, 
36. Torpedinis. I

Kaempere (Amoenit. Exot. p. 5ri.) de sinus persici Torpedine (forsan marmorata, aut saltem affini) sequentia refert: „Anus compressus egessit faeces atras terrestres cum lumbricis palmaris longitudinis albis tenuissimis." An Bothriocephalis?

37. Rajae Batis. $t$

Mant. Dub. n. 37 .

Monno in Rajac Batis ramo nervi trigemini ophthalmico reperit et pro hujus parte habuit, Conf. ra. 52 .

38. Rajae Fullonicae. $f$

Ent. 2. p. 270. n. I6.

Hab. in ventriculo Rajae Fullonicae. O. FABRI-

crus. Nil nisi Ascaris erit.

39. Rajarum Aquilae et Pastinacae. I

Ent. 2. 1. 27 1. 11. 17.

Redi cntozoar in Rajarum Aquilae et Pastinacae intestinis reperit, quae cum insequentibus ejüsdem indolis sunt.

40. Squali Squatinae. $t$

Ent. 2. p. 271 . 11. 18.

In Squali Squatinae, tractu intestinali toto REvr :entozoa reperit, quae cum antecedentibus nil nisi Bothriocephalorum articuli posteriores maturi et soluti erunt, quales saepe co. piosos' in Rajis et Squalis offendi, cum Rediana dcscriptiona satis convenientes, facile pro Monostomatibus imponentes, conf. n. 43.

41. Squali Catuli. t

Ent. 2. p. $2720^{\circ}$ n. 19.

Squali Catuli totam intestini cavitatem inter- 


\section{$-\quad 91$}

dum isdern repletam esse entozois ac quae in Argentina (n. 79.) occurrunt. Redr.

42. Squali IrIusteli. †

Ent. 2. p. 272. n. 20. p. 379. n. 15 .

Cestoideum in Squali Musteli hepate reper.

tum. REví.

43. Squali grisei. $t$

Risso (Ichthyologie de Nice, Paris, ISro, 8. p. 3צ.) intestina-sąepius magna-Fasciolarum copia obruta refert; hisce vero Bothriocephalorum articulos solutos intelligi.crediderim, conf. n. 39 et 40.

44. Squali ....

Ent.2.. p. 272.: n. 2r.

Daldorf in Squali cujusdam corde reperit, for. san Tetrarhynchum.

45. Accipenseris, Sturionis. +

Pleurorhynchus. Nav. Ent. i2. p. 283. n. 22.

Nil nisi Ophiostoma sphaerocephalum erit, par. tibus internis infra caput prolapsis, quod non raro contingit huic entozoo, cujus examen summis saepe preınitur difficultatibus,

46. Syngnathi pelagici.

Mant. 'Dub. n. 46.

Nematoideum (Capsulariam ZED.) in duorum Syngnathorum pelagicorum mesenterio Neapoli Julio reperi.

47: Muaenae Anguillae.

Ent. 2. p. 274. n. 23.

Ipse semel (Gryphiae, Majo) in intestino Muraenae Anguillae Entozoon reperi valde paradoxum, cum Distomate laticolli (n. 116.) comparandum, sed poris visui nors exbibitis, 


\section{$-19^{2}-$}

IRED : In Nuguillae maximae vesica natatoria tubercula aliquot offendit, quibus Ncmatoidea inerant.

48. Muraenae Congri. †

$\therefore$ Ent. 2. p. 275. n. 25 .

Vesiculae variarum partium entozoa continen. tes, quarum majores Revio dictae forsan Anthocephahis foetae. In Congro magno reperit.

49. Muraenae Helenae. it

Ent. 2. p. 275. n. 24.

Plurimarum partium entozoa libera et hydatidibus inclusa Renro in omnibus Marrenis, Decembro, Januario, Februario, Níartio et Aprili a se dissectis visa, de quibus judicare non ausin.?

50. Muraenae serpentis. $\mp$

Ent. 2. p. 283: n. 37. Viperae marinae.

Tubercula verminosa in Muraenae serpéntis splene a Redro reperta.

51. Fiatolae.

Ir. Mant. Dub. n.'50.

Nematoidea (an Ascarides) in Stromateos Fiatolae mesenterio Arimini Majo reperi.

52. Gadorum Aeglefini, Morhuae et virentis. $\frac{t}{b}$

Ent. 2. p. $=77$. n. 27. Garl. vir. et Morhuae.

Vesiculae nervorum et cerebri tunicis insiden. tes. entozoa minuta nematuidea continentes, Mosroo (qui pro vermibus non habuit) et Rathkio qui inmerito cum Cysticerco Cellulosae comparat', solis visae. Conf, n. 37.

53. Gadi Merluccii.

Ent. 2. p. 278. n 28. Mant. n. 53.

ReDr in appendicibus Gadi Merluccii pyloricis 


\section{$-193$}

Bothriocephalos reperisse videtur, forsan cras. sicipitem mihi dictum n. 23. Ipse in peritoneo dicti piscis Neapoli Julio Nematoidea offendi, de quorum genere incertus sum.

54. Gadi Wachniae. $f$

Mant. Dub. n. 54 .

Tilesius in Gadi Trachniae peritoneo Capsu. larias ZeDsro dictas, praeterea autem ento. zoa singularia subtrigona viridescentia in illius fossis vertebralibus reperit; an Disto. mata renalia?.

55. Gadi Chalcogramini. \&

Gadi hujus appendices, ventriculum et hepar vermibus copiosis obsideri, ex STELreri relatione manuscripta Pallas (Zoogr, asiat. ross, T. III. p, I9?) reliquit.

56. Cepolae rubescentis.

Mant. Dub. n. 56 .

In peritoneo Cepolae rubescentis aliquoties Arimini Aprili reperi, forsan Ascaridem.

57. Scorpaenae massiliensis. $t$

Risso' (Ichthyologie de Nice. Paris, 1810. 8. p. 185) in Scorpaenae massiliensis intestinis magnam copian Tentaculariarum rubellarum tenuium et longarum occurrere (et niscem voracissimum reddere) auctor est. An Echinochynchus subintelligendus est? forsan Pris. tis n. 47 .

58. Zenis Fabri.

Mant. Dub. $n, 58$.

In mesenterio Zenis Fabri Arimini Aprili Nematoidea dubia reperi, ad Zeperr Capsula. rias pertinentia. 


\section{$-194=$}

59. Zenis Apri.

Mant, Dub, n. 59 .

Consimilia entozoa in mesenterio Zenis Apri Julio Neapoli reperi.

6o. Pleuronectis Soleae.

Mant. Dub, n. 60.

Cestoideum paradoxum, quod in Pleuronectis Soleae intestinis Neapoli Julio reperi.

61. Pleuronectis Boscii.

Mant. Dub. n. 6r.

Nematoidea in mesenterio Pleuronectis Boscii Julio Neapoli reperi.

62. Spari Denticis. +

Ent. 2. p. 279. n. 29.

Tubercula verminosa. Redr.

63. Spari Boopis, Mormyri, melanuri, Alcedinis.

Mant. Dub. no, 63 .

In peritoneo piscium dictorum reperi, forsan eandem Capsulariae speciem.

64. Labri nilotici. +

Sonnixt (Voyage dans là Haute et Basse Egypte. T. 2. Paris, an 7. p.397. Vers.germ. T. 2. p. 125.) in Labri nilotici ventriculo Nematoideum reperit septendecim lineas longum utrincque acutum, albidum rubedine passim addita,

65. Sciaenae Umbrae.

Ent. 2. p. 279. n. 30. Mant. Dub, n. 65 .

Tubercula verminosa, REpr. Ipse in Sciaenae

Uimbrae peritoneo Nematoideum, forsan As- caridem Julio Neapoli reperi-

66. Percae cirrosate.

Mant. Dub. n. 66 .

In peritoneo Percae cirrosae Arimini, Majo re- 


\section{- $195-$}

peri, ad Capsulariarum vagum genus pertinentem.

67. Percae marinae.

Mant. Dub. n. 07.

Pariter in peritoneo reperi, num Capsularias,

Neapoli Junio et Julio.

68. Gasterostei aculeati. $\frac{f}{b}$

.Ent. 2. p. 279, n. 3I.

Ventriculo Gasterostei aculeati adhaerentem

vel nudum, vel vesiculis inclusum O.FA.BRIcrus reperit, forsan Hypostoma.

69. Centronoti glauci.

Mant. Dub. n. 69,

Nematoidea libera et vesiculis inclusa in peritoneo Centronoti glauci Neapoli Augusto reperi.

70. Scombri Sardae; Coliae et Scombri.

Mant. Dub. n. 70.

In peritoneo Scombri Sardae, Scombri et Coliae Neapoli Junio et Julio Nematoideum, forsan Ascaridem reperi,

71. Carancis trachuri.

Mant, Dub, n. 7 r.

In Carancis trachuri (Arimini Aprili) et imperialis (Neapoli Augusto) mesenterio reperi, cum praecedente forsan eandem speciem.

72. Triglae Hirundinis.

Mant. Dub, n. 72 .

In Triglae Hirundinis peritoneo Majo Arimin reperi forsan eandem ac sequenteis.

73. Triglae adriaticae.

Mant, Dub. n, 73. 


\section{$-196-$}

In peritoneo Triglae adriaticae Arimini Majo, Julio Neapoli reperi, forsan Capsulariam.

74. Siluri Glanidis.

Ent. 2. 1. 254. Prionoderma ascaroides, Mant. Dub, n. 73 .

Hab. In Siluri Glanidis, ventriculo Gołze Junio reperit,

75. Salmonis Lavareti. I

Ent, 2. 1, 281, n: 34.

Lavareti ventriculo extus adhaerentem $\mathrm{O}, \mathrm{FA}$ BRIcrus reperit, an Hypostoma?

75. Salmonis Eperlani. I

Ent. 2. p. 28I. n. 34.

Nematoideum plurimis locis in Eperlano repertum.

77. Salmonis Spirinchi $\dot{\dagger}$

Entozoa saepe in Salmonis Spirinchi vesica anemia óccurrentia Pallas (Zoogr. Asiat. Ross. III, p. 389.) 'Taeniolas vocat, sed in illa Farionis et Thymalli Spiroptera reperitur.

78. Esocis Lucii. I

Ent. 28\%, n. 35 .

Cestoidea alio forsan ex pisce comesto in Esocis ventriculo residua Palras reperit.

79. Argentinae Sphyraenae +

Ent. 2. p. 252. n. 36. p. 379. n. 16.

hispr in Argentinae Sphyraenae abdomine re. perit, forsan Nematoidea, Conf, n. 4r. 80. Sphyraenae Spet.

Mant, Dub, n, 79. 


\section{$=197-$}

- In peritoneo Sphyraer:ae Spet Neapoli Junio reperi.

81. Cyprini"Aspii.

Mant. Dub. n. 8I.

In Cyprini Aspii peritoneo Berolini. Junio reperi, an Filariam?

82. Cyprini rutili.

Ent. 2. p. 375. ni $r$.

In Cyprino rúcilo Hannoverae Martio reperi, an Filariam?

53. Cancri depressi. \&

Ent. 2. p. $287, \mathrm{n}, 42$.

Cavorine in Cancri depressi appendiçibus ver. triculi reperit, an Distomata?

84. Cancri Paguri. t

Ent. 2. p. 287. n. 43 .

In Pagtri vesicula ovariorum Redr reperit, an Trematoda?

85. Cancri Locustae. I

Ent. 2. p. 288. n. 44.

In vesiculis ventriculo et ovario adliaerenti-

- bus Locustäe Ruint reperit, ejusdem indolis, ac speciem praecedentem.

86. Blaptis Mortisagae. "

Henr. Maurit. Gaéde, naturae 'scrutator egregius, in Blaptis Mortisagae ventriculo multos vermes reperit conicos, quorum caput subrotundum microscopii" ope distincte vidit, conf. Ejus Beyträge zur, Anatomie der In. sécten. Altona $1815,40, \mathrm{p}, 17$.

87. Apis mellificae,

Bosc in Nouv. Bulletin de la soc. Philomat. 1812. p. 72. tab, 1. Fig, 3. Dipodium. 


\section{$\div 198=$}

Genus hocce putatitium a Boscro Dipodium dictum nil nisi Ichneumonis larvain sistit a cl. Brilarditre in Apis mellificas abdo. mine repertam.

88. Polypi vulgaris.

Ent. 2. p. 283. n. 38. Sepiae Octopodiae.

Inter ventriculi tunicas Renr reperit, quales ipse tantum in Sepia officinali observavi, nullus enim dubito, quin vermes a Rebro pessime depicti Tetrarhynchum megabothrium sistant. Ego in Polypo nil nisi Scolecem vulgarem reperi, haud vero inter ventriculi tunicas, sed in hujus cavo liberum; Scolex etiam nimis parvus est.

89. Loliginis valgaris.

Ent. 2, p. 285. n. 40. Sepiae Loliginis.

Ipse quidem Loligines entozoorum reperiendo. rum gratia saepius sed frustra secui, nihilo tamen minuş Fasciolam barbatam Linnaei, a Martino in illius intestinis repertam, Tetrarhynchum esse a me in Sepia officinali inventum persuasissimum habeo.

90. Pterotracheae aculeatae.

Ent. 2. p. 286. n. 4r.

Entozoon taenioides Forskantio ex Pterotra.' cheae ano propendens visum, nil nisi hujus ipsissimi Mollusci partem esse organicum Píron et Le eneur (Annales du Muséum d'Hist. Naturelle T, XV. p. 72, Tab. 2. fig. 8.) docuerunt. In Entozoorum catalogo ergo delendum est.

91. Helicis putris. If

Mant, Dub, n. 9r. 


\section{- 199}

In tentaculis Helicis putris LINN. Angustus Ahrens Halae Septembri corpuscula reperit, quae omnino huc facere et novum genus sistere videntur. -

92. Lumbrici terrestris.

Ent. 2. p. 288. n. 45.

Hab. inter Lumbrici terrestris cutem et tubum cibatium, certe nihil nisi Vibrio. 


\section{Conspectus Synopseos.}

Genera. Species distinctac. Dubiae. Summa.

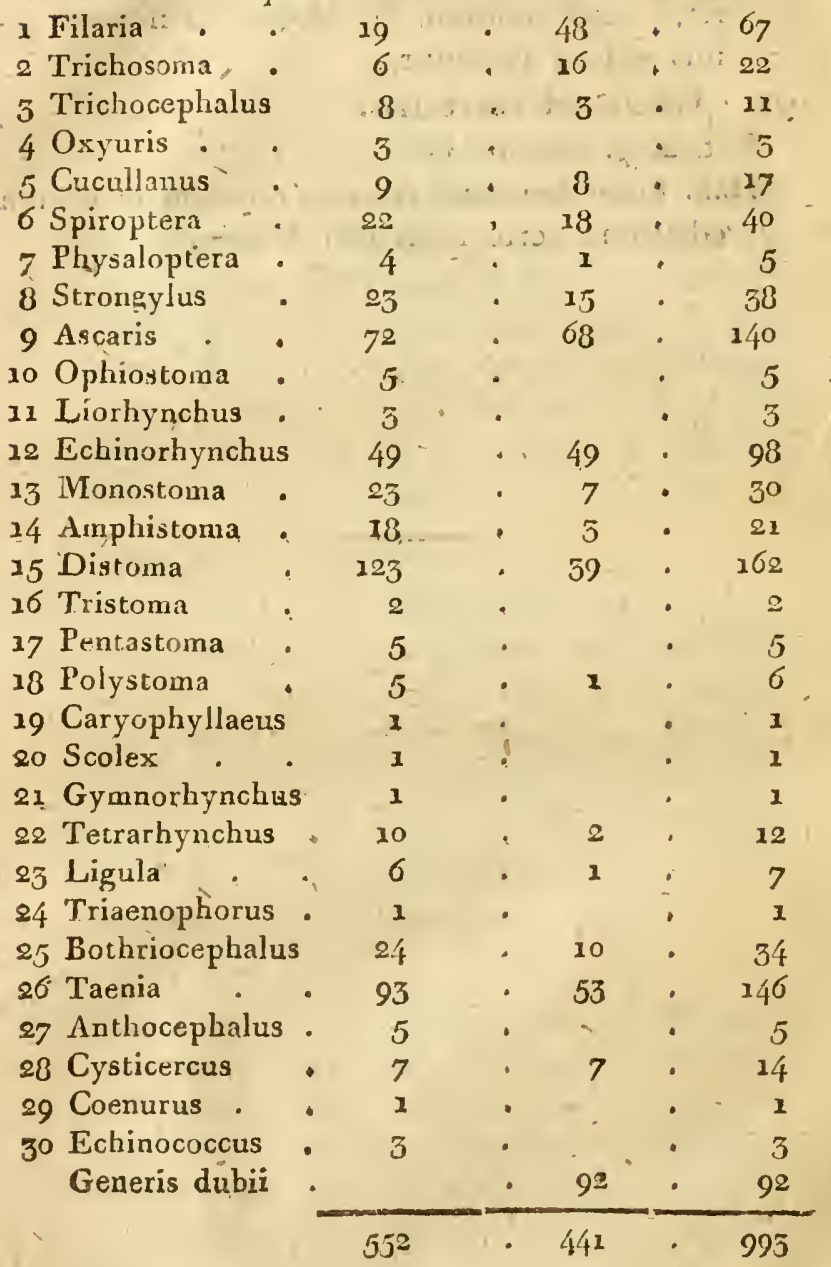




\section{Pars II.}

MantissaEntozoologiae. 
Numeri specicbus appositi illis synopseo praemissae respondent. Quibus siglum no sp. additum est, eae in Entozoorum Historia naturali non occurrunt. 


\section{Sectio prima}

\section{system at ic a}

Generum et specierum in Entozoologia traditorum emendationes, novorumque descriptiones exhibens.

\section{Ordo I. \\ Nematoidea.}

$\mathrm{O}_{\text {rdinis maxime naturalis entozoa illis reliquo- }}$ rum fabricam quoad internam magis sunt composita, ut in sectione insequente (anatomicophysiologica) exponitur; quoad externam vero saepe simpliciora videntur, quia singula ejusdem individua sexum tantum alterum, illa Trematodum et Cestoideorum autem utrumque simul proferunt, quo haec majorem partium varietatem externam exhibeant necesse sit. Inde quoque specierum Nematoideorum expositio difficilior et vix nisi utriusque sexus aut saltem masculi spe 
ciminibus ob oculos positis succedit; maribus autem tam numero minoribus, quam plerumque per breve tantum tempus obviis, illa cognitio nonnisi plurimorum naturae scrutatornm opera conjuncta demum perfici poterit.

Generum hujus ordinis in Synopsi enumeratorup characteres maximam partem boni sunt, quae singulis tamen 'adjicienda videjantur, sin. gula suo loco reperies.

\section{Genus I. Filarià.}

Genus hocce forsan dividi debuisset. Ge. nuinae Filariae sunt!longae, filiformes, stbaequales, plurimam partem molles, cute tenera facile disruptura, (plurimae nisi omnes) viviparae, ovariis longis, apicem versus, tenuibus, ceterum cras. siusculis, ipso tubo cibario crassioribus, genitali masculo simpliciore quám generibus insequentibus, absque vagina, spiculo vel solitario vel duplici, Ab lis certe differunt Entozoa Zedero Capsulariae dicta, qua etiam nomine in Catalogo Musei Viennensis locupletissimo utuntur, quo. rum vero characterem genericum me nullo modo eruere posse, ingenue fateor. Verum quidem est, multas Capsularias in spiram planam (orbi: culum sistentem) involutas reperiri, sed de omnibus lıoc neutiquam valet, ut plurimae forsan e contrario illa nota careant, eadem vero entozois ore tam orbiculari nudo quam trivalvi instructis competat. Zenerus pauca hujusmodi entozoa contemplatus exquisite spiralia, characteres facile posuit, nosmet antem plurima illorum tam spiralia quam rectiuscula cognoscendo, notas genericas bonas vix et ne vix quidem stabilire 


\section{$-205$}

valemus, ut, omnibus collectis, Capsularias ore orbiculari et corpore subaequali ad Filarias accedere, differre autem ab iisdem minore (saepe exigua) longitudine tt majori elasticitate, statuendum sit, donec meliora innotescant signa diagnostica, nomen simul melius prolatura.

Capsulariarum vero charaçteres generis sí difficultate magna laborant, illi specierum multo najore premuntur, quippe quas, speciminibus masculis rarissime obviis aut aeque simplicibus, tantopere convenire videmus, ut longe plúrimas conjungere, vel dubias seponere cogamur, Re autem hoc modo comparata, Filariam tantum Capsulariam ( $\mathrm{n}, 13$.$) inter confirmatas, illam pi-$ scium n. 52. inter dubias Filarius, tertiam vero intêr Ascarides 11. 60. enumeravi, quo hisce locis illarum memoria conservaretur; omnes reliquas ad Entozoa' generis dubii n. 46. et stqu. relegavi. Alii in his extricandis me feliciores sint, opto; quemadmodum vero Distoma appendiculatum; Caryophyllaeus et 'praesertim Scolex plurimis in piscibus saepe diversissimis semper eadem occur. runt, iia etiam illorum Capsularias plurimam partem non distingui, persuasissimum habeo.

Humulariae genus in Entozoologiae T: II. p. I. p. 13. et p. 82. aegre admissum, in Synopsi jure suppressi, qui enim entozoorum sub illo militantium oris hamuli dicebantur, aperte caudae spicula mascula dicenda sunt. Species illius duas priores inter Filarias n. 20. et 33. tertiam vero inter Trichosomata n, 5. collocatas reperies.

1. Filaria medinensis Guet.

Entozsologiam edendo nonnisi aliorum de 


\section{$-206$}

scriptiones et icones ad manus erant, postmodum autem specimina collectionis Blochiranae, quae Universitatis nostrae Museo Zoologico Regis munificentia cessit, pro lubitu examinandi cel. Lichtenstein, Collega dilectissimus copiam fecit.

Caput attenuatum, ore orbiculari exiguo. Corpus aequale, versus posteriora subincrassatum; apice caudali in duobus speciminibus subulato, inflexo, lineam longo; in tertio semitereti, acutiusculo, subincurvo, neque subulato, neque tantopere ac illis curvato. Ante apicem dictum ani orin̂cium.

Maximum horum speciminum, sed haud integrum tres pedes rhenanos attingit; crassities duas lineae tertias partes adaequat.

Caudae varietate commotus, qui pro sexus discrimine haberem, eoque magis deceptus, quia in specimine femineo proles aderat, in Syno. pseos definitione hujus Filariae specifica de utro. que sexu locutus sum, sed onnia illa specimina feminea et proligera esse, synopsi typis impressa, demum novo examine comperi.

Filariae nostrae prole quasi farctae sunt, quod si harum longttudinem illius vero minutiem spectas, foetuum multa millium millia sin. gulis tribuit, Oviductum indoles quoad particulas tantum examinari potuit, sed ab illa aliarum specierum eo recedere visa, quod turicae laeves neque foetuum velamenta fere ulla sisterentur, sed passim tantummodo inter innumeros, foetus grumosi quid adesset. Hi ut plurimum in spiram (duorum cum dimidio orbium) sed etiam in semicirculum aliosque modos convoluti, hine obtusi crassiores, illinc tenuiores, sed apice tenui libero, neque undique pellucidi, sed parte cras. 


\section{$-207$}

siori nebulosa 'utuntur, quibus a Cucullano. rum prole multum recedunt. Confer speciem octavam.

Obs. x. Quo abibit proles illa, an ab ho. mine homini communicatur, an alibi conservanda, demum hominem via incognita petens?

Obs. 2. Num Vena medinensis in America quoque, utrum solummodo in Africa et Asia oriatur, adhuc sub judice lis est; plurimi tamen originem americanam negant, etiam SavaresX novissimus hac de re scriptor (de la Fièvre jaune T. I. p. 5-12.)

Obs. 3. Angli clijusdam (Wurramson Me. dical and miscellaneous Observations relating to the Westindia. Islands T. I, Edinburgh 18:7. 8. p. 57) sententia, portionem Filariae disruptam, et in corpore relictam novum animal sistere, procul dubio rejicienda est, vermis enim talis disruptus citissime collapsus moritur; sed nostris temporibus Filariam istam animatam in dubium vocari posse, fere omnem fidem superat, et exstiterunt tamen qui illam nil nisi cellulosam vel venas emortuas esse contenderint, cl. Larrex in Bulletin de la Soc. Philom. T. 3. (an 12. 11. 83.) p. 178. et cl. Richerand (Nosographie chirurgicale Ed. III. Paris, 1812. 8. Tom. IV. p. 133). Cl. Marc in Lexico Medico (Dictionaire des sciences Médicales T. X. Paris 181i, 8, p. 24ik et sq. Dragoneau) entozoón esse quidem evincit, sed ob apicem unciformem a Filariis genere distinguit, quibus, tamen cel. Laennec, de Entozoologia optime meritus (ib. T. XIV. Paris 1816. 8. p. 493. Filaire) reddidit.

Obs. 4. De Filariae nostrae antiquitatibus conf, J. Fr. M. de Otrers. De. Vegetativis et 


\section{$-208$}

Animatis corporibus in corporibus animatis reperiundlis, Berolini I816, 8. p. 52 .

2. Filaria gracilis $\mathbf{R}$.

In Simiae Capucinae hermaphroditae (pere scilicet, simul autem uteró, tubis, ovariis instructae) abdomine: quinque reperi specimina pèritoneo involuta, immota (Januario, Berolini) aqua tepida affusa, reviviscentia.

Unicum specimen masculum, tres cum dimidio, reliqua feminea septen ad undecim pollices longa. Os rotundum, Corpus ornnium posteriore parte attenuatum, apice caudali in feminis acitiore, inflexo, cauda maris spiraliter devoluta, obtusiore. Intestinum rectun ad apicem cáudae decurrens; vasa seminalia illo tenuiora, oviducíus ampli.

Obs, In Catalogo Entozoorum Viennensi impresso Simix Paniscus et Apella, in manu. scripto etiam Sphins nominatur, quorum in al. domine, Filaria nostra reperta sit.

\section{Filaria attenuata $\mathrm{R}$.}

Entozoologiam edendo Filariam hancce nonnisi in Corvo Cornice inveneram, postmodum autem in Falconis peregrini cella aërea ventriculi nec non in illa hepatis specimina multa, omnia feminea (Novembir, Berolini) reperi, quorum longissimum duodecim cum dimidio pollices aequabat. Cel. Nixzscr eandem in Falconis pe: regrini cella pneumatica laterali vacua magna co. pia reperit, et marem et feminam sub nomine Fïlariae Tendinis nisit, illum quatuor eum dimidio pollices longum, apice caudae complanato et inflexo. In eadem ave etiam specimina plurima uichtestern reperit, et KuUg in Strigis: 


\section{$-209$}

specie incerta, quinque vel sexpollicaria, In Ca. talogo, Viennensi impresso Corsus Corax, Corone, frugilegus, Cornix, Mouedula, glandarins, Caryocatacies, Pica, Pyrrhocorax; Falco peregrinus, lanarius, subbuteo, Lithofalco; in Catalogo manuscripto praterca Falco cyanets, Filariae huic ubnoxii enumerantur.

Habetur etiam in Catalogo impresso Filaris Strigis B̈rachyoti seorsim posita, sed eandem esse suspicor, quam ab am. KIUG in Strize repertam accepi, et ab attenuata Corvorum $\in t$ Falconum nullo modo distinguere valeo.

5. Filaria unguiculata $\mathrm{R} . \mathrm{n}, \mathrm{sp}$.

Arn. Kuvg in abdomine Alaudae arvensis repertam dedit, candidam, sex pollices longam, utrinque, antrorsum tamen magis attenuatam, ca. pite obtuso, (hoc in specimine minus bene conservato), cauda reflexa unguiculata.

Cutis tenera, versus posteriorem vermis partem, rupta. Oviductus intestino fuscescente duplo crassiores, albi maculis fuscis notati, de quibus speciem insequentem conferas.

6. Filaria affinis R. n. sp.

Am. Bremser quatuor specimina misit in Fringillae nova specie Algesirae reperta, quorum duo pollicem, tertium duos curn dimidio, quartum fere tres pollices longitudine aequabant, omnia feminea.

Entozoa albida, pellucida, crassiuscula, mollia, posteriora versus vix crassiora; capite truncato, ore exiguo rotundo, calida obtusa.

Oviductus aupli crassiores in partem longam tenuiorera desimnt, quae ovula inmatura, elliptica continet, ipsi enim majora subrotunda 
foetum convolutum exhibentia fovent; quibus evacuatis albi maculis nigris crelris conspersi apparent, cotyledonibus scilicet relictis. Maculas revera cotyledones esse oviductuum habitus ovis praesentibus rel egestis diversus demonstrat; in evacuatis etiam inter cotyledones nuclas passim quoque ovulum affixum gerentes observavi. Confer speciem insequentem.

Obs. Species haec praecedenti affinis, sed cauda diversa utitur.

\section{Filaria abbreviata R. n. sp.}

Am. Brenser specimina tria mecum communicavit circa" oculum irotacillae stapazinae Aprili mense reperta.

Entozoa octo ad novem lineas louga, "crassiuscula, tenera, omnia disrupta. Capite tenuiore obtuso, ore orbiculari. Corpore aequali, cauda rotundata.

Intestintum aequale ex flavo fuscum. Oviductus duplo latiores, fere ejusdem ac entozoi amplitudinis, albi locis innumeris obscurioribu's longitudinalibus, quibus ova affiguntur (cotyledonibus): haec oblongoelliptica, medio obscura, marģinem pellucida. Oviductibus illis amplis adduntur multoties teuuiores, parte media niğra; illorum certe apices, futurae prolificationi destinati, uti etiam reliquorum animalium ovaria eodem tempore tota aequali modo son evoluta sunt.

Obs. Synopseos parte typis mandata-Brems sERUS entozoa simiinma vel cauem misit in Falconis naevii uaribus et oculis reperta, mascula et feminea. Conf. Entoz, dúb. gen, n, is, ubi fusius de his tractabitur. 
9. Filaria fusca R. n. sp.

Pleuronectes mancos novem Neapoli Junio mense secui, quorum septem aliqúot Filarias in abdomine liberas gestabant.

Entozoa duos ad quatuor pollices longa, rufescentifusca, in aqua frigida (́diei calidi, Neapoli) mox dissiliebant, ita ut intestini et oviductuum pars major vel minor, interdum fere om. nia, plerumque tamen integra, prolaberentur. Ouae aliquot diebus elapsis reperi, mox spiritui vini commisi, quo factum est, ut rupturam et pro. lapsum averterem.

!n Utrinque obtusa, posteriora versus atte. nata, ore ut videtur integro, satis magno et im. merso.

Intestinum ab ore rectum et tenue incipit, - brevi autem subito latescit et tale versus caudam prolongatur, ubi angustius factum oblique ad anum fertur; tolum fuscum est, neque, color ab ingestis oritur, sed tantum augetur; ipsa enim. ejus tunica externa-fuscescit, vasis punctisque albis insignis, Intestino prolapso cuis entozofalbida exhibetur. Oviductus tenerrimi albi pro-lem. vivam quantitate prodigiosa fovent.

3. Toetus prac omnibus, quos in entozois vidi, hoc privi habent, quod canalis eorum cibarius : fuscus jam in matris sremio translućeat, cum ce. teroquin Nematoideorum proles fere tota pellucida sit neque coloribus distinguatur, Confer spéciem insequentem.

9. Filaria, sang̨uinea R. n. sp. Tab. 1.Fig.i. Cyprinus Gibelio digitum longus Martii d. XIV. 1818. allatus est, cujus pinnae caudali Filaria inerat viva, coloris sanguinei, contorta, 
cujusque motus praesertion in utraque extrêmitate recta observabatur. 5 Color saepe hac illave parte subito magis intensus. redditus est, ac si sanguis hic subito stagnaret, dein propelleretur. .

Die XXIX. ejusdem mensis pisce.athuc vivo Filariam utroque fine tunicas pimae egressam, mortuam, partesque propendentes albas. reperi, ut integram extricare non conceduremr. Microscopio subjectam praeter intestimmi fuscum totam quantam foetubus vivis repletam observavi. Hi uti et reliquarum Filariarum majores ac illi Cucullanorum, neque undique pellucidi sunt, sed partem posteriorem (ante caudam longiusculam) opacam exhibent, quo se magis evolutos. esse, forsanque diutius gestari; testantur. Caudis facile invicem adhaerent, et materiam tantum grumosam (placentulas), neque ovula ulla vidi; quibus adnecterentur.

10. Filaria rubella $R$ n. sp.

Cel. Krug in Ranae temporariae ventriculo duas repérit Filarias aiteram tres pollices quatuorque lineas, alteram quatuor cum dimidio pol lices longam coloris pallide rosei, graciles, anrorsum. tenuiores, capite acutiusculo, cauda obtusa.

Ipse (Berolini, Octobris d. IV.) in Rana esculenta naxima, cui nernia magna dorsalis erat (pulmonem dextrum, iutestinorum et ovariorum partem continens), mesenterium et tunicam ventriculi et intestinorum externam tuberculis obsita reperi plerumque compressis, parvulis, sate inter partium dictarum tunicas qua partem látentibus.

In horum duobus maximis letuem sub. aequantibus Filariam offendi vivam, alteram quin. 
decim, aiteram octodecim lineas longam, flavorufescentem, antrorsum attenuatarn, capitis acu'tiusculi ore exiguo,' apice pustico obtuso. Intestinnm rectuin subaequale, ab ore ad apicem caudae porrectum. Genitalia conspicua non fuerunt,

In minoribus quoque tuberculis Filarias solitarias reperi, sed mutro minores et tenuiores, fuscescentes, mortuas, (indeque forsan) inaequales; num easclem, ac majores?

Num Filaria (n+5I) MLusei Viennensis sub cute Ranae esculentae reperta huc pertinet?

\section{Filaria ovata ZED.}

Am. Gaede Filariam attulit in Cyprini Go. bionis abdomine a se repértam, sed brevi interanea effundentem, quo factum est, ut entozoon corrugatum collaberetur, et pauca dignoscere liceret.

Corpus antrorsum attenuatum. Caput num vere ovatum? Cauda emarginata.

In Catalogo Entozoorum Musei Viennensis haec species tam in abdomine Cyprini Gobianis quam in hepate Cypr. Phoxini reperta enuineratur,

\section{Filaria papillosa R.}

Iu Annalibus medicis Austriacis (Medicin. Jahrbücher 'des K. K. österreichischen Staates. 2. B. 2. St. Wien. 1813. 8. S. 174-r78.) casus narratur Filariae papillosae in dextro equi oculo per aliquod tempus vivae observatae.

Oculus lucis sensu laud carebat, ceterum maxime laesus; cornea maculis multis exiguis opacis foedata; neque pupilla, neque iris conspi 
cuac; lens quoque cum capsula destructa; ut totus oculús humore nebuloso repletus videretur.

Entozoon in oculo velocissime agitabatur, albidum, crassitie fili linei duplicati, unum cum dinidio pollicem circiter longum:

$\mathrm{Ob}$ longitudinem et motum Filariam fuisse concederem, sed color ab hầc alienus est, quam semper griseam vel fuscescentem vidi, ut oculum non dissectum fuisse doleas. - Alia exempla cel. OlfÉRs refert (1. c. p. 54.) quae pariter pro Filaria militant; cl. tamen Greve (Ueber die Krankheiten der Hausthiere. Oldenburg 18r8. 8. p. 174.) quem in oculo equino vidit, Strongylum minorem suspicatur.

17. Filaria truncatula R. n. sp.

Cel. de Baer, Professor Regiomontanus, Filariam misit in Phalangii Opilionis abdómine a se repertam, in aqua autem disruptam, ut integram non acceperim.

Pars mihimet oblata fere bipollicaris, tenuissima, alba; capite truncato; ore, ni fallor, sex papillis cincto; parte posteriore paululum increscente.

Tubus cibarius rectus, in quadam ab ore distantia constrictus.

18. Filaria plicata R.

Cl. olim Hübser, Naturaescrutator Halensis, Filariam misit in Larva Bombycis Salicis a se repertam, sex cum dimidio pollices longam, gracilem, flavescenti-brunnteam, tenacissimam: capite curtinno truncaio, microscopit compositi ope, haud tamen distincte, papilloso viso; cauda crassiore obtusa.

Obs. A, I, truncata $\mathrm{n}, \mathrm{II}$, distinxi, quia 


\section{$-215=$}

caudae acumen in hac observatum nostrae non conceditur; Plicatae autern cauda eadem est, et papillae mihi visae Zidero forsan plicae dictae sunt.

19. Filaria gloliceps R. n. sp.

Neqpoli Junii d. 1X. in Uranoscopi seabri ovariis (ovis turgidis); Julii d. VIII. in Urano. scopi testiculis et in alterius ovariis, sed in utriusque tiam omniun abdominis partium peritoneo; Junii d. 'XV. in Blennii Phycidis ovariis (horum maximam partem vacuorum tunicae internae affixas) complures reperi-Filarias.

Entozoa albida vel fusca, pollicem ad unum cum dimidio pollicem longa, tenuia.

Caput rotundatum. Os orbiculare parvum, interdum valvulis quasi instructum, alias papillatum visum, aut caput obtusocostatum exhibitum. Corpus aequale. Cauda depressa obtusissima, vix capite tenuior.

Cutis tenera, qua facillime disrupta interanea prolabuntur. Intestinum fuscum seu nigrescens vasis minus fuscis recticulatum; collapsum granulatum videtur. Oviductus vacuos tantuns vidi albos. Genitalia externa in conspectum nor venerunt. An huc n, 54.?

$$
\text { Species dubiae. }
$$

20. Filaria IIominis bronchialis.

fiamularia lymphatica. Treutreno dicta, in Iominis tuberculis seu glandulis bronchorum rcperia, in cel, viri collectione, quam Dresdae 18 I 7 videre mili contigit, non anplius occurrit. Cum reliquis tamen Filariis nimis convenit, praesertim cum 11. 25. quominus seorsim enumerem, e* 
si olim separanda videhitur, character tamen t amulariae retineri nequit, gui enim orishamuli laterales dicti cerie nil nisi spicula mascula sunt.

24. Filaria Innstelarum subcutanea.

'Rosa (Lettere zoológiche. 'Pavia. I794. 4. p. 2.) Februario, Martio, Majo, sub pelle Foince praesertim slib illa dorsi multas reperit, Filarias duos ad sex pollices longas, fili linei duplicati crassitie. Amicus cel. viro se tales sub Foina. rum pelle semper invenisse retulit.

Catalogus Musei Entoz. Viennensis Filarias tantum Martis subcutaneas enumerat. Ipse tales hactenu's nondum offendi, - Confer Ent. II. 2. p. 379, n. 57.

25. Filaria Mustelarum pulmonalis.

Quam in Entozoologia (II. 2. p. 263. n. 3.) inter entozua dubia retuli, omnibus collatis huc amandari debet, speciei vigesimae quoad habitum et locum proxilla. Ipse Gryphiae Augusto in Martis pulmonibus copiosam cum prole viya rẹperi et 1 . c. descripsi,

28. Filaria Leporis pulmonalis.

Filaria pulmonalis, Froelrcir im Naturforscher T. XXIX. p. 18-20.

Copiosissimas, saepe in globulos convolu. tas in Lcporis timidi masculi, junioris, sanissimi bronchis reperit

Descrifto Frozlrchiana. Vermes quinque vel sexpollicares, vix ultra sextam lineae partem crassi, parum elastici, facillime disrumpentes. Capitis continui obtusi os non dignos. cendum erat. Corpies antrorsum magis attenua. tum, albidum, pollucidum, vase medio fusco; retrorsum sensim nec valde incrassatum; cauda 


\section{$1-217$}

acuta longitudinaliter striata, ut angulata vide. retur.

Tractns intestinalis parum tortus, fuscus, tener. Ovaria longissima, capillaria, ovulis duphi. catis (Der paria dispositis) brunneis referta. Vasa ahuc tenuiora horum ultimos fines sistere ridentur.

Auctor ob locum alienum a Filaria Leporis subcutantea diversum credit, quod facile concedo.

Obs. Num Filaria sit, dubium videtur. Strongylum retortaeformem ( $\mathrm{n}, \mathrm{I} 7$.) a Treutrero in Leporis bronchiis repertum uticue diversum hạbeo; sed ovaria tenuissima ovis per paria dispositis Trichosoma arguunt, In Catalogo Entoz. Vienn, 181 r. impresso Filaria Leporis pulmona. lis, sed in mantuscripto 1818 . mecum communicato ejus loco Capillaria pulmonalis enumeratur, quod rem conficere videtur, Capillariae enlim Trichosomatis nomine milhi dicuntur.

\section{Filaria Collurionis subcutanea.}

Rosa (Lettere Zoolog. p. 3.) Filarias decem lineas longas, molles, parum elasticas, alterain versus extremitatem tenuiores; unam in $\mathrm{Ia}$ : tere sinistro, alteram sub collo Lanii Collurionis Maji d. XIX, r790 reperit.

Num ad insequentem pertineat, in Entozoologiae Vol. Ir. P. r. p. 7r. n. 20. interrogavi, sed in Cat. Vienn. manuscripto Filaria Lanii Collurionis, minoris et pomerani subcutanea, insimul vero Filaria Collurionis pulmonalis, scorsin cnlimerantur.

33. Filaria Collurionis pulmonalis.

Zederó Tentacularia, Schranko Linguatula dicta, Hamularia cylindrica Entozool. Vol. If. 
P. I. p. 83. n. 2. procul dubio cum Entozois bronchialibus supra dictis vel ad Filarias emendanda erit, vel proprium genus efficiet hisce proximum, characteribus olim exhibitis prorsus delendis, hamuli enim oris dicti spicula caudae masculae sunt. Confer speciem praecedentern.

40. Filaria Ardeae nigrae.

RosA (Lettere Zoolog* p. 4.) in Ardeae nicrae mesenterio tres reperit Filarias intestina circumvolutas sordide albas hine subpellucidas, versus alterum extremum opacas, noyendecim ad viginti duas lineas longas, dimidiam crassas. 46. Filaria Lari.

Am, Bremser Filariam mecum communicavit sub Lari minuti masculi cute colli Aprilis d. \&4. repertam, quatuor pollices et tres lineas longam, mediocris crassitiei, albidan, versus alteram extremitatem attenuatam. Plura in specimine reddendo extricare non licuit.

52. Filaria piscium.

Entozool. Vol. If. P. I. p. 74.

In Lophii piscatorii; Gadi Aeglefmi, barbati, Brosme, Callariae, islandici, Molvae; Pleuronectis Platessoidae; P'ercae norvegicae; Salmonis arctici variis partibus ab auctoribus occurrere dicitur, sed entozoa plurima hoc nomine confusa videntur, quorum forsan nullum veram Filariam sistit.

6o. Filaria Forficulae.

Scriroedzr, Entomologus Berolinensis, AuSusto 1816 Forficulam Auriculariam attulit, cujus ¿: corpore Filaria qua partem egressa et in situ conservata et vernice obducta est., Praeparatum cgregiun, quo Filaria quasi viva sistitur, turgida et colore. naturali insignis. Partis egressae 


\section{$=\quad 219$}

extremum obtusissimum, corpus aequale crassius: culum, aliquot pollices longum, albidum.

\section{Filaria Erucarum.}

Synonymis in Eutozoolngiae Vol. II. P. T. p. 79. 11. 4I. addatur: Scrmank Reyträge zır Naturgeschichte (Leipz. 1776. 8.) p. 93. Tab. IV. fig. I. (rudis). Gordius insectorum.

Varias erucas, praesertim illam Papilionis Polychlori hisce entozois obnoxias esse. Filariam plerumque solitariam offendi, fliformem, longissimam, $\in \mathrm{x}$ flavescente albam, hinc acutam, illinc obtusam. Prope insecti valvulam caudalem ex ejusdem abdomine prorepere, quo facto et hoc et illa brevi moriantur. ScHraxk.

Passim in fructibus v. c. pyris et pomis ejusmodi Filariae reperiuntur, quarum indolem cel. Gravfinorst in epllemeridibus Vossianis (Berol. 1818-13. 26. Februar. XXVILI.) optime exposuit; Filarias ninirum esse larvarum, praesertin P'yralidis Ponrunae, et monnisi in fructibus insecto punctis occurrere.

Ejusmodi Filariam pomi in Museo Vien. nensi vidi et consinilem cel. Treutrer dedit ante complures annos a se in pyro Berganotte dicto (quoad meminerit, integro) repertam, quinque cum dimidio jollices longam, capite tenuiore obtuso, caluda crassiore acuta, corpore aecuali.

\section{Genus II. Trichosom}

Capillarias Zenerr et Musei Viennensis cum Trichouchalss in Eutozoologia conjunxerarn, et illarum speciebus tum temporis paucis cognitis conjunctio pracferenda videbatur; illis autem 
postmodum magis magisque auctis, cel. virorum vestigia premo, nomen, solummodo mutando, quo generis character, insequentis (Trichoce. phali) ratione habita, melius exprimatur. Irabitaculum Trichosomatum quam Trichocephalorum magis extensum est, et illorum toto corpore tenuissimo, insensibili fere modo retrorsum incres. cente, Trichocephali vero parte posteriore subito. et valdopere incrassata, habitus alienus redditur. Omnia autem reliqua, os et tractus intestinalis, utriusque sexus genitalia externa et interna, quin ipsa ova ovalia utrinque nodulo aucta, conve. niunt, ut divisio ommino artificialis sit,

2. Trichosoma obtusiusculum R. n. sp.

Am. Bismser specimina quatuor feminea inter Ardeae Gruir tunicas ventriculi reperta misit; marem non vidi.

Entozoa duodecim ad octodecim linéas longa, capite acutiusculo, parte anteriore capillari reliquae sensim crassiori redditae subaequali, apice caudali obtusiusculo incurvo.

3. Trichosoma obtusum $R$.

Ent. T. p. 37. n. 2. Trichocéplualus tenuissimus. Froenich in Naturforscher St. 29. p. 2I-23. Tab. I. fig. IO-12. Filaria Strigis.

Fntozoa in Strigis Bubonis intestinis coecis Julio Gryphiae a me reperta et in Entozoologia fuse descripta cum descriptione illorum a Froz. Incuro in Strigis passerinae coecis inventorum prorsus conveniunt. Ipse tamen specimina mas. cula fenineis duplo breviora, ille autem his aliyuantum longiora, observavimus.

Eadem in intestinis crassis Strigis Aluconis, 
dasypodis et Oti- repcrta esse, Catalogus Ent. Vienn. Confer specien n. 10.

4. Trichosoma inflexum R. n. sp.

Complurä specimina in intestinis Tirdi cyanei- Septeilbtis d. XVII. Ists.' Neapoli reperta cel. Bremseri benevolentiaé debico.

Entozoa , tenuissinı, pollicem plus minus longa, capite acutiusculo, posteriora versus parumper increscentia, apice caudali obtuso, in. curvo.

Maris unici minoris cauda inflexa ante apicen: processum obtulit' brevem obtusum, an-vaginam penis?

In omnibus feminis ova vidi oblonga utrin. que obtusa et pellucida, serie plerumque simplici, irregalari tamen disposita; versus caudarn ni fallor accumulata.

\section{Trichosoma longicolle $\mathrm{R}$.}

Ent. I. p. 84. n. 3. Hamularia nodulosa.

Frozicar in Naturforscher St, 2q. p. 29, 29. Filaria Tetricis. Gordium Gallinae Gozzıo dictum Znderus recte ad Capillariae genus aman. daverat, uti ex speciminibus didici ab indefesso olim Hüвxero in intestinis Phasiani Galli repertis et mecum communicatis, omnihus quidem femineis, sed toto habitu et ore simplici, minimo, punctiformi, ad seliqua Trichosonata accedentibus.

Froelichurs 1. c. specimina feininea de. scripsit Majo mense in intestinis crassis Tetraomis Tetricis a se reperta, duos cum dimidin ad tres pollices longa, conglnmerata ct inter se cbintorta. Pars antica totius entozoi tres quartas partes sibi vindicabat; caput indistinctum; catudo. 
emarginata erat. Ovula pro more disposita.

Synonymon Pepir a. cl. viro luuc relatum ad Ascaridem comparem pertinet.

Secundum Catalogum Entoz. Vienn. in crassis Phasiani Galli et colchici, Tetraonis Uro. galli et Perdicis occurrit.

\section{Trichosoma Plica R. n. sp.}

In Canis Lupi Januarii d. 22. I817 Berolini a me dissecti vesica urinaria entozoa reperi -glonere fere inextricabili convoluta, cinerascentia, parte, anteriore capillari tenuissima longissimaque sensim in partem crassiorem abeunte, apice caudali obtuso, obiter incurvo.

Coula in aliis ductibus simplici, duplici in. alis serie occurrerunt, eadem ommino forma iusignia, qua in Trichocephalis occurrunt, ovalia nimirum utroque extremo nodulum 'pellucidum sistente.i

\section{species dubiae.}

Huc etiam Filaria Leporis pulmonalis Froz. Irmo dicta, synops, n. 28, de qua supra fusius egi, amandancia videtur.

\section{Trichosoma Falconum:}

Entoz. T. II. P, 2. p. 37s. n. 7. a. Falc. Milvi. 1. 340. n. 18. Falc. Buteonis.,

Froflich in Naturforscher St. 29. p. 20. 1. 5. Tab. 1. fig. 7 -9. Fildria Milvi.

Entozoa a Froenchio in Falconis Milvi crassis reperta hic pertinere dubium nullum est.

Sesquipollicaria, tenuissina, compresso.teretia (?), parte anteriore recta versus caput atte. 
nuata, posteriore crassiore, subcompressa, ápice caudali obtuso. Ova ovalia duplici serie disposita.

Qui a specie tertia, nisi corpore forsan magis recto, distinguam, non invenio. In Caralogo Viennensi manuscripto alias ditissimo Falconis Capillaria non enumeratur, neque ipse talem de. prehendi.

\section{Trichosoma Corvorum.}

Zener (Naturgeschichte der Eingeweidewürmer p. fin. $I$. IoI, se in Ciorvo Varyocatacte Capillarias reperisse refert, sed non describit. In Catalogo Yiennensi Capillariae in intestinis Corvi Monedulac et Picae repértae habentur.

\section{Genus III. Trichocephalus.}

Hujus et praecedentis generis affinitateni supra exposui. Species ab auctoribus passim male descriptae nunc quiden naribus cognitis optime determinatae, haud numerosae videntur, et Trichocephalum echinatum, in ventriculo Lacertae apodis obvium, revera a reliquis distinctum et facile novo genere (Sclerotrichum) enumerandum si exceperis, omnes in Mammalium crassis habitant.

\section{Trichocephalus palaeformis R. n. sp.}

In Simiae ursinae pridie mortuae coero et summa coli parte specimina feminea tria unumque masculum, mortua quidem sed recentissima Septembri mense Berolini reperi.

Longitudo maris fere quindecim, feminarum 21, circiter lineas aequabat, cujus tertian 
fere partem corpus sibi sumsit. Color albus, 'corporis parte longitudinaliter cinerascente.

Os orbiculare; capitis apex minus tenuis ac Trichocephali humani, fere unguicularis. Reliqquam quoad formam cum illo conveniunt, excepto tamen genitali masculo, qû́od valde singu. lare dicendum est. Est scilicet terminale; 'vagina vero primum cyinctrica et aequalis tanden in parten palaeformem magnam et trúncatam ampliatur, ex qua stylus longissimus curvus emergit. Hic per vaginam decurrens vasculo corporis seminifero inseritur et ejusdem est cras. sitiei.

Ovula utrinçue nodulo aucta nonnisi in crassa Trichocephali parte occurrunt. Tractus intestinalis ab illo humani eo diversus est, quod magis undiique constrictus sit et fere Hirudinis intestinum referat.

Obs. 1 . Specinnina in Simize Sphingis intestinis reperta, cyuae Bremserus noster misit a meis non differmnt, sed Simia ursina et Sphinx eandem etiam speciem effngere videntur. In Museo Vlennensi praeterea specinen et masculum et temineum in Simine liatae et femineum in Simiae Subacae intestinis crassis ubiaia, pror. sus convenientia vitii. Geniale masculum in nullo satis evolutun est.

Obs. 2. Cel. Thentrem aliquot dedit Trichocephalós in Simia Satyrn Octohri 1791. a se repertos, sed Satyri loco certe $S$. Sylvanus intelligendus esc de cujus ento\%cis cel. vir in dissertatione sua estegia 179\%. Edita ioquitur; Sinia Saypus munquan Lipsiae viva visa est.

Trichocephali illi cumi palaeformi congruunt; in unico tantum genitale masculum bene 


\section{$-225$}

explicatum, terminale, a basi sensim dilatatum, apice truncato, sed ibidem trigonum videtur, quod a vario forsan entozoi statu pendet.

3. Trichocephalus affinis $R$.

Anr. Nitzsch specimina duo feminea misit, in Cervo Capreolo reperta, quorum alterum 22, alterum is lineas longum fuit, hujus paitè crassa quinque, illius sex sibi vidicante lineas; quae certe partis capillaris longitudo eximaia Trichoce. phalum affinem arguit, licet mares non vidi.

BREMSERUS specimina aliquot misit in intestino cotco Antilopes Rupicaprae inventa, mas. cula pollicern et novem lineas, feminea duos pollices et tres lintas longa, corpore totius longitudinis duas nonas partes sibi vindicante: Tota corporis ratio eadem ac Trichocephali affinis, quem ipse in Ovis et Bovis cocco Grypbiae Junio et Novembri reperi, sed in meis specininibus genitale masculum minus longum est. Hoc ceterum in altero Bremserr specimine vaginam tenutm longiusculam incurvam, ex altero apicis caudae latere egredienter, stylo non emisso; in altero consinulem sed rectam obtulit, Ova pro more.

Idem cel. vir specimina duo mascula, tertiunque femineum nisit, in Antilopes Dorcadis intestinis crassis reperta, duos pollices et quod excurrit longa, parte capillari longissinia, longitudinis totins ultra tres tertias partes sibi viudicante. Genitale masculum haud exsertum est.

4. Trichocephalus unguiculatus $\mathbf{R}$.

Anı. Braun 'Trîchocephalos plurimos misi' ante complures annos in Cuniculo fero a se re- 
pertos, cuin illis Leporis timidi prorsus convenientes.

Genitale masculum vaginam longissimam filiformerr apice truncatam sistit, ex quo styli pars brevissima emergit, ut initio hanc prattervidendo vaginam pro stylo habuerim, sed iterato examine. rem melius cognovi.

Bremserus specimen femineum dedit in Arctomydis Citilli coeco lectum, decem lineas longum, quarum septem partis capillaris sunt, fuscum, cum leporinis omnibus notis congruum, a Trich. nodoso n. 7. cui in Catalogo Entozooruna Viennensi adnumeratur, diversissimum.

5. Trichocephalus depressiusculus $R$. Quem Fromlich in Vulpe detexerat, Bremserus misit in coeco Canis familiaris repertum.

Descriptioni in Entozoologia datae quae addam, pauca sunt. Caput integrum neque emarginatum est. Os non vidi. Vesiculae colli spuriase de quibus Ent. r. II. p. 95. in Observatione locutus sum, in meis speciminibus utique desunt. Genitale masculum Jongissimum, duas lineas longum! Ex apice caudae obtusae oritur, fili instar tenuis, quod primo solum conspicitur; attentius autem contemplanti vagina ob oculos ponitur summae tenuitatis et pelluciditatis, primam circiter tertiam partem fili ambiens. Ova nondum evoluta erant.

\section{Trichocephalus crenatus $\mathrm{R}$.}

Cl. quondam HübNer specimina misit complura in intestinis crassis Suis domesticae a se reperta, hunanis nonnihil majora, vel illorum maxiinis atqualia, albida.

Feminac corpus ejusdem formae, ac Trich. humani, capite acutiusculo, interdum perforate 
viso; parte capillari transversim striata, crassa obi: ter neque constanter crenata, minime squamata, quo specimina Goezio oblata (Ent II. P. r. p. 96.) collapsa fuisse colligas. Maris corpus spirale. Genitale compositum. Apex scilicet caudae vesiculam offert lateralem, magnam, bilobam, con. tinentem: tubum fere duplo breviorem, basi angustatum, apice truncatum, ex quo emergit: filum tenuins, cylindricam vesiculam longitudine su. perans.

In Museo Viennensi non occurrit, ipse nunquam offendi, et Goezro specimina tantum duo mutila, Majo mense in Apro inverita, data sunt, Hübaerus vero copiose reperit.

\section{Trichocephalus nodosus $R$.}

Hujus etiam speciei specimen Hüsnero debeo in Mure musculo repertum, femineum, fere pollicare, parte capillari corpore incurvo nonnihil longiore.

Caput acutum est, nodulis (quos Govzrus refert) destitutum, quemadmorlum collum vesicuils cl. viro dictis caret, quas casu oriri in Ento. zoologia saepius monui.

Froeurca (Naturforscher St. 29. p. 30. n. 8.) Trichocephalum IIfuris masculum describit. Ca. pitis nodulos non vidit, colli vesiculas unilatera. les refért. Apex caudae vesicam obtulit lateralem, rotundain, majusculam, pellucidain, ex quz tubus emergit striatus, infundibuliformis, apice truncatus, flum emittens cum corporis vase (se. minali) continuum.

Musei Viennensis specimina (paucissima) in intestino coeco Muris amphibii, arvalis, Mus. 


\section{$-228-$.}

culi, Ratti et sylvatici reperta sunt. Ipse nun. quam offendi.

$$
\text { Species dubiae. }
$$

\section{Trichocephalus Lemuris.}

Spécimen femineum in intestinis crassis $L_{\boldsymbol{e}}$. muris Mongoz repertum in Mruseo Viennensi vidi et examinavi; cum Trichocephalo Simiarum palaeformi nitique congruere visum est, scd maribus nondum notis species dubia manet.

10. Trichocephalus Castoris. '

Hujus quoque specimen tantem femineum in Museo Viennensi datur.

11. Trichocephalus Cameli. \&

In intestino coeco Cameli et Dromedarii et Bactriani reperta specimina tam mascula quam feminea Catalogus. Viennensis manuscri. ptus enumerat.

\section{Genus IV. Oxyuris.}

Trichocephalum equinum Goezrr nequaquam ad genus antecedens pertinere, partemque illius crassam non posteriorem sed anteriorem esse, conspiciendo, Oxyuridis genus et nomen posui, illud quidem necessarium, hoc minus bóllum, dum maribus ignatis feminas solas prae oculis. habuerin. Tertiae autem speciei maribus cauda súbulata deest, forsanque fcminis solummodo talis cauda competit, quod nomen fingendo non sus. picatus sum. Hoc tamen plurimis receptum mutare nolui, maribusque tantopere raris, ut illi speciei primae vulgatissinae nuspiam visi sint, femiais isthoc in genere major honos debetur. 


\section{- 229}

Species tres enumeravi, reliquas duas BremSERO dictas inter Ascarides (vermicularem n. $3 r$. et of relatann n. 32.) reperies. Si harum autem caput, examine saepe iterato mihimet trivalye visum, tale non esset, cum aliis bene multis, v. c. Ascaride ltptura n. 47. et affinibus ad Oxyuridis gerus amandandae forent.

1. Oxyuris curvula $\mathrm{F}$.

Quam hactenus in Equo iantum ofiendimus, eel. Greve (Erfalhrungen etc. p. :69.) in intestinis crassis Asinorum praesertim malleo humido laborantium frequentem observavit.

\section{Oxyuris alata R. n. sp.}

Specimina tria in intestinis crassis Melis Taxì reperta Bremserus misit, omnia feminea, tres lineas longa.

Caput obtusum, antrorsum tenuius utrinque alatum, alis posteriora versus decrescentibus. $O$ i simplex or hiculare visum, absque valvularum vestigio. Corpus crassiusculum in candam subulatam excurrelis, "quae ova continet oblonga, ejuscem indolis ac illa Oxyuridis curvulae.

Obs. Habitu ad Accaridem vermicularem ac. cedit, sed valvuliśn non visis, anicum secutus sum, qui ad Oxyuridis genus refert.

3 Oxyuris ambigua R. n. sy.

Specimina plurima in intcstinis crassis Cunienli reperta Bremserus misit, et entrzocu hocce in irlis Cuniculi et domestici et feri, nec non $/ \mathrm{em}$ poris timidi occurrere Catalogus Viennensis rcfert.

Mas duas tresve lincas longus, plus minus spiralis in spiritu lavescens. Ciput attenuatum, ore orbiculari, unediocri, corpus postcriora verøus 
subincrescens; cauda pone foveam lateralem apicom tenuiorem, incurvum, ex quo semel stylum cylindricum satis longum exsertum vidi. Oesojhagus recrus, ad cardiam constrictus; ventriculus subglobosus, intestinum rectum.

Femina eluplo major, dimidium attingens pollicém, mare magis albens, Caput maris; corpus rectiusculum aut obiter curvatum, utrinque attenuatum; cauda capillaris apice subulato, quartam totius longitudisis partem sibi vindicans. Oerophagus et ventriculus maris; intestinum tenue et flavum per partem capillarem continuatum; apex subulatus diaphanus est, ut in strictura, qua ille incipit, anum adesse crediderim.

- Ova elliptica in animalculo exiguo aeque magna tamen sunt ac illa magnae Oxyuridis curvulae; sed qui hujus parte tenui tantum continentur oviductus, Oxyuridis ambiguae partem cras. sam solummodo occupant.

Obs. Speciem hanc ambiguam dixi, quia genitali masculo et ovorum situ ad Trichocephialum, reliquis autem notis ad Oxyuridem accedit et inter utrumque genus transitum oflert.

\section{Genus V. Cucullanus.}

\section{Cucullanus elegans Zedert.}

Cucullanos armatum, papillosum et coronatum ZEDERI in Entozoologia dictos ad cl. viri Cucullanum elegantem amandare coactus sum, cum nullum illos inter discrimen reperian.

Speciem hanc in ventriculo et appendicibus pyloricis Percac Luciopercae et fuviatilis, in illius etiam mesenterio saepissime varioque anni 
tempore; in Esocis Lucii summa tubi intestina. lis parte Majo bis copiose, in Gadi Lotae majoris toco intestinorum tractu copiosissimum $\mathrm{De}$. cembri, Gryphiae reperi.

Berolini Junio inense duo specimina sanguinea, tres et quod excurrit lineas longa, feminea, vulva valde exstante, alterum foetubus vivis instructum, nulla prorsus nota a Cucullano elegante discrepantia in Cyprini Aspii parte intestini superiore offendi.

Specimina quatuor in Muraenae Anguillae ventriculo et duodeno reperta, sub C. coronati nomine Hübrervs misit, sed omnibus gotis cum C. elegante convenientia. Caput utique inerme dentibus Goezro dictis caret.

O. Fr. MüLter Helminthologorum olim decus eandem speciem in Perca cernua detexit; feninam in intestinis Percae Zingel repertam Ca. talogus Viennensis mscptus refert.

Synopseos species III et XVII. huc pariter forsan pertinent.

\section{Cucullanus truncatus R. n. sp.}

In Siluri Glanidis intestinis Gryphise Octo. bri reperi et in diario Societatis Berolinensis (Magazin der Gesellsch. Naturf. Freunde Vol, VI. p. 88.) descripsi.

Mas unicus, quinque feminae milhi oblati, tres ad quinque lineas longi, primo intuitu speciei praecedenti simillimi.

Caput obtusum, cucullo ovali, longitudina-, litę striato, antice striis aliquot brevibus retrorsum flexis aucto, sive, iisdern pro uncinulis sumtis, armato, apophysi (in cuculli basi) mox aequali, mox denticulis quatuor vel quinque posticis in. 


\section{$-232-$}

signi. Vàsa cuculli lateralia qualia speciei primàe; macula lateralis pellucida quam in hac longior. Corpus ejusdem formae. Cauda maris acuta, hialata; feninae attenuata, acumine brevi truncato, vel truncato-excisa: ostio anali exquisite terminali.

Canalis intestinalis qualis Cucullano eleganti, oviductu tenuior, sed distingte ad apicem fosticurn extensus, Vulva in parte corporis fere media, magis tamen posteriore; in gravidis maxima, utrinque tumida, non emarginata, in vir. gineis exigua, Oviductns qualis eleganti; foetus vivi vero quam in hoc majores, ceterum ejusdem formae, sed absque involucris (forsan exclusioni proximi) liberi in oviductu agitabantur. In specimine virgineo oviductus corpusculis granulosis difformihus $r \in$ pletus erat.

Obs. Species variis notis, praesertim vero cueullo ovali, longiore, dentibus armato, caudaque feminae excisotruncata, a prima distinguitur.

5. Cucullanus melanocephalus R. n. sp.

In appendicibus pyloricis Scombri Sardae, inque intestinis Scombri Rochci et Coliae (semper unicum specimen, bis masculum, semel femineum) Jurio et Julio Neapoli reperi.

Cucullanus iste sex cun dimidia ad octo lineas longus, tenuis rubellus, apice caudae quám reliquum corpus intensius rubro, capite aterrimo.

Cucullus capitis solitus sed niger, quo ipsius striae minus bene in conspectum veniunt, infra eunjem apophysis vulgeris rubella vel flavescens depresso-rotundata, ab ejus vero parte anteriore utrinque vas nigrum laterale (hamulus sic dictus) decurrit ramos duos breves introrsuru mit- 


\section{$-233$}

tens, eucullo duplo longius. Tubus cibarius nihil peculiare habet. Caudac masculas dicpressae incurvae membrana utrinque lata pchlucida illam bialatam reddens. Ante apicem caudae tenuen papillárem spicula dio brevia in altero spccimine vidisse mihi visus sum. Canlda. feminte obtusa depressa. Specimen virgineúm, quo neque integro neque fisso ova vel foetus in conspectum veriehant.

Entozoa haecce vividissimo motu pollent et facillime parti admotae oris ope fortissime aff. guitur

Multos Scombros Arimini et Neapoli secui, sed nonnisi ter solitarium reperi, quem ab omni. bus reliquis Cncullanis primo intuitu distinctissimum habebis.

\section{Cucullanus foreolatus $R$.}

Quem O. Fr. MüLxtr in intestinis Gadi Morhuae, forsan tiam in illis Gadi Callariac detexit, ipse in mesenterio Blenuii Phycidis et intes. tino summo IJuraenae Helenae Junio, in peritoneo tritum Muraentarum Cassini Julio Neapoli reperi; ibidem in Helena inventum Breviserus mecum communicavit.

In Phycide marem fere octo et feminam fere novem lineas longos reperi, flavescentes, vivos.

Caput interdum foveolatum videtur, sed vere globosum est et utrinque aequaliter convexum; rasa autem sivi hamuli, varia enim specié occurrunt, cucullum cingentia, hune quasi impressum referunt sive foveolatum. Canda maris in. curva, acuta, spiculo longo acutissimo (an duplici?), ante illius apicena ex vàgina peculiari 
emergente. Canda feminae recta subulata. Ütrique sexui alae caudales desunt, neque feminae caudam mascula tenuiorem esse, Mültero concedo. Tubus intestinalis superiori parte constrictus. Ovaria cvis oblongis foeta.

Quae in Helena reperi specimina pauca, cito disrupta, tam Neapoli quam postmodum Berolini a me examinata, huc quidem facere visa sunt. Illa vero duo Musei Viennensis mecum comınunicata clubia sunt; alterum tres, sex lineas alterum excedebat, corpus utrinque acuminatum; apex caudalis incurvus erat, unciformis.

Eadem Entozoa in tribus Muraenis Cassini obvia capitis cucullum diștincte referre videbantur, peritoneo pleruinque firmiter involuta.

Obs. An Phycidis et Gadorum Cucullani a Muraenarum entozois dictis diversi?

\section{Cucullanus abbreviatus f. n. sp.}

In Pereae cirrosae magnae, 25. circa libras pendentis intestino tenui Romae Maji d. XXX. plurima reperi specimina tres ad quinque lineas longa, alba, teretia, intestinum ore fortiter pren. sitantia.

Caput obtusum, corpus posteriora versus altenuatım, apice caudali brevi acuto, fere, papil. lari. Cucullus capitis plerumque distinctus, seme] contractus partem capitis mediam ita occupavit, ut maculam rubram striatam referret. Intestinum brevi ante apicem caudae oblique terminatum. Maris unici, quem vidi, spiculum eodem loço simplex visum, forsan tamen (analogiae ratione halita) duplex, sed complicatum. Oviductus amplus serpentino ductu circa intestinum volutus. Ova magna. 


\section{$-235$}

8. Cucullanus minutus R. n. sp.

Amicissimus Brenrser specimina complura misit in Plcuronecte Passere (loco non indicato) reperta, -lineam unam, vel hujus insuper tertiam partem longa.

Caput globosum, cucullo striato lentis simplicis ope tantum discernendo; collum tenuius sensim in corpus augetur posteriora versus iterum sed minus decrescens; cauda acuta, maris inflexa, feminae recta, depressa.

Genitale masculum: spicula duo longa tenuia ante apicem caudae; pone eadem tubercuIun exiguum. Feminae vulva longius a cauda distat, oviduetus vacui, aut ovis inmaturis, materia quasi grumosa repleti.

Tubus cibarius a cucullo fusiformis, dein parum ampliatus iterumque tenuior ad posteriora progressus.

Species distinctissima.

\section{Genus VI. Spiroptera.}

Genus hocce in Catalogo Entozoorum Musei Viennensis primum Acuariae nomine stabilitum, in diario Societatis Physicae Berolinensis (Magazin der Ges. Naturf. Fr. B. VI, p. 85.) ob characterem illic propositum (Vermis teres elasticus utrrinque attenuatus, ore papilloso) neque sufficientem visum, a me impugnatum, optimis omnino adnumerandum esse, dein didici. Os quidem papillosum omnibus non est, sed omnibus tamen datur orbiculare, quo solo ab Ascaride satis superque differt, quemadmodum a Strongylo alis caudae masculae lateralibus, neque bursa terminali, facile distinguitur. Specimina mascula 


\section{- $236-$}

characterem generis exhibere, in Nematoideis (utisupra exposui) tolerari debet. Conf. Obs. I. ad speciem XIII.

Ipse olim Spiroptens tantum femineas noveram, quas ideo, charactere sufficiente non exhibito, allis generibus addidi, a quibus nunc diversas agnosco; paucas dein ipse reperi. plurimas Museo Viennensi debeo, quod singulas enumerando grata mente adnotavi.

Species numerosae sunt, singulae vero differentias specificas facile multoque facilius quam Ascarides exhibent. Nihilo tamen minus genus physalopterae huic proximum separavi, ne numero tandem obruanur, alias enim artificiosum esse facile concedo.

Spiropterae inter oesophagi et ventriculi tunicas, vel in horum tumoribus morbosis (an entozois originem debentibus?) plerumque degentes Mammalibus et Avibus potissimum propriae sunt, ut -inter species quadraginta in synopsi enumeratas, triginta quinque dicto liabitaculo conveniant; reliquarum una (i2) eodem loco sed in Amplaibio (Testudine orbiculari), altera in pisce (n. I5.) quidem sed praeter ve. sicam anemiam etiam oesophagum inhabitans inveniantur. Species n. 2, omninỏ deflectit, sub capitis cute in Falcone naevio occurrens; n. 23. vero in Hominis vesica urinsia, et 11.30 . in pedc Falconis rufipedis obviae nondum satis con. firmatae sunt.

1. Spiroptera megastoma R. n. sp.

Cl. Becklemen, Scholae Veterinariae Berolinensis Professor meritissimus in duorum Equo- 
rum tuberculis ventriculi complura reperit specimina, quae recentia mecum conmunicavit.

Mas quatuor, femina quinque vel quinque et dimidian lineas longitudine adiequant.

Entozoa tenuia, albd, capite discreto quam corpus tentiore, ore magno orbiculari, nudo sive papillis dectituto; corpore utrinque attenuato; cauda feminac rectiuscula, acuta, ano prope hujus apicem; maris caudi convoluta, apice fortiter inflexo, bialaio, stylum medium inter corpuscula rotunda emittente.

2. Spiroptera sterëura Rí. n. sp,

Bremserus ante duos et quod excurrit annos specimina duo misit inter Falconis naeviq membranam nictitantem, et bulbum oculi, uti etiam in illius meatu auditorio reperta, alterum masculum sex, alierum femineum, octo lineas longu, crassa, antrorsum magis attenuata.

Os orbiculare nudum. Canda inaris spiralis, spiram unam efficiens intortam, apice caudali tenui stricto, nodulo quasi terminato; ala utrinque tenui; ante apicem caudalem genitale mascu. lum cum vagina monophylla reflexum. Feminae cauda recta depressä, apicé rigido styliformi ver. sus extremum rotundato.

Couf. Entozoon dubii generis n, I\&.

3. Spiropteru strongylina R. n. sp.

Bremserus sex specimina misit in Suis feras ventriculo reperta, quorum duo mascula quinque circiter lineas, reliqua feminea sex ad septem lineas longa, omnia tenuia, alba.

Caput exiģuum, continuum, ore orbiculari. Corpus antrorsum potissimum attenuarum. Catrdis maris alterius spiram simplicem, alterus spiram 


\section{$-238$}

unam cum dimidia absolvens, ala utrinque lata, radiata, spiculo longissimo; apice caudae brevissimo nudo (non alato). Caudae femineae apex depressus, rectiusculus, subacutus.

Obs, Entozoa obiter inspecta facile pro Strongylis haberi possent, sed alae laterales, (neque bursa terminalis) apice caudae nudo, Spirop. teram esse evincunt. Strongyli etiam crassiores esse solent.

\section{Spiroptera nasuta $\mathrm{R}$. n. sp.}

Specimina complura in Fringillae domesticae ventriculo (cui firmiter inhaeserint) reperta B BemSERUS mecum communicavit, duas cum dimidia ad quatuor lineas longa, alba, disparia.

Marses utrinque atqualiter et parum attenuati, capite acutiusculo, ore simplici; cauda in duas tresve spiras intorta, utrinque alata, apice acutiuscalo. Spiculum non vidi.

Feminac et longiores et (potissimum) crassiores, utrinquae inflexae, antrorsum tenuiores, apicis caudalis cuspide incurvata.

Obs. An caput acutiusculum visum acumine papillan prodit? Ipse talem detegere non potui.

5. Spiroptera laticeps R. n. sp.

In Falconis Lagopodis oesophago repertas am. Firemser Spiropteras masculam et femineam, illam tenuem, quatuor, hanc crassiusculum sex et quod excurrit lineas longam.

Corpus alatum, subsagittatum, membrana ca. pitis larerali posteriora versus apice ohtuso utrinque exstante. Oris papillas non vidi Corpus antcriora versus magis attenuatam. Cauda maris fere tres spiras absolvens, membrana laterali te- 


\section{$-239$}

nui, vagina tantum penis laterali visibili. Cauda feminae obtusiuscula depressa.

6. Spiroptera alata $\mathrm{R}$.

Ent. Ir. I. p. 189. n. 51. Ascaris sagittata.

Quam am. Braun in Ardeae nigrae intestinis a se repertam misit, dein quoque a Breusero accepi inter Avis dictae tunicas ventriculi obviam, huc utique facientem.

Caput et collum, sive antica corporis pars, alata, membrana tenui, interdum postice exstante, qua illa pars sagittata redditur; semper autem hoc non contingit, ideoque speciem alatam dicere praetuli. Corpus antrorsum magis attenuatum. Cauda utriusque sexus depressa, maris tres spiras efficiens, membrana latissima ad apicem us. que extensa. Cauda feminae apice brevi depresso alato.

Obs. I. Generis character a cauda peten. dus hac specie optime innotescit.

Obs, 2, Froenich (Naturforscher St. XXIX. p. 45, n. 20. Tab, I. Fig. 9. 0. Ascaridem tenuissimam yuatuor lineas longam describit, in in. testinis crassis Ardeae cinereae repertam, sibique Asc.' parvulam dictam, quam huc pertinere crediderim, licet capitis caudaeque alas non referat. Quidquid autem sit, num eadem utrum affinis species dicatur, descriptio et figura intestini certe pessima est, neque hoc ullo in Nematoideo dividitur et duplex decurrit, sed semper simplex manet. Conf. obs, ad speciem vigesimam.

7. Spiroptera laticaudata R. n. sp.

Inter Otidis Tetracis tunicas vientriculi reperta Bremserws duo misit specimina, alterum 


\section{$-240$}

masculum, femineum alterum, hoc duodecim, illud sex lineas longum, multoque-tenuius.

Caput simplex obtusum, interdum, indistincte tamen, papillosum visum, utrinque dente obtuso exctante a corpore discreturn. Corpus utrinque, magis tamen antrorsum attenuatum. Cauda feminae apice brevi depresso acntiusculo; maris in spiram simplicem torta, membrana utrinque lata, veisus apicem rotundata, ut hujus śpecici caudam cum capiie Najae facile compararcs.

\section{S. Spiroptera bidens R. n. sp.}

Specimina sex, quorum duo mascula, inter, Meropis Apiastri tunicas ventriculi reperta BremSERUS mecum communicavit.

Mlas tres, femina sex ad octo lineas longitudine adaequant; utriusque corpus tenuissimum, filum sericum simplex aemulans.

Caput discretnm, papillosum, pone quod utrinque papilla denticuli lateralis instar exserta. Corpus utrinque magi.j tamen antrorsum attenuatum. Cauda maris spiran duplicem ofiert, pro more alata, genitali in conspectuni, non venituie. Feminae cauda apice brevi incurvo, ante quem ani orificium.

Obs, In unico specimine papillas colli non olservavi, sed caput smul alienatum erat.

9. Spiroptera bicuspis. R.

Inter Tringae helveticae tunicas ventriculi reperta am. hrevsere specimina tia misil, quo; xum แmum fermineum, ienuissinı.

Mla, duas, femina quatuor lineas aequat. $C a$ fut parvum papillosum continuum, pone quod utrinc ue cuspis, sive dens subulalus, vel horizontalis, vel retrorsum versus eminet, Curpus utrin. 


\section{$-241-$}

que sed maxime antrorsum attenuatum, parte media anterioré duplo crassiore. 'Couda maris spiras' duas offert membrana angusta utrinque fortiter revinctse; frminace apex caudalis brevis depressus et recurvus, ante quem orificium ani $\mathbf{i} \cdot$

10. Spiroptera strumisa $R$.

Ent. Il. 1. p. 193. n. 55. A scaris strumosa: In Talpae europacae ventriculo plurimis visam ipse Aprili Gryphiae reperi.

Capitis us orbiculare, pápillis cinctum, quarum Froench quatuor retulit, quaeque saepius obscurat sunt Sub basi colli subtus tenuissime alati tuherculum in utroque sexu adest. Cauda maris bis spiraliter torta, alis subcaudalibus apicem obtušmin inflexum non atingentibus; spitculım lougum inflextixi. Cauda feminae apice brevi obtuso depresso, ante quem anus.

Obs. Cel. Niтzsch caudam Ascaridis stru: mosae pro capite imposuisse, in litteris monet, sed descriptione Entozoologiae cum Spiropteris ipcis comparata, et caput et anum et spicula et colli tuberculum recte indicata invenio.

- 11. Spiroptera quadriloha R. n. sp.

In Pici vidis etsophago amanuensis noiter Berolini Junio mense quatuor specinnina reperit feminea, quae die insequente recentissina quidem sed mortua examinavi.

Femina quatuor vel quinque lineas longa, alha, crassiuccula, antrorsum attenuata, in arcurn simplicem curvata.

Caput fere illid Protei anguini, depressum, utrinque bilobum, lohis binis superioribus; totidém inferioribus, oblongis, singulorum apice pos. teriore obtuso exstante, primo intuitu bothrium 
capitis laterale bilobum, quale in Bothriocephalis occurrit, adesse fere credidissen, sed mox lo. bos quatuor distinctos vidi, nullo in Nematoideo tali modo exhibitos. Os orbiculare, papillis ex. iguis subexstantibus, haud tamen semper atque distinctis cinctum. Corpus posteriora versus increscens, apice caúdali acuto, attenuato, quan capite tamen crassiore. Vulva ad tertian entozoi quartam partem in omnibus speciminibus exstans. Ainus apici caudali proximus.

Totum corpus ovis exiguis rotundis immaturis repletum, quae cute cultello laesa effundebantur.

Species singularis, ulteriori examine dignissina.

\section{Spiroptera contorta $\boldsymbol{R}$.}

Ascaris testudinis, Ent. II. I. p. 198. n, 67. in Testudinis orbicularis ventriculo et hujus vesi. culis a Braunio detecta.

Testudo orbicularis mascula parva, cujus scutum dorsale quatuor cum dimidio, ventrale vero duos cum duabus tertiis pollicis partibus aequabat, Arimini Majo viva allata est, cujus ventriculum extus inaequalem, "passim tubercu. lis diffusis parum elevatis alienatum vidi; quo aperto Entozoa plurima in conspectum venerunt contorta, fossulis illa tubercula efficientibus tan. topere intnersis, ut caudae córporisve tantum pars promineret.

Specimina omnia ferninea, albida, duas cum dimidia ad tres cum dimidia lineas longa, antrorsum attenuata.

Caput saepe tres tantum papillas in trifolii modum positas exhibet, interdum vero illarum 


\section{$-243$}

quinque ad sex ob oculos ponuntur valde mobiles et mutabiles, ore orhiculari inedio, Cauda depressa, inflexa, apice acutiusculo.

Corpus saepe fere totum in spiras arcte sibi invicen appositas contortum. Canalis ciburius oviductibus atrentino modo circumvolutus.

Rerclini postmodum Junio mense famulus jussu meo Testudinis orbicularis ventriculum examinavit et specimina quatuct feminea reperit, a me recens examinata; ciuas tresve lineas longa, rubeila, plus minus contorta. Caput papillosum, papillis circiter scx, sed oris apex acutus saepe iu conspectum venit, an tubulus emissilis, ut Liorhynchis accedat?

13. Spiroptera Anthuris R, n. sp.

Arn, Bremser specimina misit inter Corvi frugilegi et glandarii, Coraciae Garrulae et Orioli Galbulae tunicas rentriculi repertă, ipseque liberum in Orioli intestino inferiore Florentiae Augusto mense offendi. Conf, obs, 2.

Longitudo variat; specimina mascula quatuor ad sex lineas; feminea in Coracia Garrula reperta quinque ad sex, in reliquis autem obvia octo ad quatuordecim lineas longa vidi, Cete. rum tenuissima et alba sunit.

Caput papilyis tribus munitum terminalibus, Ascaridem meitiens; Corpus medio subaequale, ceterum utrinque, magis tamen antrorsum attenuatum. Cauda maris acuta, incurva, sive spiram simplicem efficiens, alata alis parallelis latiusculis, inter quas spicklum emergit longum, cinctum vagina tri-vel tetrap!ylla, sive foliolis lutescentibus, obtusis; in diversis speciminibus plus minus exstantibus, Feminae cauda leviter 


\section{- 244}

incurva, depressa, obtusiuscula; anths lineam ab hujus apice distat. Tractus intestinalis rectus, simplex, neque tot stricturis interceptus, ac in Ascaridibus esse solet. Ovula rotundato-elliptica, linea media obscura.

Obs. I. Haec prima Brensert Acuaria fuit, quam examini subjicere licuit, et vagina penis foliolis cincta commotus fui, qui nomen genericum Anthuridis ponerem; aliis autem deinceps speciebus comparatis illam minus compositam vidi et Spiropterae nomen praetuli.

Obs. 2. 'Eadem species' teste catalogo Entozoorum Musei Viennensi typis impresso inter tunicas ventriculi Corvi Coracis, Corones, Cornicis, Caryocatactis, Picae et Pyrrhicoracis occurrit.

14. Spiroptera attenuata F. n. sp.

Am. Brenser specimina mihi duo misit inter Hirundinis urbicae tunicas yentriculi reperta, Conf. obs. 2. ..

Alterum specimen masculum tres, alterum femineum quinque lineas longitudine parumper excedebant.

Caput papillosum, apice medio in mare (certe casu) magis exserto. Corptus utrinque magis tamen posteriora versus decrescens. Cauda maris involuta aut simpliciter spiralis, alis tenui. bus, quales in Spiropteris spira caudae simplici instructis esse solet. Vagina tantum penis hoc in specimine exstat, majuscula, composita, haud explicita. Cauda feminae depressa, excavata, apice laminam tenuem angustam obtusam sistente, ante quem anus.

Obs, I, Speciei praecedenti quidem pro. 


\section{- 245}

xima, attamen praesertim cauda feminea distincta: vagina penis itidem diversa videtur.

Obs. 2. Eadem species eodem loco in $\mathbf{H I}_{\mathbf{6}}$ rundine rustica et riparia occurrit, C. E. V.

\section{Spiroptera Cystidicala R.}

Ent. I. p. I22, n, 4. Ophiostoma Cystidicola. An. Breveser et Otto multa specimina miserunt in Salmonis Farionis vesica natatoria sibi obvia. Ipse Berolini versus finem Novembris in Salmone Thymallo lato BLocrur satis copiose in vesica dicta et ita quidem reperi, ut majora specimina in hujus corpore et cauda, miInora vero versus ejusdem ductura oesophageum occurrerent; quid? quod duo in ipso oesophago inveni, nullo modo a reliquis distinguenda.

Spiropteras meas per triduím in aqua frisila vivas servavi.

Coloris sunt nivei, quinque ad octodecim lineas longze, longitudinis ratione habita valde tenues.

Os orbiculare, in vivis saepe papillis exstantibus cinctum visum. Corpuls altrorsum magis at:enuatum. Cauda maris spiram duplicem effingit; alis lateralibus mediocribus; apice tenui incurvo; spiculo satis longo. Cauda feminae crassior, depressa, obtusiuscula.

Tubns intestinalis rectus, in quibusdam anteriore parte passim obiter constrictug, quasi articulatus.

Obs. Fischerus Cystidicolam describendo, specimina inutila et putrescere incipientia prae oculis hahuit, quo factum est, ut omnia, quae de iisdem referat, falsissima sint. Cutis hujus speciei facile laccitur et ipse specinina duo la- 
cerata vidi, quae cum Fischerianis quodammodo convenirent. Longitudio quam intestino tribuit (tripla corporis) ne de oviductibus quidem valet; illud rectum, corporis longitudine.

16. Spiroptera uncinata R. n. sp.

Am, Kuvg in Anseris aomestici tuberculis oesophageis specimina satis multa Kerolini Septembri mense reperit, quae benevele mẹcum communicavit.

Mares tres quatuorve lineas longi, tenuis. simi, femirjae quatuor ad septem cum dimidia lineas emetientes crassissimae sunt, diametro transverso tertiam lineae partem adaequante.

Os orbiculare, papillis sex cinctum, quae interdum valvularum speciem exhibent. Corpus posteriora versus magis attenuatum. Cauda marit spiraliter involuta, bialata, spiculum breve emittens; feminae apex caudalis unciformis, depressus êt deflexus,

17. Spiroptera elongata R. n. sp.

Bremserves specimina tria misit inter Sternae nigrae tunicas ventriculi reperta, feminta, quorum alterum undecim, alterum septendecim, tertium octodecim lineas longa, longitudinis ratione habita tenuią, quartam sciliçet lịneae partem diametro transverso aequantia.

Caput papillosum, papillis mediis majoribus, laterålibus minoribus. Corpus retrorsum ma. gis attenuatum. Caudae apex uncinatus, deflexus, quam in specie praecedente major.

Obs. Speciei praecedenti proxima est, sed longitudinis et crassitici ratione diversa, neque speciminibus masculis comparandis utramque conjungere nolui. 
18. Spiroptera revoluta R. n. sp.

Specimina tria inter Charadrii Himantopodis tunicas ventriculi reperta Bremserus misit, quorum unuit masculum.

Mas tres, feminarum altera quinque, altera septem lineas longitudine aequant.

Caput papillosum, indistinctum. Corpus utrinque parum, magis tamen antrorsum attenua. tum. Cauda maris, bis spiraliter flexa, alis lateralibus latis, apice 'revoluta; feminae attenuata, apice depresso, sursum divaricato.

19. Spiroptera leptoptera $\mathrm{R}$.

Froflicir im Naturforscher St. XXIX. p. 36. n. I3. Ascaris anceps.

Bremserus specimina complura misit in Fal. conis Nisi ventriculo reperta, ubi etian FroEI.1Cn Aprili mense offendit; similia Falconis $\mathrm{Bu}$ tconis tunicis ventriculi implicita KLvG reperit et mecum communicavit. Spiropteras ventriculi Falconis cineracei et Tinnunculi Catalogus Entoz. Vienn. duscptus habet.

Specimina in Niso reperta mascula tres cum dimidia, feminea quatuor ad quinque cum dimidia lineas longa vidi, Froelick novem lincarum longitudinem his tribuit; in Buteone reperta feminea quinque ad septem adaequant.

Caput simplex. Os orbiculare papillis obscuris. Corpus utrinque magis tamen antrorsum attenuatum. Cauda maris spira fere duplici, membrana laterali utrinque tenuissima, ut in nulla reliquaru:n specierum tantae sit tenuitatis. Genitalia hand exscrta vidi Cauda feminea depressa, recta vel stricta, apice acuto, ante qúem anus. 


\section{$-248$}

Obs. Ascaris anceps Froelrchrm setacea, rectiusculd utrinque marginata, margine peilucidn undulato, cauda acuminata, prócul dubio huc perti. net, licet Spircpterae nostrae caput omnibus speciminibus mihi visis simpiex tt alis destitutum sit; margines autem simul undulati dici entozoa minus recentia exaninata evincunt, quibus facile cutis solvitur. Ob teriuitatem et reliqua cl. viro dicta mihi saltem Asc. anceps ad Physalopteram alatam, pariter in Falconis Nisi vertitculo obviam, reutiquan trahi posse videtur.

\section{Spiroptera euryoptera $R$.}

Frocurar im Naturforscher St. 29. p. 40 n, 10. Ascaris Collurionis.

Bremservs specimina complura inter Lanii Collurionis tunicas ventriculi reperta misit; FroE. IICH inter junioris Collurionis tunicas intestinorum Julio observavit. Eanden speciem ex La. nio Excubitore tt minore C. E. V. habet,

Specimina mea mascuia, duas cum dimidia ad tres, feminca quatuor lineas longitudine aequant. Froelichir quatuor specimina fere pollicem longa dicuntur.

Caput obtusum, minutumr, subdiscretum, papillosum. Corpus utrinque magis tamen antrorsum attenuatum. Cauda maris duas cum dimidia spiras efficit, membranis lateralibus latissimis, cum omnibus spiris, ad apicem caudae usque protensis, ut hae constantissimae sint. Spirae breviores sunt quam in Spiroptera alata (n. 6.), primoque intuitu membraíat latae canalem effingere videntur, cujus ostium apex caudalis sit. Feminae canda obtusiuscula, orificio ani ante ejusdem apicem 


\section{- 249}

tenuiorem. Ova rotundato elliptica, minora quam in Sp. Anthuride, n. 13.

Obs. Flozlacr nounisi feminas, partim dis. ruptas examinasse videtur. Ori quatuor papillas tribuit. Intestinum iterum bifidum fingit ut in Sp. alata 2n. 6. obs. 2.

\section{Spiroptera sanguinolenta $\mathrm{R}$.}

Ent. II. I, p. 242. n. 26: Strongylus L'upi.

Berolini Januarii $d_{2} 22$. in tuberculo ventriculi Canis Lupi magno magnam copiam entuzoorum reperi sanguinterum, inultaque praeterea in ventriculo, unum in duodeno libera. Januarii d. 24. in alterius Lupi tuberculis ventriculi mineribus duo iterum offendi.

Mares unum cum dimidio ad duos, feminao duos ad tres pollices longi, hae crassiores.

Caput atteruatum, haud alatum. Os magnum orbiculare, vel pluribus cinctum papillis, vel mar. gine undulatim. Corpus antrorsum magis attenuatum, maribus obiter incurvum, feminac spira. liter devolutum (in modum instrumenti, quo em. bolos educimus). Cauda maris in unam duasve spiras involuta, bialata; genitalis filiformis $\mathrm{ra}$ gina tereti breviori. Cauda feminae depressa ou. tusiuscula.

Oesophagus rectus, tubus ab eo incipiens duplo crassior, rectus, absque ventriculi mortariformis specie.

Obs. Quatuor specimina consimilia in verttriculi lupini tumoribus reperta am. Otto rnisit, talia etiam in Museo Viennensi servantur.

\section{Spiroptera obtusa $\mathbf{R}$.}

Ent. II. I. p. 17o. n. 36. Ascaris obtusa. Ipse olim in Muris Illusculi ventriculo - 
Gryphiae Januario reperi, sed recentia iterum specimina eodem loco ab am. KLug inventa plurima examinare contigit, cum meis utique con$v \in$ nientia, sex ad viginti tres lineas longa, maxima simul crassissima, diametro transverso lineam aequante. Caput vere sexpapillare, ut olim retuli; os orbiculare; quo certior fierem, anticam partem abscidi, et quas verme integro jam numeraveram singulas vidi papillas. Corpus antrorsum magis attenuatum. Cauda maris spiram duplicein effingit; apex ejusdem obtusus; alae tenues, sed ipsa caudae pars, quam includunt, concava est; spiculum filiforme, mediocris longitudinis, inflexum vel rectum; cujus vaginam nullan vidi. Caudae femineae apex conicus, neque pro gene. ris more depressus.

Tubus cibarius tenuis incipit, tum obiter curvatur, dein reçtus continuat, neque Ascaridis partes intestini ampliatas exhibet. Ova elliptica parva.

\section{Species dubiae.}

\section{2j. Spiroptera Hominis.}

W. LAwrence Case of a woman who voided-a large number of Worms by the urethra with a description of the animals. In: Medico. Chirurgical Transactions published by the Medical and Chirurgical Socicty of London. Vol. II. 11. 31. 1. 385-395. Tab. 8.

Puella Londirensis viginti quatuor annos nata anno 1 gos urinae retentione quotidianum "atheieris usum exigente lahorare incepit, variaque sed incassum malo opposuit. Tribus annis elapisis Jomannis Baranex Chirurgi cel. curae 
commissa fuit, qui cathetere applicato vermem egressum observavit, quo commotus oleum terebinthinae praeseripsit, cujus autem usum internum illa non diu tulit. Eodem vel etiam lini oleo deinceps in vesicam injecto, sed absque ullis etiam injectionibus, plurimi vermes sensim eli. minati sunt, et aegra Octobri $18 \mathrm{rr}$. quando cel. vir casum amico cel. LAWRENCE communicatum publici juris fieri curavit, malo adhuc laboravit.

Anno 1816. cel. Barnett ab am. Spiker, Bibliothecae regiae Berolinensis Custode meritissimo, tum Londini versato exhortatus litteras mandavit, quibus mulierem eodem adhuc morbo vexari me certiorem feeit, phialasque tres benevole adjecit, quarum prior ovula, securda vermes adultos, tertia juniores contineret.

I. Ovula vero sic dicta- subglobosa cum arenulis per catheterem ex vesica pauperculae educta, nequaquain talia habenda sunt. Corpuscula sunt plus minus globosa, tertiam lineae partem diametro superantia, duriuscula, forcipi comprimenti renitentia, dissecta solida visa, quominus pro hydatulis haberi possint, quales primo suspicatus sum. Coiscrementa sunt lymplatica in vesica morbosa ex humoribus alineatis ibirlem secretis, simili forsan modo ac arenulae ex lotio praecipitata.

2. Vermes magni et adulti cel, virís dicti, 1. c. descripti et tab. 8. figura inferiore delineati aeque parum ad entozoa pertinent, sed concrementa sistunt lymphatica sinubus vel ductibus fistulolis vesicae originem delentia. Fila sunt irregularia torta, trigona, tetragona, passim compressa; plerumque hinc convexa, illinc ad latera concava, et medio bicostata, inaequalia, ut filum. 
quodvis utroque extremo tenne incipiat tum sensim crassescat et medio iterum derrescat. Apices in specininibus missis haud acute terminatos vidi, quales icon sistit, sed diffluentes er plus minus laceros; lymphanque in canalibus fistulo. sis coactam passimque compressam filum inaequale efformare crediderim. Facies filorum convexa utrinque exsperala, sed tuberculorum seriem duplicem regularem fisura oblatan in meis non reperio. Longiudo. fllorum ceterum qua. tuor ad acto pollices adaequat, crassities summa lineam vix superat. Tota undique substantia homogenea, solida, spiritu forsan duriuscula reddita, nuspiam cute tecta, quod me judice argumentum crucis est. Nullum enim datur entozoon, cui cutis denegetur, quodque coctum albumen nu-, dum referat. Color flavescit.

Motun debilem undulatorium in quibusdam observatum esse, plurimos vero mortuos fuisse I. c. traditur; sed motus ille certe a temperatura znutata vel alia causa physica pependit.

3. In tertia demum phiala sex entozoa aderant nematoidea, quorum figura 1 . c. icone superiore obiter indicatur; de his autem si dicitur, aeque parum microscopii ope indagari potuisse, res certe longe aliter so habet, quod descriptio docebit.

Mas octo, femina decem lineas aequuant, tenucs, albidi, valde elastici.

Caput truncatum, passim una alterave papilla instructum visum. Os orbiculare. Corpus utrinque, praesertim tamen antrorsum attenuattum, vél parte postica sola, vel etiam anteriore spiraliter involuta. Caudu feminae crassior, apice brevissimo obtuso, tenui et pellucido. Maris 


\section{$\div 253$}

cauda apicem longiorem, tenuem g̨uidem at opacum, ni fallor, obtusum habet; casu enim meo in speciunine truncatus videtur, sed pars appendens obtusa est. Ad basin apicis ala tenuis et brevissima cum tubulo medio exiguo tereti, forsan vagina penis. Ovula distincta non vidi, sed circa tuburn intestinalem tenuem et rectum mem. branulau tenuem et punctatam tantum observare licuit.

Obs. Entozoon descriptum ad hoc genus referre cogor, et Bremserum adsentientem inveni. Forsan in opere quod de entuzois humanis molitur egregio, de speciminibus a me sibi communicatis meliora tradendo rem conficiet.

24. Spiroptera Simiae.

Ent. II. r. p. 67. n. I2. Filaria alata.

Inter Simiae Maimonis tunicas ventriculi ab am. Arbers Medico Bremensi Cel. repertam ad hoc genus pertinere nullum est dubium, De. scriptioni in Entozoologia datae nihil addere pos. sum, nisi os capitis constricti omnino orbiculare videri. Femina ceterum mihi solummodo visa speciei firmandae characterem non offert.

\section{Spiroptera Ursi Arcti.}

Am. Bremser entozoa quatuor in Ursí Arcti oesophago reperta misit, duodecim ad no. vendecim lineas longa, tenuissinıa, subgrisea, anv trorsum tenuiora.

Caput exiguum, ore orbiculari, membrana laterali (an morbose exstante) tenui. Corpus mediam versus partem aequale. Cauda depressa obtusiliscula, in tribus membrana tenui, in quarto specimine (haud tamen spiraliter convoluto, ne. que genitalibus masculis instructo) multo latori. 
Oesophagus tenuis in conspectum venit, reliquus autem tractus intestinalis ovario circumvoluto absconditus. Anus more Spiropterarum ante apicem caudae depressum et deflexum, et longiori ante illum distantia vulva prominet.

Obs. Cauda alterius speciminis latiori mem. branz quidem instructa, neque tamen spiraliter in. voluta de'genere primum me dubium reddidit; huc tamen pertinere reliqua suudent, ut specimen illud fortasse varietatem sistat. Exempla tamen specierum inter genera ceterum bene condita intermediarum in nulla Historiae naturalis parte desunt.

\section{Spiroptera Citilli.}

In Arctomydis Citilli ventriculo repertum am. Bremser entozoon misit femineum, sex cum dimidia lineas longum, utrinque magis tamen an. trorsum attenuatum; capitis ni fallor papillis sex instructum, quarum saltem tres eadem linea di. stincte vidi; mernbrana capitis laterali utrunqne tenui: apice caudali inflexo acuto papilla exigua terminato.

Spiropterae Muris obtusae n. 22, affinis videtur.

\section{3o. Spiroptera Falconis.}

Aru. Bremser specimina misit quatuor in Falconis rufipedis pede reperta, tres ad septem lineas longa.

Caput utrinque alatum, truncatum aut obtusissimum, ore, quantum videre licet, orbiculąri, evalvi. Corpus utririque magis tamen antrorsum attenuatum, majorum spiram magis planam, mino. rum protractam efficiens. Cauda depressa ob. tusa, Alae corporis tenues, caudae majores. 


\section{- 255}

Habitus utique Spiropterae, An specimina minora mascula?

\section{Genus VII. Physaloptera.}

Spircpterae proximam esse hanc exponendo concessi. bene tamen separari contenderem. Hahitus primum alienus est, ut obiter inspiciendo Physalopteram pro Strongylo haberes, a quo ta. men vesica caudae masculae laterali (infera) neque terminali facillime discinguitur. Longitudinis porro ratione habita crassities corporis multo major invenitur. Cauda denique mascula nedum. spiralis, sed ne quiden fortiter inflexa sed obiter deflexa est, ejusque alae Jaterales simul latissimae et breves sunt. Cum reliquis confundi nequit.

1. Physaloptera clausa R. n. sp. Tab. 1, Fig. 2! 3 .

Specimina multa in Erinacei europaei ven. triculo reperta Bremserus noster misit, inque bestia illa frequentem esse addidit, quam Helminthologi borealis Germaniae incolae nunquam in animali tóties dissecto reperimus.

Mascila sex ad duodecim, feminea sex ad novendecirn lineas longa, majorum diametro transverso duas lineae tertias partes aequante. Color albus.

Caput indiscretum, ore mediocri orbiculari integerrimo, in cujus fundo tuni intestinalis principium quandoque labii instar superioris et inferioris divisum, (an papillis oris retractis?) indistincte tamen conspicitur. Corpus antiursum magis attenuatum, haud exacte teres, linea sciliGet laterali magis ad inferiora ducta, quo dorsum 


\section{$-256$}

ventre magis convexum fit; linea illa tamen co. lore magis quam membrana eminente notatur. Canda maris deffexa, apice (ad lineae longitudinem) obiter incurvo, vesicula utrinque alae instar lateraliter prostante aucto, ala scilicet laterali utrinque introrsum involuta et aquam continente, unico hactenus in Nematoideis exemplo. Ultima apicis pars papillae instar eminet. Cutis vesiculae dorsalis strias aliquot horizontales et parallelas offert. Inter utramque vesiculam in apicis caudalis latere inferiori et medio quidem loco tubercultum exiguum perforatum coloris ferruginei, ex quo genitale masculim tenuissimum re: ctum satisque longum emergit. Canda feminae denressa apicem incurvum sive uncinatum et pa. pilla terninatum, alis autem prorsus destitutum sistit.

Obs. In speciebus reliquis mihimet notis alae caudae masculae in vesiculas clausas non coëunt, sed vesiculam inferam "magnam apertam elfingunt, quo nomen generi specie hac tantun cognita impositum etiam in reliquuis tolerari possit.

2. Physaloptera alata $R, n$, sp.

Specimina aliquot in Falconis Nisi ventriculo et $F$. gallici intestinis reperta BRE ySERus misit, et consimilia in $F$. pennati ventriculo inventa in Museo Viennensi vidi.

In Falcone Niso lecta, mascula duodecim ad tredecim, temineum (junius procul dubio) undecim tantum'lineas; in Falcone gallico reperta masculum scx, fenineum undecim lineas aequabant. Color albus.

Caput alatum, ala utrinque antrorsum latiore, postice evanesccnte. Os orbiculare; quatuori uti 
videtur papillis instructum, Corpus utrinque mâ. g's tamen antrorom attelluatum. Canda maris depresso incurva, ap ce obtuciucculo: alis lateralibus latis etmilar colatis, radiatis sive traniversiu striatis, striis vel plicis a florso oriundis. Alis istis récicula monep ylla inferá, exacte prioris, si aquam contineret, Effingitur. Eadem qui)que ariest papilla infera ferruginea filum genitale emitters. Cinda fenirnae recta obtusiuccula, subritepressa apice acutiusculo, brevi, ante quenr anus, -

Obs. Speciminis feminti, cujus iconem Braun miserat, in Falcone Niso reperti Ent. 2. $\mathrm{p},-373$. mertionem feci. 'Ascaris anceps FroenIcrie dicra pariter in Nisi ventuiculo reperiunda nostra $\epsilon$ st Spircpiera leptoptera n. 39.

\section{Physaloptesa abbreviata R. n. sp.}

An. Buemer specimina duo máscula, sex feminea misit, in ventriculo et intestinis Lacertae margaritaceat Algesirae (Kii-paniae) septrta.

Mascula quatuor ad quirque, ferninea sex ad novem lineas longa, omia crassiuscula, dura, albissina,

Os orbiculare, papillis liaud tamen distincte milii visis instructum. Corpus utrirque; antrorsum tamen multo magis attenuatum, Cauda maris inflexa, menbrana laterali utruque lata, turgida; tuberculo rufo getitali inficio, ut in prioribus. Feminae canda obtısa cum acumine brevissimo conico, quo deflexo truncata videtur. Ovnla ovato-eliptica, vel tt subrotunda, sed tum minora et forsan immatura, ila vcterum ambitu pellucida.

Ovula dicta quam illa Ascaridis extenuatae 


\section{- $258-$}

n. 46. in eadem Lacerta occurrentis, duplo breviora sunt.

\section{Physalop!era retusa $\mathrm{R}, \mathrm{n}$. sp.}

Am. Olfers in trium-Lacertarum Teguixin oesophago, (praesertim) ventriculo et tenuibus, Septembri et Octobri in Brasilia copiosissimas reperit et mecum communicavit, Pisonem (De Indiae utriusque re naturali èt medica. Amstelod. 1658. fol. p. 105 ) easdem jam in dicta Lacerta reperisse addendo.

Longitudo dinidium ad duos pollices aequat. Majora specimina simul crassa sunt.

Caput discretum, distincte papillosum, pa. pillis saepe duabus, mox quatuor in.conspectum venientibus. Os orbiculare. Corpus utrinque, magis tamen antrorsum attenuatum. Caridae masculae vesicz infera latissima patentissima apicem revinciens, quo in plurimis retusa vel etian emarginata conspiciatur; ceterum pro more radiata. Margo ejusdem posticus valde tumidus, laterales minus turgent. Tuberculum rufum in paucis, filum genitale in nullis exsertum vidi. Feminas multo majoris-canda apicem brevissimun obtusum utplurimum incurvurn labet.

$$
\text { Species dubia. }
$$

5. Physaloptera temuicollis $\mathbf{R}$.

Specimen unicum ftmineum, octodecim et quod excurrit lineas longun in intestiris Falconis flaliaëti repertum in ditissimo Museo Vitn. nensi examinare licuit.

Os orbiculart- Corporis pars antica (lineam longa) tepuissima, insequens subito valdeque 


\section{- 259}

augetur, ut postice lineam dimiriam diametro transversali superet. Candae apex'ar ulus

Obs. Hảbitus ab illo speritum rtliquarum utique diversus, neque huius maribus ighotis aliquid certi proteram.

\section{Genus VIII. Strongylus.}

Genus a reliquis Nematoideis hursa maris terminali penem emitteme facillime distinguendun, Mammalibus, Avibus et Amphibiis tantum proprium, magnum specierum numerum conti. nelt haud videtur. Harum confirmatas in Ento. zoologia novendecim, in Synepsi viginti tres; du. bias uroque loco quindecim enumeravi, ita ta. men ut duhiarum in En:tozoologia dictarun no. vem spiropteris, una Filariis bynopseos acces. serint.

1. Strongylus armatus R.

liuseun Viennonae hrjua specimina possi. det equina, quae ita copula jungurtur, ut bursa maris vulvam amplectatur. Conf diarium Salis. burgense M!ed. Chirurg Jan. $181:$ p. 3\%。

C1. Jusetrues Hungson (A treatise on the diseaces of Arteries and Vtins. I.ond IS:5 8. p. 569-575. Vers, germ. I. H. von den Krankheiren' der Alterien und Venen. Hannover 18:7. 8. p. $55 \tau-550$.) Strongylos in aneurysmatibus mescntericat superioris (rarius roeliacre) acini et equi satpius ohetrvavit et. illa efficere crclit, quod dub um relinquere cogor. Entozoa enim atque bene in anturysiatibus fartis gignil, quarm inter arteriarun tuniras oriri et irritarnento suo has morbidas reddere poscuut. Ipse saltem aneurysmata lraec etian vermibus protinus carentia 
Vidi, quod et aliis accidit. Icon. aneurysmatis arteriae asini mesentericae superioris a cl, viro oblata (Engraviugs intended to illustrate some of the diseasey of arteries. Lond. 1815. 4- tab. 8. fig. 2.) Inucle digna; illa Strongyli (ib. Fig. 3.) magnitudine naturali exlibita ninoris momenti, in versione libri germanica Tab., 3. Fig. 5. repetita.

Cl. Greve in libro egregio saepius citato (Erfahrungen und Becb, über die Krankheiten der Hausthiere. Oldenburg I818. 8. p. I69. sq.) aneurystrata mesenteicae Asini et Equi Strongylis pariter tribuit, illqque disrupta mortem inferre vidit. Sinülem Strongylum in equi vivi oculo observasse sibi visus est; conf, obs. In Filariam equi päpillosam, quam plưrimi oculo tribuunt.

Finor semper est Strongylus aneurysmaticus, quam ille qui in intestinis occurrit, an ideo species propria? sed hac de re Entozoologia conferatur.

\section{-3. Strongylus Gigas $R$.}

Siemato dea reliqua non solummodo magniudine superat, sed fabricam etiam valde compositam exhibet, quo Strongylum, vermibus ill. Cuvierr articulatis proximam es'e concedas. Systema nerveun, tenerrinum quidem sed distinctissimum ab OtroNé detectum; idem etiam a cel. Speinufiri repertum, qui systema respiratorium simul exposuit, cujusque praeparata egregia Ticini contemplari licuit. Haec vero in Mantissae parte secunda, anatomico-physiologica, melius dicentur.

Ipse Berolini Tamuarí d. XXII.1817. brevi antequam iter italicum suscepi, duo specimina 
feminea in Canis Lupi ảbdomine reperi mortua, quae renem dextrum excavatum et'emollitum deseruerant, coloxis sanguinei', alteruni. viginti quatuor, alterum triginta et unum pollices long, quatuor lineas diametro transversali adaequantia.

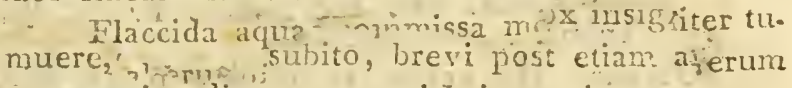
cum sonitu disrupta, omnia circumcirca sangline contaminaverunt.

Uterus simplex; ostium raginae tres pollices ab ore distat. Ova ellipitica, magna, innumen.

Tubus intestinatis cinereus multis filis cute adhaerens; utrinque corpuscula fuscescentia eadem fere semper distantia illi apposita, de qui. bus in Mantissae sectione insequente agam.

Systema nerveum quale Öro descripsit.

Obs. I, Cel Josfrit, Professor Rostochien. sis, entozoa ruagna ex hominis urethra delecta vidit, amico qui nihi mitteret dáta, sed casu perdita, hue certe pertinentia. Neque aliena credereni, quae Bobe.Moreau (Journ. de Méd. T. XLVI. I813. Maji.) cum urina hominis excreta refert.

Obs. 2. FuIYX DE azara (Voyarjes dans 1A Amérique méridionale. T. I. Paris. 8909.8. ए. 297.) Strongylum huncce in Vulpis americinas novae Agnara'Gouaz dictae rene repertum tra. dit. Amicum scilicet Don Pearo BrAs Nosed in femellae annosae rene dextro', saccum effid. ente, sex vermes vivos sanguini innatantes offen. disse, quorum maximus quindecim aequaret poljices.

4. Strongrylus papillosus R. Quem in Corvi Caryocatactis oesophago re. peri, olim cum inséquente conjunxi, cujus maren 
ignorabanı; diversum tsse nune quidem facile concedo et hur.c descriliencio probabo.

5. Strnngy!us tubifex Nrtzsni.

Ent. 1. p. 214. n. 4. sub praecedente. p. 240 n. 24. Str, mergorum. n. 25. Str.

Olfers. De veget. et anis... 56. Fig. 8-14. Str. elegaris.

Am. Nitzsch specimen masculum el feminẹum in Mergi Merganseris tuherculis oesophagais Decembri a se reperta misit, polliceul lonjitudine superantia.

Caput papillis sex conicis exatantibus mu: niturn, óstio medio nigrescente Corpur urrirque attenuatuin. parte meilia contorta maximeque infla:a, untique crenatum. Canda fomintse obtusa. Bursa maris obliqua, contracia; filum getsitale longum, rectum.

Obs. 1. Strongylua, quem in Caryocatacte reperi, medio non inflatis est, neque papillae ca. pitis post nortem habitum açৃue regularem servant; ut huic" Str. papillosi nomen olim impositum relinquam, Stronsylum autem in Colymbis, Miergis, Anatibus, Pelecanis obvịum Nrtzscho praeëunte tubificern dicain.

Obs. 2. Am. Olfers 1, c. fuse dfócripsit et a praecedente distiuxit. Bursam maris obsolete triquetram et simul amplarn refert, quồd ipse saltem sécus vidi et ab evolutione partis forsan pendet, neque cutem crispatam pellucidam naturalem dicerem. Ova vidit ovalia,

Obs. 5. Strongylum nostrum cl. Rosa (Lettere zoologiche- Pavia 17y4, 4. p. 7, n. 9.) sibi dubium obiter refert. 
Obs. 4. Strongylus horridus in carasugo Viennensi huc relatus Spiroptera nobis videtur conf. Synops. n, 35 .

\section{Strongylus filicollis $R$.}

Am. Nitzscr specimina complura in intestinis Cervi Capreoli tenuibus hyeme a se reperta inisit, feminea, omnibus notis cum illis convenientia, quae ipse in Ovibus reperi.

9. Sirongylus hypostomus R. n. sp.

Am. Bremser Strongylos complures in in. testino coeco Antilopes Rupicaprae repertos misit albos, quinque cum dimidia ad novem curn dimidia lineas longos, tenues quidom, sinul autem rigidulos.

Caput subglobosum, gibbum, sive parte nuchali exstante; ore orbiculari partim antico partim infero, integerrimo, nudo. Corpus utrinque parum attenuatum. Cauda feminae apice brevi acuto instructa, qui in nonnullis speciminibus, quorum cutis laesa fuit, reflexus et acutissimus visus est. Bursa maris deorsum angustata, ceterum truncata, radiara, patellaeformis; genitali masculo satis longo, latiusculo, lamella duplici constare viso.

Obs. Capitis bulla, qualis Strongylo armato, huic speciei pariter concessa videtur, sed dentium vestigium in nullo specimine invenire licuit. Genitale inasculum prorsus singulare; an lamellae in duobus speeiminibus masculis mecum com: municatis eodem modo comparatae vaginam tantum penis sistunt?

14. Strongylus denudatus R. n. sp.'

Aliquot specimina in Colubri tesselati pulmonibus reperta Bremserus misit, leminea, qua 


\section{- 264}

Strongyli esse, auriculdto $(1,13$.$) prox...., nustue$ dubito.

Longitudo duas ad qquatuor lineas acquat. Pars media in plurimis rupta.:

Caput nutitum (alis Str. auricularis destitu. tum), ae orbiculari, inarsine tupinidulo. Corpus utrinque, plertmque vero antrorsun magis attenuamm. Cauda plurimis subulata, unicat obusa cum longo acuminte; qualem varictatem etiam in Strengyli anricularis cauda leminer olion oliservavi, cujıs descriptionem in Entozoologia datam. conferas.

17. Strongylus retorlaeformis Zed.

Specimina in Leperis timidi tubo cibario reperta in Encozoolozia (II. 1. p. 224. n. 14.) descripsi; plurima autem cel. Treutler-dono dedit n Leporis trachea $\in$ t I ronchis Januario :790. a se inventa, quae cum descriptis praeter magnitudinem onnibus notis conyeniunt.

Quos in tractu intestinali reperi, tres ad quinque lineas, bronchiales autem quinque lineas ad unum cum dimidio pollicen longi sunt, quae certe differentia magna est.

Ceterum grisei vel fuscescentes; Bursae truncatat visae radii interdum soli superesse vi. dentur, reliqua membrana destructa. Cauda femisae acutissima depressa...

Confer Filarian Leporis pulmonalem n, 28. 18. "Sirongylus nodularis $\mathbf{R}_{2}$.

4.h. Nitzscr in shate scegetmm inter mem. branam internam et musculosum ventriculi apparatum Stzu: gy los multos Novembri reperit, quo. fum nonmulos tam masculos quam femineos beneyole mecum conmunicavit, 


\section{$-265$}

reperi, quorumque specimen inasculum tánere mihi superest, quod cum iscis comparavi. Cápic omnino idem est, neque bursàe discrimen reperio. Feminae caudam olim rectam dixi, quạe in his curvata est, sed inter tunicas ventriculi re. pertis, idenque pressis, dum mei liberi in oesophago yajabantur.'

\section{Sirongylus capitellatus R. n. sp.}

Ć́s. Treutrer specimina dio dedit in intestinis Caprimulgi europaei tenuibus Augusto a se reperta, neutrum integrum, certe tamen Stron. gylos.

Alterius pars anterior adest tres lineas longa, capite globoso; ore orbiculari éxiguo; collo terui brevi; corpore sensim aucto postice abrup. to; tubo intestinali fuscescente. Alterum specimen sex lineas longum capite et collo solis carere videtur, pars enim ejus anterior tenuis adest, quae sensim posteriora versus augetur; apex caudae incurvus et obtusus iterum decrescit,

\section{Strongylus trigonocephalus $\mathbb{R}$.}

- Scriptoribus de Strongylo in Canis familiaris ventriculo et variarum partium tumoribus obvio agentibus BCBE Moreau addendus videtur, qui $A$ scarides Cirinones in corde canis repertos refert: Journ, de Méd. 1813, Miaj. T. XLVIL.

\section{Strongylus tetragonocephalus $\mathbf{R}$.}

Cel. Treuteer specinina in Vulpis colo No. vembri meņse a se reperta mecum communicavit, quàe cum descriptione in Entozoologia data com. paravi, sed nibil addendum inveni, Cauda fe. 


\section{- ana -}

mine (quod etiam suspicatus eram) non de. prén i est.

$$
S p e c i c s \quad d u b i a e \text {. }
$$

25. Strnngylus Vulpis glandularis.

Entozoa quatdam pollicem dimidium et quod excurrit longa, crassiuscula, feninea, ore orbicuiari instructa, inque $V$ ulpis glandulis mesenterii reperta in Museo Viennensi vidi et dubie huc retuli.

26. Strongylus Vulpis oesophageus.

Am. Berenser aliquot specimina in Tulpis oesopluago r perta misit, omnia feminea, alba, quinque aci septem lineas longa, varie curvata. Os orbiculare. Corpus utrinque, magis tamen an. trorsum atrenuatum. Cauda hrevis apice tenuiore.

- Strongyli femina videtur.

\section{Strongylus Suis.}

Duo specimina in Suis bronchis inventa Bremserus misit, feminea; mascula ipse non vidit.

Alterum illorum duorlecim, alterum quindecim lineas longitudine aequant.' Caput rotundatum continuum. Cauda depressa obtusa cum acú. mine.

\section{Genus IX. Ascaris.}

Generis claracter in speciebus individua majora sistentibus probatissimus, in minoríbus passim difficilius cruitur, quae tanto saepe motu pol'lent ut vivae dijudicari nequeant, mortuae autem interdum oris partem retractam et obscuram ex- 
hibeant. Omncs idco Ascarides a me in Synopsi enumeratas hue vere pertinere, neutiquam contendere conahor, sed quoad fieri licuit singulas sedulo iterum iterumque examinavi et in parte operis ingrata multum laboris posui. Nonnullas specits BRE USER's ad Oxyuridem relegavit, quas capite mimi unal vi viso heic retinere cogor, licet genus vastissimun ${ }^{1}{ }^{\prime}$ se linenticim siriderem

Neque suldivisionis ratio a corporis parte vel utrinque aequaliter vel anteriori vel poste. riori magis atterutuata, et ab alis capitis depromta omni dubio cartet, alarum enim species cute post mortem flaccida reddita incauto imponere potest, et f'eminae majores partem corporis posteriorem interdum solito crassiorem exlibent. Meliora tamen divisionis funclamenta vix invenies et species tam numcrosas promiscue epumerare omnium. examen maxime arduum redideret. Quaedam certe animalium familiae Ascaridum etiam tribus p-culiares continent, ut ab illis forsan divisionis argumentum petendum videretur; hoc autem partim cum nostra distributione convenit, partim vero non quadrat, nam interdum animalia diversissima species Ascaridum simillimas fovent.

Specierun differentiae saepe difficillime reperiuntur, ideoque ne diversas conjungerem valde forsanque nimis cautus fui, multas tamen Entozoulogiae species meliora edoctus rescidi.

\section{Ascaris lumbricoides LivN.}

Quae anatomen hujus entozoi a cel. viris, Borano, Ortone aliisque locuplctatam spectant, in Mantissae sectione altera commodius exponentur.

Frorlici (Naturforscher St. 29. p. 33. n. 10.) Ascaridem Suum ab humana distinguit, differen. 


\section{$-268-$}

tiae autem, quas refert, nullius sum momentî, ipseque uiramque comparando zullum discrimen invenio. Genitale masculum at sesquilineam ex: sertum vidit.

\section{Ascoris resicularis Froecrou.}

Quam olim Grypliae auctumno antest nis crassis Phasiani Galli $\epsilon^{*}$ misti nec non Oti-

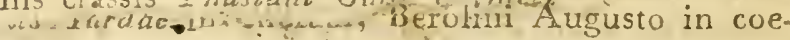
cis Pavonis cristati quinque seplimanas nati, et Anconae in illis aliquot Coturnicum reperi, Froelcr (Naturforscher St. 29. p. 48. n. 22.) in coecis Téraomis l'erdichs similiter occurrere et fere majorem quam in Plasiano colchico fieri, sed in hoc minus raram esse. Eodem loco in Thasiano nycthemero; Thmida Melsagride, Tes traone Crogallo, Bonasia et Perdice graeca repertam Cat. Enit Vienn, equmerat.

Oós: I. FHOELIGI 1, c. p. 49, n, 23, Asca. ridem gallinarum habet, setactam, cauda maris in. curva cuspidata, basi vesiculosa, ano spiculisque duobus distinctis, feminae recta longe acuminata. Forsan ab Ascaride vesiculari (Phasiani colchici) vesicularum defectu distinguit, has autem accidentales esse in Entozoologia dcmonetravi.

Obs. 2. Observationibus 'iteratis Ascarides marras in. Galli tenuibus occurrentes ab Asc. vesiculari differre didici. Conf. speciem inseguenten.

\section{4. iscaris infexa ZEn.}

Srecimina quinque $a b$ auditore quodam acceni in Phasiani Galli tennibus reperta, unum cum dindilio ad tres pollices longa, feminea, utrinque aequaliter attenuata, caudae acumine 
brevi daflexo; cum quibus specimen nagnum Ent. I. 1. 131. ols. I. relatum comparavi, et hujus quidem corporis partem posteriorem, minime autem apicem reflexum, sed hunc ut in illis de. flexum vidi.

Ab Ascaride vesiculari utique separanda, nam intestini coeci Accarides parvulae omnibus notis ab illis magnis tenuium distant, et si.ZzoE. Rus majorum quoque specimina tenella vidit, hoc nihil refert, talia enim quandoque rcperiri de. bent, cum ex ovo subito maximac fieri nequeuat, sed certe etiam minores a vesiculari differunt.

Cum Goezio itaque Ascariles magnas Pha. siani Galli ct Anatis conjungo donec forsan spe. cimina wascuia illarum discrimen exhibeant.

Feminam -sesquipollicartm in tenuibus $\mathbb{B}$ oschadis ferae Angusto repertam Heoelich (Naturf. St. 29.1. 43, n. 14.) describit.

\section{Ascáris subulara R. n. sp.}

Am. Rremser specimina nonnulla in $C a$. jirimulgi ruficollis intestinis Algesirae reperta misit, mascula septem octove, feminea novem ad décem- lineas louga.

Caput valvulis exiguis obtusis aegre discer. nendis instructum, haud discretum. Corpus plu. rimis utrinque aequaliter, quibusdarn posteriora versus tantillum magis attenuatum. Cauda maris fortiter inflexa, apice longiori subulato, bialato; spiculis duobus filiformibus acutis, elongatis. Cauda fominae recta, depressa, subulata.

Ova subrotunda foetum spiraliter convolu. tuin exhibentia, 


\section{- 270}

\section{Ascaris adunca $R$.}

In Clupeae Alosae ventriculo plurimas, paucas in illius intestinis Arimini Aprili reperi Ascarides vivas, quatuor ad octodecim lineas lon. gas, pro ratione plus minus crassas.

Maris spicula duo longa incurva observavi, ceterum ejusdem apex caudae illi feminae siniIis est.

\section{Ascaris constricta $R$.}

Quae Neapoli Junio in duorum Trachino. rum Draconum peritoneo reperi specimina caput nonnihil crassius exhibuerunt.

\section{Ascaris rotundata F. n. sp.}

In Squali Galei ventriculo $\epsilon t$ intestinis Arimini Majo praeter duo specimina mutila unum reperi integrum, femineum, octo lineas longum.

Caput rotundatum, valvulis distinctis, mediocribus; corpus utrinque atqualiter attenuatum; caudae apice acuto inflexo. Intestinum oviductibus tenuibus gyratum.

Brcmservs specimina quinque misit in Squali glauci ventriculo reperta, octo ad viginti unam lineas longa, vel aequaliter, vel artrorsum magis" attenuata, capite rotundato, caudae apice brevi acuto, ante quem in duobus speciminibus tuberculi species exhibetur.

\section{Ascaris pellucida T. n. sp.}

Cel. Treuteer Ascaridem dedit sub $U$ pu* pas Epopis tunica hepatis a se olim repertam, viginti duas lineas longam, candidissimam, pellue cidiorem quam hujus gentris species aeque man gnae reperiri solent, 
Caput nudum, valvulis magnis obtusis, dis. cretis; corpnes utrinque acqualitcr attemuatum; caudae apex obtucus, ante quem anus. ex quo corpus eminet quasi aculeis obsitum, nirsus bene taintll dignoscendum.

11. Ascaris gulosa R. n..sp.

In Testudinis tabulatae masculae ultra no. vem libras pondere aequantis, iterumque in femineae minoris coeco et colo satis multas reperi Ascarides mortuas, alisas, tre's ad quinque lineas longas, crassiusculas.

Caput valvulis tribus distinctis longiusculis; membranae lateralis, quae aderat species, accidentalis visa est, entozoa enim in'Testudinibus com: plures ante dies mortuis minus recentia erant. Corpus utrirque aeq1ıliter attenuatum. . Ciadne apcx in plurimis brevis acutus divaricatus, in paucissimis cauda recta subulata.

Tractus inlestinalis insignia; mox a capite gula incipit magia, postice ampliata, sive lànceo. lato - ovata; oespphagus illa longior, tenuis, cylindricus; ventriculur duplex subrotundus approximatus, sive proventriculus et ventriculus; intestinum cylindricum (oesophago crassius) rectiuscu. lum, passim aucium, haud procul ante! caurae apicem multo crassius factum et antrorsum directum ano terminatur.

Gcnitalia valde ambigua. Mox spicula duo pone anum pariterque antrorsum ducta vidi; mox ccrpus (vulva?) magnum angulo recto projectum, ex quo aliud emergitur antice bidentatum, et in speciminibus quidena tam cauda divaricata quam lor ga 'recta utentibus, omnibus his ergo fimintis, vario modo comparatis (conf, descript, Asc. spi. 


\section{- $272-$}

nicaudae n. 13.), masculis illis solis, quae spicu. lis utantur. Apertura genitalis ipsam ante diva. ricationem caudae.

\section{Ascaris dactyluris R. n. sp.}

In Testudinis graecae Romae Majo dissertae intestinis crassis multa millitum millia specimin:a reperi, ut maxima faccum pars iisdem constaret. Eadem copia Bremserus in compluribus T. graecis offendit, et Redi (Ent. IL. 2. p. 266. n. 9. Verm. gen. dub.) nullo non anni tempore in Tcstudinis sacco coli (i. e. coeco) vermiculos copiosissimos observavit.

Entozoa alḅa, plurimam partem pellucida, mascula exigua in spiram convoluta, vix lineam attingentia; feminea majora, duas ad duac cum dimidia lineas longa, crassiuscula, recta vel stricta.

Caput trivalve, haud discretum. Corpus utrinque aequaliter attenuatum. Cauda marinm apice brevi obtuso, depresso, ante quem spicula, pas. sim subexstantia, passim in spiropterae mudum vaginam fimbriaram egressa visa, ita tamen ut hoc dubium relinquam. Cauda feminae longa subulata;

Oesophagus rectus tenuis, in maribus pro portione corporis multo longior visus, terminatus proveintriculu subglohoso, a quo ventriculus cras. sus exoritur selisin in intestitum tenuius trans. iens. Ova cilliptico oblonga, magna, nucleo ob. scuro diviso.

15. Ascaris spinicauda Olfers. n. sp. Cel. vir qui speciei nomen imposuit, Asca. ridtes in Lacertae Teguixin intestinis crassis, prae. 
sertim coeco Septembri et Octobri in Brasília re. perit,

Specimina missa duas cum dimidia ad tres cum dimidia lineas longa.

raput mibi trivalve videtur, in illis autem, quihus cutis relaxata est, miras formas induit, ut capiti multistriato tiaram impositam diceres. Corpur maribus utrinque aequaliter, feminis retrorsum magis atteruatum tst. Canda maris incurra, apicis cuspide inflexa acuta instructa, introrsum bialata, genitali, masculo brevi subulato inter alas emergente. Cauda feminae recta subu. lata; vulva varie inodo in aliqua ab apice distantia tminte, ut mox cylind:um, mox cyathum sistat. Or Orana digestionis vulgaria.

\section{Ascaris transfuga $R, n$. sp.}

Am. Bremser specinina misit in intestinis Uisi Arcti et maritimi reperta.

Specimina in 27 . martimo reperta duos cum dimidio ad quinque cum dimidio; illa $U$. Aicti femintrea quinque ad octo, ma culum quatuor polliccs longa, pro magnitudine pius minus crassiu. scula.

Utraque, eadem, sed in illis, $U$, maritimi spicula excerta non vidi, ut Ascarides in $U_{6}$ Arcto obvias describendas sumserim.

Caput diacretum, valvulis tribus convexis, distinctissinis, alis urinque ad lineae longitudinem decurrentibus spmilanceolatis; reliquum cor7)us sulcatum, mémbrana in sulcis evanida, utringine atqualiter attenuaţum. Canda maris obtu. siuscula cum apice ṕapillari minimo, ante quem (lineae dimidiae distantia) spicula duo recta et tenuia, brevia, vix lineam dimidian aequantia. 


\section{- $274-$}

Pone haec anus. Cardd feminae obtusa apice acuto brevi, ante quem anus. In altero specini. ne caúda deformata, apice transverso.

Ols. Speciem capite alato insequentibus, corpore fortiter sulcato liumanae (lumbricoidi n. 1.) affinem, idcirco transfugam dixi.

16. Ascaris leptoptera R.

Leonem novendecim ante menses Londini natum, pridie mortum, Decembri 1817 Berolini secui, inque oesophigo sed praesertim in ventri. culo plurimas (ultra centum) reperi Ascarides, mortuas.

Alterius Leonis, Viennae mortui, pariter junioris, Ascarides satis multas am. Bremser misit, ejusdem indolis, sed passim majores, in ventriculo et intestinis repertas.

Longitudo unum ad quatuor pollices adaequat, niajora specimina simul crassiuscula.

Alae capitis, quas secundum specimina tria a cel. Schw's grrchen, Botanico Lipsiensi, in Leaena reperta et nuecum communicata, Ent. II. I. p. 137. 17. 9. lineares dixeram, in plurimis tales repéri, in majoribus tamen latescunt quin semiovata fiunt; quo commotus Bremserus leptopteram cum mystace n.'2r. conjunxit, hujus vero cauda alata, illius est nuda, neque mystacis junioris alas capitis lineares vidi, licet tale quid acci. dere ex litteris cel. Mrerem ad me datis efficiam, qui se in Fele Ascaridem invenisse refert ad leptopteram pertinentem.

In speciminibus masculis spicula vidi duo, ante apicem caudae sita, arcuata, quorum alterum altero longius est. 
17. Ascaris microptera R. n. sp.

In Canis Lupi masctuli intestinis tenuibus aliquot Ascarides mortuas reperi (Berolini, Januario) duos tresve pollices Iongas, albidas, oviductibus candidis translucentibus, in aqua cito tumidas factas. Aliquot diebus elapsis in alterius lupi oesoplago unicam reperi, pariter mortuam, cine. rascentem, oviductibus niveis, tres cum dimidio pollices longam, rectiusculam. Duo quoque spe. cimina ab am. Oтrone Vratislaviensi accepi, in Lupi tenuibus Janiario inventa, quorum alterum novem lineas, altẹrum duos pollices longa sunt.

Caput exiguum, membrana utrinque lineari brevi, qualis nec in corpore uirinque aequaliter sed parum attenuato, nec in cauda obtusa occurrit.

Obs. Áb Ascarido Leonis leptoptera alis capitis brevibus, ab Asc. Canis marginata corpore crassiore et alis tenuioribus differt, Cum $S_{p i=}$ roptera lupi sanguinolenta n, 2I. confundi nequit.

\section{Ascaris brachyoptera R. n. sp.}

Am. Bremser duo specimina misit in inte. stinis Viverrae Genettas Algesirae reperta, crassiuscula, lactea, masculum duos cum dimidio, fe. mineum tres poliices longa.

Caput discretum, valvulis tribus obtusis exiguis, ala in sperimine femineo utrinque semielliptica brevi, marginem incisa, tenuiore in mascule, sed hoc specimen cutern et capitis ct corporis passim emphysematicam ideoque defigura: tain habet. Corpus utrinque aequaliter attenuatum. Cauda muris depressa, inflexa, apice papillari brevissimo; feminae teres, apice papillari 


\section{$=276-$}

brevissimo, ante quem anus; ala revinciens caudae zulla, quio ab insequente distinguitur, cujus etiamalae capitis differunt.

\section{Ascaris Mystax ZED.}

Quam in Fele Cato domestico hactenus repereramus, Museum Viennense quoque servat in Cato fero et Lynce reperta.: Hujus Ascarides spiczile maris longa offerunt, mox rectiuscula, mox incurva,

\section{Ascaris semiteres Zen.}

Cel. Froelich (Naturforscher St. 29. p. 4l. Ascaris retusa) Ascarides a se in intestinis crassis Corvi Coroneś Augusto, et in tenuibus Trinc. gae Vanclli Septembri repertas conjungit, ut has tantum minores rocet, sed certe diversae sunt. Descriptio secundum specimina in Corone oblata a cl. viro facta esse videtur.

\section{Ascaris depressa ZED.}

Ent. II. I. p. 143. n. 13. Asc. depressa. (Falconum.) p. $1 \$ 9$. n. 52. Asc. spiralis (Strigium).

Quas in Entozoologia diversas cnumeravi, nunc quidem conjungere cogor, cauda enim in speciminihus majoribus taniurn crassior, in reli. quis corpus utrinque aerqualiter attenuatum reperitur.

Quam olim Gryphiae in Falcone Milvo Octobri, in Strige flammea Nartio et Augusto inveneram, Berolini in Falçne palumbario Augusto, in Butcone, Julio ais Armanuensi repertam recentem examinavi, et cum speciminibus comparavi in I'nlture fulzo lectis et ab am, Binzisero communicatis. 


\section{$-277$}

Haec vel minora (dimidium ad unum cum dimidio pollicem) vel majora (duos cum dimidio ad quatuor cum dinidio pollices longa) illa utrin. que, hatc postice minus attenuata. Caput in quibuscam colli tumiduli specie excipitur; nota tamen inconstans est, neque Vulturis Ascaridem ab-illa Falconum et Strigium distingnit.

Cl. Frostcrir Ascaridem Milvi (Naturf. 29. p. 34. n. I1, Tab. I, Fig. 15, I6.) Asc. Nisi (ib. p. 35. n. 12.), Asc. aequalem (Buteonis, ib. p. 37. n. 14.) et Asc. Bubonis (ib p. 39. n. 15. Tabx. Fig. I3, 14.) praeter ullam necessitatem distinxit, et cliaracteres maxime vagos posuit. Illas Milvi et Nisi in differentia specifica aequales, in descriptione autem postice crassiores dicit, et reliqua ejusdem sunt farinae, quibus exponendis supersedeo.

Catalogus Viennensis Ascarides enumerat repertas in intestinis Vulturis cinerei et fulvi; Falconis Albicillae; apivori, Buteonis, Chrysaëti, cineracei, cyanei, Lagopodis, lanarii, Milvi, naevii, Nisi, palumbarit, pennati, peregrini; ruf, Tinnuntculi; Strigis Aluconis, Bubonis, Oti.

Marsil (Danubius Pannonico-Mysicus "T. 6. p. 9. tab. 6.) valde magnam Aquilae Melanaë. bos obiter refert,

Ascaris haec plerumque in intestinis tenuibus, rarius in crassis et forsan non nisi dejicienda occurrit.

Obs. In Strigis Oti superficie intestinorum tenuium peritoneali (Berolini d. 3. Junii) corpuscula alba lentiformia vel subglobosa occurrebant, vel immersa, vel filo pendentia, lineae tertiam circiter partem diametro adaequantia. Quibus apertis vermis minutissimus sistebatur quem nu- 
dis oculis nonnisi exercitio aliquo usurpare potui, postquarn ope microscopii locum notaveram, qua reperiendus esset; teres, elasticus, in spiras arctissinas convolıtus; tandem explicatus et tum lineain dimidiam vix attingens, crassiusculus, capite olstuso, valvularum speciem exhibente, cau. da obtusa cum acumine.

An vermiculi isti sobolem Ascaridis depres. sae sistunt, cujus nidum hydatides referunt? Simile quid de Distomate crystallino n. 36. suspicor:

\section{Ascaris ensicaudata ZED,}

Ent. IT. 1. p. 145. n. 16. Asc. ensicaudata. p. 191. n. 54. Asc. lancea.

Entozoologiam edenti nonnisi Ascaridem lanceam in intestinis Turdi iliaci repertam examinare contigerat, ut ZEDERT auctoritate commo. tus illam T. Merulae ensicandatam distinguerem, dubium tamen de utriusque diversitate l, c. p. I45. obs, 2. adjicerem. Postmodum autem Octobri Gryphiae in T. Merulae tenuibus Ascaridem reperi omnino, eandem ac illam Turdi iliaci, bipollicarem, cineream intestino fusco translucente, utrinque aequaliter attenuatam, et (licet vivam) tenuissime crenulatam, membranis, Iateralibus in capite et cauda quam in reliquo corpore latioribus. Iu Catalogo Viemensi typis impresso Ascarides Turdi Merulae, viscivori; musici, torquati ct arundinacei ad ensicaudatam, illa autem Turdi iliaci ad lanceam referuntur. In Catalogo Vien. nensi inanuscripto, qui entozoorum nomina trivialia non habet, etiam Turdi saxatilis $\Lambda$ scaris enumeratur. 


\section{$-276-$}

27. Ascaris fallax R. n. $s p$.

Bremserus sperimina misit in Jacerac viridis ventriculo reperta, ipseque similia ir illius intestino sed nortua reperi, allida, mascula quatuor ar quinque, feminea quinque ad quatuordecim lineas, longa, crassitiei mediocris.

Capitis valvulae duae saepe in conspectum veniunt, tertiae interdum species sed minus distincte exorta est; illae tum majusculae obtusae seu rotundatae; interdum omnes obscurae fiunt, membrana utrinque laterali exhibita, Corpus utrinque aequaliter attenuatum. Cauda maris in: flexa, ala utrinque deflexa, feminae rectiuscula, depressa, obtusa.

Asčaridem esse suspicor; quibus vivam examinare contigerit, 'rem conficiant.

28. Ascaris nigrovenosa ZED.

Specimina satis magna in $B u$ fonis viridis pulmonibus reperta, quae Bresserus misit, cum illis a me aliis in Amphibiis inventis omnino conveniunt.

\section{Ascaris vermicularis Lixx.}

In tempus quo mares hujus speciei occurrant, Berolini 1816. inquirere incepi, sed negotiis obrutus rem nondum confeci, aliquoties saltem magnam harum Ascaridum copiam vel a pueris dejectam vel in cadavere repertam examinavi neque ullam masculam invéni. Ill. SoemMERRINGius tamen felicior fuit, cui et Bremserus tt ego specimina nostra mascula debemus.

Caput hujus speciei quoties examinaverim, fidem fere superat, nullam tamen examinandi occasiunem praetermitto, cum Brumsenus, deliciae 
nostras, os illius evalve dicendum contendat; ot tale mhi etiam in Museo. Viennensi visum, dein autem uti olim semper trivalve exhibitum sit: Valvuae ceterun in specimnibus femineis, ut, pote najoribus, facilius, et in Simią Așcaridibus, (conf. obs.) adhuc melius conspiciuntur; inter; dum retrahuntur, tumque alae capiris majores fiunt. Oxyuridem esse, idzo non concedam.

Mlas linearn superat, parte anteriore tenuiore rectiuscula, positriore crassiore spiraliter inflexa, apice caudali obtuso, ante qucm (latere vermis inferiore inflexo) tubulus brevis exstat, penis exiguam partem apice proferens, Ita salte?n in meis speciminibus tribus video. Caput quam in femina angustius.

Prolem vivam aliis dictam nullo anni $16 \mathrm{~m}$ pore reperi, sed ova oblongo-elliptica, parte media nigro-irrorata.

Obs, Brevserus specimina multa misit feminea in intestinis crassis Simiae Panisci reperta, pariter pro Oxyuride habita, quae ab humanis capite quodammodo denectuıt, reliquis vero no. tis curn illis convẹniunt.

Fere omnibus in speciminibus 'valvulae capitis tres obtusae distinctissimae sunt, ut his ma. joribus et membrana capitis laterali angustiori species forsan nova, humanae tamen maxime affinis efficiatur; sed maribus non. visis huc interim retulị. 'Ova satis magna ovato-elliptica.

\section{Ascaris obvelata $\mathbf{R}$.}

Muris Musculi tantum Ascaridem femineam, in illius, crassis Novembri a the repertam examinare licuit, sed uit specièi prateedentis ita tliam hujus valvulas capitis distincte videre milhi vi 
sus sum, quo minus Amico pro Oxynride illam habenti assentire possim.

\section{Ascaris truncata ZED.}

Ascaridem a Froexrchro in intestinis Psittaci aestivi detectam tt immerito hermephroditam dictan, cum Ascaride Columbae maculosa (n.35.) nimis congruere visam in Entozoologia (II. I. p. 165. 11. 60.) perperam suspectam reddidi, sed in Museo Vicnnensi meliora edoctus sum.

Adest ibidem primo specimen masculum in intestinis Psittaci dominicensis repertum, sesquipollicare, crassiusculum; capitis valvulis distinctis, margine laterali tenui, lineari; cauda obliqua, mucrone apicis brevi papillari, incurvo.

Adsunt porro in Musto ditissimo duo specimina femineu in intestinis Psitraci leucocephali reperta, sesquipollicaria satisque crassa, capitis mcmbrana angustissina sive lineari.

Ascaris Columbae maculosa psittacinae simillima ab ista differt: r. -membrana capitis semielliptica, neque lineari; 2. çauda mascula recta, ne. que incurva.

Spicula in G. maculosa longiora vidi, ceterumi autem ut in truncata pone anum sita sunt.

\section{Ascaris dentata ZED.}

Huebser specimina aliquot in ventricula, Bremser complura in intestinis Cyprini Barbi reperta mecun communicaverunt, alba, neque argentea, qualia ZEDER vocat.

Caput valde attenuatum, absque alis ZenEro dictis, quarumque species verme flaccido facto oboritur; valvulae exiguae, oblongae. Cauda feminae obiter inflexa, máris in spiram planam vo. 
Juta; dentes marginis caudae masculae a Zenero 'relati nil nisi crenae, sed forsan constantes sunt. Ova exigua, globos3.'

\section{Ascaris gracilescens R. n. sp.}

Arimini Aprili et Neapoli Junio in Clupeae Encrasicoli, et illic Majo in Clupeae Spratti peritoneo intestinorum multa reperi specimina; consimilia bremserus misit, in Spratti tubo intestinali reperta dicta, an jure?

Duas ad quinque lineas longa, alba vel rubella, milii viva examinata pro Ascaridibus lia. bita, Bremsero dubii generis visa.

Caput indiscretum obtusum vel truncatum, valvulis tribus latiusculis, ore tamen medio conspicuo. Corpus utrinque, magis tamen posteriora versus attenuatum, unde speciem gracilescentem dixi. Caudae apex depressus, acutus, longiuscu. lus. Intestinum rectum. Genitalia mascula non vidi.

\section{Ascaris compar SchriantK.}

Froelich (Naturforscher 29. p. 46. n, 2 r. Tab. I. Fig. 2I. Tab, 2. Fig. $1-3$ ) sub nomine Ascaridis Lagopodis descripsit; marem duos, feminam tres pollices excedere; spiculum simplex vidit, quale Ent. II. I. p. I62. obs. I. adnotavi.

Synonymon Redianum a me huc relatum forsan ad Trichosomatis speciem pertinet; Froe. xich idem ad suam Filariam Tetricis revocavit, sed de Lagopodis neque Tetricis entozois ReDr loquitur.

40. Ascaris leptocephala R. n. sp.

Am. Garde Ascaridem recentem attulit a se, in hydatide lienis, Bufonis, cruciati insidente 


\section{- 283}

Junii d. 26. Terolini repertam, albam, sesquilinearem, femineam.

Habitus fere Echinorhynchi, capite scilicet longo, tenui cliscreto, valvulis ublongis obtusis; corpus antrorsum crassius, posteriora versus sensim decrescens; cauda acuta. Tractus intestinalis simplex absque divisionibus solitis; circa eundem maculae insolitae nigrae.

Brevi post am. Otro Vratislavia duo specimina misit in Salamandrae palustris ventriculo rep,erta, quorum alterum liberun, alterum illi infixum erat, candida, crassiuscula, mascula.

Caput discretum, valvulis magnis obtusis, quarum safpe duae tantum visui exhibentur. Corpus antrorsum attenuatum; apice postico obtuso vel quasi truncato; margine inferiore apicis spiculum emittente reflexum, vel apici truncato appressum, vel remotum.

Bremserus deinceps specimina multa misit in Bufonis ignei ventriculo reperta. Unicum exiam ni fallor specimen in Protei anguini vel1triculo lectum in Museo Viennensi vidi. Conf. Entozoon dubii generis n. 35 .

Obs. Genus forte novum efficit; ob valvulas capitis Ascaridibus quidem addidi, sed quemadmoduin Nematoidea valvulis destituta in complura genera divisa sunt, ita etiam illis instructa olim plura sistent, et lubentissime ipse haec constituissem, si characteres boni ad manus essent. Confer n. 43 et sequentes.

\section{Ascaris brevicaudata ZED.}

Variis ex amphibiis comparavi cntozoa huc facientia, valvulisque capitis certe instructa, licet non semper in conspectum vemiant. was. 
In Angris, fragilis per nycthemeron spiritui vini commissi inteatino complura reperi specimina viva, dieque insequente adhue viventia, licet aqua cui inmatabant spiritu haud protinus libera erat. Omnia feminea, cum descriptione in Entozoologia data (II. I. p: 166) convenientia. Foetus vivi matre aperta cum ovis ruptis et relictis propullabant, quibus non adhaerebant, cauda licet acutissima utentes.

Cum haec Maji ditbus ultimis (Berolini) observassem, am. Gafdx Junio insequente specimina duo atulit pridie in intestinis Bufonis ignei reperta, et in aqua cui spiritus vini affusus fuit servata, motus qiuiciem expertia, at prolem vivam, quac in matrum corpore vivide agitabatur, continentia!

Reperi quoque Junio in hydatidibus Bufonis viridis mesenterio et vesicae urinariae affixis; quales Otтo circa ejusdem Bufonis oesophagım 'invenit; singulae singulas continent Ascarides.'

In Bufonis viridis recto reperta Bremserus misit, quorum duo spiculum brevissimum, neque longissimum quale ZeDERus vidit exsertnm exhibent; cauda, ut l. c. dixi, ante apicem verrucarum serie insigni.

Catalogus Viennensis hanc speciem etiam in Colubri Natricis, nec non Salamandrae atrae et maculosae intestinis repertam refert. In lac ipse quoque reperi, ut Ascaris Salamandrae Ent. II. I. p. 199. n. 68 , cum brevicaudata conjungenda sit.

\section{Ascaris echinata R. n. sp.}

Speciei maxime singularis specimina duo in Geckone Algesirae reperta Bremserus misit. 


\section{- 285}

Lineam vix excédit. Caput trivalve, valvis magnis, acutiusculis. Corpus antrorsum magis attenuatum, postice retusum, mucronem exhihens terminalem longum, tenuissimum, primum rectum dein inflexum, qualem in nulla specierum reliquarurn vidi. Tota corporis superficies seriebus aculeorum-reflexorum densis et transversis munita, qui in marginibus quam in lateribus melins dignoscuntur, illic nimirum liheri, singuli ex latiore basi exorti. Tubus cibarius rectıs; oeso. phagus tenuis brevis; proventriculus subglobosus exiguus; ventriculus triplo major, antice obtusus et crassissimus seusim in intestinum transiens.

Obs. Hriec quoque species, uti amphibiorum Ascarides variae, privi quid habet. Conf. obs, ad n. 40 .

\section{Ascaris megatyphlon $\mathbf{R}$. n. sp.}

Duas Lacertas Iguanás secui, spiritu vini servatas, quarum altera coecum foliorum residuis plenum absque entozois, altera autem idem ci-, bis vacuum at Ascaridibns refertum obtulit.

Hae dimidiam ad tres lineas longae, crasSiusculae, flaccidae, quarum plurimae femineae.

Caput valvulis tribus acutiusculis. Corpus feminae retrorsun incrassatum tandem longe subulatum; maris obtuso truncatum, genitali longo recto tenui in altero ante apicen exserto.

$\mathrm{Ob}$ partem, quam pro initio crassocum primum hahui et cum coeco variorum mamnalium et auphibiorum magno comparavi, megatypilon dixi, sed synopseos parte impressa, nomineque non amplius mutando, res ambigua viderur. Tubi intestinalis scilicet jars prima saccun longum et amplum efformat; quem excipit aeque 


\section{- 286}

longa vel in aliis speciminibus multo longior, at triplo tenuior, hasi in partem tertiam exiguam subrotundam desinens; huic apposita quarta antrorsum obtusa, crassissima, subovalis, quám insequitur quarta inflexa tenuis. Pars prima et secunda simul sumtae a (nam hoc variat) quam reliquae omnes. Partes hae diverso modo explicari possunt; olim primam ventriculum, secundam intestinum tenue, reliquas intestina crassa dixi; nunc primam pharyngem, secundam oesophagum, tertiam proven: triculum, quartam ventriculum, quintam intestinum, nelius aut saltem aeque bene dici posse concedo, alias nimirum species comparando.

45. b. Ascaris tenuicollis R. n. sp.

Cel. Tiebemaran, Anatomicorum decus, pro sua in me amicitia Ascarides complures mihi misit in Crocodili Lucii junioris, Martio I818 Heidelbergae mortui, ventriculo a se rejertas.

In hujus tuberculis duriusculis sinuosis habitant, $e x$ quibus tarien facili negotio extrahi pos. sunt; nonnullae totae albae, piurimá anteriori parte fuscescentes, quatuor ad septem lineas longae.

Caput nudum, discretum, valvulis tribus inajusculis obtusis. Corpus utrinque, praesertim tamen versus anteriora attenuatum. Canda maris incurva oblique truncata, apice brevi acutissimo terminata; ante quem spicula duo inatqualia, quorum alterum interdum latet, sita sunt; canda feminae recta depressa subulata. Membrana :audae utringue tenuis.

Obs. Amicus Crocodili tunicam ventriculi internam parvulis et copiosis abscessibus cum ex- 


\section{- $20 \%$}

crescentiis rubellis obsitam vidit. $\mathbf{P a r s}$ ventriculi cum Ascaridibus transmissa spiritu vini contra. cta nihil nisi tubercula dicta ostendit.

\section{Ascaris extenuata R. n. sp.}

Am- Bremser specimina complura misit in intestino recto Lacertae margaritaceae Algesirae reperta, omnia feminea, tres quatuorve lineas longa.

Capitis valvalae passim subconspicuae. Corpus utrinque, magis tamen antrorsum attenuatum. Cauda obtusa in longum et tenuissimum mucro. nem subito extenuata.

Tubus cibarius singularis. Oesophragus tenuis rectus brevis, subito in ventriculum subglo. bosum transiens, a quo intestinum incipit, primum crassiusculum, dein anśustius, simplex, ano satis magna distantia a lato caudae apice terminato. Vulva haud longe a capite distat, pone ventriculum sita. Oviductus crassiores, versus caudam ubi revolvuntur, in tenuiores abeunt. Ova, qualia in nullo hactenus vidi entozoo, linearia, longissima, parallelipicleda; marginibus la. teralibus aeque rectis ac antico et posico truncatis, ut si angulorum cirri adiessent, cum Squalorum ovis comparari possent. Ammion tunica externa fere triplo brevius, ovale.

Ova ista fere novum genus arguunt.

Obs. Amicus quoque vermiculos quatuor misit, vix lineam longos, capillo multo tenuiores, in spirain planam convolutos, in musculorum tuberculis (an potius hydatidibus?) Lacertae margaritaceae repertos. Horum caput trivalve, corpus antrorsum magis attenuatum, apex caudae brevis acutiusculus, Num priorum sobolem sistant? 


\section{- 288}

\section{Ascaris leptura R. n. sp.}

Bremserevs complura specimina misit in in: testinis Testudinis Mydae reperta," tres ad octo lineas longa, tenera, suhpellucida.

Caput discretum, valvulis tribus obtusis, saepe optine conspicuis, saepe, forsan ob pharyrisis partem protrisam, obscurioribus, quo antica pars nodulosa vel papillosa apparet. Corpus' a capite sensim latescit, feminae in candam longam, exquisite subulatam et rectam transiens, maris cauda crassa inflexa, acumine aeque longo et subulato ac feminae, sed fortiter reflexo.

Ante apicen istum reflexum spicula duo secta $\epsilon$ t brevia, pone quae corpus tertium cylindricum obtusum illis duplo et brevius et crassius. Oviductus ovulis oblongoovalibus repleti Tubus cibarizs oesophago longo posteriora versus sensim incrassato, tum ventriculo subrotundo parxo et intestino a crassiore apice sensim àttenuato constat.

Species cauda maris longe subulata et genitalibus masculis distinctissima.

\section{Ascaris microcephala $\mathrm{K}$.}

In Entozoologia (II. I. P. 167. n. 3r.) spe: cimen femineum descripsi ab am. Nitzscri in Ardeae comatae abdomine Majo repertum et benevolé mecum communicatum.

Arimini Aprilis d. XXIX. in Ardeae $\lambda^{\prime} y$. cíicoracis oesopliago et ventriculo complura, aliquot etiam inter ventriculi tunicas et qua partem egressa et adiposae tunicae ventriculum cingenti immissa reperi specimina viva, quorum unum masculum fuit, 


\section{- 289}

Crassitie longitudiuis ratione habita excellunt, quo ab affuibus v, c. Ase. spiculigera differunt, et similitudo quảedam curn Cucullano ascaroide Goezrr oboriatur; specimina tunicis involuta liberis tamen minus crassa sunt.

Apex caudae brevissimus incurvus, ante quem spicula valde longa, incurva, breviora tamen illis Ascaridis spiculigerae:

\section{Ascaris sulcata $\dddot{R}$. n. sp.}

Am. Breniser aliquot specimina misit in in. testinis Testudinis Mydae reperta, unum ad quatuor cuin dimidio pollices longa, pro longitudine plus minus crassa, quorum unum (e majoribus) masculum:

Caput valvulis tribus majusculis instructum; discretum. Corpus utrinque, magis tamen antrorsum attenuatum; cauda maris inflexa, feminae rectiuscula, apice (utriusque sexus) conico brea vissimo acuto, Ante hunc anus et spiculum. Unum scilicet, rectum, breve, tantum vidi; alterum tamen non deerit, sed certc latuit.

Membrana corporis nulla, sed linea utrinque impressa, unde nomen triviale desumsi.

Obs. In intestino tenui Testudinis indicas femineae versus finem Novembris; synopséos parte jami typis impressa; unicam offendi Ascaridem sex pollices longam, femineam, sordide al bam, antrorsum magis attenuatam, postice lineam crassitie superantem, capitis discreti valvulis distinctis obtusis, corpore utrinque sulcato, apice caudali brevi conico et acuto.

$\mathrm{Ob}$ corpus sulcatum neque alis instructum huc neque ad Assaridem holopteram n. 71. re. 


\section{- 290}

Fero, licet haec pariter in 'Testudine terrestri (gra€ca) reperta sit, sulcata supra descripta autem in Testudine marina occurrat.

5:. Ascaris spiculigera R.

In trium Pelecanorum Carbonum oesophago ot ventriculo Ascarides complures reperi tam masculas quam fernineas, cum illis Onocrotali in Entozoologia descriptis protinus congruentes.

Aves, Kilonii die II vel III Maji a cl. PETERSON Historiae naturalis Cultore ardentissimo glande plumbea trajectae et aquale vitae commis. sae Berolinum mandatae sunt. "Die XIV Maji alteram avem spiritu fortiter impraegnatam aperiendo Ascarides in oesophage et ventriculo rigidas et motus expertes reperi, neque in aqua frigida reviviscebant, calida vero affusa, dum tepida fiebat, omnibus vita et motus reddebantur, qui tamen fervida affusa subito evanuerunt nullo inodo revocandi. In reliquis duobus Carbonibus die instquente examinatis nonnisi vere mortuas offendi.

Eandem speciem in Pelecanis cristato et pygmaeo repertam Catalogus Viennensis habet.

54. Ascaris labiata R.

Quam in intestinis Muraenae Anguillae Gryphiae Augusto reperi (Entoz. II. I. p. I72. n. 37.), Neapoli Junio in Muraenae Congri majoris intestino infimo solitariam offendi, albam, undecim lineas longam, anteriora versus magis attenuatam, capitis valvulis maximis, cauda depressa, intesti: uis ex media corporis parte cito prolapsis.

Quam ibidem Julio in Muraenae Myri peritoneo testiculos nectente reperi solitariam, sex 
lineas longam antrorsum magis attenuatam, cauda depressa acutiuscula, dubius huc refero, quia ca- , pitis valvulae mediocres erant, neque maximae, quales in $A$. labiata inveniri solent.

Obs. Ascaris nostra in Zool. Dan. Vol.IV. p 32. Tab. 147. D. 1.2.) ab Abildgandolo descripta et delineata sistur. Voćatur Ascaris An. guillae (filiformis postice incrassata). Corpus dicitur filiforme, tres cum dinidio pollices longum, album, antice attenuatum truncatum, postice incrassatum apice setigero acuminato; lineis lateralibus aureo-viridibus, intestinis candidis. In intesinis M. Anguillae. Vixit triduo in aqua plu. riali. - Ipse neque tantam, neque talibus colori. bus splendentem vidi.

56. Ascaris crassicauda R. n. śp.

Am. Bremser specimina complur misit in intestinis Labri Tincae reperta, quinque ad qua. tuordecin lineas longa, mediocris crassitiei, alba, feminea.

Valvulae capitis tres distinctae, saepe hiantes, ut singulas numerare liciat, alias duae tertia melius conspicuae, magnae, truncatae. Corpus utrinque, magis tamen antrorsum attenuatum. Cauda obtusiuscula; interdum papillae minimae ter. minalis species adest; prope apicem caudalem tuberculum magnum, in majoribus specimini. bus quam in minoribus pro portione majus. An vulva hac in specie tantopere descendit? Conf, Asc, spinicaudam n. 13.

\section{Ascaris obtusocaudata ZED.}

Am. Oтто duas mecum comimunicavit Ascarides in Salmonis Farionis intestinis a se repertas, 


\section{- 292}

Alterain octodecim, alteram novendecim lineas longam, utramque valde crassam, parte antica tenuiore.

Talvulae oris distinctissimae. Membrana lateralis, quialem ex ZEDERI descriptione in Entozoologia retuli, utrinque in cauda quam in ca. pite magis coit. Minoris cauda apicem obliquum brevem obtulit; majoris autem speciminis apex caudalis rectus, apertura exigua perforatus.

61. Ascaris rigida $R$.

Ani. Brenser specimina tria misit in Lo. phii piscatorii tubo cibario reperta, alterum masculum duodecim, reliqua feminea quinque et undecim lineas longa, cinereo-fuscescentia, antror. sum magis attenuata.

Cauda maris incurva cuspide tenui terminata, spiculo longo simplici, Cauda feminae depressa, apice papillari.

\section{Ascaris incurva-R. n. sp.}

Dum Synopsin conscripsi nonnisi specimina duo ad manus erant, a cel. Spedalieri, Professore Ticinensi aceepta Dedit nimirum cl. vir particu. lam intestini Xiphiae Gladii in qua tubercula aderant Ascarides continentia. Hae contortae septem ad octo lineas longae, tertian lineae partem cras. sae, antrorsum magis attenuatae, capitis valvulis exiguis sed distinctis, caudae apice longiusculo semitereti acuto incurvato.

Synopseos parte impressa Xiphiam Gladium accepi sexpedalem die Octobris XXIII in Bal. tico captum, quem d. ejusdem mensis $\mathbf{X X X}$ examinavi, cujusque in branchiis, oescphago, sed praesertim in ventriculo et duodeno plurimas reperi Ascarides, aut albidas aut rubellas, unuón ad qua. 
tuor cum dimidio pollices longas, torpidas, quae aquae coinmissae, paucos citoque cessantes motus ediderunt, dum Tetrarhynchus attenuatus quem in ejusdem bestiae abdomine reperi per triduum vixit, Bothriocephali autem in recto vel hujus tuberculis obvii nullum vitae signum manife. starunt.

Ascaridis nostrae capub subdiscretum, valvulis tribus distinctissimis obtusis. Corpus utrinque magis tamen antrorsum attenuatum. Cauda maris intorta, apicis obtusi papilla minima conica, ante quem spicula duo incurva longiuscula. Cauda feminae apice longo tenuissimo subulato de. presso. Neque ova neque foetus vidi.

Obs. Specimina a cel. Spedaliert accepta ilIis a me repertis minora et habitaculo in tubercu. Iis quodammodo alienata videntur. Ipse in $\mathrm{Xi}$. phiae traberculis nonnisi Bothriocephalos, sed inferiori intestini loco, reperi, dum illa Ascarides continentia superiori occurrisse videntur. $\mathrm{Cl}_{\text {. }}$ Rrsso. (Icbthyologie de Nice p. 100.) se in Xiphiarum ventriculo copiam maximam vermium exi. guorum albidorum cellulis inclusorum reperisse refert, quod rem confirmat. Quae de irritatione horum vermium Xiphiam voracem et furiosum reddente addit, imaginationi debentur, cui, $\mathbf{c l}_{\text {. vir }}$ saepe indulget.

\section{Ascaris clavata $R$.}

Am. Gaede duas Ascarides dedit in Gadi Merlangi abdomine a se repertas, alteram decem, alteram quatuordecim lineas longas, femineas, an propriam speciem?

Caput discretum trivalve; Corpus utrinque 


\section{- 294}

magis tamen anteriora versus attenuatum; Cauda acuta, acumine satis longo.

Ipse Augusto in Gadi Aeglefini faucibus duas reperi Ascarides, alteram quindecim, alteram viginti octo lineas longas, quae capite nec tamen cauda cum clavata conveniunt, ut maribus non visis speciem dubiam relinquam.

Harum caput valvulis distinctissimis brevibus et latiusculis alaque utrinque lineari instructum; corpus utrinque magis tamen antrorsum attennatum; caudae apex obtusiusculus.

65. Ascaris collaris $R$.

Quam Neapoli in Pleuronecte manco reperi, eandem esse speciem ac illam, quam olim in Pleuronectibus Fleso et maximo inveni, crediderim.

Pl. manci hepati Ascaris haecce Capsulariarum modo orbiculum spiralem efficiens afixa erat, quo loco, verme soluto, visceri impressio cjusdem formae remansit.'

Ascaris vera est, capite truncato, corpore anteriora versus magis attenuato, cauda depressa acuta; ceterum novem lineas longa et alba.

68. Ascaris cuspidata R. n. sp.

In duarum Geotrupis Nasicornis larvarum intestino colo Berolini Máji d. IX. (n€que postea) quatuordecim reperi specimina candida, unam cum dimidio ad tres lineas longa, feminea.

Caput continuum gracilescens, ore trivalvi, valvulis exiguis, membrana utrinque tenuissima; corpus utrinque magis tamen versus anteriora attenuatum; cauda obtusa subito in cuspidem terminalem longam tenuem abiens. Cutis corporis subtiliter crenata. 
Oesophagus tenuis sensim sed parumper cras. sescens in proventriculum subglobosum desinit, qui subito in ventriculum magnum antrorsum obtusum, postice oblique decrescentem abit; intestimin parum tortuosum subaequale, nigresceńs. Oviductus ova ovalia, nigrescentia, forsan immatura continebant.

\section{0, Ascaris cephaloptera $\pi$. n. sp.}

Cel. Nestr, Musei Zoologici Florentini Director meritissinus, Ascarides duas dedit a $V_{i-}$ pera Redi recentissime oblata deorsum dejectas, albidas, alteram duos pollices totidemque lineas, alteram fere tres pollices longam, utriusque di., ametro transverso lineam dimidiam superante.

Caput exiguum, valvulis tribus minutulis; corpizs antrorsum maxime, postice parum attenuatum; apex candae obtusiusculus. Membrana capitis utrinque brevis semilanceolata, vel fere linearis; reliqui corporis nulla.

Confer speciem insequentem, cujus totum corpus membrana illa utitur, unde utriusque nomen petii,

\section{Ascaris holoptera R. n. sp.}

Am. Bremser specimina misit tria in intestinis Testudinis graecae a se reperta, quorume masculum tres cum dimidio pollices, feminea quatuor pollices et duas, quatuor pollices et octo lineas longa sunt, longitudinis ratione hábita plus minus crassa.

Caput discretum trivalve, corpus anteriora versus magis attenuatum; cauda inflexa, apice brevi papillari acuto, ante quem in specimitie 
masculo spiculum rectum et obtusum, breve. Membrana lateralis tenuis utrinque totum corpus decurrit-

Obs. Confer speciem praecedentem; sed praesertim Asc. sulcatam n. 50. in Testudine indica repertam, membranae lateralis defectu distinctam.

\section{Ascaris Serpentulus $\mathbf{R}$.}

In Entozoologia (Vol. II. I. p. I9r. n. 53.) specimen femineum descripsi ab am. Braunio mécum communicatum. Froelich (Naturforscher St. 29 p. 44. n. 19. Tab. I, Fig. I7, 18.) pariter femineum, in Ardeae cinereae intestinis tenuibus Calendis Julii a se repertum sub nomine Ascaridis Ardeae descriptum et delineatum dedit.

$$
\text { Species dubiae. }
$$

\section{Ascaris Tigridis.}

In Museo Viennensi unicum vidi specimen, fere maceratum, quinque lineas longum, capite obtuso alato, cauda acutiuscula.

\section{Ascaris Delphini.}

Henricus Julues Lebeck (Neue Schriften der Berl. Ges. Naturf. Freunde. B. 3. S. 292.) Ascarides refert plurimas vivas pollicares in ore et ventriculo Delphini gangetici repertas.

\section{Ascaris Laniorum,}

Froelich Naturforscher St. 29, p. 40, n. 16. Ascaris Collurionis.

Cl. vir in Lanii Collurionit. junioris intestinis tenuibus Julio quatuor specimina tunicis 
fortiter infixa reperit, fere pollicaria, crassiuscula, albida, quorum descriptionein dat insequentem.

Caput rubellum, emarginatum, valvulis satis distinctis, quarum quatuor adesse videntur. Corpus utrinque attenuatuin. Apex caudae brevis, acutiusculus, depresso-excavatus, ante quem anus. Membrana lateralis tenuis, crenulata, utrin'que a capite ad posteriora decurrit. - Intestimun, cujus rei jam supra exempla attuli, etiam hac in specie auctor perperam fissum et in posteriori vermis parte duplex fingit.

Catalogus Viennensis Ascarides Lanii Collurionis et $L$. minoris intestinales enumerat.

\section{Ascaris Corvi glandarii.}

Am. Отто specimen femineum misit, quod aeque parum characteres specificos obtulit, ac illa aliorum Corvorum mihi visa, semper feminea.

\section{Ascaris Picae.}

Novembri mense Gryphiae in intestinis tenuibus Corvi Picae quatuor reperi specimina feminea, bi-vel tripollicaria, crassiuscula, flavescentia, intestino fusco translucente, maculis hinc inde rubris, an accidentalibus et ex sanguine hausto oriundis.

Caput exiguum discretum, valvulis parvis, membrana laterali nullibi conspicua; Corpus utrinque actualiter attenuatum; cauda acutiuscula.

Mirrihus-non visis neque hanc neque aliorum Corvorum Ascarides distinguere valeo. 


\section{Ascaris Ciconiae.}

Rosa (Lettere zoologiche p. 4.) se in ventriculo Ciconiac nigrac inter pabulum $110 n$ digestum Ascarides duas pollicem dimidium longas reperisse refert.

\section{Ascaris Glareolae.}

In intestinis coecis Glarenlae austriacae Arimini Aprili quatuor reperi specimina, alba, sex ad octo lineas longa, viva.

Caput valvulis magnis truncatis; corpus retrorsum magis attenuatum, parte antica inflexa; cauda longa recta acuta, ante quam vulva; anus versus illius apicem. Propria videtur species.

\section{Ascaris Sternae.}

Duarum Steruarum nigrarum adhuc calentium intestina Arimini Aprili examinavi, quae extus hydatidibus exiguis .vix quartam lineae partem aequantibus obsita vidi. Harum singulae duplici constantes tunica Nematoideum spiraliter convolutum, valde exiguum, nullos motus edens obtulerunt, pro longitudine crassiusculum, cau. da obtusa cum parva papilla terminali utens, cu. jus autem caput extricare non licuit, ut dubie huc referam, Ascaridum aeque minutarum hydatides incolentium analogia commotus,

102. Ascaris Torpedinis.

Am. Bremser duo specimina misit, in Torpedinis (certe marmoratae) ventriculo reperta, tres quatuorve lineas longa, tenuissima, utrinque aeciualiter attennata, fere stricta, capite acutiusculo, valvulis haud dignoscendis, cauda recta de. pressa acutiuscula. 


\section{- 299}

106. Ascaris Orthragorisci.

In aqúa, cui Orthragorisci Molae inestina Neapoli Julii d. 26. immiseram, die insequente duas exiguas reperi Ascarides vivas, femineas.

107. Ascaris Hippocampi.

In intestino Syngnathi Hippocampi Arimini Aprili Ascaridem reperi vivam, femineam, sex lineas lengam, tenuem, utrinque aequaliter attenu. atam, caudá acuta.

108. Ascaris Centrisci.

Multos Centriscos Scolopaces Neapoli Julio examinando in duorum peritoneo singulas reperi Ascarides, parvas, crassiusculas, utrinquie aequaliter attenuatas, femineas.

109. Ascaris Ophidii barbati.

In Ophidii barbati mesenterio Neapoli Ju. nio quatuor offendi Ascarides, tres ad quinque lineas longas, albas vel rubellas, antrorsum magis attenuatas, cauda acutiuscula depressa.

\section{Ascaris Ophidii imberbis.}

In Ophidii imberbis mesenterio Neapoli Julio unicam inveni Ascaridem octo lineas longam, tenuern, capitis truncati valvulis mediocribus, corpore antrorsum magis attenuato, cauda acutiuscula depressa.

\section{Ascaris Uranoscopi.}

In aliquot Uranoscoporum scabrorum peritoneo variarum abdominis partium Neapoli Junio et Julio Ascarides reperi vividissinas, albas, tenues, quinque ad duodecim lineas longas, antror. sum magis attenuatas. 
112. Ascaris Gadi minuti.

In Gadi minuti abdomine Arimini Majo Ascaridem reperi solitariam, femineam, sesquipollicarem, antrorsum minus attenuatam, apice caudae inflexo acuto.

115. Ascaris Phycidis.

Inier Blennii Phycidis appendices pyloricas Arimini Majo duas reperi Ascarides novem lineas lungas, albas, vivas; capite parvo discreto, valvulis distinctissimis, corpore utrinque aequaliter attenuato, cauda brevi acuta divaricata, ante quam tuberculum. Canalis intestinalis rectus. Genitalia haud conspicua.

Species propria videtur, quae divaricata vocari posset.

115. Ascaris Scorpaenae.'

In intestino Scorpaená Scrofac magnae Arimini Aprili unican reperi Ascaridén vivam, albain, quindecim lineas longam, antrorsum valde attenuatam, parte postica crassiore minus decre. scente, apice caudali obtusiusculo depresso.

116. Ascaris Fabri.

In intestino Zenis Fabri Arimini Aprili Ascaridem reperi pollicarem albidam, valvulis distinctis instructam, posteriora versus magis attenuatam, laud confundendam cum Capsulariis, quas inter ejusdem piscis appendice pyloricas offendi, ad entozoa dubii generis relatas.

\section{Ascaris Linguatulae.}

In Pleuronectis Linguatulas mesenterio Neapoli Julio unicam reperi Ascaridem, capitis val- 


\section{- 301}

vulis exiguis' sed distinctis, corpore utrinque parum attenuato, cauda obtusiuscula,

119. Ascaris Maenae.

Sparorum Maenarum quatuor intestina Arimini Aprili aquae immisi, in qua die insequente sex reperi Ascarides vividissimas, a!bas, tenuissi. mas, tres quatuorve lineas longas, capite valvulis exiguis instructo, corpore retrorsum magis attenuato, cauda acuta depressa, tubo intestinali recto. Confer speciem insequentem.

120. Ascaris Boopis

In Spari Boopis peritoneo intestinali Ari. mini Majo Ascaridem reperi solitariam vivam, tres lineas longam, tenuissimam, rubeliam; capite trivalvi; corpore retrorsum magis attenuato; cau: da acuta, depressa; canali alimentario recto fusco. Cum pratcedente eadem videtur.

121. Ascaris Spari Snicre.

In Spari Spicre dicti peritoneo Neapoli Junio uitra quinquaginta reperi Ascarides, tres ad quinque lineas longas, albidas vel rubellas, capite obtuso trivalvi, corpore antrorsum magis attenuato, cauda depressa obtusiuscula. Omnes feminae vi. sae sunt, quarum nullum sexus discrimen dete. gere potui.

\section{Ascaris Cynaedi.}

In Labri Cynaedi peritoneo Neapoli Junio Ascaridem reperi exiguam, utrinque aequaliter attenuatam.

124. Ascaris Labri lusci.

In Labro lusco Neapoli Junio complura re. peri specimina, partim peritoneo intestinali inhae. 


\section{- $302-$}

rentia, partim intra hepatis tunicam latentia, viva, alba, pollicem dimidium vel integrum longa: capite truncato trivalvi, corpore antrorsum magis attenuato, apice caudae depresso acutiusculo,

125. Ascaris Novaculae.

In Coryphaenae (Labri) Novaculae peritoneo Neapoli Junio et Julio solitariam reperi, rubellam, quinque lineas longam, tenuem, valvulis oris distinctis, corpore antrorsum magis attenuato, cauda depressa acutiuscula.

126. Ascaris Sciaenae.

In Sciaenae Umbrae magnae peritoneo Spe. ziae Augusto plurimas reperi Ascarides rubellas, tres quatuorve lineas longas, retrorsum magis attenuatas, cauda acuta.

128. Asccris Mulli.

In Mulli rubescentis intestino Arimini Aprili unicam reperi Ascaridem vivam, sex lineas longann, albanl, antrorsum magis attenuatam, capitis valvulis exiguis, apice caudae depresso, obtuso; tractu cibario recto.

129. Ascaris Lyrae.

In Triglae Lyrae mensenterio Neapoli Ju. nio unicam reperi Ascaridem vivam, albam, sex lineas longam, tenuem, antrorsum magis attenu. atam, valvulis capitis distinctis, cauda depresకa acutiuscula.

154. Ascaris Sauri.

In Osmeri Sauri mesenterio Neapoli Junio tres reperi Ascarides, tres ad sex lineas longas, albidas, antrorsum magis attenuatas; cauda depressa, acutiuscula, femineas, 
136. Ascaris Argentinae.

Triginta ad quadraginta Argentinas Syhyraenas Romae Majo secando, in unicae abdomine Ascaridem reperi liberam, femineam, quindecim lineas longain, tenuem, anteriora versus magis attenuatam, capite obtuso-truncato, valvulis distinctis mediocribus, apice caudae depresso, brevi, obtusiusculo.

137. Ascaris Atherinae.

In intestino Atherinae Hepseti Neapoli Ju. nio Ascaridem offendi quatuordecim lineas longam, crassiusculam, antrorsum magis attenuatam, cauda depressa obiusa.

Alias exiguas tres ad sex lineas et quod excurrit longas, passim antrorsum crassiores, plerumque graciliores, peritoneo implicitas reperi, an ejusdem speciei?

\section{Ascaris Clupearum.}

Froelica Naturf, 29. p. 50. n. 24. Ascaris Harengum.

Ascaridem vocat filiformen, corpore aequali utrinque submarginato, cauda obtusa. Pollicem superare, crassitie pilum equinum aequare, utroque fine spiraliter involutam esse. Capitis val. vulas exiguas vix observari posse. Caudam conicam obtusissimam, apice pellucidam, vix a ca. pite distingui.

Ipse nonnisi Filariam Capsulariam n, I3, in Harengis saepissime dissectis reperi, et cl. virum illam ingenio indulgendo Ascaridem vocasse crediderim. : Qua repererit, non indicat, sed Gordium harengum BLocun citando, quem pariter ad Filerian laudavi, in illius abclomine certe offendit. 
140. Ascaris Lucani.

Froelich Naturf. p. 5r, n. 25. Ascaris-Lu. cani.

Clariss, vir ex ano Lticani Capreoli satis multas se expressisse refert Ascarides vix duas lineas longas, tenuissimas, pellucidas, cánuda. su. bulata.

Forsan ad Ascaridem cuspidatam n, 68, pertinent.

\section{Genus X. Ophiostoma.}

Genus distinctissimum, cujus tamen paucae innotuerunt species. Quaim olim Fiscurre auctoritate commotus huc revocavi Cystidicolam, antopsia innixus ad Spiropteras (n. 15.) amandavi.

1. Ophiostoma cristatum R. n. sp.

Rictularia cristata. Froelich Naturf, 29, p. 7. Tab. 1. Fig. $1-3$.

Vir clarissimus 1. c. specimina in intestinis Muris sylvatici tenuibus hoc loco dilatatis se reperisse refert, sed in litteris ad brimserem datis. hoc revocat et sese Rictularian in intestinis tenuibus .Myoxi muscardini offendisse auctor est. Specimina Musei Viennensis vere.caesarei iisdem locis in Myoxo Glire et Dryade reperta sunt.

Specimina Froelichir undecim ad tredecim. lineas longa; utrinque suibattenuata, ex cinereo alba, ni fällor, feminea.

Caput continuum, rotúindatim, bilabiatum, ore infero; labio superiorc galtato (cristato, minus bene cl. viro dicto), inferiore depresso, truncato, dentibus duobus adscendentibus, brevissimis, conicis, pellucidis (ex rictu adscendere visis) munito. Colluni ad vulvam usque denticula- 
tum. Corpus aequale post mortem crenulatum. Canda hrevis, conica, obtusa, mucrone brevi, rima exigua transversa (ani).

Tractus intestinalis pone caput dilatatus, mox contractus, tum rectus per mediam corporis partem decurrens, dein ad latus (ad inferiora po. tius?) accedens, liaud procul a cauda in saccum dilatatus, ex quo canalis brevis et tenuis ad rimam dictan abit.

Circa tubum intestinalem vasa tenuia con. torta, in quibus auctor neque prolem neque ova observavit. Vulva bilabiata, labio anteriore minore ei magis rotundato.

Os vivorum (per quinque dies observato. rum) parum mobile, labiis tantum parum vel adductis vel abductis; inortuorum diductum, apertum, dentibus visui subductis. Corpus in spiram moturn.

Obs. Clar. vir alterius tantum colli lateris denticulos refert, quales assumere vix licet; to. tum forsan collum illis obsitum est, denticulos enim asymmetricos nunquam in entozois observavi. Crenas ipse etiam tradit in collum a den: ticulis continuari, quod rem conficit.

2. Ophiostoma mucronatum $\mathbf{P}$.

In Museo Vieunensi specimen in intestinis Vespertilionis lasiopteri repertum examinavi, septendecim lineas longum, antrorsum tenuius, me. dia tamen papilla (an oris tubulo?) prominente.

Quae ibrdem vidi specinina in T.espertilione murino lecta, labiis tanturn obtusis utebantur.

3. Ophiostoma sphaerocephalum $\mathbf{h}$. Ent. II. I. p. 188, in, 50, Ascaris sphaerccephala. 
Tria tantummocto specimina feminea Gryphiae Junio in Accipenseris Sturionis intestinis crassis reperta dubius Ascaridibus arldidi, Rerolini autem eodem mense eodemque loco satis multa fèminea, pauca mascula reperi, quae huc pertinere didici, septem ad quindecim lineas longa, tenuia, caput licet rarius bile tincta, ceterum candida.

Caput discretum, superne gibbum, bilabiatum, ore transverso, labio superiore magno, tumido, bifido, inferiore pariter, sed minus profunde firso. Corpus nt:inque parum attenuatum, in femina pone medium vel ad duas totius longitu. dinis tertias partes vulva exstante distinctum. Cauda maris incurva vel inflexa, semel spiralis visa, bialata, alis striatis mediocribus; apice caud'ae tenui acuto, ante quem spicula duo stiliformia emergunt. Cauda fominae rectiuscula, depressa, acuta. Intestinum tenue. Ovarium pariter tenue, ovis oblongis farctum, vi motrice forti instructum, illaque velociter propellens.

Obs. I. Caput quo entozoa ista intestino fortiter affiguntur tam in vivis quam in mortuis exaninatu difficillimum est, ut lentibus simplicibus dualus vel tribus cor junctis potissinum usus sim. Valde crassum enin est, saepeqũe 'costatum vel multialatum (alis striatis) videtur, insimulque iritium canalis cibarii valde ampliatur. Tandem veram indolem cognovi, et Brèmserus, cui specimina misi, Ophiostoma esse cóncessit.

Obs. 2. Pleurorlyynchus cel. NaU (Schrift. d. Berlin. Narurf. Gesellsch. Freunde. B. 7. S. 47 I. Ent. II. 2. p. 273, n. 22. Synops. Entoz. dubii gen. n. 45.) capite rotundo et ovulis oblongis convenit, $\epsilon t$ tubilus oris lateralis nonnisi pars 


\section{$-307$}

prolapsa videtur. Specimen tantum inicum cl. viro ad manus fuit, sex lineas longum.

\section{Genus XI: Liorliynchus.}

Genus locce valde ambiguum. Prima enim species, Liorhynclus truncatus, quem ante viginti sex annos in Mele Taxo copiosissimun Gryphiae reperi, nulli pratterea visus est, nt novo examine illum confirmari vellem, cujus gratia genus condidi. Secunda species, ab Othone Fabricro in Phocae barbatae ventriculo reperta, maxime dubia virletur, et tertia, de qua mox fusius agam, forsan Spropteris addi posset.

\section{Liorhynchus denticulatus $R$.}

Cel. olim Hübar complura specimina misit in Muraenae Anguillae ventriculo reperta, quae examinando varia observavi, quae descriptioni Zederianae Ent. II, I. p. 249. n. 3. exhibitae addi possint.

Anticam partem ŻeDero gyratam dictam majoribus, reliquan corporis partem anteriorem denticulis minutis ct copiosissimis seriatim. munitam video, ut illi non solum in margine sed in superficie quoque corporis inveniantur. Cel, vir porro spiculum genitale cylindricum refert, sed rem magis compositam observo. Cauda maris convoluta est, apice incurvo, utrinque membrana exstante introrsum alato, quas inter alas genitale filiforme incurvum satis longum emérgit. Ovula elliptica, parva, obscura, margine tenui pellucido.

Obs. I. Speciminibus tantum mortuis exhibitis de oris tubulo emissili nil referre possum; si os orbiculare foret, speciem istam ad Spiropte. ras amandarem. 


\section{- 308 -}

Obs. 2. Cucullanum ascaroidem Goezr1; Ent. II. 2. p. 254. Prionoderma ascaroides; Synops. Entoz generis dubii n. 74. in Collectione Goezianá Ticini vidi et ad Nematoidea utique pertinere didici. Corpus crassum transversim crenatum forsan seriatim denticulatum est, caput autem ob spiritum turbidum bene distinguere non potui, neque (inspectore Musei, cel. Mangili absente) phialam aperire concessum fuit. Forsan tamen specics dictae affinis erit. 


\section{Ordo If.}

\section{Acanthocephala.}

Echinorhynchum, unicum hujus Ordinis genus al omnibus reliquis, tam ento\%ois, quam vermibus liberis facillime distinguimus. Echinorhynchus quadrirostris olim huic generi additus, dein separatus et cum aliquot speciebus affinibus a nobis in Entozoòlogia Tetrarhynchi nomine enumeratus, sed aliorum tantum anctoritate expositus fuit. Iter autem per Italiam anno 1817 facienti plurimos Tetrarbynchos vivos, et nuper quoque Berolini illos Xiphiae et Testudinis Mydae pariter vivos observare contigit, quo omne de liujus generis loco dubium disșipatum fuit. Tetrarhyı. chi enim non genere sed ordine quoque ab Eclinorhynchis differunt et Cestoideis addi debent, qua de re infra fusius tractabitur.

\section{Genus XII. Echinorhynchus.}

Quae de hujus generis anatomia post En. tozoologiae editionem innotuerunt, pauca sunt. Specien tamen reperi (Echinorlynchum vasculo. 


\section{$-310$}

sum n. 49.) cujus cutis densissimo et elegantissi-, mo vasorum rete instructa esi, quale hacterns nemini isto in ordine dictum fuit, ut lemisco. rum a proboscide pendentium vasa tantummodo cognoverimus. In sectione Mantissae insequente anatomico-physiologica relipua hùc facientia invenies.

Species plurimas satis bene distinguo, neque tantopere variare, ac amico persuasum est, admiserim; singulas tamen exponendo varietates illas semper respiciam.

\section{Echinorhynchus Gigas GozzE.}

Froelich (Naturf. St. 29. p. 74. n. 4r.) se specimina tres pollices ad pedem longa reperisee, simul autem mactatores refert expcrientia didicisse, sues rebus blandioribits vescentes paruna, glandibus autem saginatos Echinorhyuchis maxiune obnoxios esse.

2. Echinorhynchus Spirula Orfirs. N. sp. Am. Olfers praeter icones specimina duo misit in colo et coeco Simiae Rosaliae a se versus finem scptembris in Brasilia reperta, alterum decem, alterum tredecim linteas longum, hoc summa latitudine unann' cun dimidia, apice cau. dali dimidian adaeyuans.

Proboscis subglobosa, longitudine vix latitudinem superante, parte antica parumper aucta, apice intruso, zucinis duris sexfariam in quincuncem positis. Collum brevissiraum, fere nullum. Corpus tenue incipit, celcrrine in summarn crassitiem augetur, sensim posteriora versus decrescens, apice postico obtuso; ceterum obiter transversin incisum, incurvumf, unde cel. vir nornen 


\section{$-3^{11}$}

depromsisse videtur. Lemniscos figura exhibet magnos.

Obs. Manmalium generatim sumtorum Echi. norhynchi pauci, priorum autem ordinun nulli antea innotuerunt, ut Simiae Echinorhynchus mihi admirationem intulerit.

6. Echinorhynchus oligacanthus R. n. sp. Colubri'quadrilineati, quem vivun Romae acceperain, Florentiae Augusto mense mortui, intestino-inferiori, peritoneo involutus, Echinorhynclius adhaesit albus, duas lineas superans, crassiusculus, omnis vitae expers, licet coluber pridie exstinctus esset.

Proboscis subglobosa, ancinorum maximorùm seriebus tribus armata. Collum brevíssimum. Corpus antrorsum crassius, posteriora versus parum atténuatum, passimque constrictum, apice oltuso.

Num tota proboscis exserta sit, primum dubitavi, sed parte illius posteriore pellucida, nullos uncinos visui subductos crediderim.

Obs. Intestino Colubri passim puncta aiba extus adhaeserunt, quorum exarnen, num Echinorhyuchi vel aliorum entozoorum rudimenta si-. sterent, certiorem me non reddidit,

7. Echinorhynchus oligacanthoides R. n.sp.

Cel. a в Olfers Colubri-olivacei partem intestini hydatidibus innumeris obsitam, una cum Echinorhyncho harum incola, Octobri mense re. perto, hujusque iconem ex Brasilia misit.

Specimen ab amico delineatum masculum est, quatuor lineas longum; proboscide brevissima; truncata, subquadrata, mncinorum validissimorum 


\section{- $312-$}

sexfariam positorum seriebus quatuor instructa; corpore cylindrico, passim constricto; vesicula caudali ovata, fllo longissimo terminata.

Specinen transmissum fenincum tres lineas longum, praster vesicae dictae defectum et mino. res corporis stricturas, eo praesertim deflectit, quòd pars anterior crassior, caudalis vero attenuata sit.

Qua nota etiam ab Echinorhyncho praecedente recedit, cujus apparatum masculumi quoque ignoro, ut species temere miscere nolutrim, no. mine autem triviali affinitatem indigitaverim.

Obs. Coluber'olivaceus Oxfers aut nova Brasiliae species est, aut inter male descriptas la. tet. C. olivaceus, subtus albidus, capitis parte su. periore et linea dorsi longitudinali cupreis, freno oculari nigro, caudae apice subulato. Scut. 198. scutell. 307. tot. 305. cap. 14. Scutella dorsi oblonga. Boi-tiapo, cobra de cipo. T'sso Brasil. 4ä hist. nat. 279. Marcgrav. 2ifI. Olfers.

\section{Echinorhynchus tulierosus ZED.}

In Catalogo Viennensi species haec cum Ech. clavicipite n.9. conjungitur; utrumquenon vidi, sed Ech. tuberosus a Müutero diversus et describitur et delineatur, quo accedit, hunc in peritoneo, clavicipitem autem in intestinis Cyprini rutsli occurrisse; Echinorhynchus Carassii tandem (n. 98.) a Brausio detectus, et in Catalogo Viennensi pariter a reliquis Cyprinorum Echinorhynchis sejunctus, cum tuberoso maxime convenit et forsan idem cst; quibus commotus speciem supprimere non ausin. 


\section{$-.313$}

10. Echinorhynchus globulosus R.

Speciminibus, quae olim in Mfurcence dnguilla reperi, perditis num illa quae postmodum variis in piscibus offendi, exacte conveniant, pro certo quidem contendere nequeo, cum horun rol. lum hrevius reperiam, ac in. Entozoplogita cie. scripci; vix tamen differre crediderira, aut si differant, speciem maxime afïnem constituunt.

a Multa specimina in intestinis Gobii nigri saepius dissecti Arinini Aprili, Junio Neapoli reperi; proboscide ovali brevi, uncinulorum rectiusculorum seriebus octo lase munita; collo brevissimo; eorpore oblongo, retrorsum attenuato, passimi antice subventricoso, postice cylindrico. Saepe vesiculas masculas apici caudae adpensas, sed etiam ovaria eoden loco in aliis elapsa vidisse mili videor.

Quae in Gobiis quatuor nigris et in ultima quitem intestini tenuis parte, ante valvulam coli offendi, magis adhuc ad Ech. globulosum pertinere visa sunt.

b. In Spari Denticis intestinis Arimini Majo drodecim, tria Neapoli Julio réperi specinnina flavescentia vel alba, omnibus numeris cum prioribıs convenientia.

c. Idem valet de Echinorhynchis duobus in Sphyraenae $\boldsymbol{S}_{\text {pet }}$ intestinis Neapoli Junio milhinet obviis.

d. Nec non de alteris duobus ibidem Julio in intestinis Pleuronectis Linguatulae repertis.

e. In Sciaenae Umbrae magnae intestino Speziae Augusto plurima inveni specimina, unam cum dimidia ad tres lineas longa, flava vel passim alba, Proboscis ovalis, sex ad octo uncino. 


\section{- 314}

rum éxiguorum seriebus munita; collum brevissimum; corpus oblongum, nfox anteriore mox media parte incrassatum, apice obtnsiusculo.

f. Hiknserus racden multa misit specimina in intestinis Cobii Aphyac obvia, omnibus numeris cum praterlentibus quadrantia. Marium vesica caudalis glubosa passim. cullapsa ést, qualis rarius occurrit.

11. Elhinorhynchus Pumilio R. n. sp.

Speciunina nommulla in intestinis Lophii piscatorii lecta Bremserer benevolentiae debeo, lineam longa, albida, duriuccula.

Proboscis brevis, medio paululum incrassa. 1a, vel passim suiglobosa, uncinorum brevissinorum serie quadriplici aut sextuplici armata. Ciol. lum nullum. Corpus teres utrinque attenuatum; apex caudalis aut emarginatus, aut sacculo globuloso vel didymo terminatus.

Ob.s. Ipse in Lopliis saepius examinatis nunquam Echinorhynchum offendi. Speciem di. stinctami credo.

13. Echunorhynchus globotaudatus ZeD.

Am. Nitzscu iria misit specimina in Strige Alucone reperta, alterum pollicare, reliqua sesquipollicaria.

P'roboseis medio incrassasata, cujus ultima - pars uncinulornm reflexorun, longiusculorum seriebus circier viginti et quatuor munita ZEnEro pro collo imposuir. Collum nullum. Corpus cylindricum, antrorsum parum attenuatum, apici caudali in specimine minore rotundato, pápilla terininato.

14. Echinorhynchus cinctus R. n. sp. Colnbrorm atrovirentinn trium quadripeda. lium Arimini Majo examinatorum mesenterium 
et tunicam intestinorum peritonealem multis ob. sita vidi corpuscuils oblongis albidis, duas tertias circiter lineae partes aequancibus, rarius lineam attingentibus; quibus a tunica circumdante liberatis et pressione fortiori adhibita, Echinorhynchus sistebatur, lineam quarta tertiave parte superans, cujus píroboscis ovalis, collum vero cingulo aculeis armato instrucrum, corpus ovale vel oblongum, apice caudali obtusiusculo exhibita sunt. Proboscis uncinorum densissimorum duodecim ad quatuordecin seriebus, collurh vero aculeis reflexis majoribus at laxius dispositis in. structum visa.

Tandem unicum offendi Echinorhynchum libere peritoneo inhaerentem neque tunicis involutum, qui perfecte evolutus aliam obtulit speciem.

Hujus duas cum dimidia lineas longi Pro. borcis longissima, teres, in tres partes-divisa, quarum media crassior, tertia autem anteriores duas longitudine fere superavit. Incini minutissimi, denșissini, reflexi, quadraginta circiter sèriebus transversis dispositi. Collum nullum. Corjus oblongum, antrorsum angustatum, apice caudali longo tenuiore et obtuso terminatum. Corpuscula duo oblonga a proboscide exorti per magnam corporis partem translucent.

In Viperae liedi, Bononiāe Septembri 1817 acceptae et Berolini Majo 18 is mortuae mesen. terio sacculum album oblongum reperi, lineam longum, ex quo Echinorhynchum extraxi linearn vix excecientem, proboscide longa antrorsum au. cta, corpore antrorsum attenuate, parte insequente craśsiuscula retrorsum descrescente, procul dubio huc facientem. 
Nomen triviale ab habitu omnium, quos a vinculis liberavi, vulgari desumsi.

\section{Echinorhynchus bacillaris $\boldsymbol{R}$.}

Cel. olim Вrосн specimina multa in intestinis Mergi minuti reperit, sed male et descripsit et delineavit.

Longitudo pollicaris vel sesquipollicaris. Prnboscis cylindrica apicem versus aucta, sive parte posteriore (Buochro collum dicta) tenuiore, uncinorum seriebus transversis circiter triginta densissime armata. Collun nullum. Corpus cylinciricum aequale longissimum. Apex cauclae infexus obiusus, vel etiam corpuscúlo varie prominente auctus.

Qb.s. Broces caudam rectam et acutam describit et depingit, qualem in nullo suorum speciminum vidi. Blochiana enim entozoorum, quatenus superest, collectio in ihuseo Zooloyico Berolinensi servatur et phiala adest, cui ipse schedulam sua manu exaratam addidit, inesse hunc Echinorhynclum (praeterea etian Capsularias et Ech. pisci110s). Neque de speciei identitate dubium relinqui potest: ob huius Echinorhynchi magnitudinem, habiturn, proboscidem inaequalem, (proboscidem et collum Brochro sistentens), et praesertim ob ejusdem ova, qualia Blochio dicuntur ovalia, neque linearia vel elongata, viti plerum. que habentur.

\section{Echinorhynchus agilis R. n. sp.}

In intestinis Mugilis Cephali, Speziae Au. gusto mense, noven reperi specimina sesquilineam ad duas cum dimidia lineas longa, candida, stricta turgidaque, nihilo tamen minus lineis trans- 


\section{- $317 \div$}

versis densis striata, quae cum rugis Echinorhynchorum flaccidorum non comparari debent.

Proboscis exigua, clavara, subtetragona, tribus uncinorum seriebus laxe armata, quorum anti. ci maximi et satis longi, medii minores, postici minimi sunt. Collum breve. Corpnes utrinque attenuatum, parte ante medium sita reliquis cras. siore, dense striatum. Quibusdam speciminibus globulus caudalis.

Vagina proboscidis et colli duobus iiganertis longis utiur. Motus vividissirni, quantos nullo in Echinorhyncho hactenus observavi, ut summa velocitate proboscidem et collum retrahat irerum. que exserat, in Sipunculi modum Motus iste tamen cum illo Tetrarhynchi comparatus valde simplex est.

Striis transversis haud armatis singulare quid affectat.

17. Echinorhynchus fusiformis ŻD.

Catalogus Viennensis Echinorhynchos in Salmonibus Salare, Thymallo, Trutta, Farione ob. vios huc trahit; Froelich autem (Naturf, 29. p. 71-73. n. 39. Tab. 2. Fig. 12, 13.) Echinorhynchuni Farionis ab illo Truttae (proprie fusiformi Zeneri) magnitudine exigua, corpore striato, collo brevi et prohoscide subglobosa distringuit, sed cum cel. viri pace strias illas laxas accessorias haberem, et specinsina tantum pusilla, duas cum dimidia lineas longa invenit. De horum intestino quidem loquitur, sed entozoorum anatomen nosz curavit.

18. Echinorhynchus Haeruca R.

Specien Ranis vulgatissiniam in intestinis Bufonis ignei Berolini Martii die 16. reperi, Spe. 


\section{$-318$}

cimen unicum fere tres lineas longum, album, proboscide conica, uncinorum tenuium, sprielius sex munita, collo a quali et ommibus reliquis notis cum descriptione in Entozoologia (Vol. II. I. p. 265. n. 12.) data ex asse conveniens.

In Catalogo Viennensi Echinorhynchus Bufonis ignei (Ranae bombinae) propria species dí citur, et num ille Bufonis vulgaris in 'hoc parasiticus adsil, quaeritur. Qua de re in opere novissimo et egregio (Ueber lebende Würmer in lebenden Thieren. p. 2I.) am. Bremser haec habet: Ech. Haeraca, qui in Fina esculenta tantum habitat, in ventriculo Bufonis cinerei, sed cum Ranae illius junioris specimine semidigesto simul inventus est.

19. Echinorhynchus angustatus R.

Zedero praeeunte hunc (Ent, II. I. p. 266. n. 13.) ab Ech.affini (ib.p.26S.n.14.) separavi, plurimorum tamen speciminum comparatione $r \in p e-$ tita conjungendos esse didici, quemadmodum etiam Catalogus Viennensis illos conjunxit.

Troetich (Naturt. St. 29. p. 73. n. 40. Ech. candidus) specimina in Perca fluviatili reperta obiter refert.

Specimen, quod Octobri frrypliae in intestinis Siluri Glandidis reperi, silentio haud praeterẻundun videtur: candidum, tres lineas longum, tenue; proboscide recta, retrorsum vix aucta, uncinorum fortium seriebus transversis quatuordecim munita; corpore tereti, retrorsum parum attenuato; apice caudali obtuso, emarginatg, foramine quasi hiante. Ficceptaculum probosczdis lon. 'gum obscurum, a quo ductus crassus ad apicem caudae tendit; circa ductum ovula innúmera a 


\section{$-319$}

olita forma (lineari) recedentia, ovalia, an im. matura?

22. Echinorhynchus gracilis R. n. sp.

Specinina plurima in intestinis coracine garmlae Augusto reperta cel. Trevtux berievole mecum communicavit; longiturline linearn ae. quantia, vel quarta parte superantia.

Proboscis cylindrica, apice rotundata, inter. dum subclavala visạ, mncinomim exignorun serie. bus deccm ad duodecim transversis dense armata. Collum sullum, risi partem corporis antican, in: terdum strictura quodammode discretam, ita vo. care mavis. Corpus cylindricum, parte anteriore aut aequa!i aut parum inflara, posteriore attenu. ata apice obtuso. Superficits corporis variis in speciminibus poros mannos orbiculares passim marginatos exhibet. Lemmisi duo longi a pro. boscide exorti posteriora corporis petunt et satpe vasa mentiuntur.

Obs. In Catalogo Viennensi Echinorhynclio. rumi Coraciae species duae enumerantur, quo commotus dubiam n. 6 I, posui.

\section{Echinorhynchus areolatus R. n. sp.}

Am. Bremser specimen misit in intestino Motacillae atricapillae repertum, fere tres lineas longum.

Proboscis cylindrica apice rotundata, parte posteriore quasi constricta, uncinorum minutorum serichus circiter viginti transversis dense ununit: Collum nuillum aut brevissimum. Corpus cylindricum, utrinque praesertion tamen posterióra versus attenuaturn, parte caudali longa gracili obtusa. Maculae partis tenuis posticae ope microsco. 
pii conspiciuntur plurimae, formae variae, quadrangulae, triangulae, etc., quibus cauda quasi areolata redditur, quacque cum confinium (v. c. praecedentis) foraminibus rótundis non confundi debent. Ductzes opacus mediam partis posterioris longitudinem legit. - Alia Echinorhynchi species in Atricapillae omento occurrit: conf. n. 65 .

\section{Echinorhynchus tumidulus R. n. sp.}

Specimina plurima in intestinis Crotophagae Ani sive minóris, quae sa€pe multindine ob. struunt, Septembri et Octobri in Brasilia reperta am. Orfers misit, alba, duodecim ad septendecim lineas longa, mascula pro more minora.

Proboscis vix lineam longa, obiter inspecta linearis, sed attentius examinata inaequalis, medio tantillum convexiore, basi iterum increscente, sive subconica; uncinorum tenuissinorum seriebus quadragipta ef quod excurrit transversis armata. Collum nullum. Corpus cylindricum, parte anteriore ad duarum vel trium linearum longitudinem crassiore (tumidula), passim inaequali, subito in tenuiorem aequalem abeunte. Caudae ma. sculae vesicula lineam fere longa, dimidiam crassa, utrinque obtusissima, inflexa additur, in qua corpuscula duo subrotunda conspiciuntur, ductus vel vasis ope inter se et cum cauda cohaerentia. Canda feminea apicem obtusum crassiorem papilla minima terminatum et obiter inflexum habet. Ova innumera, angusta, sed praeter morem brevia et minutula; placentulae solitae non desunt.

Obs. Species liaec in Synopsi pone n. 13. enumerari debuisset; cum proboscis inaequalis di- 


\section{- 321}

cenda sit, ex basi nimirum, conica parumper au. getur et demum linearis fit.

26. Echinorhynchus transversis R. n. sp.

Singularum avium quos vidi Echirorhynchos huc facientes sigillatim recensebo.

a. Am. Brexser specimen misit in intęstino Sturui vulgaris repertum, quinque lineas superans, praeter probosciden inea dimidia longiorem, alterumque sibi esse duplo majus litteris mandavit.

Proboscis linearis, vel anticam partem tantillum crassior; uncinorum mediocrium (majorum quam in Ech, micracanthoj seriebus vigintiquatuor ad triginta dense armata; corpori transversim apposita. Collum nuilum, Corpus cylindri, cum, antrorsum tenuius, apice postico rosundato.

b. Idem amicus specimen quod examinarem mecum communicavit, in intestino Mntacillae Stapazinac Majo repertum, quinque lineas longum; proboscide duas lineae tertias partes longa, apice roturidata, transversa, uncinorum satis vali, dorum seriebus vigintiquatuor ad triginta munita; collo nullo; eorpore antrorsum attenuato, dein-. ceps crassiore, parte reliqua aequali, temuiore; apice postico obtuso.

c. Mifis quoque duo specimina in intestinis Thurdi cy.nei Septembri 1812 reperta, alterum tres cum dininidia, alterum quinque cum dinidia lineas longum, parte crassissima lineam aequante, collo nullo, proboscide corpori oblique vel transversinn imposita.

d. Addidit demum tria specimina in intestinis Turdi novae speciei obvia, quatuor ad septem lineas longa, proboscide trausversa polyacantha, 


\section{$-322$}

corpore crassiusculo et reliquis priorum notis in. signia.

Óva a forma vulgari deflectunt, solito scilicet et latiora et breviora, elliptico-lanceolata, duplici tunica constantia, quarum interna sive amnios interdum collapsa pro foetu haberi posset; sed intra hanc aliquoties foetum me vidisse mihi visus sum.

e. fo in Catalogo Viennensi idem Echino. rhynchus Turdis Merulas et sexatili pariter adscribitur.

27. Echinorhynchus micracanthus R. n. sp.

Motacillam quandam (Becafigue dictam, sed minime Ficedulam Linnaer, pro qua omnes fere aves minores in Italia venduntur) Florentiae, Au. gusto examinando in ejusdem intestinis Echino. rhynchum reperi flavum proboscide alba, mortuum, licet ave recentissima.

Corpus decem cum dimidia lineas longum, duas quintas lineae partes crassum, proboscide tertiam lineae partem longa.

Proboscis exigua, apice angustatá, sive sub. conica, corpori oblique imposita, uncinorum minutorum seriebus multis, quarum omnes non vidi, armata. Collum nullum. Corpus antrorsum incrassatum, dein aequale, apice obtuso.

Bremserus specimen misit in intestino Loxise coccothrautis repertum, septem cum dimidia li. neas longum, fere dimidiam latum, casu transver. sim rugosum.

Proboscis brevis, in majore specimine conic2, apice protracto, recta (neque corpori oblique imposita), uncinis exiguis multiplici seric disposius; in minore specimine proboscidis exigua pars 


\section{- $32 \overline{3}-$}

exseritur, quo colli brevis species oriatur, in majore non observanda. Corpus cylindricum aequale, apice posuco cobtuso.

Cbr. Speciem hanc a praecedente distinctam esse nullus dubito, ceterum variabilem.

\section{Echinorhynchus spiralis $\mathrm{R}$.}

In intestinis Ardeae minutae ab am. Nrtzsch repertuin qutm, Ent. II. I. p. 273. n. 19. descripsi, in tjusdem avis intestino tenui, Arimini Aprili reperi, septendecim lineas longum, colla. psum, ut pro Tatnia imponere potuisset, flavescentem.

Proboscis lineam dimidiam vix attingens, sedecim ad octodecim seriebus uncinorum minu. torum transversis munita; apice nudo nodulum referente. Corpus cylindricum, tortum, haud ita spirale ac illud speciminis a Nrrzsciro accepti, apice postico papillari.

\section{Echinorhynchus caudatus ŻED.}

Speciem plurimis Falcoribus propriam Froz: LICH in intestinis crassis Falconis Timnenculi repertam nomine Echinorhynchi Buteonis (Naturf. 29. p. 63.n. 33.) ita tamen descripsit, ut facile pro nova specie imporere possit. Collum nimirum pros boscidi lungitudine subaéquans, cylindricum, aequale, verrucis subtilissimis, elevatis, irregulari. bus, densis rubis obsitum, quo rubromacularunn appareat. Tale autem collum vix datur, ut non satis ab adhacrentibus purgatum examinasse videatur; secunfum auciores tian collum des st $_{\text {, }}$ probo:cis autem medio constricta habetur, cu. jus partem posteriorein $\mathrm{cl}$, vir collum vocarit. 


\section{$-324$}

50. Echinorhynchus Túba R.

Froencrer (Naturf, 29. p. 65. n، 34. Tab. 2 . Fig. I/4.) nomine Ech. Aluconis specimen descripsit in intestinis Strigis Aluconis repertum, eatenus ab illis, quae in Strige flammea reperi, defiectens, quod collo brevi instructum dicatur, cum meis collum desit. Vesicula vero lateralis apicis caudae appendiculata sexus tantum discrimen arguit, ideoque differentiam speciei non efficit.

\section{Echinorhynchus Acus R.}

Echinorhynchum lineolatum MüLLeri atictoritate commotus Ent. II. I. p. 28I. 13, 24. ab Ech. Acu ib, p. 278 n. 83. sejunxi, sed in ob. servationibus, potius conjungendos videri, addidi. Nunc utriusque utens speciminibus hoc confirmatum vidi.

Am. Gaene nimirum Echinorhynchos attulit auctumno Kilonii in Gado Merlango repertos,' quorum unicus lineolatus sive transversim interrupte plicatus fuit, et $\mathrm{cl}$. vir omnes ita compara: tos fuisse addidit, dum in Callaria reperti omnes laeves sive lincolarum expertes fuerint, quales ipse etiam in hoc pisce olim offendi. Praeter plicas illas nullo modo differunt, et istae a laxitate cutis originem trahunt, quae tamen hac in specie privi quid habet.

33. Echinorhynchus monilifornis Brews. n. $\mathrm{sp}$.

Am. 13R EMstr specimen misit in intestinis ITuris arvalis repertum, quatuor pollices et novem lineas longum, duas lineae tertias partes crassum, album.

Proboscis sublinearis, sive cylindrica, apice 
obtuso, tantillum incrassato, exigua, quartam lineae partem haud excedens, s̀extam circiter lata, uncinorum tenuissimorum seriebus circa duodecim munita. Collum nullum. Corporis teretis tres quartae partes moniliformes, sive stricturis in particulas lineam longas medioque valde exstantes divisae sunt; pars caudalis teres et aecpualis, apice tamon incurvo excepto, qui duarum stricturarum ope divisus particulam longiorem et breviorem terminalem labet.

Obs. Bellis et singularis species etiam in intestinis Criceti occurrit. : Cat, Ent, V. In opere novissimo autem (Uelser lebende Würmer in lebenden Menschen S. I8) Bremsenus refert: inter' 1995 specimina Muris arvalis scpties liunc Echinorhynchum repertum esse; praeterea bis in Mustela Putorio et semel in Falcone cineraceo para. - sitice occurrisse, Muris illius scilicet residuis simul relictis.

\section{Echinorhynchus porrigens $\mathrm{F}, 11, s p$. Tab: 1. Fig. $4-6$.}

In Entozoclogiae T. Ir. F. I. p. 30.4, n. 40. Echinorhynchos ab Huntrro repertos esse, quos cel, vir cum illis Anatis mollissinae com'paravit, retuli. Dum autem Museum Anatomicum Berolinense anno I8ro curae mea tradebatur; duas phialas reperi Echinorhynchos continentes, de quibus illustris olim Aratomicus, Јон. Theopli. Walter (Museum Anatomicum. Berolini I805. 4. p. I6I. n. I080 et IOSI:) sequentia profert: „Portio intestini jejuni ex Balaena rostrata Fabricro dicta, cujus membranae villosae vermes lumbricorum intestinalium figuram imitantes adfixi cernuntur." 
Cel. vir trahendo quosdam ab intestino removerat, sed ita quidem Echinorhynchos ex vinculis liberare non potuit. Tarn proboscis enim et receptaculum, quam colli etiam plurima pars inter tunicas intestini anfractuose, ducta latent; ubi cạut situm est, fossa quasi inter tunicas ex. aratur, materia grumosa referta.

Specimina adulta tres cum dimidio ad sex pollices longa, alba. Proboscis in omnibus speciminibus a me examinatis retracta, reque ullo modo exprimenda, sed receptaculium incidencio illam offendi inversam, lineam plus minus longam; cylindricam. Musculi retrahentes hreves, circa quos corpora duo globulis multis consiantia, neque lemnisci, quales in Ech. Gigante occurrunt. Receptaculum infundibuliforme antice fere planum, margine obtuso, duas lineas dianetro transverso superáus, retrorsum decrescens, lineam longum; cum Splachnorum. $\dot{v}$. c. vasculosi vel ampullacei apophysi comparaudum. Collum filiforme, vix tértiarii lineae partem crassum, ultra pollicen longum. Corpus anteriore parte retrorsum increșcens, dein aequalis crassitiei (circiter sesquilineae), linc inde sed iucon: stanti modo constrictum; apice caudali retuso seu foveolato; in nomnullis (maribus) papilla ex fo. vea eminente.

Corpus apertum tunicas eodem modo ac in Ech. Gigante comparatas exhibet. In masculis, qua panilla caudalis exstat, filum ex hac intus progreditur tẹnuissimum, semipollicare., Niliil ultra in animalculis diutissime spiritu vini conservalis dẹtegere licuit.

Specinina juniora adultis intermixta, pollicem plus minus longa, postice lineam dimidiam 


\section{$-327$}

crassitie aequantia, diaphana, tenera; capitis receptaculo exiguo, proboscide pariter retracta, ut foraminuli species ejusdem tantum vestigium referat.

Obs. Species insignis longicollium, quemadmodum Ech. Gigas brevicollium agmen ducit. Confer speciem insequentem.

\section{Echinorhynchts filicollis $\boldsymbol{R}$.}

Froeltch Naturf. 29. p. 70, n. 39. in tenuibus Altatis Boschadis ferae repertum nomine Ech. torquati descripsit, qui omnibus notis cum Ech. filicolli Ent. II. I. p. 283. n. 25. a me descripto convenit, et ad quem Ech. Anatis mollissimae ib. p. 304. n. 4t. pariter revocari debet.

Bremserve in Catalogo Ent. Vienn. typis impresso etiam minores Avium aquaticarum Echinorhynchos, corporis partem armaıos, cum Ecl. filicolli cónjunxir, et transitum a maximis ad minimos, ab inermibus ad armato tabula egregia nondum publici juris facta demonstrare sibimet persuasit, quod amico tamen concedere nequeo. $\mathrm{Si}$ enim, simulac specimen anceps vel intermedium occurrit, species tantopere diversas conjungere vellemus, omnes aut plurimae saltem rue. rent species.

Ech. filicollem, quem 1. c. fuse descripsi, multo facilius cum Ech, tereticolli conjungerem, ut specimina piscina in avibus post piscium illis obnoxiorum esum parasitica majora fieri et habitum quodammodo alienum (semper tamen affinem) induere statuerem, sed, re incertissima, utramque speci:m servare satius duxi.

Obs. Echinorhynchus filicollis, quemadmodum praecedens, nunquarn proboscidem exsertam, 
affert, sed in bullars sive receptaculum et ita quidem retractam sistit, ut nulla cncheiresi evolvi possit.

\section{Eohinorhynchus tereticollis $\mathbf{R}$.}

In plurimis piscrbus reperi, quos in Synopsi p. 72. recensui, ideoque non repeto.

Qui in intestino crasso Accipenseris Sturionis miṇoris A rimini Majo mihi oblati sunt plurimi, prae ceteris attentione digni habentur. Albidi erant, aurantiaci, vel carnei, duas quatuorve lineas Inngi, corpore saepe ovali, interdum orato. vel oblongó; collo longo cylindrico, saepe rugoso, rarius bulla terminato; proboscide oblonga brevi, passim clavata, duodecim ad sedecim seriebus uncinorum armata,

Specimina dicta a forma solita utique recedunt, ob collum longum aequale huc tamen referenda videntur.

Deflectit quoque specinen, quod Berolini 'Julio in 'intestino Siluri Glanidis majoris' offendi, collo scilicer aequali quidén', sed rizgoso, sivc transversim plicato, neque in aqua faciem mutante.

Confer speciem insequentem, quacum Erexi. SERvs hane conjungit.

\section{Lchinorhynchus nodulosus Scuravir.}

Am. Tiedexann multós misit Echinorhynchos in intestinis Cyprini Barbi repertos, septein ad undecim lineas lorigos, nonnullos crassitíle duas lineas attingentes "In his proboscidem éo magis clavatain videri, quo minor bulla fiat, pás. sim oblittèrata, 'visum 'mihi est.'

Brearserus hanc et praecedentem specicm 


\section{- $3^{29}$}

conjunxit et maxime affines esse concedo, 11unquam tamen Cypriai Echinorhynchum, milimet absque nominc oblatum, me cum priore cominntare posse, crediderim. Habitus enim totus, prac. sertim autem collum nimis differre videntur, quo minus conjunģam.

Si uraque species in eodem pisce v. c. Gado Lnt a occurrere dicitur, altera a piscis, esu residua et parasitica esse potest, neque hoe argumentum discrimen illud tollit. Sed praeter hos ctiam Ech. ovatum ZEDERI n. 3S. єt. Ech. sphae. ricum mili dictum n. 39. annicus conjungit. De spliaerico saltem, quem in Cotti scorpii mesenterio saepius reperi, a reliquis diversissimum esse forma, duritie, magnitudine, Joco, contendere possum; de ovalo, quem non'vidi, non ita certus sum, sed Zndar dẹscriptio diversum esse vero simile reidit, et Ech, nodulosum saepissime exa. minando nunquam talem reperi, quem cum Ech. ovato conjungerem,

\section{Echinorhynchus striatus.}

Bremserus duo specimina misit in intestinis Ardeae cinerease reperta, alterum quinque, alte rum. sex lineas longum, parte latissima lineam excédentia.

Proboscis brevis, teres, apice parum incras. sato, uncinorum mediocrium seriebus duodecian munita. Collum conicum, inermek Corporis nat's antica, levi ștrictura a collo sejuncta, conica, insequens subglobosa est, utraque autem aculeis brevibus densis: armata; reliquum corpus strictura distinctum, primo subglobosum, dein teres et tenuius, inerme, obiter vero longitudinera ve:sus sulcatum conspicitur. 


\section{$-33^{\circ}$}

Obs. Figurae Goezrr pessimae sunt; corpus armatum olim jam suspicatus sum, conf. Ent. II. I. p. 263 .

44. Echinorhynchus versicolor $R$.

Ent. II. I. p. 295. n. 33. Ech. minutus. p. 296. n. 34, Ech, constrictus. p. 238, n. 35 . Ech. collaris.

Froelich. Naturf, 29. p. 66. n. 35. Ech. Boschatis, ib. n. 36, p. 68. Ech. Anatis. p. 69. n. 37. Tab. 2. Fig. I5, 16, Ech. tenuicollis.

Entozoologiam edendo speciem hancce nondum videram, sed aliorum descriptiones secutus, tres inde species finxi, quas nunc meliora eda. ctus contraho; Ech. filicollem n. 35. autem, quo. cum Bremserus pariter conjungit, diversum ha. beo, uti supra exposui.

Novembri mense, Gryphiae in inferiore parte intestimorum tenuium Anatis Fuligulae octo reperi Echinorhynchos intestinis maxine infixos, quorum marem Ech; constrictum, feminam Ech. minutum efficere vidi.

Mas duas tresve lineas longus, candidus; proboscide mox oblonga lineari, mox ovali, uncinorum seriebus octo ad duodecim munita; vagina utplurimum brevi, tumque (sub contractione) longitudinaliter striata; semel tamen vaginam proboscide avulsa valde extensam et hac longiorem vidi. Corpus oblongum, vel utrinque, vel praescrtim anteriora versus decrescens, antica parte aculeorum exiguorum seriebus viginti ad triginta munitum. Vesica caudalis sủbglobosa, vasculasa, ope stricturae a corpore sejuncta. Ex corpore dissecto materia alba, tenuis, lente fortissima ad. 
hibita obiter grumosa, sive granulis exiguis con. stare visa effluit.

Femina duas et quod excurrit lineas longa, mare crassior, abdomine vel toto, vel maximam pariem coccineo aut potius miniato, proboscide colloque albis, Corpus plus minus, plerumque bis lortiter constrictum, parte antica (aculeorum seriebus trisinfa ad quinquaginta transversalibus armata) subovata, reliqua ovali, interdum stricturam obsoletam exhibente. Corpus ovulis lineari-ellipricia, mox pellucidis, mox punctis vagis fuscatis, farcum.

Postmodum Berolini Aprili mense in intestinis Fulicae atrae quinque reperi specimina, duas tresve lineas longa, albida; corpore oblongo, passim inciso, interdum postice saccato; probo. scide ovali multis uncinorum seriebus armata; ccllo longo tereti; hujus vagina (vel antica corporis parte) aculeis minutis tecta. Hanc prioris varietatem, Ech. collarem Schranin sistere su. spicor.

Obs. I. Froelichuos omnia specimina sua in intestinis crassis Anatis Boschadis ferae reperit, attamen tres inde species effinxit; duae priores autem, quas dixit, feminae varietates; tertia(Ech. teruicollis) marem cum vesica caudali exhibent.

Obs. \% Gozzius, qui speciem istam in Turdo Merula inventam refert, cum sequente confundit.

45. Echinorhynchus pyriformis Breuser. 11. sp.

Specimina duo in intestinis Turdi Merulae reperta Bremserus misit, sesquilineam longa, an- 
lica parte ultra duas lineae tertias pärtes crassa, allida.

Proboscis exigua, linearis, uncinis in utro. que specimitue minus bene dignoscendis; collum nullum; corporis 'fere obovati pars antica subglo. bosa, acultórum exiguorum seriebus plurimis densissime armata; pars posterior nuda versus apicem caudalem obtusiusculum decrescit.

Corpore dissecto innumera ovulorum copia sistebatur. Haec linearia, utrinque pellucida, magnitudine diversa, ut quaedam aliorum longitudine duplo triplove superarentur. Inter ovula placentulas quoque repieri, de quibus Ent. Vol, r. p. 293 egi.

Confer obs. secundam ad Speciem praecedentem.

46. Echinorhynchus Hystrix Brems. n. sp.

Am. Bremser in intestinis Pelecani Carbo2its semel unicum, deinceps autem (Nartio) plurima reperit specimina, quorurn complura benevole mecum communicavit, alba, sesquilineam ad tres lineas longa, parte crassiori lineam, tenui auten vix hujus quartam partem lata.

Proboscis cylindrica, inaequalis, parte scilicet anteriore quam reliqua, aeque longa, tenuiore, tota uncinis recurvis posteriora versus majoribus, sedecim circiter seriebus transversis dispositis armáta. Collum proboscide brevius, conicum, in: terdum cum proboscide maximam partem retractum, nudum. Corpus parte antica crassissimum, versus apicem caudalem conicum decrescens. Apice posteriore excepto corpus aculeis exiguis reflexis horret, in specimine minimo ad ulti- 


\section{$-33 j$}

num apicem protractis; in reliquis major pars nuda est.

Tria specimina mascula vesica caudali in structa, quae in duobus pellncida, in tertio (minimo) opaca fuit. In illis superior vecicae pars turgida et pellucida sacculos duos anteriores subglobosos et vasa nectentia, reliqua autem pars tunicam quasi collapsam et complicatan cxhihuit; an haec forsan ob humorem ex resica partim egressum talis facta? 'Ex apice corporis caudali ductus veșicam pettere videtiur.

\section{Echinorhynchus Pristis R.}

Lepidissimam speciem Ent. II. r. p. 299. no 36. secundum specimen descripsi, quod Gryphiae Majo in intestinis Esocis Belonis solitarium reperi, duas tertias pollicis partes longum Arimini autem Aprili in intestinis alterius Scomöri Scombri specimen bipollicare, in illis alterius duo duos pollices tribus et decem lineis superautia, et Neapoli Junio in illis ScombriColiae unicum duos polliccs novem lineis superans offendi.

Proboscis linearis, recta, líneam longa, al. ba, uncinorum validorum sericbus quaciraginta transversis munita. Collum nullum. Corpus cy. lindricum, anteriori parte in quadam a proboscide distantia paululum crassius, ceterum aequale, filiforme, lineae quartam partem diametro vix superans, dilute ruseum; parte antica tres lineas longa seriebus aculeorum fortium (crassorun) et sanguineorum obliquis, remotis, duodecim arma. Ia; apice caudali obtuso.

Obs. I. Series aculeorum corporis et anticae et posticae magis remotze sunt, 


\section{- $334-$ -}

Obs. 2. In Scombris bellem hanc speciem longissiman reperiendo, primum novàm habui, Berolini autem cum specimine Belones comparando, eandem esse didici; quo minus autem dubium supersit, speciminum italicorum descriptionem addidi. Echinorhynchus subulatus n. 48. autem, ab Hermanio in Clupea Alosa repertus utique diversus videtur, si bene descrip. tus est.

\section{Echinorhynchus vasculosus R. n. sp.}

Neapoli Junio semel solitarium in intestinis Spari (Bramae) Raji, bis in ejustem abdomine Julio, duo scilicet specimina libera primum, dein solitarium mensenterio affixum 'reperi, viva, quinque ad sex lincas longa, tenuia, partem anteriurem alba, ceteroquin rubella, elegantissima.

Proboscis ovatis, uncinorum validorum seriebus decem armata. Collum illius longitudine conicum; corporis pars antica ejustem longitudi. nis subconica, aculeis brevibus dense armata; reliquum corpus cylindricum, aequale, apice postico tenuiore, obtuso. Unicum specimen (casu) passin constrictum.

Et collum et corpus vasorum cutis rete offerunt antea in Echinorhynchis non visum, spectaculum amoenissimum. Vasa scilicet sunt lon. gitudinalia tenuia, aequalia, transversis plurimis ramosissimis ubique anzstomosantia, totzmque cu. tem perreptantia. 


\section{Species dubias.}

50. Echinorhynchus Mustelae.

In Museo Viennensi tria vidi specimina in. Mustelae vulgaris mesenterio reperta, aliquot lineas longa, crassiusctula, corpore undique laevi, proboscide retracta, quam tamen altero specimine discis:o longam et cylindricam conspicere licuit. Species procul dubio nova.

\section{Echinorhynchus Wachniae.}

Am. Trlesius (Mémoires de l'Academie de St. Petersbourg T. IL. ค. 333. et-374. Tab. XIX. Fig. 4 -7.) duas Echinorhynchi species in intestinis Gadi Thacluiae reperisse sibi visus est, quas tamen conjungere et ad Ech. Acum n, 32. amandare mallem.

\section{Echinorhynchus Sciaenae.}

In mesenterio Sciaenae Aquilae Neapoli Jinio solitarium reperi specimen, quinque lineas longum, filiforme, parte posteriori (ultima) parum incrassata, rubellum, colore versus posteriora in. tensiore.

Proboscidis exigua tantum pars in conspectum venit, uncinis parvis, uti videbatur, armata. Collum subsphaericum, an bullam obscuratam si. stens?

Si hoc foret, ad Ech. tereticollem n. 36. amandarem, sed ad rem confirmandam aliis opus est speciminibus. 


\section{$\div 336-$}

go. Echinorhynchus Atherinae.

Sex Athcrinas Hepsetos Neapoli Junio secando, in alterius intestino unicun reperi specimen duas lineas longum, album; proboscrde $\mathrm{li}$ neari recta, uncinorum mediocrium seriebus decern ad duodecim armata; collo nullo, corpore dauciformi, posteriora versus decrescente, ob. tuso.

In corpore placentas quidem, saepius dictas, nulla vero conspexi ovula. 


\section{- 337}

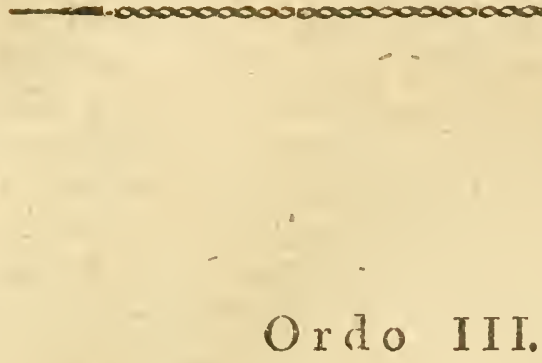

\section{Tre matod a.}

Genera pauca ordinem hunc componunt sa. tis naturalem, paucis dubis obnoxium. Nonostomata quaedan!, praesertim Hypostomata mihi dicta Caryophyllaeo affinia sunt, quo certe ill. Covienus commotus fuit, qui liunc renatodibus adderet, a me inter Cestoidea relictum, quibus v. c. Scoleci, Triaenoplıoro, utique similior vic'etur; multo facilius saltem Hypostomata Ciestodideis traderem, quain ab his Caryophyllacum remove:. rem, sed illud etiam difficultate non careret, cum varia Monostomata vera in Hypustomata trans. eant.

Ab altera parte Pentastomata aliena viden. tur, vel habitu, ut passim pro Taeniis imposue-, rint, a quibus tamen omnibus numeris recedunt, corpore minime articulato, eoque fibris, vasis, brevi omuibus partibus aliter constitu ; neque labitus eorum in . Trematodum orcline exemplo caret, cum Distomata quaedam armata magnam prodant simiitudinem. Vel vasis minus revinctis, quid? quod passim prolapsis a Trematodibus distincta videntur, quod tamen aeque parum 


\section{- 338}

concederem, nam Amphistomatum quorundam v. c. conici et subtriquetri vasa facile a cute libe rantur, et Ampl, cornuti oviductus post partum e corpore eliminari (ideoque liand revinctos esse) Ent. Vol. 1. p. 314. exposui, quo Distoma clavigerum n. 52. accedit, cujus oviductum cum cirro continuum facili negotio separavi. Cuvnerus (Regne Animal T. IV. p. 35.) certe ob nervos in Pentastomate taeniode visos, illa Nematoideis addidit, quibuscum tamen militare nequeunt. Ipse quidem nervos in specie dicta non reperi, sed forsan ob speciminis indolem minus bonam, ut hos summo viro largiar, iis autern Nematoideorum characterem non effici in aprico est. Hatc enim sunt teretia, elastica, tuboque intestinali ore et zno terminato, nec non sexu distincto utuntur; Pentastomata, androgyna, vasis nutritiis Tremato. dum more divisis instructa, depressa, et mollia sunt. Nervi forsan plurimis conceduntur Entozoorum ordinibus. Pentastomata itaque aut ordinem propriun constituant aut inter Trematoda enumerentur.

Tristomatis genus, ab.jll. viro huc relatum, utique recipiendum, disco quidem infra describendo recedens, habitu tamen, vasorum dispositione, hahitaculo convenit.

Generum quorundam affinitas magna observatur, sic v. c. Amphistomatis subtriquetri porus posterior non terminalis, sed inferus tt paululum ab apice postico remotus est, quod Bojanum, vi. rum cel. commovit, qui Distomatibus adscriberet; horum vero porus anticus saepe non exquisite anticus est, et tamen a reliquis talia non separamus. Essentiale discrimen inter Amphistoma et Disto. non interesse, lubens concedo, sed in tanta 
specierum copia, character artificialis, dummodo facilis est, exoptatus hal,eri debet, et talis me quidem judice genera dicta distinguit.

Quąenam Trematodum quorundam pars pro anteriore habenda sit, dubio non, caret. Varia Amphistomata in Entozoologia describendo posteriorem male anteriorem dixi, et quae haec circa meliora edoctus sum, hoc in opere protuli; qui Amphistomatis porus ova profert, cerie posterior est, v.c. Amph. cornuti; habitus rem non conficit, et entozoa utroque poro affixa concpiciuntur; quando itaque anatoine non aciessit, neque te observante ova deposila sunt, res incerta manet, ut errorum hoc respectu a me commissorum veniam lectores non recusaverint.

Am. Bremser, Helminthologus summus, me quoque circa Polyctoma errasse suspicatur, quod non admiserim. In Polystomate enim integerrimo (Ent. Tab. VI. Fig. 1-4) vasa ab omnibus sex poris anticis incipiunt, et $e o d \in m$ quidem modo, quo vasa $a b$ osculis Taeniae, certe capitis, oriuntur. Porus porro solitarius Polystomatis, quem posticum voco, eadem omnino ratione quibusdam Distomatibus additur, v: c.' D. caudiporo Zenis Fabri, vel D nigroflavo Orthragorisci, neque in his aliter explicari potest, poro scilicet anteriore more solito simul praesente. Tertio denique ostia anteriora complura in $\mathrm{P} \in \mathrm{n}$. tastomatibus et Cestoideis plurimis; nullo autem in entozoo ostia generationis complura dantur.

Cel. Nitzsch Distomatum a me entuieratormm quaedam, v. c. alatum, excavatum; et Am. phistomata capite discreto sub novo genere $\mathrm{Ho}_{\mathrm{o}}$ lostomum sibi dicto comprehendit; ipse singula potius genere peculiari enumerarem, sed illa si. gillatim exponendo, haec melius dicentur. 


\section{Genus XIII. Mronostoma.}

Entozoa corpore molli teretiusculo vel depresso, - hoc constituentia, notis illis quidem conveniunt, ceteroquin autem tantopere inter se differunt, ut commode in plura genera dividi possint, tquod numero specierum non magno, harurnque yaris non satis cognitis, in aliud tempus distuli.

Hypostonata primam generis sectionem sistentia pracprimis' a multis aliis distant; datur tamen transitus per Monostoma foliaceum 11. 4. quoad corporis habitum, substantiam, ei colorem album, generi alias alienum; et Monostoma cru. cibulum 11. 6. pòri antici ratione Hypostomatibus valde afñne videtur; neque mirum, Piscium Monostomata ab illis Manmalium, Avium et Anzphibiorum differre, cum Distomata similem ofierant diferentiam.

Monostomata tenuicolle n. I \% filicolie ${ }^{2}$. 15. quo etiam illud Molae dubium n. 30. pertinebit, a plurimis forma distant, et commode sejun. gi possunt, sed colli solius ratione liabita, narn. porus anticus solitarius ntique Nionostomatis est.

Variae tandem species, praesertim Monostoma ventricosum n. 19. et nuacrostonum n. 23. - ad Amphistomata amandanda videntur, sed poro postico non viśo, inter illa reliqui, Monostoma pilcatum Entozologiae autem jam inter Amphistomata (12. 12.) militat,

\section{Monostoma foliaceum R. n. sp.}

Am. Tremser specimina novem misit in Sturionis abdowine reperta, tres ad septem lineas longa, unam ad tres lata, ipseque Arimini Majo 
in Sturionis junioris, vix bipedalis, pridie in aedibus meis mortui abdowine duo reperi Monostomata viva, sed motu tantum tardo et exiguo utentia, albida, marginibus pellucidis, alterum pol- : licem, alterum octo cum dimidia lineas longùn, utrumque quatuor lineas latum.

Entozoa oblongo elliptica, utroque fine obtuso, dorso convexo, ventre plano; interdum hoc excaratur, quo margines aliàs tenuissimi et acuti fere duarum lineae tertiarum partium, corpus medium tertiac lineae partis crassiticm attingunt. In altero fine oltuso vel osculurn exiguura vel cjus loco papiliam reperi; posterior passim emarsinatur.

(In speciminibus Bremserianis interdum utrumque finem emarginatum vidi, posterior tamen utplurimum integerrimus exhibetur, anticeq̧uc corpusculum exsertum est vario se habens moda, mox cupulam, mox processum ellipticum integrum referens; corpusculo illo retracto distincte foraminulum conspicitur.)

Media maximaque corporis pars ovulis ro. tundis ovariiisque tortis repletur, quae ambiuntur vase pellucido margines ab ovariis scparante; praeterea circa locum emarginatum posticum utrinque a margine vas tenue oritur. angulo acuto Tasis illis majoribus pellucidis immissum et con. junctum, deinde in vasa dorsalia ramosa et varie anastomosantia abiens. Circa porum anticum varia vasa conveniunt et fortior canalis (an cirrus?) exinde medius oritur, sed brevi desịnit, aut saltem oculis subducitur, Cutis tenuissime reticu. lata est.

Obs. Pro Amphistomate habere non pos. sum, poro postico nunquam exhibito, licet vasa 


\section{$-342 \cdots$}

crenam sive locum enlarginatum pasticum am. bientia talem fere verosimilem reddunt. Ha': tus proprius, ut entozoa ista fere Ligulam brevem contractam referant, a cujus tamen genere omni-

- bus notis differunt.

\section{5: Monostoma orbiculare R. n. sp.}

In intestinis inferioribus spari salpae Neapoli Junio undecim, iterunque Julin quinque reperi Innostomata lineam plus minus et longa et lata, orbicularia, poro interdum tantillum orbiculum egrẹdiente, hinc convexa, illinc concava, rarius plana, albida, mortua..

Porus terminalis oblongo-ovatus, e cujus basi vas obscurum nascitur; media corporis pars corpuscula aliquot opaca continens, reliqua undique vasculis reticulata, et pulcherrimo quidem modo, ut, verme tenui et pellucido, cum Medusarum corpore quaedam similitudo exoriatur.

6. Monostoma crucibulum R. n. sp.

In intestinis Muraenae Congri maximi Ne. apoli Junio tria reperi specimina, et unum ibidem Julio in illis Muraenae Cassini, sesquiline. am longa, dimidiam lata, alba, vase dorsali flave. scenti-fusco, viva, collum longe porrigentiá.

Porus anticus exquisite terminalis, crucibu. liformis, margine antico plano utrinque exstante. Corpus depressum, dorso convexo, ventre planiusculo, elliptico-oblongum, antrorsum magis attenuatum. apice postiço acutiusculo. Ova pone porum anticum globosa, sparsa, Vasa fulva in. partibus posterioribus.

Porus valde profundus, singularis, licet an- 
ticus sit, quandam cum illo Hypostomatum similitudinem fovet.

7 Monostoma capirellatum R. 11. sp.

In intestinis Spari Salpae praesertim posterioribus Neapoli Junio et Julio multa reperi specimnina mortua, mollia, alba, capitulum quasi mentientia eoque Taeniolam referentia, duas ad quinque cum dimidia lineas longa, tertiam circiter lineae partem Lata.

Recentissima teretia, dein depressiuscula, linearia, parte postica tantillum latiore, obtusa. Ca. put rotundatum, crassiusculum, unde capituli species, haud tamen discretum. Porus anticus subglobosus (nonnihil longior quam latus), a quo incipiunt vasa plurima plurimis locis inter se anastomosantia, ut tota fere corporis pars illis obtegatur. In dorso vasa duo majora decurrunt; in posteriori parte corpuscula nonnulla subglobosa opaca medium tenent, certe ovaria, aut oviductuum partes ova ad exitum praeparantia,

\section{Monostoma lineare R. n. sp.}

Am. Bremser specimina quatuor misit in intestinis Tringae. Vanelli reperta, vix duas lineas longitudine, latidudine dimidiam adaequantia, alba, vase spirali flavo.

Porus anticus terminalis, exiguus, orbicularis. Corpus marginibus rectis lineare, apice postico obtusissimo, quam antico magis obtuso.

In media corporis linea vas spirale flavum, spirarum ductibus fere transversis, rectiasculis, approximaris; ad latera vasa alba, a capite decurrentia, versus caudam crassiora. Corpore plus 


\section{- $344-$}

minus psllucido utraque vasa egregie conspici. untur.

Öbs. In Níonostomate Tringae Vanelli pulmonali majore 11. 27. nonuisi vasa lateralia exlibentur.

\section{Minostoma verrucosum ZED,}

Specimen in intestinis Anatis Segetum re. pertum am. Brexser misit, sesquilineam longum, antice quartam, postice ultra tertiam lineae par. tein latum.

Portes anticus exiguus orhicularis. Cirrus approximatus, exsertus, longiusculus, rectus. Collum subtus concavi:m, marginibus introram flexis; corpus posteriora versus, increscens, apice postico obtuso.

Papillae sive verrucae Zenero dictae, dorsales, appressae, circiter triginta, aut ter decem, cum triplici serie sistantur, uti Froelchrus recte monuit, cum oviductibus in abdomine sitis ne. ctuntur, conf. Ent. IL. I. p. 332. obs. 3.

13. Monostoma cllipticum $R$.

Zedenus speciem istam describendo, specimen quod olim in $\boldsymbol{B}_{1}$ fonis ignci pulmonibus Julio solitarium reperit, non amplius prae oculis habuit, quo descriptio cl. viri manca et erronea exculpari debet.

Am. Gaede specimen in pulmonibus ejus. dem Bufonis Junio repertum die insequente mecum communicavit, tres lineas longum, antice tértiam, postice tres quartas lineae partes aequans.

Porus haud obliquus, qualem Zenerus describit, neque amplus, sed terminalis et medio. 


\section{$-345$}

cris, ostio orbiculari. Corpns haụd ellipticum, sed oblongum, postice dilatatum, obtusum, dorso convexiusculo, abdomine plano, marginibus rectiusculis.

Colorem: Zenerus carneum dixit, nostrum specinell candidum, vasis nigerrimis in media dextraque dorsi parte, vasculo luteo sinistiorsum conspicuis. A latere plano sive inferiori spectatum acervi ovulorum lutescentium et oviductus pariter flarus déxtrórsum, sinistrorsum vero vasculum nigrum exhibetur, locis quibusdam inter. mediis vacuis.

Am. biremser specimina quatuor misit in Bufonis cinerci pulmonibus repetta, sesquilineam ad duas cum diuidia lineas İonga, latere dorsali convexo, abdominali planiusculo, eo a specimine supra descripto diver'sa, quod antrorsum crassiora, retror-umi angustiora sunt, parte postica nígrescente, vasis ceterum nigris, ovariis lutescentibus. P’oru. anticus subiuferus est, oștio orbiculari, sG. mel longitudinali viso,

\section{IIonostoma Com R.}

Ent. 1. p. 346. n. 7. Amphistoma Cornu. Ani. Bremser duo specimina misit in $A r$. deac Nycticorácis intestinis reperta, alba, lineam plus minus longa, teretia, poro antico contracto. Gozzrus hanc speciem in intestinis Ardeac cinereae rcperit, a Zenero Distnma cornu vocatam, a nic perperam ad Amphistomata revocatan. Brevsert's mihi pro Monostomate misit, et ipse etiam porum tantum anticum vidi, in altero specunine cisturtum, sed contractum, neque ut in Groera f jura hiantem.

Hoc non coufundatur cum Amphistomate 


\section{$-346-$}

(vero) Ardearum, Holostomate cornu Nitzscrir, Syuops. n. 9.

18. Monostoma tenuicolle R. n. sp. Tab. 2. Fig $1-4$.

Cel. Bakker, Professor. Groningensis, ali. quot specimina misit inter carnes Lampridis guttati Retzir a se reperta, annon speciei sequentis ex analogia hydatidibus inclura?

Alterum specimen viginti novem, secundum viginti octo, tertium viginti tres lineas lonya, corpore tres lineas, collo summo dimidiam et quod excedit, latis. In primo specimine lon. gitudo colli ad illam corporis uti novem ad viginti; in secundo uti novem ad noyendecim; in tertio uti ocio ad quindecim se habet.

Porus anticus terminalis orbicularis parvus. Collum teres a poro increscit, ut brevi ab eo di. stantia crassissimum sit et dimidian lineam supe. ret, mox vero iterum decrescit et prope corpus tenuissimum est, lineam dimidian non attingens. Corpus colli ratione habita, crassissinum, oblon. gum, dorso concavo et contracto, abdomine ro. tundato ampliore, apice obtuso; totum cavum, vasis de quibus mox sermo erit penitus omnibus cutaneis, quo entozoon magnum in parvum spatium contrahi possit, et inter carnes obvium, harum motum non impediat.

Abdomen vasculosissimum, vasis triplicis ordinis; priora duo fusca, versus dorsum anastomosantia et hoc nudum relinquentia; alterum eorum jam in collo conspicuum, rectiusculum, in abdomine ductus transversos copiosissimos 'efficiens, irregulares, crassos, passim constrictos; secundum vasorum genus pariter fuscum, ductus 
tenuiores aequales prioribus plurimam partem parallelos, passim illos decussantes. Curn crassioribus et inaequalibus passim corpora nuajuscula, lineam duasve lineas Ionga, ovalia aut subrotun. da nectumtur, quae ovaria sunt et oxis glolosis minutis scatent, ut vasa majora oviclucrus, mino. ra seminifera crediderim. Tertium prarterea ge. nus adest, vascula sistens tenuissima albida densissima retia efficientia, ni fallor, nutritia. Collum vas fuscurn medium continet et versus basin utrinque ovarium valde tlongatum cuius vasa cum illo abdomen petunt; practerta autem collum vasis albidis scater, ut ejus tunicat tere inde conflatae sistantur; haec in abrloniue inter reliqua occurrunt; ovaria fere mediarn abdominis lineaul perunt; reliqua vasa in abdomine copiosissima, elegantissina relia efficientia.

Obs 1. Quae vasa fusca dixi, analogia spe. ciei insequentis lalita, in Monostomate vivo aut recente forsan fulva vel flavescentia sunt.

Obs. 2. Lampris guttatus Retzir, rarioribus Europae piscibus adnumerandus, est: Zeus Luna LinN. Gmel. T. 3. p. 1225, n, 7. Chrysotosus LA. CEPEDE. T. IV, p. 587 .

\section{MInnostoma filicolle R. n. sp.}

In Spari Raji interstitiis aliquot processu. um spinosorum vertebrarum dorsi accessoriorum (s. sptiriorum) et verorum vesiculas Monostoma hoc paradoxum continentes Neapoli, Julio reperi.

Vesiculae ovales,' tenui membrana constantes, individuum solitarium complicatum continent.

( . Hi rorporisque ratio ab illa speciei prae. cedicutis dilicrt, ln specimine quadraginta octo 
Iincas longo, harum triginta tres collum, sibi vindicat; in altero quadraginta et .unius linearum, lharum viginti sex; in tertio duodecim et dimidize linearum, harum decem collo debentur.

Porns obscurus, minor certe quam speciei praecedentis, in qua mox patet. Apex añticus arutiusculus, et pone eundem ostium longiuscu. lum inferiora versus adesse videtur, sed specimisia tartum mortua vidi, parte antica'flaccida, ut wihil certi hac de re proferam.

Collum longissimum, tenuissintum, aequale, vel capillare; corpus ad tres lineas crassum, oblongum dorso planiusculo, abdomine rotundato, apice 'obtuso.

Collum alsum pellucidum, ductu ovifero Inavo serpentino medio; in summa colli parte adlluc vasa minora sed obscure conspiciuntur. Cor. pus album, pellucidum, cavum, maculis ductibus. quie luteis, ova rotundato ovata continentibus, undique cinctis vasis lacteis (sive albis opacis) serpentinis copiosissimis, quo aspectus corporis fiat leñidissimus.

Obs. I. Species praecedenti maxime affrie, facili tamen negotio distinguenda: poro apicis tenuioris obscuro; collo longissimo; vasis corpóris minus frequentibus. Vasa ovigera majora "videntur, sed majora interstitia offerunt et illa secundi ordinis in hac specie parea, et cum tertiis fere coincilunt. Forsan vasa nutritia casu minus rcpleta-fuerunt.

Obs. 2. Praeter specimina completa etian collum absque corpore reperi, aliquot pollices longum, rase magno luteo spirali medio; hoc cum speciminibus in Orthragorisco Mola repertis $n, 30$, valde convenit, aut forsan idem cst. 


\section{Monostoma trigonocephalum $\mathrm{R}$.}

In Entozoologiae Tol. II. I. P. 333. n. I2. specimina quinque descripsi ab am.' Bravn in Testudinis Mydae ventriculo reperta et mecum communicata. Arimini vero Majo, in ejusdenz Testudinis crassis copiosissimum, et nuper (Berolini Octobri) in ejusdem toto tractu intestinali reperi, quo recentia describere poscim.

Lineam ad duas lineas londa, tertiam lineae partem lata, intestinis affixa albida-aut rubella; propius autem examinata: pellucida, ovariis late: ralibus albidis, margine lateris ventralis fulvo vel rubello; dorso convexo, ventre concavo.

Pars antica fere triangularis, angulis tamen posticis in vivis obtusioribus et parum exstantibus, interdum subtus productis, capite fere cordiformi. Porus globosus, anticus, ad latera ntrinque tissus. Cirrum qquen in speciminibus Braunianis observari, in recentium nullo vidi.

Semel partis posticae obtusae macula pellu. cida pro poro imponere potuisset, sed certissine talis non est.

Motus animalculi fere idem ac Distomatum.

\section{Monostoma galeatum f. n. $\mathrm{sp}$.}

In Centronoti glauci tres cum dimidio pe. des longi intestinis, praesertim anterioribus Ne. apoli Augusto pilurima reperi speci mnina lineam dimidiam longa, teretia ct tenuissima, alhitla, speciem novan distinctissimam sistentia.

Caput praesertim in mortuis discretum, sử. tetragonum, antice truncarum, subtus planum vel concavum, marginibus lateralibus et superiore papillis, sive cutis productionibus, conicis sex vol 


\section{- $350-$}

scpiem dentatum. Porus pro vario capitis motu mox inferus, mox terminalis, crucibuliforni, et fasciis quasi cutis firmioribus circumdatus; ostio mox semicirculari, mox angulato. Corpus poste. riora versus subatienuatum, passim sed haud constanter contractum, apice postico vel emarginato, vel attenuato et elongato, vel appendiculato ovali aut subovato.

Ovaria magna flava lateralia, passim etiam postice fuscescentia.

$$
\hat{s} p \text { écies dub i } a \text { e. }
$$

\section{Monostoma Vanelli.}

Am Bremser specimen misit quod Tringae $V$ anelli pulmoni extus adhaeserat, albidum, sex lineas longum, duas tertias lineae partes latitudine excedens, crassiusculum.

Corpus depressum, lineare, apice utroque obtuso. Porus alterius apicis exiguus, vix notabilis. Vasa duo recta corporis longitudinem tenent; alia adhuc in apice adesse videntur, sed obscure.

Obs. Magna certe affinitas adest cum Distomate lineari n. 8. in intestinis Tringae Vanelli obvio, ut pulmonale forsan crassitie (speciminibus majoribus) potissimum differat In Catalogo Viennensi tamen pro specie peculiari habetur.

30. Monostoma Molàe.

Orthragorisci Molae, quem Julii d. 26. Neapoli mortuum acceptram, musculi cuti dorsali substrati, post biduum examinati, entozoz obtulerunt mortua, value singularia, 
Longissima scilicét, depressa, angustissima, vase spirali luteo crassiusculo, haud tamen per totum corpus visibili, instructa.

De horum indole nihil certe proferre potuissem, nisi die insequente intèr Spari Raji processus vertebrarum dorsi spinosos Monostoma filicolle n. iy. et simul etiain hujus partem filifor. mem solam (absque corpore) offendissem, haec enim vermi Molae maxime affinis est aut eundem sistit. Mirum tamen, me in Orthragorisco Monostoma integrum non invenisse, duos licet hu. jusmodi pisces sedulo examinaverim; sed idem etiam non in omnihus Sparis Raji occurrit, qui Gymnorhynchis maxime obnoxii sunt.

\section{Genus XIV. Amplistoma.}

Inter species hujus generis haud numerosas magnum tamen invenitur discrinen, quaedam scilicet capite discreto, continuo aliae utuntur; illorum quaedam porum majorem antériori, quae. dam posteriori fine exhibent, paucissimis uterque aequalis datur. Quae capite gaudent continuo, absque mihi notá exceptione, porum anteriorem minorem offerunt.

Cl. Nitzsch in Encyclopaediae Halensis no. vae specimine indicante genus Trematodum con. stituit novum, Holo.tnmum Sihi dictum, cui, Distomata aliqunt paradoxa (alatum, Excavatum) et omnia Amplistomata capite discreto instructa inseruit, insimul vero omnium horum porum anti. cum nohis posticum audiri addidit. De multis horum forsan hoc valet, neutiquam autem de omnibus. Amphistoma enim cormutum (synops, n. 10. Ent. 


\section{$-352$}

Ir. 'I. p. 343. n. 4. Tab. V. Fig. 4-7.) maxima usi diligentia observavimus et ova poro postico eflundi vidimus, quo dubium nulluin superesse possit. Injus vero porus, anticus maximus et lobatus et ejusdem est formae, ac Amphistomatibus plurimis alter conceditur, quem idcirco anticum vocavimus, eoque magis, quippe ejustem ope intestino affigunttir, hujusque partern longe protractam mordicus tenent, quod a poro postico fieri posse, vix admiseris; cujus rei descriptionem speciem tertiarn enumerando dedi. Certe is tantum porus josticus dicendus est, quo foetura exfellitur. Si cel. vir porro-porum anticum in capitis fundo parvulum occurrere dicit, hoc in An1phistomate subclavato n. 14. saltem de postico valet in caudae fundo latescente.

In specicbus nostris tertia, sexta, septima, octava, nona, undecina et duodecina errorem circa pororum denoninationem-adesse posse, facile concedinus, ovorum exclusione observata brevi tollendum. In iisclein speciebus quod dorsun dixi, abdo'men erit.

\section{Amphistoma longicolle F. n. sp.}

Cel. Hübser specimina complura.misit in intestinis Ardeae albae, Bremserves aliqust in illis Lari ridibunti, unum in $L$. Atricilla, alterum in Arlea stellari reperta mecum communicave. runt, quatuor cum dimidia ad octo lineas longa. Caprit albun, exiguum, subcordatum, plerumque trilobum, lobis duobus posterioribus, tertio anteriore illis exccpto: saepe autem sulcus lolos postcriores dividens haud adest, tumque pars antica tantum a posteriore inajore 'distinguitur. Collum fuscescens, longissimum; ca- 
pite tenuius posteriora versus incrassatum, Corpus collo duplotriplove brevius, multo crassius vero, album, oblongum, inaequale, apice obtuso, Pori terminales, parvi, profundi, anticus inaequa. lis major, posticus orbicularis. Ova magna, elliptico-ovata, flavescentia parte posteriore (quam idcirso corpus dixi) continentur.

Ratio partium varia pro speciminum magnitudine; longitudo capitis tertiam ad duas tertias lineat partes, colli tres ad sex lineas, corporis duas ad tres lineas adaequat.

Obs. I. Specimina reliqua prorscs conveniunt, illud antem in Lari Atricillae intestinis ob. latum colore partis anterioris et posterioris albo, médiat brunneo convenit Haec autem ovis repleta erat. Caput oblongum lobis anterioribus oblongis, an integrum? Tum forsan species propria foret, neque lobulos oblongos obtusos laceratione oriri posse crediderim.

Obs. 2. Entozoa primum facile fallunt, quae pro novo genere Acanthocephalis affini haberi possent Longa enim sunt et gracilia et a filo longiusculo tunicae internae intestinorum pendent, quod microscopii ope examinatum nil nisi tuinicat villosat partem protractam et laceratam sistit. Conf. speciem tertiam.

\section{Amphistoma serpens $\mathbf{R}$. n. sp.}

Cel. Nitzscr pro sua in me amicitia specimina duo Holostomatis serpentis sibi dicti misit in intestinis Falconis Haliaëti Septembri a se reperta, alterum sex, alterum septem lineas longa.

Species praecedenti proxima, sed distincta, quam vir egregius bis copula junctam vidit. 
Caput subovatum, poro obliquo, sive subir: fero, magno, corpore collum crassitie parum superante, apice caudali truncato, poro medio eminente exıguo, in minore specimine suborbiculari, in altero lobato.

Majoris speciminis collum quam corpus multo longius, minoris autem corpus aeque longuin quam caput et collum simul sumta.

\section{Amphistoma macrocephalum $R$.}

Holostomum variabile Nrtzscr. Festucaria strigis. Froelich. Naturf. 29. p. 5I. n, 26. Féstucaria Oti. (sphalmate Otidis scriptum est) ib. p. 53. $\mathrm{n}, 27$.

In Strigis Oti tenuibus Junio mense co. piosissinum reperi, plerumque alhum, rarius bile tinctum, et interdım totum flayun, intestini parte, qua degeret, eodem colore imbuta.

Plurima specimina villos merdicus tenebant, ita ut plerumque stria rubra longitudinalis caput percurrere videretur. Capite anoto tunica intestini villosa singulari modo suclione mutata invenitur; pa:s ellim quam tenent elongata apice latiure terminatur, et simul vasa sanguinea continent, quo praeter villosam etiam cellulosam ore arreptam esse statueres, villi enim nudis oculis inspecti nunquam vasa sanguinem vehentia offer runt. Si autem porus, quo Amphistomata haec affiguntur, posticus foret, qui tantopere tunicas mutare posset, vix caperem. Nihil certi tamen inde efficere conabor.

Corporis plerumrue apice postico simpliciter perforato, initerdum tamen strictura quodammodo discreto utuntur. Ovula nullis in speciminibus 


\section{- 355}

reperi, illo itaque anni tempore jam deposita erunt.

Amphistoma, quod in intestino medio $\boldsymbol{F a l}$. conis apivori solitarium Majo reperi, apicem po. sticum pariter discretum obtulit.

5. Amphistoma isostomum R.

Amphistoma Tadornae. Entoz. II. I. p. 353. n. II,

Abildgaard Zool. Dan. Vol. IV. p. 32. Tab: 148 C. Fig. I. 2. Strigea candida, corpore inaequaliter bipartito,

Descriptio cel, viri brevissima et haec quidem: „Corpus bipartitum, pars inferior major ova. ta pellucida, dorso ovis opacis candidis foeto, ore infero sublaterali; pars superior triplo minor ovata, ore supero."

Iconibus consultis sequentia addam. "CapuE subconicum, sive oblongum versus basin incras. satum, strictura discretum a corpore versus apicem sensim attenuato. Pori integri et ejusdem magnitudinis videntur. 'Longitudo fere duas attingit lineas; corpus capite triplo longius, duplo crassius, antice linean dimidiam latum.

\section{Amphistoma gracile R. n. sp.}

Specimina tria in intestinis Mergi Mlerganseris reperta am. Breirser misit, lineam exceden. tia, tenuia.

Caput longum gracile, corpore haud cras. sius, apice lobatum, lobis variis, duobus tribusve oblongis. Corpus dorso convexo, abclomine con. cavo, utrinque attenuatum. Apex caldae discre tus, brevis, poro exiǵuo prominulo terminatus.

\section{Z 2}




\section{- $356-$}

\section{Amphistoma erraticum $\mathrm{R}$.}

I. Am. Brénser complura specinina in intestinis Colymbi arctici reperta misit, illis quae olim in Ciolymbo septentrionali (Ent. II. I. p. 344. n. 5.) offendi, paulo majora, plerumque'sesquilineam longa, dorso fuscescente. Porum posticum distincte non vidi, sed apex posticus subincisus est, incisura sive impressione exigua.

2. Idem Amicus specimina aliquot dedit in intestinis Scolopacis Ritsticolae reperta, lineam longa, spiritus vi fuscescentia,

Horum caput magnum discretum, entozoi contracti tertiam quartamve longitudinis parten sibi sumens, mux cyathum sistens, mox irregulare, magis, inaequaliter lobatum. Corpus subtus concavum, dorso convexo ovis farcto, antrorsum magis saepeque valde attenuatum; apice postico obtuso, poro parvo exciso.

Obs. Rusticolae specimina prinum ab illis Colymborum diversa habui, sed attentius collata eadem esse vidi, quo etiam pertinent, quae in Scolopace Gallinula olim offendi, et Ent. Ir. I. p. 342. obs, 4, ad Ansphistoma macrucephalum retuli.

8. Amphịstoma urnigerum R. n. sp.

Am. Bremser specimiua complura misit tam in Ranae esculentae recto, quam in illius plurimo. rum viscerum hycatidibus reperta.

Illa (intestini recti) candida, duas tresve li. neas longa, corpore tereti subaequali, in nonnullis passim constricto, apice caudali mox obtuso, mox truncato.

Porus anticus magnus pro capitis varia for a 


\section{$=357-$}

ma diversus. Hoc discretum, mox campaniforme cive urnam referens, mox infundibuliforme, margine obiter crenato, corpore multo crassius. Po. rus porticus exiguus, orbicularis, integer.

Specinina hydatides inhabitantia, quaeque y)assim ipse ex istis extraxi, lineam ad sesquilineam longa, a praecedentibus non differunt. Cor. pus hinc inde constrictum est, quod verme in parva hydatide convoluto facile explicari potest.

9. Amphistoma Cornu Nitzscir. n. sp.

I. Am. Nitzscr specimina quinque misit in intestinis Ardeae ctnereae Decembri mense a se reperta, Holostomum Cornu dicta, sesquili. nearia.

Caput discretum, corpore crassius, apice bílobum vel obiter pluries incisum, poro magno inaequali, quem tamen in mortuis haud satis distinguere possum. Corpus a capite tenuissimum incipit, sensim et tarde increscens, ut summam attigerit crassitiem versus caudam, quae iterum tenuior et recta poro exiguo integerrimo terminatur.

2. Am. Bremser aliquot specimina dedit in in. testinis Addeae Garzettae oblata, lineam cum dinidia plus minus longa; parte anteriore flavescente, reliqua fuscescente, capite passim subtrilobo, ctterum cum illis supra descriptis protinus congruenria.

Obs Non confundendum est cum Monosto. mate Cormis n. 14 parjter in Ardeis habitante, quondque Eut. II. I p. 346 n. 7. male inter Amphistomala, aut saltem inter illa, quae capite dis. creto utuntur, collocaveram: 


\section{$-358$}

\section{Amphistoma Sphacrula $\mathbf{R}$.}

Brenrekus specimina misit in intestinis Corvi Corncis reperta, cum illis quae olim eodem loco offenderam, ceterum convenientia, sed majora, ut plurima lineam attingant, et porus anticus in medio capitis multilobati in conspectum veniat, vel orbicularis, vel rimam transversam mentiens.

\section{Amphistoma pileatum R.}

Monostoma pileatum Entoz. II. I.' p. 338. n 15.

Bremserus specimina dedit in intestinis Ster. nae cantiacae reperta, sesquilineani longa, crassiuscula, cum speciminibus a me in Sterna $H i$ rundine repertis omnino eádem, sed ad Amplistoma amandanda, porus enim apicis caudalis licet exiguus utique adest.

\section{Amphistoma denticulatum R. n. sp.}

Specimina quatuor in intestinis Aicedinis Ispidae reperta al am. Bremsero accepi, linean ad sesquilineam longa, tenuia, in spiritu fu. scescentia.

Caput lanceolato-ovatum, interne poram sistens. Corpus teres, a capite tenuius incipiens, dein subaequale, apice postico rotundato, poro orbiculari, exiguo, terminali.

Ope lentis fortiter angentis corpus praesertim anteriora versus margine denticulatum conspicitur, num vero tale utrum crenatum sit, spe. ciminum recentiorum examen conficiet.

14. Ampinistoma subclaratum $\mathbf{R}$.

Quae in intestinis Ránae esculentae Berolini Octobri reperi specimina duo, per aliquot dies 


\section{- 359}

viva servavi et attente contemplatus sum, quo certior factus sum, partem attenuatam anteriorem, posterioram incrassatam esse. Apertura vero po-. sterior magna saepe margines expandit vel etiam revolvit, quo apex corporis posticus cum poro exi, uo orhiculari interdum tubulum brevem re. ferente ob oculos ponitur; Hic est, ex quo $\mathrm{Z}_{\mathbf{E}}$ percs foetus vivos egeri vidit.

Julii d. XXV. 1816. Bufonem vulgarem (cinereum) examinando, vesicamque urinariam entozoá fovere videndo, nova quilem exspectavi, sed illam aperiendo, duo hujus. Amphistomatis specimina reperi.

Confer Distoma clavigerum n. 52. quod olim cum Amph. subclavato miscui.

\section{Amphistoma truncatum R. n. sp.}

Am. Oтto, Professor Wratislaviensis, magnam horum entozoorum copiam in hepate Phocae repertam misit; ipse vero aeque copiosa in Phocae vitulinae junioris ventriculo et intéstinis temuibus (neque in hepate) Berolini Junio reperi, alba, excepta macula media antrorsum lutea, retrorsum fusca, lineam plus minus longa.

Depressiusculum; porus anticus exiguus, orbicularis; corpus a poro tenue incipit, mox late. scit et dein vel subaequale apicem posticum truncatum aut rotundatum attingit, vel ad hunc usque increscit Porus posticus magnus, margine lato subelevato.

Interdum in parte corporis media tubercú. lum exiguum, sed incerto loco. In parte poste. riore corpuscula aliquot orbicularia (ovaria vacua) satis inagna; ova immatura ante eadem, ma. 


\section{- $360-$}

joraque ovalia ad latera; neque ratura, neque cirrum vidi.

16. Amphistoma unguiculatum R. n. sp. Quatuor Salamandyas palustres (duas taeniatas sive mares, totidem femineas) Martio Bero. lini secando, entozoa non offendi; illarum autem intestina aquae committendo, in hac quinque vel sex reperi Amphistomata, lineam dimidian longitudine plus minus aequantia, depressa, angusta, alba,

Oblonga, marginibus mox integerrimis, mox corporis parte anteriore ope stricturae intercepta. Porus anticus terminalis, exiguus, capitis partem anticam mediam occupans, unguen fere referens. Posticus major videtur, apice caudali saepe emarginato.

Microscopio subjectum Amplistoma nebulosum, ovariis non distinctis, neque aliis partibus internis dignoscendis exhibetur.

17. Amphistoma conicum $\mathrm{r}$.

Cel. Trevtler specimen unicum a se $\mathbf{A u}$ gusto mense in Ovis domesticac ingluvie repertum dedit, quod ab Amphistomate bovino et cervino nullo modo distinguere valeo, duas cum dimidia lineas longum, postice ultra dimidiam lineae partem diametro transverso adaequans.

Entozoologiam edendo porum posticum pro antico habui, cum illius ope intestinis infixum viderem.

18. Amphistoma subtriquetrum R. n. sp.

Specimina in Míuseo Anatornico Berolinensi reperi, quibus additum erat, in Castoris Fibri tenui. 
bus reperta fuisse. Deinceps vero ab am. Brevsero et cel. Вojano, professore Wilnensi accepi, ipseque Mrajo mense in coeco et colo Castoris reperi, albida, duas ad tres cum dimidia lineas longa.

Corpus a poro antico terminali exiguo et orbiculari posteriora versus increscit, obtuse terminatum, poro postico haud terminali, sed quartam - lincat partem ab apice caudali distans, inferus, amplus, profundus, ostii margine acutiusculo in. tus verso. Dorsum convexum, passin in speciminibus diutius servatis sul,carinatam visum; abdomen planum, unde nomen triviale desumsi. Ova flavescentia praesertim ad latera accumulata sunt.

Cel. Basanus in litteris monuit, haud Amphistoma єsse, sed Distoma (amphistomoides sibi dicium), Bresseri's vero Amphistomatibus ser. vandum contendit. Cumqque parus̀ posticus licet inferus, apice postico proximus sit, inter Amphistomata r liuquo; sunt enim pariter Distomata plurima quorum porus anterior baud terminalis sed inferus cit, nihilo tamen minus anticus dica. tur. Hujuis tamen Entozoi ope Distomata et Am. phistamata conjungi, nullus negabit. Confer Di. stoma microsoma n. 80.

Quae Bojanes, vir celeherrimus, de hujus speciei anatome in litteris retulit, omnia in speciminibus recentissimis comprobata vidi. A poro scilicet antico utrinque vas nutritium decurrit; media parte uriucque sexus genitalia occurrunt; porus pocricus musculosus coecus. Rervorum vestrgia nulla vidi, 


\section{$-362$}

\section{Amphistoma Falconis peregrini.}

In Catalogo Entozoorum Musei Viennensis Amphistoma Falconis peregrmi ad Amphistoma macrocephatum n. 3. revocatur; quod ipse tamen in illius intestilis ienuihus Novembri mense Be. rolini copiose reperi, distinctum visum est.

sperimina mea linean plus minus longa, albida, partem anticam haud subito discretam (uti macrocephalo comperit) sedlevi sinu sensim ex. orto obiter distinchar et ovalem offerunt, ostio exiguo; parte caudali depressa, margine inaequali, poro majore obscuro.

\section{Genus X.V. Distoma.}

Genus hoc omnia reliqua v. c. Echinorhynchum, Ascaridem, quin ipsam 'Taeniam'specierum' numero superat, harum autem characteres plerumque faciles, saepeque optimos indicat, ut numerus ille magnus nullum excitet tatdium.

Pauca forsan genere separari possent, v. c. Distomata excavatum, n. 82, spathaceum, n. 83. spatulatum, n. 84. alatum, n. 96. a. Nitzschio ad Holostomata refereuda, sed D. excisum 11. 45. inter haec et reliqua jam transitum efficit, variis. que collum passim, aut motu vulgari planum redditur.

Echinata, quibus caput reniforme ant saltem discretun est, aegrius tamen, sejungi possent, religua enim his accedunt; neque inermia ab armatis gentratim sumtis avelli possunt, cum colli corporisclue aculei saepe parvi et cognitu difficiles occirra:ut.

Subdivisiones interdum fallere possunt, quod 


\section{$-363-$}

enim recens examịnatum teres visum sit Distoma, spiritu vini servatum, forsan depressum exhilse. tur; ipse tamen quoad fieri potuerit, semper statum recentem prae oculis habui. Pororum ratio utplurinum species affines colligit, interdum ramen species valde affines pororum rationem diversam sistunt. Distomata piscina prae reliquis poris amplis suglobosis dignoscumtur, sunt tamen illa inter etiam minoribus t planioribus instructa; reliquarum classium Distomata minus differunt. Habitaculum simile v, c. vesica urinaria saepe diversarum classium species aftines reddit. Successores olim hisce de rebus egregia proferent:

\section{Distoma hepaticum Arilng.}

Species mammalibus plurimis communis, multoque pluribus, quam in Entozoologia enumerare licuit.

Specimen in IIalmaturi gigantei (Kanguri) hepate repertum in Museo Viennensi vidi, ab ovino neutiquam discrepans; ibidem adest in $C_{u}$ nicnli hepate lectum. In Sciuri europaei hepate, hydatide inclusum cel. Targionr Tozzettr ofiendit, ovino simillimum (Brera Suppl. p. 95.). In Camelo Bactriano et Antilope Kevella, Cat. Ent. Vienn. manuscriptus, In Autilopes Corinnae oeșophago reperta ill. Sormmerring mihi dedit sperimina tria, quorum duo minora, "tertium majus, vulgaris formae.

Phoca barbata propriam Distomatis specien hepate fovet, D. tenuicolle, n. 5. In Erinacei hylatide hepatis peculiaris quoque species, $n$. pusillum, n. 50. occurrit, 11 teriore tamen examine diguum. 'Quadrumanae, Ferae, Cetacea hactenus 
hepatis Distomata nulla protulerunt; de Cetaceis nihil certi proferri potest, speciminibus eorundem nimis pancis examinatis; Feris utique deesse videntur; in Quadrumanis forsan, velut in homine, rarius occurrunt.

Synonymis addi possunt sequentia:

REDr Esperienze intorno agl' insetti. p. ${ }^{\top} \mathrm{I} 4 \mathrm{~s}$. cum ic. Verme del fegato de' montoni o ca. strati,

Sanf. Gottu. Garelin Reise durch Rufsland Th. 3. p. 49r. In hepate ovis orientalis.

Froelicir Naturf. 29. p. 55. n. 28. Fasciola hcpatica. Specimina polkicein longa, novem lineas lata in Cervi hepate Martio reperit et cun bovinis et ovinis convenire refert.

Quaedam alia in Mantissae sectione tertia, bibliographica, dicentur.

\section{Distoma dendriticum R. n. sp.}

Maximam speciminum copiam cel, SpedaIIERT in intestinis Xiphiae Gladii reperit et benevole mecum communicavit; ipse duobus in Gladiis ne unicum quidem offendi.

Entozoz plana, alba, vasis fuscescentibus, rarius oblonga, plerumque ovato lanceolata, aut lanceolato-ovata, unam cum, dimiclia, vel duas, rarius tres lineas longa, latissima parte fere lineam attingentia.

Pori semighobosi, mediocres, ventralis major. Pars antica angustissima, sensim versus Corpus ampliata, vix quartan totius longitudinis partem sibi sumens. Nargines corporis integri; "pex posticus aut rotundatus, aut attenuatus et plus minus actutus.

Cirrus in nulio specimine exsertus est, sed 
pone porum anteriorem macula rotunda opaca illius receptaculum indicat, uti maculae quaedam opacae (pariter tamen alhae) majores pone porum ventralem earlem directione obviae ovaria sistunt. Vasa triplicia sunt: primum magnum ra. mosissimun fuscum, fere Ju:ıgermanniam tamarisciformen referens, mediam maximamque corporis partem sibi tindicat; ad latera ınedii corporis porro vasa minora fusca, pariter ramosissima dantur; inter illa vero a poro antico usque ad finem caudalem utrinque vas simplex album cursu undulato exhibetur. A pori antici media parte ad cirri receptaculum vas brevisimum rectum et albun decurrit, quod maximi fusci forsan initiam erit, unde illa tria tantum numeravi. Confer speciem insequentem.

5. Distoma tenuicolle R. n. sp.

Magnam copian in hepate Plocue barbatce Lipsiae 178s Aprili mense a se repertam cel. Tréuter bono dedit.

Entozoa oblonga, plana, alba, medio fuscescentia, tres cum dimidia ad quatuor cum dimidia lineas longa, ad summum dimidiam lata. it

Pori semiglobosi mediocres, ventralis paulo major. Pars inter poros (collum) lineam longa, angusia, sensim versus porum ventralem la. tescens; corporis margines rectiusculi; callda obtusiuscula, apice passim papillari vel perforato viso.

A poro antico utrinque linea diaphana ad pori ventralis latera decurrit; pone hunc vasa majora fusca racemosa, ovis ellipticis exiguis re. ferı; in posteriore lineae medianae parté aliquot maculae orbiculares sive ovaria vacua; ad latera 


\section{- 366}

vascula fusca, ramosissima, ramis brevissimis, qui oviductus ovulorum immaturorum sistunt. Inter. haec lateralia ed medium utrinque vas illud pellucidum ad caudae apicem utrinque continųatur. In unico specirnine cirrum longum tenuem filiformen videre inili visus sum.

\section{Distoma carnosum R. n. sp.}

In aqua cui Spari Denticis majoris intestina Arimini Majo immiceram septem reperi Di. stonata mortua, alteroque die vivum illius in veutriculo, villosae affixum. In minore Dentice Neapoli Julio specimina duo exhibita suut.

Sesquilineam ad duas lineas longa, parte postica fere lineam-lata, recentia ruhella, postmo. dum alba parte posteriore sordide àlba aut flavescente, quem colorem etian vivum illud ob. tulit.

Pori globosi, anticus inferus, exiguus; ventralis quadruplo major, maxime prominens, margine tumido. Collum vivi conicum, mortuorum retractum, fere nullum. Corpus ovatum, dorso convexo, ventre plano, ovulis globosis turgidum. Cirrum exsertum non vidi.

Species distinctissima, quae cum reliquarum nulla confundi potest.

\section{Distoma hians $\mathrm{R}$.}

In Oesophago Ardeae nigrae Gryphiae Septembri quadraginta reperi specimina, utroque poro inter plicas oesophageas affixa, sanguinea, in aqua demum alba, quorum quaedam serlecim per horas vixerunt. Plerumque convoluta erant. Curitracta duas ad quatuor, extensa, collo praesertim attenuato, ultra sex lineas longa. 


\section{$-\quad 367$}

\section{Distoma lucipetum R. n. sp.}

Am. Brevserms specimina misit aliquot sub alterius oculi membrana nictitante Lari slanci Aprili, aliaque sub utriusque oculi memt ana dicta Lari fusci Majo reperia. Leco certe inserlito, quo lucem petere possint, entuzois lere semper denegatam.

Duas ad tres cum dimilia lineas longa, ul. tra dimidiam lata, depressa, alida ribore pone porum ventralem aduixto.

Pori orbiculares, anticur inferma, ventralis remotus, duplo major, apertura illius semper, hujus plerumque orbiculari, interdum longiori quam lata, subelliptica. Collum, sive pars inter poros sita, reliçuo corpore angustius, oblongum, nar. gine convexo. Corpus latizs, circa'porun ven. tralem crassissimuin, versus posteriora decrescens, apice cauciali obtuso:

A poro antico utrinque exoritur vas temue flavescens, totam vermis longitudinem emetiens. Pone illum corpusculum datur exiguum op̉acum, 2 quo itidem utrinque vas enascitur, inter illa decurrens.- Mediam inter poros partem cirrus te, net rigidulus, dimidian circiter lineam longus, plerumque rectus, rarius apice reflexus. Pone porum ventralem vasa plurima racemosa (osiductus) lineis passim horizontalibus, aliisque decus. santibus inter vasa longitudinalia conspiciuntur. 'Tandem aliquot corpora oblonga, magna, linean medianam tenentia, ovaria evacuata (aut immatu. ris repleta) sequuntur.

10. Distoma pulchellum R. n. sp.

Aliquoties in intestinis Labri cynaedi Neapoli Junio reperi solitarium, vivum, Semel sex ejusmodi 


\section{$-368$}

Labros me praesente prope insulam Nisidan captos, Neapoli adhuc vividos, brevi post mortuos, hora elapsa secui et octo reperi Distomata, omnia mortta, curn piscina alioquin vitae satis tenacia sint.

Entozoa plerumque lineam dimidiam, raro integram longa, alba, macula media flava Depressa, elliptica, antrorsum plermmque magis, attenuata; poris globosis inferis, remotis, ventrali duplo majore.

$V$ as dorsale flavum, spirale. Ovaria utrinque valde racemosa, adspectu lepidissimo.

\section{Distoma holostomum R. n. sp.}

Bremserus specimen misit in intestinis Ralli equatici repertum, elegantissimum, sesquilinean longun, dimicliam latum.

Pori haximi, anticus terminalis orbicularis, ventralis major apertura transversim ellipțica. Corpus oblongum, antrorsum latius, truncatum, retrorsum angustius, apice postico abtusiusculo; dorso convexiusculo, ventre plano. Vasa late. ralia obscura; racemus medius longitudinalis.

Species distinctissima.

\section{Distoma transversale $\mathrm{h}$.}

In ventriculo Cobitidis fossilis Berolini Aprili mense specinen reperi lineam excedens, cum descriptione Ent. Il. I. p. 36r.'n. 7. a me datá conveniens, ut pauca tantum addam.

Quibusdam entozoi motibus pori anterioris minoris margo dilatatus in conspectum venit, aliis tollebatur. Cirrum non vidi Pars postica, ad latus ipsius apicis emarginata fuit, 


\section{$-369$}

14. Distoma tumidulum R. n. $\mathrm{sp}$.

Bremserus specimina quatucr misit in intestinis Syngnathi Hiprocampi (in quo saepius examinato nunquam reperi) obvia, lineam plus minus longa.

Depresso-plana, linearia, utrinque obtusa Porus anticus terminalis, globosus, minor; voritralis mediam fere totius longitudinis partem occupantis, valdeque protuberantis (unde nomen desumsi) apertura transversa. Ante hunc tuber. culum exiguum, cirri certe receptaculum. Ovula ad partis posterioris latera digesta sunt.

Obs. A. Distomate labiato n. 78. Syngnathi pelagici diversissimum videtur; quandam fovet similitudinem D, gubbosi n, 7I., sed hoc teres est et poros alienos offert.

16. Distoma polymorphum $\mathbf{R}_{\text {。 }}$

Num huc pertinet Distoma anguillae, corpore clavato, Amirdgard Zuol. Dani Vol, IV. p. 26. Tab, 142. Fig. 7-10,

Cel. vir ultra trecenta specimina in Anguil. lae Helgolandiat abdiomine reperit, quorum descriptionem dat insequentem. "Corpus clavatum, sordide album, collo extensili, apice incrassato, ore apicis laterali. Pars infcrior corporis lata crassa, poro ventrali tumido marginato. Intestinum contortum viride in parte posteriore cora poris."

Variis notis a D. polymorpho Ent. II $\mathbf{r}$ p. 363. n. 9. quod ipse in intestinis Anguillae copiosum reperi, differt, primum loco, cun abdo. minale sit; dein magnitudine, nam figura septima Distoma magnitudine naturali referre dicta qua- 


\section{- $370-$}

tuor cum dimidia lineas superat, dum specimina mihi visa tertiam dimidiamque lineae parten adhequant; in meis denique vas viride non ob. servavi.

Forsan itaque mea pusilla, haec adulta fuerunt, aut propriam speciem sistunt.

18. Distoma caudiporum R. n. sp.

In intestino Zenis Fabri Arimini Aprili unicum reperi Distoma sesquilineam longum, valde extensile, album parte media fulva, supra convexum, subtus planum.

Porıs anticur inferus, globosus, liians apertura orbiculari mediocri; clausus rimam longitudinalem exhibens; ventralis remotus, duplo major, globosus, aperturae orbicularis margine tumido. Collum vase lacteo antice transverso, tum ad latera utrinque posteriora versus decurrènte instructum, cirro non visibili. Corpus subellipticum collo duplo latins, cauda retracta truncatum; ovariis cincinnatis refertis ovis globosis; microscopio subjectum vitreum, areolatum visum. Cauda retractilis corpore multo tenuior, extensi. lis, crenata, longa, apice excavato porum mentiente, qùo fit ut protracta et mota alienum habitum induat.

Obs Ad hanc sectionem pertinere visum est, vereor,tamen ne Distomati appendiculato $n_{\text {. }}$ 85, nimis affine sit.

\section{Distoma cygnoides ZED.}

Plerumque candidum est, vidi etiam parte anteriore alha, posteriore flava.

In vesica urinaria Ranae esculentae plurimis visam; in illa Zufonis ignei Gaede, Naturaescru. 
tator indefessus, Berolini Junii d. r6. duo, et d. 26. octo specimina reperit exigua, lineam vix superantia, candida, pellucida, ovis tamen ellipticis foeta, quae passim, dum illa contemplatus sum, protrudebantur.

\section{Distoma cymhiforme R. n. sp.}

In Testudinis Mydae vesica urinaria Arimini Majo quatuor reperi Distomata duas cum dimidia ad tres cum dimidia lineas longa, antica parte dimidiam, postica integram lata, dorso convexo; ventre concavo, vividissima.

Pori globosi, remoti, anticus inferus, veno tralis duplo major. Collum vaide elongandum, oblongum; angustum; pone porum ventralem crena utrinque conspicitur, quo illud discernitur a corpore ovato, postice obtuso.

Collum cum poris candidum; pellucidım. Utrinque pone porum ventralem et crenam dictam macula stellata subrotunda, lactea, margini corporis vicina, ganglia illa sisténs lateralia quae cel. Отто in Distomate hepatico invenisse vult, a' gangliis tamen mihi aliena visa. In medio cor. pore macula violacea, margine albido cincta (vas spirale); pone quam ovaria viridescentia con. gesta.

\section{1: Distoma folium Otfers n. sp.}

Olfers de vegetativis et animatis. p. 45. Fig. 15. D. folium. Cél. vir complura specimina in vesica urinaria Erocis Lacii Julio et Augusto Berolini reperit et mecum communicavit, tertiam ad dimidiam linexe partem longa, alba, depressa.

Pori orbiculares, anticur terminalis, minor; ventralis vivo passim protruditur. Collum rectum 


\section{T

tenue; corpus subrotundum seu ovatum, dimiciam vermis longitudinem latitudine attingens; quo commotus speciem $D$, Lagenulam in adversariis vocaveram, alio tamen nomine ab inventore po. sito, illud suppressi. Figura cl. viri Distoma valde elongatum sistit, quale certe vivum sistitur. Corpus undique ovis farctum.

Obs. I. Species Distomati seriali n. 22, proxima, quae pariter in organis uropoëticis occurrere videtur, forsan in ureteribus Salmonis alpini, cujus in sanguine dorsali (renes significante) oc. currere $O$. Fabricinls auctor est.

Obs, 2. Cel. ab Olfers pluries specimina duo per inferiores corporis partes, potissimum circa porum ventralrm cohaerere vidit, quorum alterum moribundi instar segnes tantum motus edidit, alterum vero alacre, huc et illuc collum elongatum protendens, alterum secum-traxit, ita forsan coitum absolvens,

\section{Distoma divergens $\mathbf{F}$,}

In intestinis Blennii Gattorugine Arimini Aprili duo reperi specimina mortua, duas tertias lineae partes longa, alba, macula fulva, depressiuscula.

Pori globosi, anticus inferus, ventralis du. plo major. Collum antrorsum attenuatum, in cujus hasi cirrus incurvus. Corpus latius, obtusim. Ova fusca ad latera partis posterioris.

Ejusdem Distomatis forsan adultioris specimina undecin in intestinis Blennii tentaculati Arimini Majo reperi, flavescentia puncto postico fulvo, dimidiam ad duas tertias lineae partes longa. In his ova tam ad latera partis anterioris, quam in tota parte postica. 


\section{$-375-$}

Aliquot diebus elapsis ibidem tria specimina in intestinis Blennii cornuti offendi, prorsus, convenientia. Vasa in dorso valde crassa, fusca, procul dubio ovaria sistunt.

\section{Distoma fasciatum R. n. sp.}

In intestino, Labri Tincae Neapoli Junio. -quinque specimina inveni viva, unam ad unam cum duabus tertiis lineae partibus longa, tertiam lata, depressiuscula, albida, macula media lutea.

Pori globosi, apertura orbiculari, anticus terminalis, ventralis remotus duplo major. Cor: pus subellipticum obtusum, colli parte antica plerumque valde attenuata.

Vas dorsale flavum; ovaria valde ramosa lateralia per totum corpus utrinque decurrentia; vas. tertiun antice transversum utrinque inter ovaria posteriora versús ductum; praeterea autem (a. quo nomen desumsi) vas quartum inter dicta medium obtusum et satis amplum, pone porum ventralem exortum, dein rectum et lineare ad apicen posticum fertur, corpuscula sacciformia Distomatibus vulgaria transiens.

Quae in intestinis Labri melopis Neapoli Julio reperi Distomata septem, viva, duas tertias linéa partes ad sesquilineam longa, oblongoovata, plana, hava, macula fulva, eo solum a praecedentibus recedebant, quod corpus repandum sive passin constrictum offerrent.

Quae in intestinis aliquot Percarnm marinarum mascularum eodem mense Neapoli reperi specimina tria mortua (piscibus vix ante horam apud me mortuis), albiela maculá exigua lutea protinus cum illis in Labro Tinca repertis con. 


\section{- 374 -}

veniunt. - Confer speciem n. 26, et 27, affines, ni fallor tamen diversas.

\section{Distoma sinuatum R. n. sp.}

Multis Ophidiis imiberbibus entozoorum caussa frusta examinatis, tandem tribus iterum Julio Neapoli dissectis duo repcri Disromata, lintam cum huius tertia parte longa, quartam circiter lata, albida, macula media rufescente satis magna.

Pori suborbiculares, medio riter distantes, ventralis major. Collum et corpus depressum, illud conicum, hoc posteriora versus auctum, apice rotundato, totins Distomatis marginilus sinuatis, integerrimis. Ova lateralia fusca congesta. Vas spirale fulvum. Confer speciem praecedentem et insequentem.

\section{Distoma fulvum R. n. $: \mathrm{sp}$}

In intestinis Gadi Molvae Neapoli Junio unicum reperi specimen, lineam excedens, depres. sum, fulvum.

Pari semiglobosi : marginati, ventralis distans major. Inter poros tuberculum parvum. Corpus crassius sive latius, ovale, collo tenuiore. Vas utrinque longitudinale album, fuscumque latius a poro ventrali, ad apicem caudae. Ova nulla aderant, sed areae mediae vacuae.

Subinde margo denticulatus visus est, re tamen attentius spectata corpus undique laeve sistitur. Singulari modo cauda antrorsum refletitur, quo porum ventralem tegat, retractilis tamen non est.

Quod Bremserus misit specimen in intestinis Gadi mediterranei Aprili repertum, lineam cum quarta hujus parte longum, fasciam quidem 


\section{- 375}

mediam haud exhibuit, ceterum, autem prorsus convenit, neque illam in recentioribus desiderari crediderim. Confer species n. 25. et 26.

28. Uistoma oxy ephalum R. n. sp.

Froeligh Naturf, 29: St. S. 56. n. 29. Tab.2. Fig. 8, 9, Fasciola appendiculata (Boschadis).

Nrtzsch in litteris: Disioma inerme.

Cel. Nitzsch specimina undecin misit Julio mense in intestinis:Anatis Boschadis ferae re: perta, tres quatuorve lineas longa; Breirserus vero duo in illis Anatis clypeatae lecta, tres lineas longa, tertiam lineae partem' latitudine ex. cedentia.

Porus anticus apicis anterioris acuti valde exiguus, inferus, orbicularis. Collum ovale latiusculum breve, cirro brevi rectiúsculo subexstante. Porus ventralis valde magnus et tumidus, apertura orbiculari. Corpus planum lineare, apice postico obtusiusculo. Ovorum racemi longi' utrinque latera tenent; media corporis pars pone porum ventralem vasculosa, versus posteriora pellucida. Plura in speciminibus spiritu vini servatis extricare mihi non licuit.

Obs. I. Species a Froelichio primum recognita, a Zenero ad Distoma echinatum relata fuit, quem illius speciminibus non visis Ent. II. I. p. 419. obs. I. secutus sum. Horum autem examine instituto, ab echinato n. 106. omnibus numeris recedere, bene vidi.

Obs. 2. Froelichus 1. c. Corpus lanceolatum, subtus planum, supra subconvexum, ad margines subcrenatum, rubellum. Porns anticus mininus, margine tumidulo. Pone illum tuberculum exiguum flavum, ex quo cirrus brevis, ob. tusus, serpentinus prodit. Portıs ventralis magnus 
prominens tumidus. Ab eodem canalis retrorsum ducitur. Ovula ad latera congésta.- Apex posticus obtusus, stbpellucidus, ostio indistincto instructus, ex quo caudula brevis oblonga, plana, in vaginam (ope iustrunenti pressorii visibilem) retractilis exseritur.

Appendicem istam, a quo cl, vir nomen petiit, in nullo meorum speciminum video, neque tamen negarem; alia autem specie (n. 85.) dudum ita nominata, nomen mutandum erat.

Obr. 3. Num etiam in Colymbo cristato oc. currit? Conf. n. r1 3 .

29. Distoma complanatum R. n. sp.

Am. Rosenthal, Professor Berolingensis, in Ardeae cintereae oesophago Aprili mense specimina quatuor reperit, recenter mecun communicata.

Entozoa unam cum dimidia ad duas cum dimidia lineas longa, vix dimidiam lata, alba punctis nigris varia.

Corpus oblongum, tenue, antrorsum parum, attenuatum, marginibus obtusiusculis, postice obtusum. Portes anticus terminalis ad inferiora paululum vergens, margine tumido, apertura orbiculari, magna. Porus ventralis lineae quartam partem ab illo distans, major, margine tumido, apertura nox triangulari, mox oblonga. Ovaria utrinque decurrunt, vase fusco permeante, anteapicem corporis posticum coëunte. Cirrum non vidi

Obs. Species affinis tam D. hianti n. 7., quam D.heterostomati n. 50 ; ab hoc tamen cor. poris forma et pororum ratione, ab illo corpore tenui, albo, poris aliter comparatis recedit.

31. Distoma albicolle R, n. sp.

Am. Bremser specimina misit sex in hepate 


\section{- 377}

et cystide fellea: Falconis pennati reperta, duas cum dimidia ad tres lineas longa, latissina parto anteriore alba, reliqua fuscescente.

Apex anticus tenuis, collum breve subit la. tescens, transiens in corporis partem anticam la. tissimam, a qua reliqua sensim attenuatur, termi. nata apice caudali obtusiusculo. Pori semiglo. bosi, anticus terminalis, ventralis duplo major, haud longe distans, margine tumidulo. Cirrum non vidi.

Obs. Species haec D. macrouro n. 30. (Ent; II. I. p. 372.' n. 15. D. longicauda) in Cornicis hepate et vesicula fellea reperiendo proxima; poris tamen aliter constitutis utrumque conjungere non ausus sum.

\section{Distoma Naja R.}

Ent. II. I. p. 434. n, 7 r. Distomã Colubri Natricis pulmonale.

Abildganrd Zool, Dan. Vol. IV .p. 34. Talb, 15I. Fig. A. 1. 2. Fasciola longicollis.

In pulmone Colubri Natricis valde magni, diu ab optimo Hrm, Medico celeberrimo, servati, quinque reperi specimina, quinque ad no. vem lineas longa, fuscoalboque variegata, depressa.

Pars antica ovalis, utrinque obtusa, lineam longa, dimidiam lata, poros continens, subito transit in corporis partem anteriorem angustissimam, quatuor lineas longam; haec qua maxime angusta est, iterum increscit, ut pars posterior (etiam quatuor lineas longa) tandem aeque lata fiat ac collum, et obtusa terninetur.

Pori globosi, magni, approximati, ventralis duplo major. Pone porum anticum pars exigua 
șubglobosa, ejusdem quasi appendix, 'a qua vasculi exigua oriuntur, visui sexper non oblata, uirinque circa porum ventralem posteriora versus decurrentia, tandem hinc inde pellucentia, procul dubio mitrientia. Ante porum ventralem cirri apex semel exsertus, acutiusculus. In parte corporis teniui vas dorsale șubspirale album ex. hibetur supra porun, ventraleț antrorsum: ductum, in cirrum abiens; positriora versus idem vas fuscescit, ultimam corporis partem percurrit et hujus maximam partem sibi vindicat, certe oviductus. Ovula oblongo-ovalia, matura, sul,fusca, reliqua alba. I'trinque prope oviductum medium vascula materiam grumosam ferentia, an seminifera? Unirum receptaculum (ovarium) orhiculare in corporis latioris facii parte media,

Obs. Amildgand in pulmone Colubri Na. tricis sex menses absque nutrimento degentis tandemque mortui duo reperit specimina pollicaria, postice lineam lata. Forma externa figuris obla. tis hene exprimitur, descriptio autem et icones vasa etc. sistentes pessimae sunt. Partem pone porum ventralem sitan collum vocat; Distoma, quiod ubivis depressum est, cylindricum refert, et quae bujus farinae sunt reliqua.

\section{Disıoma variegatum R. n. sp.}

Am. Bremser multa specimina misit in $R a$ nae esculentae pulmonibus reperta, ubi ipse quoque Berolin: Octobri semel legi.

Entozoa duo ad septem, plerumque quatuor aut quinque lineas longa, majorum pars anterior dimidiam, posterior integram lineam lata, depressa, marginibus obtusis.

Pori orbiculares remoti, anticus major, ven- 
tralis ostio exiguo. Collum dimidiam fere totius veruis longitudinem sibi sumens, duplo angustius, quam corpus apice obtuso terminatum. Cir. rum exsertum non vidi, Ovula exigua rotundatoelliptica.

Color valde variegatus; ductibus oviferis fuscescentibus, medio spirali nigrescente; vasis dorsalibus tenuibus nigris maculisque corporis albis subito nigrescentibus. Motus segnis.

Obs. Distoma cylindraceum n. 66. quod ipse in Ranae temporariae tantum. pulmonibus reperi, a Zedero et Braunio, 'viris cel. etiam in R. esculentae pulmonibus occurrere dicitur, cum huic autem proprium sit Distoma pulmonale, illos aut Rianas aut Distomata confudisse, fere su. spicarer,

\section{Distoma capitellatum R.}

Tres Uranoscopos scabros Arimini Aprili et Majo, duos Junio Neapoli secui et in cmnium vesica fellea aliquot reperi Distomata, depressa, sesquilinecm ad duas lineas longa, parte latissima tertiam lineae partem attingentia; aut alha macula media viridi vaseque spirali brunneo variegata, aut poris et cauda exceptis tota viridia.

Pori globosi, anticus feré duplo major, discretus, caput referens; ventralis remotus, ut spatium intermedium teriam totius longitudinis partem sibi vindicet. Corpus a poro antico sensim latescit, ut circa porum ventralem làtissimum sit, tum sensim, magis tamen, iterum decrescit, parte ultima, sive cauda subito tenuiore facta, lineari, apice obtuso, Cirrum non vidi.

Vas spirale brunneum pori ventralis parti posticae adhaeret, multos gyros efficiens, dein- 
ceps in latere sinistro decurrens, caudam non atingens.

O3s. Uranoscopus unicus hactenus piscis est, cujus in vesica fellea Entozoa reperta sint; nung̨uam pisces secando illam inapertam reliqui, sed.hospites non inveni; Uranoscopi vesica e contra semper tales obtulit; sed non praetereun. dum est, ejus bilem quoque a vulgari piscinae labilu recedere, semper turbidam occurrere, semper sedimentum et membranulas spurias contine. xe; cystidem amplam esse, magnoq̨ue ductu ventriculuin petere, quae itaque majus forsan officium subit. Sed Uxanoscopus generatim valde verminosus est, et locis quirlem alienis etiam Filarian globicipitem n, 19. fovet; Ascaridem n. 11 . practerea peritoneo et Distoma fallax n. II 5. ventriculo alit,

Confer D, crystallinum n. 36 .

36. Distoma crystallinum $\mathrm{R} . \mathrm{n}, \mathrm{sp}$.

Am. Garde, nunc Professor Leodiensis, in Ranae temporariae vesicula fellea (cujus bilis flavescens, solitoque pellucidior fuit) Berolini Maji die VIII. solitarium reperit ultra duas tertias lineae partes longum, quartam latum, quod pro sua in me benevolentia recens attulit.?

Distoma depressiusculum, album, pellucidum (unde nomen) macula pone porum ventralcm rufa; vivi forma mutabilis, mortui subellip. tica, aut ellipticoovata, parte antica exigua con. tracta, apice postico obtuso.

Pori globosi, remoti, anticus major. Ovária haud bene distincta.

Idem autem Amicus Maji die XXX. sexaginta Ranis esculentis examinatis in unicae vesi- 
cula fellea aut potius ductu cystico quinque hujusmodi Distomata offendit, quorum ova maturiora colorem eatenus mutaverant, ut corpus album lituris flavis et rufescentibus pictum esset.

Obs. I. Cel. Gaede in Rianae temporariae (cujus vesiculae Distoma inerat supra descriptum) hepate hydatides aliquot sphaericas reperit, illius margini acuto partim immersas, partim prominulas, arenulam referentes, linean dimidiam circiter diametro aequantes, quas hepati adluuc inhaerentes mecum communicavit.

Hydatidibus singulis Distoma inerat vivum, maxime mutabile; illis apertis tunica sive vesica externa detracta fuit, quo facto animalculum tu, nica interna inclusum multo melius visui se pfaebuit. Interna tunica, licet verinem liberum contineret, detrahi non potuit, ut materiam internam fere pro albumine haberem, nisi forsan ob exiguitatem tota capi non potuit tunica interna, aut plurimis constat stratis; plurimis/enim moleculis cultelli acutissimi ope ablatis, semper aliquid restabät.

Distoma polymorphum, vividum, agilissimum, crystallinum, subovatum, poris subglobosis, antico majore, quod pro illius supra de. scripti prole haberem. Hora quinta matutina repertum, hora undecima et dein iterum sexta vespertina a me observatum, motus edere continuavit.

Obs. 2. Tanae esculentac, cujus Distomata supra notavi, hydatides pariter aderant et quidem mesenterii, plurimae, arenulae magnitudine, sin. gulae idem Distoma parvulum foventes vividisa simum.

Obs. 3. Junio insequente optimus GAEDE 
hepar et pulmones Bufonis ignei, nec non mesenterium et peritoneum hepatis Bufon's viridis iisdem omnino hydatidibus Distoma includentibus obsita dedit.

Obs. 4. Ipse Berolini Augusto mense Viperae Beri superfiriem cordis et praeserim hujus apicem ejusmodi hydatidibus globosis, quartam circiter lineae partem diametro' aequantibus ob. sitos et singulis idem Distoma contineri observavi."

Decembri mense iterum alterius Viperae Beri totam cordis superficiem talibus hydatidibus onustam vidi, sed aliis negotiis avocatus easclem non examinavi. Me autem in utriusque Viperae reliquis partibus nutlas tales hydatides reperire potuisse notatu dignum est.

\section{Distoma maculosum $\grave{\mathrm{R}}$.}

Quod Ent. II. I. p. 374. n. 17. descripsi Distoma Hirundinum, postmodum Gryphiae Septembri mense in intestinis Caprimulgi europaei offendi, quod comparationis gratia describan.

Depressiusculum, lineam vix superans; corpus oblongum continuum, sive collo non discreto, margine undique repando, obtusiusculo; apice utroque obtuso, postico subtruncato et ob margines repandos subdiscreto. Pori globosi; approximati, anticus inferus, major, apertura mox orbiculari, mox subtriquetra visa, illa 'ventralis orbiculari, utraque exigua, hujus tamen minore.

Corpus album, speculis duobus mediis (ovariis evacuatis) ovulis aliquot ellipticis minimis, immaturis, sparsis; margines ovulis aggregatis obscuriores; vas medium spirale fulvum a poro 


\section{- 383 -}

medio ad apicem posticum sensim gracilescens protenditur.

Animalculum per quatuordecim horas aquae commissum tandem examinavi, quo color alienus explicatur.

\section{Distoma Linguatula R. n, sp.}

Am. a (novae Brasiliae speciei, conf. obs.) parte irtestiri anteriore Augusto mense reperta nisit, lineam ad sesquilineam longa, tertiam lincae partem lata, depressa,

Entozoon ellipticum, utplurimum ntrinque obtusum, interdum poro antico paululum exstante, quo pars anterior tenuior fiat, alias antrorsum parumper, sed inconstanti modo larius. Pori orbiculares, anticus major. Ante porum ventralem exiguum tuberculum genitale conspicitur fere aeque magnum.

Vasa mutrientia utrinque ad latera decurrunt; generationi destinata duplici ordine spirali medium tenent, omnia fusca, verme albido illo. rum copia fuscescente viso.

Obs. Rana brasiliensis in qua cel. vir Distoma locce elegantissimum detexit utique nova videtur: Supra sordide viridis, subtus albidior; palmae pollice superne, duobusque digitis insequentibus interne fuscis.

\section{Distoma micrococcum R. n. sp.}

In intestinis Glareolae austriacae Arimini Aprili magnam speciminum copiam reperi, omnium tenellorum, conf, obs,

Entozoa minutissima, arenulae granulum referentia, quartam lineae partem longa, ostavam 


\section{- 384}

ad,duodecimam lata; alba, puncto medio fusco; plerumque obovata, interdum elliptica, quando. que medio contracta, vel ventre concavo, dorsoque gibbo tarde procedentia; plürima motu carebant.

Porns anticus inferus subglobosus, raro pro. fundus visus, apertura exigua, saepe oblonga, satpius orbiculari, major Poro ventrali, mediam corporis partem tenente, apertura orbiculari in. structo. Cirrus forma vasis magni torti transparere visus.

Maculae variae córporis orbiculares (specula auctorum, sive ovaria vacua) microscopii ope in conspectum venientes, passim porum ventralem obscurum reddiderunt, ut ipsa foros mentirentur. Ovula parte posteriori ad latera comparuerunt pauca, sphaerica.

Obs. Am. Premser tria specimina misit in ejusdem avis intestinis reperta, quorum alterum bene diguoscendum lineam excedit; oblongum, depressiusculum; poro antico majore, aperturz longitudinali; poro ventrali apertura orbiculari; ovariis racemosis lateralibus.

\section{Distoma fuscatum R. n. sp.}

In intestinis tenuibus aliquot Coturnicum Anconae Majo complura reperi Distomata viva, aqua cito moritura, sesquilineam longa, quartam. circiter lineae partem lata, alba, macula dorsi longitudinali media nigrescente.

Corpus oblongum, biconvexum sive depressiusculum; plerumque caput reliquo corpore crassius, rarius discretum; cauda plerumque continua, rarius strictura sejuncta, vel crassior; uterque apex obtusus. 


\section{- 385}

Porus anticus, inferus, duplo major, ostio oblongo, ventralis orhiculari. Ante hunc tuberculım. cirri receptaculum, ostio, uti videbatur, -oblongo. Vas dorsale spirale, ova ad latera digesta.

Obs. In altero specimine porum ventralem valde exstantem et solito majorem vidi.

\section{Distoma ringens R. n.-sp.}

Bremser specimina tria in intestinis Pici tri. dactyli, reperta misit lineam longa, media parte ultrd teriam lineae partem lata, depressa, cras. siuscula, ideoque opaca, quo minus interna perscrutari potuerim.

Porus anticus maximus, exquisite terminalis margine tumido. Tentralis remotus, varius, altero in specimine exiguus illoque multo minor; in secundo minor quidem, sed mediocris; in tertio fere aeque magnus, sed margine non tumido. Cormus cblongum ventrali latere subconcavum, dorsali convexo; apice postico obtuso.

\section{Distoma crassicolle R.}

In duarum Salamandrarum maçnlatarum intestinis a pyloro usque ad anum sparsa reperi specimina viginti quinque; stcundum Froetrcmus in recto Salam. atrae, in hujus et macula. tae recto secundum, Catalogum Viennensem oc, currere dicitur,

Entozoa lineam ad sesquilineam longa, quartam teriamve lineae partem lata, albida, posteriora versus plus minus cinerra, ovis sphaericis in postica parte accumulatis, interdum vero duas horum series porum ventralem ambire vidi.

Porus anticus termmalis globosus; ventralis $\mathrm{B} b$ 


\section{- 386}

distans minor (tam porus quam ostium minora); rarius ostiolunin inter utrumque porum in conspectum venit, cujus tamen cirrum exsertum non vidi. Pars antica sive collumi utphurinum antrorsum subangustatum, interdum retrorsum attenua. turn; apice antico semper obtuso, Corpuls circa et pone porum ventralem latissinum, tum decrescens, apice postico obtusiusculo. Hunc FroeLICi quam anteriorem latiorem refert, quod in hisce speciminibus saltem non villi.

47. Distoma tereticolle R. Tab. 2. Fig. 5.

De excrescentiis in hujus corpore observa. tis et delineatis confer sectionem Mantissae insequenteni.

Aliud Lucii Distoma inter dubia 17. r.58. occurrit, forsanque D. truncatum 11. 159. A BILD: GAARDio dictum D, tereticolle mutilum est.

\section{Distoma gelatinosum R. n. sp.}

Testudinis Myáé intestinis Arimini Majo squae commissis, in hac novem reperi Distomata, quae pri um pro muco gelatinoso ab intestinis soluto habui.

Enturua sex ad decem lineas longa, dimidiam ad duas tertias lineat partes lata, depres. siuscula, utrinque passim contracta vel subcrenata, antrorsum magis attenuata, utrinque obtusa, plurimam partem diaphana, alba, stria longitudi. nali media aliquantulúm in flavum vergente.

Porus anticus (capite discreto) globosus, major, ventralis remotus, ejusdem formae, fere

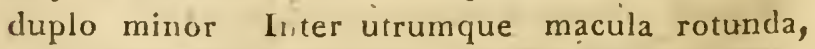
exig11a, cirri rectptaculum. A poro antico vas tenue, simplex oritur, quod ubi porum ventra. 


\section{- 387 -}

lem attingit, dividitur, tt hunc ambit, dein rectum utrinque ad posteriora decurrit, in' ultima autem corporis parte anastomosi jurictum iterum simplex fit, quo facile pro nervo imponere pos. sit. Inter vasa recta ovaria oblonga vasis intercepra, et ova valde exigua anteriori parte con. gesta. Ad latera vasorum rectorum exierua, omnia pellucida sunt.

Motum non observavi nisi in partis ante. rioris apice interdum extenso et erécto.

49. Distoma megastomun R. n sp.

- In Squali Galai ventriculo Arimini Majo triginta reperi Distomata, quorum viginti tria adulta tres cum dimidia ad quatuor lineas longa, ultra lineam lata; septem minora vero sisquilineam longa, vix dimidiam lineam lata; omnia el. liprico oblonga, alba, parte postica adultorum fla. vescente.

- Corpus oblongum, depressum, a latere dor. sali convexum, abdomine arlultorum excavato. Pars anterior sive collum a parte posteriore, sive corpore stricte sic dicto, parumper latiore, sinu exiguo distinguitur. UTterque apex rotundatus.

Pori globosi, magni, apertura orbiculari, margine tumido; anticus inferus, major, ventralis remotus, in junioribus totius vermis medium te$n \in n s$, in adultis autem pars posterior ad anteriorem uti quatuor ad ties cómparata. Cirri receptaculum maculam albam opacain et rotundam inter poros sistit. In adultis ova pone "porum ventralem utrinque longitudinaliter digesta; pone eundem porurn vas dorsale transversum erstiris flavi, quod reflexum utrinçue ad posteriora recte decurrit. - Species elegans. 


\section{$-388$}

\section{Distoma heterostomum $\mathrm{R}$.}

Rosa Lettere zoolog. p. 5. n, 4. Fasciola epatica dell' Ardea purpurea.

In duarum Ardearum purpurearum Julio occisarum ore, ad latera linguze et sub eadem, Distomata reperit fortissime affixa, plana, figura folii laurini, antice paululum truncata; tres lineas longa, unam medio lata; transversim striata (annulata minus bene vacat), alba, linea utrinque obscura longitudinali.

Cum Distomate hepatico male quidem miscuit, sed quae de colore, vasis (linea), et loco refert, illa illustrant, quae de specimine Ardeae purpureae oesophageo a cel. quondam ANDr. JuRINE mecum communicato, mortuo et varia celante Ent. II. x. p. 38I. n. 24. retuli.

Striae corporis privi quid habent, sed ista quidem species plurimis notis ab aftinibus dificert. In appendice Distoma, dicetur in Ardeae cujusdam brasiliensis faucibus occurrens.

51. Distoma mentulátum R. n. sp.

In tribus Colubri Natricis speciminibus femineis Berolini Julio et duabus Lacertis maculatis Arimini Aprili ego, et am Gaene in Lacerta agili Berolini Julio, complura reperimus Distomata intestinum tenue occupantia, quae sígillatim referam.

I. Illa Colmbri Natricis unam ad tres lineas longa, linearia, depressa, utrinque obtusa, rubella. .

Porus anticus major apertura oblonga; ven. tralis remotus, multo minor, apertura orbiculari. 


\section{$-389$}

Cirrus haud visus. Ovula immatura et recepta. cula ovorum orbicularia vacua, Vas posticum subspirale.

Apicem posticum primo angustatum observavi, sed nutam inconstantem esse didici.

2. Specimina in Lacerta agili obvia lineam aì sesquilineam longa, quartam tertiamve lineae nartem lata, depressiuscula, recentia rubella, post -nycthemeron alba lateribus flavicantibus, vase po. stico obiter spirali rubro vel fulvo.

Corpus oblongum, antice obtusum, postice bhusiusculum, narginibus lateralibus rectiusculis; interdum pars antica, interdum postica constricta, saepe nulla. Porus anticus a ventrali remotus, fere diplo major, uterque globosus;' illius aper. tura oblonga, hujus orbiculari.

Partes lateralès ovulis flavent, quae coacervata vas mediun rúbrum reddunt, quod serpen. tino ductu supra porum ventralem adscendit, ibique cirro jungitur cylindrico, longissimo, spirali, a quo nomen desumsi.

3. Quae in Lacertis maculatis (Lezard ta. cheté Daudin?) reperi : depressa, aíhida, lineam vix excecientia, oblongoelliptica, utrinque obtusa, posteriora versus magis attenuata.

Ports anticus oblongus, apertura pariter oblonga, major; ventralis globosus, apertura orbiculari. Inter utrumque cirrus longus tenuis exserius. Ovula ad latera digesta.

Confer speciem insequentem alfinem, sed distinctam.

52. Distcma clavigerum $\mathrm{R}, \mathrm{n}, \mathrm{sp}$.

$A m, A B$ Olfers in intestinis tenuibus $B a$ - 
fonis viridis Berolini Julio magnum speciminum numérum reperir el merum communicavit.

Viva rubella, 'mortua alba, lituris posticis flavescemtibus; longitúliné linean raro tantillum excedentia, media pare fere dimidian lata. Ovato-elliptica, exiremiate antica angustiore, dorso convexo, rentre plano. P'orïs orbicularibus subremolis, venrati duplorminore.

Arl latus sisistrum ventralis pori cirrus sin. gularis, qualem nunquam vidi, strictus scilicet, clnate instar a latere pori ad marginem vermis obiqque antrorsum ductus, et capitulo perforato hunc excedens. Verme aperto ovulorum ellipticorum opia innumera effusa est, insimul autem oviductus longus ovis farctus in conspectum venit, in cirrum illum transiens; ut utrumque cohacrentem vini spiritui immiscrim, quae separatio in, aliis Distopnatibus non successit. Plurima praeterea corporis pars ovis farcta, passim tamen maculae spthaericae postice cum oviductibus ob. servantur.

Obr 1. Dubius primo factus sum, an corpus illud magnum clavaeforme cirrus utrum hujus theca vel vagina sit, sed in omnibus reliquis Distorsatibus cirrus rudus est.

Obs, 2. Hoc procul dubio est Distoma, quod Froelich (Naturf. St. 25. p. 69. Tab. 3. Fig 7. 8. Fasciola Ranae) Junio mense in Rana temporaria detexit, quodque in plurimis Batrachiis nbvium Caralogus Viennensis typis impressus restituit, cum ipse non visum, Zenero praeeunte, cum Amphistomate subclavato (Ent. II. I. p. 39s) perperam conjunxissem. Froelichius céterum cirrum latentem aut tantillum exsertum tantum vidit, speciminibus forsan minus evolutis. 


\section{- 390}

Dextrum vocat, verme a latere abdominali spe. ctato, quod insolitum salten est.

53 Distoma Squamula R. n. sp.

Am Bremserus complura misit specimina in intestinis Mustelae Putorii reperta, alba, marula media brunnea.

Longitudo lineam dimidiam utplurimum tan. tillum superat, latitudo duas tertias aut tres lineae quartas, partes adaequat. Corpus depressuin, margine antico convexo, lateralibus rotundatis, posteriore vel rectiusculo vel saepe sublunato. Haud raro totum corpus pentagonum videtur, marginibus duobus anterioribus (a poro antico oriundis), toticlem lateralibus, quinto postico. Rarius apex medius papillaris in margine postico lunatim exciso eminet.

Portus anticus terminalis exiguus, orbicularis, ventralis si vere datur, remotus et illi aequa. lis $\in$ st.

Ovaria fusca ramosisšima latera vermis amplissima sibi suinunt; inter illa media parte (pone porum ventralem aut ejus speciem) ductus obscurus et variae maculae orbiculares.

Obs. Vermis ob latitudinem longitudine majorem ab omnibus reliquis speciebus recedens miliı quidérn Monostoma videtur; aliquoties enim pori quidem ventralis speciem quandam obscuram, illum autein nunquain distincte observavi, BremSERUM tamen, qui certe vivum vidit, Distoma vocantem secutus sum, quoties enim de re observata agitur, affirmans hujus majorem fidem facit: quam ob causam etian speciem n, 6o. Anico Monostoma visam Distomatibus tamen addere non haesitavi. 
6o. Distoma trilobum R. n. sp.

Brevsert's noster specimina quatuor in in. testinis Pelecani Carbonis reperta mecum comnunicavit,

Entozoa lineam dimidiam longa, depressa, antice lati-siena, obiter inspecta truncata visa, sensim posteriora versus ẹl ad apifem obtusum usque attenuata. P'ri orbiculares exigui atquales, anticus terminalis, ventralis parum distans, immersus.

Corpus antice utrinque eminet, ut poro antico cum his eminentiis lateralibus computato margo anterior trilobus videatur. Ceterum superficies dorsalis convexiuscula, ventralis divisa in anteriorem concavam, posieriorem plauam; illa porum ventralem immersum gerit.

Obs. Porus hicce verıtralis facile visui subducitur, quo factum cst, ut Amicus entozoon istud pro Morostumate habeat, nec nisi examine repetito de illius indole convictus fuerim. Confer observationem ad speciern n. 53. Cel. Nitzscri Distoma trilobum forsan Holostomatibus suis ad. deret.

\section{2. "Distoma isostomum R. n. sp.}

Am. Отто Majo mense, Astacos fuviatiles secando in alterius ductilus biliferis Distoma reperit rubellum; valde mobile, quod benevole mecum communicavit,

Entozoon spiritu vini albidum factum, lineam cum quarta hujus parte longum, quartain latum, utrinque obtusum, versus posteriora parum décrescens, dorso convexiusculo, ventre plano. 
Pori aequales, globosi, mediocres, nudis oculis conspicui; anticus terminalis, ventralis remotissimus, mediam totius partcm occupars. Keque cirrum, neque hujus receptaculum vidi.

Pone porum ventralem linea media pellucida recta, ad cujus latera, marginem versus, ductus flexuosi pariter pellucidi conspiciuntur, oviductus forsan vacui, ova enim unllibi vidi.

Obs. I. Cel, Garus (Lehrbuch der Zootomie. Leipzig 18!8. 8." "p. 5t.) se vere anni ISI vermes gangliis posterioribus compthrium Asta. corum fluviatiliuna firmiter inhaerentes reperisse refert, sesqquilineam longos, Linguatulae similes, quos ulterius non descripsit, forsan ad nostrum Distoma pertinentes. Ipse multos, am. Rosz:that plurimos Astacos secuimus recentisimos, sed munquan ejusmodi quid observavinus. In Gadis verines nervis insidentes frequentes obseŕvati sunt,

Obs. z: Inter ducelas bilifuros ejusúem Asta." ci, qui Distoma pratbuit, Orro tria quoque re. perit corpora minuta, nigricantia, daza, asperrima, quorum alterum adluc in eadera pliala cum Distomate servo, spiritu non mutatum,

\section{Distoma irroratum K. n. sp.}

In Testudinis Mydae ventriculo Arimini Majo tredecim reperi Distomata, duas ad lics cum dimidia lineas longa, dimidiam ad duas taitias lineae partes lata, sublinearia, utrinque obıusa, depressiuscula, dorso convexo, ventre plano; poris carneis, corpore niveo, lacteobrunneoque variegato, pictura dorsi ramosa alhids; vividissima.

Pori, ob colorem ab illo corporis alienam v́alde singulares, sunt globosi, aequales, tertiam 


\section{- $394-$}

circiter totius longitndinis partem distantes; an. ticu! interdum major sistitur, valdeque dilatari porest, quo apertura transversa fit; ventralis apertura major videmr. Cirrus in nullo exsertus.

Marula albila totum dorsum decurrit, varie ramosa, et in inferiori corporis latere illius partes ad latura quoque in conspectum veniunt. Postica pars brunnea est.

\section{Distoma clavatum $\mathrm{R}$ :}

Synopsi typis mandata speciem hanc insignem videre nondum contigit, postmodum autem am. De Chasisso ex itinere circa orbem redux duo $n t c c u m$ communicavit specimina in Scombri

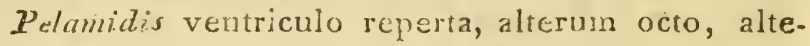
rum lecem et quod excurrit lineas longa, parte anteriore vix unam, postrema duas superantia lineas.

Pori subglobosi, anticus subinferus, ventralis parum distans maxinus, illo plus quam duplo major, fere lineam superans, ore hiante. Neque cirrus neque hujus receptaculum, sed iuter poros tantum vas transversum fuscum utrinque reflexum et posteriora versus decurrens, exhibelur.

Collum in corpus sensim abit porum aucturn, sed cylindricum subdequale continuatum, ucquedum subito caudam magnam globosam eificiat.

Totius vermis superficies elegantissime plieata, plica tamen nulla circulum absolvenie sed in novas transeunte, ut tota cutis unicárn plicam gyris innumeris et tenuissimis saepissimeque duplicatis ethiciat. Singulae itaque plicae in alias divisas saepe minores ad apicem caudae usque abeunt, cujus nuflum vidi orificium, sed ultimae 


\section{- 395}

quasi plicae terminum subrotundum, planum, aut scutellurn si ita dicere mavis.

Diflerentiam in Synopsi ponendo de rugis locutus sum, ejus vero loco plicaé dicantur; denticulos cutis suspicatus sum, nullos tamen reperi.

Entozoon in spiritu vini debili conserva. tum molle, colores tamen non retinuit, sed totum cinereum est.

Obs. I. Icones Tilesir Ent. II. I. p. 39 r. n. 33. nitidissimas dixi, sed verissimae etiam sunt, alioque temporc cum Bremserr thesauro pulcherrimo publici juris fient.

Obs. 2. Distoma Coryphaenae 1. c. durbius separavi, specimina vero, quae ab am. Olfer,s, Na. turaescrutatore lynceo accepi, in Coryphaera Equiseli reperta, infra describenda, plurinum? distant, quod etiam de omnihus Distomatibus valet, in Scombris Scombro, Colia, et trachuro a me repertis, infra exponendis.

Obs. 3. Synonymis addantur Brteardiere it. 1. p. 46 . qui nostram speciem in Pelamidis ventriculo repertit, et obiter describit; et Plangue (Bibl. de Méd. T X. p. 993. Tab. 22I.) Garsini observationem trausscribens.

\section{Distoma gibbosium R.}

Ent. II. I. p. 399. n. 39. D. gibloosum.

Am. Bremser tria specimina misit in iniestinis Esocis Belones reperta, ab illo, quod 1. c. Tab. VI. Fig. 8. delineatum dedi, eo diversa, quod totus venter haud promineat; sed tantummodo porus ventralis. Illud gravidum, haec virginea videntur.

Alba, parte postica fuscescente, ubi nimirum ovula exigua lateralia collecta sunt. Teretia sunt, 


\section{$-396$}

poro ventrali pedunculato, pedunculo brevi crasso. Porum anticum haud bene vidi.

Misit etiam Distonata tria in intestinis $\mathrm{Co}_{0}$. lyndi arctici reperta', duas tertias lineae partes longa, minus bene conservata, quae ex pisce co. mesto in Colymbo residua et parasitica, forsanque huc pertinentia crederem.

Pori fere latent, anticus foraminis exigui specie quudammodo notabilis; ventralis pedunculatus, verme fere furcato; utriusque pori basis parti pellucidiori insidet, ad cujus latera locus obscurus, Ciauda obtusiuscula, Corpus teres videtui.

\section{Distoma furcatum Brerro. n. sp.}

Brenserus specimina duo in intestinis Mulli Surmuleti lecta misit; ipse quinque in illis Mulli ruvescentis Arimini Aprili, unicum in illis Gadi Molvae Neapoli Junio reperi, Aba, sesquilineam ad quatuor cum dimidia lineas longa, vix quartam lineae partem lata, filiformia.

Corpus teres, aequale, aut antrorsum attenuatum tenuissimum, postice obtusiusculum, aníce collo quasi duplici utens; poro scilicet ventrali pedunculato, pedunculo longo, quam collum passim longiore, mox antrorsum directo, mox divaricato, quo vermis furcatus appareat.

Porus anticus terminalis longior quam latus, apertura quasi excisa; ventralis paulo major, ce. terum conșimilis. Interdum sinu, qua collum et pedunculus pori ventralis conveniunt, porulus tertius arlesse videtur, cirri receptaculum, quem nunquam exsertum vidi. Corpus passim corpuscula oblongo-elliptica obscura vel etiam alio in individuo vacua et pellucida, in linea mediana, par- 


\section{- 397}

tim ovula sphaerica nigrescentia, exhibet, quibus ultima corporis pars tota repletur.

73. Distoma fractum R. n. sp.

In intestinis Spari Salpae multa specimina Junio et Julio Neapoli offendi, albovirescentia, sesquilineam ad duas cum dimidia lineas longa, zrassa.

Corpus teres, utrinque acutiusculum, medio quasi fractum, poro scilicet ventrâlí cum abdominis parte prótruso et dorso eo loco emarginato vel sinum offerente. Crassissima pauca porum non protrusum exhibuerunt. Abciomen collo tenuius.

Porus anticus inferus, oblongus, profundus; ventralis remotus subglobosus, major. Semel cirrum brevern inter poros exsertum vidi. Ova subglobosa, posticam partem occupantia.

Plura ob entozoorum crassitiem observare non licuit.

\section{Distoma genu R. n. sp.}

In intestinis Labri lu'ci tam tenuibus quam potissimum recto Neapoli Junio bis copiose of. fendi.

Distoma hocce lineam et quod excurrit longum, tertiam circiter partem latum, albidum, me. dio tulvum.

Corpus teres, crassum, fractum, abdomine scilicet propendente, utrinque obtusiusculum. Collum longum versus porum ventralem auctum, a quo corpus ad caudam attenuatur. Porls allticils inferus, semiglobosus; ventralis subglobosus, longior tanen yuam latus, illo major Cirrus simplex, brevis, ante porum ventralem situs, quando. 


\section{- 398}

que prominet. Totum corpus supra et pone po. rum ventralem ovis farctum est.

\section{Distoma ventricosum R. n. sp.}

Arimini Aprili in ventriculo Clupeae Alosae plurima reperi Distomata appendiculata $12,8 \%$, in illius antem intestino reliquo (appendicibus exceptis) summam copiam Distomatum huc facien. tium, vivorum, alborum, postica tamen parte virideścentium.

Haec sunt dimidiam ad duas tertias lineae partes longa, teretia, qua porus ventralis situs est, ventre próninente; apice postico obtuso. Pori globosi, anticus terminalis minór.

Ante porum ventralem cirrus exiguus adesse visus est rectus. Pone illum corpus, quod in nullo antea observaveram Distomate, deinceps autem in specie insequente iterum reperi: glo. buli nimirum duo, sub- rxicroscopio nigri, nigris pedunculis (illos longitudine parum superantibus) angulo acuto vel obtuso postice conjunctis insidebant, varia directione obvenientes; utplurimum globuli exacte sphaerici, interdum minores et in. aequales, forsanque collapsi, ut peculiarem ovariorum formam indicare videantur. Ob crassitiem vermis autem vasa anteriora haud bene discerni potuerunt; postica pars ovis globosis viridibus. repleta est.

\section{Distoma baccigerum R. n. sp.}

Atherinis Hepsetis sex Nẹpoli Junii d. X. dissectis, in aqua, cui illarum intestina inıniseram, complura reperi Distomata, dimidian ad duas tertias lineae partem longa, quartam tertiam. 


\section{- 393}

ve lata, crassiuscula, ovata, alba, macula postica flavescunte.

Pori orhiculares, anticus minor, apertura utriusque orbiculari. Aute pirum ventratem remotum cirri receptaculum. Macula postica lintea ovaria sistit, ante hanc sloli nigri pedummat, de quibus speciem pratectentem describrolo fu. sius egi. Globi tamen has in sprcie'porun venl. tralén magis ambiunt, quae praecedenti quidem affinis, corporis forma satis differt.

\section{Distoma Ascidia R. n. sp.}

In aqua cui Spari Bodplis intestina Majo Arimini, Neapoli Junio immiseram, conplura offendi Distomata tertiam dimidiamve lineae par. tem longa, teretia, alha macula favescente

Pori globosi, inferi, approximati; ventralis duplo major, interdum tantopere prominens, quem prolapsum dicere posses.

- Collum breve corpore tenuius, hoc medio increscens, vel apice postico obtuso, passin per. forato, vel uti Neapoli vidi appendiculatum et caudam tenuem oblongam obtusituscularn $\epsilon$ mittens. In speciminibus vivis Arimini repertis interdum collum prolongatuin; dorsun cuncavum, ventre valde prominente, quo fere ad D. gibbosum n. 7 t. accedat; ova fusca te verme expanso maculae pellucidiores.

Haec in mortuis illis Neapoli lectis non vidi, bene autem observavi vas dorsale antice transversum, utrinque tum longitudisaliter et rectum posteriora versus decurrens, ubi tandem coire visum est.

Consimile in intestino $S_{\text {frari Pari Nearoli }}$ Julio reperi, lineam dimidam lowyum, quartam 


\section{$-400$}

latm, albidum, oblongo-ovatum, posteriora versus latesceus, apice postico rotundato Pori orhiculares, mediocriter distantes, ventralis major. Ova utrinque ad latera cumulata, ápice postico racuo et pellucido. An huc faciens?

Ascidiam habitu optime refert.

\section{Distoma labiatum $\mathrm{R}, \mathrm{n} . \mathrm{sp}$.}

In Syngnathi pelagici superficie hepatis con. cava Neapoli Julio mense unicum reperi Disto. ma peritonei tunica hydatidem mentiente inclua sum, vivum.

Lineam dimidiam longum, pro varia extensione triplo vel quintuplo angustius, teres, alou bidum.

Pori orbiculares, ventralis major, protrusus; anticus inferus, saepe labio superiore quasi addito, unde nomen desumsi; cute scilicet plicata poró retracto, antice vacua relicta. Corpus collo extensili crassius, postice obtusissinum.

Vas ante porum ventralem transversim, dein utrinque posteriora versus decurrit, sed fere moniliforme aut punctis insigne.

\section{Distom:a apertum R. $\mathrm{n}, \mathrm{sp}$.}

In Apogonis sive Mulli imberbis intestinis Neapoli Junio tria reperi specimina dimitiam lineam superantia, quartam circiter latissima pirte attingentia, albida, mortua.

Pori globosi, anticus duplo minor. Corjines collo crassius, apice postico acutiusculo, pone vel ctian sinul ante porum ventralem constrictum.

Julio insequente in alterius Apogonis inte. stinis duo reper Distomata, sed r centiara, line. am longa, albida, tertia, coluo conico tenuiore 


\section{- $401-$}

quam corpus cylindricum obtusum, apice in utro. que specinine quasi hiante, vel aperto; an forsan cauda appendiculata (mibi non visa) retracta? Ova per series longitudinales in abdomine digesta; vas dorsi spirale pro more. Cirri receptaculum inter utrumque porum, ipse non observabatur.

\section{Distoma microsoma R. n. sp.}

In aqua cui Percarım marinarum duarum intestina Neapoli Julio commisi, tria reperi specimina albida, posteriora versus flavicantia, tere. tia, lineam ad sesquilineam longa, quartam circiter lineae partem crassa, viva, motus vulgares edentia.

Pori inferi globosi remotissimi; anticus infra apicem anteriorem r biusiusculum; ventralis triplo major in parte posteriore latissima situs, tantopere ab illo remotus, ut ab apice caudaif obtuso vix quartam totius longitudinis partem in. termediam haheat. Collum sive pars longissima tres quartas partes sibi sumens a poro ventrali sensim antrorsum attenuatur. Cirrus haud exsertus. Pone porum anticum vas transversum nigrum utrinque rectum ad porum posteriorem decurrens, pone quem maculae duae ellipticae magnae obscurae et ovula congesta.

Obs. Distoma certe dicendum, licet non ne. gandum sit, si porus magis retrocederet, Amphistoma fore. Confer Amphistoma subtriquetrum n. 18.

81. Distoma globulus R. n. sp.

In Anatis Fuligulae intestinis tenuibus spe-

$\mathrm{C} \varepsilon$ 
cimina innumera, certe ultra mille, Gryphiae Novembri merse reperi, arenulas mentientia.

Albida, parte media flavicante, forma mox globosa, mox pyriformi, lineae tertian qua tumve partern diametris attingentia.

Corpus plerumque contractum, tumqque vel subglobosum, vel ovatum; aut plus minus extensum, tumque abdomine, vel etiam, rarius tamen, dorso maxime gibbum, extremitate vel utraque vel antica solummodo attenuata. Poris anticus inferus, subglobosus, eadem onnino forma quam Distomatis gibbosi, Entoz. Tab. VI. Fig. ४. Porus ventralis distans, major, varius visui exhibi- tus; saepe ventris tumidi extremum inferius excavatum.

Pone porum aniicum processis mox brevior, mox longior, raro tamen in conspectum venit, speciminibus plurimis mortuis. Cirri vicibus sine dubio fungitur, at crassiusculus et longitudinaliter striatus a forma vulgari recedit. Vasa distincta won vidi, sed corpus ovalis majusculis ellipticis farctum fuit, quae saepe maximan, etiam anteriorem partem replcnt. Rarius ovaria vacua maculas pellucidas majores obtulerunt.

Distomati gilbboso n. ji. affine, speciem tamen propriam sistit.

\section{Distoma excavatum $\mathfrak{h}$.}

Differentia specifica in Synopsi, ab illa quam in Ent. II. r. p. 399. n. 40. dedi, eo recedit, quod partem anteriorcm collum, pusteriorem corpus dixi, cum in Entozoologia illam corpus, hanc caudam vocaverim Pars inter poros ubveniens autem, longitudinis ratione nulla habita, semper 


\section{$-403$}

collum dicenda est. reterum nihil mutandun reperio, Confer specien insequentem valde affinem.

\section{Distomà spathaceum h. n. sp.}

Specimina quatuor in intestinis Lari glauci reperta Bremserus benevole communicavit, lineam vis superantia, examinatu difficillima, lentis simplicis ope tantum bene dignoscenda.

Pars antica, sive collum, spatham referens, ovalis, plana txcavata, poro antico obscuro, a quo tamen vasa decurrentia facilius conspiciuntur; in basi colli excavati porus ventralis satis magnus, orbicularis situs est, quem etiam altero in speci. mine recinato protrusum vidi. Ad latera marginis anterioris in altero specinine apices duo opaci.

Corpus, collo sesqui vel duplo longius, dorso convexo ovis farcto, sed corpusculis simul in conspectum venitutihus majoribus aliquot, aut ovaris vacuis Distomatum siltmnibus; abdomine excavato. Foramen apicis postici nullum vidi.

Obs. Speciei praecerlenti proxima est, sed ratione partium mutata differt. ivirzschrus Holosto. matibus adnumeraret, sed ab Amphistosnatibus buc pariter revocatis nimis distat.

\section{Distoma spatulatum R. n. sp.}

An. Brenser specimen misit in intestinis Ardeac minutae repertum, quatuor cum dimidia lineas longum, parte anteriore alba, posteriore fuscescente.

Collum planum, tenue, sensim antrorsum increscens, maryinibus lateralibus subinflexis, antico latissimo truncato. Corpus teres, Iongissi- 


\section{- $404,-$}

mum, requale, apice postico acutiusculo. Porus anticus terminalis, exiguus; ventrális triplo ma. jor, distans, utriusque apertura orbiculari.

Obs. I. Corporis excrescentiae duae acutiu. sculae obliquae irregulares, prồculdubio praeternaturales et ejusdem indolis, ac illae, quas in Distomate Lucii tereticolli observavi et Tab. II. Fig. 5. delineatas dedi.

Obs. 2. Species ista Nitzschro pariter Ho. lostoma foret ac praecedentes, sed ab Amphisto; matibus, quibuscum conjungit, nimis differt.

\section{Distoma appendiculatum $\mathrm{h}$.}

Ent. II. I. p. 400. n. 4I. D. appendiculatum. p. 404. n. 44. D. crenatum. p. 437. $\mathrm{n} .77$. D. Clupere rhenanae.

Entozoologiam conscribendo Distomata in Clupea Alosa et Gasterosteo aculeato Gryphiae mihimet obvia, intermediis mon visis distincta liabui, postmodum autem iter in Italiam faciens plurimis in piscibus tamque varia reperi, ut crrorem facile agnoverim.

Color plerumque totius Distomatis albus excepta macula media fulva vel rufescente, majore vel minore, pro diversa ovariorum evolutione; passim autem pars posterior insimul fuscescit, qualem specimina in Torpeainis marmoratae et Pleuronectis maximi ventriculo reperta monstrarunt; neque raro totum. Distorna flavescens, aut fulvum parte postica flavescente, aut rubellum ex. hibetur.

Saepe lineam longa; nunquam duas lineas (nisi fortirer extensa) superare vidi.

Semper teretia sunt, collo interdum sub mo. tu subtus excavato; passim magis vel minus cre. 


\section{$-405$}

Inata, rarius crenulata, rarissime absque crenis corporeque tantum constricto occurrunt.

Corporis forna maxime varia, prouti cauda appendiculata aut exserta aut retracta est. Saepe hrevissinum, et subovatum aut ovale, vel postice truncatum vel etiam perforatum videtur, cauda autem enissa illa species deletur. Cingulum, de quo in Entozoologia egi, passim quale ibidem de: lineatum dedi, in conspectum venit.

Pori globosi, apertura orbiculari; ventralis generatim antico duplo major est.

Saepe vas dorsale fuscum exhibetur pone porum anticum transiersale, dein reflexum utrinque posteriora versus decurrens, interdum solito latius. Vasa posteriora cincinnata.

Specininibus, quae in ventriculo Ophidiorum barbatorum reperi, Corpuscula oblonga opaca érant, qualia ceteris Distomatibus vulgaria esse solent: alias non vidi. Ova nunquam observavi, conf. speciem insequentem. Cirrum solummodo in speciminibus offendi, quae Pleurvnectes maximi, Arimini extus sibi adhàerentia plitulerunt, brevem, rectun, inter poros fere medium.

Obs. 1. Entozoologiam edendo Clupeam Alod sam Rhenanam a marina perperain distinxi, ideoque Distoma in illa ab Hermanno repertum practer necessitatem separayi. Eandem omnino Alosam in Adriatico quam in Baltico, et in utraque idem Distoma reperi, quid? quod Larius lacus apsam pariter fovere videtur.

Obs. 2. Raja clavata Majo mense-Arimini a piscatoribus allata est, cujus -dorso undecin hujuș Distomatis specimina inhaeserunt, cuịus vero tubus cibarius entozois penitus caruit; forsan aliis piscibus in piscina illi impositis Dișto.l 


\section{$-406 \div$}

mata huc advenerant; Torpedo tamen Rajae generi proxima illa ventriculo fovet.

Obs. 3. In Accipenteris Sturionis vesica na. tatoria uuum, tria in ventriculo reperi specimina (Majo, Arimini), ut harum partium commercium, observatione in Spiroptera Cystidicola facta comprobatum, non multum impediri concedas.

Obs. 4 Am. Bremser specimina tria misit in Plenronecte Passere reperta, valde exigua, forsan spiritu vini contracta, ceteroquin non diversa.

\section{Distoma affine R. n. $\mathrm{sp}$ :}

In intestinis Percae cirrosae Arimini Majo duo reperi specimina, contracta vix tertiam lineae partem, expansa lineam attingentia, teretia, favescentia, postice obscuriora, viva.

Pori globosi, ventralis major. Corpus ovatum, apice prolongato obtuso. Vas fuccum in collo transversum utrinque reflexum versus po. steriora decurrit.

Ovula, qualia in Distomatibus hactenus non vidi, elliptica scilicet puncto medio fusco, ceterum ad posteriora cumulata, ut caudam repleant.

Obs. An species hac non solummodo praecedenti affinis sed eadem? In D. appendiculato autern nunquam ova deprehendi, quod neutiquam horum defectui, sed vel horum ad partes intimas relationi, vel Distomatis cuti circa ovaria magis opacae tribui debet.

87. Distoma rufoviride $R$. n. sp.

In Muraenae Congri valde magni ventriculo $\mathrm{Ne}$ apoli Junio mense octo reperi specimina, tres lineas 


\section{$-407$}

plerumque longitudine superantia, dimidiam lata, teretia, viridia parte media rufescente.

Porus anticus inferus, ventrali duplo triplo. ve minor, uterque semiglobosus, apertura orbiculari, illins quandoque transversa, seu rimain potius sistente. Collum breve, haud discretum, posicriora versus auctum, tuberculum gerens, ex quo cirrus brevis rectus emergit. Corpus duplici modo occurrit, vel cauda retracta tumque crassius apice valde obtuso, vel exserta, tumque a poro ventrali posteriora versus sensim attenuatum, apice caudali longo tereti discreto.

Vas dorsale rufescens valde complicatum vel gyrosum; ovula posteriore parte collecta in speciminibus a me examinatis nondum evoluta massam deformem viriclem sistunt.

Species ceterum cum insequente forsan con. jungenda erit.

\section{Distoma grandiporum R. n. sp.}

In Muraenae Helenae ventriculo specimina quatuor, quintum in ejusdem faucibus Neapoli Junio reperi, sesquilineam longa, tertiam ad dimidiam lineae partem lata, teretia, viridescentia, vasis dorsalibus fulvis, viva.

Pori semiglobosi, anticus inferus exiguus, ventralis maximus, ostio amplissimo. Collum pro more variabile, interdum lon'gissimum. Corpus cauda exserta posteriora versus attenuatum; retracta obcusissimum.

Porum ventralem vix ulla in specie tantum visli, quo uti et magnitudine simul minore com. motus sum, qui Distoma hocce a praecedente distinguerem. Conjungendum tamen erit; BremsrRus enim' specimen misit pariter in Helene 


\section{$-408$}

Septembri mense Neapoli repertum, meis majus, duas scilicet cum dimidia lineas longum, poro ventrali majore, vermis tamen ratione habita minime tanto, ac in supra descriptis vidi. Color speciminis transmissi spiritus ope mutatus, albus, ovulis ad latera congestis' flavis, vasis et areis tam mediis quam etiam apicis caudalis albis.

89. Distoma pallens R. n. sp.

In intestino Spari Auratae Neapoli calendis Augusti specinina reperi octo, unam duasve lineas longa, tertiam lineae partem lata, lactea macula sublutea media, saepe vix notabili.

Portus anticus inferus subgiobosus, apertura saepius oblonga visa; ventralis duplo major, apertura transversa. Collum teres latitudine corpori aequale, neque discretum. Corpus oblongum, pqstice rotundatum, depressiusculum. Ova utrinque serie magna longitudinali congesta, maculis intermediis (in linea mediana sitis) orbicularibus, pel. Iucidis, Latera quasi pulverulenta apparent. Cirrus brevis rectus truncatus.

Obs. Summa certe Distomatis insequentis affinitas, a quo tamen poris aliter constitutis differt; utraque tamen species ad prinam sectionem, ob corpus depressiusculum, melius forsan Ieferretur, quod Synopsi typis impressa demum observo.

\section{Distoma areolatum $\mathrm{R}$.}

\section{Ent. II. 1. p. 40I. n. 42.}

Quod Otho Fabricius, auctor meritissimus in Pleuronecte Platessa detexit, ipse Neapoli Junio in intestinis Pleuronectum sex mancorum re. peri, vivum, elegantissimum. 


\section{$-409$}

Specimina mea Fabricianis majora, lineam ad duas cum dimidia lineas longa, quartam tertianıve lineae partem lata, viridescentia, pulverulenta quasi, areolis sive maculis o:bicularibus tri. bus quatuorve mediis pellucioiis.

Corpus depressiusculum neque teres (ut cum specie ratcedente melius ad sectionem praegressani amandaretur) utrinque obtusum, plerum. que postice atteiluatum, passim constrictum.

Pori globosi saepius aeqúales, interdum anlicus tantillum major videtur; hic ceterum inferus, cute capitis ante eundem sita galeam quasi efficiente. Ventralis in junioribus magis, in majoribus minus remotus; hujus ceteroquin apertura orbiculari, illius oblonga.

\section{Distoma gracilescens R. n. sp.}

Bremserus complura specimina misit in intestinis Lophii piscatorii detecta, in quibus ipse quoque, Tergesti Aprili mense reperi; quae autem de recentibus notavi, casu perdidi, ut de. scriptio illa spiritu vini servata adumbret.

Lineam ad sesquilineam longa, quartain 1 . neae partem lata, teretia, retrorsum gracilescentia, alba, parte media fusco - irrorata.

Pori globosi, anticus major, inferus; semel ventralis, alias exiguus, sed obscuro modo magnus visus est. Corpus passirn crenatum; col. luin et hujus pars anterior ejusdem crassitiei; pasterior obtusa tenuior, apice caudali brevi retracto.

Ova immatura globosa. Alute apicem candalem retractum interne globulus fuscus cure ovariis conveniens pellucet. 


\section{Distoma torulosum R. n. sp.}

In aqua, cui Siluri Glanidis, intestina Gry. phiae Octubri immiseram, unicum reperi specimen duas lineas longum, tertiam lineae partem latum.

Teres, inaequale, sive hinc inde constrictum, ideoque torulosum; retrorsum sensim attenuatum, antice obtusum, postice obtusiusculum, albidum.

Porus anticus inferus, ventralis remotus, uterque subglobosus, tumidulus, ostio orbiculari; anticus ventrali aliquantulum major. Inter poros vasa duo longitudinalia; in parte posteriore vas spirale, in quo humorem inoveri observavi, Ovula utrinque digesta, immatura et minima.

\section{Distoma tubarium R. n. $s p$,}

In intestinis Sciacnae Umbrae magnae recentissimae versus finem Augusti Speziae viginti et quod excurrit specimina reperi mortua, lineam vel sesquilineam longa, tenuia, alba, vase dorsali migricante.

Pori hemisphacrici, anticus duplo major, terminalis. Corpus teres, a poro antico ad ventratem sensim auctum, pone hunc iterum sensim attersuatum, apice postico obtusiusculo.

Utrinque in corporis parte posteriore ductus lateralis rectus et amplus decurrit, qui ante porum ve'itralem curvatur et opposito contiguus fit, necue tamen anastomosat. Inter quos -vas dorsi sinirle et ovaria orbicialaria pellucent.

Species tubis illis sive ductihus latis, alio in Distomate nunqua in visis distinctissimia. 


\section{Distoma fliforme R. n. sp.}

In intestinis Cepolae Taeniae Arimini Aprili aliquot reperi specimina, duas vel duas cum'dimidia lineas longa, tenuissima, utrinque obtusa, viva.

Porus anticus major, ohlongus, ventralis distans, apertura orbiculari. Collum teres album, quartam totius longitudinis partem sibi vindicans. Corpus teres aequale, flavidum, óvulis rotundis transparentibus, Cirrus haud emergit, maculam opacam longitudinalem pone porum anticum efficiens.

\section{Distoma excisum R. n, sp.}

Multa speçimina in Scombrí Scombri ven. triculo Aprili et bis Majo Armini rèperi viva, alba, parte media tlavo-irrorata.

Lorgitudo plerumque tres lineas adaequat, varia tamen quatuor, unum post mortem quinque lineas attingebant; crassities tertian lineae partem excedit.

Porus anticus profundus, fere infmolibuliformis, antrorsum ampliatus, fundo contracto, ir:ferioris marginis parte antica excisa, sive porus inferiora versus emarginatus est. Ventrulis ad duas tertias lineae partél distans, globosus, autico fere duplo minor.

Collum supra convexum, infra concavim. Corpns teres inaequale, animalculo vivo fortiter cre:atum, ut fere marcines versus aculeaturn vi. dtakur; in mortuis àuten crenis omnino destiutuin et late hine inde contractum, apice caudali aigusurite oicusiusculu. 


\section{$-412-$.}

In specimine maximo cirrus rectus, antrorsum infra collum et ad partem pori antici excisam usque porrectus, adesse videtur. Ovaria fla. vescentia, sed alia etiam vasa alba inter illa ob oculos veniunt, forsan nutritia.

Cum his congruunt, quae aliquoties Junio et Julio Neapoli plus minus copirsa in ventricu. lo et intestinis Sccmbri Coliae reperi, valde varia, duas ad sex lineas longa, quin ad decem lineas extensa, albida maculeis luteis, vel etiam majorum speciminum fuscis. Pori, corpus crenatum, reliqua eaciern; cirrum tamen in his nun. quam vidi; caudo exsertilis, mox brevior, mox longissima.

Obs. I. Hanc speciem cum Distnmate Coryphaenae n. I 53. conjungere non dubitarem, nisi specimina copiosa ab am. $A B$ Olferś in ventri. culo Coryphaenae Equiselis reperta' porum anticum integrum exhiberent. Distoma clavatum $n$. 65. in Scombri, Pelamidis ventriculo habitans, ab exciso maxime alienum est.

Obs. 2. In aqua cui Coliae intestina immisi Junio et Julio minora quoque Distomata nonnulla reperi, quae primum pro $D$. excisi prole habui, ideoque in Synopsi non seorsim indicavi, quae propriaul tamen effingent speciem, uti plurimi pisces duobus obnoxii sunt Distomatibus, quorum alterum ventriculum, alterum intestina tentet.

Alba sunt, lineam non excedentia, teretia, parte postica tenuiore, sed haud appendiculata, vasis oaricis lateralibus racemosis.

96. Distoma alatum $R$.

guod olim in Vulpe tantum offendimus, 


\section{$-413-$}

Berolini Januario mense maxima copia in Canis Lapi ventriculo, rarius in duodeno et initio jejuni, neque in ceteris intestinis reperi, sesquilineam longum.

In alio Lupo èdem mense dissecto ne unicum quidem specimen vidi.

\section{Distoma fuscescens R. ก. sp.}

In acjua, cui Spari Denticis intestina Majo Arimini immiseram, unum, alterumque specimen in ejus ventriculo, postea etiam solitarium Neapoli Julio reperi.

Distomata viva alba macula media fusce. scente, duas fere lineas longa, quartam circiter lineae partem lata, teretia.

Pori globosi, distantes, aequales, interdurn tamen anticus terminalis aliquantulum major visus est. Collum corpore paululum crassius. Cotpus aequale postice obtusiusculum.

Aste porum ventralem utrinque vas album crassum conspicitur posteriora versus decurtetss; in media autem parte vas spirale rubellum (vel etiam nigrum) quod rectiusculum pone porum ventralem incipit, mox spiris arctis et fere trans. versis posteriora versus continuatum, demum la. xius et tanden simplex desinit.

\section{Distoma-laureatum ZED.}

Synonymis Ent. II. I. p. 413. 2. 49, allatis addatur Froelich Naturf. Sc. 29. p. 62, n. 32. Tab. 2. Fig. 4. 5. Fasciola Farionis, quan vir cl. notis inconstantibus et rejiciendis a Fasciola truttat olim sibi dicta distingure tentat. In Fos. rionis intestinis crassis versus finem Octobris sztis copiose reperit. 


\section{Distoma lineare $R$.}

"Quod Gryphiae anno 1792. Octobri mense in duorum Gallorum gallinaceorum juniorum intestinis crassis reperi, mihi postea non oblatum est, neque in Museo Viennensi ditissimo occurrit.

I lem autem videtur, quod in trachea variarum Ordinis gallinacei avium Angli te Americani offenderunt. Qua de re conferatur: Account of a species of Fasciola which intests the trachea of the Poultry, with a niode of cure. By George Montagu in Transact. Societ. Wernerian. T. r. n. XII. p. 194-199. Tab. VIL. Fig. 4.

Distoma trachea (sic!) auctori dictum, teres postice attenuatum acutum, rubrum interantis albis, pollicem fere longum, latitudine lineam dimidiam non excedere. Porum ventralem longissine pedunculatum (pedunculo- quam collo longiore) infundibuliformem, minorem pого antico, obiter sexpartito.

Speciem hanc a pharynge (an larynge?) per tracheam decurrere; affigi ope pori veutralis pe: dinculati, loco, qua adhaereat, semper inflammato; inflammationem, haud'vero Distoma, ad pulınones descendere. Urinam loco aquae pulti vel farinae admixtam morbo mederi.

Se r806-rsor (quo hac de re commenta. riolum hunc conscripsit) morbum istum pullorum gallinaceorum observasse verminosum. Ab amico se accepisse, in Phasianis colchicis tenellis Distoma iracheale, quando plumae sexum prodere incipiunt, irequeris occurrere; addit eliam te idem in Purdicis trachea reperisse, alterumque speci- 


\section{- $\quad 15$}

men parte postica ex trachea perforata propendisse.

Editores Actorum adnotant cl. Wiesevtian, Anatomiae Professorem Baltimoriensem in America Septentrionali nalum idem, eodem etiam ibidem ac in Ánglia nomine veniens (Gape, ab oscitatione frequente cuin nuchae extensione, suffocationis anxietate productis) observasse, quod junioribus etiam snatibu, infestum est. Distoma idern, sed poro. ventrali haud peelunculato ab illò inventum esse, in Merical and Physical Journal I799. Vol. II. p. 204. descriptum et delineatum.

De morbo dicto apud Nostrates non audi. vi, neque Occonomi neque Veterinarii postmodum a me interrogati illum hoverunt. Non au. tem detrit, sed cum aliis certe confustus est.

Cum cel. Winsenthal pórum ventralem ses. silem observaverit, quem cl. Montagu longissime pedunculatun dicit, nullus dubito, quin ìistomata mea linearia, quinque ad scpteni lincas longa, rubella, poro anico papillis sex cincto, huc faciant. Conf. Ent. I1. 1. p. 4:4. n. 50.

\section{Distoma acanthoides R. n. sp.}

'In intestino tenui Phocae vitulinae junioris, Berolini Junio mense mortuae et receus allatae duo reperi specinina alba, quorum alterum duas lineas tertianve, alierum unam et tres quàrtas lineat partes longa, tertian lata fuerunt.

corpus depressum, sublintare, apice postico attenuato. Pori orbiculares, anticus teminalis exiguus, capite subdisereto, tx lata basi cunico, echunis rectis cincto, aliis lattralibus posticis ac. cumulatis, Porus ventralis multo major, profun- 
dus. Cirrus longus crassiusculus flexuosus, Ovzula immatura utrinque lateralia.

105. Distona bilobum R. n. sp.

Am. Bremser aliquot specimina in intestinis Tantali Falcinelli reperta misit, quatuor ad quinque cum dimidia lineas longa, dimidiam ad duas tertias lineae partes lata, speciem nitidam facilli: meque distinguendam sistentia.

Caput bilobum, lobis semilunaribus, quo. tum discus planus spinosus, margo externus convexus spinis majoribus, ciliatus invenitur. Qua 'lobi medio conyeniunt, porus anticus exiguus inferus; ventralis parum distans multo major et profundus, uterque orbicularis. Corpus lineare planum, apice postico obtusiusculo. Cirrus non exsertus.

\section{Distoma echinatum ZED.}

Ent. II. I. p; 418 n. 52. D. echinatum. p.' 432. n. 67. D. gruis.

Am. Nitzsch bis bina misit specimina in intestinis Ardeae Gruis auctumno a se reperta, sex septemve lineas longa, quorum alterum cirrum emiserat recurvum brevem poro ventrali fere appressum, 'nullo modo a D. echinato Anatum distinguenda, ut D gruis Ent. 1. c. delendum sit.

BreMSERUS noster pariter specimina non. nulla misit in intestinis. Gruis reperta, tres ad quinque lineas longa, capite minore, quam in Nitzschianis exhibito. Ab examine Distomatis avocatus, alterum specimen casu sub microscopio reliqui, quod siccatum et vitro inhaerens echino. rum indolem egregie demonstravit. Marginem capitis anticum convexum quatuordecim echini 


\section{$-417$}

tenent alterne prostantes et recedentes; tum ad latera aliquor alterni occurrunt, majorquie copia demun postice urinque cumulata hahelur.

Idem Amicus aliquot misil specimina in $A r$. deae comatae intestinis chvia, lineam plus minus longa, corpore depresso angucto sublineari, capite reniformi echinis armatu, poro ventrali ma. jore, huc certe facientia.

Idem valet de speciminibus, quàe optimus vir in intestinis Pelecani Carbonis reperta, sesquilin yam ad duas lineas longa, conpluza inecum communicavit, nulla proraus nota distinguenda.

- Pari modo conveniunt, quae am. Klug dedit, in Anseris intestinis a se reperta.

A pud Froezichicir. (Naturf. 20. St. p. 58. n. 30. Yab. 2. Fig. 6. 7.) hate pecies in Anatis Boschadis ferae intestiuisu lecta Fasciola revoluta audit, quat probe distinguatur a clarissimi viri Fasciola appendiculata, sive nostro - Distomate oxycephaio 20.28.

\section{Distoma uncinatum $\mathrm{Z} E \mathrm{D}$.}

Huc revocanda Fasciola crenata Froelichrt Natur. St. 29 p 00. n. 31. Tab. 2. Fig. 10. 1t,

Aliquot specimina in intestmis crassis Fulicae Chloropondis Wajo reperit, fere tres lineas longa, duas terias lineie partes lata, Zederiano specimine ergo minora.

Frorlich collim corpore aliquintum angustius refert, quod ZenERo latius dicitur, forcanque a specominum magnitudine et attate pendet. Echinos autem capitis Froelich perperam pro poris venditat caput perforantibus; in sperife praecedente strias vocaverat. Margines corporis te. 
nuissime crenatos refert, icone autem tales non sistit.

108. Distoma militare R.

Specinien in intestinis Ralli Porzanae repertum, quatuor lineas longum, angustum, am. B REAstrus misit, nomine triviali non addito; in Catd. logo Viennensi typis impresso autem idem nncinatum, vocatur. Forsan ZeDerI Distoma ita dictum (in Chloropode repertum, mihique non visum) cum illo, quod militare vocavi, idem est, specimine nimirum ZEDERI varietatem offerente, alias enim distinguantur necesse est.

Collum enim corpore neutiquam latius est, corporis autern maxima pars aculeis rectis minimis obsita, quos ZEDERus uncinatum describendo tacet.

109. Distoma echinocephalum R. n. sp.

Cel. Treutler Distomata complura dedit Aúgusto mense anni 1789 in intestino recto $\mathrm{Fal}$ conis Milvi a se reperta, sesquilineam plus minus longa, angusta.

Bene licet conservata indaganti tamen non satisfecerunt, adeo ut num vere nova dicatur spe. cies, utrum alii cuidam D. armatorum speciei po. tius addenda sit, dubius maneam.

Caput corpore angustius subrotundum, cin-ctum echinis recris faciem eandem ac in D. echinato exhibentibus. Porus anticus exiguus ventrali multo minor. Corpus prope hunc parum neque semper latescit, ceterum lineare, angustum, posteriora versus parum attenuatum, apice obtuso. V'asa duo longitudinalia, aliaque minus distincta in media corporis parte, quae tamen privi quid habere videntur. 


\section{- 419}

112. Distoma denticulatum $R$.

Am. Bremser specimina quatuor misit in intestinis Sternae Cantiacae reperta, lineam vix excedentia, capitis collique denticulis mox magis appressis vel exsantibus facileque observandis, utique ad D. denticulatum pertinentia, quod ipse in Sterna Hirundine, affini ave reperi et Ent, II, I. p. 424. n. 57. descripsi。

\section{Distoma spinulosum. $\mathrm{R}$.}

Am. Bremser nonnulla misit specimina tertiam dimidiamve lineae partem longa, fuscescen. tia, in intestinis Colymbi cristati lecta, dubic huc revocatá.

Alterius capnt subconicum echinatum, echinis ad latera congestis; poru ventralis major; corpus terts aequale, postice óbtusum, quod D. spinuloso a me in Colymbo septentrionali te in Laris naevio et cinerario reperto, Ent. II. 1. p. 425. n. 58. descripto haud male respondet.

Reliqua vero specimina deflectunt. Horum Caput exiguum, indistinctum; abdomen versus porum ventralem praeter modum tumidum; reli. quum corpus tenuius, ad latera ovariornm race. mos more solito exlibens; cirro non exserto.

Obs. An haec specimina gravida, utrum ad D. oxycephalum n. 28. pertinent?

114. Distoma ferox R.

Quod Entozoologian conscribendo in inte. stinis Ardene Ciconiae tanium $\mathrm{i}_{\mathrm{p}} \mathrm{se}^{\mathrm{r}} \mathrm{reper} \in \mathrm{ram}$, postmodum Gryphiae Septembri mense in illis A. nigrae frequens offendi. Singulis incestinorum tuberculis unum plurave specimina inerant, unam 
ad tres lineas longa, postice crassiora, neque collo, uti alias vidi, globoso, conf. Ent. II. ı. p. 426. n. 59. Cum nulla alia nota discrepent, tt specimina olim Aprili et Julio invenerim, discrimen forsan $a b$ aetate aut a partu pendet.

115. Distoma fallax R. n. sp.

In Uranoscopi scabri Junio Neapoli vivi dissecti ventriculo duas per horas aguae commisso aliquot specimina reperi mortua, vase dorsali nigro instructa, quorum ab examine illa haud illustrante avocatus sum. Julio insequente duos iterum secui Uranoscopos recentissimos et in intestinis aquae commissis Distoma vivum reperi tres lineas longum, in aqua vividissimum, in ventriculis complura sesquilineam longa, tandem unicurn quinque lineâs a daequans.

Mínorum porum quidem anticum vidi, de ventrali dubius relictus sum; tandem specimen maximum se evoluit, quo Distoma esse agnovi.

Porus anticus minor subglobosus, echinis rectis cinctus. Collum cylindricum breve. Porus ventralis globosus abdominis hoc loco turnidi apici insidens. Corpus reliquum teres, subaequale, apice postico tenuiore in maximo specimine fere perforato viso, in reliquis obtusissimo, an appen. dice retracta?

Vas dorsale nigrum spirale in omnibus speciminibus conspicuum; in magno illo oviductus maximus vacuus, specula sic dicta efficiens.,

An exigua illa specimina, in quorum nullo capitis chinos, in paucis vero pori ventralis ve. stiginm obscurum reperi, majoris sobolem sistunt? Vase dorsali saltem et habitu conveniunt. 


\section{$=421 \div$}

\section{Distoma laticolle R, n. sp:}

In Carancis trachuri (Scombri Linn.) intestinis Arimini Aprilis d, XXVI. sex reperi specimina viva, alba, puncto medio flavo instructa, sciquilineann longa, quartam. lineae partem lata, mola collum latissimum expandentia. Vespera quale reperi, ulterius examinare non licuit, subsequente enim mane mortua et contracta fuerunt, quorum tantum ova fuscescentia corporis partem posticam replentia distinguere possem.

Aliquot diebus elapsis in Trachuro unicum reperi specinen mortuum, sed explanatum, poris splineroidalibus, diametro longitudinali majore, quo minus spliaerici dici possint, ventrali majore, anLico echinorum corona cincto. Collum latum planum utrinque aculeis senis rectis distantibus armatum. Corpus aequale, apice postico obtuso, teruiore.

In duobus iterum Trachuris Neapoli Julio, mense Distomata haec reperi, sed valde tenella, vix linean dimiliam attingentia, nullum echinorum vestigitum prodentia.

Obs. Num Entozoon paradoxum in intestino Anguillae semel a me repertum et Ent. IF' 2.p. 27 . n. 23. i. descriptum, buc pertinet? Synops. p. 19I. n. 47 Conf. Distona hispidum, n. 120.

\section{Distoma exasperatum R. n. sp.}

Am. Bremser specimen misit in intestinis Soricis Eremitae repertum, fuscescens, duas lineas longum, antice dimidiam crassum.

Corpus teres, posteriora versus sensim attenuatum, apice antico rotundato, posteriore cmar. ginato; superficie tota transversius striata, vel mi- 
croscopio adhihito, tenuissimis aculeis rectis obsila, pratsertim ad margines pori ventralis. $\boldsymbol{P}$ orus arlicue magis inferus quam terminalis; ventralis multo najor; utriusque apertura transversa, an post mortem talis facta?. Inter poros cirrus cornu spirale crasciusculum albidum referens.

Obs. Species distinctissima, nєque cum Distomare Vépertilionum, neque cum illo Mustelarum, Erinacel et Melis, omnia licet aculeata sint, conveniens.

\section{Disloma cristatum R. n. sp.}

In duorum Stromatum Fiatolaruin ventriculo Ariminí Majo decem reperi specimina teretia, torulosa, alba, parte media viridescente, duas ad tres cum dimidia lineas longa, duas tertias lineae part: $\mathbf{s}$ diametro transverso raro excedentia, motu yulgari Distomatum. piscinorum vividissimo utentia.

Pori subaequales, anticus longior; ventralis approximatus, subglobosus, fere major. Antica pars inflexa, quo porus anticus ventrali inferior fit; in nucha tuherculum exstat, quod nomini ansam dedit. Corpus circa porum ventralam crassissimum, brevi post subito contrahitur, ut reliqua pars multo tenuior sit, caudam longam mentiens. Sup rficies tota excepto ultino partis tenuioris apice aculeis obtusiusculis obsita; nuchae tuberculum pariter aculeatim. Vasa anteriora postice angulo acuto conveniunt, vas quasi furcatum sistentia; praterea oviductuum gyri minus bene dignoscendi in conspectum veniunt.

Obs. Habitus Distomati appendiculato affinis, a quo tamen aculeis, nuchae tuberculo, co. 


\section{$-423$}

lore plurimum differt. A specie insequente po. roruin ratione praesertim recedit.

120. Distoma hispidum Abildgand.

Ent. II. I. p. 435, 17. 74. D. Sturionis,

In intestino Sturionis minozis, quatuordecim tantum pollices longi, Arimini Majo vivi al. iati, duodecim reperi specimina, duas cum dinaidia ad quatuor lineas longa, teretia, motu vividissimo pro more pollentia, alba.

Pori globosi, anticus minor. Nucha, colJum et anterior corporis pars aculeis reflexis hispida, ita tamen ut aculei nuchae sacpe magni et discreti in conspectum veniant, et collum dilatatixm duodenos circa echinos majores utrinque offerat, neque locus, qua pars inermis incipiat, certus videatur. Mox enim dimidia tantum totius pars armata, mox ultima tantum spinis est libera. Nítlia in corporis parte ovorum receptacula magna ovalia; utrinque ad latera ova globosa conspici. untur. Cirrus tortus obscure tantum exhibetur.

In Sturionis majoris crasso Berolini Junio quatuor reperi specimina vira, quatuor lineas longa, partem anticam (sesquilineam circa emetientem) alba, reliquam flava vel flavoirrorata. Hispida. Porus terminalis exstans, in mortuis quan. doque fere major visus. Pars posterior ovis repleta rotundis flavis, vaseque spirali medio pa. riter fiavo notabilis.

Prexrerus etiam specimina complura misit in intestinis Sturionis reperta, unam ad tres lineas longa, fuscescentia (certe spiritus vi), teretia, undique hispida, spinulis rectis vel re. flexis. Porus ventralis antico terminali multo major; collum quibusdam contractum et tumi 
dum, aliis extensum corpore tenuius, versus po. sterioz'a crassiusculo,

Obs Speciem in Entozoologia ex Vtrorgrt tantum indice mutuatam dubias inter collocivi, utpote non descripian; distincta tamen est apecies, etiam a D. laticolli n. I 16 . et D. cristato $n$. Ir9. quod cuique descriptiones comparanti patebit; insequenti forsan fimis affinis. Vibolzgrus eandem in Sturionis ventriculo repertam relert.

121. Distoma scahrum ZED.

Quod Müuzerus in Gadi barbati ventriculo reperit, ipse in intestinis Gadi .Holvae (majoris quam illius, in quo D. furcatum et fulvum, Ne. apoli Junio offendi.

Sprcimina complura, sesquilineam Ionga, teretia, allsa. Pori glohosi, ventralis major, 1 : tt Mülneres antico aequalem dixe:ir; hic terminalis, corona echinorum densorum - rectorun cinctus. Collum infra hunc constrictum, quo caput discretum fiat, ceterum ad porum ventralen u^que hispidum. Corpus laeve, collo parum crascius, saepe pone porum posticum majori vel minore parte tenuis; apex posterior obtusus.

Ova corporis partem posteriorem replent; vas in collo transversum utrinque retrorsum decurrit, praterea vas adcst mediun; nigrescens autem, quale Mium.rents habet, non vidi.

Obs. Specics ista praecedenti valcie affuis, echinorum corona, corpore mudo, et habitu distinguenda videtur.

122. Distoma contortum R. n. sp.

In Orthrogoriscorim Molarum duarum branchiis Neapoli Juho mense vigiuti et quod cx- 
currit Distomata offendi, die insequente (aqua saepius renovata) adtuc viventia, quin vividissiina, simular tangerentur, in'annulos vehementissime se contorqueritia, et spiritui vini immissa, pariter spiralia manentia. Quam ob caussam longindo atgre determinari potuit, plurima tamen pollicem superant. Pars antica alta, posterior (pone porm ventralem incipiens) carneo flavesctus, lateribus albidis; vase dorsali utringue tenui fuscescente a poro antico aci caudam decurrente, que fit ut verme convoluto et colio circa porun auticum flexo, vasa duo circulum duplicem. elfingert videantur.

Pori subglobosi, ventralis duplo major, plerumque peciunculo brevi et tenui, interdum valde clongato insidens. Collum longum, latere dorsali convexo, ventrali obiter concavo, undique aculeis exiguis, lente tamen simnlici bene observancis, ad porum ventralem usque ubsitum. Corputs teres longissimum, posteriora versus attenuatum. Circum non vidi.

Obs, 1. Nullo in Distomate tantam contractilitatcm -vidi, quantam haec species exhibet, sed branchiis piscis rigidis, continuo motu agitatis, et aqua marina alluendis afixa majori quidem $v \dot{i}$ contractionis et tenaciori vita indiget.

Obs. 2. De discrimine inter hanc et insequentem speciem eodem in pisce obviam infra serno erit.

123. Distoma nigroflarum $\mathrm{R}$.

Ent. II. 2. p. 257. Schisturus paradoxus.

Iisdem in OrLhragoriscis MIolis, qui praecedentem obtulerunt speciem, etiam hanc reperi. Plurima specimina ventriculo et intestinis, 
duo vel tria faucibus prope branchia inerant, pauca duas, rmulta tres ad sex lineas longa, yuae pollicem superabant, rarissima, unum tamen pollicem et undecin lineas aequabat; hoc tenuius, reliqua corpore crassiore.

Color generalis quidem albus est, sed albedinis praeter poros vix quidquam vivis in con. spectum venit, vase dorsali ramosissimo crasso, ramis pariter crassis et brevibus, nigrescentibus, oyiductibus vero luteis plurimum contortuplicatis, annulos quasi effingentibus, latera et postremam corporis partem sibi sumentibus.

Pori subglobosi, ventralis multo major, apertura saepe longitudinali, pedunculatus, pedunculo saepe satis longo. Collum breve conicum, cujus pars antica cum capite aculeis minimis obsita. Córpus circa porum ventralem saepe tumidum (et dorsali et ventrali latere), dein cylindricum, apice obtuso apertura instructo, quae saepe materian fuscam eflundit. Cutis mollis, totusque vermis flaccidus, post mortem rectiusculus aut simpliciter curvatus exhibetur. Cirrum non. vidi.

Obs. I. Species haec a praecedente plurimum differt, loco, colore, mollitie, collo conico, n€que supra convexo subtus concavo. Quae per anuum spiritu couservata sunt, eodem adhuc modo facile discingui possunt specimina; praecedentis enim speciei contorta, duriuscula, haec molliora, rectiuscula, colore illorum albo, horum obscuro, collo in utrisque diverso.

Obs. 2. ReDr, viri cel. vermem paradoxum, in ventriculo et intestinis Orthragorisci Molae repertum huc pertinte crediderim. Plurima saltem satis bene quadrant, neque descriptionem 
exactam suo tempore dabant, sed obiter haec illave inlicabant. Vermem inveriendo, porum anticuin collo et ventralem pedunculo insidentes pro cauda duplici habuit, cujus orificimn utrumque apertura genitalis diceretur. Orificium intermedium pro ano veniens forsan cirri receptacu. lum fuit, Si autem figura ab ill viro oblata vere magnitudinem naturąlem exhibet, specimina gigantea vidit, De illorum motu rilit relert mortua igitur fuisse videntur, quod colorem albidum iisdem tributum explicaret. Interanta descripta, explicatione Rediana seposita, illa Distomatum sic satis exhibent neque ad alius ordinis entozoa referri possunt.

Bremserius, Helminthologorum decus, Distomate furcato n. $7 \%$ detecto, Schisturum ad idem genus revocandum esse in litteris monuit, neque negandum est, illud licet maxime pusillum hunc optime referre.

\section{Distoma Wachniae.}

Trumsius (Mém. de l'Ac. des Sriences de St. Petersbourg T. II. p. 363. et 374. Tab. XIX. Fig. 8-10.) Distoma in Gadi Machniae intes!inis repertum obiter refert, quod forsan ad $D$. scabrum n. I2I. pertinet. Ejusdem antem piscis Distoma forsan renale vidit, quod inter entozoa dubii generis n. 54. Synops. p. 195. relinquere coactus sum.

\section{Genus XVI. Tristoma.}

Cel. olim La Marminare, qui serunciam speciem detexit, ejusdem et descriptionem et figle. ras dedit, locum vero in Systemace Naturat 1ion 


\section{$-428$}

adnotavit, illam obiter insectum vocando. Cel. Bosc de ejusdem loco valde dubius, crustaceis tamen suctoriis afinem credidit et barbaro nomine Capsalae distinxit. Col. Oжrм in Historiae Naturalis Enchiridio ditissimo eandem illarn spe. ciem nomine generico, qiod etiam Caryophyllaeo olim fuit impositum, Phylline insignivit; de loco auten pariter dubius fuisse videtur, primum enim p. 1\$2. Lernaearum, deinceps autem p. 370. Hirudinum familiae addidit, quid? quod Hirudines a Tristomate maxime alienas, Hirudinem Hippoglossi et ir. grossam, ad idem Phyllines genus retulit.

' Non possum, quin ill. Cuvierus sequar, qui prinam speciem dictegendo, genus et nomine congruo anxit et Tremarodibus addidit. Poros aut horum saltem posteriorem discoideum et radiatum canale sensibili destitui nihil refert, nam Distomatis porus ventralis pariter impervius est, quin Pentastomatis porus impar tantum oris vicibus fungitur. Proboscis, quam La Martanrere dixit, forsan cirrus fuit, qua de re infra scrmo erit. Qund mili autem rem conficere videtur, Tristomatis vasorum retia illis Trematodum mire respondent, neque intestina, cl, viro in specie secunda dicta, ab oviductibus sejungenda credidissem.

1. 'Tristoma coccinem Cuvier, n, sp. Tab. 1. Fig. 7,8 .

Cevier Regne anima! T. IV. p. 42. Tab. 15. Fig. 10. Tristuma coccineum.

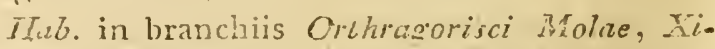
phine Cladii, alicrumque maris mediterranei piscium, Crviero teste; ipse nunquan repeli. 
Pollicem magnituiine aequare vel superare et coloris esse lacte rubri l c. refert; quae ill. vir pro sua in rue benerolentia mandavit, specimina duo, srisea sunt, dorso pallidiore, abdomine obscuriore ad Vasorum anbitum fuscescente; altcrim novem lineàs et longum et latum, alterum fere stptem longum, sex cum dimidia latum.

Corpus utrinque planum, suborbiculare, marginis anterioris lobulo medio distincto, posterioris incisura duas lineas longa, quo corpus antice trilobum, postice hilobum vocari possit, marginibus loborum rotundatis, ceterum toto in ambitu tenuibus. Ad lobuli anterioris incisuras (in latere inferiori) uiringue ports orbicularis lineanz dimidian diametris adaequans, laevis, obiter concavus, imperforatus, basi tantum media exigua affixus, ambitu toto libero. Hos inter poros et nonnihil quidem magis ad posteriora foramon conspicitur, ex quo corpusculum crasciuscilum obtusum errinet, quod nullo modo exricare potui, analogiae autem ratione habita, facilins pro cirro, quam pro proboscide haberem, qualis secundae speciei tribuitur.

In postericre abdominis parie et a margine quitem anteriore ad quatuor cum duabus tertiis lintearum et a posteriore ad unius com rluabus lineae tertiis partibus distartian Orbiculus conspicitur radiatzs, in minore specimine duas lineas, in majore duas insupce lineae tertias partes longus et latus. Fjus in centro discus consfyicitur orbicularis (in utrogue specimine lineam lon. gus et latus) a quo septem rarii elevati marginem externum crassiusculnm, subinflexum et fere undulatum petunt; radiorum impar antrorsum di. rectus, ceterum omnes aequali distantia symme. 


\section{- 430}

trice positi. Superficies disci et loca inter radios laeves granulata sunt, granulis exiguis elevatis. Orhiculus disco solummodo affixus, reliqua parte circun circd libera.

l’ori antici molles uti tota substantia, orbiculo quódammodo excepto, qui duriusculus, neque tamen durior est, quam variorum entozoorum variae partes.

Vasa totum corpus perreptant et retia efficiunt, quorum fasciculi ramosi ubique peripheriam petunt, et sigillatim terminantur ut tenuis tantum margo illis carens et pellucidus sit. Nullam praeterea partem neque vasorum centrum quoddam detegere licuit.

2. Tristoma maculatum R. n. sp. Tab. 1. Fig. 9. 10 .

La Martintere in Journ. de Physique Septbr. 1787. p. 207. Tab, 2. Fig. 4, 5 .

Iofm in Voyage de la Perouse autour du monde. T.IV.Paris. 1798. 8. p. 79. Tab.20. Fig.4.5.

Bosc in Nouv. Bull. de la Soc. Philom. 18I I. p. 384. Capsala Martinieri.

Oren Lehrbuch der Naturgeschichte. Leipz. 1815. 8. Th. III, I. Abth. p. 182, et 370. Phylline Diodontis.

La Mantintere Comes illustris Navarchae La Perouse Tristoma hocce corpori Diodontis, quem inter Nootka et Monterey Californiae saepius offendit, afíxum vidit.

Magnitucio eadem-ac speciei prioris, aut paullo major videtur. Coloris est surdide albi, dorso maculis consperso exiguis ovalibus lividis, aut aucioris verbis utendo, colorem faecis vinire. ferentibus (de couleur de lie de vin). 


\section{- 431}

Forma, pori antici, posticus radiatus aut septemcostatus, exacte speciei prioris. Ope porórum corporibus adhaeret. Proboscis inter poros anteriores, antrorsum aculeolis (pointes) aspera, quos auctor tot suspicatur ostia, quibus piscium, quorum corpus tenet, sanguinem hauriat. Subtus corriplures intestinorum gyri translucent, qui parvo receptaculo fere quadrangulo inmittuntur. Ipse haec aliter explicarem et intestina oviductus, rcceptaculum autem ovarium dicerem. Num p o. boscis potius cirrus sit, incertum rclinquo, cum nullius Trematodis cirrum asperum vel aculeatum viderim; reliquorum autem animalium pene saepe acruleis munito, idem etiam de hujus gene. ris phallo valere posset.

Mox anterioribus, mox posteriore utexdo poris animal progreditur, se autem convolvendo aquae fundum petit, La Martiniere teste, quod reliquis Trematodibus analogum est, ideoque autem substantiam ćartilagineam, quam illi tribuit, vix admiserim.

Obs, I. Cel, vir superiorem proboscidis partem aculeatam refert, sed etiarn poros anticos ipse superiores vocat, licet inferiores sint, ut vermen erectum certe sibi finxerit; prohoscidem idcirco antrorsum aculeatam dixi, qualem etian icones referunt.

Obs. 2. Cel, OKEN 1. c. p. 847. n. 1S2. icones in Diario physico exhibitas illis Hodoepo. rici Perousiani praeferendas esse refert, quod memoriae lapsu factum esse videtur, nam Diarii tabula neque proboscidis indolem, neque ovidu. ctus exprimit, qúo commotus Hodoèporici figu. ras recudi curavi. 


\section{Genus XVII. Pentastoma.}

De Pentastomatis genere non ad Cestoidea, sed ad Trenlatoda pertinente, haec generatim sumta exponendo, locutus sum. Species periparcae vicientur, attamen a Polystomate, cujus sui. divisionem olim efficiebant, utpote nimis diversae, separandae fuerunt. Species quatuor priorcs simillimae, quid? quod secunda, tertia et quarta vix sufficienter distingui possunt, cum duas po. steriores autern non viderim, Easdem supprimere non ausim. Quinta naxime divera, notas tamen proprit generis non continet, quod illam tradendo illustrabo.

\section{Pentastoma taenioides $\mathrm{R}$.}

Ent. r. p. 44!. n. I. Polystoma (Pentástoma) taenioides, Taenia lanceolata Crabertis.

In Múséo Viennensi tria vidi specimina in Canis Lupi sinubus frontalibus reperta, yuor $\mathrm{m}$ alterum octo lineas, secundum policem et duas lineas, tertium tres pollices et quatuor lineas longitudine aequabant, hoc simul crassiusculum, ceterum nullo modo a caninis diversa. In Sc'ola Veterinaria tam Berolinensi, quam Viennensi. in equorum sinubus frontalibus nunquam repe úm est, yuo commoti Brimserus et ego lere genéri canino soli tribuissemus, sed nuper testis egregius Chaberti exsurgit. cel. Greve, qui (Ueber die Krankheiten der Hausthiere. Oldenhurg. I813. 5. 194.) se in Muli cellulis ethmoidalibus iden reperisse refert.

Quae anatomen hujus specici spectant, in Mantissae sectione insequente traclam. 


\section{$-433$}

3. Pentastoma emarginatum R. $n$. sp.

Boss in Nouv. Bulletin de la Soc. Philo. mat. rsur. n. 44. p. 269. Tab. 2. Fig. $x$. Tetragulus Çaviae.

Cel quondam Le Gatlors in Caviae Cobayae pulmonibus quadraginta et quod excurrit specimira Parisis $\$ 810$ reperit, cum cel Bosc communicata, qui l. c. descripsit.

Dimidiam ad seqquilineam longâ, vix quartam lineae partem lata, lactei coloris, corporis utroque apice emarginato.

Porus medins sive $O$ s simplex, orbiculare, magnum. Poros laterales Boscivs tacet, et uncinos tantum refert ex isdem oriundos, quos valde magnos delineari curavit, et corneos et basi cras. siusculos describit, qui in Pentastomatibus taeniode et denticulato milhi solummodo visis valde tenues sunt. Cel vir de ejusmodi rebus tamen minus sollicitus esse solet, ut figurae magnam fidem non tribuam.

Corpns depressiusculum, retrorsum decrescens, transversim seriatim denticulatum, antice emarginatum, apice postico anum terminalem $\in \mathbf{x}$. libente.

Obs. 1. Cl. vir Tetragulum sihi dictum in ipsa pulmonis substantia occurrere, et cava, quibus inhaeret, margine prominulo et rubro di. gnoscí.

Obs. 2. In Porcello indico antea nunquam entozoa reperta sunt, et Naturaescrutatoribus $\mathrm{Mu}$ sei Viennensis vere Iynceis hanc circa rem non contigit quam mihi esse felicioribus. Cum cel. 


\section{- 43 年-}

autein LE Gatlors saepissime experimentis suis cuniculos subjecerit, et Pentastoma hoc illi in Leporis pulmonibus occurrenti, P. serrato n. 4. simillimum sit, an forsan Cuniculi pulınonibus Pentastomata haecce inhaeserint, fere dubium moverem. Confer observationcm in Taeniam festivam $n \cdot \%$.

5. Pentastoma proboscideum R.

Ent. II. 1, p. 433. n. 69. Distoma Crotali Durissi.

Humbord in Recueil d'Observations de Zoologie et d'Anatomie comparée. Fasc. 5. et 6. Paris. 1809. 4. n. XIlI, p. 298-304. Tab. 26. Porocephalus Crotali.

Rudol.phr in: Magazin der Berlin. Gesellsch. Naturforsch. Freunde B. VI. p. 106, n, 63. Po. lystoma (Pentastoma) proboscideum.

Quos ill. Alexander ais Humiboldt in Cu- manae Crotalo Durisso detexit vermes, primum pro Echinorlyynchis, dein pro Distomatis specie armata habuit (quo commotus in Entozoologia Distomatibus dubiis addidi), postmodum autem Porocephali nomine descripsit et delineavit, huc uique referendi sunt.

Specimina in Durissi pulmone et abdominé reperit sesquipollicaria ad bipollicaria, unam duas. ve lineas lata; maximum duos cum dimidio pollices longum, antice fere quatuor lineas, postice fere unam latum; ex albo flavescentia, versus extrema albidiora; proboscide exserta et a latere visa larvas Bombycis Mori referentia. 
Ex ill. viri descriptione: Corpus cylindricum, subclavatum, postice obtusum, pellucidion, transverse rugosum, margine subcrenulato. Cóllum nullum. Proboscis ventralis, antice praemorsa, oris leporini in modum fissa, subemarginata. Aculei quinque fuscescentes, retractiles, uncinati pone os in parte inferiori corporis poriti. Clia. ractere generico iidem aculei arutuci retractiles, in foveis sub proboscicie siti dicuncur. Anum non vidit.

Oviductus longissimos rel. vir pro intestino, vas dorsale album, anteriora versus fissum, pro nervo habet.

Obs. Species tam magnitudine, qua primae hujus gentris speciei soli cedit, tt corpore tere. ti, in reliquis plano vel depresso, insignis; quin. que praeterea uncinis instructa dicitur, quorum reliquae quatuor tantum exhibent, ut illius ostium medium pariter intrme haberem. Num proboscis vera sit, maxime dubitum videtur, cum iconibus saltem non exprimatur, neque reliquis concessa sit speciebus. Icon apicem anticum emarginatum refert, et proboscis ill virc dicta nil nisi pars poris anterior et pro more extensi. lis videtur.

Si-autem re vera quintus adesset aculeus sive uncinus, idcirco genus novum non exstru. erem. c is.

\section{Genus XVIII. Pólystoma.}

Paucae species, quarum illae homini trihutae suspectae sunt, genus maxime naturale etfin. 


\section{$-436^{\circ}=$}

gunt; néque Polystomatis duplicati poros ostio duplici instructos, reliquis convenientibus, idnti facerem, ut pronovi generis charactere habcrem. Verum quidem est, nullius praterea Trematodis porum biforem inventurn esse, seid inter Cestòidea bothria divisa rara non sunt, neque genctis notam exhiberit. Ipse saltem naturae scrútator, "cuùi Polystoma Thynni sibi dictum detexit, vix pro no. vo genere habuit, dun liujus notncin illi imponeret. Lasankius quidem in animalium non vertebratorum historia distinxit, sed alia agendo, nam Pentastomata et Polystomáta Linguatulae nomine olim his imposito conjungit, Polystuma Thynni et Porocephalum autem qua propria ge. nera $a b$ his separat.

\section{Polystoma ocellatum R. n. sp.}

\section{Arimini Majo Testudinis orbicularis palato} duos firmissime inhaerere vidi vermiculos rubros, quos mox Polystomata agnovi, speciei primae (P. integerrimo; in Ranae vèsica urinaria habitanti) proxima," attamen diversa.

Longitudo sesquilineam aequat, latitudo dimidiam lineam superat. Color carieus, verme contracto magis intensus, vasis nimirum sanguirei coloris pellucentibus tum' 'magis' approximatis, apice caudali albo. Forma corporis poly morpha, plerumque ovata, apice postico addito; dorsum convexum, abdomen vel concavum, vel planum.

Pori sex antici subglobosi, eodem quidem modo ac in $\mathbf{P}$. integerrimo dispositi, ceterum autem diversi, Margo illorum anterior plus, mi- 


\section{$-437-$}

nus contractus; externus replicari potest, tum circulus exiguus conspicitur, quem in $\mathbf{P}$. integer rimo non vidi. Pori a latere spectati majus ad. huc discrimen offerunt, singuli enim tum tribus partibus sibi impositis constare videntur, quarum infina orbiculum supra dictum sistit, cujus ope portis corpori nectitur et versatilis fit; secunda jaulto latior, tertiaque sive summa et latissima porum et hujus marginem sistunt. In media inter omnes poros parte eaque anteriore punctum est, 3. quo cutis inter singulos plicam acutam profert extrorsum in cute evanescentem. Hamulos, qui P. Integerrimo conceduntur, hac in specie non vidi,

Porus ventralis caudae, quam apici anterior: propior, exiguus; caudalis illo major, quam pori antici tamen minor, a latere fissus, fere iners aut parúm mutabilis, saepe riman fere sistens, cum illa Distomatis appendiculati comparanda. Semel etiam partem fere consimilem ad exitum paratam, sed dein visui obductam contemplatis sum. Ante porum caudalem utrinque macula pellucida, ocellaris, quaé primum porum mentiebatur, unde nomen triviale desumsi. Vasa ob vermis opacitatem rarius in dorso vidi, neque prosequi potui. Ova in dorso ad latera congesta conspici. untur.

\section{Polystoma Pinguicola ZẼ.}

Cel. Treutreri éntozoorum collectionem Dresdae 18r7. perlustrando in hane speciem praeprimis inquisivi, sed humanorum Vermium a cl, viro olin descriptorum nullus superfuit, Phi. 
alam quidem benevolé mecum communicavit, quae" Pinguicolam forsan contineret, sëd ean. dem Berolini attentissinie perscrutando non nisi corpirscutur nigrum contracam et dưrum reperi, quod omuem organisationis notam dene. gavit.

4. Polvstoma duplicatum R. n. sp. Tab. 2: Fig. 6.

IA Roche in Nouv. Bull. de la Soc. Philom. ix 1. n 44. p. 27r. Tab. 2. Fig. 3. Poly. stoma Tliynini.

Cel. vir in insularum Balearium majore Polysomathece Scombri Thynni branchiis pororum ope fortiter inlaerens reperit, molle, laeve, gristum, duos circa centimetros (sed mensurae lirearis a $\mathrm{cl}$ viro in tabula datae ratione habita, sépten tantum lineas) longum.

Depressum, oblongum, pone partem anteriorem oltusam et antrorsum increscentem, con. strictum, corpore subovali, apice postico tenui obtusiusculo. Pori sex anteriores inferi, in figu. ra lunatim positi, singuli septo transverso in duo cava divisi, quorum quodvis basi foramen exhi. bet, cl. viro, cum alia apertura oris vicibus fun. gens desit, pro isto merito habitum. Reliquis Polystomatibus tanturn sex, huic autem duodecim ora concessa sunt. Inter poros inedios duo tuliercula conica, aut tentacula brevissima, aegre visibilia. Apicis postici rimam longitudinalem inferam pro ano habet.

Germina hujus entozoi sub branchiarum 


\section{- 439}

thynni cute propria deponi suspicatur, quia vermiculi sub eadem tumores parvos griseos et ova. les efficiunt.

Obs. Mirum est porum ventralem in specie prima et secunda mihimet visum, in tertia TreuT. IERo pariter dictum, a cl, viro non relatum esse, Num vere deest? 


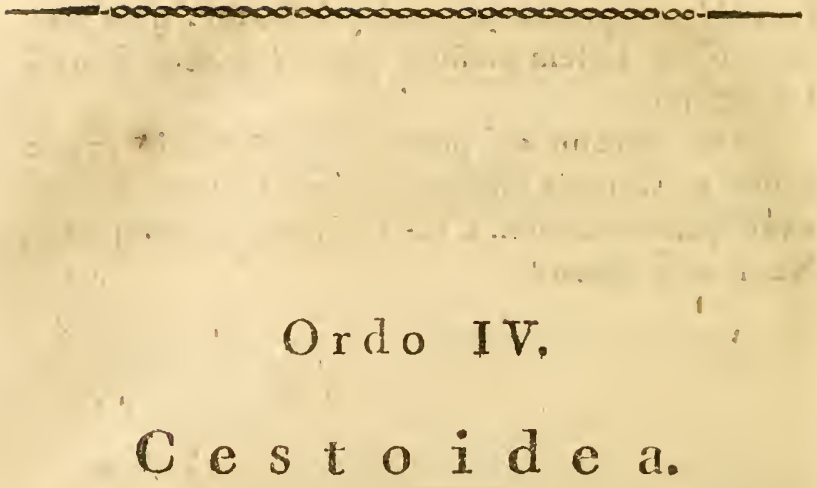

Quae in Entozoologia de hoc ordine retuIi, repetere nolo, nam gentribus licet quibusdam addiric, earlem civisionis ratio in articulata et tion articulata valet; duos tanien indt non exstruxi ordines, cum genera articulatorum illis relicuorum sint proxima et variis modis transitum offerant. De simpliciorihus, quae Tremato. dibus magis afinnia sunt, ordịndum est,

\section{Genus XIX. Caryophyllacus.}

Cel, Zenenur, cui Helminthologia plurima debet, in Entozoologia secutus marem et feminam ab illo distinctos retuli, licet ipse individuorum discrimen sexuale nunquam repererim; res autem magis magisque dubia facıa est, et Caryophyllaeúm nonnisi androgynum habere possum. Tanta quidem specimina, ac cel. vir oculis usur. pavit, ipse nunquam vidi, sed analogia nimis obstat, quo minus illi adsentire possim. ZEDErus autem solus cirrum vel lemniscum exsertum vidisse vidétur. 
1. Caryophyllaeus mutabilis $R$.

Praeter pisces in Synopsi enumeratos, etiam, Cyprimun Dobulam nominare possum, cujus Caryophyllaeos nulfa nota discingueridos aucrumna Isı. Berolini recentes examinare contigit.

Cel ar Orfars (De Vegetativis et Anima. tis p. 41.) se Februario mense in proventricula Mergi Verganseris duos Caryophyllaẹos invenisse referl, in cavo parietis abnormi nidulantes, pollicern circiter longos, antice lineam quarta parte superantes, postice dimidiam aequantes. Caput val. de incrassatum, multifimbriatum, microscopio subjectum radicis fasciculatae formarn exhibuit; ore inter fimbrias medio; corpus depressum postice sensin atteruatum, lateribus integris. Hactenus Amicus. Ipse Carycphyllaeum fuisse vix admitterem, sed eundem potius haberem vermem pa. radoxum, quem olim in Colymbi septentrionalis ventriculo reperi: conf. Entoz. If. 2. p. $266 . n_{*}$ 8. Synops, gen, dub, p. 188. n. 24.

\section{Genus XX. Scolex.}

Entozoologiam edendo Scolecem verum nondum videram, pisces maris Baltici licet quam. plurimos cultello subjecissem. Italiam autem petendo, tam in Adriatici, quam in Mediterranei maris piscibus illum saepissime et passin fre. quentissime reperi. Quo factum est, ut omnia de hujus generis constantia rnox dissiparentur dubia. Nullum forsan datur animalculum magis proteiforme; mille modis variat, ut incautus facile complures in species dilacerare posset; qui autem saepius observat, facile habitum ejusden 


\section{$\therefore 442$}

sibi familiarem reddet. Est praeterea vivi nola fallere nescia, passim mox, saepe autem post examen per horam protractum vix observabilis, cùm certo Scolecis situ tantum pateat: puncta duo volo corporis albi sanguinea, saepe fulgentia, qualia nullis in entozois aliis videre licuit, quaeque in Gobii minuti Scolece vasa duo rubra parallela pone caput incipientia et retrorsum ducta, in corpore autem Evanida, effingere observavi. In reliquorum piscium Scolecibus puncta illa num quam tantopere elongata vel explicita vidi.

1. Scolex polymorphus $R$.

Specialia quaedam de variorum piscium Scolecibus referam, dicta quo melius illustrentur.

In intestino magni Lophii piscatorii Tergesti Aprili quam plurimos reperi, et omnes figuras a Müllero oblatas bonas esse, multo plures vero addi posse, agnovi. Os terminale est, bothria vero quatuor ad latera circum circa disposita et tantopere versatilia, ut minima quidem parte affigantur necesse sit.

In Ophidiis sex baróatis Arimini Majo intestina Scoblecibus referissima reperi, ut illa ape. riendo hi tabulam tegerent, et aqua quibus eadem immisi vermibus scateret,

- Stromateos Fiatolae ibidem eodem mense dissecti omnes mesenterii et peritonei ventriculum et intestina induentis partea Scoleces fovebant, quos primo intuitu pro Echinorhynchis parvulis habui.

In Labro luseo, Neapoli, Junio, tam nudum intestinalem, quam vesicula inclusum in hepate aliisque abdominis partibus peritoneo involutis reperi. 


\section{$-443=$}

Specimina quae Aprili mense Arimini - in intestinis Polypi vulgaris (Sepiae octopodiae) et Gobii nigri nec non ventriculo et intestino Tor. pedinis marmoratae; quae ibidem Majo tam in intestinis Gadi Merluccii et Squali Acanthiae quam in peritoneo Pleuronectis naximi; vel Neapoli Junio in intestinis Scorpaenae Porci, Spari Boo. pis, Apognini, Clupene Encrasicoli, et ibidem Au. gusto in illis Cotti Gobii referi, nullo modo a Müllerianis differunt.

Quod Arimini Majo in intestino Gobii minuti offendi specimen, uti supra retuli, pun. cta sanguinea in vasorum formam elongata ob. tulit.

Quem Neapoli Julio mense in intestino Spari novi ibidem Schiandra dicti reperi solita. rium, solito majorem, et fere duas lineas longum, passim contractum, a vulgari Scolece tamen di. versum non haberem. Quam facile enim alienas formas induit, sequens etiam docet observatio.

Pridie Calendas Augusti Neapoli in intestino Lepadogastris Gouani Scolecem offendi lineam dimidiam longum. cujus caput subrotundum bothria nunquarn bene conspicua exhibuit, apicis autem quandoque partem parvam subglobosam antice dentatam emisit; cuius vero corpus rectum lineare interdum utrinque tali modo contractum fuit, quo moniliforme appareret. Ipsis dein Calendis in alio Lepadogastre Scolecem inveni yividiorem, qui puncta sanguinea, bothria etc. obtulit.

Quos in Ophidiis reperi, saepe inter se cohaerere vidi, ut alter Scolex alterius majoris apici calndae rectus affixus esset, -alter autem alterum 


\section{$-444$}

medio teneret et sic porro. Lophii etiam Scoleces Distomatibus saepe inhaerere vidi, Num inter Entozoa etiam bellum obtinet?

Obs. Cel. ar Ozfers (De vegetativis et animatis p. I09. Fig. 17-20) in Gasterostei aculeati recto vermiculos reperit, tertian lineae partem longos, pentastomos, quos novo genere compre: hendit, et Rhytelminthi Taeniolae nomine enume: rat. Cum cel. viri autem pace veras Taeniolas habeo, forsanque Taeniae filicollis sobolem, quem? zdmodum Scolex tetrastomus olim mihi dictus Taeniae longicollis prolem sistit, et Taenia Linea pariter talem exhibet articulis destitutam, tạndem evolvendam, quarn conferas.

Genus $X X I . G$ gmnorhynchus.

Genus corpore depresso continuo, longis. simo; colli receptaculo subgloboso; capite bothriis duobus bipartitis et proboscidibus quatuor nudis instructo, $a b$ omnibus reliquis Cestoideis recedit, neque Scolecem esse, Cuviero, Viro summo concesserim. Cui sententiae probandae descriptio insequens sufficiet.

1. Gymnorhynchus reptans R. n. sp.

Cuvis Regne Animal T. IV. p. 48 . Scolex Gigas.

In Spari Raji carnibus a capite ad caudam. uaque omnibus, quás longissinus perreptat, Ne. apoli Junio et Julio semper reperi.

Vermis longitudinem tripedalem attingit, lineam ad duas lineas latus, crassus, albus excepto colli receptaculo flavescente, sạtis difficile inte- 
ger extricandus, 'cum carnes Spari gyris multiplicibus saepeque praeter opinionem-ductis teneat.

i) Caput cim proboscinibus seśquilineam lon. gum, subtetragozum, fere Bothriocephali, bothriis duobus planiusculis bipartitis instructum, antice (horum ex margine uti videtur) proboscides emittens capite longiores, tetragonas (angulis rotundatis), externe papillis minimis rotundis creberrime obsitas, apice perforatas, inermes sive unci-nis destitutas. Collum capite aliquoties longius desinens in Receptaculum (capitis et colli) quatuor ad quinque lineas longum, tres latum, sphae'xoideum vel inverse' ovatum, plerumque flavum, caput abscondens et emitiens. Corpus pone receptaculüm uiplurimum contractum, dein mägnam. partem subaequale, depressiusculum vel teretiusculum, passim. constrictum, tum sensim tenuius factum, ultimo apice tenuissimo, angustissimo, obtusiusculo, saepe flavo terminatur.

Tota Gymnorhynchi substantia mollis, hb' mogenea, neque corpore discisso vel disru. pto organiorum intersiorum, vel ovorum speciem prodit.

Obs. Entozoà singularia in 'Sorice (MYygale), Erinaceo, Falcone et Strige reperta, Synops. gen. dub. p. '185. n. 8 p. 186.' n, 9. 'p."187. 11. 19. et 20. habitu quidefn ad Gyinnorhynchim accedunt, illorum autem caput ignoratur, ut comparatio nondum locum inveriat.

\section{Genus XXII. Tetrarhyncíus.}

Redr, vir summus Tetrarhynchum primus descripsit, et in Argentina Sphyraena quidem re- 
pertum, mihimet Argentinis plurimis Romae dis. sectis non visum. Goezıo dein contigit, qui aliam speciem in Salmone Salare obviam exami. naret, sed cum haec $\in$ minoribus, neque alia illi species oblata sit, indolem ejusdem perspictre non potnit et Echinorhynchis addidit, quem Guelinus in chaotica sua farragine secutus RE. Dranum qunque animalculum illis immiscuit. Tertia dein species a cel. Bosc in Coryphaena Hippuride detecta et pro novo gentre Tentacularia sibi dicto habita et obiter descripta est Quartam quidem speciem AmrdogaArd in Gado Mlurhua vidit neque tamen descripsit.

Ipse Entozoologiam edendo nullam hujus generis speciem videram, et Tetrarhynchos mibi dictos, quales descriptos inveni, relinquere co. actus fui. Aliquot ante annos vero am. Gaene, nunc Professor Leodiensis, specimina obtulit speciei tertiae in $X_{i}$ phiae branchiis a cel. F1scher, Anatomico Kiloniensi reperta, et Tetrarliynchum Cestoideis neque Acanthocephalis adnumerandum esse mox vidi. Ill. Cuvare, qui Cestoideis Ligulan solam relinquit, qua de re hanc descri. bendo tractabitur, Tetrarhynchum etiam Taenioideis suis merito adscripsit. In Italia Tetrarhynchos quam plurimos vivos examinare mihi contigit, et cum divitiae divitiis facile addantur, redux Berolini tales iterum vivos reperi.

$\mathrm{Ob}$ proboscides uncinatas solas genus hocce olim Echinorhynchis additum fuit, postmodum autem Bothriocephali corollati organa consimilia reperi et ill. Cuvier genus Floriceps sibi dictum, graeco nomine mihi Anthocephalus vocatum, detegendo, hujus iterum affiritatem maximam cum Tetrarhynchis aguovit, Caput, bothriis instru. 


\section{- 447 -}

ctum, corpusque solidum et molle, vividissimis variisque motibus aptum, ab Echinorhynchis penitus recedunt.

Anthocephali mihi dicti complures nisi omnes ad Cystica pertinent, ut a Tetrarhynclis cystide caudali destitutis separari opus fuerit. Ho. thriocephali quidam Tetrarhynrlis pariter altines dantur, sed corpore articulato nimis difierunt.

Proboscidun singularum singula receptacula deliritata dedi. Plura autem anatome hactsnus non docuit. Corpus enilis substantia molli repletum videtur, totumque tantopere mobile ut summopere contrahi et extendi queat.- Ovaria vel ova nonrlum vidi, nisi haec forsan in specie sexta. Memorahilis autem pars huic prae ceteris Cestoideis concessa. videtur, papilla scilicet caudalis, vel sirrplex vel duplex, quam pratsertim in specie septima motu continuo agitatam vidi. Qui Tetrarhynchum vivum illiusqque par. tis motus non vidit, genus vix dijudicabit.

Species hactenus piscibus solis adscriptae sunt, ipse tamen alteram (septimam) etiam in Testudine, alteram (sextam) etiam in Sepia of. fendi.

1. Tetrarhynchus megacephalus R. n. sp. Tab. 2. Fig. 7, 8.

In pariete abdominis dorsali et anteriori Squali stellaris Neapoli. Julio unicum reperi specimen, albidum, septem lineas longum, latitudine capitis tres, corporis luas atquante.

Caput conicum. Proboscides subulatae, sive antrorsum atsmatae, teretes, uncinis duris digitum radentibus plurimas per serics dispositis, 


\section{$-448$}

Bothria duo lateralia, subóvata, marginata, linea eninente longitudinali divisa, marginibus tumidiusculis, mutabilia, nunquam tamen quatuor bo. thria mentientia. Corpus depressum lineare, rugosum, capite duplo longius, marginibus lateralibus obtusis, papilla terminatum parvula.

Proboscides mox hae, mox illae retrahun. tur. Interdum Tetrarhynchus margini innititur, tum caput sagittatum et bothria marginalia videntur. Papilla post mortem retracta fuit, quo cauda truncata medio perforata videtur.

2. Tetrarhynchus grossus R. n. sp. Tab.2。 Fig. 9. 10.

Am. Truesius specimen misit, crijus habitacu. lum indicare non potuit, majoris tamen piscis erit incola, et cum aliis vermibus piscinis eadem phiala quoque servatus fuit.

Cum acciperem, Tetrarliynchum nondum videraț, ut specimen hocce proboscidibus retractis diu the vexaverit.

Planum, et crassum est, marginibus rectis obtusis; sedecim lineas longum, quorum capurt sibi quatuor et dimidiam vindicat; corpus postice tres lineas, antice (uti et caput) duas latum.

Caput ovale corpore crassius, bothrio utriuśque lateris solitario angustissino, sive lintari, linea longitudinali eminente diviso. Corpus antrorsum parum angustatum, elongatum, postice obtusum, papilla media suborbiculari termina. tum, utraque superficie rugosum.

Rugae consimiles capitis, aeque bene ac illae corporis, post mortem aut sub morte natae sunt, quod specie insequente didici 


\section{-449 .}

Capnt quatuor thecas longiusculas, obiter incurvas, et duras continet, singulas ductu tenuiore pendentes, proboscides includentes. Apparatom musculosum, qualis Echinorhynchis conceditur, nullum vidi. Corpzes discissum eodem modo se hahet ac articulus Taeniae cultello divicus, neque ova neque, alias partes intermas vidi.

Species certe magna est, quae contracta se-o decim lineas super t, et bothriis linearibus ab affinibus praescrtim distat,

3. Tetrarhynchus attentiatus $\mathbf{R}$. h. sp.: : Specimina a cel. Fischer, Professore Kilos niensi in Xiphiae Gladif branchis reperta optimi

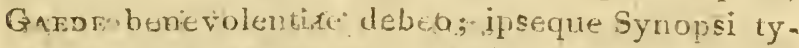
pis mandara specinen solitarium in Gladii pariete abdominis dorşali offendí. Pisčis, die Octobris XXIMr. hn. Baltico captus, die XXX. ejusdem mensis a me Berolini examinatus Tetrarhynchum candidicsimuli vivŭm obtulit, queñ per triduum talem aqua servavi.

Speciminitm acceptorum alterum septendecim. alterumx viginti tres lincas longum, capite duas ireave linea lońgo, 'duas 'latb', corpore lineam ad sescrilifieam ${ }^{1}$ lató. Quod lipéreperi, contras cttum póllicem, "quanco sé extendebat tres pollí ces longtun'fuit, Lignlan aemiłl luiss. 'Motus maxiinc varius. mox cérpus articulatum visum, saepe crispium, parte ivedia 'vel et posteriore sinul pas sin' fortiter constrictumi. Post mortern specimen aquae rimis bitr 'commissum rugosum, et quasi tuiberculosimn factun est. $\therefore$ mas:

Capin obtusum, corpore. crassius et latius, Tif 
bothrio utrinque laterali, obconico, sive antroisum latiori, poskice acuto, linea elevata diviso, minus prolundo. Proboscides durae, breves, cy. lindricae, obtusze, uncinorum seriebus plurimis munitae. Corpus terue, sublineare, postice decrescens, papilla terminatum, quam mox oblongam simplicem, mox ovatam, vel ovalem et dis. cretam vidi, organon 'forte generationi dicatum.

Bothria quidem motu, profundiora reddi possunt, sed generatim quam in reliquis speciebus minus profunda sunt: Proboscides nunquam retrahi yel, longiores reddi observavi.

\section{Tetrarhynchus discophorus R. n. sp.}

In Rrama (Sparo) Raji Neapoli Junio reperi, et majorum quidem speciminum unum in branchiis, duo in ventriculo, nonnulla minora autem inter hujus tunicas, omnia candidissima.

Majora contracta duas, extensa et proboscidibus exsertis quatuor lineas longitudine adaequant, ultra lineam lata; minora lineam vix sú. perant.

Caput magnum rotundatum., Bothria lateralia magna, orbicularia, septo medio longitudinali divisa, apice proboscides emittentia; his re: tractis bothria exserta, saepe quasi pedunculata, exsertis autem sessilia et complanata. Probosci. des teretes, multis uncinorum seriebus armatae. Corpus contractum bothriis latitudine parum cedens, neque longius, rugosum et crassiusculum, apice caudali emarginato; idem extensum capite angustius, planum, lineare, apice obtuso. 


\section{- 451}

5. Tetrarhynchus tenuicollis R. n. sp.

In Pleuronectis Pegosae (aut huic affinis, Ari. mini Majo a piscatoribus oblati) tunica ventriculi externa globulum vidi album, grani piperis ininoris magnitudine, quo aperto Tetrarhynchum offendi, quatuòr lineas longum, parte antica tenui, postica lineam lata, vivum.

Caput subcordatum, bothriis bilobis quadriauriculatum; proboscides filiformes tenuissimae, uncinis recurvis ternatis longa serie verticillatae, bothriorum apicibua emiscae Collum teres po. stice attenuatum. Corpus ovatum, utrinque convexum, crenatum.

Specimina duo simillima, sed multo minora, in Lophii piscatorii peritoneo ventriculun induente Romae Majo reperi, quae die insequente, cum accuratius examinare vellem, in aqua difflue. re vidi.

6. Tetrarhynchus megabothrius R, n, sp Tab. 2. Fig. 14.

Inter quatuor Sepiarum officinalium Arimini Aprili dissectarum tunicas ventriculi primi (rarius oesophagi) externam et mediam, extus pun. ctum album referentes, complura reperi specimina, lineam vel duas insuper lineae tertias partes longa, antice dimidiam lata, postice decrescentia, depressiuscula, candida.

Caput maximum, sub motu polymorphum, mox hic, mox illic inflatum, antice truncatum vel e contra rotundatum, celerrimeque figuram varians; tranquillunn, vel animalculi mortui, antice rotundatum, postice subsaşittatum, bothriis bilobis, maximis, exstantihus. I'roboscides longae, filiformes, uncinorum plurimis seriebus armatae, 


\section{$-452=1$}

mox omnés, mox una alterave tantum-exsertac. Corpus rectum, si caprat spectas, cxiguum, lioc angustius'saepeque brevius, papilla temuionc tcF. minatum, undique corpusculis rotundis, an oyis globosis? repleturn.

Neapali Junio, inter Siombri Sardae tunicag ventriculi complura, intestini autem unum, reperi specimina, proboscidibus non computatis line. an et qu’ed excurrit longa.

Probosides in his passim totae terctes, interdum autem versus anteviora quadrangulae visáe sunt. Bochria singula biloba sexcostata vidi, quo totus Tetrarhynchus antice costatus appare. at. Si proboscides rctractae sunt, eorum loco puncta quatuor obscura in conspectum veniunt; si emitti incipiunt, interdum apice subglobosae videntul,

Totum corpus cura capite et papilla caudali granulosum, aut corpusculis subrotundis, fere ovalibus referium videtur. Ova totum Tetra. rhynchur replẹe fere fidem superat, ut de illis dubius relictis sim.

Obs. Termis dubius Ent. I1. 3. 1.284. 11. 39. a cl. DICQUEMARE inter membranas Sepiae viscera involvenies repertis, pollicaris dicitur, alias huc irahi posset; Tetrarhynchus saltem videtur.

Fasciolam barbatam Lrímier, a MTartivo Rolandsan in intestinis Loliginis repertam, Ent. II. 2. p. 235. n. 40. huc pertinere, nou dubito; Jetrartyucho enim non satis a muco libera. to proboscides plures quam quatuor adesse virezstur.

De verne autem, quem Redr in Polypi tu. berculis ventriculi repertum describit, niliil pro- 


\section{- $453-$}

ferre valeo, cum descriptio et figura nimis rudes sint, et ipse tantum in Polypi ventriculo Scolecem repererim, quem certe non vult. Forsan tamen Tetrarhynchus fuit, locus saltem optine converiit.

7. Tetrarhynchus macrobothrius R., Tab. 2. Fig. $11-13$.

Ent. II. I. p. 320, n. 2, T. papillosus.

Descriptione speciminum a cel. BosG in plurimis Coryphaenac Hippuridis partibus, a cel. Trussio inter Scombri Pelamidis musculos repertorum, in Entozoologia usus sum. Berolini autem Octobri mense inter tunicas ventriculi Testudinis Mydae specimen vivum reperi, et nonnulla ab am Orfers in Coryphaenae Equiselis (neque Hip- puridis, uti in Synopsi suspicatus sum) abdomine - tam libera, quam in liydatidibus, Decembri mense reperta, transmissa sunt.

Testudinis Mydae ventriculum apcriendo foramen reperi, exitum canalis, in quo Spiropteram suspicatus suras, sed praeter opinionem Tetra. rhynchum offendi, pro more candidum, quatuor cum dimidia lincas longum, fere duas lineae tertias partes latum, subtetragonum.

Bothria primum vix in conspectum venizunt, sed totus vermis costatus videtur, examine autem continuato, bothria longissima, costata, adpressa, lateralia exhibentur, marginibus latiusculis tantum cum postica çorporis parte laevibus. Proboscides uncinis ternatis armatae. Papilla caudalis biloba, lobis profundis, obtusis, continuo motu vel diductis vel adductis, vel partem retractis.

Specimina Olfersiana cum descripto proti- 


\section{$-454$}

nus congruunt, duas sum dimidia ad tres cum dimidia lineas longa, subtetragona, duas lineae tertias partes lata; spiritu vini fuscescentia reddita, duriuscula.

Apex posticus papilla kiloba retracta obtusissimus, apertura ininina.

Obs. Tentacularia a Boscro, vira cel. in Bull. de la Soc. Pinlom. rm97. n. 2. p. 9 fig. r. detecła, obiter tantum delincata est; TrLesrr figurac Entoz. Tah. VII. Fig. 3-9. elegantiores quidem, haul tamen suíncientes, quare novas addidi. Prohoseidum apices passim papillae speciem induunt, praesertim quando exsę̧i incipiunt.

8. Tetrarliynchus appendiculatus R.

Ent. II. 1. p. 3r8. n. 1. Tab. VII. Fig. Io, Ir, 12. nomine dicto Echinorhyıchum quadrirostrem Goezir ex cel. viri mente descripsi et ejusdein icones repetii, Viennae autem Martio 1817. specimina duo Goeziana ex hujus collectione (nunc Ticini servata) depromta inspicere licuit, oculo tamen huic generi nondum familiari.

Alterum specimen prohoscidibus non computatis tres lineas longum appendice minore, al. terum dinidia linea brevius appendice majore uticur, quae papillam valde elongatam obtusi. usculam sistit, quam reliquae species breviorem exhihent. Proboscides longiusculae, tenues. Bo. thria lateralia longa costata, quam in praecedente specie tamen breviora.

Tetrarhyncho macrobothrio affinis, sed diversus est.

9. Tetrarhynchus scolecinus R. n. sp. In duobus Squalis sicllaribus, masculo et 
femineo, Neapoli Julio mense, carnes praesertim caudae horum Tetrarhynchorum copia incredibili scatentes offendi.

Primum singulos hydatide contineri species exorta est, sed aquae commissos convolutos, at nudos cognovi. Albi, contracti, vix linearn longi, sponte vero ultra tres lineas extendebantur. Corpus teretiusculum, vario modo constrictum, parte antica summopere mobili, ut primum Sco. lecem adtesse crediderim. Tandem plurimi bo. thria duo ovalia explicuere vivido motu agitata, inter quae proboscides retractas et vaginis commis. sas trans corpus diaphanum pellucere vidi. Has etiam exsertas dein, sed rarissime observavi, neque alia est species, cujus proboscides tam raro emittuntur. Pressione vero ipsas promovere fru. stra tentatur. Caput ceterum exiguum, probosci. dibus verticillatis brevius. Corpus elongatum, depressum, passim constrictum, papilla brevi terminatum.

Postmodum (Neapoli, Julio) in Squali Centrinas musculis prope pharyngem duo reperi specimina vesiculis flavescentibus inclusa. Proboscides retractae per collum pelluce. bant. ${ }^{4}$ Botniria duo ovalia. Corpus contractum obovatum, extensum oblongum, collo tenuiore. Alterum duas lineas, alterum capite non explicato lineam longun erat.

Augusto mense ibidem ad marginem pinnae Rajae oxyrhyuchae pectoralis, et latere quidem inferiore, vesiculam reperi aliquot lineas longam, trans cutem pellucentem, qua aperta Tetrarhyn̨chum conspexi duas cum dimidia lineas longum, bothriis oblongo-rotundatis, collo tellui, corpore ováto-oblongo, apice postico acutiusculo. 


\section{$-456$}

10. Tetrarhynchus gracilis R. n. sp.

Novem Annzodytes Cicerelos, quo nomine Rafinesoue Scmalz jure ab Amm. Tóbia distin. xit, Neapoli Julio examinavi et in singulorum intestino complures reperi Tetrarbynchos, caput saepe absconcientes.

Vormiculi, albi, depressiusculi vcl teretes, lineares, duas ad quinque lineas longi. Caput bothriis duobus lateralibus, lato-cllipticis. Proboscides longae, uncinis armatae, retractae trans col. lum pellucentes. Corpus mox linc inde, mox nullibi constrictum. Caput cetcrun breve; collum longum, interdum continuum, interdum ope stricturae a corpore discretum. Apex posticus acutiusculus,

Stricturae nullibi tantae sunt, quae articulos sistant, vel vermem bothriocephaium arguant, Triaenophoro proximus est.

$$
\text { species dubia e. }
$$

1.1. Tetrarhynchus Squali."

La Nartinere in Voyage de la Pérouse T. IV. Paris 174\%. 3. p. 84. Tab. 20. Fig. 9. 20. Hirudinis species.

Bosc in Nouv, Bullet. de la Soc. Philomat. I811. p. 384. Hepatoxylon Squali.

La Martiniere illustris navarchae LA Perouse comes infelix in Squali, cujus species non dicitur, hepate vermem reperit, lujus substantiam ultra pollicem dimidium intrantem, album, pollice longiortm.

Caput magnum, proboscidibus quatuor hrevibus, duriusculis; armatis, quibus singulis si figu 
rae monae et verbis auctoris fides habenda est, singula subjiciuntur bothria oblonga, parva; quorum itaque utriusque lateris bina forent; figura decina tamen, caput ab antica facie delineatuna. sistens, bothria cujusvis lateris confluentia exlibet, ut biloba forsan sint. Corpus depressum antice ad tres lineas latum, retrorsum angustatum, apice postico acuto; annulatum dictum (cun! annulis sive articulis Taeniae comparatum) forsan tantum rugosum fuit, quale in specic tertia mihinet insa quandoque visum est, ut hanc figuram satis bene redderet.

Obs. Proboscides crassae, breves, totiusque cornoris hobitus, quo minus speciem hanc ad. Xoh-riucplali genus referam, vetant. Cel. Bosc Tentaculariae genus a se conditum oblitus, liac specie novum (nomine maxime incongruo) ex. struxisse videtur.

12. Tetrarhynchus Pleuronectis maximi.

Tetrariynchus lingualis. Crvier Regne Animal. T. IV. p. 46. Tab. XV. Fig. 6. 7.

Cel. vir speciem hanc sub linsua Pleuronectis maximi et complurium piscium frequenier oceurrere, quo tamen loce ipse nullo in pisce Tetrarhynchum reperi, licet partis illius examen. nunquam practermicerim. Figurae, quae vivura delineatum sistere dicuntur, Tetradhynchum no-' rem lineas longum, antice tres lineás et quod excurrit latum exhibent, corpore botiris specta. tis brevi, papilli caudali exigua. Habitus ommi. no' est spcciei sixtae, mili semper pusillae visae, probusciles t.a.t lungac sisuntur, quae majoribus breviomes et ciassiures sunt. An itaque ma. s.siturio aucia, $" 1 \cdots$ n naturalis, figuris redditur? 


\section{$-458$}

13. Tetrârhynchus Argentinąe.

Ent. II. I. p. 322. n. 3. T. elongatus.

Cel. ReDI in Argentiviae Sphyraenae abdo. mine reperit.

14. Tetrarhynchus Morhuae.

Ent. Ir. 1. p. 324. n. 4. T. Morhuae.

Cel. Amindganizd in Gado Morhua offendit. Species n. 13. et 14. memoriae lapsu in Synopsi non enumeravi.

\section{Ginus XXIII. Liguia.}

Ligulae Piscium olim solae cognitae fabricam simplicissimam exhibuerunt, neque Avium Ligulae Вцоснго et Gozzio visae magis compositam probarunt; ipse in speciminibus Ligularum variis in avibus repertarum Entozoologiam edendo nonnisi ovaria inveni; postmodum etiam in avium Ligulis genitalia marcula, sive lemuiscos, detegere milị contigit; Bresserus tandem iṇ Pelecani Carbonis Ligula caput detexit, illi Bothriocephalorum simplicium simile.

In piscium Ligulis nihil detegitur, nisi forsan Ligula Truttae excipienda est, de quo tamen dubitare licet Neque observatoribus illud vitio vertendum est, summa enim sedulitate et sacpis. sime illas examinavi et dissecui, sed nunquam genitalia vel alias partes discimiles reperi. Neque aviurn Ligulae semper, aut totae ab illis differunt. Saepe pisciris sinillimae, durae et compactae discissae substantiam totam homogeneam, alias autem hanc illamve tantum fortem alteratam, ovariis et lemniscis insignem, reliquas vero pari 


\section{- 459}

modo simplices offerunt; rarissime omnia evoluta sunt. Confer observationes in specimina spęciei tertiae in Pelecanis reperta.

Quibus commotus Ligulas piscinas primo tantum vitze stadio contineri, ab avibus autem deglutitas alterum subire, et legitima metamorphosi ovaria et lemniscos seusin explicare crediderim. Bremserus me haec circa haeréseos taxat, non possum tamen, quin hypothesin meam proferam, mihi saltem probabilem visam et discrimina Ligularum, satis explicantem.

Amicus scilicet ovaria Ligularum semper adesse credit, piscinarum illa autem ob corpus crassum et durum haud in conspectum venire; in avibus vero Ligulas attenuari et calore macerari, quo ovaria patefiant, Ipse vero Ligulas piscinas saepe summa diligentia examinavi et secui, ipse easdem passin summopere extenuatas offendi aut reddidi, neque tamen ovariorum ullum exhibitum est vestigitum.

Ligulae in piscibus et animalibus, quae his vescuntur, tantum occurrunt; in piscibus abdominales, in phoca et avibus sunt intestinales, quod hypothesin meam auget.

Adsunt quidem vermes aliis locis obvii, sim. plicitate Ligulis affines, qui nondum tamen satis examinati, ideoque inter generis dubii Entozoa (n. 8. 9. 19. et 20.) relicti fuerunt.

Ligulae in pisciculi Cyprino Barbo affinis abslomine obviae Italis nomine Macaroni piatti edules et in deliciis sunt.

1. Ligula uniserialis $R$. Specinina tria in Falcone Albicilla reperta 
in Museo Viennensi vidi; quorum primum vi: girti pollices longum, parte priori non evolutum, sive piscinum, dein, ut in Ent. II. 2. p. 12. n。 צ. descripsi, ovariis solitariis mediis, passim rima transversa notatis; secundum tredecim pollices Jongum, consimile; tertium tres pollices longun tetum piscimum erat.

Obs. Bremserus Braunium, a quo speciem hanc in Falcone fulvo repertam accepi, non in hoc sed in Falcone Albicilla reperisse suspicatur, quia Ligulae vel in piscibus ipsis, vel in animalibus his vescentibus occurrant. Falco fulvus tamen fuisse potest, qui avium marinarum comestarum ope Ligulis obnoxius factus sit. Neque, - sos hypolhetice tantmu relicua animalia a Ligulis libera habere, reticendum est.

\section{Ligula alternans $\mathrm{R}$.}

In Müseo Viemnensi specimina vidi plurima in Laro parasitico reperta. Evolutorum ovaria vage alterna et pars antica fere articulata.

Quae ibidem vidi plurima in Laro ridibuido lecta, plerumque ovaria altema, interdun fere opposita cxhibuerunt. Adcrant etiam specimina alba, pellucida, ovariis carcutia.

Mussei laudati, nunquam vēro satis laudandi specimina in Luaro glatco reperta piscinorum niodo haud evoluta, lata et multistriata erant.

Quac cel. Nitzsca in intestinis Lari cani senioris Novembri reperta misit specimina duo, nondum evoluta sed piscinis similima sunt.

3. Ligula interrupia $\mathbf{L}$. Specinen magnum in Mergo Mergansere 
Novembri mense reperum Nixzschrus mecum communicavit, sed piscinis penitus simile, cras. sum, linea media impressa.

Inter specimina in IIfergo Albeilo lecta, quae Museum Viennense servat, frustula aliquot villi; fere consumta, 'nivea, ovaris indistinctis alternis. Alia longiora, linearia, tres ad sex pollices lomga, sesquilineam ad duas lineas lata; ovariis op. positis utehantur minoribus et transversis; alee rum tamen frustulum ovaria opposita majora et rotunda obtulit.

- Speciminum plurimorum in Mergo serratore repertorum quaedam ibidem observavi ovariis lemniscatis, qualia primurn in sequente detexi specié.

In Pelecani pygmaè oesophago et ventrie culo repert? duo ibiden contemplatus sum specimina. quorum aiterum decen, quatuor polliccs alterum longum erat. Irajus hinc piscinum, tum loco lineae unitus mediae utrinque linea separata adfuit, passim vesiculas referens, neque tamen, ovaria, sed forsan horum rudimenta (opposita) sistens.

Notatu dignum censeo, specimen in Peleca. ni Carbonis ventriculo repertum, latum et cras. sum omnino ac Ligulam 'Bramae, ibiclem mihi occurrisse; alterum autem in ejusdem avis intestinis inventum, viginti quinque pollices longurn hinc tenuius, utrinque angustius factim, nondum tamen ovariis instructum esse. Hoc pro nica sal'tem miliat hypothesi.

Postmodum am. Braxser duo spécimina mecum communicavit versus Martii mensis firem 1818. in Pelecano Carbone reperta, quorum alte. 


\section{$-462$}

runı octo pollices longum, antica parte satis indistincta, postica valde attenuatum, ulterius non evolutum; quorum alterum autem sex pollices longum caput distinctum obtulit, illi Bothriocephali solidi vel nodosi simillimum, fere triangulare, apice antico acutiusculo, lateribus planis (dorsali et ventrali) fovea media 'longitudinali exaratis, quo liarum fundo pelluceant. Corporis latitudo pone caput cito augetur, ut summa latitudo tres lineas superet, tum magis magisque an. gustantur, fine caudali valde tenui. Corpus passim obiter crenatum est, et ovariorum quoddam sed leve' vesigium exhibet, quo similitudo quaedam Bothriocephali exoriatur.

Amicus simul icones misit alterius speciminis, cujus bothria adhuc distinctiora šint, et bene quidem delineata vidi, sed cum transmisso omnino convenientia.

Num Pelecani Ligulae huc vel ad speciem praecedentem pertineant, incertum quidem est, sed species illa vesicularum oppositarum in $\mathbf{L i}$ gula Pelecani pygmaei notata, huc potius referendas esse, persuasit.

\section{- 4. Ligula sparsa R. Tab. 3. Fig. 1.}

Am. Gaede, nunc Professor Leodiensis, Berolini Junii d. XXVII. I8I ${ }^{\circ}$. in intestinis Colymbi cristati Ligulas plurimas reperit et recentes mecum communicavit.

Tres ad sex pollices longae, candidae, ova. riorum linea nigrescente. Adultaruin extrema pro more crassiuscula, reliquum corpus tenuius; aliis aeque magnis haud attenuatum fuit.

Ovaria foramina habent impressa, transver- 


\section{- $403-$}

salia; serie simplici, eadem tamen irregulari dis. posita, ut ovaria mox magis dextrorsum, mox sinistrorsum magis spectent et fere pro alternis lia. beri possint. Unico in specimine ex ovariorum foraminibus leminisci, sive orsana mascula propen. debant, brevia, teretia, recta, acutinscula, qu lia antea non visa sunt, gaure in tabula tertia dclineari curavi.

Quae ipse olim in Colymbn cristato rcperi specimina, piscinis simillima; quae in Museo Viennensi vidi, eadem ex ave dtpromta, plurima, evoluta seriem ovariorum solitariam obrulerunt, ipsa antice tantopere articulos initara ut incauto imponere potuissent; re attentius spectata auteth corpus solidum, cute anterioris partis plicata, ob. servatur.

Quae ibidem vidi in Colymbo subcristato lecta, pari modo antice articulorum sed nullam ovariorum speciem obtulerunt. Spccinen 2 Bremsero tránsmissum eundem habitum prae se fert, sed apicem anticum fissum simul exhibet.

Aderant etiam in dicto Museo specimina in Colymbo septentrionali et arctico inventa, plurima, tres ad sex et quod excurrit pollices longa, quo. rum evoluta ovariis utehantur plerumque sim. plici serie digestis, raro alternis, rarius oppositis, ceterum exiguis, approxinatis, subtransversis.

Reliquae hac sub specie enumerandae $\mathrm{Li}$. gulae mihi pariter in Nuseo Viennensi. visae sunt.

Ligulae in Ardea Egretta repertae frustula plurima aliquot pollices longa, piscina; alia teruiora facta ovariorum serie $f \in r \in$ orbicularium, vel transverse ovalium irregulari, mox solitaria, mox 


\section{- $464-$}

alternante. In plurimorum ovariorum medio punctum prominens, an lemniscus?

Ligulae Ardeae Nycticoracis specimēn decem pollices excerlens, duas tresve lineas latum, an: tice crassiusculum, ceterum attenuatum, ovariorum ovalium transversalium serie solitaria irregulari, vel passim fere alternorum. In opposito latere. linea longitudinalis impressa adfuit.

Speciminum in Fulica chloropode repertorum fraşmenta aliquot, tres ad quinque cum dimirio. poilices longa, duas circiter lineas lata, attenuari. iacipientia; ovariis riman referentibus transver. sam, serie unica dispositis vel passim alternis.

Iigulae Sternae nigrae specinina complura, tres ad quinque pollices longa, vel piscina, vel ovariorum serie solitaria aut alternante; illud Sternae Hirnudinis tres cum dimidio pollices longurn, lineas duas latum, , piscinum, crasstm. absque ovariorum indicio; fragmentum veropracterea ejus. dem aderat pollicare, duas tertias line ae partes, latum, depressum, apice obtusiusculo discreto.

Ligula Anatis Boschadís ferae quinque cum dimidio pollices longa, ties lineas lata, margine. undulato, piscina, hinc ope linene mediae exarata, illine tamen ovariorum vestigia olsiter in. dicat.

Ligulas huic speciei tributas, omnes jure huc rélatas esse, non jurarem.

\section{Ligula nodosa $\mathbf{k}$.}

Ob puncta linea mediae inscripta speciem hancce ovariis evolutis instructam Ent. II. 2. p. 17. suspicans sum; ulteriori tamen exanini ver; mem Salmonis Truttae commendatum velifn, ne; 


\section{$-465-$}

que hujus solius piscis Ligulas, casque exiguas dictas, quatuor nimirum cum dimidio pollices lon. gas, linean dinidiam latas, tantoperea reliquis piscinis recedere crediderim.

\section{Ligula simplicissima $R$.}

Singulorum fere pisciim Ligulas ab auctoribus relatas $Z$ Enerr, observatoris alias egresii vestigia premendo, in Intōzoologia seorsim enu. meravi; plurima autem deinceps comparare licuit specimina, neque notas ullas, quibus distinguerem, constantes reperi, quare in Synopsi p. 134. n. 6. eadem specie compreluendi.

Cel. Morechint, cujus memoria Physicis aeque grata est ac Medicis, dum eundem Romae visitavi, piscium mentionem injecit in lacu Neapolitano (Lago Fucino dicto) habitantium, qui vermes continerent, incolis edules. Novitate adductus viros optimos Boer et Kunde, Medicos practicos Berolinenses, tunc iter per Italiam faćientes, adhortatus sum, qui mihi pisces et vernes compararent. Aliquot septimanis elapsis tam pisces quam duas horum Ligulas Neapolin attulertint. Pisces ex cl. Morecrins et amicorum relatione Lascr et Lascagna, verines autem Ma. caroni piatti vocantur, ut pisces his comnendabiles Lasca con macaroni emtoribus laudentur. Pisciculus Cyprini species est, Barbo affinis, a cel. Risso (Ichthyologie de Nice p. $36 r$ n. 2.) immerito pro C. Bulatmai habitus, mihi potius nova species visus, quan alio loco describam. Ligularum ceterum altera duos pollices et decem, altera duos pollices et underim tineas longa, utraque duas cum dimidia lineas lata, nulla prorsus nota a minoribus Ligulae piscinae speciminibus deflectens. 
Minimum quod vidi specimén am. Gaene Berolini dedit ibidem Junio in Gobione a se repertum, novem lineas longum, hinc obtusum line. an, illinc acutiusculum lineam dimidiam latum, latere altero plano albicante, convexiusculo autem altero et flavicante.

Quae in Cyprino Gibelione Julio mense Be! rolini reperi specimina duo, abdomen partim egressa fuerunt. Alterum ejusdem piscis specimen duos pollices totidemque lineas longum, li. neam ad 'sesquilineam latum, primo intuitu ovariis instructum visum est, sed examine instituto illa penitus deesse demonstrabatur.

In Museo Viennensi specimina in Perca Lucioperca et Ruviatili, Siluro Glanide, 'Esoce Lucin, Cyprinis Alburno, Brama, Carassio et erythrophthalino reperta vidi, de quibus nonnulla referre liceat.

Ésocis Lncii Ligula sex pöllices İ́nga, altero extreno fere tres lineas, altero vix sesquilineam lata, crassinscula, medio quasi emollita et absorptione attenuata, attamen nullibi ovariorum vestigium offerens, sed toturn animal praeter lineam longitudinalem insculptain nihil memorabile monstravit.

Percae Luciopercae. Ligula fere bipollicaris, incompleta, utramque versus extremitatem parum emollita, ceterum duriuscula, ubivis simplex.

Percae fluviatilis Ligula quinque pollicaris, ultra duas lineas lata, altero extremo integro, altero difflzente. Illic fere caput adesse dixeris, apices enin duo sunt ovati sibi invicem appres. si vel convergentes in apice partis integrae. 
7. Ligula crispa $R$.

In Phocae vitulinae junioris Junio mense Berolini nortuae, die insequente a me dissectae intestinis tenuibus unica adfuit Ligula, vitae $\in x$. pers, alba, quindecinn pollices longa, parte antica (ápice deficiente) tenui, lineam. non excedente, postica parum latiore, media tres lineas aequante, margine latcrali passin undulato Sub. stantia mollis, homngenea; parii anticae tamen fossulae mediae insunt, ovariorum futurorum for. san loca prodentes

Cyprinorum Ligulae, ł. c. illa Bramae, sae. pe margines undulatcs offerunt, et Phoca illa piscibus saginata fuit; specimen descriptum itaque adventicinm et a Cyprino relictuni esse posse; facile concedarn.

\section{Genus XXIV. Triaenophorus.}

Taeniam nodulosam Auctorum a Taeniis reliquis distinguendo, in Observationibus meis, anno 1793. editis, Tricuspidáriae vel Triaenophori nomen generi novo imponendum proposui. Illud postmodum servavi, et Ent. IL. I. p. 25. defendi, sed nimis malum retinere non possuin; quo commotus alterum nomen; graecum et sine dubio melius refromsi, quod dum non de alterius nomine mutando agitur, veiniam inveniet

\section{Triaenophorus nodulosus $\stackrel{\mathrm{R}}{\text {. }}$}

Tricuspidaria nodulosa Ent. II. 2. p. 32.

Specimina parva in, intestinis Gasterostei aculeati in aedihus meis Berolini ab am. GaEve reperta examinavi, pusilla, sex lineas longa, di-

\section{$\mathrm{G} g$ ?}




\section{$-468$}

midiam lata, utrinque obtusiuscula, altero fine parum attenuata. Caput distinctum non vidi.

Compluribus Synguthis Hippocantpis Ari. mini Majo racentissime morthis a me examina. tis, in aqua, cui. illorum intestina inmisi, tria spe. cimina visa, quinque ad septem lineas longa, Jineae tertiam partem (ubique acqualiter) lata, plana, alba, articulis subquadratis vel subcampanu. laris primum constare visa, sed attentius examinata crenas tantum prodiderunt mediam corporis partem ron attingentes, alterumque specimen is fere omnino destitutum fuit. Ceterum omnia un dique punctis conspersa, an ovulis scatentias. Caput quidem non vidi, attamen huc referrem.

Bremservs duo specimina misit in Cotti Go. bionis hepate reperta, quorum altcrum fine cau. dali singulari modo contracto instructum est, quale quid Pallassio pro capire secundo imposuisse anicùs suspicatur.

De Cysticercis Lotac, Percae, Lucii, olim dictis at summo jure huc referendis, Cysticerci genus illustrando agam.

\section{Genus XXV. Bothriocephalus:}

Bothriocephali genus stabiliendo nonnisi species duobus bothriis vel lateralibus, vel marginalibus instructas prae oculis habui, quae optime, facillime scilicet, a Taeniis distingui potuerünt, quarum omnes osculis quatuor utuntur. Alias deinceps Bothriocephali species vidi quatuor quidem insignes bothriis, his autem ab osculis Taeniarum plus minus profundis, ad Trematodum poros accedentibus, tantopere diversas, ut zeque fa. 


\section{$-469$}

cile sejungerentur. Tandem autem species $(\mathbb{B}$. auriculatus, $\mathrm{n}, 18$ in Torpedine liabitans) acces. sit, crijus quidem locus dubiis premitur. Corporis cnim fabrica penitus eadem, ac specierum arrnatarum, quae summo jure Bothriocephalis adnumerantur; capitis autem adultae organa suctoria neque Bothriocephalo, neque Taeniis bene competunt, et fere tantum, sed obiter cum illis Tatriae festivae $n_{*} 7$. conveniunt; junioris autem eadem bothria videntur. Cum habitus vero, praesertim. àriculorum evolutorum, ovaria et articulorum foramina cum iilis Bothricephalorum armatorum conveniant in Rajis et Squalis reperiundorum, et illa species pariter in Pisce Cartilagineo reperiatur, inter Bothriocephalos eam reliqui. Qui autem in Torpedine Taeniam se in. venisse sibi videtur, neque inter meas reperiat, Animalium indice in usum vocato, facile rem componet.

Complura ceterum genera facile Bothriocephalis meis condi possent;' Dibothrius species. I-15; Tetrabothrius, iterum facile dividendus, species 16-19; Onchobothrius syecies 20-22; Rhynchobothrius species 23,24 . continentes. Numero autem specierum exiguo, et affinitate inter plurimas summa, genera tantopere cumulare nolui, divisiones tamen non neglexi.

Uti Bothriocephalorum dibothriorum et $\mathrm{Li}$. gularmu affinitas magña est, ita forsan, modo his non alieno, Bothriocephalus solidus n. rif. in avibus alterum vitae stadium absolvit et $B$, no. dosus n. 15. fit.

\section{Bothriocephalus latus Brenserry:}

Taeniaun latam Linvari (Ent. II. 2. p+ 70. 
11. 'T.), cujus caput diutissime nos latuit, ad Bo. thriocephali genus pertinere Bremserve detexit, eoque Helminthologiam valdopere auxit. Descriptionem, et icones hujus speciei nitidas in Amici novissimo opere: Ucber lebende IVürmer im lebenden Menrchen reperies. Specimen latissimum. (pollicem latum) Blechianae collectionis in Museo Zoologico Berolinensi servatur.

Caput alterum, quod Bremseri benevolen'tia examinare contigit, duas tertias lineat partes longum. quartam latum, ad latera sulcatum, sulcis Bntria sistentibus et antrorsum convenientibus. Alterius vero speciminis bothria non convenerunt, séd singula superne clausa fuerunt. Collum vel nullum vel brevissimum. Articuli partis anterioris angustissimi et brevissimi fere rugas tantum sistunt. "De reliquis in Entozoologia 1. c. egi.

Varietatem vero, aut monstrositatem, si ma. vis, Amicus laudatus reperit, non praetereundam. Specimeri scilicet in Museo caesareo servatum et egregie delincatum articulos quosdam forainina lateranlia duo, eadem linea transversa sita exhi. bentes offert.

2. Bothriocephalus plicatus R. n, sp. Tab. 3. Fig. 2.

Species distinctissima a Penco, fugiente calamo, ita tamen descripta, ut Mǘlero, ZEDEp̧o et GMfirio pro Echinorhyncho imponere non dehuisset, conf. Ent. II. 2. p. 3าg. Obs.,

Ipse illam primum in intestino recto Xipliae Gladii diu in spiritu vini çonservato et partim maceratam reperi; deinceps specimen ab cel. SPE- 


\section{$-471$}

narrerr accepi, Tab. 3. Fig. 2. delineatum; tandem specinina mortua quldern sed recentia et integerrima in recto Xiphiae, Octobri mense in Baltico capti, et septimana elapsa Berolini a me dissecti, oflendi; candida, formae maximae variae, tam in his, quae libera in recti cavo, quam in il. lis, quat inter recti tunicas plus minus latent et funiculos anfractuosos et duriusculos habitant.

De illis, quae libera reperi, primum agam. Caput depressnm, oblongo ovatum, disco antico plano, marginibus obtusis; bothrio utriuque laterali oblongo. Collum nullum. Corpus vel a capite teinue incipit et ad posteriorem partem senșim latescit uibiterum decrescit, vel ad apicem posticum obtusum usque augetur, vel pars antica te. nuis t aequalis mant et postica lanceolata vel plus min us orbicularis est. specimina reperi pollicem ad sex pullices longa, parte postica tum tres ar quinque lintas lata; adest specimen ses. quipollicare, cujus pars antica semipollicaris lineam latitudine vix superat, cujus autem pars postica pollicem longa octo lineas lata est. Recens tota, vel macerala qua partem articulos' transversos Taeniae plicatae (equi) exhibet, unde no$m \in n$ desurnsi. Articuli nimirum antici vel rugaeformes, vel hrevissimi cuneati, tum in maceratis sensim distinctiores, passim infundibuliformes, vel elongati, mediam partem recti, marginibus posticis exstantibus et pendulis; reliqui transversim positi ut margines liberi (alias antici et postici) hac in specie superiores et inferiores sint, insimul undulati et crispi, vel vario modo pli. cati.

Quae inter tunicas intestini se insinuarunt illis callosis factis saepe tantopere pressae sunt, 


\section{- $472-$}

ut qua partem teretia et articulorum expertia facta sint, quale spccimen a cel. Siemalieri acceptum delineari curavi, et qualia ipse dein reperi, sed vario modo, mox parum, mox multum, hac illave prite, constricta.

Obs. Synopsis dum prelo sudabat, specimina recentia demun reperi; naceratis olim repertij bothria capitis marcinalia esse videbantur, quod dein stcus vidi; illud quidem in Synopsi corrigere, lorúl autem mutare non potui. Ne: que caput compressum et subsagitatum est, quale in Synopsi dixi, nisi a margine spectatur; a la, tere visum depressum tt ublongo ovaitum est.

3 Bothriocephalus claviceps $\mathrm{R}$.

In intestinis Mhuracnse Carrimi Neapoli Julio duo fragmenta reperi "tes lineas longa, latiuscula, articulis subquadratis, marginibus rotundatis; singulorum articulorum parie media ovarium continente, liuc forsan referenda.

4. Bothrioceplatus proboscideus $\mathrm{R}$.

Specimina maxima in intestinis Salmonis Huchonis reperta, quae Brenserus mecum com'municavit, ad specien insequentem (praesertim parte anteriore) transitum faciunt.

Dẹsiderarissinus Paztas Zoográph. Asiatico Poss. T. III. P. 346. Salmonis Huchonis intestinum pimatum et appendices Taeniis refertissima dixit. Ines ib. p. 409. Ascarides et Taeniolas in ventriculo Salmonis Omul frequentes, coecis inliaerentes, eaque saepe perfodientes refert; forsan has ab illis non diversas, 


\section{Bothriocephalus infundibuliformis $\mathrm{R}$.}

Quae Bremserues misit specimina, in inte: stino et appendicibus pyloricis Salmonis Salvelini et alpini reperta, a specie praecedente utique di. versa sunt.

Caput et bothria lateralia oblonga sunt. Collum nullum. Articuli antici breves, tum lon. giores ZEDERo minus bene infundibuliformes dicti, oblongi potius, postice parum latiores.

Quae Amicus misit in appendicibus Salmnnis Thymalli pyloricis reperta, a Eothriocephalo aliena et ad Taenian longicollem pertinere crediderim.

Specimina antem sub nomine Bothriocephali proboscidei ab optimo viro accepta (et in Ifuchone quidem reperta) teneo maxima, quae parte anteriore cum B. iufundibuliformi satis conveniunt, ceteruin quiclem B. proboscideum argunt.

7. Bothriocephalus microcephalus R.n. sp. Duos Orthragoriscos Molas Neapoli Julii d. 26. secui, quorum minoris recentissimi ventriculus, anteriór intestini pars et branchiae Bothriocephalos continebant plurimos, plerumque sex ad decem, rarius tres ad quatuor, unicum duodecim pollices longos, ad summum duas lineas la. tos, albidos, aqua saepius renovata altero adhuc die vivos servatos, Major Orthragoriscus aliquot tantum tenella et exigua specimina obtulit bran. chialia et intestinalia, unumque magium.

Caput sagittatun exiguum, papilla termina. tum, bothriis ovalibus lateralibus. Collum nullum. Articuli priores aliquot brevissimi, tum longiores, subquadrati, angulis tamen posticis ex. 


\section{$-474=$}

stantibus, quo ista pars serrata spectetur; plurimi deinceps hrevissimi, sensim latiores, angulis posticis obtusatis; réliqui angustiores; ultimus aćutus. Ovaria unum duove corpuscula rotunda articuli merii effingunt, a quo ductus pellucidus, aliquantulum posteriora versus inclinatus, medi. am marginis partem, ita tamen perforat, ut fora. mina marginalia váge alterna sint. Specininis maxini supra dicti dimidia pars ovariis explicatis utens minus crassa, anterior "e 'contra tota. opaca fuit.

Specinina tenella sesquilineam, tres ad sex lineas lorga, fere tertia sunt, et capita (pro omnium pullorum more) ratione majorum speriminum labita, masna exhibent. Minimorum articuli male distinguntur; speciminis tres lineas longi articulos-triginta sex numeravi.

\section{Bothriocephalus gramularis $R$.}

Zenerus speciem istam in Cyprino detexit, cujus speciem ignorabat; Bremserus autem illius fragmenta mecum communicavit in intestinis $C y$ prini Phoxini reperta, quae computata sedecim lineas adaequant.

Articuli crassiusculi et reliqua Zenero dicła satis conveniunt, sed oscula duo magna subglobosa (neque bothria) in anteriore capiris latere vidisse milii visus sum, ut Taeuiam potius haberem, et torulosam quidem, a me in Cyprino Jise repertam.

Rem istam certam dicerem, nisi Bremsert auctoritas obstaret, qui pro Bothriocephalo misit.

1o. Bothriocephalus Rectangulum R.

Am. Buemser quatuor specimina misit, quin. 


\section{- 475}

decim, sedecim, septendecim et triginta lineas. longa, in intestinis Cyprini Barbi lecta.

Caput bothriis lateralibus (neque rnarginalibus, qualia in Entozoologia dicuntur) profundis sagitatum, iisdem applanatis ovatum. Articuli antici brevissimi, fere rugaeformes, sensim majores, dein subquadrati, angulis obrusis, passimque breviores aut longiores, ultimus longus apice obtusiusculo terminatus.

Arriculis (praeter minores anticos) ostium medium lateris inferioris. Ovula in articulis sparsa. Striae qnidem passin in hisce occurrunt, sed ductum illum fractum, a quo species nomen tulit, specimina diutius spiritu vini servata non exhibuerunt.

\section{Bothriocephalus punctatus $R$.}

In Torpedinis ocellatae (vulgaris Risso) ventriculo, Neapoli, Junio, specimen capite orbum reperi, ceterum bene conservatum, insinul vero reliquias exigui Pleuronectis semidigesti, quo il. lum casu arlfuisse credas.

Ibidem eodem mense in Gadi minuti intestino specimen reperi, duos pollices longun. Ca. put angulis lateralibus anterioribus exstans, medio obscurum, bothria, verme piscis licet recentissini jam mortuo, distincta non sistens. Articuli antici angusti, subcampanúlati, insequentes brevissimi, tum subquadrati, scmper tamen latio-e quam longi. Ovaria media sparsa, nigre. scerica, posteriora pellucida, an evactuata?

Qualem Gryphiae, talcm ctiam Arimini (A priit) in intèstinis Plcuroncctis maximi copiosum reperi. In aliquot Pleuronecibus Pegosis ibrdem eodem mense frequentem vidi. Adcrant 


\section{$-476$}

etiam Bothriocephali tenelli, Scolecem mentientes, capite tamen bene expresso. In intestinis Pleuronectis Boscii Neapoli Julio fragmenta reperi punctis fuscis dignoscenda.

Ibidem eodem mense in Pleuronectis Rihom. $\tilde{b} i$ intestino anteriore et appendicum altera hujus Bothriocephali fragmenta et specimen capite instructum quindecim tineas longum. Mllorum quaedain unam vel unam cum dimidia, alterum autem duas lineas fatum, punctis ovariorum binis, de quibus Entozool. Vol. Ir. p. 52 et 53. egi :

Bresiserus specimina misit in Pleuronecte Solea reperta, sed contracta, quae minus bene dijudicari possint. Caput utique Bothriocephali punctati, sed articuli antici solito latiores visi sunt.

\section{Bothriocephalus angustatus R. n. sp.}

Specimina duo in intestinis Scorpaenae Scrofae reperta Bremserus misit, quae novam specien arguere videntur.

Caput longissimum et angustissimum, $\mathbb{Z}_{0}$ thriis lateralibus linearibus. 'Collum nullum. Articuli anteriores elongati, angustissimi, cuneati, sive parte posteriore sensim latiore, angulis posticis exstantibus; qui insequuntur magis obtusati brevioresçile redditi sunt, tum infundibuliformes, tanflem sulsqualrati sisturitur. Ovaria speciei praecedentis, cui haec etiam valde affinis cet.

\section{Bothriocephalus crassiceps R. n. sp.}

Neapoli Julio mense in Gadi Merluccii unajoris duodeno sex reperi specimina capite instructa, tres lineas ad rnos-pollices longa, majora lineam lata, alba; ovariis fuscescentibus.' 


\section{$-477$}

Caput magnum subghobosum, antrorsum parumper attenuatum. Bothria marginalia ciblonga profunda et magna in vivis; in mortuis bollhrii ostium parvum anticurn adesse videtur. Collum nullum. Articuli breves, margine posteriore incrassato utrinque exsiante, quo corpus serratum fiat. Articuli ceterum inaequales, ut passim angustiores et longiores intercurrant. Ov:l vel ovalia vel ovata, forsan secundum inajorem ma. turitatis gradum.

A B. punctato diversissimus, licet ovaria lateralia fuscescant, sed haec ipsa etiam in $\mathbf{B}$. crassicipite quuam, in B. punctato majora sunt,

\section{Bothriocephalus solidus $\mathrm{P}$.}

Desideratissimus Pateas (Ent. IT. 2. p. 6r. Obs. 6. Gasterosteos Spream habitantes Botinriocephalo non olinoxios esse retulit, sed nune quidem res alio se habet modo, et atstate quotannis copiosissimum afferunt, passinque aliquot dies in aqua puteali vivum servo.

Interdum pars postica ope stricturae apicens. obovatum petiolatum sistit. In nonnullis artictilorum singulorum oscula media, rotunda, satis ınagna, sed nunquam ova observavi.

Specimina per aliquot dies in aqua viva servata tantopere elongantur et explicantur ut Bothriocephalo nodoso simillima fiant, neque amplius Arindgandio contradicerem, qui eandem speciem credit.

Inse eodem modo Gasterostei B. solidum sterilem in avibus nodosum et fertilem fieri, ac Ligulae in avium intestino explicantur, crediclerim, Quo accedit, Viennenses Helmintuologos 


\section{$-478$}

'meritissimos nunquam in avibus aquaticis Bothri. ocephalum reperisse, quem Bloch, Abildgara Braun et Ego saepissime et frequentissimum in iisdem offendimus; sed illis Gasterosteus deest, apud nos vulgatissimus.

16. Bothriocephalus màcrocephalus $R$.

Quem in Entozoologia dibothrium (bothriis singulis , bipartitis) dixi, meliore jure tetrabothrium dicerdum esse, examine repetito didici.

17. Bothriocephalus cylindraceus R. n. sp.

Am. Bremserus aliquot specimina misit in intestinis Lari Atricillae reperta, quorum unum capite instructum est.

Vermes obiter inspecti Nematoideum refe. runt, teretiusculi scilicet aut parum depressi, antrorsum tenuissimi, duodecim ad quindecim lineas longi, postice dimidiam lati; capize Bothriocephali macrocephali at multoties minore et tam exiguo, ut oculis nudis puncrum tantum apprareat, et lente simplici vix sufficienter examinari pos. sit, cum illius facillime oculis ron armatis examinetur.

Capjut subtetragonum, depressum, antice truncatum; bothriis lateralibus utrinque duobus; marginibus (dextro et sinistro) crassis excavatis. Collum capite triplo longius, antrorsum dilatatum, dein sensim angustatum. Articuli priores brevis. sini, rugaeformes; insequentes sensim latiores, crassiusculi, breves, rotundati; tum stubquadrati altero in specimine paucis angustioribus interrupti; tandem campanulatus, quales forsan plures istis speciminibus desiderantur.

Fragmenta simul adfuerunt multo tenuiora, quorum unum tamen pari modo interruptum et 


\section{- 479}

qua partem crassiusculum vidi; duo praeterea brevia articulis crassis, sed minus contractis, duas tertias lineae partes lata.

Idem Amicus aliquot specimina dedit in intestinis Lari glauci Maji die sexto reperta, pollicem ad quaiuor pollices longa, partim cylindracea, partim depressa. Dutorum quae capite instructa fuerunt, unim omnino idem ac specimen in Laro Atricilla repertum obtulit; alterius antem caput tantopere retractum et abbreviatmin, ut illud Bothriocephalorum piscinorum fere referret.

18. Bothriocephalus auriculatus R. n. sp. Arimini Aprili in Torpedinis marmoratae majoris intestino crasso, muco copiosissimo re pleto, inter valvulas complurà reperi specimina mortua quatuor ad sex pollices Ionga, praeter partem ultimam arriculis longioribus instructam, aliquot pollices, longam, semper disjunctam, ut vermis octo ad decem pullices attingere vidcatur, qui latitudine articulorum brevium inean supe. rat, lacteique coloris est. Neapoli funio specimen magnum in eodem pisce reperi.

Caput, vitreae fere pellucidatis, quatuor constat cyathis: antrorsum maxime dilatatis, margineque libero opaco crassiore instructis, posteriora versus attenuatis ut, basi exigua omnes quatuor confluant in collum breve, angustum, vasis longitudinalibus instructum. Arkiculipriores breves, angusti, sensim latiores et generatim majores facti, vasis duobus continuatis; tum articuli sensim longiores at simul angustiores, parte media pellucida. Articulorum ultimorum latera opaca ovigera' marginibus alterie medio retusis vel emarginatis. Ova globosa.

Romae Majo in Squali, forsan Galei, crassis 


\section{$-480$}

intestinis nonnulla specimina minora reperi, quo. rum bothria mox ut in praecedentibus profundiora, mox altem parun concava, reliquarum specierum bothriis similiora, margine undulato aut crispo, fovea media oblonga urebantur. Omnia uti specimina supra descripta inermia fuerunt.

Am. Brtirseres specimina tam in Torpedine quam in Squalo Galeo lecta misit, cum illis a me in Torpedine repertis convenientia.

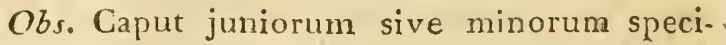
minum illi Bothriocephalorum affinium analogum,, corpus, praesertim autem articuli pesteriores, omnibus numeris cum his convenientia, quo minus speriem istam Taeniis adderem, me jure commoverunt. Forsan autem novum genus variis piscium cartilagineorum entozois articulatis effingi posset. - Hujus ceterum speciei specimina transmissa quandoque uncinorum capitis species exorta est, qualem in recentibus nun. quam vidi.

\section{Bothriocephalus tumidulus R. n. sp.}

Am. Bremser fragmentum misit quinque lineas longum, in intestinis Rajae Pastinacae repertum.

Caput absconditum bothriis utrinque duobus ovalibus tumentibns, quorun singula linea elevata longitudinali media dividuntur, ceterum striis transversis tota teguntur. Collum (an capitis pars?) breve, versus corpus decrescens. Articuli priores angustissimi, elongati; insequentes parum latiores, breviores, subquadrati angulis obusis; reliqui ex affinibus divinandi desiderantur.

Fragmentum tantum vidi exiguum; caput 


\section{$-481$}

autem inerme, bothriis transversim modo maxime regulari striatis tumens, tantopere ab illo reliquorum differt, ut seorsim enumerare cogar. An uncini tantum retracti sunt?

\section{Bothriocephalus coronatus $\mathbf{R}$.}

Species ab optimo Braunio in intestinis Rajae Batis detecta, in Entozoologia secundum cl. viri figuras ohiter tantum descripta: Ent. II. 2. p. 213. n. 116. Tab. X. Fig. 7-10. Taenia Rajae Batis.

Ipse hanc postmodum in Italia frequentissimam reperi, $\in t$ singulas observationes, qurbus majorem fidem faciam, sigillation referam.

1. Primum Arimini Apriti in Torpedinis marmoratae sais nignae intestino crasso d duo fragmenta reperi, alterum quatuor, alterum sex lineas longa, capillum tenuilate superantia, viva.

Caput bothrits quatuor ovalibus antrorsum approximatis, retrorsum divergentibus instructum; singula uncinum bis bifurcatum, qualem Entozoologiae Tab. X. Fig. 9. retert, apici antico adnexum gerentia. Collum longissimum, ita ut alterius speciminis nihil praeterea adesset, alterius quinque lineas longurn paucos exciperet articulos subquadrangulos, vix duplo latiores quam longos.

2. Multa dein specimina viva in Squalt stellaris intestino crasso Arimini Majo reperi, quatuor ad octo pollices longa, multosque praeteres articulos posteriores solutos.

Capitis bothria transversim costata, quandoque postice foramen sistere videbautur, quale quid Braunio imposuisat viderur, qui Entoz. Tab. X, Fig. S, foramen bothrii medii duplicatum 
reldidit. Uncini dichotomi antici, ramis quam in Figura citata magis expansis. Collum depressum, capite tenuius, mox tale incipiens, neque ut in specie priore antrorsum dilatatum, ut pro capitis parte haberi possit, elongaturn, a corpore discre. tum. Articuli priores rugaeformes; sensim tum longiores facti et subquadrati; dein longiores quam lati; campanulati; subrotundi; tandem longissimi, oblongo elliptici. Horum oviductus transversus saepe lemniscum emittens, ordine vage ; terno. Vasa duo longitudinalia mox in corporis initio conspicua sunt, posteriora versus decur. rentia.

5. Neapoli Junio inter Torpedinis ocellatae valvulas intestim crassi cochlidiales plurima reperi fragmenta aliquot lineas ad tres polliceslonga. In speciminis bipollicaris collo longo vasa quatuor longitudinalia vidi, qualia in illo Taeniarum saepius occurrmbt.

4. Ibirlem Junio et Julio in Torpedinis marmoratae crasso multa specimina parva reperi vi$\mathrm{va}$, in quibus bothria quatuor capitis distincta, neque duo biloba dicenda bene vidi.

5. Ibidem Julio in Squali stellaris fere tripeclalis intestino crasso innuineran copiam ho. rum vermium reperi, sex ad octo pollices, quin pedem et ultra longorum, insinul vero articulos plurimos posticos solutos.

Hamulós cápitis reliqua substantia duriores et uigrescentes vidi. Articulorum series sex in. sequentes reperi: r. brevissimorum et angusto. rum; 2. brevissimorum et latiorum; 3. sensim Jongioruin; 4. ellipticorum; 5. lanceolato-oblongorum; 6. oblongorum seu linearium. 


\section{- 483}

Articuli maturi soluti fere in nullius piscis Lrviaer Rajis vel Squalis adnumerandi crasso in. testino desunt, modo maxime vario configurati, antice quasi capitulo (anterioris articuli parti po. sticae inserendo) instructi, quo facillime pro En. tozoo tertice classis imponere possint, nisi ostium marginale naturam indicaret.

Ex articuli soluti foramine marginali genitale masculum filiforme vagina instructum, apice longissimo et tenuissimo utens, velociter et motu quasi peristaltico egreliens observavi.

6. Bremserus etiam specimina misit, in intestinis Squali Squatinae Et Rajae Pastinacae re. perta, curn dictis protinus convenientia.

\section{Bothriocephalus uncinatus R. n. sp.}

In intestino crasso Squali Galei Arimini Majo specimina duo vix septem lineas longa, et teriii caput reperi.

Caput quadrangulum, magnum, bothriis tu. midulis transversim costatis, papillis quatuor an. ticis; uncinis validissimis octo simpliciter furcatis, binis ex singulis papillis oriundis, Collum brevissimum, capite paruín angustius. Articuli priores rugaeformes, collo latiores; mox majores subquadranguli; postici fére campanulati.

Obs. Hamuli fere iidlem sunt ac Triaeno. phori sed caput et articuli ab hoc speciem no. stram aburde distinguunt. A specie praecedente pariter diversa videtur, ob hamulos simpliciores duplici numero praesentes, ob collum breve, ob articulos ultimos in speciminibus pusillis, quae idcirco fere integra viderentur, campanulatos. 
22. Bothriocephalus venicillatus R. 2. sp.

In ejusdem Squali Galei Arimini Majo dis. secti crasso, quo prateclentem, etiam hanc speciem omnium elegantissimam reperi.

Specimina pauca legi, tres ad quatuor polli. ces longa, unicum capite instructum, quo etiam post mortem intestino firmissime inhaesit. Aliquot diebus elapsis iterum in Galeo offendi, sed capite destitutum.

Caput exiguum tetragionum, uncinis furcatis majoribus instructam, qui mihi quidem cjusdem indolis ac speciei praecedentis visi sunt, cum vero caput penitus a muco liberare non concessit, num octo bifurcati, vel quatuor bis bifurcati adsint, pro certo contendere néqueo. Collum nullum; Corporis vero pars antica filo sericeo simplici haud crassior est, quo facile' pro Collo imponere possit. Articuli priores longissini lineares, marginis postici laciniis sex angustissimis acutis retrorsum spectantibus articuli insequentis partem anteriorem circumdantes; qui sequuntur articuii fere infun. dibuliformes laciniis posticis minus et tenuibus et longis; tum brevissimi, bacillares, laciniis quatuor emarginatis utentes, quae incauto pro octo simplicibus imponere possunt; insequentium articulorum longiorum et angustiorum laciniae ad latera pratsertim, exstant, ut verme inflexo pro spinis haberi queant; tum articuli campanulati laciniis posticis brevissimis obtusis; ultimi denique harum expertes maxine elongati, oblongo elliptici, vel sublineares, marginibus lateralibus cras. sioribus, opacis, disco medio pellucente, osculis marginalibus alternis, rarius conspicuis. 


\section{$-485$}

Iaciniae vel fimbriae verticillatae caulem plantae foliis stellatis instructum in memoriam re. vocantes huic speciei soli competunt, eamque miro modo exoruaut.

Vermes recentes albi sunt, per aliquot horas autem aquae commissi, articulorum majorum mar. gines vel etiam lineam mediam (ovarium) virides exhibent, quemadmodum color niger ovariis Bothriocephali punctati etiam post aliquod tempus accedere solet, cont. Ent. II. 2. p. 53 . obs. 5 .

\section{Bothriocephalus, corollatus $\mathbf{R}$.}

In Rajae Rubi maximi, octoginta ad centum libras pondere aequantis ventriculo. Arimini $\mathbf{V a}$. jo decem reperi capita, plerumque sola, rarius uno alterove articulo illis addito.

Proboscides ab illis Tetrarhynchorum di. stingui nequeunt, qui ad Bothriocephalos acce. dunt, attamen ab his diversi sunt, et papilla caudali, articulos caudae nunquam accessuros esse probant.

Romae eodem mense in intestinis crassis Squali, forsan Galei, complura specimina reperi, quorum unum duos pollices longum fuit, $a$ put Tetrarhynchi, sed angustius, ac olinn Parisiis reperi, bothrii.s quatuor instructum; Collum longum; Articuli anteriores rugaeformes, reliqui elliptico oblongi osculis marginalibus, Articuli posteriores plurini soluti pro more simul aderant, qui per nycthemeron aquae commissi avaria nigrescentia exhibuerunt.

Alterum specimen omnes proboscides retractas in collo transparentes obtulit, quod dein in 


\section{$-486$}

Tetrarhynchis sed potissimum in Anthocephalis saepius vidi.

\section{- Genus XXVI. Taenia.}

Quae de generis amplissimi subdivisione Entoz. II. I. p. 40. et sq. protuli, repetere nolo, sed meliora proferre etiau nunc temporis mili non conceditur. Taeniae inermes bene dividi possunt, cum aliarum caput rostello destitutum, aliarum illo instructum sit Armatarum autem omnium caput rostellatum exhibetur, neque ab osculis marginalibus vel lemniscis cerias tarundem sectiones formare licet, cum species ceteroquin diversae his passim conveniant.

Si de familiis Taeniarum, secundum quae inhabitant animalia, exstruendis cogitas, plurimis animaibus Taeniam tan armatam quam inermem esse, ne obliviscaris, alias enim species maxime heterogeneas misceres. Sic v. c. Ferarum Taeniae armatae ab illarum inermibus toto coélo re. cedunt.

Novum reperi plurimarum discrimen, me quidem judice, plurimi faciendum, propriaque monographia dignum. Ova scilicet Tąeniarum diversarum saepe maxime differre vidi, quo commotus, nonnulla in tabula hujus operis tertia delineari curavi. Si ova magna Taeniae columbinae armatae cum illis fere centies minoribus speciei columbinae incrmis comparas; aut quorundam velamen externum allantoidem mammalium nonnullorum forma sua sistere vides; aut modum cogitas, quo paucissima (magna) et alterius Tacniae (parva) innumera ovariis affixa 


\section{$-487-$}

sint, diversissimum, ipsiusque illorum numeri diversitatem tantan; rem examine dignissimam esse concedes. Taeniis armatis ceterum generatim ova majora et pauciora quam inermibus esse videntur, it illae ferarum minus copiosarum le. ges sequantur.

1. Taenia expansa $\mathrm{R}$.

Am. Nitzsch ante aliquot annos se plus quam triginta Capreolorum intestina examinasse at in unico tantum Martio mense Taeniam reperisse scripsit, cujus etiam dein parten posteriorem misit, quam ab illa Taeniae expansae nullo modo distinguere possum, cumque reliqui Capreoli Entozoa a me examini subjecta cum ovinis congruant, illius Taeniam pariter huc revocare non 'dubitọ.

Am. Bremser specimen capite instructum inque intestinis Antilopes Rupicaprae repertum. mandavit, quod cum T. expansa omnibus numeris convenit. Simul autem Taeniae tenellae fragmenta fere teretiuscula eademque satis longa eodem loco reperta misit, qualia me inter T. expansas ovinas reperisse non memini.

Idem Amicus speçimen sẹdecim pollices longum et aliquet fragmenta ab Antilope Dorca. de mascula Martio mense dejecta misit.

Passim macerata et mutata fuit Taenia, cujus tamen caput, pars antica tenuissima, articulorum majorum bene conservati, quinque lineas lati, horum canales laterales, brevi omnia T. expansam indicant.

Pars quidem vermis magis contracta fuit, quam in ovina vidi, quod tamen uti in casu pri. ore praeternaturale crediderim. 


\section{$-488$}

Altera pars tantopere macerata fuit, ut sub. stantiam tenuem mediam omnibus articulis ccm. munem, utriusque lateris externam huic appositam singulis propriam ease, dignoscere possis, quod antea aeque bene non vidi.

\section{Taenia pectinata Goeze.}

Cel Tremtrer specimen capite instructum Januario $m$ nse in Lepore timido repertum mecum communicavit. Caput obtusum brevissimum oscula antica habet. Reliquis addenda non reperi.

Cel. Froelich (Naturf. 29. p. 77. n. 43. Tab. II. Fig. 17-20.) Taeniam Marmotae sibi dictam a leporina distinctann visam ita descripsit, ut eandem esse pateat.

- In aliquot Marmotarum intestinis copiosam reperit; in adulta (Julii d.' 28 ) quinquaginta circiter specimina reperit, quorum quinque ad septem inferdum convolura intestinum extenderunt.

Specimina vidit, quorum articuli postici medio perforati erant, aliis individuis illos penetrantibus.

\section{Taenia lanceolata Goeze.}

Berolini Aprili mense in Colymbi subcristati iritestino medio Taenias duas reperi mortuas, alteram majorein et crassiorem, neque ovariis, neque lemniscis insignem, capite orbam, alteran minorem, quam nisi illa collo instructa, haec vero, uti mox dictetur, maxime evoluta fuis. set. pro illius parte habuissem. Longitudo illius septeIn cum dimadio, hujus sex pollices; latitudo summa illius quinque, hujus tres lineas aequabat. 


\section{$-489$}

Speciminis minoris caput, fere quale Bro. cro Tab. I. Fig. 6. sistitur, sed osculis anterioribus.

Gaput scilicet ovale, osculis quatuor orbicularibus exiguis anticis instructum Collum brevissimum et tenuissimum, filiforme. Corporis pars antica angísta, cito latescens, parte reliqua subzequali. Prater partem anteriorem (dimidium pollicem circiter longam) totum reliquum corpus singulorum articulorum lemniscos utrin. que exserit longiusculos, passim acutos, plerum. que apicem dilatatos, semen emittentes, interdum ex foramine marginali emergere visos, plerumque autem marginis ipsius propagines.

Capite et lemniscis non visis facile pro Bo. thriocephalo nodoso juniore, cujus ovaria nondum evoluta sint, imponere posset. Am. BREM. ser fragmenta, in C. subcristato reperta, capice destituta, at lemniscis oppositis insignia misit, quae specimine descripto non viso certissime pro nova specie habuissem.

Obs. r. Cl. Rosa (Lettere zoolog. p. 6.) Taenias in recto Anatis (cristatam vocat) repertas ita describit, ut huc trahi possint. Pollicem aut sesquipollicem longas, lintam dimidiam circiter latas, collo tenuiore, capite majnisulo ob. tuso quadriosculato, albas, depressas, annulis brevissimis serratas dicit.

$\mathrm{Ob}_{5}$, 2. Cel. Schranx in Faunae Boicae $\mathrm{T}$. [II. P. II. p. 238, n. 3145. Taeniarn lanceolatam Gallinae et p.239.'n. 3144. T. lanceolatam Anseris habet, de illa auiern dubitare licet. Hujus vero lemnisci nequaquam nigri, sed aeque albi sunt ac re- 
liqua corporis substantia; et si cl. Froeltcr tales vidit, hoc accicientale fuit,

\section{Taenia plicata $\mathbf{R}_{\text {. }}$}

Catalogus Viennensis typis impressus hanc et Taeniam perfoliatam Goezur conjunxerat, sed ulteriore exatnine instituto Bremserus utramque diversam este agnovit.

De Taeniarum osculis capitis in Entozoologia (Vol. .. p. 365.) fusius egi, meque variis in Tatniis canales ab illis exortos vidisse retuli; idem et postea contigit. Non successit tamen experinentum nuper (Januario Isig) variis cum speciminibus $T$. plicatae magnis et recentissimis visis institutum, quarum vasa cpe pororum mercurio replere tentavi. Sed alio forsan tempore suc. cedet, nam Distomatis liepatici vasa alias val-1 de bibula passim tamen mércurium non recipiunt.

\section{Taenia festiva R. n. sp.}

In Halmaturi gigantei (in Nova Hollandia. geniti) ductibus hejaticis et vesicula fellea tria reperta sunt specimina, quorum alterun BrEMSERUS transmisit.

Longitudo octo ad decem pollices, latitudo duas ad tres lineas aequat; substantia tenuis, pel. lucida.

Caput tetragonum, antrorsum incrassatum, osculis, quatuor anticis subrotundis approximatis. Collum nullum. Articuli anteriores angusti, rugaeformes, omnes reliqui bacillares (breves, latissini, subaequales), marginibus lateralibus acutis, posteriora spectantibus, plerumque papillis auctis. Ovaria lateralia bacillaria opaca linea media totius 
vermis pellucida distinguntur; ipsorum apices au. teu tam extrorsum quam introrsum (versus lineam medianam) magis turgent, ut pars intermedia ova-riorum tentior passin fere evanescat et de quadruplici ovariorum serie cogitari possit. Ova globosa, parva, copiosissina; fluorum magnitudinis ratione. hahita, magnum inter chorion et amnion adest interstitium.

Animal lepidissimum cum Bothriocephalo verticillato Cestoideorum elegantiac. Caput Taeniae festivae majusculum, si vulgares Tatnias sumis, equinarun autem ratione labita, perparvum.

Obs. Malmaturus, in quo Taeniae descriptae et Distoma hepaticum reperta sunt, in Nova Hollandial genitus et pater fllorum fuit, quos Cialesa. rcum theriotroplieum Schoenbrunnense continet Halmaturos. In his nunquam Entozoa reperta sunt. Ita etiam Gaviae Cobayae in Furopa propreatac entozois fure carent, conf. Obs, secunAam in Pentastoma eprarginatum n. 3. p. 43.3.

\section{Taenia omphalodes Ierrmann.}

Specimina in intestinis Mluris amphibii reperta Bremserus noster misit due, tres quatuorve pollices longa, posteriora versus lineam latitudine superantia, tertiumque tenellum, angustissimum, capitis osculis fere solis protrusis et reliquo capite fere nullo.

Idem dedit specimina complura in intestinis Muris arvalis inventa, duos ad quinique pollices longa, postice passim duas lineas lata.

Caput obconicum sive subtetragontm antrorsun dilatatum, osculis profundis hemisphaeri- 
cls totam superficiem anticam sibi vindicantibus; collum mox breviusculum, mox capite pluries longius. Articuli plurimis speciminibus omnes breves et lati. Inirum adest specimen circa tripollicare, cujus articuli anterioreg breves sensim laiores fiunt, iuscquentihus longitudinis ratioṇe hahita brevibus, ultimis autem longis, triplo lon. gioribus que m latis, subcuneatis, marginibus lateralibus fere undulatis.

Obs. I. Plurinis, praesertim Mammalium Taeniis articuli uitimi elongati esse sulent, at spe. ciminibus illis masnis nulli tales sunt, omnes autem majores articuli orulis exiguis rotundis pleni, cum elongati rulto minores illis careant. Hoc in casn itaque articuli elongati varietatem potius sistere videntur.

Obs 2. Differentia in Synopsi oblata secundum descriptionem nostram, praesertim circa collum, corrigenda $\in$ rit.

\section{Taenia crenata Gozze.}

Omnes, quas hactenus videram Picorum Taenias, ad T. crateriformem 11. 91. pertinere, et ' $\mathbf{T}$ '. crenatam rostello retracto partein illius anteriorem sistere collegi; specimina vero duo, in intestinis Pici ILartii reperta, quae Brexsezus misit, me iterum dubium reddiderunt.

Caput glubosum. Rostellim nullum ant penitus retracium. Collum longiusculum. Articul' omnes breves, angulo postiro utrinque exstante; priores autem angustissimi (capillares simul sumti. mox latis celerrime quidem increscentibus excipiuntur.

Utrumque specimen hac nota convenit, quae T. crateriformi non comnetit, nisi casu forsan 
vermes tantopere coniracti fuerunt, cujus rei exemplum in Taenia polymorpha n. 37. vidi.

\section{Taenia opuntioides h. n. sp.}

Speciem istam, cujus caput non vidi. dubius propono, neque iamen supprimere volui.

Inter Taenias Lupi marginatás ah am. OtTone Professore Wratislaviensi transmissas fragmenta vidi Taeniae cum $\mathrm{T}$. cucumrina et litterata plurimum convenientis, et ipse pariter inter Taenias narginatas Januario mense $B$ rolini a me repertas consimilia offendi, quae af ilas vix pertinebunt. et qqucmadmodum Canic, Vulpes, Felis etc. ita etiam Lupus latuiam armatam marginatam) et $\boldsymbol{T}$. incrmem (opuntioidem) fovere videtur. Catalogo Viennensi manuscrip:o in usum vocato Lupi Taeniam armatam et inermom enumerari video, quod rem conficit.

Taenia rostra: articulis subinfundibuliformibus, reliquis elliptico-ovatis; maturis aut ovis farctis levissimo nexu inter se cohaerentibus; osculis marginalibus alternis.

Affinibus articuli minus laxe cohaerent, ut tantopere quam huic solutos alibi non viderim.

\section{Taenia dendritica Goeze.}

Cel. Nitzscir specimina mecum communicavit in intestinis Sciuri europaci Decembri re. perta, duos ad octo pollices longa.

Caput subglobosum, osculis orbicularibus remotis, collo brevi, cum articulis anterioribus fere capillare (ad quinque linearum longitudinem); hi ceterum brevissimi, cuneiformes; tum articula se. quuntur sensim sensimque longiores. 
Specimen, quod cel. Treutler, Augusto mense in Sciuri jejuno repertum, dono dedit, capite globoso parvo, osculis subglobosis profundis, collo longiusculo angusto et plano utitur.

18. Taenia difformis R. n. sp.

In Cribulo canoro (versus finem intestini tenuis) aliquot specimina Majo mense Berolini reperi, quorum unum in intestino liberum, reliqua intestinis fortiter infixa deprehendi: tres ad sex pollices longa, summa latitudine lineam vix attingentia, candida.

Caput subglobosum, osculis mediis suborbicularibus (diametro longitudinali paulisper excedente) marginatis, distantibus, magnis, planis. Collum nullum. Articuli anteriores diffornies, mox hoc, mox illo latere irregulari modo exstantes; tum breves plurimi, marginis postici angulis obtusiusculis, prominentibus; insequentibus sensim majoribus; tum iterum longa serie aequalibus, brevibus, margine postico obtuso exstantibus; reliquis paucis angustioribus, quorum non. nulli ultimorum fere inversi, ut margo posterior anteriore brevior sit. Articuli anteriores plani, posteriores convexiusculi, tota superficie punctati visi, undique scilicet ovulis farcti.

Obs. Froeticil (Naturforscher St. 29. p. 83. n. 48.) hanc speciem, in intestinis Cuculi tenuibus repertam, sub nomine Taeniae brevicollis descripsit, et rostrum ejusdem brevissimum obtusum et muticum retulit, quale ipse quidem non vidi, ideoque vero non negarem.

19. Taenia angustata R. n..sp.

Am, Breyser specimina duo in intestinis 


\section{$-495$}

Melis Taxi reperta misit, alterum septem, alterum novem lintas longun, hoc capite intructum, utrumque valde angustum.

Caput discretum, obcordatum, mediocre, osculis magnis orbicularibus. Collum longum (ad duas lineas), depressum, tenue, versus caput pau. lulum increscens, ceterum satis aequale. Articuli anteriores collo tantillum latiores, breves, senvim aucti, omnes plus minus quadrati, angulis rotun. datis; reliqui oblongi, inaequales, altero latere passin vél exstantes vel contracti; ulimus pro more apice decrescente obtusiusculo terminatus. An rostellum retractum latuit?

\section{Taenia longicollis $\mathrm{R}$.}

Specimina in Salmonibus Eperlanis Berolini reperi, tres, sex ad octodecim lineas longa, cum descriptione in Entozoologia data prorsus convenientia, neque possum, quin Scolecem tetrasto. mum (ib. II. 2. p. b. n. 3.) pro tjusdem prole hạbeam.

likemserus specimina in appendicibus $\mathrm{Sal}$. monis Thymalli pyloricis reperta misit, quat huc neque ad Botlırioceptalum pertinte crediclerin. Schrankilus (Fauna Boica T. III. P. II. p. 242. n. 315.3.) Taeniam Renkinam habet, hue pro. cul dubio amandandam, Sahno Wartmanni enim, cui T. longicollis est, Germaniae australi Renken audit.

Eadem Taenia Salmonum hydatidibus hepa. tis inclusa in Entozoologia immerito intu! Cysticercos relata est, quod in Synopsi correxi.

24. Taenia dispar Goezz.

Cujus descriptionem apud atictores nunquam 


\section{- $496-$}

bene intellexi, eam tandem Speziae Septembri mense in Gechonis vulgaris, Lacértae Stellionis Linn. duodeno et parte intestini tenuis proxima copiose reperi.

Capitis oscnla quatuor aperta subbemisphaerica, clausa autem orbicularia videntur, motu ceterum, tardo uruntur. Canales quatuor ab isdem exorti posteriora versus vergunt; brevi autem visui subducuntur. Collum longissimum, duos vel duos cum dimidio pollices longum. Articuli anteriores suborbiculares. Posteriores lineares hisce speciminibus non erant.

Descriptione data omnis ambiguitas tollitur. Membrana articulos obvolvens rulla adest, sed collum longissimum continuum, quod longitudine sua certe imposuit. Articuli hujus quam reliquarum specierum aeque parum tunica communi circumciantur; neque collum unquam articulatum fieri crediderim.

Ovaria lutescentia, glohosa, sparsa in articulis liberis, horum autem foramina rulla vidi.

Bremserus specinina misit in Bufouibus ci. nereo et viridi nec non in Hyla arborea reperta.

Illa Bufonis cinerei maxima, alterum quatuor pollices longum, reliquis omnibus crassius; quae Bufo viridis obtulit, tenerrima, parte anteriore longissima, diaphana, qualem in sequentibus non vidi; illa demun Hylae duos ad tres pollicé longa, articulos maxime varios sistunt, posteriores plerumque longi, recti, interdum elliptici. Membrana involvens ceterum nulla.

25. Taenia tuberculata $R, n$. sp. Specimina duo Algesirae in Lacerta $\left(\mathrm{n}_{\mathrm{t}} \mathrm{IX}.\right)$ 


\section{- 497 -}

reperta Bremserus transmicit, quorum alterum ses. quipollicare, alterum tripollicare, tertium ad duas lineae tertias partes lata.

Caput globoso-ovatum, oscilis quatuor an. ticis orbicularibus mediocribus, quintoque minore internerlio. Collim brevissinum, fere nullum. Articuli plani, subquadrati, latiores quam longi, marginibus rectis tenuibus, ut articulos nonnisi luci oppositos distinguere possis. Foramina articulorum, etiam primorum, marginalia tuberculo. rum instar prominentia, margine scilicet elevato instructa, eoque valde prostantia, vage alterna, ut mox complura hujus, mox unum alterunve oppositi lateris sint, ordine incerto. Ovaria indi stincta; maculas saltem orbiculares exiguuas tan. tum-passim in articulis vidi, quibus dissectis ovula nulla reperi.

Species antecedenti procul dubio affinis, collo tamen brevissimo et foraminibus tubercu. losis distinctissima.

\section{Taenia osculata Gozze.}

Entozoologian edendo duplicem hujus Tae. niae formam, mihi tum temporis constantem 'vi. sám prae oculis habui, quo commotus $T$. calycinam mihi dictam ab osculata distinxi. Postmo. dum vero Gryphiae Octobri nueuse Siluri Glanidis quinquaginta tres libras pondere aequantis in. testina examinando, Taenias plurinas reperi in. ter $\mathrm{T}$. osculatam et calycinam et ita quidem in. ternedias, ut nullum amplius discrimen superes. set. Tres quatuorve pedes tongae collo mox longiore mox breviore utebantur; articuli lineis longitudinalibus, rarius (postetiores) etiam trans. versis insignes; aquae commissae quasi gelatino. 
sae reddebantur, aliquot diés vivae conservatae. Taenia calycina ideoque rescindenda est,

27. Taenia sphaerophora $R$.

Am. Bremser specimina misit in intestinis Scolopacis Arquatae reperta, cum descriptione Ento7. II. 2, p. 119. n. 26. data omnino conveniertia. Rostellum magnum quidem est, sed spiritu vini contractum, quo ejusdem osculum visui subtrahitur. - In his quidem ova non reperi, in speciminibus olim a me lectis ova rotunda invenio, velamentis bicaudatis destituta, quibus illa speciei insequentis utuntur, ceterum vasis racematim inhaerentia.

28. Taenia variabilis R. 'Tab. 3. Fig. 3-6.

Haec cum praecedente aliisque speciebus mihi enumeratis in Catalogo Viennensi typis impresso conjungitur, cui tamen assentire nequeo; sphaerophorae enim rostellian magnum et collum longum, variabili autem hoc brevissimum, illud exiguum est; utriusque etiam ova differunt.

Frozitch (Naturf, 29. p. 86. n. 50. Tab. 2. Fig: 23-25.) Taeniam stentoream describit in intestinis Tringae hypoleticae repertam, quam huc traherem, licet vir cel. armatam dicat. Ipse saltem uncinulorum coronam ad basin rostelli nunquam vidi, qualem retulit, et errorem quendam subrepsisse suspicor. Árticuli cl. viro hypocrateriformes dicti melius infundibuliformes dicentur.

Fragmenta, quae Buemserus mecum communicavit in Tringa hypoleuca obvia, capite qui- 


\section{- 499}

dem destituta sunt, articulorum autem ratione ha. bita luuc amandari debent.

In intestinis Tringae Glareolae reperta, quae idem Anicus misit, pariter huc facere videntur.

Alterum specimen copite instructum, exiguo, rotundo; rostelio tentello; collo fere nullo, aut capiris tantum longiturinem acquante; articulis primum brevissimis, dcin sensim majoribus et longioribus. Eo tamen specinen privi quid ha. bet, primo, quod articuli priores breves maculam mediam obscuram, quanı alias posteriores tantum sistunt; secundo quod articuli insequentes sub. campanulati (neque infundibuliformes) vasa lateralia satis magna, et foramina alterna optinie exhibent. Maxime itaque evolutum est.

Cel. Nrmzsch etiam fragmenta dedit, in Tringa Glareola lecta, quorum articuli anteriores bre. ves, insequentes mox longiores, reliqui longissi$\mathrm{mi}$, passim ultra duas lineas longi, tertiam lineae partem vix lati sunt. Amicus quidem ad T. sphaerophoram retulit, sed huc potius referrem, nisi ،caput ct collum aliena sint.

Specimina quae Brsmserus noster dedit in intestinis Scolopacis subarquatae et Sc. Calidris reperta, capite, collo breví, et articulorum indole huc pariter referenda sunt.

Idem valet de speciminibus tribus ab Eo. dem acceptis, quorum maximum quadripollicare, quaeque in intestinis Scolopacis Gallingoiuis inventa sunt, capite, collo brevissimo, articulis prioribus brevissimis, tum valde elongatis et tenuis. simis, tandem latis plus minus campanulatis, convenientia.

Horum ultimi semisoluti et delabi incipien. 
Tes incredibili copia ovorum singularium scate. bant. Illis apertis primum omnia vasis tenuiss:mis referta videbantur, re autem attentius spectata ovula exigua velamento latissimo ct brevissimo utrinque in apicein capillarem abeunte, allantoidem et amion ovalia media parte circumdante, efformari vidi.

Comparationis gratia speciminum in Tringa Vanello a me repertorum articulos maturos examinavi et eadem omnino ovula reperi.

Comparavi quoque ova Taeniae porosae n. 90. affinis, sed armatae, formamque analogam, sed magnitudinem illorum multoties majorem, numerum autem multo minorem quam in T variabili observavi, conf. Tab. III, Fig. 7 , 8. et speciem insequentem.

\section{Taenia laevigata f. n. sp.}

Am. Bremser specimina triz in intestinis Charadrii Hiaticulae-reperta misit.

Captit subglobosum, osculis magnis, rostello cylindrico obtuso, Colium elongatum, capite multoties longins, latiusculum, planum, abiens in articulos latiores quam longos, sensin longiores, passim ordine tamén incerto angustioribus interceptos, quos longissimi tandem sequuntur (duplo longiores quam lati); angulis omnium articulorum rotundatis.

Altero in specimine hi quidem, sed obtusi, exstant. Omnes ceterum articuli majores ovis rotundis dispersis plena, absque appendicibus lateralibus, quae praecedentis speciei ovulis conceduntur.

Hisce velamen esse, ceterarum Taeniarum 
ovis denegatum, vix statuendum est: sed istis forsan illud tantopere reliquis tunicis accedit, ut eadenin simul forma utens non animadvertatur; in T. porosa, T, variabili certeque aliis bene, multis forma et magnitudine recedens, facillime sub oculos cadat.

-In Catalogo Viennensi typis impresso cum insequente et praecedente cónjungitur, sed qua milhi saltem visa sunt specimina diversas arguunt species, licet affines.

\section{Taenia amphitricha R. n. sp.}

- Amicus saepissime laudatus etiam hujus specimina misit in intestinis Tringae alpinae re. perta, unum ad quinque pollices longa, latitudine summa lineam superantia.

Caput duplici forma occurrit, rostello scilicet retracto inflatum, superficie antica truncata, exserto subrotundum. Rostellym capite longius, cylindricum, apice in nodulum abeunte. Oscula magna media, neque antica ut in $T$. variabili, neque exstantia uti plerumque in $\mathbf{T}$. sphaerophora et Filo exhibentur. Collum capite alíquoties longius, antrorsum dilatatum. Articuli anteriores angustissimi vel omnes contracti, parum longiores, vel etiam breviores quam lati, vel valde extensi, duploque longiores, margine tamen po. stico semper convenientes crasso recto, apicibus lateralibus exstante, disco articulorum pellucido, vasis lateralibus saepe transparentibus. Inter-. dum margo posticus valde tumet. Illos sequuntur articuli consimiles, sed majores et pro por. tione breviores, sensim quoad latitudinem augendi, brevissimi tamen servati. Tum latitudo iterum sensim tantopere decrescit, ut pars posterior 
eademque longa iterum capillaris fiat, articulis simul posticis illis anicis margine postico tumido similibus, sacpe tamen magis campanulatis, frarginibus lateralibus et anteriore rotundatis.

- Lemnisci alterni, recti, cylindrici arriculis tam latioribus quarm angustioribus competunt. Singulorum dnctus transversus non aequalis medius, uti alias esse solet, sed oblique retrorsum fertur et ita lemnisco jungitur.

31. Taenia mutabilis R.n. sp.

Am. Olfers frizgmenta complura, quae Taeriam aliquor pollices lonsam elfictre possent, in int stinis Ćretophagae dui, Septembri et Octobri a se in Biasilia reperta, una cum icone Taeniae misit. Hanc inıegram nunquam offendit.

Caput icone subglobosum sistitur, apice eminente truncato, annon rostello retracto? Osculas elliptica mediam capitis partem occupantia. Collum rapite triplo longius. Articuli priores transversi, brevisimi, insequentes angustiores, simul vero etian longiores; quos sequuntur subcampanulai, tandemque campanulati, margine postico tumido, sensim latiores, foraminibus marginalibus alternis.

Articulos plurimum variare Olfers addit.

\section{Taenia cyathiformis Froelucu.}

Saepius hanc majorem capite orbam reperi, sic v. c. in quinque Rirundinibus rusticis et riparia una, quas adhuc calentes Arimini Aprili secui; cujus quidem rei causa me penitus fugit. Exiguas praeterea in $\mathbf{H}$. riparia reperi capite in. structas, quarum altera hoc rostellatum, rostello capitellato, exhibuit, Specimen tenellum in $I_{\text {. }}$, 


\section{- $503-$}

apode repertum cel. Nitzsch mecim communicavit.

Am. Brfmser specimina nonnulla dedit in Hirundinis Helkae intestinis reperta, pollicem superantia, formae duplicis.

Alterum maxime explicatum, tenue, angustum: capite subgloboso, rostelii tantum particula cminente; collo plano longiusculo; articulis anterioribus brevibus, sensim in campanulatos reliquos transeuntibus.

Duo specimina crassissima, attamen depres$\mathrm{sa}$, contractissima; capite cordiformi; rostello integro exserto obtusissimo; collo haud conspicuo; articulis omuibus brevissimis, forman tamen cyathiformem, praesertim posteriori vermis parte, arguentịbus,

Fragmentum Taeniae a cel. Treutler Au. gusto mense in intestinis tenuibus Caprinnlgi eu. ropaei repertum et benevole mecum communicatum, num liuc pertineat, dubium videtur.

\section{Taenia infundibuliformis Gózze.}

Froelich (Naturforscher 29. p. 82. n. 46.) speciunina pusilla vix pollicem aequantia in $\mathbf{B o}$. schade fera reperta refert.

Rosa (Lettere zoolog. p. 6.) frustula refert Taeniae scirrho ni fallor intestini recti Gallinae im. plicita.

36. Taenia yaginata $R, n$. sp.

Bremsfrus specimina misit complura in in: testinis Charadrii Himantopodis reperta, et majora et minora.

Illa tres ad septeni pollices longa, anteriora 


\section{- 504}

versus quartam lineae partem lata, cito increscen. tia, ut latitudo posterioris partis duas tresve line. as aequet, crassiuscula.

Caput exiguum, depressum, utrinque margine positico tumido, antice al gustissimum, latera rotundatum. Rostelli valde exigui specits, noduli instar, rarius tantum habetur. Oscula in parte capitis posteriore tumidula orcurrunt, Orbicularia. Collum nullum. Articuli omnes breves, anteriores iidemque longe plurimi, brevissimi dicendi, latitudine quam longitudine multo majore, angulis posticis acutis; posteriores demum, illorumque ratione habita pauciores suhquadrati, angulis po. sticis non exstantibus, margine tamen posteriore articuli praegressi anteriorem insequentis te. gente.

Articulorum anteriorum márgines laterales aequales; reliquorum autem omnes lemiscum tereten acutum vel obtusinsculum brevem et duriusculum ex foramine rotundo protruso, vaginam lemnisci sistente, exacte alternantes emittunt. Ovula magna, chorio rotundo; annio oblongo exiguo, magno spatio ab illo distante.

Minores Taeniae angustissimae, planiusculae, unum duosve pollices longae, capite consimili, sed rostello majore, subcylindrico instructae. $A r$ biculi quorundam speciminum omnes serrati, margine postico exstante; alis oblongi intercedunt et posteriores passim subquadrati sunt, angulis obtusissimis, An illorum soboles utrum species distincta?

Prosa (Lettere Zoolog. p. 5.) obiter de Cha. radrii Himantopodis Taenia loquitur, fila tamen ex foraminibus marginalibus vage alternis pro. pendentia,longiuscula refert 


\section{$-505^{\circ}-$}

37. Taenia polymorpha R. n. sp.

Am. Bresser specimina complura benevole mecum communicavit in intestinis Recurvirostrae Avocetlae reperta, maxime inter se discrepantia; quo factum est ut in Catalogo Collectionis Caesareae Viennensis typis impresso duae hujus Avis Taeniae enumerentur; in manuscripto autem'serius confecto unica tantum illius Taenia et jure quidem habetur, nam maxime ludit, nuspiam autem transitus deest, neque varietatum character certus dandus erit. Singulas hasce enumerem, insimul autem capiti semper oscula esse orbicu. laria, ideoque haec non repeti, aldam.

Taeniae minores unum duosve pollices lon. gae capite utuntur angulato, latiore quam longo; rostello vel retracto, vel parum exstante, tumque subgloboso; collo mediccri, versus caput late. scente, articnlis anterioribus angustis et brevissi. mis, sensim paullo latioribas, nunquam tamen la. titudine lineam attingentibus.

Taeniae latiores vel magnae, vel parvae, hae caput angulatum exhibent, rastello obscuro: collum nullum vel exiguum; articuli latescunt lineamque superant, longo tamen itinere tales redditi. Ipsae tres et quod excurrit pollices longae.

Tertia forma latiores et breviores, citissime latescentes sistit, pollicem vix excedentes; capite angulato, rostellato; rostello quasi strictura diviso, inermi; articulis latitudine lineam superantibus.

Ouarta varietas Taenias sistit tres, quatuor et quod excurrit pollices longas, postice tres quatuorve lineas latas, Harum cajus discoideum, 
vermis ratione habita parvum; rostellum exsertum. non vidi; coilun nullum aut exiguum; articuli cito latescunt.

Omres ceteroquin articuli hatiores quam longi ansulos posticos óbtusos exstantes habent; illi mximi specininis maxime contracti articulos fere raeniae plicatae transversos referunt.

Leminirci oppositi, recurvi, durius uli, angu. lo articuli postico longiores, in magni illius speciminis parie antẹiore Iongissimi, filiformes, penduli.

Ova valde exilia, quae, 'cum Taenia crateriformis a'rticulis maturis instructa ad manus esset, cum hujus ovis comparavi valde magnis. Istorum centum $\epsilon t$ quinquaginta circiter aream lineae quadratae implent, quae ovorum Taeniae polymorphae mille et ducenta vel sexcenta capit. E contra autem ova illa $\mathbf{T}$. crateriformis magna embryonem non exhihent, quem exilia illa sub oculos ponunt. Sunt nimirum formae fere ovatae, et amnion continent ovale ab obtusiore ori extremo magis remotum; hoc vero embryonem includit linearem parte triplici constantem, apice nimirum subgloboso, parte media longiore, tertia longissima.

\section{Tacnia bacillaris Gozze.}

Haec in Catalogo Viennensi cum T. fila. mentosa Goezir conjungitur, neque haec nisi lem. niscis exsertis diversa videtur, quos mihi 1 amen nunquarn observare contigit, licet illam saepissime invenerim.

\section{Taenia sphenocephala $R$.}

Brearserus specimina nonnulla misit in in- 


\section{- 507}

testinis Columbae Turturis reperta', quorum alterum capite iustructum.

Caput minime cuneatum, sed potius triangulare. Rostellun cylindricum. Collum versus caput ampliaturn, ceterum lineare, longissimum. Articuli omnes breves, praesertim anteriores. Posteriorum passim denticulus aut lemnisci brevis quatdam species exstare videtur. Majorum ceterun articulormm macula media orbicularis opaca. Iidem singuli aliquot millia ovorum valde exiguorum, rotundorum, orhiculo medio opaco, contuent, quae illis Taeniae Columbae domesticae brasiliensis certe octogies minora sunt. Conf. Tab, 3. Fig. I9, et 20.

Idem Amicus specimina complura dedit in intestinis Columbae Liviac Majo mense reperta, de quibus, num huc faciant, dubius sum.

Taeniae sunt bi - vel tripollicares, anteriore parte capillares, posteriori lineam duasve lineas attingentes.

Caput unico tantum est specimini et quidem valde lato, sed tali modo comparatum, ut num penitus evolutum sit, merito dubites. Obliquum scilicet est' et antice complanatum, osculis ceteroquin orbicularibus satis bene conspicuis. Collum brevissimum. Partis anterioris capillaris articuli priores serie plus minus longa brevissimi, fere rugatformes, lineis longitulinalibue, vel vasis nutritiis, duobus tribusve pellucidis in conspectum venientibus; tum angustissimi, illis angustiores, subinfundibuliformes; partis insequentis-saepe su. biso latioris factae articuli breves marginibus lateralibus obtusis, angulis posticis acutiusculis, incumbentibus. 
Articulorum latissimorum maculae rotundae opacae duae, dextra et sinistra, ovaria sistentes; mi. nus latorum saepe alterius lateris maculae tantum adsunt, qualem inaequalitatem me antea observasse non credo. Ad utrumque latus vas longitudinale decurrit. Neque ova ovariis exprimere, neque articulis dissectis illa videre contigit, Foramina articulorum aeque parum. vidi.

Num hatc propriam sistit speciem, incertum quidem est, ațtamen sistere stispicarer,

40. Taenia platycephala $\mathbf{R}$.

Quae Gryphiae reperta in Entozoologia (II. 2.p.94. n.10.) descripsi specimina, illis minus eva. luta fuerunt, quae Berolini Decembri mense in tenuibus Lusciniac offéndi.

Horum scilicet alteri capitis rostellum exser. tum, breve, obtusums ; Articuli quoque posteriores aderąnt elongati, infundibuliformes.

Bremserus specimen, quod inspicerem, mecum communicavit, egregium, in intestinis Motacillae stapazinae repertum, exiguum quidem et tres tantum linẹas longum, sed ęo notabile, quod:

rostellum cylindricum, obcusum, longius exsertum esset, quam antea vidi, quodque articuli posteriores passinz lemıliscis uterentur crassis, brevibus, obțusis, ẹusdem lateris, Ceterum bene convenit.

Idem Amicus specimina dedit in intestinis Emberizae melanocephalae lecta, du, os vel duos cum dimidio pollices longa, rostello retracto, nullis notis diversa.

Alia quoque dedit in Ifotacilla nisoria reperta, huc pariter facere visa. 


\section{$-509$}

In plurimis practerea Ayiculis occurrit, quas duce Catalogo Viennensi in Synopsi enumeravi, etiau in Alauda, uti olin mihi visum est.

41. Taenia angulata $\mathrm{R}$.

Taeniam maculatam Batschro dictam Entoz. II. உ. p. 132. n. 34, a Brocuro minus bene descriptam separavi, sed Catalogi Viennensis aucto. ritate commotus cum $\mathrm{T}$. angulata conjungo. Au. ctores olim Taeniarum partem anteriorem sacpe collum vocaverunt, licet articulata sit, quod confusionem ciet; si enim verbis fides non habenda est, cognitio omnis tollitur.

Bremserus specimina aliquot misit, in Turdi nova specie (sibi octava) reperta, quorum alteruin caput quidem exhibet, sed rostello retracto. Collum breve. Articuli anteriores mox breviores, mox multo longioribus intercepti sistuntur. Hoc a mortis ratióne derivarem, nam ejusdem Taeniae articulos mox valde elongatos, mox valde contra. ctos saepe vidi et passim retuli; quemadmodum humanorum etiam cadaverum iris mox contracta, mox dilatata, musculi mox duri, mox flaccidi reperiuntur, et in plurimis partibus diversitas obtitinet. Res autem non pratervidenda est, ne falsa specie inducti, quae conjuncta manere debent, separemus.

44. Taenia tenuirostris R. $n+s p$.

Am. Bremserus aliquot specimina misit in intestinis Mergi Serratoris reperta, quorum alterum capite instructum, postice sesquilinean latum.

Caput subrotundum parvum; ro tellum gracile versus apicem obtusum increscens; inerme. Collum mediocre. Articuli anteriores angusti, 
brevissimi; insequentes longiores; reliqui angulis posticis acutis, quo corpus serratum redditur.

Quod idem amicus wisit in intestinis Mergi Merganseris repertum paululum deflectit. Caput scilicet magis tvolutum, fere cordiforme, est; osculis mediis orbicularibus; rostello ceterum gracili clavato. Corpus idem; neque negandum, pratter articulos anteriores longiores, hoc optime convenire cum Tatnia trilineata 11.87 . quae autein rostello majore, eodemque armato distinguitur.

Eandem quoque Taeniam et satis magnam in intestinis Mergi Albelli Berolini Majo reperi, sed capite destitutam.

\section{Taenia inversa R. n. sp.}

Specimina nonnulla in intestinis Sternae nigrae reperta Brenseri benevolentiae debeo, vix pollicaria, posteriora versus dimidiam fere line. am lata.

Caput subrotundum, osculis orbicularibus anticis, rostello exiguo, tenui, saepe nodulum tantum referente, oblongo, obtuso. Collum brevissimum. Articuli priores aliquot brevissimi; tum angustiores saepe, serie diversa et capillari, plus minus longa, interdum angustissimi, singuli basi latiores, versus apicem attenuati; sensim iterum latiores, qui maximam corporis partem sibi sumunt; reliqui tandem elongati; ultimus apice acutiusculo.

Articulorum omnium latiorum, tam anteriorum, quam reliquorum anguli postici obtusi, vel utrinque rotundati, vel angulis anticis magis antrorsum flexis, quo directio articulorum fere inversa videtur. 
Specimina tenella, quae mili ad manus fuerunt, Taeniam istam forsan haud satis illustrant, cujus itaque descriptionem successoribus commendo.

46. Taenia rapititaris $R$.

An. Breverar cornplura specimina misit in intestinis Colymbi auriti Jecta. conte quiden iner$m i$, neque lemitsis instiuia, halitu tamen, stois colli, articulorum ratione Taeniat mullistrictate nimis accecitntia.

Misit quocire atia tenuisima, in intestinis Colymbi subcristati reptita, capile destitula, de quibus num at $T$. capillarem, utrum ad $T$. rnultistriatam faciani, contendere non ausing, sed utraque certe conjungenda erit, ut illiua, quae capil. laris vocata sit, aculeorum corona non deficiac sed lateat.

\section{Taenia capitellata $\mathrm{R}$.}

Speciei elegantissimae ab ÁmildgaArdo detectae am. Bremser specimina nonnulla dedit in intestinis Colymbi arctici reperta, duos ad quatuor pollices longa, posteriora versus duas tertias lineae partes lata.

Caput subglobosum, quandoque subcorda. tum, osculis mediis orbicularibus. Rostellum retractum nodulum parvum referens; exsertum longum filiforme, nodulo subrotundo terminatum, nudis oculis conspiciendo. Collum breve, antrorsum in caput dilatatum, posteriora versus decrescens et lintare, neque annuli illud cingentis, Abinganarbro dicti, speciem vidi, nisi collum alterius speciminis apice his constricum cel. viro pro tali imposuit, Articuli anteriores satis longat 
serie angusti, angulis posticis exstantibus; dein sensim breviores et latiores, neque alios vel cam. panulatos ab Abrtdgandio relatos vidi. Singuli breves, antrorsum contracti, angulis posterioribus exstantes, quo corpus serratum redditur.

Lemnisci, cel, viro non visi, articulorum majorum sunt unilaterales, lingulati, sive subelliptici, vel passim subclavati, recti.

\section{Taenia fllum Goeze.}

Am Brenser specinina misit in intestinis Scolopacis Rusticolae. reperta, capite subglohoso, rostello, collo longo, articulis omnibus brevibus acutis corpus serratum reddentibus huc, neque ad sphacrophoram vel variabilem facientia.

Misit quidem alias Taenias pariter in Rusticola repertas, articulis quibusdam longis ad $T$. variabilem accedentes, sed collum longum et capue nostram Taeniam evincunt. Confer quae haec circa ad T. angulatam n. 4I. observavi;

Specimina porro mecum communicavit in intestinis Tringae ochropodis reperta, pollicem ad sex pollices longa. Hisce caput plus minus ratundum, saepe angulis obtusis lateralibus; rostellum cylindricum obtusum vel rotundatum; collum longum; articuli omnes breves, serrati, po. steriores latiores, margine postico passim tumidu10. Hae a $\mathbf{T}$. variabili quidem diversissimae, sed ad $T$. sphacrophoram quodammodo accedunt.

Taeniarum in intestinis Tringae pugnacis re. pertarurn fragmenta Brevser et Nitzsch, viri cel. mecum communicarunt, sed capite destituta, reliquis autem notis $T$, filum indicantia, 


\section{$-513-$}

50, Taenia microcephala R. n. sp.

Am. Bremser specimina complura misit in intestinis Tantali Falcinelli reperta, unum duosvé pollices longa, postice linean dimidiam vel integram lata,

Caput breve, continuum; rostellum cylindricum obtusum, interdum capite longius porrectum; osculis maximam capitis parvi partem sibi sumentibus. - Collum longum, interdum media parte capite latius, postice attenuatura. 'Articuli conformes breves, marginibus lateralibus rotundatis, angulis posticis acutiusculis reflexis.

Plurima specimina virginea, articulo postico rotundato. Majora specimina lineam mediam ob. scuram exhibent, $a b$ ovariis, procul dubio derivandam; sed neque in illis, quibus articuli secedunt, ova vel lemniscos observavi.

\section{Taenia Linea GoezE.}

Anconae Majo mense septem Coturnices ex: aminavi, quarum sex Taenias obtulerunt,.. vario modo comparatas.

Duabus in Coturnicibus specimina reperi complures pollices longa, tenuissima, diaphana. In tertia specimina maxima, postice sesquilineam lata, versus coeca et initium recti, minora in tenuibus offendi. In ave quarta nomnisi prolem reperi, quae facilé pro novo genere imponere posset, neque a vermibus dubii generis n. 23. in Perdice saxatili repertis, diversa videtur. Vermiculi scilicet villosae intestinorum tenuium firmiter inhaerebant, duas circiter lineas longi; Taeniae vel capite et colli parte solummodo, vel etiam articulis aliquot rugaeformibus constantes,

\section{$\mathrm{Kk}$}


quo nullum originis dubium superesset. Taeniolis istis caput insimul magis rotundatum fuit, cum magnis et liberis fere polygonum sit.

Quae altero die in quinta et sextá Coturnice reperi specimina, haud aeque lata fuerunt, sed articulos posteriores multos, longiores et campà. nulatos exhibuerunt. Offendi etiam specimina brevia vel magis compacta, capite majore, collo breviore, articulis anterioribus solito longioribus;

Obs. Quemadmodum vermiculos in Perdice saxatili obvios, Taeniae capite et colli parte formatos, fere novo genere Tetrathyridii enumeras. sem, nisi observationes praemissae rem explicuis. sent, ita olim Taenias longicolles pusillas pro Scolece tetrastomo vendidi; et am. AB OLFERS, qui Gasterostei Taeniolas Rhytelminthi Taeniolae nomine describeret et delinearet, pariter inductus est. - Confer observationes in Scolecem.

\section{Taenia elliptica B̈Arscri.}

Quod olim súspicatụs sum, Taeniam scilicet cuneicipitem ZeDERI, Entoz. II. \&. p. 143. n. 44. ad T. ellipticam ib. p. 195. n. 76. pertinere, neque hanc armatam esse, speciminibus comprobatum vidi in intestinis Felis Cati repertis et ab am. Bremsero benevole mecum communicatis.

His enim adsunt omnia, quae Zederus de cuneicipite refert, caput scilicet variabile, mox subglubosum, mox cuneiforme, sed praeterea articuli dantur postici, qui Zederianis speciminibus defuerunt, quo Taeniam cuneicipitem esse Taeniam ellipticam mancam, efficitur.

\section{Taenia globifera BAtscr.'}

Species ista summopere variat, meque mul. 


\section{- 515-}

tis licet ejusdem speciminibus vicis, capitis bonam ideam habeo; neque ova distiricte vidi. Biochrr figura utique falsa viletur, quae totos articulos ovis repletos sistit; Goezri forsan icon (Tab. XXXíl. A. Fig. 16.) melior, semel salt m ejusmodi quid ob òculos venit, sacculus scilicet minuta corpora gestiens.

Froelich (Nalurf. 29. p. 80, n. 44. T. glo. bulosa) caput subrotundum tuberosum vocat, quod non bene capio, ceterum Taeniam bene descripsit in Falconis Milvi crassis intestinis a se repertamí. Qua forsan auctoritate Tatnia flagellum Goxzir n. 67. in Milvo reperta in Catalogo Viennensi hŭc trahitur, ipse enim Milvi Taeniam non enumerat in Museo inveniendani. Hinc illam suo loco dubius reliqui, rieque supprimere ausus sumi'

Am。 Gaxde hi intestinis Tinniunculi specimen quatuor cum dinidio pollices Berolini offendit et recenș attulit. Hujus Caput antice obtusatum, rostello breví obtuso auctum, postice sub. sagittatum; collum breve; articuli anteriores angustissimi, brevissini; tum latiores, passim tanen minoribus intercepti; degin longiores; omnes mar. gine postico plus minus tumido, utrinque exstantes; ultimi laxi elliptico ovati, âuî subellip urci, vel subrotundi (hi breviores) tuinica media taintum colracrentes.

Specimen in Briteone repertum quod am. Brexiser misit, aliquot pollices longum, caput oblongum exhibet, diculis posticis vaide prominulis; parte anteriore (an rostello?) magna oblonga obtusa.

Specimina duo, quaé itidem Bremsero de. beo, in Falcoue lagopode lecta iterum differunt; 


\section{$-516-$}

alterius caput osculis prominulis, partem anteriorem brevem attenuataín obtusiusculam hałset; rostello nullo conspicuo; alterius capnt fere tri'gonum, neque osculis, neque rostello dignø. scendis.

In specininibus ab eodem Aimico missis êt in Falcone lanario repertis, capite destitutis arti. culi mágni maculam mediam orbicularem exhibént, certe ovarium; sed ova non adsunt.

Neque magnis speciminibus, in Falcone rufo lectis, capite destitutis, neque articulis turgidis laxis, facile secedentibus, speciminum in $F$. $l a$ gopode repertorum; ova sunt, in quae sedulo inquisivi.

\section{Taenia brevicollis R, n. sp.}

Am. Gaede, nunc Professor Leodiensis, Berolini Junio mense in intestinis Arustelae ermineae Taeniam reperit solitariam, sex pollices longam, candidam.

Caput subórbiculare, discretum; rostelló exiguo, inermi, acutiusculo; osculis orbicularibus, mediocribus. Collum strictum a capite distinctum; hoc latius, brevissimum." Articuli anteriores an. gusti, brevissimi, inaequales, laxiusculi; insequentes majores, breves, sensim subquadrati; reliquí elongati, angustiores, fere parallelipipedi, cras. siusculi.

Articulorum omnium majorum, tam quadratorum, quam longorum foraminia vage alterna, in tubi speciem protracta; ex guibus in articulis elongatis lemnisci propendent lineares, longi, apice parum latiori, obtuso, posteriora spectante instructi.

Obs. Rrimo intuitu fere ad T. intermediam 
a. 7r. retulissem, quae autem eapite, rostello armato, collo, articulis anterioribus plurimum differt. T. brevicolli etiam ovaria dendritica non sunt, quae in intermedia observantur. Insequentibus utique magis affinis est.

\section{Taenia tenuicollis $\mathbf{R}$.}

Ent. II. 2. p. 196, n, 77. Taenia Putorite p. 197. 11. 78. T. Inustelae vulgaris,

Am. Bremser specimina rnecum communicavit, tam in Mustela vulgari, quam in Putorio reperta, inter se haud diversa.

Caput parvum, subrotundum, rostello inermi, recto, truncato, brevi; osculis orbicularibus mediocribus. Collum satis longum, tenue. Articuli priores breves, obtusati, sensim subcuneati, majores; reliqui longiores, angustioresque, foraminibus vage alternis. Ultimi elongatorum duas fere lineas adaequant.

Obs. Speciei praecedenti valde affinis, capite colloque tamen distincta. Cum T. intermedia $\mathrm{n}_{\text {。 }}$ 7r. etiam quaedam obtinet similitudo, sed collum longum hanc tollit.

\section{Taenia obtusata R. n. sp.}

Am. Brearser specimina misit in Vespertilionis murini tubo intestinali reperta, quatuor ad octo. lineas longa, parte antcriori capillari, posteriori tertiam circiter lineae partem lata.

Caput subrotındum, latius quam longum; osculis subglobosis profundis; rostello nullo quidem in specinine exserto, passim tamen distinctissimo, pellucente, exiguo, tenui, inermi. Collum breve. Articnli omnes marginibus convexis, angulis, obtusatis vel oblitteratis; rarius campani- 


\section{$-518=$}

formes; antici longiores quam lati, postici latiores. Majorum articulorum foramina alterna tumidula, Ovula sparsa. Lemniscos non vidi,

Distincta ceria species,

\section{Taenia candelabraria Goeze.}

Am. Breysero Taenias aliquot debeo in intestinis Strigis brachyoti repertas, aliquot polli. ces longas, quarun altera capite instructa est.

Caput majusculum, oscula suborbicularia, posticam ejus partem sibi vindicantia; rottellum retractum pellucens in capitis parte anteriore obtusa tlongata. Collurn satis longum et latiusculum. Articuli anteriores brevissimi, rugaeformes; sen. sim majores facti, breves, subcaneati, vasis longiturinalibus trarsparentibus; tum magis nagisque elongati in formam campanulatam abeuntes, plerumque medio constricti; vel et longiores bis constricti, angulị posticis exstantibus @ii tandem retrahuntur, ut articuli marginibus rectiusculis utantur, cute tenui, pellucida instructi, cujus ope canalis medius cum ovario (candelabrum Goẹ. zII) conspiciantur.

Arțiculorum majorum scilicet parti posticae corpus transverse ellipticum opacum inest, quod ovarium sąciforme crederen, licet ova hujus Taeniae non vịderim, passim enimi illud corpus punctatum apparet, neque in reliquo articulo ova adsunt; ex hoc sacco ductus rectus antrorsum ducitur in articuli parte antica in cupulan dilata. lus, yna cum ovario articuli praegressi iterun colaetere videtur. Ex ductu longitudinali anten, haud exacte in ejus medio, sed paululum ante hoc, canalis transversus ad foranen articuli marginalc tesidit. Corpora corta non vidi, quae 


\section{- 519}

loç ductus medii in figuris Goezianis (Tab. XXXII. B.) Exhibeutur, et cupula ibidem nimis magna si. stitur; foramina autem vage alterna, canales trans. versi et ovarium in parte articuli posteriore il. I̦ic penitus omittuntur.

Species insequenti affinis, sed certe diversa.

\section{Taenia parallelipipeda $R$.}

Specimina aliquot Majo mense in intestinis Lanii minoris reperta ab am. Bremsero accepi, duos ad quinque poilices longa.

Caput subglobosum, vel etiam subovatum, rostello brevi, crassiusculo, obtuso. Collum brevissimum vel breve. Articuli anteriores quales Ent. II. 2. p. 377. descripsi, posteriores semper medio constricti. Omnium cutis crassa, neque ut in specie praecedente, pellucida; candelabri Goeziani (ovarii cum oviductu) vestigium nullum. Ova nulla in conspectum'venerunt.

Specimina mea in Collurione reperta et l.c.

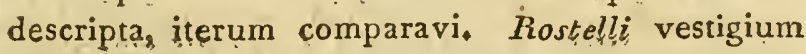
utique adest. Artiçulos anteriores quam in $\mathrm{T}$. candelabraria magis rotundatos reperio, pósteriores non ita pellucentes, undique ovulis elliptico-rotundis repletos, neque ovario sacciformi instructos, magis elorigatos.

Species lraec a praecedente ergo distinctis. sima, qua de re in Synopsi inmerito dubitavi.

Rosa (Lettere zoolog. p. 6.) Taeniam in Lanio Excubitore repertam obiter refert.

62. Taenia farcíminalis BATscrr.

Froelich (Naturf. 29. p. 82. n. 47.) specimina Taeniae infundibuliformis 11. 33. q̣uadripol 


\section{$-520-$}

licaria articulis irregularibus ludentia perperam huc refert.

\section{Taenia stylosa R.}

In Catalogo Viennensi typis impresso haee cum 'Taeniis undulata n. 83. et serpentulo n. 89. conjungitur, ipse tamen examine repetito omnes tres distinctissimas haßeo; hanc inermen, illas armatas quidem, sed collo et articulis diversas.

66. Taenia oligotoma Nitzscri, n. sp.

Taeniolss quatuor in intestinis Sternae fise sipeaiis Junio repertas am. Nitzsch misit, sesquilineam ad duas lineas longas, antice angustiores, postice quartam lineae partem latas.

Caput subglobosum, crassiusculum, discretum; asculis orbicularibus magnis illud fere totum sibi vindicantibus, quo media capitis parte fere se attingant; fuscescentibus, reliquo horum speciminum colore albido. Rostellum breve subglobosum, apophysi brevi insidens, omnibus exsertum., Collum brevissimum, articulo primo majus, capite latius Articuli priores sex octove brevissimi; tum sensim lọgiores, latitudine tamen longitudinem aliquoties superante; tandem majores sex octove diversis speciminibus diversi, aut prioribus mere majores, aut angustiores et fere campanulati, aut rotundati.

Nonnulli certe articuli, forsan elongati, deficiunt, species tamen distincta videtur.

\section{Taenia flagellum Goezz.}

In Catalogo Viennensi haec ad T. globiferam n. 53. jure relata videtur, ibidem antem cum plurimorum quidem Falconum, neque tamen Milvi 


\section{$-521$}

Taenià enumeretur, illam delere non ausủs sum, sed dubiam relinquo.

\section{Taenia Malleus GoezE.}

Cl. Froeurch (Naturf. 29. p. 13. Tab. T. Fig. 4-6.) genus novum fingit, Fimbriaria sibi dictum, cujus characterem sequentem offert: „Vermis elongatus, planus, subarticulatus; caput obscurum membrana pellucida fimbriato-plicata, antice truncata et ampliata occultatum."

Hujus species enumerat duas, alteram scilicet: Fimbriariam mitram: corpore articulato, articulis utrinque emarginatis, capitis fimbria bilaba, plicata, lobo lanceolato prolixo. Alteram autem, Fimbriariam malleum, corpore subcontinuo, transversin striato, capitis fimbria subintegra, malleiformi, altero latere in lobum conicolanceolatum prolixo,

Illam in Anate Boschade fera, hanc in Anate domestica occurrere refert.

Mihimet ar êem Taeniae malleatae suspectae sunt, ut reliquarum Taeniarum monstra crediderim. Variis in Anatibus repertae sunt, et ipse in Penclope offendi, séd semel etiam Taeniam malleatam in $P$ ico, loco certe inopinato, inveni,

Species salten a'cl, viro stabilitas non majoris ponderis habeo, quam genus ipsum. Taeniae enim sunt, altero fíne alienatae, illarumque monstrositates quam plurimae occurrunt, quarum in specie insequente mentio fiet.

Hypothesis autem mea si experientia com probaretur, species ista delenda foret, ut singulis 


\section{$-522=$}

speciebus, quae monstrositati huic obnoxiae sunt, illa in observationibus adderetur:

69. Taenia Solium LinN?

Olim in cadaveribus rarius occurrere cre. didi, quod dein secus vidi. Hyeme enim praeteriı cum Medicinze studioso cuidam intestino. rum examen in theatro anatomico Berolinensi commendarem, brevissimo tempore tribus in cadaveribus Taeniae repertạ sunt, easque, quam vulgo assumitur, mulıo frequentius in homine adesse, sed innoxias praesentiam non indiçare, persuasum habeo.

- In Museo Viennensi specimen vidi, cujus articuli majores perforati sunt, foramine medio mox rotundo, mox magis rhomboidẹ, minore vel majore, nunquam autem tanto, ac Masars De Cazellfys delineavit, qua de re Ent. I. p. I05 n. 363. conferatur, Plerumque articuli maturị toti solvuntur et vermes cucurbitinos sistunt, passim tamen ovaria sola decedere videntur, quo Taeniae fenestratae oriuntur. Alio in specimine foramina articulum inferiori magis loco trajiciebant.

Priore in specimine foramina marginalia ariculorum breviorum tubulos sistúnt, quos hac in specie tantopere protractos antẹ non, vidi.

Adest etiam-in Museo Caesareo monstrositas Taeniae maxime singularis, articulorum nem. pe duplicitas antea non observata. Alter articulorum margo lateralis simplex est brevi autem in sluos articulos, sibi incumbentes abit, quoruin margines laterales (illi simplici oppositi) liberi sunt. Articuli bini sibi impositi eatenus minus bene dici possunt, quia margine altero simplici 
conveniunt, et utraque pars duplici tunica dum constat, pars libera quatuor paginas habet, qua coalescunt autem tunicae internae cessant. Fo. ramina marginalia maximam partem unilateralia sunt et in margine simplici tantum occurrunt. Haec circa tamen varicttaes obtinent sequentes: 1 . margo simplex foramine non utitur sed alter li. berorum; aut 2. margo simplex et alter libero. rum; aut 3. margo simplex et uterque liberorum foramine utitur. Duo etiam adsunt articuli, quorum margo simplex foranine duplici, superiore et inferiore instruitur. Hujus Taeniae ca. put deesse maxime dolendum est, forsan eodem modo duplicatum est, ac $T$. crassicolli $n, 75$. ibidem servatae observatur.

Quod in Entozoologia contra Taeniam commendavi Oleum Chaberti, a Bremséro etiam sumino usu adhibetur, et in opere novissimo egregio de Entozois vivis in homine vivo com. mendatur; idem etiam a Medicorum Berolinensi. um Principe, cel. Heim in usum vocatur, qui specimina capite instructa mecum communicavit illius ope depulsa.

\section{Taenia marginata Batscr.}

Am, Oтто specimina complura misit, et ipse quam plurima in duorum Luporum intestinis tenuibus Berolini Januario mense reperi, pedem duosve pedes longitudine superantia, summa la. titudine fere tres lineas aequante.

Caput subrotundum, discretum, magnitudine aucta a Comite DE Bokrs apud Goezium (Tab. XXIL. A. Fig, I-5.) optime delineatun. Collum illi affinium simillimum, neque brevius, yquale in figura laudata occurrit, nisi haec circa varietas obtinet. 


\section{$-524-$}

Reliqua descriptio in Entozoologia (II. 2. p. 165.n. 57.) oblata sufficit.

Speciem inermem, T. opuntioidem, pariter if Lupo $t$ t cum hac promiscue sed rarius occurrentem, supra n. 15 . indicavi.

74. Taenia laticollis 'R. n. sp.

Specimina duo in intestinis Felis Lyncis reperta JREMSElevs mecum communicavit, alțerum duos, alterum tres pollices longa, postice lạtitudine lineam tertia parte superantia.

Caput crass̄um, discretum, lineam dimịdiam longum, rostcllo uncinisque validis, minoribus tamen quam in $\mathrm{T}$. crassicolli; osculis majoribus magisque exstantibus. Collum sesquilineam longum, crașsiusculum, capite articulisque prioribus parum tantum angustius. Articuli anteriores brevissimi, insequentes sensim majores, subquadrati, vel oblongiusculi, marginibus lateralibus plus minus convexis, postico rectiusçulo. Foramina marginalia vage alterna valde protrusa, tubum truncatum sistentia, quam in speciebus affinibus majorem.

\section{I aenia crassicóllis $\mathbf{R}$.}

Hujus varietates et monstrositates rarissimas Museum Caesareum Viennense continet.

Specimen ninirum adest, cujus caput sex oscula exhibet, et anterior corporis pars bipolli. caris (tanta enim solummodo datur; ope incisurae profundae divisa et triquetra facta est. Reliquam partem desiderari dolendum est, forsan énim duplicata fuit, uti Taenia Solium supra descripta, quam conferas.

Ipse tamen Taeniam canis prismaticam pos- 


\section{$-525$}

sideo, cujus caput vulgari modo comparatum est. Cont, Ent. II. 2. p. 102. obs. 2.

Specimen porro $T$. crassicollis ibiđèm servatur, capite normali, articulis tantopere contractis, ut Taeniam Anseris lanceolatam referat。

78. Taenia quadrata $\mathrm{R}$. n. $\mathrm{sp}$.

A $m$, Brevsérús specimina misit in intestinis Mruscicapae atricapillae et collaris réperta, speciem distinctam sistentia, Taeniis Mammalium armatịs affinem.

Caput facie antica quadrata, rostello. brevi. zincinulis satis magnis reflexis armato. Collum nullum. Articuli anteriores brevissimi, reliqui longiores, margine postico tumidulo, angulis utrinque obtusis exstante, Foramina marginalia vage alterna.

In nonuullis articulis speciminis in Muscicapa atricapilla reperti lemniscus brevissimus emi. net; specimini majusculo autem in M. collari le. cto articulus est, cujus ex foramine leanniscus longus filiformis propendet.

\section{Taenia acuta $\mathrm{H} . \mathrm{n}, \mathrm{sp}$.}

Specimina in intestinis Vespertilionis lasiopteri reperta Bremseri benevolentiae debeo; quorum unum semipollicare capite instruitur.

Caput subglobosum; osculis quatior orbicu. laribus exiguis mediis; rostello magno; valido, apice uncinulis mediocribus armato. Collum capite aliquoties longius rectum et aequale. Articuli anteriores rugaeformes, brevissimi, latitudine collum aequantes. Insequentes magis contracti, marginibus lateralibus convexis; angulis posticis acutis: turn alii ejusdem formae, sed latiores, si 


\section{- $526-$}

longiores forent, campanulati dicendi; dein alii marginibus lateralibus concavis, angulis posticis semper acutis; tandem latissimi (speciminis ses. quipollicaris, capite destituti) subcampanulati, marginibus tamen lateralibus variis, mox convexis, mox rectiusculis, mox concavis; angulis posticis acutis.

In media articulorum majorum parte macula opaca subrotunda, ovarium indicans. Margines. unius lảteris saepe foramen tumidulum monstrant, ex quo passim filum tenue, flaccidum simul pro. pendet.

Obs. Taenia obtusata Vespertilionis murini n. 59. dicta ab ista valdopere differt, forsanque Taenia Vespertilionis auriti n. 94. inter dubias enumerata ad alterutram pertinet, nisi Vespertilionibus, uti plurimis manmalibus, Taenia tam inermis, quams armata est, quod tempus docebit.

\section{Taenia multistriata $\mathbb{R}$.}

Bremservis specimina nonnulla misit, in intestinis Colymbi minuti reperta, quae cum descriptione mea Ent.II.2. p. 183. n.6\%. oblata exasse conveniunt.

Nitzschius duo specimina mandavit eadem in ave Augusto mense reperta, valde contracta, an forsan viva spiritui vini immissa? Hisce coilum capillare evanuit et antica párs crassiuscula. ot rugosa apparet; reliqua pro more.

Taenia capillaris n. 46. huc forsan referenla. A reliquis distinctissima, etiam ab insequen. te, quam conferas. 


\section{$-527-$}

85. Taenia inflata $R, n$, sp.

Am. Brevser specimina complura mecumn conimunicavit in intestinis Fulicae utrae reperta, unum ad tres pollices longa, parte anteriore capillari fere pollicem longa, plana, posteriore crassiuscula, duas rertias lineae paries lata.

Caput magnum, obovatim aut fere clava. tuni; osculis orbirularibus magnis anticis; rostello valiclo, subgloboso apice uncinorum reflexorum corona simplici armato. Collum longum, tenuis. simum, passiin in nonnullis inflatum, ut his lo. cis triplo crássitis sit. Articuli priores longa se. rie brevissimi et angustissimi; tum majores cito increscentes; quo fit ut pars capillaris a reliqua saepe maxime distinguatur et huic addita vide. atur. Arriculi omnes reliqui brevissimi, angulis posticis acutis, brevibus, posteriora versus directis.

Partis látioris, hunquam àutem latissimae et crassissimae, articuli lemniscis instructi unilateralibus, tenuissimis, saepe articuli latitudinen su. perantibus. Conf. Taenia scolecind n. 93.

Articuli ultimi crassi repleti ovis quorum velamentum externum utrinque in diverticulum excurrit longissimum et tenuissimum, tenuius quidem ac in alfinibus observavi; conf. Taeniam variabilem n. 2S, et porosam n. 90.

Striae colli quaiuor, corporis quinque lon. giturlinales fuscae. Loca colli inflata aliis in Taeniis nunquam vidi. Speciei pratcedenti haec valde aftinis est, capite tamen, rustello, lemniscis satis distincta.

86. Taenia sinuosa $R$.

Am Nixzsch specimen misit in Anate Bo. 
schade fera Julio repertum, postice lineàm supe rans, cum descriptione mea Ent. II. 2. p. I84. n. 68. oblata conveniens praeter uncinos rostelli obscure tantum exhibitos*

An Taenia laevis no 42. huc referri debet?

87. Taenia trilineata BATSCIr.

Hujus specimina inermia olim tantum vide. ram, ut Ent. II. 2. p. 136. n. 38. inter. ineimes = enumeraverim. Clariss. Froelich (Naturf. 29. P. 84. n. 46. Tab. 2. Fig. 21, 22. Taenia longirostris) ipsan quidem armatam suspicatur, sed uncinulos fon vidit, unde rostellum subinerme vocat. Est tamen armata, quod specimina me docuerunt ab am. Nrtzschio in Anatis Boschadis Jerae intestinis coecis Junio et Novembri reperta, quae sub nomine Taeniae furcigerae sIBI dictae vel T. lon. girostris Frofirchr' 'necum communicavit.

Rostellum cylindricum, elongatum, apice uncinis longiusculis tenuibus armatum. Articulorumi apices postici reflexi sunt. Reliqua cum descriptione olim 1, c. data conveniunt.

88. Taenia undulata $\mathrm{R}$.

Froecich (Naturf. 29. p.8r. n. 45.T. undula.) in intestinis crassis Corvi Coracis repertam obiter describit, et cum Taenia confundit in Anatis Boschadis ferae crassis obvia. Taeniae Coracis unum cum dimidio ad quatuor, pollices longae fuerunt.

Cel. Nixzscr specimen mecurn communicavit in Coraice Martio mense a se repertum, duos cum dimidio pollices longum, antice lineae tertiam partem, postice sesquilineam latuin. Caput quadratum, antrorsum rostellum emit- 
tens, cujus uncinulos obiter tantum vidi. Collum nullum. Articuli. ad unum omnes latitudine lon. gitudixem superant, Lemnisci non aderant.

89. Taenia Serpentulus Scirrairm.

Haec et praecedens cum in Catalogo Vien. nensi typis impresso conjungantur, specimina mea sedulo iterum comparavi, sed distinctas esse, denuo observavi, Differentiae specificae in Syn. opsi p. 167. oblatae idem probabunt.

90. Taenia porosa R. Tab. 3 Fig. 7. 8 .

Speciei insignis a me in Laro cinerario re. pertae Ent. II. 2. p. 190. 'n. 7r. Tab. X. Fig. I. descriptionem dedi, quam aliis speciminibus ab am. Bremsero acceptis nunc augere datur.

I. Plurima primum huc refero in Laro mi. nuto inventa, formae variae.

Majora specimina aliquot pollices longa; capite orbata, articulis prioribus brevibus, nox longa serie elongatis, conicis vel infundibulifor. mibus, vel tandem campanulatis,- reliquis subquadratis, brevioribus quam latis, angulis obtusis.

Alia specimina, multoque plura, exigua, tres ad quinque lineas longa; capite subrotundo; oscu. lis magnis (quae speciei nomen dederunt); ro. stello conico infra apicem armato; saepeque foramen capitis vidi, in quod rostellum retrahitur. Collum antice latum, tum tenuius, breve. Articuli plus minus breves, irregulares, passim intersupti.

Inter specimina illa quaedam adsunt candi. dissima, lata, partem Taeniae posticam rantum si- 
stentia, quae nudis oculis muco obducta viderentur. Re autem attentius et microscopii ope exáminata, articuli illi ovis repleti ad latera et praesertim angulum posticum versus utrinque hiabant et ova fundebant maxima, quae Táb. 3. Fig.7.S. - delinata dedi. Horum velamentum externum iransversum elliptico lanceolatum, saepe in diver. ticula tenuia abiens, tunica ovi secunda rotunda triplo et quod excurrit latius, longitudine autem media illam parum superans; tertia autem tunica sive intima globosa est uti secunda, sed hac multo minor. Varia ova his similia sed minóra passim tetigi, de quibus Taeniam variabilem n. 28 . et $\mathbf{T}$. inflatam $n .85$. conferas.

2. Specimen in Laro fusco repertum accepi, illis simile, sed capite destitutum.

3. Aliquot specimina in Laro ridibundo le. cta. Horun alterum bipollicare, capite instru. ctum, quale olim delineatum dedi, et praecedentia conjungens. Articuli subquadrati pone angulum anticum foramina vage alterna exhiberit, quorum plurima lemniscumi brevem, acutum, antrorsum directum emittunt.

(Cum hisce specimen simul aderat Taeniae variabilis, capite rotundo, rostello tenui, collo et articulis prioribus brevissimis, reliquis longis et angustis instructum, ut Laro praeter $\mathbf{T}$, armatam (porosam) etiam inermis (variabilis) adesse videatur.)

4. Taenia Lari cani ab am. Braun reperta, inter dubias n. 136. collocata, forsan Taeniam porosam capite evoluto sistit, quale observare mihi nondum contigit. 


\section{$-531$}

91. Taenia crateriformis Gozze. Tab. 3. Fig. 9.

In Pici viridis intestinis Amanuensis noster Berolini Junio quinque specimina reperit, quatuor ad quinque pollices longa.

Caput rotundun. Rosteillum cylindricum apice globoso, uncinulorum corona (passim obscura) cincto. Oscula orbicularia magra. Collum longiusculum, longitudine varia. Articuli anteriores rugaeformes, angusti; insequentes magis unagisque distincti, breves tamen; sensin deinceps majores, tandem margine postico tumido utrinque exstantes; ultimi crassiores, ovis referi, saepe his effusis fere rhomboidei visi. Foramina articulorum marginalia opposita.

Ova hujus Taeniae globosa et maxima sunt, singula enim sextain circiter partem areae micro-metri, quae vigesimam quintam lineae quádratae partem adaequat, sibi vindicant, vel centesimam et quinquagesimam lineae quadratae partem magnitudine attingunt. Tunica externa ab interna valdopere distat, uti Gofze Tab. 3I. B. Fig, 18. satis bene expressit.

Am. Bremserus etiam specimina misit in Pico viridi reperta, quorum rostellum capitis retractum est, quae ceterum autem cum descriptis conveniunt, simul autem explicant, quare Catalogus Viennensis typis impressus Taeniam crateriformem ad $T$. crenatam, neque hanc ad illam refert, quod ipse praefero. Speciniña enim Musei caesarei rostrum armatum non protulerunt, quod ipse vidi.

Cel. Treutler in Upupae Epopis abdomine, circa renes, Majo mense Taeniam reperit conn- 
plures pollices longam, capite destitutam, ovaris autem et reliquis notis huc facientem, quam benevole mecum communicavit.

\section{Taenia longirostris $\mathrm{R}$. n . sp.}

, In. Glareolae austriacae intestino summo Arimini Aprili Taenias et Taeniólas offendi immotas, calida affusa reviviscentes, motu illarum cito evanescente, harum satis diu remanente.

Taeniolae multae adfuerunt, duas ad qua. tuor lineas longae. Harum caput varium, mox cordatum, mox fere pyramidatum; osculis orbicularibus, passim etiam oblongis, Restellum mox simplex, teres, longum, acutum, mox nodulo instructum uncinorum corona armato. Collum brevissimum. Articuli priores brevissimi; reliqui parum majores, latiores quam longi, vasis qua. tuer longitudinalibus.

Majora specimina bi-vel tripollicaria, capito et priore corporis parte destituta articulos anticos subquadratos, insequentes campanulatos, tum infundibuliformes, demum elongatos, passim fere parallelipipedos obtulerunt.

Alia specimina, neque tamen completa, ab am. Bremsiero accepi, Horum caput subrotunduim, osculis magnis, rostello longo vel longissimo, sed uncinulis' non exsertis. Collum brevissinum. Articuli primum perbreves, saepe longa serie, tum campanulati vel subcampanulati, et tandem longiores.' Neque ovila neque lemniscos vidi.

93. Taenia scolecina $R, n$. sp.

Speciei singularis in Pelecani Carbonis in: testinis a se detectae specimina plurica am+ Brzar, 


\section{$-533$}

srr misit, unam ad duas cum dimidia lineas lon. $\mathrm{ga}_{2}$ angustissima.

Capnt corpore duplo latius, polymorplum, subglobosum, vel obcordatum, vel parte anteri. ore eademque latiore ope stricturae a posteriori subovata distincta; tetrastomum, duobus plerum. que oscuits tantum in conspectum venientibus. Rostellum saepissime retractum, macisla capitis anteriore obscura illud prodente; unico in specinine distinctissine armatum, uncinulis satis magnis.

Articuli anterioris, majorisque simul corpo. ris partis, brevissimi, priores rugaeformes; marginibus satis aequalibus, lemniscis unilateralibus brevibus truncatis, mox copiosis, mox potius spar. sis, saepius ubique latentibus.

Posterior brevissinaque corporis pars articulis constat et latioribus et longioribus, mox aequalibus, contiguis, mox singulis versus angulos rotundatis et laxius inter se cohaerentibus; interdum iisdém altero latere exstantibus et quam anterior pars duplo latioribus; semel paucis tantum hoc modo altero latere exstantibus et quasi gibbum ibidem efficiéntibus.

Hi ceterum articuli, qui vel laxius colrae. rent, vel extenduntur passimque gibbi fiunt, ovu* lis onusti apparent exiguis et rotundis.

Vermis iste singularis spadici multurum florum instar anteriori parte masculus, posteriorí femineus videtur, dum alias Taeniae articulos siugulos androgynos exhibent, nisi forte Taenia inflata n. S5. et affines luc pertinent. Reẹ ulteriore incquisitione dignissima, in sectione sequente uberius illustranda erit. 


\section{- $534=$ \\ species dub iac.}

101. Taenia Myoxi.

Taeniae in intestinis Myoxi Gliris repertae am. Brimser fragmenta mecum communicavit pollicem dimidium ad tres pollices longa, capite destituta, articulis instructa breviusculis, versus angulos rotundatis, passim sed absque ordine angustioribus, posticis ova rotunda ferentibus, novam uti videtur speciem prodentibus. Speciminibus in Museo Caesareo servatis caput pariter deest.

125. Taenia Unguicula Braun.

Am. Bremser fragmentum Taeniae in intestinis Ardeae Ciconiae repertum misit tripollicare, capite destitutum, et speciminibus in Ḿfusęo Viennensi servatis hoc pariter deesse, simul adnotavit.

Articuli fragmenti nostri priores satis longa serie longi, antrorsum angustati, posteriora versus increscentes, margine postico valde tumido, angulis utrinque exstantes; hi sensim in subcampanulatos abeunt, forarninibus alternis instructos; ultimi laxe cohaerentes, subquadrati, aut sublunati, margine postico exciso, angulis posticis reflexis.

Fragmenta Taeniae in Ardcae cinereae intestinis ab am. Braun detecta," et Ent. II. 2. p. 207. n. 97. descripta eandem speciem efficere videntur. In Appendice duae novae Taeniarum species in Ardeis Brasilianis repertae dicentur, quarum neutra luc facit.

137. Taenia Tadornae.

T. Tadornae: plana capite quadriosculari, medio aculeato. Zool. Dan, Vol. IV. p. 3I. Tab. I 148. Fig. B. I. 2. 
Inter minimas hujus generis. Corpus aequale. Caput truncatum, angulatum, osculis quatuor majoribus in angulis, aculeo brevissimo in centro capitis. Segmenta corporis brevissima, utrinque poris evacuatoriis in angulo antico.

In intestinis Anatis Tadornae invenit Amrov. GAARD.

Quae Zoologia Danica refert, verbotenus reddidi, iis autem species minime efficitur. Altera figura teste Taenia semipollicaris fuit, et secundum alteram virginea et integra fuisse vide. tur, nam ultimi articuli rotundati foramen est medium terminale. Foramina ceterum opposita, quae speciem novam arguunt, figura illa male redduntur, non enim vere marginalia sunt, sed ad latera marginis' utriusque in latere Taeniae inferiore occurrunt.

Speciei ulterior expositio Holminthologis Borealibus incumbit. 


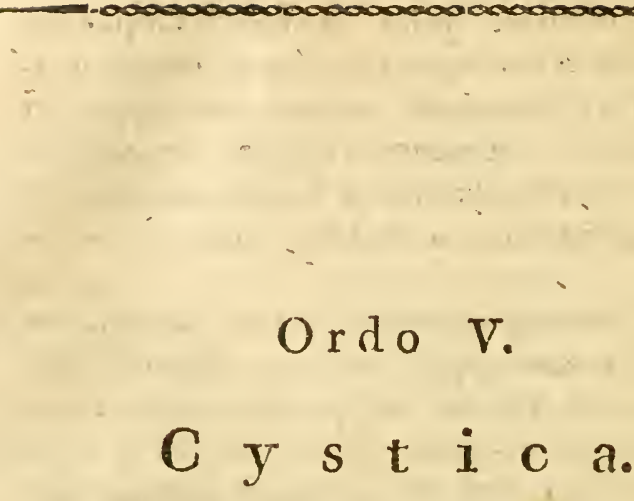

Genera huc facientia plurimis quidem notis ad Cestoidea referri possunt, vesica tamen caudali et vivendi ratione nimis ab his distant, quo minus conjungi possint. Cysticercus fasciolaris ve. sica caudali abrupta pro Taenia facillime imponi potest, et tantillum aberat, quominus ipse tali spccimine in collectione Brasiliensi occurrente inductus fuissem; differt tamen essentialiter, nam articuli non evolvuntur, neque unquam ovaria vel lemniscos, in iisdem observavimus. Eadem Coenuro, egregius adhuc, quoâd figuram proban. tur. Hujus enim vermiculi, Taeniis proximi, toti inverti possunt, quod illis articulatis nunquam continget. Hicce tamen Character Omnibus Cy: sticis vix communis est, de Cysticerco fasciolari saltem, num inverti possit, merito dubitatúr. Echi. nococcus fere nihil nisi Taeniae caput sistit.

Praeter illos in Entozoologia varii vermes Aliorum auctoritate male ad Cysticercos relati sunt, qui vel ad Triaenophorum, vel ad Bothrio. cephalos, vel ad Taenias pertinent, hisque in Syn. opsi nunc redditi sunt. Ill autem Cuvier no. 
vum genus detexit, Cestoideorum' alteri, Tetrarhyncho scilicet, proximum; dum hoc autem apice caudali in vesicam abit, corpus certe etiam in. terna mutatum est. Hocce genus, cel, viro Floriceps dictum, quod nomen retinui, at graece reddidi (Anthocephalus), lacunam systematis naturalis replet, alias genera Taeniarum solummodo aemula, nunc autem etiam Tetrarhynchis et Bothriocephalis (rhynchobothriis) analoga offerentis. Hos quidem a cel. viro pariter ad Floricipitem revocatos, utpote articulatos, neque cysticos, rescidi,

\section{Geлus XXVII, Anthocephilus.}

Speciés omnes a me huc bene relatas esse, nullus contenderem, nullam tamen absque exa. mine, quoad fieri potuit accurato, huc retuli. Ut modum, quo vesicae cauda immissa sit, cogno. scerem, diuturno labore opus fuit, sed in specie prima, rem satis perspectam me habere, persua. sus sum. Illustris Cuvier ipse rem admittit, sed dubiam reperit, speciemque sacco membranaceo involvi refert, qui quoquo modo corpori connexus videatur (qui paroit tenir à son corps d'une inanière quelquorique).

1. Anthocephalus elongatus R. n. sp. Tah. 3. Fig. $12-17$.

i. Duorum Orthragoriscortm Molarum Julio Neapoli examinatorum hepata vermibus vesicu. laribus to:am quoad superficiem perreptata reperi, quo gyri albi pellucerent, qui intera tamen $\epsilon$ tiam penetrabant, longissimi, et difficillimé integri ex. trahurstur. 
Majoris insuper Orthragorisci ascitici interstititia inter intestina vesicis duris et elasticis, pollicaribus, vel semipollicaribus passim repleta et tẹla laxa revincta; quae vesicae facile integrae solvuntur.

His apertis vesicae tenerae, utplurimum clavatae, pollicares vel sesquipollicares; superne duas lineas transversim einetientes, vivo motu vigen. tes in conspectum veniunt. Accuratius inspectae in utroque latere vas longitudinale serpentinum exhibent, quod in apice crasso convenit, loco pellucido ni fallor perforato. Vesica aperta humorem tenacem, substantiae oculi vitreae analogum, et vermen, quem, ni cautus sis̄, pro Tetrarkyncho habebis.

Caput Tetrarhynchi bothriis duobus ovalibus, lineaın longis, et proboscidibus quatuor rectis satis validis, lineam superantibus; collum ultrá septem lineas longum teres, lineam crassum; corpus discretum, depressum, aeque longum, apice postico perforato.

Vesica autem majori diligentia aperta, vermem eacum colaerere, et to quidern loco quo apex posticus perforatus videtur; ut illa ex fovea vesica replicata eundem involvat. Inde etiam vesiculae motus explicatur.

Vermes hepatici specie non differunt, sed Joco olpportuno, in hepatis molli substantia valde prolongantur, neque vesica dura adest. Alterum specimen sédecim pollices longum est.

Quae praegrediuntur examen mie docuit Neapoli institutum; Berolini autem, ut icones pararentur, entozoa iterum sollicite observavi, et vesicam internam duplicem esse vidi, laminis pluriman partem sibi incumbentibus, et circa ver. 


\section{- $539-$}

mis caudam facilius separandis, Intimá oblongà est vesica caudalis, et clavata huic exterior. Inde etiam explicatur, qui in hepate absque vesica extima dura vermes persistere possunt; duplici enim gaudentibus motus liber conceditur, licet vesica externe adhaereat. In recentibus autem laminae certé valde sibi inhaerere possunt, ut internam Neapoli praeterviderim,

2. In Centronoti glauci vix pedalis abdomine Neapoli Julio mense cystidem reperi cylindricam sex lineas longam, fere lineam latam, turgidam, pellucidan, altero fine opaco, excepto, qui caput retractum arguebat, quod tamen extricare non licuit.

Calendis Augusti autem ibidem Centrono. tus tres cum dimidio pedes longus allatus est, cujus in abdomine specimina majora reperi et omnino eadem, ac quae aliquot ante dies in $\mathrm{Or}$ thragorisco inveneram, ut de his nullum dubium sit.

3. In Sciaenae Aquilae mesenterio Neapoli Junio dnodecim et quod excurrit reperi hydatides, tres ad undecim lineas longas, unam alteramve latas, utrinque obtusas. Tunica externa albiḍa, mesenterio ope cellulosac revincta, firma et elastica, qua aperta etiam hydatides formam suam retineant. Externa tunica prudenter aperta aqua nulla effluit, sed nova in conspectum venit hydatis candidissima, pellucida, altero extremo opa. ca. Pressione adhibita aqua ex eadem exsilit limpida et macula illa opaca in corporis capitisque spẹciem evolvitur. Cysticum esse vermem evictus quidem, sed dum hunc ante Anthocephalos Molae et Centronoti observavi, de genere dubius fui. Nunc hoc quidem certum est, num 


\section{- $540 \rightarrow$}

eadem autem sit Anthocephali species, contendere nón possum, magnitudo tamen eandem esse suadet.

Obs. Quem ill. Cuvine (Regne Animal T. IV. p. 19o. Tab, XV. Fig. I. 2. Floriceps) delineari curavit, ex hepate Orthragorisci Molae de. surntus, et utique noster est. Quem p. 45. autem habet, Bothriocephalus corollatus, huc non pert?net, utpote arriculatus, vesica caudali destitutus. Cel. Viri genus proprie Bothriocephalos rhynchobothrios complectitur, ita ut p. $\$ 6$. Tetrarliynchos a Floricipite minori articulorum copia distingui tradat, neque vesiculae caudalis, neque. Anthocephali nostri (paucis articulis' constaintis) mentionem faciat, ut liunc serius detexisse vi. deatur.

\section{Anthocephalus gracilis F. n. sp.}

'Scombri Rochei Rissoxis peritoneo intestinorum vesiculas inhaerentes reperi Neapoli Junio merse. Harum major lentem superabat, reliquae linean haud attingebant, duplici constare visae umica. Julio insequente iterum Scombrum Rochei secui, compluribus et majoribus hydatidibus obnoxium, praesertim testiculis, sed etiam pericardio et ventriculo laxe adhaerentibus, lineam ad matuor cum dimiclia lineam longis, his ultra lineam crassis. Vermes tres ad sex lineas longi sunt.

Tunica hydatidis extima firma, saepe splen. dens, aut coloris grisei; secunda tenuissima, sectione praecedente praetervisa; tertia minus téunis, candida ceterum uti secunda, oblonga, utro. que apice intruso (quo perforata videretur) aqua. linnidissima repleta. Haec est vesica caudalis 
Anthocephali mox describendi, qui tamen pressione adhibita, non ex altero apice, sed ex vesica medio rupta emissus est.

Caput bialatum bothriis auricularibus, profundis, utrinque solitariis, postice distantibus, mu. tabilibus; pro3oscidibus quatuor dense aculeatis. Collum teres postcriora versus parum increscens, intus reccptacula proboscitium continens, quae retractae per collum translucent.' Corpus colli longitudine, sed tenuius, ccterum cum eodem continuum neque articulo separatum, apice postico cum vesica caudali colıeret, qua amota distincte perforatum est, margine foraminis prominulo, mu tabili.

Versus finem Julii Ncapoli eandem omnino speciem in mesenterio Bramae (Spari) Raji of fendi.

\section{Anthocephalus Granulum R.n. sp.}

Carancis trachuri ventriculo et appendicibus pyloricis cerpuscula plurima fuscescentia, lentifor-, mia, lineae dimidiam tertiamve partern diametro aequantia adhaerere vidi, Arimini, Aprili mense. Ibidem aliquoties Majo in abdomine Spari Alcen diuis copiose, et minori copia Neapoli Julio in mesenterio Scombri Coliae majoris offendi.

Tunica externa fuscescens facile secedit, tum. que saccus albus compaŕet firmior, difficilius aperiendus, granulis quasi repletus, Quo aperto ver. mis sistitur exilissimus, albus, vividissimus, granulosus.

Caput cordatum sub motu bothriis quatuor instructum videtur, dein tamen verme immoto, horum tantum esse duo, didici. Proboscides longae uncinis ternis verticillatae, mox setractae et 
per collum translucentes, mox exsertae. Collum oblongurn, capite multo tenuius, mox laeve, mox longitudinaliter costatum. Corpus ovatum aut obovatum.

Obs. I. Am. Otfers eandem omnino spèciem sub Tetrarhynchi (in Scombri parvi cujus. dam abdomine lecti) nomine misit.

Obs. 2. Nexum hujus vermis et vesiculae ambientis non vicli, ideoque diu pro Tetrarlyncho habui; ob hydatidem duplicem (aut forsan triplicem) tamen dubius huc retuli, cum Tetrarhynchi vel liberi, vel inter tunicas simplices aut tubercula inclusi occurrant, ideoque cystici dici nequeant.

\section{Anthocephalus macrourus R. n. sp.}

Am. ab Olfers specimina in Spari Lusitanis Cherubinho dicti hepate inde corroso, et (vel libera? vel) in hydatidibus inter viscera occurrentibus Julio et Augusto in Brasilia reperta, horum que icones benevole mecum communicavit.

Caput bothriis quatuor distinctis, subovals, concavis, singulis apice proboscidem emittentibus longam, uncinis ternis spiraliter positis armatam. Collum, longum, capite tenuius, probuscidum thecas translucentes continens, abiens in corpus'breve, ovale, in quod caput et collum retrahi possunt, quodque vesica caudali terminatur cylindrica longissima, duos circa pollices longa, dum recepraculum aliquot (tres) lineas adaequet, neque reliqua pars pollicem dimidium attingat.

Obs. r. Anicus vermem, sibi Tetrarhynchum dictum, pro Cuvieri Floricipite habet, cui certe affinis est, sed a quo tamen diversum esse descriptio speciei primae, dibothriae etc, docet. 
Obs. 2. Iden 'Amicus aliquot specimina in abdomine Triglae fasciatae Augusto mense in Brasilia reperta una cum horum iconibus misit, de quibus utrum, huc faciant, an propriam sistant speciem, incertus sum.

Bothria quátuór videntur (neque duo biloba), ovalia, exigua, contracta et convexiuscula; proboscides quales priores uncinis ternis spiraliter armatae. Caput lineam superat. "Collum dias circiter lineas longum, téres. Corpus lincam sh. perans ovale. Vesica caudalis collapsa, depressa, cum toto verme ope spiritus fuscescens reddita, sesquipollicaris vel cum toto verme bipollicaris.

\section{Anthocephalus interruptus R. n. sp.}

Am. aв Orfers specimina in abdomine Trichinri lepturi hepati et intestinis adhaerentia Octo. bri mense in Brasilia "reperta, uti illa priorum, pro Tetrarhynchis dedit, certe hydatidibus inclusä 'quaś non transmisit.

Vermes albi, pollicem dimidium longi, capite bothriis quatuor ovalibus expansis, ad prae. cedentem speciem-in Sparo repertam maxime accedunt. Proboscides ejusdem indolis. Collum tenue breve; corpris ovatum breve ope partis tenuioris in vesicam caudalem oblongam saepe constrictam abiens. Specimini, cujus proboscides retractae sunt, collum vix in conspectum venit, sed totnm corpus absque ordine saepius constrictum est.

Amicus speciem istam a praecedente vir diversam credit, ego tamen neque in hac neque in prioribus vesicam caudalem tali modo interceptam vidi, 


\section{- $544-$ \\ Genus XXVIII. Cysticercus.}

Species genus hocce constituentes minus numerosae, sed optime convenientes videntur. Quem olim minus evolutum adeoque inermem reperi, Cysticercum fistularem, dein eodem modo ac reliquos armatum vidi, ut illud etiam discrimen iolleretur. Qui maxime differe videtur, Cysticercus Delphini, nondum satis exploratus est, forsan autem novum genus efformat; qua de re illum exponendo sermo erit.

Num Cysticerci vesica caudalis obliterari possit, quaestio me judice negari debet, ut species Zedero Vesicarias dictas me recte suppressisse et ad Triaenophorum, Bothriocephalos et Taenias amandasse, mihi videor. Si Cysticercus Leporis variabilis n. I3. dictus huc vere pertivet, pars ejus postica, mihi complanata visa, certe tamen vesica collapsa erit. Habitus hujus vesicae caudalis maxime alienus in Cysticerco crispo n. 8. occurrit. Passim antem duae conjunctae Cysticercum bicipitem effingunt, cujus rei exempla species tertia, quinta et octava offerunt.

\section{Cysticercus fasciolaris $\mathbf{R}$.}

Eandem omnino speciem in Muris Ratti hepate in Brasilia repertam am. OlFers trans. misit.

\section{Cysticercus fistularis $\mathbf{R}$.}

Specimen Entoz. II. 2. p. 218. 13, 2. descri: ptum uncinorum coronam non exhibuit, Majo anni 181r. autem aliud in Equi, abdomine in Schola Veterinaria Berolinensi repertum' est, quod cel. ReCKLebex pro sua in me benevolentia 


\section{$-545-$}

recens mecum communicavit, solito more ar. matum.

3. Cysticercus tenuicollis R. Tab. 3. Fig. 18 :

Synopsi jam typis impressa speciem hanc in mesenterio Simiae Maimonis Januario, et Februario in abdomine Simiae Aygulae reperi.

Specimen in Maimone occurrens, vivum, vesica externa globosá liberatum. et calidae im: missum, motus vulgares edidit, tres pollices lon. gum; vesica caudali collapsa basi duos pollices lata. Corpus, sive pars rugosa; angusta, tenuis, quatuor tanturn lineas longum. Caput tetragonum osculis et rostello vulgari modo comparatis nigricantibus. Collum brevissimum; neque tan. tae tenuitatis ac in Cysticerco vitulino.

Specimen in Aygula obvium vix sesquipollicare, vesica caudali turgida octo lineas lata: Reliqua exacte prioris, etiam coronae et osculo: ruin color nigricans.

Aliud oblatum est in Simice cujusdam ine: senterio repertum, praeter opinionem curiosum: Dum enim vesicam externam aperio et Cysticercurn extraho, liujus cystidem caudalèn valde la. tam niiror, sed re attentius inspecta, illum bicipitem invenio, qualeni Tab. 3. Fig. Is. sistit. Utrinque scilicet corpus conspicitur rugosum in vesicam abiens, ut liujus rei nullum sit 'dubium; licet capita in specimine diutius spiritu vini ser. vato extricare non contigerit.

In Museo Viennensi specimen servatur in. ter diaphragma et hepar Simiae Sabaeae repertum; quod fere triceps videtur, sed pars magis producta, corpus anaximum mentlens, indurata et irre- 
gukaris est, reliquae minores vero parum prominent, ut rem dubiam relinquam.

Am. Klug 'specimen magnum dedit in ab. domine Sciuri vulgaris á se repertum, huc omni. no referendum; et am. Arbers nuper duo trans. misit in Cervo Tarando sibi obvia, quorum quidem caput extricare nequeo, quorum autem habitus omnino idem est.

\section{Cysticercus cellulosae $\mathbf{R}$.}

- Nonus vertitur annus, ex quo cum Collega conjunctissimo, ill. K KaPE, theatro anatomico Berolinensi praeesse contigit, in quo singulis annis circa ducenta et quinquaginta cadavera humana secantur, quorum quaterna aut quina quotannis Cysticercum Cellulosae plus ininus copiose offerunt. Utplurimum cadavera Leucoplegmaticorum, neque tamen Ascite vel Anasarca laborantium, illi obnoxia sunt.' Plerumque in musculis; $\dot{v}$. c. glutaeis, iliaco-psoa, extensoribus cruris, sed etiam licet rarius in cerebro occurrunt. Semel mulieris adipe flava tenuiore abundantis cadaver a no. bis dissectum est, cujus musculi plurimos, et ipsae cordis trabeculae, quas sollicite liunc in finem examinavi, tres exhibuerunt Cysticercos; ipsius autem cerebrum, quod in Museo Regio Anatomico servandum curavi, pariter illis scatet, ut in corpore striato; inqúe medulla oblongata aeque be. ne quam inter gyros et in. substantia medullari occurrant. Symptomata, quae Cysticerci praesen. tiam in vivis prodant, ex defunctorum historia morbi enodare, hactentis non con'cessum fuit.

Viri cel. HrmLx Universitatis Gottingensis, et Florman Lundensis Professores meritissimi eundem Cysticercum in homine observatum illw- 


\section{$-547$}

strarunt, hic etiam de suillo egreģia retulit, de quibus Mantissae pars tertia bibliographica con. sulatur.

Dilectiss. Brenser aliquot specimina misit in Simia Cepho reperta, et illorum viginti in ejus cerebro, sex ad octo autem inter capicis musculos occurrisse, simul litteris mandavit.

Cysticercum humanum nunquam vivum reperi, sed saepe recentissimum, et dummodo spiritu non contractus est, caput ejusdem leni inter digitos pressione, a corpore antrorsum directa, fa. cile semper elicitur. Nullum ejusderm ab illo Si. miae et Suis discrimen video.

Doebelius in Practica Venatoria (Ed. III. Lips. fol. 1785. p. 24. b.) sues feros Cysticercis sive Finnis destitui, forsan primus contendit, quod dein pro certo habitum est; sed cel. J. Fr. NrEMavi (Handbuch der Staatsarzneyirissenschaft. Th. II. Leipz. 1813. 8. p. 366.) sues feros illis pariter obnoxios esse refert, et ni- fallor eandem rem etiam alibi legi, rationi maxime consentane: am, aliae enim ferae hydatidibus non carent.

\section{Cysticercus longicollis R. r. sp.}

Specimina complura in Muris arvalis tho. race reperta Bremserus mecum communicavit, quoruin pauca caput collunique exserta obtulerunt.

Caput discretuin, osculis orbicularibus àngularibus, rostello brevissimo lato, uncinulorum validorum corona duplici armato. Colluin capite corporeque tenuius, quam hoc longixs, cum ca. pite lineam longum, depressumi. Corpins rugosum, flaccidum, reliquo verme obscurius, fere granulis refertum visum, sed apertum tamen ovula: 


\section{- 548}

non exhibens (qualia etiam in reliquis hactenus desiderantur speciebus), lineam dimidiam et quorl excurrit longum, vesica caudali angustius, depressum. Vesica catdalis maxime variabilis, "tplurimum ovalis aut subglobosa, ad duas tertias lineae partes longa. Interdum vesicae unus alterve appendix brevis posticus est, et in Museo Viennensi specimina etiam vidi, quorum vesicae caudales parte postica confluunt, quuo vermis $b i$ ceps efficiatur.

Vidi etiam icones elegantissimas in Museo Viennensi paratas, speciem lanc lepidissimam egregie illustrantes.

\section{Cysticercus pisiformis ZED.}

Froelicir (Naturf, 29, p. 75. ṅ, 42. Hydàtula pisiformịs) speciem hancce copiosissime $\mathrm{Ju}$ lio mense in Leporis plurimis partibus reperit, hepate, pulmone, liene, mesenterio, ovario, utero etc.

\section{Cysticercus sphaerocephalus R. n. sp.}

Hujus tria specimina in Museo Viennensî vidi, in Lemuris Mongoz variis abdominis partibus lecta.

Primum in ejusdem omento repertum, ni failor, bipollicare, caput exhibuit rotundatum, an. tice valde convexum, neque pro reliquarum specierum more tetragonum antice complanatum. Oscula orbicularia simul ab uncinulorum corona magis retrorsum cedentia, ac ilias fit. Corpus brevissimum rugosum, capite parum longius, Vesica caudalis maxima, oblongo-ovalis.

Specimen alterum quatuor pollices et octo lineas longuna, quod vesicae urinariae adhaesit, 


\section{- 549}

caput quoque rotundatum et antice tantopere convexum, ut fere conicum diceres, exhibuit; idem tamen versus oscula fere tetragonum factum. Wesica caudalis passim stricturis intercepta.

Tertium specimen hepati adhaerens, tripollicare, easdem. vesicae caudalis stricturas monstravit; de capite utpote retracto nihil referre licet.

Utrum species insequens distincta, an hujus potius varietas sit, determinare non ausim. In Catalogo Viennensi, tam typis impresso, quam manuscripto conjunguntur.

\section{Species. dubiae.}

8. Cysticercus crispus $\mathrm{R}$.

Habitat in Lemuris Mougnz cavo tharacis, costarum pleurac inhaerens. Míuseum Viẹ?nense.

Nihil praeter Scolecem tantopere formam mutare posse, quam hunc Cysticercum, certe confitendum $\epsilon s t$, dum reliquae hujus generis species maxime simplices sunt et ferre nullas varietates exliibent.

1. Specimina caput evolutum sistentia sariora hoc tetragonum; oscula orbicularia angularia anticá; rosteilum vulgare. De collo vix sermo esse potest. Corpus breve antrorsim attenuatum, pro more rugosura. Vesica eaudalis vel longa tenuis, compressa, crispa; vel etian brevior et simul lation, in plurimas partes transversas, varias, passim divisas, undulatas et crispas desinens.

2. Specimina caput abscondentia mox: $l b$ minam pellucicsam parvam, polymorpharn, v. c. sublunatam, quadrangularem, vel quincquangula. 


\section{- 550}

rem, angulis acutis vel obtusis prosilientibus instructam, hac illave parte maculan opacam (ca. put et corpus retractum) exhibentem; mox corpus longa parte pellucidum, hinc opacum referunt; et qui membranulas illas crispas vel collapsas solas videret, pro Cysticerco vix haberet.

Duo specimina in Museo Viennensi, vario tamen modo duplicata, ita nimirum, ut vesiculae basi confluant, capita (aut potius loca opaca illa prodentia, nam ipsa non explicantur) utrinque semotissinua sint.

Laminae exiguae saepe unam alteramve tan. tum, vel eliam tres, quatuor lineas longitudine et latitudine attingunt. Specinen majus, cujus mensu. ras notavi, capite explicato, octo lineas longum, latissima vesicae crispae parte tres lineas aequabat. Maximum, quod in Museo catsareo vidi, duos pollices longitudine excedit, latitudine unam ad tres lineas aequat.

Longitudo ista quandam praecedentis speciei similitudịnem prodit, omnia reliqua autem aliena sunt, cumque multa animalia duobus obnoxia sint Cysticercis, et facies illa crispa nulli praeterea contingat, hanc speciem, donec certiora exhibeantur, separatim enumerandam esse duxi.

\section{Cysticercus Leporis variabilis.}

Am. Brevser specimina complura misit in Leporis, variabilis mesenterio reperta, unam ad tres lineas longa, tertiam dimidianve linẹe partern lata.

Singula corpus sistunt planum mox oblon. gum, mox obovatum, parte antica opaca, capite forsan ịbịdem curn parte rugosa retracto, ita ut 
corpus planum Fesicam forsan caudalem coll: psam aut pressione mutatam exhibeát. Si Amicus ron misisset harum rerum peritissimus, pro Cysticerco non habuissem.

\section{Cysticercus Delphini.}

A'miciss. de Chanrsso ex itinere circa orbem redux specimina aliquot mihi desideratissima pro sua in me benevolentia attulit, sed spiritu nimis debili male conservata, ita ut nihil nisi vesica externa dura (solito durior et coriacea) restiterit, Cysticercus ipse illa aperta difflueret, et unicus formam tantum oblongo-oyatam, parte antica (corpore et capite retracto) crassa, obiter exhibuerit, neque spiritz:m vini fortiorem affundendo hunc servare potui. Cysticerco autem diffluente, interna vesicae pars filamenta satis longa et tenuia continere visa est, quae microscopio subjecta utrinque tenuissime crenulatata, aut articulis rugaeformibus constare dixeris. Talia reliquis in Cysticercis nullus vidit. Hujus itaque species examen maxime commendatum volo.

\section{Genus XXX. Echinococcus.}

Auctores plurimos et egregios, inter quos Bremserus eminet, hydatides corporis animalis ad unam omnes vivas et animatas vocare video, neque tamen ab eorundem partibus stare possumi. Mihi quiden ea tandem hydatis animal vivum vocatur, quae vitam propriam degit, uti Cysticerci, Coenuri etc. Quae autem Organismi alieni (v. c. humani) particulam efficit, animal me judice dici nequit, Mortua non est, quamdiu organis. 


\section{$-552-$}

mi partem' sistit, uti etiam ulcus, pustula, efflorescentia; sed haec ideo non sunt animalia.

Hydropem saccatum fere quotannis semel, rarius bis observo, sed hydatides quas ille offert, vitae nullum signum offerre milhi videntur, neque Entozoa in isdem reperio; sed sedimentum granulosum parietibus hydatidum intus irregulari modo adhaerens. Sed Anatomiam pathologicam editurus hąc de re fusius agam.

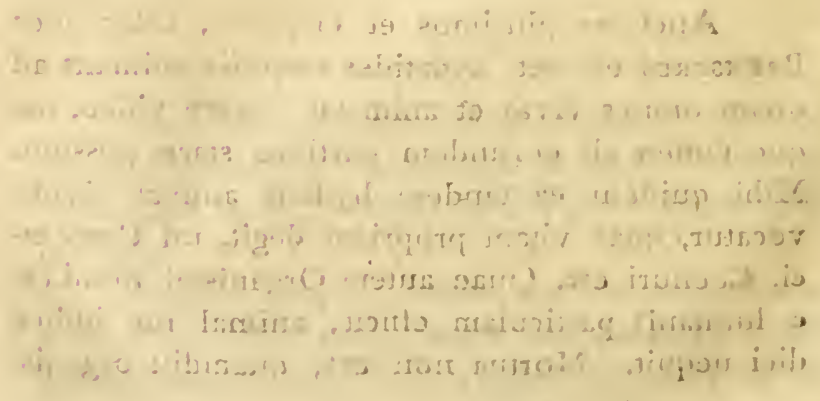




\section{VI.}

\section{Entozoa vel Generis dubii, vel fictitia.}

Vermes aliquot Hominis putatitios in Entozoologia, uti etiam Synopsi p. 184. retuli, quibus novus addi posset, Lumbricus scilicet terrestris vulgatissimus a cl. Stiebelio (cujus Diacanthum l. c. ramentum vegetabile declaravi) aegrae adscriptus, quam magnetice tetigit, et quae majora miracula adhuc pollicita, est, cujus autem fraudem dein detectam esse, cel. Doeblinger litteris suis me certiorem reddidit, gui etiam lunbricum mecum communicavit.

Medicos juniores artis suac historia non imbui vere dolendum est, omnia enim miracula, omnes nugae, omnes fraudes, quibus incurii ca. piuntur, ab. illa diu refutata sunt, neque agyrtae, circumforanei, thaumaturgi, aliique impostores nostris temporibus vi majori pollent, vel artent fraudulentam diutius tegunt, sed brevi cognoscun. tur, contemnuntur, prosternantur, et ab historia demum rejiciuntur.

\section{Vespertilionis auriti.}

Duo hujus specimina in Musèo Viennerrsi 
vidi, pollicaria, tenuissima, capite obtuso, haud tamen satis bene conservato, quo ejusdem fabrica paieat. Alterius cauda diffluebat, alterius vero inflexa, acuta, fere cuspidata est. Forsan Stron. gylus.

\section{Vespertilionis discoloris.}

Entozoa nematoidea hinc crassiora, illine tenuiora, in Museo Viennensi vidi, forsan Trichosomata, sed certi quid efficere non licuit.

\section{Vulpis.}

Am. Bremser Nematoideum misit, in Canis Iulpis glandula mesenterii repertum, quatuor lineas longum, crassitiei mediocris, album.

Capitis obtusi os orbiculare, cinctum, nti videtur, papillis, compluribus depressis, haud exstantibus. Corpus utrinque, magis tamen antrorsum attenuatum, media circiter parte emargina. tum, ac si cupula intrusa esset. Apex caudae papilliformis brevis inflexus.

An Strongyli femina?

\section{Erinacei.}

Am. Truesius Entozoa singularia misit, quae in hydatidibus occurrisse retulit costis Erinacei europaei adhaerentibus.

Cestoidea utrum cystica sint, alii dijudicent. Vermes sunt complures, duos ad sex et quod excurrit pollices longi, forsan autem dum plures particulae ad eundem referendae sunt, multo longiores. Latitudo plerumque dimidiam vel integram, passim duas lineas aequat, sed loca dantur quae octavam lineae partem:non superant. Substantia plerumque tenuis aequalis, qualis Ligu- 
lae avicularis, valde complanatae; sed particulae etiam dantur, praesertim quaedam initiales crassiusculae, rugosae, quae talis Ligulae partem haud evolutam referunt, idque eo magis, cum ejusmodi pars apicem acutiusculum vel emarginatum sistat, qualem Ligulae pariter exhibent.

Cum Mygales vel Soricis moschati vermibus olim sub Ligula descriptis, Ent. II. 2. p. 24. n. 9. nec non Falconis et Strigis vermibus n. 19. et 20. infra exponendis, hi forsan conjungendi sunt.

\section{Falconis naevii.}

Synopseos parte impressa Bremserus, entozoa misit in oculis et naribus Falconis naevii reperta, pri. mo intuitu et aliis occupato nova milhi visa, quae idcirco dubiis adderem, postmodum autem, specierum catalogum formando, errorem animadverti, et illa nihil nisi Filariam abbreviatam Synops. n. 7. Mantiss, p. 2 io. descriptam sistere, plurimum autem a Spiroptera stereura n. 2. ib. p. 237 recedere vidi. Cum haec autem loco dicto in Falcone naevio, illa vero in Mtotacilla stapazina occurrere relata sit, amicum iterum adii, qui vermes se a viro accepisse scripsit, pelles avium in Museo Viennensi referciente, illudque habitaculum indicante. Hoc tamen certe aut circa Fila. riam, aut circa Spiropteram erronee indicatum erit, nam Falco et Motacilla iisdem parasitis vix et tali praesertim loco obnoxii erunt.

Entozoa illa interim describam, cum Filaria abbreviata l. c. descripta feminea tantum fuerit.

Omnia specimina transversim disrupta fin. erunt, quod Filariis facile, Spiropteris vero haud 
accidere solet, mollia, alba; mascula sex ad sep. tem, feminea octo et quod excurrit lineas longa, longitudinis ratione habita valde crassa.

Caput acutiusculum, ore (forsan ob cuiem disruptam) haud bene dignoscendo.' Corpus retrorsum parumper decrescens. Cauda maris spiram simpilicem efficiens, apice brevissimo acuto, filo genitali incurvo, alis vix dignoscendis. Can. da feninae recta, apice brevissimo, depresso, acuto, ante quem inus. Oviductus ampli, quales in Filariis descripsi variis, parique modo lineis onscuris, sive cotyledonibus instructi, quibus ova inhaerent.

\section{Falconis.}

Am. Trlesius Entozoa misit, quae cum Filariis circa renes et ovarium Falconis in itinere circa orbem Julii mensis d. XXI. reperit.

Glomus inextricabile vermium aliquot pollices longorum, lineam vel sesquilineam latorum, pellucidorum, tenuium, Ligulam avicularem valde angustam rếerentium, altero apice crassiore transversim rugoso vel emarginato vel obtusiusculo, altero vero diffluente aut abrupto.

Summa certe Ligulae affinitas. Confer spe. ciem insequentem.

\section{Strigis Accipitrinae.}

Am. Nitzsciz se a cl. Naumani, Ornitholo. so meritissimo verines duos accepisse, ante aliquot septimanas sub pelle'lypochondriorum Strigis accpitrinae sive nisoriae ab illo repertos, caput abscondentes, litteris mandavit, alterumque simul transmisit.

Transmissi longitudo quatuor circa pollices, 


\section{$-\quad 557$}

latitudo lineain aequat. Caput pari modo retractum, ac Ligulis esse solet, apice antico emarginato parteque hunc excipiente latiore, crassiore, transversim rugoso, reliquo corpore laevi, depres. so aut fere plano, passim constricto, nullibi articulato.

Hujus, uti prioris ét specieruni gen: dub $\mathrm{n}_{\text {. }}$ 8. et 9. summa certe cum Ligulis affinitas. In Appendice species dicetur in Ardea caerulea reperta, huc pariter faciens, .

\section{Lanii pomerani.}

Specimen in Lanii pomerani ábdomine repertum am. Bremser misit, fere quinque lineas longum, pone apicem anticum duas tertias lineae partes, versus apicem posticum tertiam latum, depresso planum.

Apex anticus angुustus, tenuis, brevis, absque osculis conspicuis; pars insequens latissima, tuin corpus satis aequale, apice postico obtuso. Totum vero articulis obtusis, nondum satis evo. Jutis, saltem passim minus distinclis divisuna, An. Taenia?

\section{Fringillae domesticằe.}

Am. Bremser septem vermes misit in Fritio gillae domesticae ventriculo, cui fortiter inhaeserint, repertos, duas clim dimidia ad tres lineas longos, crassiusculos, in annuli speciem contra. ctos, albos, femineos.

Caput indistinctum, ut nihil nisi apex exiguus acutus papilliformis in conspectum veniat; corpus subaequale; parte posteriore quam anteri. ore magis attenuata; caudae apex acuminatus sex individuis exiguus, septimo valde magnus, $O e \cdot$ 


\section{- 558}

sophaguls tenuis in ventriculum crássiorem, ob. lungum abit; reliqua tractus intesinalis non trans. lucent. Ova elliptica, parte media oblonga ni. grescente.

An femina Pliysalopterae?

\section{Perdicis samatilis.}

Bremserus duos vermiculos misit in Perdicis saxatilis abdomine repertos, alterum duas cum dimidia, alterum tres lineas lohgum, summa latitudine lineam aequantes, candidos.

Caprit Taeniae depressum, osculis scilicet quatuor orbicularibus, marginatis, approximatis, utriusque lateris binis. Corpus depressum, hinc convexiusculum, lanceolatum, parte ánteriore fere articulatum visum, postica tamen parte articulis omnino destitutà.

Amicus animalcula ista motus miros edi. disse adnotavit, quae novo generi adscriberet. Quam sententiam certe meam fecissem, nisi post. modum in Coturnice easdem bestiolas, ipsissimas vero etiam in Taeniam transeuntes vidissem, qua de re Taenia Linea n, 5I, conferatur. Confer etiam dubii generis vermes mox describendos $n$. 27 . et 28 .

\section{Lacertae viridis.}

Specimina sex in Lacertae viridis tuberculis hepatis reperta Brenserts misit, novum forsan genus efficientia.

Vermes unam cum quarta lineàe parte ad duas lineas longi, tertiam ad dinidiam lineae partern lati, depressí, transversim plicati, capite mox subrotundo, osculis Taeniae duobus appressis instructo, mox oscula singula separata et fere pe- 
dicellata erigens, ut oculos Crustaceorum referant.

Qui mox enumerabuntur, vermiculi in $\mathbf{L a}$ certa murali reperti; magnitudine minore tantum differunt.

\section{Lacertae müralis.}

Specimina complura in tuberculis Lacerta muralis hepaticis obvia Birmsenus misit, tertiam vel dimidiam lineae parteïn longa, pro ratione lata, plana, plicata, ostiolis duobus anticis, exacte illa Lacertae viridis referentia, sed constanter mi. nora, ideoque speciem propriam sistera visa.

Genere hovo, Dithyridii nomine insignilo; entozoa ista prope Scolecem enumerassem, nisi eorundem cum Taeniolis Perdicis saxatilis (Verm. dub. gen. n. 23, ) affinitas magna dubium movisset, an haec pariter foetus sistant? In Ranarum saltem hydatidibus hepatis foetum Distomatis cry: stallini (Mantiss. p. 380.) observavi. Conf. dubs gen. n. 35 .

\section{Colubri olivacei.}

Cel. aв. Olfers aliquot specimina feminea Nematoidei in Colubri olivacei ventriculo in Brasilia Octobri mense reperta, una cum horum icone misit, quibus tamen genus efficere non possum.

\section{Protei anginini.}

In aqua, cui intestina trium Proteorum an. guinorum recens mortuorum, Arimini Aprili, immiseram, quatuor reperi Nematoidea, tres vel quatuor lineas longa, tenuissima, candida, péllu. cida, ut facile visum fugiant. 


\section{$-560-$}

Capitis os medium papillis compluribus hemisphaericis cinctum. Corpus antrorsum satis attenuatum, posteriora versus parum decrescit, aut idem volumen servat. Cauda incurva, apice subulato longiusculo, anie quem anus. Intestinum recte decurrit, in collo yentriculi quandam speciem effingit, granulatum.

Omnium trium Proteorum intestina passim hydatidibus solitariis, vel etiam aggregatis obsita; yauciores in hepate reperi. In quarto Proteo romnia prorsus viscera iis scatebant.

Niliil nisi materiam caseosam continere primum visae sunt, neque oculis vel nudis vel lente simplici armatis vermes detegere potui. Tandem microscopii beneficio minutissimos, cum Cuculla: norum prole microscopica comparandos observa. vi, capite obtuso, cauda longa subulata instructos, vividissimos. In parvis hydatidibus unum duosve tântum, in majore autem, eademque globosa, lineam dimidiam diametro superante, magnum horum nunerum et simul exuvias majoris vermis reperi, quem ad illos supra descriptos traherem, licet corporis tunicam vacuam solam sisteret.

Fydatides itaque expositae liorum vermium nidi sunt, quemadmodum Distomati crystallino similes contingunt, conf. p. 330 . Forsan etiam Áscarides, incisa Synops. p. 46. n० 4r. et pusilla ib. n. 42. hydatidum incolze et perexiguae non: zisi foetus sunt.

\section{Rajae Batis.}

Cel. Alex. Moxro (Observations on the structure and functions of the Nervous System. Eininb, 1783. fol.) aliquot vesiculas Nematoidea exigua continentes in Rajae Batis ramulis rami 


\section{$-561$}

ophtlalmici quintí paris reperit, corpuscula sphae. roidalia sibi dicta, et pro nervorum partibus ha. bita Tab. XILI. Fig. I4. delineari curavit, quae versionis Érmanicae (Bemerkuigen - über die Structur und Verrichtungen des Nervensystems von Alex, Moxro. Leipz. 1787. 4.) Tab, VIII, Fig. 10. redduntur.

Easclem omnino vesiculas verminosas in medulla spinali et nervis Gadi Morhuae observatas Tab, XXXII, (Version, german. Tab, XI. Fig. $1-3$.$) , et in nervis Gacii Aegle/mi visas$ Tab. XXXII. et XXXIV. delineatas dedit.

Omnes tres tabulae iterum redeunt in cel. viri Ópus ichthyologicum: The structare and physiology of the fishes. Jdinb. 1785. fol. numeris tantum mutatis, sunt enim heic Tab. $3 \mathrm{I}-33$.

Ultimae duae tabulae autem (ad Gadum Aea glefinum facientes) Schnemert, viri summi ver. sione germanica: Vergleichung des Baues und der Plyysiologic der Fische. Leipz; 1787. 4, Tab. 22. et 23. tantum repetuntur, cum prima in versione operis neurologici reddita sit.

Ipse hactenus neque in Rajis, neque in $\mathrm{Ga}$ dis vermiculos istos reperi. Confer Verm, dub. ge11. 11, 52, Synops, p. 192, Ent, II. 2, P. 277. 11. 27 .

\section{Syngnathi pelagici.}

In Syngnathi pelagici mesenterio Neapoli Julio sex reperi Filarias (vel Capsularias) albas, aliquot linens longas, in spiram planam orbicularem convolutas, teretes, atquales, capitis ori uti videbatur orbiculari, cauda acutiuscula.

Eodem die in altero Syngnatho pelagico duos ejusmodi vermes reperi. 
51. Fiatolae.

Inter Stromateor Fiatolae appendices pylo. ricas, gyrosque intestinorum, Arimini, Aprili, Nematoidea reperi complura, brevia, quinque ad octo lineas longa, utrinque aequaliter attenuata, capite obcuso papillari, cauda acuiiuscula depressa; motu vulgari utentia; forsan Ascarides femineas.

\section{Gadi Merluccii.}

Neapoli Julio in peritoneo Gadi ITerluccii tres reperi exiguos vermes teretes, vivos, capite obtuso, cauda acutissima; forsan Ascarides femineas.

\section{Gadi Wachniae.}

Tilesius (Mém. de l'Acad. des Sč. de St. Petersbourg, T. II. 1810. 4. p. 374).

Capsularias refert in peritoneo Gadi Wachniae repertas, Tab, XIX, Fig. '2. et 3. delineatas;

praeterea vero (Tab. XIX. Fig. Ir - I3. Tab. XX.) Entozoa valde singularia, qualia hactenus nulli dicta sunt, viridescentia, subtriangularia, in fossis vertebrarum prope insertionem. costarum jacentia. In situ naturali vermes muco obductos esse, et quasi nidum mucosum in fossis parare, medioque impressos videri; exemtis vero hocce cavun centrale ex papillis duabus verosimiliter ortum esse.

Aliquot (3-4) lineas longi et maximo diametro lati videntur; ego quidem consimiles non vidi, neque descriptionem supra redditam, neque figuras intelligo. An forsan Trematoda renalia sunt? 


\section{$-563-$}

56. Cepolae rubescentis.

I. Arimini versus finem Aprilis inter Cepo. lace rubescentis appendices pyloricas, et reliquis tractus intestinalis partibus inhaerentia reperi $\mathrm{Ne}$ matoidea circiter triginta, cinerascentia, duas ad quatuor lineas longa, tenuissima. Caput trinode, interdum papilla media instructum visum, truncatum. Corpus utrinqne parum, magis tamen po. steriora versus attenuatum. Canda acuta depres. sa. Specinina reliquis duplo minora mascula forsan sunt, sed spiculis non exsertis. Microsco. pii ope canalis medius obscurus totumque corpus percurrens, sed niliil ultra conspicitur.

2. In alius Cepolae rubescentis peritoneo $\mathrm{Ne}$. matoideum reperi rubellum, pollicem dimidium longum, utrinque obtusum, antice crassius, inte. stinis prolapsis. Hujus caput compluribus valvulis instructum visum, Viviparum erat, foetus illis Cucullani similes.

An hoc individuum prioribus magis evo. lutum?

\section{Zenis Fabri.}

Inter appendices Zenis Fabri pyloricas Arimini Aprili quam plurimos reperi vermes teretes, albos, tres quatuorve lineas ad pollicem usque longos, tenues, vividissimos, femineos,

Caput obtusum, papillatum, neque trivalve; corpus utrinque, praecipue tamen posteriora versus attenuatum, cauda depressa, acuta. Cana. lis medius opacus. 


\section{Zenis Apri. .}

Novem Zenes Apros Neapoli Julio aperien. do decern reperi Nematoidea peritonealia, parva, partim rubella, plurima tamen alba, viva, feminéa.

\section{6o, Pleuronectis Soleae.}

Neapoli Julio in intcstinis Pleuronectis So. leae duos reperi vermes cestoideos, fere pollica. res, cum tertii fragmento.

Vivi caput illud Bothriocephali visum est, sed dum mortui examinare volui, retractum in; veni, apice corporis retuso, Corpus inarticulatun, utrinque vase pellucido utens, parte media punctata.

Berolini novo subjeci examini, et habitum ab illo Bothriocephali alienum reperi; articulorum nullam speciem; pùnctorum mediorum seriem longitudinalem, maculis hinc inde majori: bus pellucidis. Caput exiguum, contractum, sub. globosum.

Dicta ad ฐ̧enus dijudicandum non suffi• ciunt.

\section{Pleuronectis Boscii.}

In triun Pleuronectum Boscii mesenterio Neapoli Julio complura reperi Nematoidea feminea, partim alba, partim rubella, maguitudine valde diversa.

\section{Sparorum.}

In Spari Alcedinis peritoneo Arimini Majo Nematoidea (Capsularias) reperi quatuor ad sex 


\section{$-565$}

lineas longa, tcnuissima; capite obtuso, corpore retrorsum magis attenuato, cauda subulata recta, Intestinum rectum,

Neapoli Junio in peritoneo complurium Sparorum Boopum copiosa reperi rubella, posteriora versus magis attenuata, aliquot lincas longa, vividissima, uti praecedentia et insecquentia feminea.

Consimilia Neapoli Julio in feritoneo Spari nelanuri ei Momyri, reperi, tres quatuorve lineas longa.

\section{Sciaenae Umbrae.}

In Sciaenae Unbrae peritoneo Ncapoli Julio Nematoideuni reperi solitàrium, quatuor lineas longum, tenuissimum, antroristum magis attenuatum.

\section{Percae cirrosae.}

In peritoneo intestinali duarum Percarum cirrosarum Arimini Majo tres ofiendi vermes te. retes, vivos, quinque arl sex lineas longos, albidos, utrinque aequaliter attenuatos, capite an papilloso, utrum valvulis exiguis instructo? Cauda brevi, obtusa.

\section{Percae marinae.}

- In peritoneo Percae marinac Neapoli Junio et Julio Capsularias ZEDERo dictas reperi pluzimas, femineas.

69 . Centronoti glauci.

In Centronoti glauci peritonco Neapoli Augusto mense Nematoidca reperi octo, quatuar al 
sex lineas longa, alba, capite an trinodi, cauda adunca,

Talia quoque vesiculis inclusa offendi fuscescentibus, quae intestinis adhaerebant.

70. Scoribrorum.

In peritoneo Scombri Scombri Arimini Majo Nematcidea illis Cepolae n. 56. et Carancis n. $7 \mathbf{I}$. simillima.

Eadem quoque in peritoneo quatuor Scombrorum Coliarum et Scombri Sardae, Neapoli Ju. . nio et Julio.

\section{Carancis trachuri.}

In Carance trachuro Arimini Aprili et in specie majore, quam imperialem vocant, Neapoli Augusto consimilia reperi, ac in Scombris n. 70, et Cepola n. 56 .

\section{Triglae Hirundinis.}

In hujus peritoneo duas Capsularias femineas exiguas Arimini Majo reperi, Confer inse. quentem.

\section{Triglae adriaticae.}

Inter quatuor Triglas adriaticas Arimini Majo examinatas una Capsulariam obtulit peritonei albam, sex lineas longam, utrinque vix attenuatam, capite discreto exiguo, papillis (quinque?) cincto, cauda acuta depressa.

Neapoli Julio in T. adriaticae peritoneo quinque reperi specimina utrinque, magis tamen antrorsum attenuata, tres ad sex lineas Ionga, al$\mathrm{ba}$, capitis valvulis obscuris. 


\section{$-567$}

74. Siluri Glanidis.

Cucullanum ascaroidem GoEzir, in Entozool. II. 2. p. 254. dubio Prionodermatis genere enumeratmm, in Museo Ticinensi, quod Goezianam Entozoorum collectionem possidet, servatum vili, sed examinare non potui. Mrusei enim Dire"tore, cel. Mangribr absente, lagenulam, quae vernes dubios continebat, aperire non licuit; spiritu attem turbido facto vermes obiner tantum vide$r$ contigit. Nematoidea esse tamen certus sum, cassiuscula, transversin striata; forsan Liorhynsio Anguillae denticulato p. 307. affinia sunt.

\section{So. Sphyraenae Spet.}

In hujus abdomine Neapoli Jınio Capsularia, quasdarn reperi pusillas, rubellas, fęminẹas.

\section{:1. Cyprini Aspii.}

In Cyprini Aspii magni peritoneo Berolini Junio mense quatuor reperi vermes teretes, mortuos

Tria specimina rufa fuerunt, quartum magis pallitum. Illa fere bipollicaria, et mesenterio tantopere implicita, ut vix solvere potuerim. Cor. pus naequale fuit, forsán ob cutem emphysema. tican et secedentem, neque certi quid eruere potui. Ob mollitiem et colorem Filarias tamen suspi:or.

\section{Helicis putris.}

Abhandlung über Würmer, welche in einer EIdschnecke entdeckt worden sind. Von August Ahrexs. In: Magazin der Gesellsch. 
Naturf. Freunde zu Berlin. IV. 1, n, 37. S. 292296. Tab. IX. Fig. 12-19.

-Mantissae hujus agmen vermes claudunt maxime memorabiles, quos cl. Anress Scptembris d. XI. anno r810. Malae Saxonum in Helice putri populi folio insidente offendit, quosque Entomo. tomus meritissimus, cel. Ramnomr l. c. compro bavit, certe rariores; nam ex quo observationet laudatas legii: complures Helices putres, sed frt stra examinavi.

Specimina quatuor, reperta sunt, quorun duo prirnum in tentaculis Helicis majoribus, tortium in ejusdem tentaculo minore, yuartum sib - collo occurrerunt, quae locum mutando, alias illius partes sibi vindicarunt, et Helice Octolris die primo emortua, in ejus corpore pariter mortua reperta sunt.

Vermes pollicem longi, clavati, apice aassiore rotundato, tenuiore pellucido, deliquesente, an integro? Color albidus, apicis crassiorb tasnen macula ferruginea splenderis, larvarum ori acmule, organis tamen suctoriis vel ore-nor observandis; macula illa ferruginea duabus minoribus nigris instructa; aliae maculae nigrae eevatae partem anteriorem cingunt; reliquum corpus lituris viridescentibus insigne; cute molli filrosa, transversim annulata.

Corpus. ex cel. Rampohr observatione centum et viginti ovis ovalibus repletum, interne albis, substantia viridescente cinctis et tunica de. mum firma circumdatis.

Obs. Virum saepius laudatum corpora haec pro entozois neque pro larvis insectorum habere 


\section{- $569-$}

rei magnam quidem fidem facit, ideoque ipse in eandem sententiam abii, sed dubia semper nora exsurgunt. Tales enim colores, tales maculae elevatae, corpus tanturn tam pauca ova conti. nens, horum indoles et color ab entozois valde aliena, sunt. Rem ergo in medio relinquam. 


\section{Sectio secunda}

\section{Anatomico-Physiologica.}

$\mathrm{O}_{\mathrm{m}}$ pus hocce moliendo, praeter Entozoa, quae Synopsi et Mantissae parte zoographica cnumeravi, nulla quae ádderenn ad manus erant, ide. oque illius partem, quam nunc adgredior, anato. mico-physiologicam maxime augere constitueram, sed Mantissae aliquot plagulis vix impressis, En. toozorum Brasiliensium collectio advenit magna (secunda), quam cel. An Olfers nobis misit, majorque clariss. Nattereri cura Museo Caesareo Viennensi mandata, cujus beneficio plurima mecum communicata sunt, quos thesauros occasione data rublici juris facere religioni habui. Omne itaque tempus, quod munus et operis editio per. miscrunt, animalculis illis Brasilianis examinan. iis et describendis impendi, quae appendice sive Mantissa, altera sistam, et duplicis indicis ope cum reliquis conjungam.

Ne liber antem nimis incresceret, istam et insequentem Mantissae sectionem, quoad fieri potuit, rescidi. Nihilo tamen minus pauca, quae lue loco exposui, magnum rei incrementum satis superque probabunt. 


\section{$-57$ \\ C a $\mathrm{p}$ u $\mathrm{t}$ I.

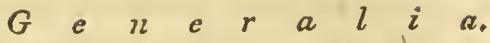

Historiac naturalis studio quo longius incumbo, eo magis Animalium evertebratorum in duas magnas classes divisio Irnwaeana veritate sua omnibus illis classificationibus praeferenda videtur, quae Mollussca et Zoophyta inter Crustacca, Arachinidea et Insecta intermedia esse volunt. Hacc porro, omnibus rite consideratis, prino, quo Irsaneus posuit iocn, relinquenda videntur, utpote rei maxime essentialis, sensorii communis et systematis nervei evolutione, adeoque sensibus, arteque et industria ceteris praestantiora.

Cephalopoila Cuvier Piscibus proxima esse contendenti facile hoc concedercm, sèd quernadmodium inter animalia vertebrata series genuina, catenam dicere amabant, ab homine ad piscium ultimos descenders non observatur, ita etiam inter $\in$ vertebrata talis exspectari non debet, neque invenitur; sed a Piscibus ab altera parte Crusta. cea, Arachnidea et Insecta, ab altera vero stant Mollusca, Aunulata et Zoophyta.

Vermcs sic dictos Linnaeanos inter se valdopere differre, mox sub oculos cadit, sed generalis tamen quidam habitus omnes inter observatur, et Hydrae pusillae brachia fere eaden sunt ac Sepiae; Botryllorum aninaleula Ascidiarum, Infusoria varia Entozoorum, Annulata Cirropo. dum, Planarias Hirudinum aemula invenintus, alia sexcenta ut taceam, interque Mollusca et $\not 20$. ophyta liseliatorum multa transitum permittunt. Pari modo Crustacea ope Arachnidum cun In- 
sectis conjuncta sunt; inter Vermes et Insects Irsaser nullus datur transitus, nisi minus curandus ille per Cirropoda et Annulata, quaé systemate quidem nerveo obiter spectato convenire videntur, neque tamen conveniunt, Insecti enim systema nerveum quam Hirudinis et Lumbrici mulio magis compositum est, reliquis autem omnibus maxime differunt.

Entozooruin nervos ignorando, eadem pro. prium inter Vermes Linnaeanos "ordinern constituere mini persuasi, nervis autem detectis de illo sermo esse nequit. Entozoa ergo Neniatoidea Annulatis, familia tamen separata, addi debent, Lernaeam autem ejustlem tribus esse, uti ill. GuVhen refert, vix admitterem; Nemertes Gordio proximus videtur, sed illum non nisi siccatum vidi. Reliqui Entozoorum Ordines Radiatorum sive Zoophytorum regnum chaoticum intrant, cu. jus distributio ea tantum bona erit, quae systema plantarum naturale imitata, stbordines et familias bene limitatas ponit.

Illa autem distributio multorum examen ac. curatius postulat, neque tam cito perficietur. Acarthocephali v. c. inter Radiata genus affine non inveniunt, idcoque familiam peculiarcm effor. mant. Sipunculus quidem obiter spectatus illis accedere videtur, tubo tamen oris filigero retractili (ante Raxánum Patavinum igno:o, a me postmodum. Neapoli saepissime in vivis speciminibus observato) et tractu intestinali Holothuriis proximus est, quam Echinorhynchus multo magis compositus. Quem optimus Parreries olim dixit Echinorhynchum scutatum vel clype. atzm (Tavole per servire alla classificazione e conoscciza degli animali del Sterano Anprea 
Revirr. Padova i 807 , fol. Tab. 6.), dein talem non amplius admisit, et Schreiberium vocavit; cel, autem Ranzaxi Thalusscina scutatum dixit (Opuscoli Scientifici di Bologna. Fasc. Il, 1817. 4. p. II2.), animat ulteriore examine dignissi. mun, ab Eutczois autem maxime alienum.

Trematodur: affinitas passim vária viretur, nondum autem probata est. Planariae saltem, quas ill. Cuvier iis addit, Cèstoideis non articulatis magis affines milii videntur, et qunan cel. Niтzscu (Beytrag zur Infusorienkunde Halle is:7. 3. p. 7 et sequ. Tab. r.) Cercariae cphemerae et Distomatis reperit similitudinem, ea fere non major est, quam illa, quas inter habitum Hydrae tt Cephalopodis obtinet.

Cestoideis simplicibus Planariae nonnullae utique affines videntur, articulatis, affinia extra animalium corpora non repériuntur.

Idem de Cysticis valet. Animalculum enim singulare, quod in 'Tethyos superície parasiticum vivit, et a cel. Raynerio Hydatula varia olim rocatum fuit, dein novo generi merito adscriptum, cui nomen nondum imposuerat, cum hos duos ante annos in cel, viri familiaritatem venirem. Ipse hoc Neapoli saepissime, passimque Tethy; inhaerens a piscatoribus accepi et Phoenlicurlins varium dixi, Corpus $\in \mathrm{i}$, est obovatum, antice ob. tusissimum, apice posteriore attenuato vel conico, simplici aut duplici; dorso convexo variegatum, sive griseum lineis flavicantilus anastomoticis re. ticulatum, ventre plano album, apicibus caudalibus ruberrimis. Cute externa detracta, alteram fibris muscularibus constantem "et in utreque cor. 'poris latere ganglium detexi, a quo nervi in fibras musculares radiatim emittantur. - Intra tunicam 
dictam musculosam nilil nisi substantia albuminosa tenax conspicitur, et intestina et vasa aeçne parım vidi, quam aperturam ullam externam. Abrominis totins ope Tethyi adhaeret, utrum per cutem vero resorbeat, num apices purpurci ope aperturae visum fugientis nutrimentum hauriant, aliis exponendum relinquo. Quidinid autem sit, ad Cystica certe epizoon istud non pertinet.

Entozoa autem si inter religuos vermes Sy. stematis gentralis distribui debent, privi tamen semper quid labent, et ob habiaculum peculiare non habere nequeunt. Hinc ex quo in Entozoorum historiam naturalem praelectiones publicas habere incepi, eorundem conspectum cum Fauna vel Flora regionis peculiaris comparare solitus sum. Quemadmodum vero, qui Faunae cuidam illustrandae yires suas impendit, illius ambitum valde augebit et multa specialia optime inquirere potest, eo tamen nunquann sibi satisfaciet, lisi Zoologiarn generatim amplectitur, ita, etiam Er. tozoorum studium assiduum ad illorum genera et species stabilienda maxime necessarium est, sed aliorum vermium historian quo magis simul promovemus, omuiui nexum eo facilius intelliginus, omniumque vitae rationem comparare valemus,

\section{C a $\mathrm{p}$ u $\mathrm{t}$ II.}

De nervis Entozoorum.

§. I. Strongylus Gigas in Lupi renibus ohvius ab am. Orrone recenter examinatus syste. ma nerveum obtulit, a cel, viro (Magazin der Gesellsch. Naturf, Freunde B. VII, Berlin I8I6. 


\section{$-\quad 575$}

y. 225. Tab. V. Fig. I.) descriptum et delinea. tum. Ganglio obiongo versus os sub oesophago oriri, tenuius sub intestino decurrere, gangliis quam plurimis, $\epsilon \mathrm{x}$ guihus nervi oriantur, instrui et simili tanien garglio, ac illo capitis in apice caudae terminari refert. Milii Januario 1817 tam. dem consigit, eundem strongylum eoden loco reperire, et nervum circa oesophagi initium an. nulum efformantem planiusculurn, ceterum autem modo a cel, viro descripto sub intestino decurrere vidi. Eundem nervum postea in Museo Ticinensi a cel. Spedacieri in Strongylo renis canini egregie praeparatum vidi. Summa hujus et systematis nervei Annulatorum analogia quidern est, sed animal majusculum a Lumbrico, Hirudine aliisque multo minoribus, at magis compositis, non solum funiculo nerveo ipso majore, sed tiam gangliis horumque nervis magis distinctis, superatur, quo inferior ejusdem in systemate locus simul evincitur.

De hujus itaque entozoi nervis dubium superesse nequit, utinam de illis reliquorum idem dic $\in$ re possemus.

§. 2. Ascaridi lumbricoidi cl. Отто (1. c. p. 227. Tab. VI. Fig. 6.) nervum duplicem, abdominalem et dorsalem tribuit, quorum tamen fines tam anteriores quam posteriores non observavit. Mihi autem utrinque in cute ipsa evanescere, ileoque pro nervis minus bene ha. beri videntur, quo accedit, singulos nimis inter se remotos, neque ulla parte liberos, neque instar Strongyli nervi a, cute removendos esse. Tanta etiarn in re gravissima Strongyli et Ascaridis differentia vix admitti potest, cum reliquis organis plurimum conveniant; OттоNis autem 


\section{$-576$}

hypothesi adınissa, Ascaridi multo minori duo concederentur nervi, quorum singuli Strongylino nnajores forent. Locus tandem dorsalis alterius - nervi vix evertebratis competit; de nervo recurrente insectorum enim sermo fieri nequit, pror. sus alieno. Conferas quae $\S$. 4. hujus Capitis de Pentastomatis nervis retuli.

\section{§. 3. Distomatis hepatici systema nerveum} 2 cel. RAMDOHR, Entomotomo meritissimo (Berlin. Gesellsch. Naturf. Freunde B. VI. p. 'izo. Tab. 3. Fig. 5, 6.) descriptum et delineatum me quidem judice nullo modo admitti potest. Cere. bri speciem describit dorsalem, versus basin pori anterioris, ante ovaria et inter vasa duo nutrientia majora sitam, abeuntem in processum nodulo posteriore versus ductum excretorium ovariorum occurrente terminatum; ex ipso cercbro ante processum oriri nervum, mox in duos ranos divisum, supra ovaria per totam corporis longitudi. nem decurrentes. Отто l, c. cerebrum illud cir. rum esse reconditum, nervos illos vasa nutritia suspicatur; Gaede (Obss, de insect, et verm. structura. Kil. I817.4. p.Io.) cerebrum PandorsIo dictum pro-vase seminifero labet; ipse variis Distomatibus de novo dissectis, Otroxi ad. sentirem; si enim pars cirri egressa, est, rciiqui basis interna cerebrum illud forsan nentiri po. tuit.

Nervis istis rejectis, cel. Otтo (Magazin B. VIr. p. 228. Tab. VI. Fig. 7-10) alios statuit, quos am. Ganne l.c. bene pro vasis habet. Vasa ncmpe tenuia hateralia ramosa illius nervi sunt, et qua illa cum transverso conjunguntur, in utro๕luc ergo angulo, ganglium supponit, guod certissime non existit, sed angulum vasorum conve- 
nientium tantillum ampliatum sistit. Plerumque vas istud transversum, uti et laterale illud utrinque inter nutritia, majora disci et ovigera marginis decurrens, alba et decolora sunt, sed vasis marginis fuscis illa pro nervis habita aeque fu. sca reddita video. Sunt mili specimina, quorum vasa ista colorem hus nigrescentem in spiritu vini conservarunt, nuo de gangliis et nervis cel. viri certissime judicare valeo,' Varietas etiam illa inter occurrit, ubi vas transversum altero in latere duplex est, et nervi sic dicti rami pulcher. rime repleti sunt.

Amplisisomatis conici et subtriquetri anato. men instituendo, sollicite in nervos inquisivi, sed nullos reperi, neque cel. Bosanus, qui $\mathbf{A}$. subtriquetrum cultello subjecit, et quae reperit, bentrule necum communicavit, nervorum mentionem fecit. Monostoma tenuicolle, vere gigan. teum, si species affines comparas, cujusque vasa Táb. 2. Fig. 1-4. delineata dedi, nervorum ne minimum quidem vestigium nffert.

5. 4. Pentastomatis tacnioidis ganglium ce. rebrale et fila duo nervea inter omuia Entozoa optime conspici, ill. Cuvier (Regne aninal T. IV.p. 35. not. 2.) auctor est, neque, si veri nervi sunt, eos facillime sub oculos cadere, negari potest. In utroque scilicet abdominis latere, I ovariis reclinatis, nervus Cuvnero dictus, albus, linearis, a fibris cutis nuscularibus longitudinalibus facile distinguendus, conspicitur, qui per totam longitudinem decurrit et versus os utrinque filis longis coire mihi visus est. Haec tamen forsan ob speciminis minus bene servati indolem non satis perspexi; fila enim, in quae nervos illos antice coibant, a nervis aliena visa sunt, neque gangli- 


\section{$-578$}

um distinctum inveni. Habitus nervorum dicto. rum illis Ascaridi tributis satis similis reperitur, sed loco recedunt, Ascaridis einim ex OtToxis mente alter dorsalis, alter abdominalis est, Pen. tastomatis autem uterque abdominalis. Argumen tum tandem superest, minoris quidem ponderis, si cultellus rem absolvit, nunc autem non praetereundum. Pentastomatis enim nervi illos Strongyli superarent, qui ad Nematoidea pertinet, ideoque loco alteriori gaudet; forsan autem anatome speciminum recentiun a cel. viro instituta, eum meliorà docuir, et animal illud paradoxum inter plures ordines quasi medium esse, fateri debèmus.

5. 5. Haec fere sunt, quae de'nervis Entozoorum referam, BRERAM enim solida historiae naturalis cognitione penitus destitutum, omnia miscentem, aut pro lubitu fingentem non curo; qui Taeniarum autem ovaria pro singulorum articu. lorum ganglio nervos radiatim emittente ven'dita, et alia plurima $\in$ jusdam farinat quaerit,illius Supplementa (p. 33. et sq.) adeat. Frustra autem nervorum Taeniae ab eodem fictorum icones exspectas, hae enim nimis facile refutari potuissent. Ita etiam, dum Ascaridis lumbricoidis ganglium cerebrale et systema nerveum per minutissima describit, nihil horum delineat, sed figuram MAN. grlanam, nervos Lumbrici terestris sistentem Tab. 3. Fig. 20. repetit, et eandem rem esse, contendere non erubescit. Si hoc autem fuisset, nunquam certe de Ascaridis nervis lis agitata fuis. set, Lumbrici enim nervos omnes uno ore concedunt, neque ad illos probandos Brerae cultrum magnum in usum vocant. 


\section{$\div 579$}

\section{C a $\mathrm{p}$ u $\mathrm{t}$ I I I.}

\section{De Organis reproductionis.}

\$. I. Reproductionem entozois omnibus simplicem, horum tamen diversis ordinibus, diverso gradu talem dari, non est quoc' moneam. Pauca quoque generatim sunt, quae huic capiti addenda habeo, horum autem nonnulla lectoris attentione digna fore spero.

5. 2. Nematoideorum tubus cibarius ob ore ad anum usque cuti adnectitur, quod plurimis locis filis copiosissimis et tenerrimis fit, ab intestino cutem petentibus; anteriori tamen illius parte, in Strongylo Gigánte, loco filorum, nexum vero mesenterio absolvi video. Ipse fila illa partim pro vasis habui, nutritionem peragentibus, absorbentibus et secernentibus - (nutrientibus) quod Entozoologiae T. r. p. 25I, claris verbis exposui. Pro organis respiratoriis vero non habui, neque nunc habeo.

Otto autem l. c. p. 224. et Spedaliert, praep,arata sua qui Ticini milimet monstravit, viri cel. fila illa cum insectorum tracheis comparando, respirationi eadem inservire persuasi sunt, neque filorum nudis oculis spectatorum habitum illis satis convenientem esse, negari potest. Ea. dem tamen cultello vel microscopio subjecta alie. nam faciem exhibent et optime cum tela nostra cellulosa comparantur; distincte etiam anteriora versus in mesenterium abeunt, quod Holothuriis, Sipunculo et affinibus non detst, simili etiam modo ac Strongylus vel alia Nematoidea, cute disrupta, subito intestina effundentibus, quibus. 
cum vero nostri vermes variis notis maxime co. veniunt. Similia etiam fila Echinorhynchi lem. niscos revincunt, quod nostram sententiam maxime confirmat, illi enim aperte orgànà nutritia sunt, neque reliquis locis fila in Echinorhyncho occurrunt, ubi nihil revinciendum est, et nutritio alia locum habet, uti in paragrapho insequente exponitur.

Si fila illa tracheace forent, Nematoidea hac ratione adhuc insecta superarant, nam innumeris locis cutem petunt, cujus foramina cl. Oтто videre sibi visus est, dum insectorum stigmata pauca sunt, sed foramina illa mihi quidem nihil nisi loca teneriora, reliquis partibus ablatis, haud tamen jerforata videntur, Respiratio si Nematoideis vel etiam reliquis Entozois conceditur, mo. do omnibus Vermibus Linnaeanis alieno, et Insectis solis competente, certissime non perficitur, sed simpliciori et cutis quidem rpe, quae mágis composita est, ac vulgo creditur.

Strongyli saltem et Ascaridis lumbricoidis cutis vesiculis exiguis scatet, ab OtTONe l. c. p. 224. circa illum, et a cel. Bojayo (Russ. Samml. II. IV. p. 552.) circa hanc expositis, quarum foisan humor trans cutem decarbonisatur, vel alio modo mutatur.

Illas praeter vesiculas, in "Strongylo giganteo, sed in hoc solo, organa reperi singularia, quae ab Ortore non dicta esse mireris. Utrinque scilicet ad latera tubi cibarii, partem maxime anticam et posticam si exceperis, corpuscula fusca quam plurima, mox solitaria, mox copiosius dispersa occurrunt, quae Januario 1817 in speciminibus Strongyli gigantei femineis duobus recentibus primum vidi, dein autem, ejuslem anni - 
Séptembri mense, in Museo Ticinensi apud cel. SPEDALIERI iterum observavi, in spiritu colorem conservantia. Singuia minuta, inaequalia, microscopii ope granulis constare mihimet visa, cuti laxe inhaerentia, fere pro hepatis rudimentis haherem; cel. Sred,urerr, ni falloz, ad branchias pertinere visạ.

Cel. houarts 1. c. quadam a capite Ascaridis lumbricoidis distantia prope vasa lateralia utrinque binos fasciculos villosos obscure coloratos occurrere refert, haud exacte sibi oppositos, semper tamen binos, et ad pollicem dimidium circa inter se distantes. Partes has nunquam de. sirleravi, sed nonnisi albas reperi, et forsan justo. minoris habui; ipse saltem cum genitalia non tantopere antrorsum ducantur, et anterior tubi intestinalis pars ibidem posita sit, vulgarern cutis apparatum hoc loco in ejus, utpote fortius agentis, usum auctum esse suspicatus sum. Cel. Vir autem neque de his, neque de reliquis partibus a se descriptis sententiam suam adjecit, quod uti. que dolendum est.

In Strongyli intestinum tubum ovigerum minime immitti, uti cel. Oxro retulit, in Capitis in. sequentis $\$$. I. exponam.

Forma tractus intestinalis maxime variat, multaeque praesertin Ascarides illius discrimine notari possent, ut figurae illas varietates calligen. tes exoptatae forent. Conferas v. gr. descriptiones Ascaridun gulosae p.' 27. . dactyluridis p. 272. echinatae et megatyphli p. 285. extenuatae p. 287. datas.

1. 2. Echinorhynchum vasculosum, supra p. 334. descriptum Neapoli reperiendo, vasa corporis Echinorhynchi cutanea mihi primum visa esse, faten. 
dum est, quae absque praeparatione equidem in reliquis non patent, facillima tamen encheiresi sub oculos pani possunt. Echinorlizynchus Gigas si per aliquot dies aquae et dein spiritui vini minus forti committitur, tota ejus cutis inverti et facillime integra detrahi potest, quales etiam passim Echinorhynchi casu-reperiuntur, quorum cutis externa, plus minus infiala, a musculosa distat, ut verme obiter spectato, de tubo intestinali trans= lucente cogitare posses, quod etiam Helminthalogis quibusdain imposuit.

Echinorhynchi Gigantis autem partem cutisquamlibet, luci oppositam, si ab interno latere ope lentis simplicis spectas, vasorum retia elegantissina reperies, undique vesiculis pellucidis exilibus et rotundis cohaerentia, quae centra quasi sunt ramulorum anastomosantium, aut forsan simpliciter vasorum coëuntium dilatationes, quales vasa Testudinis Mydae lymphatica mesenterii ope mercurii injiciendo nuper vidi, et ibi contra auctorum opinionem (́prope intestina ipsa) valvulas statuerem.

Absorptio cutanea Echinorhynchorum vivorum his vasis facillime explicatur, illa mortiorum autem fere aeque facilis pororum cutaneorum illis saepe maximorum ope perficiatur, necessè est, de quibus Entoz. II. I. p. 256. obs. I. $\epsilon$ gi.

Olim vasorum cutaneorum retia elegantissima tantum in bulla Echinorhynchi flicollis vidi et Ent. II. I. p. 284. obs. I. retuli.

Lemniscos longos Echinorhynchi Gigantis, Entoz. I. p. 253. sq. fuse descriptos, iisdem fi!is tenerrimis ac intestinum Nematoidleorum revinctos video, quod ill. Cuvieri sententiam, qui illos pro intestinis coecis labet, utique comproiare 
videtur, licet vasorum ramosorum, magnis saccu• lis vel receptaculis unitorum, apparatus in iisciem conspiciendus ab intestinis alienus si:; milique saltem lemnisci nuncluam turgidi vel teretes, sed semper complanati visi sunt, dum intestina coeca Pentastomatis cel. viro dicta turgida reperio, idemque de illis Amphistomatis dicendum est.

Fac autem lemniscos organą esse secundaria, quáe ex humore per cutem absorpto et in cavum commune delato, assimilanda resorbeant, vel lemniscos ab anteriore parte circa organa motoria proboscidis et hanc jpsam nutritioni prospicere, dum reliquis cutis actio sufficiat, aut siniilia, quae magis arrideant; nutritioni praeesse sem. per statuendum erit.

1. 5. Trematodum vasa comparando, triplicia saltem reperiec, ovigera, seminifera et nutritia; de illis in capite insequente agam, de his autem quaedam nunc proferre liceat. Vasa nutritia sacpe ad unum omnia valde minuta sunt, quod de Mnnostomatibus saltem valet maximis, vesiculas referentibus et transitum quasi ad $\mathbf{C y}$ stica praeparantibus, guorum vasa ovifera ovariis magnis comnexis distinctissima et maxima, nutritia valile parva sunt, qualia Tạbulae secundae Fig. tertia et quarta sistunt. Aliorum, praesertim Distomatis hepatici vasa nutritia, a poro anteriore orimula, valde magnia sunt, et magnis apicibus liberis terminare videntur, qualia mercurio injecta ab Orrose 1. c. Tab. VI. Fig. 9. bene delineata sistuntur; successu tanen feliciore gavi. sus, mercurium illis ex apicibus in vasa minoris ordinis supcrifciem tegentia transire videbis, re. tia effingentia multo tenuiora.

Tremalodum reliquorum vasa nutritria sip: 


\section{- 584}

gulis generibus peculiaria, nune quidem exponere non valeo. Pentastomati Cuvrerus, vir summus, tubum intestinalem rectum adscribit, idteque et ob systema nerveum Nematoideis addit; specimen autem, quod dissecui, me dubium reliquit; ab anteriori enim parte duo intestinula cotca sunt complicata, posteriora versus canalis tenuis albus decurrit, sed non -cuti affixus, qualis Nematoideis esse solet, sed undique ovariis circum. volutus, neque ejusdem fines cognoścere potui; quain ob causam rem in medio relinquam. Plurima etian circa Pentastomatis, sed praesertim Polystomatis vasa -in Entozoologin exposui; confer quoque sectionis praered. p. 33.3. ubi de quatstione, quaenam Polystomatis pars antica sit, tusius egi. Tristomatis vasa, qualia oculis nudis sistuntur, Tab. x. Fig. 7. x. delineari curavi; neque lentis simplicis ope plus videre contigit.

\$. 4. Bremserus, Helninthologorum decus, Bothriocephalos non per capitis bothria, sed per os medium haurire contendit, neque hoc in $B$ : punctato adesse negari potest, an omnibus vero sit, valde dubium videtur. In B. auriculdto iale frustra quaeritur, vasa autem colli reperimus, qualia ab osculis capitis oriunda multis in Taeniis videmus, conf. p. 479.

Cel. Orfers (de veget, et anim. p. 35.) articulos. Taeniae singulos ope absorptionis cutaneze perparum, maxime autem ope osculi marginalis nutriri contendit, sed osculum hoc vere ad genitalia pertinere in capite insequente evincam. Si cl. vir absorptionem cutaneam minoris aestumat, hac de re non litigabo, sed res alio modo explicari potest. Annon enim ad vasa linearia nutrientia, utrinque longitudinaliçer decurrentia, si 


\section{$-585$}

articulus solutus est, in utroque ejus fine uirin. que hiantia, absorbendi officium deferri posset? - Ipse saltem, quid huic sententiae obstet, non video.

5. 5. Quoad Cystica observationem in Cy. sticerco Delphini, male tanen conservato, factam solummodo referam: crijus'scilicet in vesica caudali deliquescente fila longissima, tenuissima, plana reperi, quae microscopio subjecta fere crispata, forsan ob macerationem, visa sunt, et huc forte faciunt.

\section{C a p u $t$ I V.}

De organis generationis, ovis hornmque nidis.

S. I. Nematoideoram genitalia mascula externa, praeter illa Strongyli et quarundam Ascarid $z u m$, alias satis simplicia visa sunt; nunc vero genera duo, Plhysalopterae et Spiropterae, accedunt, quorum organa externa alio quiciem modo, sed aeque composita sunt. Uti enim Strongylo. bursa terminalis est, variis cutis procluctionibus, sive lobis (aut phyllis) constans, quae coitu locum habente vulvam amplectuntur, ut penis filiformis bursae medium tenens vaginam eo profundius intrare possit, ita etiam Physalopterae et Spiro. pterae vesica lateralis alata et turgida vulvam lateraliter obtegit, quo spiculum vaginae immittant. Museum Viennense vere Caesareum Strongylos Equi et Cuniculi feri coitus vinculis morte non liberatos inter thesauri helminthologici rariora diutius jam possedit, sed nunc etiam Physalopte. ras Simiae Rosalize, a Natterezo ex Brasilia transmissas, morteque non disjunctas possidet, 
quarum icones Bremserus misit; quibus exemplis dicta optime probantur. Physaloptera vesicam caudae masculam Tab. I. Fig. 3. delineari curavi et de illius et Spiropterae genitalibus p. 256. et p. 243. sq. pluribus egi, quae brevitatis caussa nunc omilto.

Strongyli Gigantis uterum simplicem, neque in modum Ascaridis lumbricoidis bicornem, esse, Отто 1. c. p. 232. recte observarit. Quae cl, vir $a u t \in m$ de oviductus simplicis fine intestino recto inmisso refert, minime locum babere virli. Oviductus scilicet coecus terminatur et apex iste ope filorum vulgarium intestino et tunicae corporis interuae adnectitur. Locus, qua terminatur, incertus esse videtur, ham in specimine, quod ipse examinavi, tres pollices ab apice ver. mis caudali distabat, cl. Otro autem ad lineae ab ano distantiam terminari refert. Qvarium apice suo intestino recto immitti res nimis monstrosa foret!

Strongylum pene et utero simplici, Ascaridem pene duplici et utero bicorni instructos comparando, forsan a pene simplici ad uterum simplicem, a duplici ad bicornem apud Nematoidea concludi posse, hypothesin movi, quam cito deletain virli. Physalopterae retusae (in Lacerta Teguixin habitantis) pene simplicissimo utentis feninam enim aperiendo, praeter opinionem ute. rum bicornem offendi.

5. 2. Echinorhynchum Gigantem saepissime, sed semper femineum accepi, quare organorun ejusdem masculorum descriptio a cel. Nitzscr (Allgemeine Encyclopädie von Ersch und Gruber. I. Thl. Leipz. 1818. 4. p. 242.) publici jưris facta mihimet acceptissima fuit. Duos reperit te- 


\section{- 587 -}

sticulos magnos, cucumeriformes, eadem serie et parva distantia, ut posterior fere mediam vermis partem occuparet, cuti musculosae ope filorum vasculosorum affixos, quorum ductus seminiferi tenues in vesiculam seminalem elongatam abierunt, quaternis utrinque lobis sive diverticulis in. structan, et ope tuluuli sive ductus excretorii brevis et crassi vesicula dilatata in apice postico Echinorhynchi terminabatur. Hanc vesiculam saepe inversam protrudi, et sub coitu eandem apicem acutiorem corporis leminei amplecti suspicatur; si autem globuli in protrusa observentur, hos vesiculae seminaiis diverticula, certe au. tem testiculos non esse, addit. Hoc omnino ex cl. viri descriptione evincitur, cum testiculi superiori loco alfixi sint; sed sacculum clausum propendentem, saepe partis ope tenuissimae corpori adhaerentem et flaccidum vulvam femineam amplecti posse, vix admitteres.

In Ech. 'oligacanthoide (Mantiss, p. 31r.) am. Orfers filum longissinum cx vesicula caudaé ovali exsertum vidit, et ipse in corpore Ech. porrigentis (ib. p. 326.) filum, longiusculum reperi, ain vesiculam seminalem collapsam? An haec inve:ca coitus tempore protruditur, aut eodem peracto, forsan vitae termino, prolabitur? Ipse tanten vesiculam Echinorhynchorum masculorum saepissime protrusam, at nunquam filum tale egressum vidi.

Oinnes ceterum Echinorhynchorım species, quoar omnia, a cel. Nitzsch relata, cum Ech. giganteo convenire, nullus contendet. Sic v. c. vesiculae Ech. globulosi aliorumque globulos vasorum ope inter se nexas, ceterum autem undique liberos oflerunt, qui cl. viri descriptione non 
explicantur; non enim lobi vesiculae seminalis esse possunt, quae ipsa visui subtrahitur. Interdum etiam vesicula illa propendens ipsa lobata est, vel didyma.

Ovaria, quibus Echinorhynchórurn ova continerentur, non adsunt, sed haec libera in abdomi. ne natant, interque ea corpuscula reperiuntur, quae pro cotyledonibus aut placentulis habui, rquorumqque" ope antea ova cuti adfixa crederem; "partibus saltem, quae in Nematoideis tales certissime sunt, maxine affines inveniuntur. Ovarii majo$r$ is speciem a tunica corporis interna oriri posse, quaudo externa ab eadem soluta est, facile con. cipitur, eodem modo corpus a tunica externa emphysematica cinctum pro tubo intestinali imposuit.

5. 3. Cel. Nitzsen (Beitrag zur Infusarienkunde p. 7 , et p. Il.) Distomatis porum ventralem hoc nomine indignum et merum esse acetabulum suctorium statuit, quod, si pori fabricam musculosam et fundum clausum, ipsumque interdum in Distomatibus, praesertim piscinis prolabi spectas, utique verum videtur. Ipse quoque nun. quam mercurii vin ejus fundum vincere posse observavi,-sed auctoritas GoezIr, qui (Naturgesch. der Eingew. p. 170.) alterius Distomatis hepatici cirrum alterius poro ventrali immissum observasse vult, quominus illum pro mero acetabulo haberem, vetuit, et am. Olfers (De veget. et animat. p. 45.) simile quid in Distomate folio vidisse sibi videtur. Cel. tamen Gaene (Obss. de insect. et verm, structura p. Ir.) Goezium hallucinatum esse et Distomata' casu invicem adhraésisse suspicatur, cumque ovula, quod ipse saepius distincte vidi, non per porum ventralem, 
sed per cirri apicem emittantuir, Distomata nor. androgyna, sed hermaphrodita vocanda esse con. tendit. Adherere sibi entozoa parvula saepe vidi, etiam Distomata, vel et speciei et generis nomine diversa animalcula, v. C. Scolecem; sed nunquan Distomatum copnlam observavi. Ni. liilo tamen ininus rem magis dubiam haberem, nisi anatome illam confirmaret. Nullum enim unquam vas in porum clausum, distinctissime au. tem oviductum in cirrum abire video, quod plurima Distomata in Entozoologia describendo no. tavi, cujusque rei exemplum maxime luculentum in Mantissa P. 390. dedi, ubi Distomatis clavigeri oviductum cum cirro continuum cultelli ope ex illius corpore solutum retuli; sed D. Najae etiam descriptionem conferas p. 379. oblatam.

Neque Amphistomatum porus posticus to. tus ovis emitiendis impenditur, sed ipse vel coe. cus est (v. c. in A. conico, subtriquetro), vel po. rum baseos minorem continet (ut in $A$. subclavato), qui illo negotio fungitur, et cirrum forsan emitrit, qui tarnen passim fere deficere videtur, uti A.' cornuto, quod ova eflundere vidi (Entz. r. p. 298. C. Tab. V. Fig, 4.), aut qui passin lo. cum dissitum occupat, uti in D. conico. Omuium tamen specierum structuran hanc circa rem minime cognoscimus, ideoque pori nomen retinerem, magis latam notionem involvens, dum illud acetabuli nimis strictum est, et quando pars ipsa, quae ova emittit, ab eo non excluditur, rejicien. dum videtur.

5. 4. Cestoideorum genera pauca sunt, quorum organa genitalia bene cognoscimus- $\mathrm{De} \mathrm{C}_{\alpha}$ ryophyllaeo, cui optimus ZEDlR sexus distinctos adscripsit, postea dubius factus sum, at androgy- 


\section{- 590}

num vel hermaphroditum potius haberem, rem tamen ulteriori examini commendaren. Ligulae, cujus olim ovaria, postmodum etiam genitalia mascula detegere mihi contigit, quae p.463. descripsi et Tab. 3. Fig. I. delineata dedi.

Circa Taeniae genitalia cel. ar Otrers (De veget, et anim. p. 32, et 35.) sententiam prótulif, quam meam facere nequeo et accuratius dilucidabo, quod amicus amico non in malam partem vertat.

Lemniscum (utinam eum cirrum dixissem) ad genitalia pertinere negat, et rostellum esse vult, cujus ope articuli singuli nutrinentum ca. piant; et si minimis vel minoribus 'Taeniis organa talia desint, illis ob exiguam corporis molem oscula capitis sufficere, addit. Goezir'm autem, qui (Naturgsch. d. Eingeww. p: 38r. Tab. 29. Fig. 7.) semet ovula Taeniae lanceolatae per lemniscos effusa vidisse testatur, errasse suspicatur.

Ipse quidem ova ipsis cirris effundi nunquam vidi, et plurimarum Taeniarum ova alio modo emitti facile concedo, illos tamen ad gene. rationem facere nullus dubito. Majoribus enim articulis tantum dantur, neque semper adsunt, ut plurimarum Taeniarum haec organa nos penicus fugiant, quarundam rarius conspiciantur; si vero adsunt, ova etiam, vel adesse, vel nuper adfuisse non dubites. Ova enim licet utplurinum, nou semper tamen in ipsis articulis cirrigeris occulrunt; Taenia scolecina (n. 93.. Mantiss. p. 532.) saltem articulorum seriem alteram cirris instructarn, insequentem ovigeram exhibet, neque Taeniae inflatae (n. 85. p. 527.) articuli latissimi ovigéri, sed praecedentes cirros promunt. 
Cirri ipsi, sive lemnisci, maxime inter se differunt, nullibi autem majores, sed nondum exsertos, quam in Anseris Taenia sinuosa vidi, de qua in appendice agam.

Atque parum Taeniarum foramina marginalia, vel lateralia Bothriocephalorum et Ligula. rum pro organis nutritiis habeo, non enim nisi partibus evolutis conceduntur, quo fit ut $\mathrm{Piscium}$ Ligulis omnino denegentur, inter Avium Ligulas autem cirri quam rarissimi sint, quod argunertum validum censeo.

Cestoidea ope foraminum illorum se afugere posse, certe nitil contra nostram sententiam probat, sed e' contra eahdem affirmat.' Distomatum cirro porus plerumque vicinus est, quo animal hujus ope simul affigatur, et simili modo vermes cucurbitini, quibus utpote eliminandis nutrimentum superflum est, ope foraminis papillo. si affixi ova tutiore loco deponere possent. Ad nutritionem autem articulorum singulorum et so. lutorum, si illa indigerent, explicandam, foramina vasorum longitudinalium hiantia, de quibus in Capite praecedente locutus sum, melius in usum vocarentur.

Argunenta non contemnenda me attulisse crediderim, analogiain tamen non praeteribo, in ejustnodi rebus nunquam negligendam. Animalia composita, sit venia verbo, umnium partium capita, aut ora eadem proferunt, neque primariae tantum caput formae peculiaris conceditur; quod Zoophyta abunde testantur, sed Coenurus etiam omnium corpusculorum caput semper idem exhibet. Qui ergo animal fingi posset capite instructum, quod partibus brevi amissuris singulis capita novae formae gigneret? Neque ad articulos 


\section{$-59^{2}$}

refugere possumus, nam Ligúlae non articulatae, sed ovariis multiplicatis instructae, horum ques. que singulorum cirris singulis utuntur. Has autem sine ovariorum foraminibus et cirris longis. simas vivere, nulli ignoramus. Quemadmodum vero, Nemiatoideorum multa pene duplici utuntur, ita etiam Ta€nias nonnullas foraminibus et cirris articulorum oppositis instructae occurrunt; articu. lis autem revera parvis citoque perituris duo capita concedi, ipse vix concederes. Me autem ex Taeniae porosae (n. go.) articulis utrinque postice liantibus ova effusa vidisse, supra p. 530. retuli.

Dicta autem, si foramina et lemniscos ad generationis, organa pertinere forsan evincunt, Cestoideorum singulae partes utrum hermaphroditae, an androgyrae vocandae sint, non demon. strant. Lemnisci tamen et ovaria, non iisdem articulis semper simul evoluta, forsan animal hermaphroditum, partes autem singulas androgynas probarent.

5. 5. Ova quod attinet Entozoorum, semper illorum naturae indagandae operam navavi, nuperis tanien temporibus, successu incitatus, huic studio magis incubui.

Omnia reliquorum animalium velamenta ovi Entozois quoque concedi, Taeniae saltem docent, quarum singulae ovorum tunicae saepius forma discrepant, ideoque facilius sub oculos cadunt. Tabulae tertiae buic libro adjectae figurae nonaullae Taeniarum ova sistentes hoc probant; olin quiden nonnisi Taenias videram, quarum tunica, quoad formam Allantoidi similis, extima fuit, sed postinodum, etiam Taenia longiceps innotuit, in intestinis Orioli cristati Brasiliae 
a cl. Natterero detecta, inque Appendice describenda, cujus ova Chorion ovale. Allantoidem autem nomine dignam, appendicitus liheris totum Anniou non circumdaniem. lioc veru fere globosum sistunt. Conf. Tab. 3. Fig. 2 I.

Figurae allatae ovorum quoad magnitudinem et formam discrimen summum obtinent, et maxime notabile, neque hoc tamen Taeniis solis proprium, licet iisdem magis solenne. Conferas quaeso, quae de Taeniarum ovis p. 486. de illis Ascaridis extenuatae p. 287. Echinorhynchi bacillaris p. 3r6. et tumiduli p. 320. in Mantissa; de illis Pentastomatis taenioidis autem Entoz. II. I. p. 443. Tab. XII. Fig. 12. exposui, quaeque hic repetere non licet.

Filarias Mantissae describendo, harum ova placentulis linearibus brevibus affixa retuli, quod oviductibus quidem fere evacuatis optime conspicitur. Simili certe modo omnes Nematoide. orum oviductus ova adnexa gerunt, matura vero sensim solvuntur et versus locum, qua excernen. da sint, feruntur.

De Echinorhynchis supra egi. Distonatum ova immatura racemis lateralibus servari, soluta demum, quando matura sunt, aut semine con. spergenda, versus porum' ventralem cumulantur, cirri ope egeruntur. Varis tamen Trematodibus, ipsis etiam Distomatibus, optime inter haec cognitis, varia dubia supersunt, quae alterius speciei tamen assicluo studio vinci possent, quod tironibus maxime commendo et dissertationis inauguralis egregium foret thema.

Taenias quod attinet ovis majoribus insignes, quorum pauca totum articulum replent, quorumque mensuram passim in Mantissae sectione prae. 
cedente Jedi, haec distinctissime vasculo tenui ramoso per articulum ramos extendenti singula seorsim baccarum a instar affixa vidi, minora vero ova vasis sive ductibus gregatim adhaerent.

Ova deposita citissime evolvi variis argumentis in Entozoologia probavi, nuper quoque Ascaridis in Rana brasiliensii a cl. Natterro detectae specimina examinando, nil nisi feminas adultas, duas circiter lineas longas, passim ovis maximis gravidas, et prolem (pariter femineam) lintam dimidiam ad duas tertias lineae partes longam reperi, quae certe incrementum celerrimum cepit, dun quaedan Ascarides ovis adhuc onustae repertae sunt. Saepe etiain Entozoa spe. cici magnae valde parva, ideoque tenella, ovis tamen scatere vidi.

Prolem autem entozoorum, aliorum anima. lium instar, capite saepe magno uti, Taeniae et Bothriocephali praesertim probant, conf. v, c. B. microcephalum p. 473.

\$. 6. Non omnium quidem Entozoorum ova nude, sed multa intra partium tunicas, vel sub cute organi externa, hydatidem mentiente, deponi videntur. Sic certe Spiropterae plurimaé ovula inter ventriculi vel intestinorum tunicas, qua ipsae saepissime plus minus latent, secura relinquunt. Bothiriocephalus plicatus in Xiphiae Gladii recto habitans, hujusque flujdo innatans, cui ova non committere posset nisi mox peritu. $\mathrm{ra}$, saepissine inter recti tunicas plurimam partein absconditur, quas forsan ova conservandi gratia penetrat. Tetrarhynchi pari modo saepe inter tunicas latent, uti de' $T$. discophoro p. 450. retuli. Praesertim autem quae de hydatidibus 


\section{- 595}

Distomatis crystallini prolem continentibus $\mathrm{p}$.

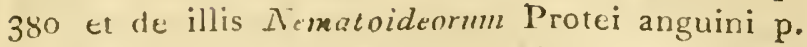
556. exposui, attentione digna videntur, brevitatis causa hic omittenda. Ascarides vel alia Nematoidea hydatidibus inclusa; qualia passim enu. meravi, ex specierum numero ideo forsan rele. gari possent, cum non nisi sobolem immaturam sistant.

\section{C a p u $\quad$ t V.}

Dc Entozoorum vita, varietatibus, monstris et morbis.

§. 1. De vita Entozoorum fugaci in Opere majori fusius egi, et multa illius exempla attuli; in Italia tamen uteridionali fugacitatem illam multo majorem et decompositionem morti proxi. mam, aliis autem arimalibus communem vidi. Pisces vix emortuos omni splendore et colorum varietate privari, $\operatorname{mox}$ squalore obtegi et ad noctem lucem phosphoricam emittere, mirabundus saepissime observavi. In avibus vero vel piscibus vix occisis entozoa saepe mortua offendi, et nonuisi aqua frequentissime mutata, quorun. dam vitam diutius strvavi, Haec circa conferas, quae de Ascaride Sternae p. 298. de Distomate pulchello p. 367. de D. fasciato p. 373. et de $D$. contorto p. 424. retuli.

Vitae tenacis e contra exempla Entozois regionum septentrionalium non ita rara sunt; satis memorabile tamen Ascaridis spiculigerae p. 290. dedi, quae in ave per duodecim forsan dies spi. ritu vini forti impraegnata, asphyctica quidem re. perta, sed in vitam revocata fuit; nec non do 


\section{$-596-$}

Tetrarhyncho attentato p. 449. quem (Ascaridibus et Bothriocephalis intestinorum mortuis) in Xiphiae abdomine, hepate licet putrido undique difflucnre, vividum reperi et per triduum in aque servavi.

Spiritus vini exigua copia aquae additus Entozoorum vitae non insidiosus videtur, sed aquam conservando, illis utilis frerj potest. Neapoli saltern quam in Coryphaena Novacula minus recenti ad vesperam reperi Ascaridem, neque microscopio subjicere potui, quo altero die examinare possem, aquae spiritu vini impraegnatae immisi, et praeter opinionem mane vivam reperi. Praesertim autem legi merentur, quae de Ascuride brevicaudata et hujus prole a spiritu non laesis p. 284.. relata sunt.

5. 2. Num Entozois nonnullis vita non codem loco absolvatur, sed evolutio alibi contingat, quaestio aqud me exorta $a b$ am. Bremsero, Helminthologorum decore, quidem rejecta et haereseos macula notata est, uti in Mantissae parte praecedentc retuli. Non possum tamen, quin rem mihi valde probabilem visam etiam hoc loco proferam, cuun Anatomicorum et Plysiologorum nonnulli forsan hanc tantum Mantissae sectionem legerint, quorum tamen istam circa rem judicio carere nollem.

Ligulam piscitm scilicet abdominalem nunquam nisi lomogeneam et simplicissimam, illam autem avium aquaticarum, piscium esu degentium, intestinalem, mox uti in piscibus occurrit, mox plus minus mutatam offendimus, cui génitalia feninea, mascula, quin ipsum caput evoluta: sint. Piscinarum vero Ligularum maceratione vel anatomica disquisitione nilil organorum forsan latentiuan detegitur, quominus Ligulas illas 


\section{$-597$}

avium calore tantum extenuari et haec in conspectun venire contendas; sed vera une judice fit mutatio, secundo Ligulae stadio vitae debita, quaeque primo, dum piscina est, ficri neguit. Nou cnim diversa esse animalia co proluatur, quodliggulae integrae, vel eaedem qua parten cum I iscinis ex ásse convenientes, novi ergo hospitcs, in avihus reperiantur. Forzan etiam Ligulae idcirco tantum certum tempus in piscibus durare possunt, et altero stadio proximae pisces perforando evadere tentant.

Sinile quid Botliriocephalo solido, in Gasterostei aculeati abdomine degenti contingere crediderim, qui avium intestinis receptus demum evolvatur et genitalibus instruatur, quae priore in stadio nunquam observantur. Bothriocephalits noclosus avium aquaticarum, qui ex eo fit, uti Avium Ligula duplici ergo statu occurrit; si brevi ex pisce advenerit, nondum evolutus, sed simplex, et deinceps genitalibus instructus. Ita etiam explicatur, quare Aves Aquaticae Germaniae septentrionalis et Daniae, ubi Gasterosteus vulgatissimus est, Dothriocephalo scatent; in Austria vero et regionibus australibus, quibus Gasterosteus deest, eaedem aves aquaticae illum vermem nunquam exhibent,

Hypothetica esse concedo, veri similitudine tamen carere non'videntur.

Filariam medinensem hominis cutem trajicere, hujus beneficio fieri videtur, ne verne post partum aut alia causa mortuo et inter partes sensibìles corrumpendo laedatur, sed de illo elininando cogitet. Viviparam illam esse, et quemadmodum p. 206. retuli, millenis pullorum nnillibus scatere, nos omnino in admirationem rapit, for- 


\section{- $598-$}

san autem matris eliminatione proli innumera fiunt obstacula,-quae illius copia tantum vincantur.

5. 3. Eodem etiam loco, prater illas primae aetatis, de quibus egi, mutationes etiam se: rius accidere videntur; quat ohservatio Bremsero nostro debetur. Taeniam Solinm satpius absque uncinulorum corona occurrere vidit, et longam Taeniam canis serratam possidet, illa pariter destitutam, uti in libro novissimo: Ueber lebende Würmer im lebenden Mersschen p. rol. retulit. Sed longe antea Echinorhyuchos avium aquaticarum observando, illos stnsim echinis proboscidis et corporis aculeis privari vidit, quod pertinaciter negavi, neque Malitissam edendo de Echinorhyncho filicolli admisi; sed postea Amicus specimina mecum communicavit Ech. sphaerocephali sibi dicti, in Haematopodis Ostralegi et Lari cujusdam intéstinis a cl. Natterero in Brasilia reperta, 'quorum juniora proboscide neque bulla instructa, corpus anteriora versus aculeata, adulta autem corpore fere laevi et proboscide in bullam señsim deundatam mutăta obserrantur, neque transitus ullo ınodo negandus est. Quibus visis manus victas dare cogor.

Triaenophori (Tricuspidariae) aculeos capitis numero variare, Entoz. II, 2. p. 3h. obs. 3. re. tuli. Num Bothriocephali nonnulli aculeos deponant, incertum est.

5. 4. Mlonstra inter Entozoa minus rara videntur, licet nonnulla dubiis adhuc premantur, ideoque dubie tantum referenda.

Huc praesertim Triaenophori (Tricuspidasiae) nodulosi bicipites, sive utroque fine, caput gerentes pertinent, quós desideratissimus Pallas inter minora illorum specimina aliquoties se vi. 
vidisse (N. Nord. Beytr. I. I. p. 94.) retulit, milit quidem (Entoz. L. p. 340.) dubii visi, cum animaiculi vulgatissimi uullus praeterea illam mon. strositatem viderit, neque tale monstrum biceps dein divisum fore, duosque redditurum esse ver. mes, hypothesis ill. viri arrideret. Venerabilis . tamen senex, cujus olim familiaritem me iniisse, fauscissimis adnuméro, quibus Deus, vitam mean beavit, de Triaenoplioris mecum locutus, se bicipites tales certissime vidisse asseveravit.

Cysticerci bicipites passim a me in sectione praecedente relati sunt. Simiae Cysticercus, forsan tennicollis, collectionis meae, quem Tab.3. Fig. 8. delineatum dedi et p. 545 . descripsi, vesica latiorte exortus nullam difficultatem habet, sed monstris duplicibus animalium vertebratorum se adjungit. - Idern de Cyscicerco Lemuris Mongoz crispo dicendum est, cujus duo specimina bicipitia in Museo Caesareo Vịnnensi vidi. Quod ibidem autem servatur Cysticerci in Simia Sabaea reperti valde ambiguum videtur, nimis saltem contractum est, quin bene dijudicari possit.

Duplicitatem aliam capitis, capitibus quasi duobus coalitis et se penetrantibus, in Taenia crassicalli Felis domestici observatam p. 524. retuli, cujus specimen in Museo laudato pariter servatur, capitis osculis sex instructum, corpore prismatico. Hoc illa cum monstrositate tamen necessario non conjunctum esse, Taenia collectionis meae demonstrat cucumerina, quam in cane repertam cel. Braun mecum communicavit, quaeque corpore prismatico, capite tamen simplici utitur.

Duplicitatem articulorum sibi incumbentium alteroque margine cohaerentium in Taenia So. 
lio a BREMSERo repertam, supra p.,522. retuli, nunc autem figuras illas illustrantes, quae Amici librum egregium exornant, Tab. III. Fig. 12-14. vidisse contigit, quaeque rem optime ob oculos ponunt.

N. Tinor ecrte duplicitas est, neque tamen praetereuncia, in Dothriocephalo lato occurcens, cujus specinen in Miluseo Caesareo servatur et a Bremsero Tab. Il. Fig. 9 et 12 . optime sistitur, ubi nimirunı articuli foraminibus-lateralibus duplicatis ncurtur.

Monstrositatis per excessum, quam dicunt, aut dupliciatis plus ininus extensae exempla sunt, quae bactenus dedi. Illius per defecturn exem. pla facilius fursan pratervidentur, ut specimina imperfecta vel mutila censeautur; Treutierus tamen, vir cel. (Auctarium ad Helminthologiam h. c. Lips. 1793. 4. p. 17. Tab. 3. Fig. 5. 6) Ascariden lumbricoidem duabus tantum capitis valvulis utentem, praeterea vero parte anteriore incurva abnormen refert, huc forsan facientem.

Monstris tandem Taenias malleatas adnumero, quarum pars anterior quasi fracta caput non evolvit, quaeque non solum in Anatibus occur. runt, sed quarum specimen etiam in Pico reperi, uti p. 52r, exposui.

\$. 5. Status quidan cutis Entozoorum morbosus saepius mihi oblatıs, quem non omitte:em, dignus visus est. Distomatum scilicet corpus passim excrescentias duriusculas exhibet, teretiusculas, plerumque exiguas, solitarias, aut complures dispersas, quales Tab. 2. Fig. 5 in corpore $D i$ stomatis Lucii terelicollis occurrentes dclineari curavi, et p. 404. obs. 2. etiam de D. spatulato retuli. Similes certe excrescentias am. Olfers 


\section{$-601$}

(De veget. et anim. P. 59. obs. r.) in Strongylo tubifice oisservavit, curn non penetrantes, sed cutis solius esse referat; eaedem autem a me observatis multo longiores fuerunt, unam scilicet ad tres lineas longae!

Cutis laesioni topicae adscriberem, cum illius e contra status emphysematicus̀ vel tunicas externam et internam disjungat, $v \in l$ cutis partes hinc inde in parvas vesiculas elévet, quae auctoribus variis imposuerunt, quo naturales haberent et-specierum nomina et characteres inde depromerent, quod Ascarides, Trichocephalos, aliosque expo. nendo passim illustravi. 


\section{Sectio tertia}

\section{bibliographica.}

Libros, cum Entozuologiam ederem, mihi non visos, aut nondum publici juris factos, quorum postea notitiam habui, illius Bibliothecat et ita quidem addam, ut numeros ejusdem circa veteteres simpliciter retineam, litteris autem illis ad. ditis, hovi insigniantur et qua inserantur, simul pateat. Rationes autem, quae sectionem praecedentem, hanc etiam et fere magis restringere ju. bent,

\section{A. $S u b s$ i d i a.}

I0. b. Notitia collectionis insignis vermium intestiualium et exhortatio ad commercium litterarium, quo illa perticiatur, et scientiae atque ama. toribus reddatur communiter proficua. Naturaescrutatoribus generatim, specialiter autem Enthelminthologis dicata ab. Administratione Reg. Caes. Muset Histortae Naturalis Viennea. sis. Junio 1811. 31. p. in 4. *

Etiain germanice: Nachricht von einer bo trächtlichen Sammlung thierisçher Eingeweide- 
würmer etc. Von der K. K. Naturalienkabinets. Direction in Wien. 3T. p. in 4. *

Libellus tam paucis plagulis constans, tot et tanta continens, praeter hunc, quo felicissimo quidem omine Bibliothecam inauguror, in omni historiae naturalis ambitu certe non datur secun. dus. Anno 1806. quo Carolus a Schrefbers, Vir cel. Musei Imperialis Director constitutus fuit, hoc, humanis aliquot exceptis, entozois penitus caruit, et quinque annis elapsis, quando Notitia laudata publici juris facta est, omnibus hujus generis collectionibus palmam praeripuit, ita ut vix unquam $a b$ alia attingeretur. Tanti vero viri sub auspicis, Musèi Inspector, Josephus Nat. terer, hujusque alter filius Josephus, Custos Musei, huic augendo et exornando summo ardore, suinma industria se adcingunt, dum alter filius Johasnes, omnium Bonorum laude dignissinus, nobsque exoptatissimus, Austriam, Hungariam, Jtaliam peragravit, plurimaque detexit et collegit, quid? quod dein Brasiliam salutavit et largam ibi messern facit, qua noster etiam liber plurimum gaudet. Partis vero helminthologicae cura specialis Bremsero commissa fuit, viro, qui ingenio, fervore, assidluitate, humanitate, integritate plurimos antecellit; nemini cedit.

Rerum. autem tam exiguo tempore paratarum copia immensa si admirationem tuam mo$v \in t$, studium cel. virorum illi impensum, quod eandem moveat, magis dignum est. Animalium enim circa vermes investigatorum numerus fere omnem fidem superat, cujus rei aliquot exempla dabo. Specimina scilicet examinarunt Vesperti. lionis murini 147, lasiopteri 213; Canis 119; Fe. lis 92, Mustelae vulgaris 307; Tálpae 375; Eri- 


\section{- 60.}

nacei 88; Ratti Iro; Musculi III8; Muris arvalis 1563; Cirilli 6r; Sciuri 96; Leporis 209: Cuniculi I6f; Falconis lagopodis 273; Buteonis 202; Tinnunculi 158; Strigis Oti I30; Collurionis 2I3; Corvi frugilegi 356, glandarii' 357, Picae I15; quae et Ranac esculentae 1272. et Hylae arbo. reae 2:37. heic loci dicta sufficiant. Ita façturn est, ut de singulorim animalium entozois, horum frequentia, terpore, locis, plurima innotuerint, quae alio modo innotescere nequeant.

Continet vero libellus aureus praeter Musei brevem historian 1 . Indicem animalium dissectorum, horum numero et entozorum repertorum tam nominibus, quam locis additis; neque illa excluduntur animalia, in quibus nulla cel. viris oblata sunt, v. c. Caviae Cobayae specinina 38. vermibus protinus libera. 2. Indicem systematicum Entozoorum Collectionis, enumeratis primum speciebus in Entozoologia a nobis descriptis, novis, illas numero haud raro superantibus, additis. 3. Demum Indicem Entozoorum a nobis enune. ratorum, Museo autem desideratorum.

\section{B. $S$ y s t e m a $t a$.}

26. b. Le Regne Auimal distribué d'après son organisation, pour servir de base à l'histoire maturelle des animaux et d'introduction d'ana. tomie comparée, Par Cuvrer T. I-IV. Pa. ris. 1817.8 . cum figg, * Iiber optimis, quibus Historia naturalis gaudet, adnumerandus, quique mihi in Italia versanti bibliothecae instar fuit; neque animalia in Europa meridionali ilegentia ullo ab auctore tantopere illustrata referies. Entozoa varia illarum regionum et no- 


\section{$-605$}

tatu quidem maxima digna primus et ante nos so!ns dixit v. c. Tristoma coccineum; Scolecem Gigantern (Gymnorbynchum nobis), Floricipitem (Antliocéphálum elongatum nobis), 20tra-

"rhjonchuin lingualem etc. Entoxor duos in or. dines inagnos distribuit, Cavitarius et Parenchy. matosns; illi Nematoidea nobis dicta et praeterea Pentastoma (F́rionoderma), Leruacam, et Nemertem (Gordis quodammodo afinem); hi reliquos nostros ordines amplectuntur, divisi in familiam T. Acanthocephalorum, 2. Tremato. dum, quo et Caryophyllaeum et Planariam; 3. Taenioideorum, quo Taeniam, Tireuspidlariam, Bothriocephalum, Floricipitem, Tetrarihynchnm, Cysticercum, Coenurum, Scolecem; 4. Cestoideorum; quo solam Ligulam refert. Si cel, Vir Bremser measquic observationes de Ligulis minus ac vulgo creditur, simplicibus hoc volumine prolatas novisset, certissime a Tricuspidaria, Bothriocephalo ctc. non separasset. De singulis ceterum stuo loco egi.

29. b. Histoire naturelle des Animaux sans ver. tébres. Par de Lamarcr. Paris. r815-18. T. I-V. in 8. * Nova liaec editio plurimum aucta et nonclum absoluta. Cel, vir Entozoorum cognitione nimis destituitur practica, quin libri auctorum ei sufficere possent; plurina itaque male miscuit et idem genus aliquoties in utrum. que ordinem et varias sectiones relegavit.' $\mathbf{L i}$ brum liunc edendo, humeris suis non prospexit, quod ut probem, Vermizem silsi Tomo tertio dictorum distributionem referam, nollium et ri. gidorum; tertius enim ordo hispidorum entozos non spectat, sed Naidem, Stylariam, Tubificem amplectitur. Mosciuar tres sunt sectiones x. $V_{e}$ 


\section{- 606-}

siculares: Ditrachyceras, Hydatis, Hydatigena, (utrumque genus Cysticercos sistit), Coenurus; Echinococcus; 2. Planulares: Taenia, Bothriocephalus, Tricuspidaria, Ligula, Linguatula (Polystomata et Pentastomata), Polystoma (dupli. catum nobis dictum), Planaria, Fasciola (Disto. ma); 3. Heteromorphi: Monostoma, Amphisioma, Caryophyllaeus, Tentacularia (Tetrarhynchus), Scolex, Tetragulus (Pentastoma), Sagittula (particula piscis! conf, Ent. I. p. Itig. n, 623. Bastiani.). Rigmorum unica est sectio: Echino. rhynchus, Porocephalus (Pentastoma), Liorhyn. chus, Strongylus, Ascaris, Fissula (Ophiostoma), Trichocephalus, Oxyuris, Hamularia, Filaria, Gordius, Quae certe classificatio nullo funda. mento utitur.

32. b. Oken's Lehrbuch der Naturgeschichte. Dritter Theil, Zoologie. Erste Abtheilung, Fleischlose Thiere, Leipzig. 1815. 8. * Cel. Viri ordines et familias nonnisi numeris reddere possum, cum illorum nomina germanica non intelligam, neque latina is addiderit, et numeris ipsi debeantur; distributionis autem simplex enarratio comparationem cum aliorum classificationibus permittet. Ordinss I. Familiae I. Genera: Echinococcus; Coenurus; Cysticercus. Famil. II. Taenia; Bothriocephalus (cur Oken et Lamarck Botryocephalum scribant, me quidem fugit, cum non ab uvis, sed a foveis capitis nomen acceperit); Rhytis (Bothr. nodosus et solidus). Famil. III. Ligula, Tricuspidaria, Prionoderma (Cucullanus ascaroides Gorzir, certissime Nematoideum). ORD. II. Famil. I. Genera: Polystoma (Pentastoma complectens), Scolex (Monostoma, Caryophyllaeum, Scolecem, Amphistoma complectens); 


\section{$-607$}

Distoma (r. in animalihus viventia: Fasciola; 2. Extra eadern: Planaria). Familia II. Poro. cephalus (Pentastoma); Echinorlyynchus; Tetrarhynchus. Familia III. Phylline (Trrstoma); Schisturus, (Leruaeae et Schisturus); Lernaea (L Gadi et Tristoma supra Phylliue dictum). Ord. IIL. Fanı. I. Hamularia; Liorhynchus; Cucullanus. Famil II. Gordius (Filarias, Gordios veros, Vibriones complectens); Trichocephalus; Oxyuris. Famil. III. Sirongylus; Ophiostoma (quo et animal fictitiun a. D. Bruyere descriptum, infra n, 624.b. dicendum refertur); - Ascaris.

Cel. viros, ingenio quantocunque polleant, specierum autem cognitione destitutos, neque genera, neque familias, nequ ordines (semper. que ascendendo minus) bene condere posse, Orexius et Lamarckjus testantur.

32. c. De Vegetativis et Animatis Corporibus in corporibus animatis reperiundis Commentarius. Auctore I. Er. M. DE Olfers, Med, et Chirurg. Doct. Pars I. Cum, tab. atitea. Berol. 18t6. 8. p. I12. * Dissertatio viri nohis con. junctissimi, ingenio, erúditione, assiduitate, candore commendatissini, Pegis Legationi Brasiliensi a Secretis, quem Deus incolumen redu. cat. Partem libri efflorescentias cutis et epizoa tractantem, utpote huc non facientem transeo, et cel. viri tantum Entozoorum systema referam. Dividuntur in I. Phytotheria: a. Cryptozoa. 1. Chaos. b. Gyminodela: a. a. cy. stica. 2. Echinoroccus. 3.-Coenurus. 4. Cysticercus. b. b. ibera. a, alyidota: 5. Tae. nia. 6. Bothrior phalus. B. cestoidea: 7. Tricuspidaria. 8, Ligula. II. Morphamorbaea. a. 


\section{$-608$}

corpore ligulato, 9. Caryophyllaeus. ro. Scolex. b. corpore subcarnoso, II. Polystoma. I2. Distoma. 13. Amphistoma, 14. Monostoma. c. corpore sacciformi. 15. Echinorhy'nchus. 16. Tetrarhynchus. III. Henninthes. Ore a. tubuloso. 17. Liorhynchus. b. punctiformi. 18 . Filaria. 19. Trichocephalus, c. anguloso aut amplo orbiculari. 20. Oxyuris. 21. Cucullanus, 22. Strongylus, d. valvuloso. 23. Ophiostoma. 24. Ascaris, Multa certe ingeniose distributa, Felminthum tamen familiae inter se, quam illae reliquorum ordinum, multo minus differunt, et fere praeter necessitatem ad. ditae sunt. Echinorhynchi cum Trematodibus codem ordine militare nequeunt. Aeque paxum Chaos, Cystica et Taenias etc. eodem or. dine comprehenderem, et subordines etian Amicus admisit. Nonnullas species a se detectas et descriptas addidit, v. c. Distoma folium Esocis Lucii vesicam urinariam habitans, aliaque, singula suo loco in Synopsi et Mantissa relata.

\section{Observationes miscellaneae.}

65. b. Mémoires sur quelques Insectes par M. de ia Martinutre, Naturaliste, qui voyage avec M. de lA Perrouse. In Olsss. sur la physique par Rozier T. XXXI. Paris. 1787. 4. 1\%. $207-209$. Suite $\mathrm{p} .264-6 . *$ Redeunt in Hodoeporicum illustris at infelicis Navarcliae. Inter alia, huc non pertinentia, Tristoma maculatum Diodontis et 'Tetrarhynchus Squali describuntur et delineantur, quae sun loco in Nantissa dixi; quaeque a Boscro repetuntur: Sur deux nouveaux genres de vers, In: Nouv. 
Bulletin de la soc. Philom. Isrr. n. 5 r. p.384.* Prius Capsala, afterum Hepatoxylun vocatur. 69. b. Beyträgt zur Naturge achichte der Einge. weidewirmer von I. Alors Froelich, Physicus in Eiwangen. In: Naturforscher. St. XXIX. (1802) p. 5- qri. Tabb 5. 2. * Quo rasu cl. viri dissertationem tertiam, duas priores in Entozoologia enumerando, pratervidere po. tuerim, me omnino latet, nisi forsan Diarii illius exmplar, quo Gryphiae tisus sum, hoc volumine caruit. Multas obarvationes refert, quas singulas Mantissae debito loco inserui.

Ante 7\%. Lettere zoologiche ossia osservazioni sopra diverai animali dirette al Conte Commeno dall Abb. Vincenzo Rosar Pavia 1794. 27. p. 4. * Priorea septern paginae huc fa. cientes Filarias Foinae, Collurionis. Ciconiae nigrae, Strongylum tulificem, Distoma hetero: stomum et aliquot laenias obiter referunt.

84. b. Erster Nachtrag zu meiner Naturgeschichte der Eingeweideürmer von Kant Asmmon Ru. noxpur. In: Magazin der Gesellschalt Naturf. Freunde zu Berlin. Vol Vl is 4. 4. p. 83II. Entozoologiae Sippleinentum, quod totum in Míantissam redit, praeter Froelicliana, quae. dam nova, v. c. Echinorhynchum Balaenae porrigentem continens.

\section{F a u n a e.}

89. Entozoologiam erlendo Zoorogiae Danica Volumen quartum non virteram, quod post. modum accepi, cujusque omuia huc facientia, 
Abildgaardir posthuma, in Synopsi et Mantissa suo loco retuli.

91. Fauna Buica von Franz von Paula Schrank. Etiam haec Entozoologiam Edenti defuit. In tertii ultimique Voluminis. Parte secunda p. 177 - 248 . Vermes intestinales ordine quidem minus bono recensentur: Ligula, Trichocephalus, Linguatula (Hamularia), Caryophyllaeus, Ascaris, Cucullanus, Strongylus, Uncinaria (Strongylus), Festucaria (Monostoma et Distoma alatum), Distoma, Echinorhynchus, Hydatula, Hygroma (Cysticercus Cellulosae etc.), Taenia. Species valde miscentur. Quae cel. viro propria sunt, aliis locis jam publici juris fecit, unde depromta Entozoologiae in serui.

\section{E. Scriptores de Vermibus Humanis.}

I38. JoACH. Jungir Historiam vermium non visam retuli, postmodum vidi, sed ad entozoa non pertinet.

142. Brescruar de Lumbricis legi, tritissima Veterum placita referentem.

750. Clericani Operis versionem Anglicam ex Dryandro attuleram, postea mili visam. Tabulae libro octava forma edito tres additae sunt in folio, omnes uti videtur Clericr figuras continentes.

159. Litteras Bianchini (p. 254. in 8.) nunc possideo, minoris momenti. De origine ex Vallisnieri mente, nidos vermium in coeco esse; contra Morealium febres petechiales a lumbricis derivantem. 


\section{$-611$}

16r. Comm, de Vermibus in C. H. et Anthel. minthico priori anno invento a JoH. Godorr, Fischerio. Stadae. 175r. 24. p. í 8. * Tta in. scriptio, quam olim a Boemnero mutuatam dedi, corrigenda est. Juglandis laudes. Larvae cum urina excretae.

167. Мiсн. Jo. Bittermann Diss, de vermibus. Vindob. ${ }^{7} 76.5 \%$ p. in 8. * Ita inścriptio ex Plovcoveto desumta corrigatur. Generalia continte. Ventriculum et intestina solam fere vermium sedem esse. Extrinsecus afferri.

169. Palmerr Tentamen de Vermibus intestino. rum, in Thesauro Diss. Edinensium Vol IIr. p. $34-55 *$ recusum legi, sed tabulas meo in specimine desidero. Quae ipse vidit, paucissima sunt, Taeniae tamen vasa injecit. Is auctor est, ex quo Angli plurimum hauriunt. 193. b. De vermibus intestinalibus. Praes. Berne.

Christ. Otto ז. Joh. Sam. Gaebler. Traj. ad Viadr. 1800, 1\% p. in 8. * Ita inscriptio Plovcquetr mutanda. Ceterum nullius momenti est.

195. b. Memorie fisico-mediche sopra i principali vermi del corpo umano vivente e le cosi dette malattie verminose, per servire di supplimerto e di continuazione alle Lezioni di Vat. Lutgi Brera. Crema 1811. XI. et 452. p. in 4. Tabb. V. * Lectionum cl. viri Entoz. Vol. I. p. 6r. n. 195. relatarum supplementum, luce vix dignum. Auctor historiat naturalis et anatomiae ignarus, utriusque heroa (invita Minerva) se praestare vult, ideoque plurima fingit et absona cumulat. Non possum quin elegantissimum cl. viri systema referam, quod certe sine pari est. Ordo I, Puestosomi, Vermes Plani. Gen 
I. Taenia, spec. I. armata, 2. inermis. Gen, 2. Fasciola, spec. I. intestirulis (Ligula), 2. hepatica (Distoma). Gen. 3. Linguatula Spec. I. Hexathyridium Pinguicola. 2. Hex. Verarum. 3. Cercosoma (Larva Muscae pendulae). Or. Do II. Fischiosomi, Vermes vesiculares. Gen. I. Eremita. spec. I, Fisch. globosum, sp. 2. Fisch, piriforme, sp. 3. Ditrachicerosoma (nomen enim Dirrachyceras Sulzerr cl, viro nondum satis longum fuit, quo sexpedale illud eligeret, verme ipso decies longius). Gen. 2. So. ciales. Spec. Fischiosoma polvcephalum. Gen. 3. Capsulares. spec. I. Fina muscularis, 2, F. hepatica (Hydrops saccatus hepatis). 3. Fina visceralis. 4, F. hydatoides (hydatides cum urina excretae). Ordo III. Ascaridi, Gen. I. Microsomi. Spec. I. Trichocephalus, 2. Ascaris vermicularis, 3. Asc. stephanostoma (larva muscae). 4. Asc, conostomà (pariter muscae larva). Gen. 2. Megalosomi: spec. I. Ascaris lumbricoides, Ordo IV. Linomortr, Filiformes. Gen. I. Anchilocefali. spec. r. Hamularia lymphatica, Gen. 2. Foxocefali. spec. I. Gordius aquaticus. spec, 2. Filaria medinen. sis. Ordo V. Amaurosomi, corpore vix visibili. Gen. I. Membranacei. Spec, Cercaria temax. Gen. 2. Chaos, sp. I. Chaos intestinale. 2. infusorio-spermaticum.

Quae retuli, inaudita sunt, v. c. genus alterum inagnó, parvo alterum constitui, utrique Ascaridem inseri, alteri maxime hetcrogenea simul immisceri; Larvas insectorum cum vermibus conjungi et quae sunt reliqua, omnium qui legant, risum moventia. De Anatome ficta in Mantissae parte secunda locutus sum, et hoc tases 
tum addam, experimentum a cl. viro p. rs6. sq. relatum, ubi ova Ascaridis ab infante dejecta, in scatola sibi transmissa et siccata canis abdomini immisit (quod emplastro bene clausisse vult), et vigintitribus diebus elapsis hoc ascaridum millibus perreptatum vidit, cl. Professoris ignorantiarn maxime prodere. Nulla Entozoorum ova tanta sunt, neque maximi, ac ille eadem fingit; nunquam entozoorum ova composita sunt, nul. lumque datur Nematoideum; cujus ova ita conglomerata sunt; nullius entozoi ova siccata corpora dura sistunt, quae tali modo scatola trans. mitti possent. Tota Ascaris cum ovorum multis milibus siccata, muci particulam diaphanarn sistit, objecto, cui imposit afuerit, inhaerens et quae cl. vir pro ovis labuit, concrementa forsan fuerunt lymphatica, quorum magna copia formis maxime variis saepe dejicitur. Corporibus hisce ahdomini canis immissis, vulneris certe cura debita non est habita, quo muscae ova sua ibidem 'deposuerint, non enim Ascarides, quas se in $\mathrm{Ca}$ nis abdomine vidisse gloriatur, sed muscarum larvas vidit, quod ipsius descriptione patet, et ipse certe tale quid suspicatus esi, nam illarum icones non tradit, - Hisce dudum conscriptis BremseRUM ill. JACQUIN auctoritate innixum corpuscula illa pro seminibus Fragariae, Ascarides vero pariter pro larvis habere video.

Honinem istum plurima ex Entozoologia. tres annos ante ejus supplementum publici juris facta furtim depromsisse, illamqne semel tantum (et forsan praeter voluntatem) p. 232 not. 369 . citasse, silentio praeteriissem, nisi in Diario quod edit (Nuovi commentari di Medicina e di Chirurgia Pad. 1818. August. n. 16.) ex insidiis me 
aggrederetur et plagii a se commissi me suspe. ctum reddere videretur. Opus meum laudat, sed annum, quo editum sit, tacet, et in nota $p$. 162. me neque Zevianx dissertationem infra $n$. dicendam, neque suum Supplementum in Bibliotheca Voluminis primi enumerasse monet. Hoc autem 1808 , Zeviant vero dissertatio 1809 , et BrerAE supplementum demum iba I comparuit, quod retulisse sufficiat.

195. c. Jo. Nepomuk Anton Gelinek Diss, de Entozois homini familiaribus. Prag. 1812. 46. F. 4. tab. 1. * Compilatio oscitanter facta, ita ut indicem nostrum vermium humanorum Ent. II. 2. p. 295. transscribendo, in quo Strongylus Gigas casu omissus est, illum pariter omittat. 195. d. A Treatise on Worms and other Animals which infest the human-body; with the most speedy, safe and pleasant means of cure. By T. Bradley. London 1813. XIX et 215. p. in 8. tabb. 3. Minoris momenti. HooPERO fere solo utitur. De foraminibus Taeniae Solii marginalibus p. 7\%. saepe pluribus instrui refert osculis, meque raro ternis aut quaternis, quod memoriae lapsu scriptum videtur, tot enim foramina nullus vidit, et bina jam rara sunt.

195. e. Jo, Tréd, Kalcker Diss. de Vermibus Intestinorum. Lugd. Bat, 1817. p. 24. in 4. Nullius momenti. Nisi cel. Prnet, Parisiensis, nomen in Dissertatione occurreret, a quo scilicet Bцоснтит, Medicum Suecum (sic!) de vermibus scripsisse hausit, illam ante quinquaginta $v \in l$ sexaginta annos conscriptam crederes, nam Van Doeveren novissimus est Helmintholo. gus, quem ipse legit. 
195. f. Dr. Brkaser über lebende Würmer im lebenden Menschen. Ein Bucl für ausübende Acrzté. Mit nach der Natur gezeichneten Ab. bildungen auf vier Tafeln. Nebst einem Anhang über P’seudo-Helminthen. Wien rsı. XII. et $28 \%$ p. in. 4 . cum tabb. IV. majoribus, quinta minore.* Liber egregius, qui brevi onnium in manu erit, et sui parem non agnoscit, neque facile inveniet. Muli quidem auctors ingenio utuntur, saepe magno, sed magna zon perficiunt, cum alia, aut non satis agendo, res pertractent, et ii solummodo eximị dicendi sunt, qui ingenio excitati, labore nunquam fatigati, omnibus ceteris neque sibi tamen satisfaciunt, quibusque si ullus unquam, Bremserus certe adnumerandus est.

Capite prino de formatione corporum organicorum in cóporibus organicis agit, eamque primitivan, quam bene dicit (alias spontaneam vel organicam dictám), esse evincit, multaque utilia simul adstruit. C. secundo Systema Ento. zoorum. C. 3. Intestinas:a: Trichocephalus; Oxyuris vernicularis; Asc. lumbr. Bothriocephalus latus; Taenia Solium. Tar paucas inter species tot per secula observatas cel. vir Ox. vermicula. ris marem primus descripsit, el $\mathbf{T}$. latam Linnaer a Solio genere differre pariter prinius detexit. $C$. 4. De causis entozoa intestinalia producentibus, ubi varia egregia afferuntur. 'C. 5. De diagnosi vermiun intestinalium, et morbis iis debitorum. C. 6. De Anthelminthicis. C. 7. De speciali methodo anthelmintica singulis vermibus opponen. da, longae quidem experientiae fructu. C. 8. De Filaria medinensi; Hamularia; Strongylo giganteo. C. 9. de Distornate hepatico; Polystomate 
Pinguicola. C. ro. De Entozois cysticis. C. II Formulae medicinales. C. 12. Appendix de Pseudohelminthibus, Ditrachycerate, Stephanostomate eic. Inriex alphabeticus Scriproruin locupletissi. mus. Librum saris laudare noon possumus, tot propria continentem, quot rarissime libri continent, sed tabularum intritio facienda est omnibus ceteris entozoorum iconibus longe praefetendarum, quatque propriis rel viri observationi. bus innituntur Fundus, tabularum nigér, cui figurae quasi innatant, partes pellucidas egregio morio redit, neque solummodo naturalis singulorum habitus perfectissime traditur, sed varieta. tes, sed monstrosa etiam sistuntur, antea nun. quam observata. Naturaescrutaribus ergo quam medicis liber a eque carus erit, quem tabulis instructum me nimis sero accepisse doleo, quin figuras ad species nostras laudarc potuerim.

F. De Animalium domesticorum vermibus.

203. b. Abhandlung über die Würmer in den Lungen und der Leber, und das Klaúenweh der schafe. Von Hieronymus Waidinger.' Wien und Triest 18.8 125 p. in 12. * De Ovis Strongylo bronchiali et Distomate hepatico expertus loquitur Veterinarius.

204. b. Lirfakrungen und Beobachtungen über die Krantheiten der Hausthiere im Vergleich mit den Krankheiten des Menschen. Ein Beitrag zur versicichenden Pathologie und Chirurgie für Aerzte und Thierarzte von BerN. mard Anton Greve, Erstes Bäridchen. Oiden. burg 18I8, XV. et 210, p. "in 8: * Variis de morbis cl, auctor utilissimas observationes re- 
fert, suique magnam spem excitat, eoque laetiorem, Veferinarii de scientiae suaé incremento tantopere solliciti quo rariores sint. Capite vero XVIl p $165-184$. speciatim de aninalium domesticorum Entozois agit. Aneurysmata Equorum a Strongylis in is reperiundis derivat; Strongylum Gigantem in renibus Tauri et Canis, Pentastoma taenioides in Muli sinubus frontalibus offendit, quod restimonium magni facio, nam praeter Chabektum nullus in Equo reperire potuit, in Cane non ita rarum, et in Lupo a Bresseno repertum.

\section{G. Monographiae Vermiun.}

Ascaris. 159. b. Commentationum in Artrr Ami-

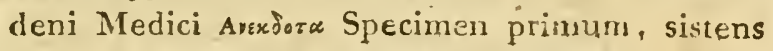
libri IX. Cap. XLI. rię, Asxaefiow. Praes, Gabr. Bonsdorff r. Joh. Magnus a Tengstroes. Aboac. 1817. 59. p. in 4. * Generalibus de Entozois praemissis, uberrimus exhibetur Capitis illius Commentarius.

Distoma hepaticum. 315. b. Darstellung und Ansichten der itzt herschenden Rindvieh - und besonders Schafkrankheit, von dem gemeinen Mann die Fäule genannt. Von Scmubt. Halle $1816.24 \mathrm{p}$. in 8. * Distoma in ductibús hepatis biliariis et extra hosce occurrere, qiuod non explicat. Ipse in hepate nunquam extra-ductus illos vidi Interdum Distomata in cystide fellea occurrere, cujus biliș aquea sit. Liber nullius momenti,

Distoma linecre. 318. b. Account of a species of Fasciola which infest the trachea of the poultry, with a mode of cure. By Georce 
Montagu in Transact. Soc. Wernerian. T. I. n. XIl. p. ra $-19 \%$. Tab. 7. Fig. 4. * Fuse p. 4/4. retuli, quod repetere nolo.

In Diario oeconomico Halensi: Der Land. und Hauswirth. De Januar. 22. 1819. p. «3, 24. Ueber das häufige Sterben der Gänse. * Anseres juniores Junio et Julio frequentissime perire et Hirudines majores et minores, milii granum ad duos pollices aequantes, tum autem valde attenuatas in anserum naribus et capite, saepe plurimas reperiri, narratur, quod ad idem Distoma. referrem.

Pentastoma proboscideum. 32r. b. Sur un ver intestin trouvé dans les poumons du serpent a someites de Cumana, par Alex. DE HurBOLD'T, in ill. viri Recueil d' Obss, de Zoolo. grie et d'Anatomie comparée. Paris 18 Ir. 4.p. 299-304. Tab. 26. * In Mantissa p. 434. fuse descriptum, sed illa impressa inter entozoa quae cl. Natterer in Brasilia reperit, offendi, et quidem in Crocodili scleropis pulmonibus lectum, qua de re in Appendice agam.

Pentastoma emarginatum. 321, c. Sur un nouveau genre dans la classe des vers intestinaux, nommé Tetragule par M. Bosc. In: Nouv. Bulletin des sc. par la soc. philom. Paris. Maj. I811. n. 44. p. 269. Tab. 2. Fig, 1. * Omnia p. 433. notavi.

Polystoma duplicatum. 52I. d. Sur deux animaux vivants sur les branchies des poissons, par M. F. Delaroche. Ib. p. 270-2. * Alterum Aniunalium Chondracanthus, alterum vero hoc Po. lystoma, Tab. 2. Fig. 3. delineatum. Omnia huc facientia $\mathrm{p}$ 438. reddidinus.

Taenia. 34ı. SArr. Erssis Diss, de Taenia secun- 
da Pratert recusa est in: Halder Disp, medico-pract. Vol. III. p. 43-460. *

362. Dissertationis Bedper inscriptioni a Dryasdro mutuatae nihil addi potest; illam dein accepi, ne minimi quidem momenti.

376. Lengsfendi opusculum Entozoologia edita accepi. Complures Taeniae species fingit, quas cum $\mathbf{T}$. lata et $\mathbf{T}$. solio figuris tabulae arljectae plurimis reddit, sed omnes ad $\mathbf{T}$. solium pertinent. Alterius caput (Fig. 6.) monstrosum refert. Ceterum arcani commendandi gratia scripsit.

377. b. Jacobr Reinlein Animadversiones circa ortum, incrementum, causas, symptomata et curam Taeniae latae in intestinis humanis nidu. lantis, casibus practicis illustratae. Vienn. $181 \mathrm{r}$. XIV et I97. p. in 8. cum tab. I. "

Vers. germ. Jаков Reindeis's Bemerkungen üher den Ursprung, die Entwicklung, die Ursachen, Symptome und Heilart des breiten Bandwurms in den Gedärmen des Menschen, Durch practische Fälle erörtert. A. d. lat. Wien I8I2. XII. et 203 . p. in 8. 'Tab. I. *

Taeniam latam nonnisi titulus habet, textus et figurae T. Solium referunt, hujus vero caput trinode cum valido unco, et partes articulorum singulorum pessime sistuntur. Liber ceterum practicici argumenti, variique casus relati attentione digni sunt, praesertim ille viri sexagenarii, qui diaeta mutata et ferculis e lacte paratis potis. simum usus leucophlegmatia et Taenía laborare incepit, qua expulsa et ad pristinam diaetam revertens belle se habuit.

377. c. Diss, exhibens Taeniarum specierum in universum, et methodorum variarum, quibus 
haęc species, quae hominem incolit, expellatur enumerationem, annexa singularis morbi historia, feminae Taenia laborantis, auct. Gum. Erx. Leonh. Katrobay. Vtatislar. ISig. 32. p. 4.* Taeniarum Entozoologia duce enumeratio, anthelminthicorum index, casus Taeniosae parum memorabilis.

Cyrticercus celtulosae. 402. Quám in Entozoolo. gia dedi inscriptio posterior est; prior laec: Taeniae hydatigenae in plexu choroideo nuper inventze historiae. Inseruntur quaedam obss. circa vernes intestinales. Diss. inaug. praes. Jo. Gotrlob Hasio, aut Jo. Leoni. Frscherus Tips. 1789. 44. p. in 4. tab. 1, " Omnia praeter icunculam inscriptionis eadem, ut forma quarta largiori textus margine reddatur.

403. b. Beobachtung und Beschreibung des Finnenwurms bey dem Menschen von Kare HinIx. In: Hufeland's und Himly's Journal. De cember 1809 . p. iL6-152. tabb. 3. * Cadaveris humani.Cysticerci tam cerebri quam muscli. lorum egregiis figuris sistuntur, microscopio etiam in usum vocato.

403. c. Berattelse om Dynt funnen hos Menniskan af Arv. H. Florman, In: Vetensk, Acad. Nya Handlingar. T. XXYI. 1810. P. 179-182, * Duo specimina in cadaveris viri sexagenarii pectorali majore, neque alia aibi reperit. 4n3. d. Dynt (Cysticercus cellulosae s. Taenia Finua) funnen i hjernan pà ett yrsukt swin, at A. H, Florman. In Kongl, Vet. Ac. Handlingar för isr 5 . Stockholm is 5.8 . p. 132-36.* Observatio maxime memorabiils, suis scilicet annum nati, vertiginosi, sinistrorsum in circulos acti, qui semper minores describerentur. 
Bestia se suadente mactata, amicus plurimos inter colti musculos, multos in pia matre et substantia corticali, paucòs in medullari, sed viginci Cysticoreos solutos, mullibi affixas in ventriculo laterali dextro reperit! Vertigo suis hoc modo certe facile explicata, sed quid Cysticer. cos a vinculis solverit, nisi ipsa forsan eorun. dem multitudo, me prorinus fugit. Plexui enim choroideo non adhatsisse, cel. vir expres. sis verbis refert. Exemplum sine pari.

Sus vertiginosus rarus; musculum vertigino. sum, aeque rarum Entoz. 1. p. 125. n. 426 dixi; sed quatuor ante annos Testudinem graecam vertiginosam, certe rariorem his meis oculis inspexi, cujus tamen mali caussam in cadavere non reperi.

H. Scripta ad Entozoorum anatomen et physiologiam spectantia.

521. Diss. Goeschenri, cujus inscriptionem ab Hefrtero mutuatam dedi, nunc quidem possideo, et legi: generationem aequivocam s spontaneam contra sacram scripturam esse. 529. Bianchi de generatione librum Entozoologia edita demum legere contigit, et Bu.ERAE quidem fratrem natu majorem illum dicerem, qui eadem verborum copia aeque pauca sistit, credulitate sua idem taedium facit, et saepissime fumum vendit.

534. Posewitzrn dissertatiunculam accepi, de qua Doerivgir judicium meum facio.

535. b. Slancio sulla genealogia della terra e sulla costruzione dinamica della organizzazione 
- seguito da una ricerca sull origine dei vermi abitanii le interiora degli aninuali, di Gusepre Gavtier r. Jena in Sassonia 1905. 135. p. in 8. * Sub dedicatione legitur: Novarra li 13. Giugno 1815 . Somnia de mutationibus animalium ex necessitáte, adfectibus etc. De homine bipede facto. De Entozois a p. 77. ad finem agitur. Mutari, commutari, Propria non admittit.

535. c. Joh. Andr. v. Scherrer. Ueber den Ursprung der Eingeweidewürmer. Medicinische Jahrbücher des K. K. Oestreich. Siaates. B. 3. St. 2. Wien 1815. 8. p. 83-116. *

535. d. Idem. "Ueber Helminthographie. Ib. B.

3. St. 4. I8I6. p. II7-13I. *

535. e. Insm. Topologie der Eingeweidewürmer.

ib. B. 4. St, 1. 1817. p. 65-89**

535. f. Eususda Bemerkungen über meine Topo. logie der Eingeweidewürmer. Ib. 4. B. St. 2. 1817. p. $164-170$. *

Dissertationes $\mathrm{c}-\mathrm{f}$ dictae ejusdem sunt argumenti, ideoque simul recensendae. Cl. auctor Entozoa ex infusoriis oriri statuit, sed in tela cellulosa, cujus ad hanc generationem partes summas facit, ut saepe infusoria reponi videantur, nam hydatides, cel. Brandis aliorumque modo, ex cellulosa formari et has alias in formas trans. itum expliçare gloriatur. Tela singulorum animalium cellulosa singularum partium diversa, singulos etiain illarum vermes diversos nasci, nimisque numerosos fore, quam qui singuli nominibus trivialibus instrui possent, ut melius a partibus animalis dicerentur v. c. Ascaris intestinalis lupi etc. neque descriptiones singulorum tantopere commendandas esse, cum nimis varient, 


\section{- 623 -}

ideoque ejusdem loci entozoa non semper eadem forent. Taudem ex Vienneusi nostroque indice topologiae speciem excerpsit, qua Entozoorum originem ex cellulosa probare sibi persuasit.

Ci. virum omni hujus sturii practica cognitione destitui, dolendum est, ingenio enir solo et aliquot catalogos percurrendo haec res certissime non absolvitur. Viginti octo anni elapsi sunt, ex quo Entozoologiae horas subsecivas, saepeque integros menses sacravi, eo aurm, me perparum hac de re scire, et plurimum siudii su. peresse dilici. Si nosmet cl. viro habitaculum entozoorum indicando non satisfacimus, hoc eo. dem ex fonte derivandlum est; quia crim res istas protinus ignorat, etiam luce mieridiana clariora illi obscura sunt. 'Tempus non anplius est, quo de rebus experientia longoque usu addiscendis ex tripode judicatur. Quia falsiscima ex hypothesi omnium locorum entozoa propria credit, multitudinem illorum tantam fore hallucinatur, quae nomina trivialia et specierum descriptiones excludat, eo autem, licet inscius, totum studium subvertit et inutile reddit. Attentissima singularum specierum contemplatione, illarum tantum vitae rationem et affinium nexum intelligimus et ad altiora ascendere valemus, Specierum studiúm si neglexisset Bremserus, Echinorliynchorum mutationes, Taeniarum varietates, $T$ latan $B o$. triocephalum esse et sexcenta alia ignoraremus. Omne historiae naturalis studium specierum cognitione innititur, neque earundem multitudo terrere potest, sed numerus exiguus. Quamdiu paucas cognoscimus, genericas relationes, distributiones specierum multaque alia ignoramus, singulae enim absque nexu sunt, Plurimis autem 
cognitis unitas habetur, et historiae naturalis stu. dium tum tantum facillimum erit, quando familiae per singula bene dispositae sunt, ut tuto per easdem incedere valeamus. - Quae non satis bene cognosco, conjungere, saepe forsan nimis timishis fui, is tamen timor, quam incerta conjun. gendi audacia minus nocebit; inde vero tot species dubias meo in opere occurrere explicatur; centum forsan piscium Ascarides et Filariae eaedem sunt, sed re tantum comprobata successores hoc enuntient. Quibus enim differentiae nota deest, ea non differre, quis negabit, sed examine ¿epetito hoc tantum addiscitur. Et quae omnium cl. viri querelarum basis est hypothetica, vermium ex tela cellulosa origo, de qua multa verba faciamus, vix digna est. Omnia enim, quae nosmet de rerum origine proferre valemus, partim tantum vera, partim incerta sunt, ideoque vocibus generalioribus utamur, quae facilius tolerari pos. sunt, quippe falsum, quod is inest, modestia simul excusant. Inde etiam dissimilationis vox maxime arrisit, et illa in organo quodam oborta, Entozoa oriri, tolerari potest; -quaenam autem partes secedant, aut dissinilentur, ignoramus; de tela cellulosa ergo si sermo fit, hujus dissimilationem aeque parum cognoscimus; et illam ipsam, non partes conteutas dissinilatione entozoa proferre, quis quaeso mortalium effabit? Entozoa tandem infusoriis originem debere, non majori veri similitudine, quam illa utitur hypothesis, secundam quam homo ex simia, aves ripariae ex gallinaceis et sic porro enati sunt. Nulla hanc transformationem prohant, et quae Goeze, quae Blocı́, aliique detexerunt entożoa, nosmet tadem offendirnus, sed eorundem de- 
scriptiones saepe vix ullae, aut insufficientes, ver. bis vagis datae sunt, illorumque icones non raro malae ab aliis male descriptae malum auxerunt. Si autem vocibus iisdem eodem semper sensu utimur, haec facile superantur impedimenta, certe non tanta, quam cl. viro experientia destituto et obiter rem agenti visa sunt. Sed vela contraham; necesse est, et dicta forsan ad Helminthologos exculpandos sufficiant.

535. g. Ueber das Nervensystem der Eingeweidewürmer von A. Oтто. In Berlin. Naturf. Freunde Magazin B. VII. S. 223-233. Tab, V. VI. * Observationes laude dignissimae, quas in sectione praecedente singulas recensui. 535. h. Diss, sistens Observationes quasdam de insectorum vermiumque structura. Hexr. Maurit, Gaede, Kiliae 1817. 20. p. 4. * Am Au. ctor p. 8-13. Anatomen Distomatis hepatici tractat et cel. Ortonis et Ramdohr observationes recenset, uti in sect. praeced. retuli.

535. i. Bemerkungen aus dem Gebiet der verglei. chenden Anatomie von L. H. Bojanus. In Rușs. Sammlung für Naturwissenschaft und Heilkunst. II. rv. Riga und Leipz. 181 7. 8. * Inter plurimas et egregias, cel. viri observationes, illae de fabrica Ascaridis lumbricoidis, quam vulgo creditur, magis composita, qua de re su. pra egi, p. 55I-553. leguntur.

\section{Scripta de morbis verminosis.}

559. Weinschenkir Diss. (28, p. 4.) cujus inscriptionem Hefrerero debueram, dein legi et minoris momenti esse vidi.

588. b. The morbid anatomy of the human gul. 
let, stomacli and intestines by Alex Monro. Edinb. 18! 1. 8. * p. $649-663$, de vermibus agit, qui tubum intestinalem infestantur; Taeniam Solium, dentatam et latam habet, $a b$ hac T. latam Hooperi distinguit, sed omnia quàe de vermibus refert, nullius ponderis sunt.

588. c. Case of a Woman, who voided a large number of Worms by the Urethra, with a description of the animals. By Liwrence. Reàd Nov. 12. 18זr. In Medico-Chirugical Traus. actions published by the Medical and Chirurgical Society of London. Vol. II. Ed. 3. Lond. I817. 8. p. 385-395. Tab. VIII.* Ca. sus a cl. Barrett observatus, quem supra $\mathrm{p}$. 250-253. de Spiroptera humana scribendo recensui.

588. d. Die Wurmkrankheiten, Darstellung ihrer Entstehung, Fortpflanzung, des Aufenthalts der Wurmgattungen, die wir im menschlichen Körper antreffen $u$, s. w. Von Arbrecit. Hamburg und Altona, s. a. 67. pr in 8.* Me: dicastri liber futilis.

588. e. Diss. de morbis verminosis praes, Jo, CAR. Frid. Leunio. auct. Gotrtob Aug. Hergeseli. Lips. 1818. 22. p. 4. " Nota refert.

588. f. Würmer in der Leber einer Wahnsinnigen, eine Krankengeschichte nebst Sectionsbericht von Dr. Hayner. In' Nasse's Zeitschrift für psychische Aerzte. I. 4. p. 514-520.* In mulieris vesanae, mortem ex fame metuentis, ductibus hepatis biliferis valde extensis septem Ascarides lumbricoides, octava partim in duodeno partim in ductu choledocho, undecim in ventri. culo, in tenuibus, potissimum duodeno et jejuno, 


\section{- $627-$}

triginta et quod excurrit, parva tandem in ductu pancreatico repertae sunt. De contentis statuque intestinorum niliil refertur, sed aegra longius diarrhoea laboravit aquosa, faeculenta, et causa certe peculiaris in intestino ipso aut ejus contentis quaerenda est, qua vermes omnes sursum et in loca aliena pulsi sunt. In cada. vere feminae periodice maniacae idem auctor Ascaridem in ductu choledocho offendit. Ego hoc nunquam vidi, quaedam autem exempla vermium in hepate et pancreate repertorum Entoz. I. p. 138 et 139. dedi, quorum alterum pariter ad Maniacum facit. Facilius tamen in Maniacis, plerumque modo maxime irregulari viventibus et mox omnia ingerentibus, mox diu esurientibus vermes in loca insolita ferri, quam maniam ipsam ab illis derivarem, praesertim si haec longius perdurat, nam vita Asca. ridum longa non est, et tali loco adhuc citius perirent, quo malum tolleretur, mortuae et col. lapsae enim ex ductibus mox putrefactae effluerent.

588. g. Cases shewing the coincidence of Worms in the Intestines with Haemoptysis and remarks an the probability of the two affections having a connexion with each other.' By Natr. RumsEx. In Medico Chirurgical Transactions publ. by the Med. and Chirurg. Society of Londom. Vol. IX. P. 2. Lond. 1818. p. 389-404.* Note subjoined, ib. p. 485-487. * Auctor vermibus nimia mala tribuens, etiam epidemica, casus aliquot refert haemoptysin ab illis exortann vix probantes. 
K. De Entozois vel dubiis vel fictis.

624. b. Lettre de M. Bruyere à M. Thounv. In Rozier Obss. sur la Physique. Août 1787. p. I09-II. Tab. I. Fig. 8. * Nihil forsan nisi concrementum lymphaticum, cujus maxillas et oculos auctor credulus describit, cujusque partem posticam non integram esse dolet. Entozoon saltem non est, ab homine ceterum dejectum.

624. c. Vermi del cuore vivi e veri. Memoria del D. Grov. Verardo Zeviani, ricevuta li 4 . Gennajo Isos. In Memorie di Matematica e di Fisica della Societá italiana della scienze. T. XIV. P. 2. Verona 1809، 4. p. $152-160$. * Auctor in canis ventriculo cordis sinistro quatuor reperisse vult vermes teretes, tenaces, gla. bros, flavicantes, tenues, quorum bini dimidium, bini integrum pedem longi fuerunt. Caput, collum aliageve partes discerni non potuerunt, illius loco duae particulae linguiformes, quas labia credit; caudam acutam fuisse. Ex alterius parte anteriore filum album digiti transversi longitudine propendisse, quod pro foetu habet. Auctor historiae naturalis ignorantissimus fuit, uti descriptio evincit, neque utrum concrementa polyposa, an Filarias viderit, certum est; capitis enim illorum motum, tali ob. servatori visum magni non facio.

624. d. Abhandlung über Würmer, welche in einer Erdschnecke entdeckt worden sind. Von August Ahress. In Berlin. Gesellsch. Naturforsch. Freunde Magazin B. IV. p. 292-296. Tab. IX. Fig. 12-19. * Entozoa aut Insecti 


\section{- $629-$}

larvàe in Helice putri reperta, de quibus $\mathrm{p}$. 567. sq. fusius egi.

624. e. Dissertatio supra n.588. c. dicta huc quo.

que trahi posset, cum praeter Spiropteram concrementa lymphatica pro entozois habita describat.

624. f. Description du Dipodium, genre nouveau de Vers intestinaux par M. Bosc. In Nouv. Bulletin de la Sóc. Philom. Mrai 1812. p. 72. Tab, I. Fig. 3, De Ichneumonis larva serA10 est, quam in Apis mellificae abdomine repertam cl, vir pro novo Entozoorum genere habet.

624. g. Beiträge zur Anatomie der Insekten von Hisnr, Morttz Gaede. Altona 1815. 4." Entozoa in Blaptis Mortisagae ventriculo reperta, conica, capite rotundo instructa p. 17. refert. 624. h. Dyacanthus (sic) polycephalus, ein Intestinalwurm des Menschen. Beobachtet und beschrieben von Dr. Streber. In Mieckex's Deutschem Archiv der Physiologie B, 3. H. 2. Halle und Berlin 1817. 8. S. 174-179. Tab. I. Fig. I-5. * Ramentum est vegetabile, for. san uvae pedicellus, uti p. 184. exposui. 



\section{Pars III.}

Appendix et Indices. 


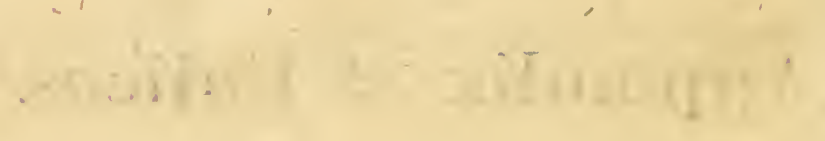




\section{A p pendix}

s i v e

\section{Mantissa Altera.}

Synopsin conscribendo, me tam cito tantum; ae Appendix continet, novorum thesaurum Helmin. thologis oblaturum esse, nullus quidem suspica. tus sum. Gratius vero et exoptatius nihil accidere potuisset, et dum Orfers et Natterer, virorum cel. collectionibus tantis simul uti conces. sum fuit, entozoa exotica generatim sumta quodammodo dijudicare licet. Multa Europaea Brasilianis communia vel affinia observamus, ut de familiis animalium ad horum parasitas conclusio ex analogia desumta saepissime valere videatur. Genera Entozoorum nova neque inter Brasiliana neque inter alia exotica hactenus a me perlustrata reperi. Quorundam praesertim in Mammalium et Avium, sed etiam aliorum in animalium generibus peculiaribus habitantium habitus alienus et formae insolitae; plurima tamen reliquorum a formis notis parum deflectunt. Neque regionum exoticarum animalia majorem specierum numerum continere 


\section{- 634 -}

videntur, ipse saltem in Italia aeque multas ni plures reperi, Americanorum, Afrorum etc. Taenias aliosque vermes nos omnino ignorare aegre fero, sed omnes amicos qui alios orbis partes vi. sitarunt, in id inquirant, etiam atque etiam rogavi, si enim Russis et Helvetis Bothriocephalus neque Taenia, reliquis Europaeis haec neque ille competit, utrum eadem an alia entozoa aliis houninis speciebus adscribenda sint, scitu est dignissimum.

\section{A. Nematoidca.}

1. Filaria obtuso-caudata R. n. sp. inter Synops. species n. 3 et 4 . inserenda.

F. gracilis, retrorsum parum attenuata, capite acu. tiusculo, apice caudali maris obtusissimo.

Hab. In thorace Pici lineati Natterer in Brasilia reperit.

Specimen a Bremsero accepi quindecim lineas lon. gum, dilute fuscescens. Caput acutiusculum, ore orbiculari nudo. Corpus tenue, retrorsum parum attenuatum, apice caudali obtusissimo, brevissimo, ante quem spicula duo inaequalia, apice caudali aliquoties longiora.

2. Filaria cystica R. n. sp. inter Synops, n. 8. et 9.

F. crassiuscula, fusca, utrinque obtusa, caudae fe. mineae crassioris apice brevi papilliformi.

Hab. Orreks Novembri mense in Brasilia Syltbranchum laticaudem secando, inter hujus peritoneum et musculos abdominis quinque vesiculas offendit, phaseoli semen magnitudine ae- 
quantes, quarum quatuor crassioribus utebantur tunicis, quinta pellucida erat. Altera ex vesicula meatus in substantian duxit musculorum. Singulae sero cruento repletae singulas continebant Filarias, altera illarum excepta, quae Filariam majorem et minorem obtulit.

Filarias tres et quartae fragmentum accepi. Illae fuscescentes, duriusculae, tenaciusculae (quo satis a F. fusca recedunt'), mediocris crassitiei, tres, quatuorve pollices longae. Corpus aequale, parte antica parum tenuiore. Os orbiculare distinctissimum. Carida obtusa cum papilla minima, in quam intestinum tenuissimum abire vidi.

3. Filaria Alcedinis. Pone n. 33. Synops:

Olfers duas Filarias misit in Alcedinis n. II. silhi dictae aludomine Februario in Brasilia repertas, fuscescentes: Alterum specimen tredecim, alterum (si partes disruptae unum efficiunt) viginti octo lineas longum. Os orbiculare magnum nudúm. Catuda crassior conica, obtusiu: scula, perforata.

$\therefore \quad$ Aliud specimen a Natterero in abdomine Alcedinis n. 82. in Brasilia repertum, flavescens; quatuordecim lineas longum, antice valde attennatum, ore obscuro; cauda conica obtusiuscula crassiore.

Propria certe species, cujus tamen differentia specifica speciminibus istis inaequalibus non efficitur.

4. Filaria Motacillae. Pone Synops. n. 38.

Specimen in Muscicapae n. 144. abdomine a Natterero in Brasilia repertum Bremserus misit album, fere bipollicare, mediocris crassitiei, 


\section{$-636$}

parte posteriore tenuiore, utrinque obtusum; ore punctum referente, vix visibili; ano ante bre. vem caudae apicem depressum prominulo.

5. Filaria Ardearum. Pone Synops. n. 40. Aliquot specimina duos ad quatuor cum di. midio pollices longa Orfers Aprili mense in Brasilia et in ventriculo quidem Ardeae sibi $n$. IV dictae reperit. Corpus aequale utrinque obtusiusculum, cauda crassiore. Os orbiculare nudum.

IDEM ibidem Mrajo specimina in Ardea n. VI. reperit, de quibus sequentia adnotavit. In oesophago, ventriculo et cavo abdominis Filariae plurimae, quae canales cartilagineos a ventriculo ad hepar effinxerant, hoc perforantes; intestina dein circumvolvunt; alize quoçue in sacculo ade: rant, humore fuscescente repleto, cujus ope ven. triculus cum osse sacro cohaesit.

Filaria altera major fusca, haud integra; altera minor, alba, utrinque obtusa, versus caua. dam crassior; haec duos, illa tres superat pollices, utraque mollis; tertia et quarta sesquipollicares, albae, tenues. Primae ova immatura valde exigua, subelliptica.

6. Trichosoma Crypturi. Ante Synops. n. 18

IIab. In Brasiliae Crypturi (sive Tinami n. 172.) intestinis, Natterer.

Specimina Bremsero debita feminea, quatuor ad sex lineas longa, tenuissima. Mares Nistrerer non transmisit.

Caput discretum nodulum rcferens., Corpo. ris pars antica (sive collum breve) tenuior, reli- 


\section{- 637 -}

quum aequale usque ad apicem caudae longum, capite colloque tenuiorem, depressum, styliformem.

Obs. Corpus breve et aequale a Trichoso. mate alienum, nimis tenue tamen est, quin vermem pro Spiropterae fernina habeas.

7. Trichocephalus contortus R.n. sp. Pone , Synops. n. 3 .

T. - Parte capillari longiore corpore maris subspirali, feminae contorto, vagina penis brevi truncata.

Duos Mures capenses (Georhychos InLrGERI) spiritu vini servatos, at recens ex Capite bonae spei a desideratissimo Bergro, cui terra sit levis, transmíssos secando, in alterius coeco quatuor hujus Trichocephali distinctissimi reperi specimina, quorum unicum masculum, hoc octodecim, feminea vigintiduas lineas longa.

Corpus maris vix spirale dicendum, sed obiter convolutum, carnei coloris; feminarum varie contortum, neque, ut in reliquis inermibus esse solet, rectiusculum, vel incurvum; oviductu nigro translucente variegatum. Pars capillaris ra. tione corporis habita brevis; in mare ad corpus uti decem ad octo, in femina uti duodecim ad decem se habet.

Pars capillaris antice tenuissima, posteriore parte sensim, at parum aucta, fere subito in corpus multoties crassius abit; si hoc fortiter contortum est, interno flexurae latere plicas offert, quae obiter inspectae pro verrucis imponere possent, sed nilil constantis habent. Corpus maris apice postico obtusissimo terminatur, ante quem lateris abdominalis vagina eminet penis, tubulum bre- 
vem Iatiusculum truncatum sistens, ex quo filum tenue incurvum emergit. Cauda feminae attenuata acutiusculá. Ova pro more elliptica utrinque no. dulo insignia

8. Trichocephalus minutus R. n. sp. Pone Synops. n. 6.

T. parte capillari longissima, capite acutiusculo, corpore maris spirali obtuso, postice attenuato, feminae incurvo papillato.

IIab. In Didelphidis Cayopollin coeco Decembri mense Orfers in Brasilia reperit, speciminá mascula tria, feminea complura, octo ad decem lineas longa, albida.

Pars capillaris ad corpus in maribus uti sex ad duo, in feminis uti septem ad tria (et quod excurrit) se habet. $O$ s indistinctum. Apex anterior acutiusculus. Pars capillaris more solito in corpus abit; maribus obiter spirale, versus apicem obtusum posteriorem attenuatum, genitali non exserto; feminis incurvun, apice postico in papil. Iam protracto, Ova binodia solita.

Obs. Species partium ratione et corpore maris postice attenuato certe distincta, genitalium li. cet fabrica ignoretur.

9. Trichocephalus gracilis R. n. sp. Pone Synops. n. 10.

Aliquot specimina feminea in Caviae Agnti coeco Septembri mense a se in Brasilia reperta Olfers transmisit, albida, parte capillari in du. obus versus anteriora fuscescente, unum cum tribus pollicis quartis partibus, ad duos pollices long?. Caput acuturn. Corpus gracile, obiter in 


\section{$-639$}

curvum, postice obtusiusculum, valde longum, ut fere dimidiam totius longitudinis partem sibi vin. dicet.

Species certe nova, quam, maribus incognitis, dubiis tamen addidi.

10. Trichocephalus gibbosus R. n. sp. Pone Synops, n. 11.

Speciei mirabilis, forsanque novum genus constituentis specimina duo cl. Porr, Med. D. Museo Viennensi ex Brasilia misit, et in phiala quidem, quae vermes vesicae felleae Scombri Thynni, prope Aequatorem 1817 capti, contineret, et in qua praeterea Cucullani melanocepltali et Anthocephali fuerunt, de quibus infra sermo erit.

Specimen alterum duos cum dimidio, alterum tres cum dimiclio pollices longum; utriusque corpus novem lineas aequat, ut differentia a parte capillari pendeat, quae in neutro tamen integra videtur.

Cutis fusca, corporis transversim rugosa. Partis capillaris apex in altero specimine fissus, in altero (minore) parte tenuiore truncata auctus, vix tamen integer; pars anterior ceterum, quam Trichocephalis inermibus esse solet, minus tenuis, fere quintam lineae partem (micrometro adhibito) adaequans, aequalis et nonnisi, ubi corpori inseritur, paululum incrassata. Corpus utriusque speciminis ad lineam dinnidiam infra colli insertionem in gibbum abit brevem, ut ibidem tres quintas lineae partes crassum sit; tum corpus iterum contrahitur, versus medium increscens et duas lineace quintas partes adatquans; dein vero decrescens, 


\section{$-640$}

apice postico quintam lineae partem longo, tenuis. simo, subulato, ante quem ani orificium parvum, rotundum, immersum.

Corpus apertum cutem duplicem, extimam firmissimam et duram monstravit. Intus materiam conspexi grumosam fuscam, quae microscopio subjecta foetuum numerum prodidit ingentem, et simul ova nondum matura, foetum tamen com. plicatum indicantia, fornae subglobosae.

Foctus parte altera crassiuscula, altera tenuissime subulata, sed quam illa bis vel ter breviori utuntur, ut is et adultis longe alia partium ista. rum ratio contingat. Plurimis quidem foetubus Ioco, qua tenuis pars incipit, crassior utrinque eminentia notatur, sed non omnibus, ut gibbum feminis tantum privum et forsan vulvam prominentem (licet nostris speciminibus omnino clausam) eo indicari suspicarer.

Obs. Species certe singularis, nisi cutis du. ra, gibbus, foetus vivi novum genus arguunt; si vere piscina est, res explicatur, nam omnes Trichocephali inermes habitu convenientes in Mammalibus degunt, et qui in Ophisauro reperitur, Trich. echinatus aeque aberrat. Hujus corpus aperui, num pariter foetus vivos contineret, sed specimen meum in Entozool. II. 1. p. 98. descriptum masculum videtur, materiam enim albam grumosam continet, quae microscopio subjecta adspectum servavit, neque foetus neque ova obtulit. An Trich. gibbosus tamen armatus erit?

11. Cucullanus melanocephalus $\mathbf{R}$. Synops. p. 20. n. 5. Mantiss. p. 232. Elegantissimae speciei, quam ipse Neapoli in Scombris Colia, Rochei et Sarda reperi, speci- 


\section{- 641}

mina etiam communicavit am. Brenser in intestinis Scombri Thymii prope Aequatorem Octo. bri mense ícapti a Nattenero et Pohlio reper. ta, a meis haud recedentia.

12. Spiroptera gracilis R. n. sp. Pone Synops. n. 3 .

Sp. Ore orbiculari nudo, caudae femineae apice depresso acuto, masculae spira subtriplici, alis

- latiusculis, pene longo.

Ilab. In intestinis Bradypodis tridactyli NarTERER in Brasilia detexit.

Specimina tres ad sex lineas longa, tenuis. sima, Trichosomatis instar, contorta. Caput an. teriora versus magis attenuatum. Cauda maris in spiram subtriplicem ahit, arctissime involutam, ut apicem non viderim; flo genitali longe ex. serto; membrana alari latiuscula, saepe obscura. Canda feminae depressa acuta.

A Trichosomate cauda mascula facile distin. guitur; sed femina etiam longitudine milsore, et corpore medio tantum obscuro, neque ovorum serie duplici repleto, ab illo genere recedit.

'13. Spiroptera denudata R. n. sp. Pone Synops, n. 4.

Sp. capite rotundato, ore nudo, caudae feminéae apice obtuso, depresso, masculae spira duplici laxa.

Hab. In intestinis Tanagrae n. 124. Natteren in Brasilia reperit.

Mas fere quinque, femina fere septem lineas attingit. Caput rotundatum, simplex, absque papillis, oris 'apertura non dignoscenda. Corpus antrorsum magis sed parum attenuatum, Cauda 


\section{$-642-$}

maris spiram duplicem solutam sive laxam eff cit, membranis revincientibus tenuissimum tan tum marginem sistentibus, quo fere denudata fit; apex nudus. Canda feminae obtusa, depressa, Ova ad hujus tubum intestinalem utrinque pel. lucent minima sphaerica.

14. Spiroptera acutissina. Pone antecedentem.

Sp. capitis ore orbiculari nùdo, caudae femineae apice acutissimo, masculae spira duplici lato. alata.

Hab. In ventriculo Falconis n. III. Olfers Februario mense in Brasilia reperit.

Mas quatuor, femina sex cum dimidia lineas adaequant. Caput ore orbiculari, mihi saltem nudo et papillis destituto viso. Corpus posteriora versus minus, sed generatim parum attenuatum. Candae masculae spira duplex, membrana alari utrinque lata, apicem obtusum' nudum non attingente; genitale non exsertum; cauda feminae re cta, depressa, acutissima.

Obs. Si papillae capitis, quas non vidi, ades. sent, pone speciem n. I'́. collocari deberet, cuí affinis quidem est, a qua tamen facile distin. guitur.

15. Spiroptera Ralli. Pone Synops. n. 37 Inter Ralli n. 18o. tunicas ventriculi NatréRER in Brasilia reperit.

Specimina duas ad quinque lineas longa, valdé tenuia, feminea. Caput obtusum, duorum simplex, tertii utrinque ala semilanceolata instructum. Corpus subaequale. Cauda capite crassior, obtusiuscula, depressa, inflexa. 
Specimen quociue accepi in intestinis Ralli 'n. Ig' a Nitterero in Brasilia repertum, quinque lineas longum, fernineum, quod dubie tantum huc refiero.

Hujus caput obtusum papillam vel apicem medium acutiusculum profert. Corpus subaequa. le. Cauda capite crassior, obtusiuscula, depressa, inflexa.

In prioribus saltem capitis papillam non vidi, ceterum conveniunt.

\section{Physaloptera clausa $\mathrm{R}_{\text {. }}$}

Synops. p. 29. n. I. Mantiss. p. 255.

In Erinacei europaei masculi ventriculo Be. rolini Martii d. XXVI, I8rg. quindecin reperi Physalopteras, quarum sex masculae, novem ad triginta lineas longas, minores albas, majores ru. bellas, ventriculo fortiter infixas, quasque per triduum ayua trigida passim commutata vivas con. servavi.

Quietarum capitis obtusi os orbiculare, mo: tis autem pars interna, plerumque bilabiata visa, proboscidis brevissimae instar exseritur et continuo agitatur. Corpus antrorsum "attenuàtum, motuque multo magis attenuari potest, ac in mortuis cernitur. Candae masculae vesica lateralis multo majoribus plicis aut vasis lateralibus, sed paucioribus utitur, 'quan' Tab. I. Fig. 3. delineatae sunt. Caudae fentheae apex obtusiusculus brevis, incurvus. Tulva prominens bilabiata ad finem primae corporis tertiae partis, Ova inmatura, aut non foecundata potius, ovalia, parva, in. numera, quae fortissima etiam microscopii com. positi lente adhibita tota pellucida fuerunt. 


\section{$-6.44$}

\section{Physaloptera turgida R. n. sp. Pone}

- Synops. n. 1.

Pliys. Ore nudo, caudae masculae vesica plana. utrinque turgida, femineae obtusissimae apice inflexo.

Flab. In Didelphidis Cayopollin ventriculo Decenibri mense OLFERS in Brasilia reperit.

Specinina mascula duo, totidem accepi feminea, illa octo ad decem, haec tredecim ad quindecim lineas longa, illa ultra dimidiam, haec fere lineam crassa.

Caput continuum, ore orbiculari, nudo; papillas, saltem non vidi, sed cutis, forsan maceratione, passim antice producta, illarum aemula est. Corpuls antrorsum satis, retrorsurn parum attenuatum. Cauda mascula vesicam offert ab illa reliquarum inflexa diversissimam, planam, rectam, ovato-lanceolatam, marginibus lateralibus aqua turgidis clausis, ad apicem caudae retusum vel fere emarginatum decurrentibus, ibique conve $\downarrow$ nientibus, parte inter illos concava, antrorsum tuberculum rufum exhibente; filo non emisso. Cauda feninae obtusissima, apice brevissimo quasi intruso et inflexo.

Specimen quoque Brxusero debeo mascu. Ium in Didelphidis n. 7. intestinis tenuibus a Natrerero in Brasilia repertum, cum descriptis protinus conveniens.

18. Physaloptera dilatata R. n. sp. Pone praecedentem inserenda.

Plyys. capite alato, caudae masculae vesica latissima aperta, apicem haud attingente, femineae apice obtusiusculo depresso. 


\section{$-645$}

Fab. In Simiae Rosaliae ventriculo Natrerer in Brasilia reperit.

Specimina mascula tres, ad tres cum dimidia, feminea sex et quod excurrit lineas longa, parte postica crassiuscula. Caput obtusiusculum, ala utrinque laterali, antice truncata et latiore, brevi in corpore evanescente. Corpus ab anteriore ad posteriorem partem increscit, hujus apice iterum decrescente. Candae masculae vesica latissima, aperta, antice obtusissima, apicem caudae nop a:tingente, qui costae instar decurrit. Caudac femineae apex obtusiusculus depressus.

Ubi feminae prima corporis tertia pars desinit, hoc circuin circa impressum, et subtus fovea magna orbicularis conspicitur, coitus nuper trans. acti vestigium certissimum. Bremserus, Helmin. thologus oculatissimus, hoc omnibus in feminis observavit, $\epsilon$ t Physalopterarum harum coitus vir1culis morte non solutarum iconem transmisil. Impressio circularis a costa vesicae corpus am. plectentis media derivanda est, quod icon probat a cel. viro publici juris facicnda, Spiculum exser. tum non vidi.

\section{Physaloptera alata R.}

\section{Synops, p. 29, n. 2. Mantiss, p. 256.}

Quam in Falconibus curopaeis repertam 1. c. descripsi, am. Ozfers quoque in Falconis n. II. ventriculo Novembri mense in Brasilia reperit.

Specimina duo accepi; alterum $m$ sculum, quindecim lineas longum, ore orbiculari, sexpapillato, uti videtur, meinbrana laterali in colla evanescente. Caudae vesicula contracta, aut minus expansa. Alterum " femineum sex tantum in- 


\section{- 646}

neas longum, cauda obtusiuscula, depressa conveniens, alis autem capitis retrorsum latioribus subito cessantibus, et caput sagittatum reddentibus recedens. Specimen certe atatis tenuioris fuit, quod longitudo exigua probat, ut illi forsan varietas haec adscribenda sit.

20. Physaloptera strongylina R. n. sp. Pone Synops. n. 2.

Phys. capite papillato, caudae masculae vesica apice reclinata, femineae apice obtuso, depresso.

Hab. In ventriculo et intestinis Cuculi seniculi Nattrier in Brasilia detexit.

Specimina albida, crassitiei mediocris. Mas tres cum dimidio lineas longus in ventricuio Cuculi, partion inter illius tunicas latens; feminae in illius intestinis occurrentes septem ad ncvem. lineas longae. Caput papillatum videtur. Corpus utrinque parum attenuatum. Cauda maris apice vesicae lateralis fere reclinato bursam Strongyli mentitur, et a Physaloptera ad hunc transitum. facit; sed vesica non est terminalis, tuberculum etiam hujus abdominale rufum, ex qua stylus crassiusculus et longus emergit; quae rem dubiis liberant. Cauda feminae reçta, obtusa, depressa, sive subtus complanata.

\section{Physaloptera retusa $\mathrm{F}$.}

Synops. p. 30. n. 4. Mantiss. p. 258.

Specimina quoque accepi a Natterero in Lacertae Teguixin intestinis reperta', cum descriptione 1. c. convenientia, vesicula caudali quan. doque patentissima, nullius spiculo exserto.

Specimen praeterea masculum a Natrerero 


\section{- 647 -}

in Stellionis n. I. intestinis (cum altero masculo et unico femineo) repertum Bremserus misit, quinque lineas longum; apice vesicae caudalis fere emarginato se huc pertinere probans.

22. Physaloptera tenuicollis $\mathrm{R}$.

- Synops. p. 30. n. 5. Mantiss. p. 258.

Speciem istam recte ad hoc'genus relatam ésse, Phýsuloptera primae speciei viva me docuit, collum saepe pari modo extenuans.

25. Physaloptera saginata $\mathbf{R}$. n. sp. Pone Synops. n. 5 .

In Strigis intestinis Natrerer in Brasilia reperit, sed tantummodo specimina feminea, quorum Bremserus duo transmisit speciem distinctam probantia, dilute fuscescentia, extremis albis, peilucidis, alterum fere quindecim, altcruin fere viginti lineas longa, lineam diametro transverso attingentia, quae crassities illis nomen dedit.

Os transversim elliptico.rotundatum, diame. tro scilicet transverso longiore, pharyngis parte eminente oris aperturam claudente, Corpus fortiter annulatum, sive transversim et densissime striatum, antrorsum magis attenuatum, apice pustico obtusissimo.

24. Strongylus costatus R, n. sp. Pone Synops. n. 2.

Str. capite obtuso costato, bursa maris subtriloba, cauda feminae obtusa.

Hab. In intestinis Colubri n. 15. Natrerer in Brasilia reperit.

Specinen et masculum et femineum Bremserr optimi benevolentiae debeo, illud frere quin. 
que, hoc fere sex lineas longum, crassitiei mediocris.

Caput bullam armatán continere videtur, costatam, costis ni fallor octo, latiusculis, depressiusculis. Corpus anteriora versus magis attenu. atum. Bursa maris subtriloba, lobis duobus majoribus lateralibus, tertio inferiore, breviore. Cauda feminae obtusa, ante cujus apicem (ad lineae distantiam) tuberculum subovatum. Ova rotunda pellucent.

25. Strongylus galeatus R. n. sp. Pone Synops n. 5 .

Str. capite rotundato galeato, bursa maris monophylla crenata, cauda feminae mucronata. Fab. In intestinis Lacertae Teguixin Natterer in Brasilia reperit.

- Specimina mascula duas vix superantia lineas, feminea fere tres lineas longa, illisque crassiora.

Caput in vivis dijudicandum erit. Ipse du. orum dierum horas subsecivas huic speciei examinandae impendi, interior tamen capitis fabrica me fugit; rotundatum est, ala membranacea trans. ycrsa, brevi at lata, nuchae imposita, galeam Mambrini, qua incomparabilis Heros Don Quixo: te caput tribus Anticyris insanabile tegere solitus fuit, in memoriam revocante. Capiti ceterum bulla inesse videtur dura, qualis Strongylis armato et dentato conceditur, eadem vero fibris lon. gitudinalibus affixa, quas microscopii neque com. positi, neque simplicis ope bene intelligere potui; species est, ac si bulla fasciis et transversis et longitudinalibus, aut papillis forsan exstantibus uteretur. Corpus infra caput contracturn est, po- 


\section{- 649}

steriora versus minus attenuatum. Cauda maris terminatur bursa versus dorsım gibba, latere ventrali haud exstante, monophylla, crenata rotius quam lobata, vasis lateralibus pro more insigni, filo genitali laud exserto. Canula feminae mucronata, mucrone brevi, ante quem auss, cui intestinum oblique inseritur. Haud longe ab ano tuberculum exstat vulvae simplex vel duplex, magnopere protrusum.

26. Strongylus subauricularis R, n. sp. Pone Synops. n. 13.

Str. capite truncato nudo, bursa maris biloba, cauda feminae acuta.

Hab. In intestinis Ranae musicae Natterer in Brasilia, reperit.

Specimina yuatuor ad sex lineas longa, tenuia. Caput trumctum, pipilarmm speciem, attamen haud distinciam prate se ferens, nudum, semel (émplnysematice) alatum visurn. Corporis pars antèrior ralie tennis, insequens subaequalis, reliqua (minus tamen yuam antrorsum) decrescens. Bursa nuaris biloba, radiata, Feminae cauda acuta depressa.

Species Str. auriculari alfinis, sed diversa visa; nomine tamen affinitatem indigitavi.

27. Strongylus leptocephalus F. n. sp. Pone Synops. 11, 19.

Str. capitis ore orbiculari, bursa maris obliqua multilobata, cauda feminae acutiusçula.

Hab. In intestinis crassis Bradypodis tridactyli feminei Olfers Majo mense in Brasilia de. texit.

Specimina plurima, sed submacerata accepi, 
cum in intestinis crassis reperta sint, forsan mortua et dejicienda Mascula sex, feminea sex ad quindecim lineas longa, crassitiei mediocris, extremitate vel utraque, vel antica sola saepe fuscescentia, ceterum alba.

Caput cum collo brevi tenue, ore orbiculari nudo. Corpus posteriora versus minus attenuatum. Bursa maris, uti videtur, multilobata, filó genitali longo. Canda feminae apice utitur brevi acutiusculo, Ova oblongo-ovalia, ammio ejusdem ac chorion formae, passin a b hoc solito magis distante.

Cutis plurimis speciminibus emphysematica et crenata; idem etiam bursae accidit, quo ejus. dem lobi obscuri reddantur. Collum satpe tortum et plurimis quidem eodem modo. Feminae majores minus deformatae sunt. Num color utriusque apicis fuscus naturalis sit?

\section{Ascaris elongata R. n. sp. Pone Syn- ops. n. 1.}

Asc. capite nudo, corpore gracili utrinque ae. qualiter attenuato, cauda feminae acuta depressa.

Hab. In intestinis Simiae Beelzebul Januario mense Olfers in Brasilia reperit.

Specimen accepi unicum, fenineum, duos pollices longum, quartam lineae partem latum, rectiusculum, albiduin, primo adspectu facile pro Echinorhyncho imponens. Caput exiguum valvulis tribus distinctissimis. Corpus utrinque parum et aequaliter, aut tantillum posteriora versus magis attenuatum, ut discrimen nullius momenti sit. Cauda depressa et acuta. 


\section{- $651-$}

29 Ascaris strongylina R. n. sp. Pone Sýnops. n. 3 .

Asc. capite nudo, corpore utrinque aequaliter atteriuato, cauda maris alata, mucronata, feminat subulata.

Hab. In intestinis Crypturi (Tinami no 17r.) et Tetraonis Uru (173) Natterer in Brasilia reperit.

Specimina Crypturi duas ad tres lineas longa. Caput distincte trivalve, valvulis oblongiusculis. Corpus utrinque satis aequaliter attenuatum. Caucla maris obtusissinia mucrone terminata, qui a dorsali latere rectus et tenuissimus excurrit, alis subtus rotundatis, basin mucronis attingentibus. Cauda feminae recta longe et teliuissime subulata.

Specimina feminea in Tetraone Uru reperta tres et quod excurrit lineas longa, caudam illis ex Crypturo descriptis fere longius subulatam offerunt, ceteruń omnino eadem.

30. Ascaris osculata $\mathbf{R}$.

Ent. 1, p. 135. n. 7. Synops, p. 39. n'. 9. sed locum pone Synops, n, 72 . invenire debu. isset.

Quae olim descripsi specimina, in Phocae vitulinae ventriculo a me reperta, valde pusilla fuerunt; majora postmodum cel. Baxker misit, codem loco lecta, sesquipollicem ad duos poliicus longa, cute vero emphysematica corporeque contracto deformia. Nuper vero specinina mandavit hremserus noster, in intestinis. Phocae gróell. landicae inventa quorum mascula quindecin, feminea octodecim ad vigintiquatuor lintas longa 


\section{$\div 652=$}

sunt, haec lineam crassa, òmnia optime conservata.

Caput valvulis majusculis marginatis, suborbicularibus, a quibus nomendesumsi, instructum. Corpus posteriora versus minus attenuatum; cauda crassa, maris inflexa, apice brevissima acuto, antè quem spicula duo longissima, incurva; cauda feminae recta obtusa cum brevissimo et tenui apice acuto. Mcmbrana lateralis in apice colli latior obtusiuscula, tum tenuis decurrens, brevi cenuissima et fere invisihilis.

Specimina mea pusilla comparavi, et horum quoque reperi, quibus pars posterior crassior est, reliqua conveniunt.

\section{Ascaris'spinicauda OLfers.}

Synops. p. 40. n. I3. Mantiss. p. 272.

Specimina tria femina, quae Natrerer sub palpebris Scinci_n. 9. in Brasilia libera invenit, a Bremseró transmissa, aut huc pertinent, aut speciem maxime affinem sistunt. Aliquot- lineas longa, capite alterius trivalvi, reliquorum non dignoscendo, cute scilicet emphysematica, deformi. Carida quidem quam spinicaudae minus subulata videtur, sed specimina manca sunt; alterius vero ante apicem similis adest cyathus, ac in illa de. scripsi.

Specimina in intestinis crassis Lacertae Teguixin ab am. OleErs Septembri et Octobri reperta 1. c. adumbravi, sed transmisit etiam specimen, in illius coeco Decembri mense a se inventum, de quo, num huc faciat, dubius sum. Fe. mineum est, decem lineas longum, crassiusculum, retrorsum fere magis attenuatum; capitis valvu. 


\section{$-653=$}

lis distinctis; cauda non subulata, seri apice depresso acuto, ante quem anus conspicitur.

32. Ascaris mascula R. n. sp. Pone Synop's. n. 13.

Asc. cajitie nudo, corpore utrinque aequaliter attenuato, cauda maris inflexa obtusiuscula, feminae recta, mucronata.

Hab. In intestinis Colubri n. 16. Natueuer in Brasilia reperit.

Specinina tria tres ad quatuor lineas longa, quorum untm masculum, femineis crassius, sed non ovigeris, ut alio forsan' tempore feminde praevaleant. 'Rem singularem visam nomine tri. viali notavi.

Caput nudum, ceterum non dignoscendum, quo a specie insequerite satis affivi distinguitur. Corptes maris retrorsum fere crassius, feminarum (an semper?) utrinque aequaliter attenuatum. Cauda maris inflexa, apice obtusiusculo brevi, ante quem spicula duo crassa, brevia, inflexa. Cauda feminae acuta, mucronata, rectá, Ova non exhibita sunt.

\section{Ascaris unguiculata R. n. sp. Pone: - praecedentem inserenda.}

Asc. capite nudo, corpore aequaliter attenuato, cauda maris unguiculata, feininae recta, subulata.

Hab. In intestinis Amphisbaenae n. 30. NatteRER in Brasilia reperit.

Specimina dimidiam ad duas lineas longa. Caput valvulis satis bene dignoscendis instructum, attenuatum, nudum. Corpus utrinque fere aequaliter decrescens; discrimine saltern vario et 
exiguo. Caucla maris a corporé crena distincta, ex qua spiculum breve emergit, apicém satis longurn habet incurvum, sensim decrescentem, acutum, unguem avis rapacis bene referentem. Cauda feminae recta, depressa, subulata.

Oesophagus longus, basi ampliatus; ventriculus subrotundus; intestinum apice obtusum, tum attenuatum. Ova quidem rotunda'sed haud distincte vidi.

34. Ascaris foecunda R. n. sp. Pone Synops. n. 14.

Asc. capite nudo, corpore utrinque fere aequa. liter attenuato, cauda feminae obtusa longe cuspidata.

Ha3. In intestinis crassis Hylae n. 4. et intestinis Ranae comutae Natterer in Brasilia reperit.

Specimina in Hyla inventa, omnia feminea, adulta sesquilineam ad duas lineas, proles dimidiam ad integram longa, cute plurimam partem emphysematica. Caput nudum passim trivalve visum, alias obscurum. Corpus media parte antrorsum paululum crassius, ceterum fere aequali. ter attenuatum. Cauda obtusa, longe cuspirlata, cuspide tenui aequali. Ova oblongo : elliptica, maximis, quae inter Nematoidea vidi, adnumeranda, licet mater exigua sit, quam ob ova et prolern magna foecundam vocavi.

Specimina Fanae cornutae debita, vix lineam longa, tenella, prolem sistentia, feminea, cauda acutissima, cuin praecedentis prole convenire visa. 
35. Ascaris depresea ZED.

Synops, p. 42. n. 24. Mantiss. p. 276.

Quae in omnibus Falconibus nostratibus oc. currit, etiam brasilianis non deest, ipse-saltem speciminum transmissorum, ommium quidem $f_{e}$ mineornm, nullum discrimen detegere potui.

Olfers primum duo specimina'misit, qua. tuor pollices et novem lineas longa, retrorsim crassiora, albida, in Falconis n. III, intestinis te. nuibus Februario reperta.

Alia duo deinceps accepi, alterum sesquipollicare, alterum d'uos cum dirnidio pollices lon. ga, quae Natterer in Falconis magnirostris intestinis offendit.

\section{Ascaris auriculata R. n. sp. Pone Syn-} ops. n. 30.

Asc. capitis obtusi alis vesicularibins, corpore utrinque aequaliter attemato, cauda acuta, maris inflexa, feminae divaricata.

Hab. In intestinis Colubri n. 20. NATterer in Brasilia reperit.

Specimina complura inascula, unum femine. um a Bremsero acrepi, sex ad undecim lineas longa. Caput obtusum, valvulis tribus oblique introrsum ductis, latis, brevibus, alisque lateralibus magnis, ornnium speciminnum vesicularibus. Corpus utrinque aequaliter atenuatum. Cauda maris fortiter innexa, apice brevi acuto, ante quean spicula duo longissima. Caudae femineae apex brevis acutus divaricatus. 


\section{$-656$}

37. Ascaris veligera $\Gamma$. n. sp. Pone Synops. n. 32 .

Asc. Capitis depressi alis lato-lanceolatis, cor- pore antice crassiore, cauda maris obtusa inflexa, feminae altenuata acutiuscula.

Ilab. In coeco Leporis brasiliensis Otrers Apriii, et NAtterer in Brasilia invenerunt.

Specimina Olfersiana omnia feminea, recta aut stricta, scptem lineas'superantia, Nattereriana mascula quaner lincas, feminea pariter septem. longa, sed plus minus convoluta.

Capitis depressi valvulae exiguae, de quibus fère dubins factus fuissem, tanclem in nonnuillis bene milis exlibitae. Alae capitis lato-lanccolatae. Corpas antrersum minus attenuatum. Cauda mascula inflexa, alis latolanceolatis inštructa, quae ejus apicern non attingunt.' Spicula lraud exserti. Cauda feminae nuda, non subu. lata, uti videri posset, sed tantum attenuata, apice terminata acutiusculo. Ová óblónga majuscula, amnio a chorio satis remoto.

38. Ascaris retusa R. $n \cdot s p$. Pone praecedentem. $3^{2}, \mathrm{~b}$.

'Asc- Capitis retusi alis tenuissimis, corpore antice crassiore, cauda inaris obiter incurva, fe. minne recta, acrita.

Hiab. In Dasypodis novemcincti crassis horumque tuberculis 'Namterer in Brasilia reperit: Specimina masculum tres, feminea tres quatuorve lineas longa. Caput continuum, tenuius, retusum, alis tenuissinis, valvulis obscuris, forsan appressis; Burersino enim circa hoc genus diff̣ciliori Ascaris dicitui. Corpus posterior versus 


\section{$-657=$}

magis attenuatum. Canda maris obiter íncurva, apice brevi acuto, spiculis non exsertis. Cauda feminac depressa, acuta; brevi ante ejus apicem anuls. Vulva in altero specimine ad primam ver. mis tertiam partem, in altero pone duas totius quintas partes eminentiam effornat acutiusculam.

Natterer in tuberculis intestinorum cras. sorum singulis humore albo repletis vermem so. litarium reperit.

39. Ascaris reclinata R. n. sp. Pone priort m. 32, c.

Asc. capite obtuso alato, cauda maris mucro. nata incurva, feminae subulata reclinata.

Flab. "In intestinis Cirntophagae majoris Natte. RER in Brasilia detexis.

Specinen, accepi masculum fere quatuor, femineum fere sex lineas longum

Maris eaput obrusurn, valvulis inconspicuis, alis vel nullis vel tenuissimis; corpus retrorsum magis attenuatum; cauda obiter incurva, apice subito in mucronem tenuissimum vel capillarem abiens, ante quen olae partis inflexae; has inter spiculum brevissimum, rectum; posteriore loco et brevi ante mucronem tuberculum adest inaequale, vix naturale.

Feminae caput valvulis expansis quasi dentatis, ala utrinque laterali semilauceolata, in antica corporis parte 'evantescente', forsan post mortem aucta. Corpus posteriora versus magis attenuatum. Cauda stibulata, apice tenui capillari, reclinata vel reflexa; 'qua reflectitur, partis cushvexae tuberculo exstante, num ano?

40. Ascaris truncata ZED.

Synops. p. 45. n, 36. Mańtiss. p. 28r, 


\section{- $658-$}

Specimina duo feminea Olfers transmisit in intestinis Psittaci aestivi var. Januario mense a se reperta, decem lineas longa, crassitie non eadem, membrana capitis vix notabili.

Specimen quoque accepi femineum, crassiusculum, quindecim lineas longum, quod Natterer in intestinis Psittaci $\mathrm{n}$. 35, in Brasilia re. perit.

41. Ascaris tentaculata R. n. sp. Ante Synops. n. 37.

Asc. Capitis nudi valvulis oblongis obtusis, corpore antice crassiore, cauda maris incurva mucronata, feminae stricta subulata.

Hab. In intestino coeco Didelplidis Cayopollin Olfers Decembri mense; in illo D. Oouaiquiqua dictae (forsan ejusdem speciei ?) NAT. terer in Brasilia repererunt.

Specimina utplurimum tres, rarius sex ad octo lineas longa, albida,

Caput nudum, 'trivalve, valvulis ablongis, obtusis, discretis, distin̋ctissimis. Corpus rectum, antice minus decrescens. Ciauda maris incurva crassiuscula, apice tenui mucronato, ante quem spicula duo a forma solita valde recedentia, teretia, longa, inflexa, obtusa tentaculis simillima, quorum alterum saepe latet. Cauda feminae stricta, longe subulata.

Corpus passim intestina effundit. Oesophagus rectù; ventriculus mortariformis, dein intestinum rotundatum, sensim decrescens, Ova ma. tura non vidi. 


\section{$-659$}

42. Ascaris forcipata R. n. sp. Pane praecedentem.

Asc. Capitis nudi valvulis obtusis, corpore antice crassiore, cauda mucronata, maris inflexa, feminae recta.

Hab. In complurium avium Brasiliensium intestinis NatTerer reperit, quas singulas recensebo.

A. Specimina in intestinis Cuculorum seniculi, naevii et Tiugazu reperta, flavicantia, duas ad quatuor lineas Jonga; alterum masculum fere quinque adaequat.

Caput nudum, valvulis vix dignoscendis. Corpus plerumque, etiam feminae xetrorsum magis attenuatum, passim maribus pars posterior crassior. Cauda maris inflexa, alis rentubus instructa, acuta, apice mucronato, ante quem spicula praelonga foliacea, forcipis laminas referentia, passin media parte in orbiculi specien dilatata; alias aequalia. Cauda.feminae acuta, mu. cronata, recta.

Ad basin spiculorum anteriorem intestina alterius maris prolapsa, loco certe insolito. Ante caudam feminae pariter prolapsus intestini et simul oviductus, illo parum crassioris, ova rotunda majuscula continentis, qualia etiam corpus pellu. cidum exhibet, Septem inter specimina unicum femineum.

B. Specimina in intestinis Bucconis n. 79. inventa, tres quatuorve lineas longa; inter tredecim duo feminea.

Capitis valvulae neque horum bene in conspectum veniunt. Spicula longissima, passim foliacea vel laminam duplicem referentia, saepe- 
Inedio incrassata, haùd tamen orbiculum referentia.

C. Specimina quatuor, quorum unum masculum, in intestinis Caprimulgi' Urutau reperta, tres quatuorve lineas longa.

Horum unum caput trivalve exhibuit, valvulis obtusis, Corpus ceterum, spicula longa foliacea, ovaria prolapsa, ova, omnia eadem. Caudae masculce apex reflexus est, quor etiam in altero specimine in Cuculo reperto observavi et accidentale putarir.

D. Am. Bremser specimina, quae inspicerem, quoque mecum communicavit masculum et femineum in intestinis Caprimulgi n. 94. reperta, sex et septern lineas longa, ergo majora, ceterumi eadem.

E. Eadem quoque Nematoidea in intestinis Caprimulgi Bacaurau dicti a NATterero detecta esse, litteris miandạvit.

Obs. Cuculi et Bucconis Ascaridem eandem csse, Tactus vidi, cum ipsam vero in CaprimulEis ctiam'obviam noverim, hoc primum anomalum visum est " sed avibus dictis : victus idem, et Cuculi et Caprimulgi, ergo etiam hujus et Bucconis' similitudinem quandam esse,' negari nequit. Si specie's tamen minus distincta foret, error forsan obtinerel, sed Ascaris, quam descripsi, forcipata áb omnibus reíiquis tantopere recedit, ut fere de genere dubitarem, et valvularum speciem semel tantum visam non magni haberem, nísi genitalia mascula'specici praecedentis, certissime Ascaridis, analogiam offerrent, et habitus hujus esset generis, - 


\section{$-661-$}

43. Ascaris uncinata R. n. sp. Pone Synops. n. 42 a

Asc. capitis nudi valvulis longiusculis obtusis, corpore antrorsum crassiore, caudae mascu. lae inflexae apice attenuato, feminecie rectae acutiusculo,

Iab. In intestinis Caviae Apereac et coeco $C_{a-}$ viae Pacae NAtrerer in Brasilia detexit.

Specimen masculum septem lineas longum in Aperea; alterumque sex, et femineum octo lineas longum, crassiora in Paca reperta, am. Bremser pro una eademque specie rectissime de. clarata transmisit.

Caput discretum, valvulis longiusculis obtusis. Corpus in masculis distincte posteriora versus minus attenuatum, in femineo autem (non ovigera tamen) utrinque fere aequaliter decrescens. Cauda maris fortiter inflexa, apice longiusculo attenuato acutiusculo, unciformi; ipsa in illius flexura (speciminis in Aperea reperti) tubercula duo obtusiuscula eminent; in illo Pacae autem complura et minora tubercula sub oculos cadunt. Spicula non exscrta. Cauda feminae acutiuscula, recta.

Obs. Species valvulis et cauda maris singulari modo attenuata, neque tarnen mucronata, fa. cile distinguenda.

44. Ascaris alienata R. n. sp. Pone Synops. n. 50 .

Asc. Capitis valvulis obtusis, corpore retrorsum crassiore, caudac masculae inflexae, femineae rectiusculae apicc brevi obtusiusculo. Ilab. In intestinis Nasuae rufae junioris Ozreks Januario mense in Brasilia detexit. 
Specimen masculum accepi sedecim, femineum (si duo fragmenta unius sunt partes) viginti duas lineas longum.

Caput trivalve, valvulis reliquo verme pellucidioribus, obtusis, distinctissimis. Corpus antrorsum magis attenuatum. Canda mascula subtus concava, inflexa, apice brevissimo, tenui, obtusiusculo, diaphano, ante quem spiculum breve, quod facile simplex habeas, sed attentius examinatum, duplex laminis apicem versus tantillum distantibus. Cauda feminae rectiuscula, obtusissima, apice brevissimo obtusiusculo diaphano, ante quem rima transversa, Omnium speciminum prolapsus intestinorum observatur.

Ala tenuissima capitis masculi primum adesse visa, sed examine continuato species illa subIata est. Reliquis vero ferarum Ascaridibus: alatis, hanc alis destitutam merito alienatam. dixị.

\section{Ascaris spiculigera $\mathfrak{R}$.}

Synops. p. 4S. 11. 5r. Mrantiss. p. 290.

Specimina plurima sex lineas ad duos poìEices longa in oesophago et ventriculo Pelecani (Haliei) brasiliensis Aprili et Majo a se reperta Orfers transmisit, nulla quidem nota ab illis discrepantia, quae ipse in Carbone reperi. Spiculum saepius unum, rarius, utrùmque exsertum est. Ovula rotunda, amnio et chorio approximatis. Specimina tria parva a Natteriero ista in ave seperta non differre vidi.

Magnam etiam harum Ascaridum copiam Natterer in oesophago et ventriculo Pelecani Aquilac in Brasilia offendit, dimidium ad unum 


\section{- 663 -}

eum dimidio pollicem longa, omníbus notis convenientia.

\section{Ascaris cephaloptera R.}

Synops. p. 52, n. 7o. Mantiss. p. 295.

Cel. Doellamger specimina quinque misit, quorum unum in ventriculo, reliqua in intestinis tenuibus Colubri quadrilineati reperta sunt, albissima, unum masculum octodecim, reliqua feminea ad viginti tress lineas. longa.

Capitis membrana, 1. c. dicta in quatuor speciminibus distincta, quinti obscura. Cauda maris crassa incurva apice obtusiusculo brevissimo, ante quem spiculum breve rectum, Illa femina obtusa, fere obtusissima.

\section{Ascaris serpentulus R.}

Synops. p. 53. n, 72, Mantiss, p. 296.

Hanc in Ardea cinerea repertam descripsi. Am. Olfees variis in Ardeis brasilianis offendit, et Natterer in intestinis Ardeae Nycticoracis in Brasilia invenit.

Speciminum, quae OlFers, in ventriculo $\mathrm{Ar}$ deae scapularis Majo reperit, mascula duo sunt, octo ad novem, feminea complura, sex ad octo lineas longa, haec crassiora, cauda recta, illorum inflexa, spiculis duobus. brevissimis ante apicem caudae brevissimum exsertis.

Idem specimen misit masculum decem, et femineum novem lineas longum in Ardeae $11 . \mathrm{II}$. ventriculo Martio mense in Brasilia reperta, crassiuscula, spiculis longis exsertis.

Idem tria feminea in ventriculo Arcleae n. IV. Majo reperta; et complura practurea in $A r$ déa n. VI. oesophagò et ventriculo reperta, pas- 
sim magna. Femina magna fere tres pollices attingit. Hujus ova, uti supra n. 45 illa Asc. spiculigerae descripsi, in Halieo brașiliensi repertae, rotunda, interstitio inter chorion et amnion parvo, sed illa serpentuli majora sunt. Spicula longiuscula incurva inasculiș compluribus. exserta.

Ascarides tres masculae, sex ad septem, femineae, quindeciu lineas longae a Natterero in Nycticorace repertae, quas huc, quam ad Asc. microcephalain Synops. n. 48. referre mallem, cum alae capitis vestigium adsit; aut Bremserum secutus Asc.microcephalam et serpentulum (illis quiden visis) conjungerem. Spiculum parum ex. sertum.

\section{Ascaris Martis. Pone n. 76.}

Amanuensis in Mustelae Martis intestinis tenuibus Decembri mense Berolini tria reperit specimina mortua, quae recens examinavi, tres quatuorve lineas longa, tenuia, alba, feminea.

Caput truncatum, trivalve; corpus posteriora versus magis attenuatum. Cauda longe subulata. Oesophagus basi ampliatus, ventriculus mortari tomis.

Bremsero forsan Oxyuris foret. Ascaris, quam Gorze in Marte reperit, ad majores pertinere et Afrstaci similis dicitur, ideo huc non facit.

49. Ascaris Colymborum.

Svnops. p. 55. n. 97 .

Specimen in intestinis Colymbi (Podicipe-

dis) n. 196, a NAtterero in Brasilia repertum accepi, quinque lineas longum, femineum, crassiusculum. Valvulae distinctae, Corpus retror 


\section{$-665=$}

sum crassius, Caudad apex brevis acutus, ante quem anus.

$$
\text { B. Acanthocephal a. }
$$

50. Echinorhynchus microcephalus R. n. sp. Pone Synops. n. ?.

Ecl. Proboscide minuta subglobosa, collo nula lo, corpore longissirno, inaequali, antrorsum tenuissimo.

Hab. In intestinis Didelphidis Cayopollin Decembri mense Otrers in Brasilia offendit.

Specimen tripollicare, a spiritu fuscescens, Proboscis subglobosa, exigua, octavam lineae partem tantum adaequans, echinorum reflexorum mediocrium seriebus sex transversis munita, Col* lum nullum. Corpus longissimum, teres, anteriori parte tenuissinum; tum sensim increscens et lineam latitudine excedens, passin constrictum; dein subaequale, apice posteriore ohtuso,

\section{Echinorhynchus Spirula Orfers.}

\section{Synops. p+ 63. n. 2. Mantiss. p. 3 I0.}

a. Quem in Sinia Rosalia repertum 1, $c$. descripsi, dein etiam accepi a Natrerero, eodem loco inventum, nec non ab eadem Simia dejectum, Specimina 6, 9, 12 lineas Ionga. Dejectum (cum aliis tribus, uti Brenserus litteris mandavit) nigrum est, ideoque jam in Simiae corpore nortuum, alias enim verrnes dejecti' nigri non esse solent, sic aliquot ante dies Echinorhynchum a Rana esculenta dejecturn albissinum reperi.

b. Natterer quoque specimen hujus Echinorhynchi bremsero teste in coeco Simice Apel- 


\section{$-666$}

tae offendit, huc faciens, sedecim lineăs longum, album, proboscidis parte exserta echinis fortibus armata.

c. Natrtererum etiam indefessum Echinó. rhynchi a se in intestinis Viverrae Naricae reperti specimina tria transmisisse, Brevserus retulit, quorum minimum unum, maximum tres cum dimidio pollices. adlaequat. Quod cel. vir mecum commnnicavit, septendecim lineas longum, omni-

- tous notis cum Ech. Spirula convenire milhi videtur. Proboscis eadem, exigua, echinis anterioribus fortioribus, reliquis sensim minoribus, sexfariam senis. Collum proboscidis longitudine, ambo simul sumta vix lineam dimidiam longa. Corpus ejusdern omnino formae, minus. annulatum aut incisum, quam specimina in Simiis reperta minora, sed majus aeque laeve est.

Collum Simiae Echinorhynchis minus exsertum, et si discrimen adesset, quod vix conce. derem, proboscide Echinorhynchi Naricae tantillum minore, colloque tantillum longiore ha. beretur.

\section{Echinorhynchus oligacanthuides R:}

\section{Synops. p. 64. n. 7. Mantiss. p. 3I T.}

Quem Olfers intestinis Colubri olivacei sibi dicti adhaerentem, Natrerer in illis Colubri n. 17. (forsan ejusdem) a se in Brasilia repertum transmisit. Specimen a Bremsero mecum communicatum tres lineas longum, nullo modo ab illo l. c. descripto recedit, nisi quod Natterero teste in intestinis oblatum sit.

\section{Echinorhynchus tumidulus $R$.}

$$
\text { Synops, p. 69, n. 25. Mantiss. p. } 320 \text {. }
$$


Speciem in intestinis Crotophagae Ani ab am. Olfers repertan l. c. descripsi et pone n. 13. Synopseos melius enumerari retuii.

Eandem postmodum a Natterero in Cro. tophaga dicto loco repertam accepi; vesica cau. dalis et his vero et insequentibus Nattererianis deest, ut Otfers specinnina sua alio tempore invenisse videatur.

Natterer specimina misit in intestinis Falconis Albicollis a se in Brasilia reperta; et $\mathrm{OL}_{\text {L }}$ FERs consimilia, in Falconis ne. In intestinis cras. sis Novembri sibimet obvia.

Unicum etiam specimen septem lineas lon. gum, vesica caudali instructum, cum praecedentibus omnino idem OLFers transmisit in intestinis crassis Cuculi n, I. Novembri mense a se re; pertum.

Obs. Nisi species ista distinctissima ideoque cognitu facillima foret, eanden in avibus aeque diversis occurrere vix admitteres.

\section{Echinorhynchus caudatus ZED.}

Synops. p: 50. n. 29. Mantiss., p. 323 .

Bremserus specimina aliquot transmisit a Natterero in intestinis Falconis magnirostris in Brasilia reperta, sex octove lineas longa, collapsa, constricta, Taenias mentientia.

Nullius proboscis integra, sed duorum tan. tum ejus particula exserta, echinis tenuissimis ar. mata. Corpus lineare, passim anteriora versus paullo crassius, apice postico rotundato, vel extrema parte inflexo.

Proboscidis parte tantum visa, de specie non omnino certus sum. 
55. Echinorhynchus terebra R.n. sp. ante Synops. n. 32.

Ech. Proboscide cylindrica, uncinorum se. riebus circiter sexaginta, collo nullo, corpore longissime.

Hab.Cel. DE Cranrisso ex itinere circa orbem facto redux particulam dedit ventriculi Scombri Jelanidis Echinorhynchis obsitam, fortissime inhaerentibus, ut inucum intestinaium amovendo corpus eorundem facile detralseretur, proboscide relicta.

Specimina octo ad duodecim lineas longa, quartam circiter lineac partem lata; diu spiritu debili conservata colorem plus minus rubrum ob. tulerunt, apice postico passim nigro.

Proboscis longissima, apicem versus plerumque tantillum incrassata, vel clavatae aemula, interdum cylindrica aequalis, aut parte postica constricta, echinorum tenuium longiusculorum seriebus sexaginta ad octoginta transversis. instructa. Collum nullum. Corpus primum cylindricum aequale; deinceps omribus tali modo incisum, ut pro Taeniis facillime imponant: ipse saltem nunquam Echinorhynchum vidi, tantopere crenatum, quem fere- pinnatifinm dicas; apex posticus iterum integerrimus obtusus.

Apex iste niger quandoque vesiculam refert masculam, re tamen attentius examinata, cuticulam ante cundem solutam, ideoque minus obscuram, illum autem ob hanc relictam obscuriorem esse vidi. Hisce enim in specininibus quam in aliis apice nigro destitutis aẹue bene ova offendi, oblonga, utrinque attenuata et acuta. obs. Aliis in Scombris et in affini Cory- 


\section{$-669$}

phaena Hippuride cum Ech, Pristis occurrat, infra n. 58. dicendus, hunc forsan deformatum Echinorhynchi terebrae habitum induere suspica. tus sum, et proboscidem convenire negari nequit. Terebrat tamen corpus aculeis caret, quos epidermidis jactura simul delapsos esse, statuendum fo. ret, sed illa etiam et tamen inermis aliis adest. Ech. Pristim pluries reperi, semper tamen parte posteriore laevi, neque pinatifida; semper porro liberum et in intestinis, neque tantopere affixum et in ventriculo offendi. Seorsim ergo enumerare coactus sum.

56. Echinorhynchus mutabilis R. n. sp. Pone Synops. n. 43.

Ech. Proboscide ad basin aucta, collo co. nico inermi, corporis parte anteriore aculeata, posteriore sulcata.

Hab. Otrers specimina, in intestinis tenuibus Ardeae n. r. Februario, Aprili et Majo, et in illis Ardeae n. 2. Martio a se reperta transmisit; alia vero in intestinis Ardeae Egrettae, Nycticoracis et virescentis, Plataleae Ajajae et Sternae minutae a Natterero in Brasilia reperta Museo Vieunensi debeo.

Specimina, unam cum dimidiam ad quinque lineas longa, proboscide excepta Ech. striatum ex asse referentia, cujus vero illa pars antrorsum, sive apicem versus aucta, basin versus tenuior et aequalis est.

Ech. mutabilis, proboscis basi crassiore utitur et dein (fere subito) antrorsum attenuatur. Collum conicum inerme. Corporis pars anterior om. nibus in speciminibus (etiam in illo Plataleae) aculeata, reliqua tenuior nuda. 


\section{- $670-$}

Inter specimina quae Otfers Mrajo mense in Ardea n. I. reperit, nonnulla mascula occurrunt, quorum vesiea caudalis vel turgida, subglo. bosa et pellucida, insimulque longitudinaliter striata (an vasculosa), vel collapsa et opaca sistiur.

Specincn autem femineum a Natterero in Ardea Nycticorace repertum ova obtulit magna, quae prolem distinctam amnio inclusain exhibere videntur, a chorio satis remotam.

Praeter statum evolutum descriptum, alius saepissime occurrit, quo vel proboscis et collum, vel anterior etian corporis pars plus minus retracta sunt, ut vermiculos videas polymorphos, passim Amphistoma referentes. Interdum pro. boscis emergit, sed collum et corporis pars occul. tantur, ut hujus aculeorum, pauci vel nulli in conspectum veniant. Rarius corpore tenui proboscidis receptaculo prolapso defigurati habentur.

Obs. Num Echinorhynchus striatus, quem tantum evolutum vidi, eodem modo varius re. peritur?

57. Echinorhynchus sphaerocephalus Brems. n. sp. Pone Synops. n. 44.

Ech. juniar: proboscide subglobosa, collo longo corporisque parte posteriore nudis, anteriore aculeata. Senilis: proboscide nulla, bulla rotunda corporisque parte anteriore parum armatis, reliqua et collo nudis.

Hab. In intestinis Haematopodis ostralegi et Lari n. I99. Natterer in Brasilia detexit.

a. Ech. Haematopodis. Plurima specimina duas cum dimidia, unum septem, alterum novem lineas attingunt.

Minorum: Proboscis globosa, vel subglobosa 
(antica parte tum minus convexa), echinorum mediocrium seriebus plurimis dense armata. Collum longum, capite triplo vel quadruplo longius, fere duplo tenuius, mox cylindricum, mox basi tantil. lum auctum, inerme. Corpus in tres partes divisum, quarum prior collo primum aequalis sensim vero aucta, tenuissime aculeata observatur; se. cunda subito valde incrassata iterumque cito decrescerss, uti tertia inearís, brevis, obtusa, inermes sunt,

Unicum tantum specimen aeque parvum. proboscidis loco bullam magnam seriebus longitudinalibus armatam obtulit.

Magnornm: Proboscis nulla,' sed bulla terminalis magna, rotunda, parum armata. Collum tenue filiforme, alterius speciminis antrorsum ineașlem partes uti juniorum divisum, iquarum anterior parum aculeata est.

b. Ech. Lari. Speciminc parva duas lịneàs et quod excurrit longa et proboscidem et corporis partem auteriorem armata sunt. Magnu decem vel undecim lineas lonģa proboscidema nullam, bullam magnam parumper armatam et corpus laeve offerunt.

Obs. I. Quemadmodum plantas spontaneas (praesertim sibiricas, v.c. Potentillas, Astragalos) in hortis sensim majores fieri et pilis denudari; quemadmodum vesicae felleae tunicam internam. humore collecto, extenuatam et dilatatam sensim laevissimam fieri, aut cervicem uteri et vaginam plicis demum destitui videmus, ita etiam probo. scidem Echinorhynchorum quorundam juniorum, forsan magis circumvagantium armatissimam, seniorum sedem capitis inter tunicás intestinorum certam, et forsan nunquam mutandam nactorum, 
sensin echinis denudatam et in bullam, rasis ditissirnam, corpus rrajus factum facilius nutrientem commutari observanus. Bremservs vir oculatissimus, rerumque copia summa adjutus, lloc in Echinorhyncho versicolore (Synops. n. 4q.) juni. ore, et Ech. filicolli (Synops. n. 35.) seniore, ideoque Ech. polymorphi nomine ab Eodem conjunctis, primus observavit, sed me adversarium invenit, Sanctis certe nunquam adnumerandum, cum mihimet nondum probata, nullius, neque amici, auctoritate commotus concedam, sed simul ac milhi probara sint, lubentissime mea faciam. Ita nunc factum est, ut Echinorhynchos juniores cum senioribus, armatos illos sensim in denudatos transientes, Lari eidem intestini parti promi. scue inhaerere viderim, quod antea Ech. filicollem et versicolorem observando mihi nunquam contigit.

Obs. 2. Echinorhynchos quosdam piscinos (Ech. tereticollem et nodulosum, n. 36. et 37.) tam proboscide quam bulla instructos esse, praecedentibus spectatitis utique parodoxum videtur. Echinorhynchus porrigens Ealacnae (n, 34), cujus etiam specinina tenella proboscidem mihi non obtulerunt, pariter examine ulteriore di. gnus est.

\section{Echinorhynchus Pristis $R$.}

Synops. p. 75. n. 47. Mantiss. p. 333.

Quem in Esocis Belones et Scombrorum intestinis a me repertum descripsi, Brearser quoque mecum communicavit, in intestinis Coryphaenae Hippuridis a NitTerero lectum.

Specimen fere septem lineas longum, qúa. rum sesquilineam proboscis sibi vindicat; haec 
antrorsum paululum jucta et rotundata, ceterum eadem est ac reliquorum. Corporis aculei fortes, diaphani, triangulares cosia media dignoscenda, musci folia quasi referentes.

Num Echinorhynchus Terebra supra n. 50. dictus, in Scombri Pelamidis ventriculo repertus nostrum maceratione defiguratum sistit?

59. Echinorhynchus Orioli cristati. Pone Synops. n. 62.

In intestinis Orioli cristati Nattzras in Brasilia reperit.

Specimina quae vidi, sesquilineam longa, sed pollicare quoque Museo tsse Birensers litteris mandavit; alha, proboscide corporisque parte anteriore retractis, margine anitriole corpores ampliato, reliquo apicem posticum versus tenuiore, obtusiusculo, Ova exigua, elliptica.

6o. Echinorhynchus Emberizae. Pone Syna ops. n. 63.

Natterer in intestinis Emberizae Brasilianae (seu Ticutica) reperit. Specimen quatuor cum dimidia lineas longum vidi, cujus autem uti reliquorum duorum a $\mathrm{cl}$, viro lectorum proboscis retracta est.

61. Echinorhynchus Tanagrae. Ante Syn. ops. n. 64 .

Specimen in intestinis Tanagrae n. 3. OrExRs in Brasilia reperit et transmisit, quindecim lineas longun, fere tres quartas lineae partes la. tum. Proboscis pluriman partem retracta, ut apicis echinis exiguis muniti tantillum promineat,

Uu 
Collum breve, latum, conicum. Corpus cylindricum antrorsum et versus caudam obtusam cras. situs.

$$
\text { C. Trematoda. }
$$

62. Amphistoma unciforme R. n. sp. Pone Synops. n. 19.

Specimen lineam excedens accepi, a NaTTERERO in intestinis Orioli cristati in Brasilia repertum et pro Amphistomate transmissum.

Teres, capite continuo, parte infra idem contractá, poro antico orbiculari. Corpore a poro sensim auctum, dein iterum decrescens, in caudam incurvam obtusiusculam, cujus porum ipse reperire non possum, Natrererus tamen certe vidit, cum speciem huic generi tribuat. Dorsum convexius, abdomen planiusculum.

\section{Amphistoma Tanagrae, Pone praece- dentem.}

Amphistomata in Tanagrae Tatoa bursa Farrrcro dicta se reperisse, nobisque in lagenula numero II. notata transmisisse Olfers litteris mandavit, ubi ipse non reperi; forsan autem in lagenula $n_{*}$ I. notata adfuerunt, quam et lagenulam NAtre. rertanam, Ascarides et Echinorhynchos Falconis albicollis continentem, neque inscriptione sua Amphistomata promittentem, simul examinando, ex inopinato Amphistomata mihi sub oculos ca. dunt, quae licet ob dicta sedis incertae, attamen non praetereunda sunt, ne bursam $F_{\text {Abrici }}$ Trematodibus obnoxiam esse (confer Distoma ovatum Synops. p. 93. n, s.) de avibus Europaeis solis dicatur. 
Specimina dimidiam ad duas tertias lineae partes longa, alba, medio flavo irrorasa. Corporis pars anteriur dorso excavato, ahdomine convexo, apice. autico tenui, cujus porum non vidi; posterior teres, poro maguo hiante.

64. Amphistoma Sylviae. Ante Synops. n. 20.

Natterer in intestinis Sylviae cyaneae in Brasilia reperit.

Specimina duo lineam dimiliam superantia, tenuia, augusta, depresa, ope ctricturae mediae in duas partes subaequales discreta. Ad larera et versus alterum apicem (certe ídeo cauda. lem) ovila obscura ajgregata, reliquum corpus griseum.

Poros distincte non vidi, Bremservs Dístomatibus sed aeque dubius addit, ac ipse huc re fero; num forsan tenellum et ad Distoma defle. ctens infra n. 68. aicendum pertinet?

\section{Distoma tubulatum R. n. sp. Pone Syn- ops. n. 6.}

D. depressum, elliptico-ovatum, poris sema globosis, ventrali majore, prominente.

Hab. Specimina complura in intestino Mus raenae $\mathrm{n}$. X. Ol. Olfers Augusto mense in Brasilia reperit.

Lineam ad sesquilineam Ionga, tertiam lineae partem laticudine superantia, crassiuscula, depressa, antrorsum albida, postice fuscescentia. Pori semiglobosi vel in vivis forsan subglobosi. ventralis major, tubuli modo omnium speciminum ex corpore prominenś. Collum versusspo. $\mathrm{Uu} 2$ 


\section{$-676$}

rum ventralem íncrescens, corpus sensim aucturn, apice postico obtuso.

Obs. Speciei huic nıultae affines sunt, v. c. Distoma carnosum Synops, n. $\mathbf{b}, \mathbf{D}$, divergens, $n$. 24. D. pallens $n$, 89 , etc, propria tamen haec videtur.

66. Distoma canaliculatum R. n. sp. Pone Synops. n. 28.

D. elongatum, dorso convexo, ventre concavo, poris orbicularibus non profundis, ventrali majore.

Flab. In intestinis Siernae n. 102, NATTERIR in Brasilia reperit.

Specimina duo mecum communicata, quorum alterum quinque, alterım fere septem lineas longum, tertiam circiter lineae partem lata, couvoluta.

Pori orbiculares, minime profundi, approximati; ventralis antico multo major. Collum breve, dorso convexum ventrali latere parum exca. vatum. Corpus longissimum, canaliculatum, dorso convexo, ventre valde concavo, marginibus lateralibus involutic, apice postico acutiusculo.

Vas dorsi spirale brunneum, corpus cinereum, collum àbưm.

Species.quam insequens aeque singularis, ad Distomata partim teretia, partim excavata, transitum faciens.

67. Distoma grande R. n. sp. Pone prae. cedentem.

D. elongatum dorso concavo, ventre convexo, collo rotundato, poro antico orbiculari exiguo, ventrali maximo transverso. 


\section{- 677}

Hab. Speciem distinctissimam et bellem Nat. TERER in intestinis Platalea Ajajae in Braa silia itexic。

Specimina duo, quae Bremserus misit, pola licem longa, sesquilinearn ad duas lineas rata, crassa, coloris cainei, spiritu forsan mutati,

Pornes anticus terminalis, orbicularis, exiguus, parum excavatus, vix sextam lineae partem emetiens; ventralis approximatus, maximus, trans. versim ellipticus, diametro longitudinali duas hineae tertias partes, transverşa lineam aequante, mangine, anteriore rectiusculo, posteriore rotundato, profundus, quasi ductus ex eo substantiam partis corporis posterioris peteret. Cirrus acutus, brevissimus, octavan lineae partem longus, a pori ventralis margine anteriore, antrorsum direetus, collo appressus. Apex anticus acutus, a quo collum rotundatum ampliatųm, dimidiam li. neam longum, ad porum ventralem descendens. Corpus ad caudam obtusain usque utrinque rectum decurrit, dorso concavo, in altero specimine minus excavato, abdomine convexo. Alterum specimen rectiusculum, alierum involutuin.

68. Distoma defectens R, n. sp. Pone Synops. n. 29.

D. depressoplanum, sublanceolatum, poris inferis, antico orbiculari, ventrali oblongo inzjore.

Hab. In intestinis Sylviae n. 163. NAtTerer in Brasilia reperit.

Specimen, quod, Museo Viennensi šebeo, spiritu vini nigerrimum factum, sesquilineam lorr- 
gum, tertiam lineae partem latum, sublanceola. tum, depressoplanum

Pori inferi, anticus orbicularis, ventralis ob. longus, sive longitudinaliter ellipticus, major. Collum a poro antico ad ventralem augetur, ubi utrinque crena distinguitur; corpus poteriora ver. sus angustatur, apice postico aculiusculo.

Obs. Distoma hocce ab : avicularuma nostratium Distomaribus eo defiectit, quod his porus anticus (neque yentralis) et: major et oblongus sit. Confer Amphistoma supra n. 64. dictum, huc forsan faciens, sed cujus poros videre non contigit.

69. Distoma pyxidatum Brems. n. sp. Ante Synops. n. 33.

D. depressum, elongafum, angustissimum, poro antico obconico, ventrali subgloboso, illo -. majore.

Hab. In intestinis Crocodilis Scleropis NatTerer in Brasilia detexit.

Specimina complura Museo Viennensi debeo, quinque ad undecin lineas longa, depressa, antice cum collo plana, angustissima.

Porus anticus terminaiis obconicus, a Bremo sero nostro curn pyxide bene scomparatus, ab apertura antica orbiculari hiante posteriora ver. sus sensim tenuior factus; ventralis multo minor, subglobosus, uterque flavus. Collmm planum, breve, ut cum poris lineam tanthm àlaequet; ipsum utrinque supra hasin pori antici adscendit, quo liaec alata appareat. Inter poros macula flava, cirri certe receptaculum. Collum et antica corporis pars alba et diaphana, haec angustissima, vix octavam lineae partem lata, plana, majoribus 


\section{$-679$}

speciminibus quatuor ad quinque lineas longa; tum corpus sensim augetur, ut depressiusculum et quartam lineae partem sensim latum fiat, apice obtusiusculo; pars ista depressa vas, spirale nigrescens, spiris copiosissimis, et posteriora versus orbiculos tres continet brunneos.

Species distinctissima, Colubri Natricis ta. men Distomati n. 32, affinis, aliarumque forsan affinitas major, uti Grocodili et Crotali Pentasto. ma idem esto,

\section{Distoma Linguatuliz.}

Synops. p. 100. n. 38. Mantiss. p. 383.

Quod in Ranae.n. sp, ab. am, Olfers in Brasilia repertum 1. c. descripsi, Natterer quoque in Ranae musicae n. 6. (forsan ejusdem, p. 383. adumbratae) intestinis a se repertum transmisit, neque, quod descriptioni datae adderem, of. fendi.

71. Distoma Monas R. n. sp. Pone Synops. n. 38.

D. Depressum, subovale, poris maximis, antico majore oblongo, ventrali orbiculari

Hab. In intestinis Amphisbachae. n, 30. NATteRER in Brasilia reperit.

Specimina plurima Museo Viennensi debeo tertiam circiter lineae partem longa, sextam lata, ex cinereo flava. Depressa, utrinque obtu. sa, mox ovalia, mox obovata, parte postica plus minus contracta, apice postico saepe retuso, vel obiter emarginato. Pori valde magni, remoti, inferi, margine tumido; anticus orbicularis. Cirrus non exsertus. Ovorum immaturorum racemi late. rales, ova matura medium tonent. 
72. Distoma marginatum R. n. sp. Pone Synops. n. 50 .

D. oblongum, supra convexiusculum, subtus planum, pori antici majoris apertura orbicu. lari, ventralis triquetra.

Hab. In Ardeae n. VI. faucibus specimina palato $\mathrm{t}$ linguae lateribus gregatim adhaerentia Orfers noster Majo mense in Brasilia reperit*

Tres, quatuor et quod excurrit lineas longa, antice tres quartas lineae partes, postice sesquilineam lata; oblonga, utrinque obtusa, parte anteriore (collo et antica corporis parte) angustiore, reliqua latiore; marginibus rotundatis; dorso convexiusculo, latere ventrali plano.

Parns anticus inferus, margine lato plano, postice emarginato, fere reniformis, apertura parva rotunda, non profunda; ventralis remotus, fere orbicularis, crassus, apertura triquetra profundá, majore quam illa prioris, sed ambitus totius pori anteriori major est.

Pane porum ventralem et in media corporis parte punctum impressum, rarius in conspectum veniens, ubi cirrus latet, quem in specimine idcirco a me siccato etiam translucere vidi. Cirrus posticus quidem rarus, sed adest etiam D. heterostomati n. 50. et nostrum reliquis Ardearum Distomatibus inermibus maxime affine invenitur, v. c. D. hianti n. 7, sed praesertim 12, complanato $\mathrm{n}, 29$.

A poro antico posteriora versus vasa duo decurrunt obscura, ova utrinque in corpore disposita sunt, sed ob speciminum crassitiem-interna minus bene dignoscuntur. 
73. Distoma repandum R. n. sp. Pone Synops. n. 51 ,

D. depressum, oblongum, postice repandum, pori antici majoris apertura longitudinali, ven. tralis orbiculari.

Hab. NAtterer in intestinis Ranlae $\mathrm{n}$, $\mathrm{r}$. in Brasilia reperit.

Specimina duas, vel duas cum dimidia lineas loriga, depressa, formae maxime variabilis, mox oblonga, marginibus lateralibus rectiusculis, utrinque obtusiuscula, mox parte anteriore temuiore, posteriore multo latiore, saepe repanda, vel vage sinuata.

Pori remoti, anticus major, apertura oblonga, ventralis orbiculari. Cirri exigua species poro sinistrorsum admota translucet. Color albus, vase redio rel flavo, vel fuscescente; lateralibus plerumque flavis.

Obs. Species ista D. mentulato n. $5 \mathrm{I}$, in intestinis Colubri Natricis et Lacertarum obvio, fere nimis affinis est, ob magnitudinem tamen majoren, cirrum uti videtur minorem, et partem posteriorem repandan pro tenpore stjunxi.

74. Distoma cochleariforme R. n. sp. Pone Synops n. 52 .

D. depressum, lineare, collo excavito, poris orbicularibus, anteriore majore.

Hab. In intestinis Pelecani Aquilae, Sternae mimutae, St.? cantiacae et Steruae n, 102. Nat. TERER in Brasilia reperit.

a. SE, ecinen in $P$. Aquila repertun ferc quatuor lineas longunt.

Pori orbiculares, anticus major, apertura 


\section{- $682-$}

rransversa vișa, Collum dorso convexum, latere inferiori concavum, ovale vel ovatum, corpore Tatius, ut illud duas lineae tertias partes, hoc dimidiam latum sit, dum hoc tres, illud unam lon. gum observatur. In basi colli porus ventralis medius, sed lente tantum simplici, verme luci opposito, dignoscendus, microșcopio subjectus latet. Corpus lineare, depressiusculum, apice po. stico obtusiusculo, ductu spirali, aliisque vasis passim pellucentibus.

b. Specinina in intestinis Sternarum reperta; omnino eadem videntur, sed minora, unam cum dimidia, ad duas cum dimidia lineas longa.

Porus anticus major, ventralis in basi colli situs, uterque orbicularis. Collum mox brevius, mox longius, nunquam corporis longitudine, interdum isto fere duplo brevius, sed latius, exca. vatum. Corpus lineare, depressum, passim obiter excavatum, orbiculis aliquot sive speculis insigne, unde corpus albuin maculis aliquot brunneis notatur.

Obs. I. Species Distomati planicolli, in $\mathrm{Pe}$ lecano Sula reperto, infra n. 82, dicendo utique affine, sed hoc armatum est et collo longissimo utitur.

Obs, 2. Distomata collo excavato et corpore tereti instructa haud rara sunt, rariora vero, quae collo concavo et corpore depresso, uti hoc, vel quae collo et corpore concavis notantur, ut $\mathbf{D}$. canaliculatum supra n. 60. dictum.

\section{Distoma clavatum $\mathrm{R}$.}

Synops. p. ro6. n. 65. Mantiss, p. 394.

Specimina duo a cl. Porr, Med. Doct. in Scombri Thyni 1817 prope aequatorem capti ven 


\section{- $683-$}

triculo reperta Brersenus misit, novem alterum, alterum dccem lintas longa, spiritu forsan oim avolato siccata, valde dura saltem, praeterea autem a descriptione p. 394. data non recedentia.

76. Distoma incomtum R. n. sp. Pone
Synops. n. 65 .

D. teres, collo longissimo rugoso, corpore co. nico breviore, poris inferis, ventrali maximo. Hab. In intestinis Chaetodontis n. 54. Olfers Decembri mense in Brasilia reperit.

Specimina quinque duas cum dimidia ad quatuor lineas longa, ultra lineạn crassa, ad pos um medium fere sesquilinean lata, spiritu fu. sca reddita.

Porus anticus inferus transversus, rimam medio contractam sistens; ventralis remotissimus, maximus, prominens, hemisphaericus. Collum (pars inter poros) teres aut depressiusculum, ret1 orsum increscens, transversim striatum, longis. simum, ultra dimidiam totius longitudinis partem sibi vindicans; corpus prope porum ventralem crassissimum, pone hunc ad apicem obtusum us. que attenuatum, sive conicum.

\section{Dis̄toma furcatum Brems.}

\section{Synops. 1. 107. n. 72. Mantiss. p. 396. \\ Specimina duo, a Nattrizero in Coryphae.} nae Hippuridis intestinis reperta Bremserus misit, -quatuor cum dinidia ad quinque lineas longa, valde diaphana, ut vasa majura dorsalia serpentina fusca, et minora versus receptaculım cirri (in sinu inter collum et pedunculum pori ventra. lis) perbene viderentur. 


\section{- $684-$}

Ceterum cum descriptione 1. c. data conve. niunt. .

78. Distoma tornatum R. n. sp. Pone Synops. 1. 88 .

D. teres, corpore antrorsum attenuato, caudà longissima gracilescente, poris subglobosis, ventrali inajore.

7F6b Olfers maximam copiam in ventriculo Coryphatnae Equiselis Decembri mense reperit; in illo Coryph. Hippuridis Natterer invenit.

A. specimina Olfersiana plerumque quatuor, ғаго quinque vel sex, rarissime septem lineas Jonga, utplurimum pellucida, alba vase maculisque luteis, ideoque obiter inspecta flavicantia vi. sa, abdomine passim fusco-irrnrato.

Corpur teres, antrorsum tenuius, breve, plerumque discretum a cauda tenuiore, passim con. stricta (vel contracta fere moniliformi), corpore duplo triplove longiore, obtusiuscula. Interdum 1:on discretum, sed-inaequale et tenuius factum ad apicem continuatur. Pori subglobosi, anticus inlerus minor, ventralis saepe duplo major, pas. sim corpore divaricalo protrusus. Interdum po. rus anterior inflexus et absconditus est; semel cirri recti et brevis vestigium videre mihi visus suin.

Aliquoties unam alieramve corporis excre. scentiam observavi, qualem $\mathrm{p} 600$. \$i 5 . retuli.

13. Specimina Nattereriana utplurimum minora, passim minima, alia tamen aєque inagna ac descripta. Pauca pellucida, ut citius forsan ६piritui immissa sint. Quaedam divaricata, ventrali poro valde protruso. Interdum Distomate 


\section{$-685$}

brevissimo reddito, pars postica obtusa, brevis, et crassissina facta est.

$O b$. I. Summa horum et Distomatis excisi n. 95. in Scombris minoribus reperiundi similitudo, cujus autem porus anticus terminalis, basi excisus et ventrali major observatur, ut conjungi nequeant.

O3s. 2. Distoma Coryphaenae Synops. n. 153. inter duhia relatum deleri potrst; Fasciola enim fusca Boscir ad D. clavatum atpra 1s. 75. enumeratum, $F$. Coryphoenae et condata autem huc facere videntur, nisi illa etiam hujus varietatem sistit.

\section{Distoma lineare $\mathrm{R}$.}

Synops. p. 113. n. 10:. Mantiss. p. $4 \mathrm{r} 4$.

In Diario Halensi Oeconomico (Der Landund Hauswirth. Halle is h, n. 3 Jan. 2. p. 23.) de morte anserum srmo ést, Julio ad Octobrem usque frequente, Hirudinibus effecta, in naribus imis ad cerehrum usque reperiundis. Hirudines passim triginta ad quinquagin:a uno in capite re. periri, magnitudine seminis milii contracta, majora expansa duos pollices longas esse.

Hirudines fuisse, vix admitteres, et Distoma lineare, uti p. 414. retuli, non solum avibus gallinaceis, sed etiam anatibus infestum facilius accusares.

\section{Distoma echinatum ZED.}

Synóps. p. 115. n. Toti. Mantiss. p. 416.

Quod in Pelecanis, Anatibus et Ardeis variis in Europá repertum novimus, NatTERER quoque in intestinis Ardeae Nycticoracis in 3rasiliz offendit, 
Specimina transmissa duas cum dimidia lineas longa, angusta, tenuia, albida, macula media rufescente, tam capite reniformi, quam echinis, poris etc. cum descriptis conveniunt.

\section{Distoma coronatum R. n. sp. Pone Synops. n. 116.}

D. depresso-planum, collo excavato, capite subreniformi, echinis cincto.

Hab. In intestinis tenuibus Didelphidis? vir. ginianae Natterer in Brasilia detexit.

Specimina aliquot Museo Viennensi debi. ta, quae examinavi, duas ad quaruor lineas longa, majora lineam dimidiam latitudine superantia.

Caput saepe discretum, transversum, fere reniforme, echinis rectis ad latera pori antici inferi, orbicularic; num talibus etiam ante eundem? Collum subovale, dorso convexum, subtus concavum, fine posteriore tuberculum, cirri receptaculum exhibens. Collum corpori quasi appensum, et qua utrumque convenit porus obsicrvatur ventralis antico major, subglobosus, apertura orbiculari, passim valde contracta. Corpus depressoplanum, subtiliter transversim striatum, posteriora versus attenuatum, apice acutiusculo.

Unico specimini, loco coronae capitis, prope porum anteriorem processus teretiusculus clavaeformis appositus est, quem non intelligo.

82. Distoma planicolle $\mathbf{R}$ n. sp. Pone praeced. inserenda.

D. depressum, collo planiusculo lengissimo, poris orbicularibus, antico majore echinis cincto. 


\section{- 687 -}

Hab. In intestinis Pelecani Sulae Näterer in

Brasilia reperit.

Specimina complura, lineam longitudine vix adtingentia, quartam lineae partem lata, oblongo. ovalia,

Pori orbiculares; anticus major, terminalis, - echinorum reflexorum corona cinctus; ventralis remotus (ad duas tertias totius longitudinis partes), multo minor. Collum longissimum, lineare, diaphanum, dorso convexo, latere ventrali obiter excavato, ut fere planum, ejusque basi porus ventralis immissus sit. Corpus breve, collo duplo brevius, depressum, lineare, apice obtuso. Pone porum posticum ovaria translucent.

Obs. Affinitas magna Distomatis cochleariformis supra n. 74. dicti in Pelecano Aquila et Sternis obvii, sed inermis, collo breviori, et poro utroque orbiculari instructi, adeoque facile distin. guendi.

83. Pentastoma proboscideum $R$.

Synops. p. 124. n. 5. Mantiss. p. 434.

Quod in Crotalo Durisio ab ill. Arexandro a B Humbold detectum 1. c. retuli, Natterer quoque in Crocodili scleropis pulmonibus in $\mathrm{Bra}$ silia reperit.

Specimen a Museo Viennensi benevole mecum communicatum tres lineas longitudine superans, crassiore parte duas lineae tertias latum, dorso convexiusculo, ventre planiusculo seu depresśo. Duo alia specimina simul reperta sunt, quorum mensuras ignoro.

Apex anticus latior obtusus, cum parva cutis prominentia, quae nullam proboscidis fert similitudinepp, Oscula antica infera quinque Iuna. 


\section{$-688$}

tin: posita, quorum bina utrinque lateralia hamu. lum parrum fuscum emittunt, quintum intermediun quam latcralium duo anteriora paululum magis retrocedens, nullum exserens hamulum, sed papilla alba clausum apparet, cute forsan pori interna pro. minula. Corpus plicis transversis laxis rugosum, parte posteriore, lineam longa, angustiore, ad latera quam reliquum corpus magis crenata. Apex posticus obtusus foraminulum continere videtur.

Obs. I. Crocodilum et Crotalum idem Pentastoma pulmonibus fovere non mirum, cum Equus, Asinus, Canis et Lupus idem Pentastoma tzenioides sinubus frontalibus contineant; forsanque Caprae, Leporis et Cobayae Pentastomata a me dubie seorsim enumerata pariter unam constituant speciem.

Obs. 2. Dubia quae p. 535. circa descriptionem ab ill. Alexanuro ab Humbolot datame labui, vermis examine confirmata sunt.

$$
\text { D. Cesto } i d e a_{\text {. }}
$$

\section{Gymncrhynchus reptans $\boldsymbol{R}$.}

\section{Synops. p. 129, n. I. Mantiss, p. 444.}

G. Cuvier, Vir illustris, cuin litieris nuper (Mariii 1819. d. XXIII.) datis pro sua in me benevolentia Gymnorlynchi inter Bramae Raji cárnes obvii, Bothriocephali ventricosi sibi dicti (olim Scolecis gigantei) figuram misit, sed corpore non articulato a Bothriocephalis mihi quidem áieni visi, qua de re Mantissa 1. c. conferatur.

85. Tetrarhynchus discophorus $R$. Sjnops. p. 130. n. 4. Mantiss. p. 450. Specimina Coryphenae Hippuridis hepati inherentia Natrexk reperit. 


\section{- 689}

Quod accepi, fere septem lineas Iongum est. Caput cum proboscidibus breviluus, fortibus, ro. tundatis, echinis duris obsitis, fere duas liricas longum. Bothrium latoellipticum sive suborbiculare lineam longitudine superat. Corpus attenuatum, apex posticus integer non eat.

Obs. Brfisservs speciem insequentem eodem in pisce obviam pro hujus prole haber videtur, sed bothriorum et proboscidum ratio diversas esse probant.

\section{Tetrarhynchus macrobothrius $\mathbf{R}$.}

Synops, p. I3I. n. 7. Mantiss, p. 453.

Inter Coryphaenae Hippuridis tunicas ven. triculi Natterer reperit.

Spècimina quáe. Bremserus nisit, partim qualia Tab. II. Fig. II-I3. delineata dedi, et 1 . c. descripsi', partim duplo minora, figura varia, corpore ovato, vel ovali, nunquam autem bothriis exstantibus (quales in T. megathothrio Sepiae officinalis occurrunt), sed appressis et elon. gatis.

Obs. Tetrarhynchorum istorum forma tantopere aberrante, T. macrobothrii specimina iiscum comparavi, et Teträrhynchum, quem in Scombro Sarda reperi et ad $T$, megabothrium retuli, huc amandarem.

87. Taenia diminuta R. n. sp. Pone Synops. n. 9 .

T. capite obconico, collo longo, tenui; articulis anticis bevissimis, reliquis subcuneatis ob. tusis.

IIab. In intestinis Muris Ratti gravidi Olfers in Brasilia reperit. 


\section{- 690 -}

Specimina sex ad novem pollices et quod excurrit longa, nam fragmentis computatis forsan longiora, Caput obconicum, osculis anticis ma. jusculis, exacte T. omphalodis, quam Mantiss, p. 49r. descripsi, sed multo minus, unde nomen triviale desumsi. Collum longum tenue. Articuli priores rugaeformes, sensió inajores, semper tamen longitudinis ratione habita breves, angulis obtusis; posteriores passim irregulares, sed bre. ves. Ova subglobosa, mediocria.

Obs. 'Omphalodem 'dixerim, nisi caput multo minus, ova majora forent; haec forsan in $\mathrm{Om}$. phalode crescere posse contenderes, sed caput non augebitur.

88. Taenia cruciata R. n. sp. Pone Synops. n. 10.

T. capite linea cruciata diviso, collo nullo, ar. ticulis anterioribus brevibus, reliquis monililiformibus.

Hab. In intestinis Pici lineati Natterer in Bra. silia reperit.

Specimina majora novem ad quindecim, minora et teneilla unam ad sex lineas longa.

Caprit ab antica parte spectatum linea cruciata divisum, oscula magna angularia, nullum vero rostellum obtulit, A latere spectatum caput interdum 'transversum ret irregulare, plerumque latum breve et emarginatum 'exhibitum est. 'ColZum nullum, minorum tamen articuli anteriores angustissimi colli speciem reddunt falsam. Articuli priores brevissimi saisque longa serie simul summopere contracti, quo veruis valde crassus fit. Insequentes varii, magis magisque evoluti laxi, plus minus moniliformes. Articulorum pars 


\section{$-691$}

antica tenuior, media magis plana, utrinque eminens crassior, margine postico tumido. Articuli majores passim altero margine crenain exlibent. Alterius specinninis virginei articuli omnes breves, ultimus autem longior et apice perforatus,

Ova in nullo yidi specimine.

89. "Taenia Jonqiceps R. $n$ sp. Ante Synops. n. 11. Tab. III. Fig. 21.

T. capitis elongati osculis anticis, collo nullo, articulis brevissimis, inaequentibus cuneatis longloribus, reliquic latissimis.

Hab. In intestinis Orioli cristati. NatTerer in

Brasilia reperit.

Specimina aliquot Museo Tiennenci debeo octo ad octodecim lineas longa, quorum unum capite instructum test.

Caput ovali oblongum, antice complanatum, osculis angularibus adeoque anticis, licet caput elongatum sit. Collum nullum. Articuli antici brevissimi; insequentes sensim parum longiores, antrorsum angustati, crassiusculi, margine posticoplus minus tuniido; sensim láti fiunt, ad sesquilintam usque, ceterum breves, angulis posticis appressis, parum exstantibus, marginibus lateralibus rotundalis,

Ova qualia hactenus desideraveram, quorum scilicet tunica media (neque externa) allantoidis figuram referret, neque tamen totum Amnion cingeret, sed, partem liberam relinqueret. Ova ceterum non ad maxiura referenda, majuscula ta. men sunt. Conf. Tab, Lr, Fig. 2 r. et Mantiss, p. 592 , S. 5 .

\section{$\mathrm{X} \times 2$}


90. Taenia racemosa $R, n$. sp. Pone Synops. n. 52.

T. capite obconico; collo brevì, angustissimó, articulis planis, elongatis, foraminibus thi cyi nalibus alternis prominulis.

Hab. In intestinis Colubri n. 20. specimen sex. pollicare et fragmenta hujus Taeniae Natromek in Brasilia reperit.

Caput antrorsum dilatatum, sive obconicurn, osculis orbicularibus, aut hemisphaericis, narit satis profunda videntur, anticis. Collum breve, angustissimum. Articuli, quos vidi, plani, tenues, elongati, lineam ad sesquilineam longi, tertio lineae parte iatiores, foraminibus marginalibus al. ternis, prominentibus.

Ovaria singulorum articulorum lineam me. diam fere totam sibi vindicant, ad cujus latera utrinque maculae exiguae, opacae ita digestae sunt, ut ovaria. racemosa appareant.

Obs. Caput Taeniae omplaalodis Synops. n. 9. articuli vero $T$ tuberculatae 31.25 . ut fragmenta, quae vidi, Taeniam illis intermediam reddant.

\section{Taenia cyathiformis Froelicí.}

- Synops. p. 152, n. 32. Mantiss. p. 502 .

A. Specimina, quae Natterer in intestinis Hirundinis n. 95. in Brasilia reperit, iconem Taeniae cyathiformis a Froecrcmro (Naturf. St. 25. Tab. III.) exhibitam optime reddentia, unum ad tres pollices longa, candidissima.

Caput subrotundum, vel subrhombeum, rostello obtuso, inermi. Collum varium, plerumque brevissimum. Articuli anteriores mox breviori, mox longioni serie breves, sensin magis exstan- 


\section{- 693 -}

tes, tum cyathiformes margine postico undique liberc exstante et insequentis articuli partem antican cingente, qui illi quasi immissus est.

Unicum specimén, neque magnum, èo deflectit, quod articuli longiores mediu fortiter consiricti sint. Quaedam specimina valde crassa, sed nullius ova ex!libita sunt.

B. Specimina, quate NATterer in intestinis Mirmainis rupestris in Hispaniạ reperit, singularia. neque tamen hujus generis unica. Valde contracia scilicet et triangularia, ut specimen tres cun dimidia lineas longum, antice acutissimum, postice sesquilineam latum sit. Caput non exser. tum. Articuli omnes brevissimi.

92. Taenia campanulata F. n. sp. Pone Synops, n. $3^{2}$.

T. capite subrhombea, rostello obtuso, collo brevi, articulis anticis brevissimis, reliquiș campanulatis.

FIab. In intestinis Muscicapae n. I28. et Muscicapae audacis NatTERr.R in B̦rasilia reperit. A. Specimina in RTuscicapa $\mathrm{n}$. 128. obvił, unum duosve pollices longa, candidissima.

Caput subrhombeum, osculis interdum ad latera maxime prominulis, parte capitis antica mox magis, mox minus protracta et obtusa, bis partem tenuem exiguam exserente, semel foramen anticum exhibere visa, utrum rostello, an hujus basi? Collum breve. Articuli anteriores brevissimi, rugaeformes; insequentes sensim majores, angulis posticis exstantibus, sensimque transeuntes in campanulatos, qui maximam partem efficiunt. Ovaria vel ora nullo in specimi. ne vidi. 


\section{- 694 -}

Candor surnmus uti $\mathbf{T}$. cyathiformi brasilianae supra n. 9r. A. descriptae, cui etiam ipsa proxima est, sed capite diverso utitur et ar'iruli posteriores mere campanulati surit, neque singuli margine postico libero singulos insequentes am. biunt.

B. Specimen in Muscicapa audace repertum capite destituitur. Articuli priores rugaeformes, insequentes breves, sed angulis posticis exstantibus, subcampanulati. - Vere campanulati huic specimini desunt, quod etiam colore utitur fuscescente; nililo tamen minus pro eadem specie habeo.

\section{Taenia vaginata $R$.}

Syrops p. 153. n. 36. Mantiss. p. 503 .

Quam in Europae Charadrio Himanlopode repertam 1. c. descripsi, NatTener in intestinis Ch radrii melanopteri sive Himantopodis melanopteri? (an ab illo diversi), et in intestinis Tringae n. 187. in Brasilia reperit.

A. Himantopodis. Duo specimina adulta et complura tenella; illa ultra duos pollices longa, alterum fere duas lineas latum, alterum has superans. Caput fere maceratum, haud bene dijudicandum; reliqua exacte $T$. vaginatae. Speciminibus tenellis caput bene visibile, rortello exiguo instructum, articulis anticis passim longiusculis, subinfundibuliformibus.

B Tringae. Speciminum, quae vidi, alterum tres quartas pollicis partes, alterum duos pollices et tres lineas longum, utrumque pastice linicam latitudine superans; Museo' sexpollicare servari, BrEMSER, litteris mandavit. ('aput parvun, osculis orbicularibus, rostello exiguo, Collum nullum. 


\section{- 695}

Articuli speciminis majoris magis explicati, minoris breviores, Ceterum uti l. co descripsi,

Hujus vero lemnisci plurimi (non omnes) ab antea visis eo differunt, quod solito longiores infra apicem corpusculo rotundo, aucti, vel ipsi ibidem ampliati sint. Nisi a singulari corporis conditione pendet, speciei novae characterem bo. num offerret.

9.4. Taenia sphaerocephala $R_{0} n_{0}$ sp. Ante Synops. n. 38 .

T. capite rostelloque subglobosis, collo breviusculo, articulis brevissimis, lemniscis brevi. bus acutiusculis.

Hab. In toto, intestino tenui Chrysochlori capensis, (Talpae aureae) spiritu vini servati fragmenta plurima reperi.

Specimina, aliquot lineas ad duos pollices longa, summa latitudine lineam attingentia. $\mathrm{Ca}$ put subglobosum, roskello, mox retracto, mox plus minus exserto, tumque sugloboso. Collum breviusculum, Articuli priores rugaeformes, serie fere pollicem dimidium aequante; tum magis, di. stincti, brevissimi tamen, ạngusti, angulis latẹralị. bus acutis, serie longiore; sensim transeuntes in majores, bacillares, tandem marginibus lateralibus rotundatis, angulis posticis exstantibus, quos, nisi brevitas obstaret, campanulatos dicerem

Lemnisci breves, acutiusculi plerumque ex angulorum parte postica, interdum etiam ex eorundem parte media vel antica egrediuntur. Ova minoris magnitudinis, globosa, margine pellucido, nucleo obscuro, ejusdem formae. 
95. Taenia pyramidata R. n. sp. Pone Synops. n. 38.

T. capite subrhombeo, antrorsum protracto, collo brevi, articulis brevissimis, insequentibus inaequalihus rotundatis, illis lemniscatis. Hab.' In inlestinis Turdi rog. Natterer in Brasilia reperit.

Fragmenta accepi lineam et quod excurrit longa. Caput alteri specimini exserturn, subrhombeun, antrorsim protractuin, angustatum et truncatum, quort itaque pyramidatum vocari possit. Antice pars latet, forsan costellum anmalum, qiod si. foret: prope Syı.ops. n. 93. inserenda esset. Collum breve. Articuli anteriores hrevissini, capillos ransversim positos refuentes, uno latere lemniscati, lemniscis brevibus, recis, obtusis.

Alia frasmenta ariculos breviores quam latos, descriptis tamen multo longiores, inaequales, at semper rotundatos exhibent.

- Lemnisci articulorum brevissimorum et unilaterales, neque insequentium majorum, rarissimi sunt et affinitatem $\mathbf{T}$. scolecinae $n, 93$, arguunt.

96. Taenia unilateralis R. n. sp. Pone Synops. n. 47.

T. capite brevissimo, rostello capitato, collo nullo, articulis cuneatis uno latere lemniscatis.

Hab. In intestinis Ardeae virescentis et Egrcttae Natrerer in Brasilia reperit,

A. Ardeae virescentis. Taeniam fere septem pollices lohgam, parte antica destitutam, hanc seorsim, et Taeniolas, illius forsan prolem, accepi. Fragmentum maximum articulos obtulit cu- 


\section{$-697=$}

neatos, marginum tamen angulis posticis parum exstantibus, tenues, quorum maximi lineam et tres quartas partes lati, quartam vel tertian lineae partem longi. sunt, singuli eodem latere lemni. scum brevern, linearem, truncatum emittentes.

Fragmentum minus: caput brevissimum, osculis orbicularibus anticis, rostello antice subgloboso. Collum nullum. Articuli anteriores an. gustiores, quam in fragmento descripto.

Taeniolarum altera capite pyrannidato, ro. stello longiusculo, articulis cuneatis angustissinis. Alsera capite acuto, cordato, collo brevissimo vel nullo, Reliquae Taeniolae, omnes lineam plus minus longae, capite carent.

Taenia magna lemniscos quidem exsertos, sed ariculos ovis vacuos obtulit, articuli ovigeri ergo jam stcesserunt, vel ova per lemniscos eli. mirsata fuerunt, si hoc admittas.

B. Specimen in Egretta repertum, quod accepi, bipollicare, antice capillare, postice duas lineae tertias parres latum, capite destituitur, articulis ornnibus cuneatis, verme acute serrato.

Obs. Ardeae virescentis et Egrettae Taenias dictas easdam esse nullus dubito, valdopere autem differunt a Taenia aurita in Ardea caerulea reperta, infra n. 98. dicenda, neque minus a Taenia Unguicula (Synops. p. 17.3. n. 125. Mantiss. p. 534) in Ardeis Europaeis obvia.

\section{Taenia crassipora R. n. sp. Pone Syn-} ops. n. 58 .

T. capite tetragono, rostello obtuso, collo plano breviusculo, articulorum brevium antrorsurn angustiorum angulis rotundatis. 


\section{- 698.}

Eab. In intestinis Viverrae Naricae Natterer in Brasilia reperit.

Specimina complura unum cum dimidio ad quatuor cum dimidio pollices longa.

Caput majusculum tetragonum, ab antica ad posticam partem compressum; osculis angularibus magnis. crassis, apertura oblonga; rostello intermedio brevi, obtuso, nullo in specimine vel Aevissimum uncinorum vestigium prodens, Collum planum, pellucidum, vasa linearia longitudi. nalia exhibens, ad latera crispum, et capite et corpore angustius, plerumque valde breve, in specimine majore ad lineam dimidiam extensum. Arkiculi conformes breves, antrorsum angustiores, cito latescentes, passim media constricti, marginibus lateralibus angulisque rotundatis; posteriores lineam dimidiam latitudine superant, Ova desunt, pariter forsan articuli elongati posteriores, affinibus solennes deerunt.

98. Taenia aurita R. n. sp. Pone Synops. n. 78 .

T. capitis subrhombei osculis mediis, rostello obtuso, collo nullo, articulis brevissimis, insequentibus cuneatis, reliquis oblongis, forasninibus marginalibus alternis

Hab. Otfers in intestinis Ardeae n. IV. Martio, Natrerer in illis Ardeae caeruleae, in Brasilia repererunt.

A. Specimina Nattereriana. Alterum novem lineas langum, postice dimidiam latum, crassum, alterum tres lineas longum, forsan fragmentum.

Caput subrhombeum, ab articulis scilicet increscens in partem, quam oscula tenent, ab his decrescens in rostellum validum, cujus uncini ob- 


\section{- 699}

scure exhibentur. Oscula singula protracta et au. ricularıı modo exstantia. Collum nullum. Ar. ticuli primi (ad lineae longitudinern) angustissimi, tum sensim latiores, omnes vero brevissimi, quo corpus crassum annulatum videatur, angulis articulorum obtusissimis.

Fragmentum alterum in apicem tenuem excurrit capite destitutum.

B. Specimina Olfersiana magis completa. Duo bipollicaria capite instructa, tertium tripollicare illo destitutum, praeterea autem complura fragmenta, praesertim partis posterioris.

Caput utique rbombeum, oscula prominentia, non tantopere tamen ac in specimine Nattereriano; uncinorum fortium corona distincta. Collum nullum. Articuli plurimi subcuneati, antice brevissimi, dein passim longiores, ultimi fragmen. tis tantum semipollicaribus vel pollicaribus exhibiti, oblongi, tenues, angulis contractis; foramiui bus marginalibus alternis, singulis parti articuli anticae proximis, neque medium ejus marginem tenentibus. Ovula in posteriori mediaque articulorum parte sparsa, pauca, vasis ante illa vario modo convolutis et ductum ad foramen marginale transmittentibus.

\section{9. 'Taenia sinuosa R.}

Synops. p. 166. n. 86. Mantiss. p. 527.

A. Specimina aliquot, unum ad tres pollices longa, a Natterero in intestinis Anatis 197. in Brasilia reperta Museo Viennensi debeo buc facere visa.

Caput subglobosum, aut etiam subrhombeum. Rostellum subclavatum unico exsertum, api. cis aucti uncinulis obscure tantum a latere visis. 
Collum longiusculum. Artiouli omnes breves acuit.

Specimen alterum valde contactum et car. nosum, ut tenuissima pars a crassa et latá fere subito excipiatur.

Specimen etiam adest aliquot pollices lon. gum, fere tres lineas laum, reliquis triplo, latius, articulis omuibus brevibus, capite retracto, sed parte antica tali modo excisa, ut pro Bothriocephalo nodoso juniore fere haberem, séd nullius articculi nodulus vel ovarii vestigium exhibetur.

B Cel Broscne, Veterinarius meritissimus, olim Viennensis, nunc Drestensis, specimina in intestinis Anseris saginati a se reperta, duas species constitucre sibi visa, dum his edendis occupatus sum, benevole mecum communicavit, quae varios Helminthologorum lapsus egregie illustrant.

Caput rotundum, rostellum cylindricuin lon. gum pellucere sinens, osculis orbicularibus; quod inaeniam fasciatam Synops. 11. 48. aut crenatam ZeDerr reddit. Collum breve, ad $T$. sinuosam. spectans; sed ob partem corporis tenuissimam, quae sequitur, etiam T. lacvem Synops, n. 42. sistit. Articuli anteriores brevissimi, rügaeformes, reliqui majores, angulis acutis rectis, lineis tribus longitudinalibus tranślucentibus. Ita specimina virginea comparata sunt, tr. trilineatum Synops. $\mathrm{n}$. 87. prodentia.

Articuli aliorum speciminum magis evoluti, angulos reflexos, parum exstantes habent, ductus iransversos maximos, unilaterales et ovaria tria sacciformia continentes. - Pars gravida macerata lemniscum ad basin articuli aperiri optime denonstrat. 


\section{$-701$}

Adest etiam particula tenuis, macerari incipiens, cujus articuli anteriores magis elongati sunt; qualibus certe factum est, ut To infundibuliformis Synops. n. 33. anseri adscripta sit.

Obs. Bressstrus certe specimina similia, inermia virlit, qui in Catalogo Musei Viennensis typis impresso Taeniain laєvem, trilineatam, fa. sciatam et gracilem una specie colligat, Taeniam sinuosam autem inter non visas enumeret.

\$oo. I aenia megacantha R. n. sp. Pone Synops. n. 91 .

T. capite subgloboso, rostello cylindrico capitato, collo brevissimo, articulis prioribus brevissimis, reliquis subcampanulatis.

Hab. in intestinis Caprimulgorum.

A. Olfers fragmenta misit in intestinis $\mathrm{Ca}$. primulgi n. I. Februario mense in Brasilia reper. ta, quae computata novem lineas adaequant, articulis posticis circa duas lineae tertias partes Jatis.

Caput subglolosum, osculis fere anticis. Ro. stellum cylindricun, crassiusculum, apice rotundato (subgloboso) alictum, uncinorum reflexorum, fortium et longorum serie simplici arinatum. Cols lum nullum aut brevissimum. Articuli priores breves, sensim majores, ultimi subcampanulati, medio tamen saepe constricti. Omnicim margo posticus tumidus, angulis obtusis exstantibus, substantia crassiuscula, quo vermis depressus fiat. Ova non adsunt,

B. Fragrnenta in intestinis Caprimulgi n. 14. a Natterero in Bracilia lecta, Bremsziass man. davit, aliquot lineas longa, planiuscula, articulos quoad majores plana, 


\section{- 702}

Caput rotundatum, transversum, osculis orbicularibus anticis. Rostellum tenuissinum cylin. dricum, apice globulum referente inermem. ollum brevissimum. Articuli priores brevissimi, in. sequentes maiores, breves tamen, margine laterali rotundato, angulis haud exstantibus; reliqui sıbcampanulati longi, marginibus lateralibus parum convexis, angulis posticis brevissimis acutis.

C. Tria denique fragmenta lineam vix superantia, a Natterero in intestinis Ciaprimulgi Urutau in Brasilia reperta, capite et aliquot articulis constantia.

Alterum rostello longo, ad latera quasi in. ciso, collo hrevi, articulis aliquot obtusis. Secundum rostello brevi subgloboso. Tertium absque capite, articulis rotundatis.

D. Cel. Treutler, Professor Dresdensis Taeniae fragmentum dedit in intestinis Caprimulgi europaei tenuibus a se repertum, fére novem li. neas longum, dimidiam latum, posterioren vermis partem sistens. Articulis scilicet çonstat latioribus quam longis, margine posteriore turnicio, utrinque exstante, laterali utrinque rotundato, sive subcampanulatis.

Obs. Specimina descripta quidem variis notis inter se differunt, non tanti tamen habendis, ut pro diversis speciebus habeantur. Rostellum speciminum B. $t$ t C. minus et inerme, sed tjusdern formae ac in A. ut illa juniora videantur. Sptcimen $D$. capite non viso rerte dijudicari ne. quit, articuli tamen subcampanulati $\in t$ locus conveniunt:

101. Taenia crassula R. n. sp. Pone Synops. n. 92. Tab. 1!!. Fig. 19.

T. capite ovali, rostello obtuso, collo longi- 


\section{$-703-$}

usculo, tenui, articulis prioribus brevissimis, insequentibus auctis, reliquis subinfundibuliformibus, depressis.

Hab. In intestiuis Columbae domesticae ex Africae oris in Brasiliam allatae INovembri mense Olfers reperit.

Specimina aliquot duodecim ad quindecim poliices longa, aliorumque fragmenta.

Caput ovale, osculis orbicularibus majusculis, illius medium tenentibus, Rosiellum mox subglohosum, uncinis retractis, mox obtusum, fere truncatum, uncinis brevibus, saepe obscuris, armatum. Collum satis longum, tenue, strias pellucidas offert longitudinales, "transiens in Articulos angustos, breves, passim inaequales, longa serie tales 'continuatos; sensim dilatatos, semper breves, angulis obtusis. Tum majores sequuntur ejusdem formae, at paulo longiores et latissimi, fere duas linea's lati; tandem angustiores et longiores, subinfundibuliformes, marginibus obtusis, uti praecedentes satis crassi, multo crassiores quam illi Taeniae sphenocephalae in Columba Turture seperiundae.

Articuli ovis repleti sunt paucis, sed maximis, quorum singula lineae octavam pariem longa, duodecimam lata sunt, cum ova Taeniae sphenocephalae pusilla (at singulis articulis innu. mera) octoginta ad centum lineae quadratae area capiantur. Forma illorum varia est, plerumque ovalis, sed etiam ovata, et quandoque licet tarius, triangularis. Anulion quasi uvam refert, ut foetus forsan complicatus transluceat.

Totus articulus rete vasorum tenuium sistit, quibus ova inhaerent, ut passim etiam ova vase longo stipata, nonnunquam ramoso, promantur. 


\section{- 704 -}

Linicum quod sciam exémplum, ubi vasa cèstoidei solvi possunt.

102. Taenia Capito R. n. sp. Ante Synops. 11. 93 .

T. capite globoso, rostello obtuso, collo brevissimo, articulis prioribus brevibus, latis, re. liquis longioribus angustatis.

Hab. In intestinis Plataleae Ajajae NatTerer in Brasilia reperit.

Specimina, quae Mruseo Viennensi debeo, unam ar! tres lineas longa sunt, speciem distinclissimm prodentia.

Caput magnum, depresso globosum, osculis magnis orbicularibus, rostello valido obtuso, uncizlorum fortium corona armato. Totam coronam nunquam vidi, sed magnos tantum hujus illiusve lateris, reliquis obtectis. Collum brevissimum. Articuli priores breves, lati, collo parum latiores, quo: rum quatuordecim numeravi; insequentes his aliquoties longiores, sed angustiores, pauciores (septem, octo) irregulares. Alterius speciminis articulus ultimus unciformis, an defornis?

103. Taenia Strigis. Pone Synops n. 110.

In intestinis Strigis n. I6. Natterer in Brasilia reperit.

Specimen fere bipollicare, antice tenuissi: mum, reliquam partem satis aequale, dimidiarm fere lineam latum, cui caput et articuli anteriores. desunt.

Articuli priores fragmenti sunt angustissimi, tonuisșimi, longiusculi, subinfundibuliformes; insequentes sensim breviores, retrorsum.. latiores, margine postico crasso, hique ninaximam vermis 


\section{$-705$}

partem efficiunt; posteriora, versus iterum elongaıtur et subquadrati fiunt; ultimi pauci moniliformes.

Neque lemniscos, "neque ovaria, neque ova vidi. Species ceterum a Strigium nostratium Taenia candelabraria Synops. n. 6o. diversissina est,

10\%. Taenia Dendrocolaptis, Pone Synops. n. 116.

In intestinis Dendrocolaptis (Picucule) NAT. TERER in Brasilia reperit.

Fragmentum accepi duas tertias lineae par tes longum. Craput subrotundum, osculis latera. libus promineritibus, rostello parvo subgloboso. Collum tenut, capite aliquoties longius. Articuli primi collo haud latiores, obtusi, brevissimi. Re. liqua desunt.

105. Taenia Turdi. Ante Synops. n. 117.

Olfers fragmentum sesquipollicare in intestinis telluibus Turdi n, III. Majo in Brasilia repertum misit.

Praeter caput etiam corporis pars deesse videtur. Omnes articuli latiores, quam longi, marginibus lateralibus rotundatis, angulis posticis obtusiusculis, plus minus exstantibus. An adulta Taenia, cujus proles supra n. 95. descripta sit?

\section{Taenia Motacillae provincialis, Pone} Synops. n. 120.

Fragmenta in intestinis Motacillae provincialis Natterer in Hispania reperit, speciem elegantissimam sistentia.

Quaedam fragmenta articulos proferunt illis Taeniae cyathiformis proximos; alia autem 
longos campanulatos, medio contractos, angulis posticis tumidis rotundatis, longitudine varia; alia denique oblongos marginibus rotundatis, autrorsum contractos, angulis posticis brevissimis, fert rectis.

107. Taenia Motacillae brasil. Pone praecedentem.

Oxfers speciminum aliquot fragmenta pollicaria ad bipollicaria, duorumque tantum capita, in intestinis Motacillae n, I. in Brasilia Martio mense reperta misit.

Caput subrhombeum, osculis orbicularibus anterioribus, rosiello obtusiusculo, vix toto $\mathrm{ex}$ serto, an armato? Collum breve. Articuli priores rugaeformes, tum sensim majores. sed breves, angulis acutiusiulis, lineis lateralibus pellucidis; dein subinfundibuliformes, pratcedentibus longe plures, angulis posticis obtusis exstantibus.

108. Taenia Motacillae cyaneae. Ante Synops. n. 121.

Specimina duo tenella a NatTerero in in. testinis Motacillae cyaneae in Brasilia reperta $\mathrm{Mu}$ seo Viennensi debeo, lineam longa, angustissima, sive lintearia.

Caput majusculum subglobosum, rostello re. tracto. Collum an longissimum? An articuli priores tantopere tenues ut praetervideantur? Ultima saltem speciminum parte umbra quaedam articulos tantum indicare videtur.

109. Taenia Scolopacis. Pone Synops. n. 126

Specimina plurima in Scolopacis n. 188. 


\section{- $707-$}

? Gallinaginis intestinis Natter rit, prolem sistentia lepidissimam, unam duasve lineas longam. -

Quaedam absque articulorum specie, hrevia, angustissima, capite majusculo, subrotundo vel rhombeo, osculis exstantibus, rostello cylindrico, semel vaginato viso. Alia articulis brevissimis, antice rugaeformibus, postice majoribus, angulis exstantibus, capite priorum, aut passim rostello minore. Plurima articulis anticis longis, infundibuliformibus, angustissimis, satis multis; paucis latioribus brevibus; haec omnia capite destituta. Pauca capite et articulis mox insequentibus infundibuliformibus longis simul instructa, sed tum horum pauci; caput vero osculis fere auriculas referentibus, exstantibus, rostello longo, cylindrico, collo brevi.

Nimia prolis istius varietas speciem deter. minare vetat. An tamen ad $\mathbf{T}$. variabilem per. tineret?

110. Taenia Tringae. An Synops. n. 130.?

Specimina capite destituta Natterer in intestinis Tringue Interpretis in Brasilia reperit, quorum duo Museo Viennensi debeo, sex ad octo lineas longa, tertiam circiter lineae partem lata,' speciem propriam arguentia.

Pars anterior submacerata longa non articu. lata, surnmae tenuitatis, án pars Taenize continua, cui articulorum laminae imponuntur? Tum (in specimine minore) articuli sequuntur satis long? serie infundibuliformes, et tandem breves, lati, utrinque obtusi. Altero in speciuine partem il. lam capillarem non articulatam excipiunt articuli paucissimi angusti, hos vero sequuntur articuli 


\section{- 708}

subito lati facti, breves, utrinque obtusiusculi ae-. quales, sive bacillares. Ultimi sunt lemniscati, lemniscis unilateralibus, rectis, longiusculis, acutis, vaginatis.

111. Taenia Charadrii. Pone Synops.n. 130.

Fragmentum Taeniae in intestinis Charadrii, collaris Januario in Brasilia repertum Olfers transmisit, sesquilineare, articulis septendecim constans, quorum quatuordecim minutissimi, singuli antrorsum tenuiores, marginis postici angulis obtusis; tres posteriores majores sunt, angulis oblitteratis, ultimi duo foraminibus marginalibus al. ternis prominulis。

112. Taenia Sternae. An Synops, n. 132.

Specimina nonnulla in Sternae $\mathrm{n}$. 102. inte. stinis a Natterero in Brasilia reperta Bremserus misit, elegantissima.

Alterum specimen posteriorem Taeniae partem sistens, decèm pollicés longum, alterum huic anteriorem continens quatuor pollices aequat, reliqua minora, ut integrum forsan satis magnum sit. Caput deest. Quaedam minorum speciminum articulis brevissimis incipiunt, sensim sed parum elongandis; quos sequuntur angustissimi et longissimi, dein subcuneati, convexi, marginibus lateralibus rotundatis, postico recto crispo exstante, quo parum cohaereant.

Alteri specimiui series articulorum longorum et angustorum lopgissima, capillo multo tepuior. 
113. Taenia Pelecani Aquilae Pone Synops. n. 136.

Fragmenta in intestinis Pelecani Aquilae a Natterero in Brasilia reperta Bremserus mecum communicavit, alterum fere pollicare, alterum fere septem lineas longum, forsan ad idem specimen facientia, tertian lineae partem summa latitudine attingentia.

Articuli brevissimi, obtusissimi, crassi, ma. gnam partem subaequales, parte antica (majoris fragmenti) subito angustissima facta, articulis obtusis, consimilibus, sed longioribus et fere qua. dratis.

\section{Taenia Colubri, Pone Synops. $n, 140$.}

Fragmenta in intestinis Colubri n. 12, a Natrerero in Brasilia reperta Museo Viennensi debeo lineam circiter longa, duodecim ad octode. cim articulis constantia, latioribus quam longis, subcuneatis, angulis plus minus exstantibus, c2. pite destituta.

Utrum vere colubrina, an ex ate quadam deglutita forsan residua?

$$
\text { E. Cystic } a_{*}^{2}
$$

15. Anthocephalus, elongatus, $\mathrm{f}_{\text {i }}$

Synops. p. 177. n. I.'Mantiss. p.!537.

A. : Specimina aliquot inaperta in Scomỏr Thiyrni vesica fellea (potius hepate, aut intes'il- lius tunicas) a cl. Porr reperta, Bzknseleus mecum communicavit, quibus suspensa mane aper. tis Anthocerhalum nostrum offendi.'

I3. Specimen tres cum dirnidio pollices longum, sub tunica intestirì exteña Silurần. 5,2 
NATTEkRo repertum, quod examinarem concessum est, quodque cum Anthocephali elongati specininibus in Orthragorisci hepate obviis conjungerem, proboscidibus armatis, parte infra caput inflata, corpore longo depresso, optime conve. niens, nullaque nota recedens.

C. In ejusdem Siluri abdomine prope rectuin vesica reperta est, quam inapertam vidi, ovalem, eandem omnino, ac qualem supra p. 537 in Sciaena Aquila lectam descripsi, ét in qua vermem translucentem vidi.

D. Ill. Cuvier pro sua in me henevolentia Floricipitis saccati sibi dicti, in hepate Orthrago. risci Molae reperti iconem sua manu paratam misit, quae specimen valde elongatum egregie-reddit, cun in op re saepius laudato Regne Animal

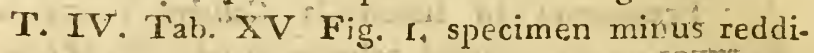
derit, quale Tạb. III. Fig. 12-17. delineari curavi. Specie differre non crediderim.

\section{F. Entozoa dubii vel Ordinis vel Generis.}

116. Felis Cati feri. Pone Synops: n. 7 . - p. 185.

In Museo Viennensi Nematoideum singu. lare vidi, inter Felis Cati feri tunicas ventriculi repertum, quod in Synopsi memoriae lapsu omí. si, ideoque hoc loco referam.

Maxima pars inter tunicas latuit, minor emersit, utraque etiam verme vinculis caute soluto diversa oblata est. Longitudo sex linearum, di. ameter transversus lineae dimidiae.

Caput discretum exiguum, tuberculis duobus mediis seu anticis, quinque, minoribus lateralibus. Pars corporis anterior exserta albida, aculeis re. 
otis minutis undique obsita; pars tunicis implicita mollior, flavescens, rugis transversis laxis, nullibi a:matis instructa. Apex caudae fere ovatus, crassior.

An Liorhynchi species?

117. Falconis albicollis. Pone Synops: n. 19. p. 187.

Nernatoidei specimina tria accépi, a NatteRERO in intestinis Falconis albicollis in Brasilia repecta, tres quatuorve lineas longa, alba, feminea, capite truncato (obscure trivalvi viso), cau* da tenuiore acuta depressa.

118. Strigis. Pone Synops. n. 20. p. 187.

Nematoidei dubii specimina duo transmissa sunt, a Natrerero in intestinis strigis n. 16. in Brasilia repèrta, tres lineas longa, tenuissima; femineá.

Os rotundum videtur. Corpus antrorsum minus, postice maxime attenuatum, vel subulatum, mucrone capillari brevi, Forsan Strongylus aut Spiroptera.

119. Turdi, Pone Synops. n. 21. p. 187.

Nematoidei, forsan Spiropterae, duo specimina accepi a Natterero in intestinis Turdi n. 109. in Brasilia reperta, feminea, aliquot lineas longa, maxime tenuia, capite obtuso, cauda obtusiuscula, depressa et inflexa.

120. Ardeae caeruleae. Pone Syuops. и. 23. p. 188.

Inter Ardeae caeruleae pellem et musculos colli et thoracis Natxerer in Brasilia tres repe- 
rit vermes, valdopere sese elongantes ef contrat. hentes, quorum specimen media utens magnita. 1, dine Museo Viennensi debeo.

Vermis fere novern lineas longus, dimidiam ad duas tertias lineae partes latus, crassiusculus et depressus, albus. Pars antica, qualis Ligulae, apice emarginato, ceterum transversim rugosa, sublanceolata, duas lineas longa, transiens in reliquam subaequalem, magis gelatinosam, depres. sam, postice deliqueșcentem.

Obs. Partem istam posticam cystide terminatam fuisse, vix crederem, eoque minus cum vermes loco dicto nudi reperti videantur. Ejusdem ceterum generis procul dubio sunt, ac vermes Erinacei (Synops. p. 185. ní 8. Mantiss. p. 554), Mygales moschatae (Synops. p. 186. n. 9.), Falconis (Synops. p. 187.n. 19. Mant. p. 556.) et Strigis accipitrinae (Synops. p. [87. n. 20. Mant. p. 556.) enumerati, Ligulae generi proximi.

121. Boae caninae. Pone Synops, n. 29. p. is8.

Boae caninae majoris, spiritu vini servatze intestino hydatides pisum aequantes', multoque - minores inhaerentes reperi, quibtis singula tine, rant Nematoidea, aliçuot lineas ad pollicem longa, forsan Ascarides femineae.

122. Colubri. Pone Synops. n. 31. p. 189.

Nematoideum a Natterero in intestinis Colubri n. 17 . in Brasilia repertum accepi, fuscéscens, quatuordecim lineas longum, capitis ore milî́ quidem orbiculari viso, corpore subacquali, apice caudae brevi papilliformi, divaricato, sub q̨uo anus conspicitur. 
Pro Ascaride feminea transmissa est, sed valvulas capitis non vidi.

123. Ranae. Pone Synops. n. 34. p. 189.

A. Nematoidei, forsan Ascaridis specimina tria in intestinis Ranae n.' 1 . 2 NAtteanro in Brasilia reperta Brevservs transmisit, feminea quinque ad decem lineas longa, antrorsum magis attenuata; capite obscuro, an trivalvi? Cauda depressa, obtusa, papilla exigua acuta terminata.

13. Specimina accepi a Natterero in intestino Rance musicale 13.6 . in Brasilia reperta, feminea, alterum septem, alterum duodecim lineas longa, antrorsum magis attenuata, cauda papillata, capite forsan trivalvi, cujus duas saltem eminen. tias vidi.

\section{Triglae. Pone Synops, n. 73. p. 195.}

In Triglae n. r9. abdomine insestinis et ova. riis adhaerentem (utrum liberum, an vesica inclusum?) Natrerer in Brasilia reperit.

Specimen transmissum album, tredecim lineas longum, valde singulare.

Pars antica, tetragona, depressa, fere duas lineas longa, undique clausa visa, alia excipitur filiformi pariter duas lineas longa; relicyuum cor. pus novem lineas longum, depressum, antice for. titer utrinque incisum, vel crenatum, neqque ta. men articulatum, posteriori et aeque longa parte àequale, apice obrusiusculo.

Parte antica tetraçona cultelii ope aperta, caput sub oculos cccilit tetáabothrium (proboscin dibus retractis) collumque rugnsunn.

Am. Burmsenis pirt Bothriocephalo misit, huic vero gentri receptaculum, quale descripsi, 


\section{- $714-$}

caput et collum abscondens, non conceditur, oinniumque Bothriocephalorum corpus articula. tum est

Anthocephalum potius crediderim, vel Gym. norhynchum, Conf. Anthocephalus macrourus (dubius) in Trigla fasciata a cel, Olfers repertus, supra p. 543. descriptus. 


\section{Index systematicus}

animalium, in quibus Entozoa hactenus reperta sunt, locis qua occurrunt simul indicatis.

Siglorum partes Entozois obnoxias indicantium explicatio.

a. Abdominis cavum.

ad. Adeps generatin.

ap. appendices pyloricae.

art. arteriae.

au. aures.

2x. axillne,

bra. branchiae pisciom.

bro. broncli eorumque ramificationes.

bu. bursa avium FABricro dicta.

c. contextus cellulosus generatim,

Ba, inter carnes.

cav. cavernulae sive cryptae $\checkmark . C_{\text {, }}$ caudales.

caud. cauda.

cer. cerebrum.

co. cor.

col. intestinum colon.

coll. collum.

corp. corpori extus adhaerere.

cox. coxa. 


\section{$-.7616$}

cr. intestina crassa generatim.

du: duodenum.

e. veimem parti siglo addito indicatae extus adhaexere:

f. femur.

fau. fauces.

fell. vesica felles.

fr. sinus frontales.

gen. genitalia externav

gl. glandulae conglobatae sive ly'mphaticasd

lep. hepar.

Hyd, vermem hydatide contineri.

hyp.- lyy pochondria.

i. intestina generatim, parte $\mathrm{ab}$ auctoribus non indicata, vel verpibus in işdera rajis.

ingu. inguina.

li. sub lingua.

mes. mesenterium:

mu. musculi.

na. vesica natatoria.

nas. cavum nasi.

ne. nervi.

nict. sub membrana oculi nictitante:

oc. oculus.

oe. oesophagus.

om. ómentum.

ov. ovaria.

pa. pancreas.

palp. sub palpebris?

pe. pes.

peli. vermem sub pelle occurrere, vel varis locis, ubi ni. liil, vel certa parte, ubi hujus siglun additur.

per. pericardium.

pi. peritoneum.

pirm. pinnae piscium.

pl. pleura.

pu. pulmones.

rect, intestinum rectum.

ren. renes.

sp. splen. 


\section{$-717$}

t. intestina tenuia.

te. testiculus.

tent, tentacula.

th. thoracis cavum.

tra. tracliea.

ir. i. tractus intestinorum.

tub. vermen tuberculo contineri, cujus sedes semper indicatur.

tun. vermem inter partis siglo indicatae tunicas latere.

ty. tynupari cavum.

ur. vesica urinaıia, quae a nomullis immerito amphi

biis denegatır, cum finidum illa contentum acido urico instruatur, idemque calculos gignat urina. rios.

ut. uterus.

ซ. ventriculus,

ve. venae.

vert, inter vertebrarum processus vel prope earundem coro pora occurrere.

ves. Vesica partis siglo indicatae accessoria. vi. viscera generatim.

Si vermis tam partíbus quibusdam liber inest, quam hydatide vel tuberculo continetur, hoc linea siglis interjecta indicatur.

Nomine generico Entozoi tantum exhibito, illud quidem innotuisse, speciem autem nondum confirmatam esse, declaratur.

Quibns etian genus, vel ipse ordo nondum tuto ad. signatur, dubia (Dub.) vocata sunt.

Numerus si duplex additus est, prior paginam, alter synopseos numerum indicat.

Litteris apx numero additis, vermem hoo numero in appendice p. 634 . incipiente prostare indigitavi.

Stella entozoi noxaini anteposita, illud collection zaea sęrvari notavi. 


\section{$-718$}

Paucissinis sigla habitaculum indicantia, utpote ab anctoribus non relata, addi non potuerunt, illud tamen entozois affuibus collatis, facile invenietur.

Animalia entozois obnoxia enumerando, quonam ho: rum systemate potisoimum uterer, diu haesitavi, Linnaeanun tamen, passim emendatum, quo Entozoologiam edendo usus"sum, comparationis gratia etiam nunc adhibere, ubi non de Systemate animalium, sed de conspectu Entozoorum agitur, commodissimum visum est. Fandem ob rationem multorum generum subdivisiones, vel genera secundaria 'suppressi, singulorumque generum species, quas systematice vel serie debital enumerare vires nieas superas. set, ordine alphabetico enumeravi. 


\section{Classis 1. Mammalia.}

\section{Ordo I. Bimanus,}

\section{i. Homo.}

* Filaria medirensis. c. mu. oc. 3. 1. - (bronclialis). gl bro. 7.20.

* Trichocephalus dispar. cr. 16. 1.

- Spiroptera. ves. ur. 27. 23.Strongylus Gigas. ren. 3r. 3 .

* Ascaris lumbricoides. t. 371 .

* - vermicularis. rect. col. ger. 44. 31.

* Distoma hepaticum. fell. 92. 1.

Polystoma pinguicola. tub. or, 125. 3 . (venarum). vel. 126. 5 .

* Bothriocephalus latus. t. 136. 1.

* Taenia Solium. $t$ 162.69.

- Cysticercus cellulosae. hyd, mu. cer. 180, 4. (visceralis) vi, 1९1.9.

Echinococcus. hyd. hep. ? vi. 183, 1. Dub. Diceras rude. i. 184, 1.

\section{Ordo i1. Quadrumana.}

Filaria gracilis. a. 3. 2.

2. Simia Apella.

Echinorhynchus Spirula coec. 51. Apx.

3. Simia Aygula.

* C5sticercus tenuicollis. hyd. pi. Iso. 3 .

- Ascaris elongata. i. 28. A px.

4. Simia Bélzebul.

* Filaria gracilis. a. 3. 2.

5. Simia Capucina.

6. Simia Cephus.

* Cysticercus cellulosae. cer. ma 190. 4.

Ecbinococcus. hyd. pi. Pl. 185. 2.

7. Simia Cynomolgus. 


\section{- $720^{\circ}-$}

\&. Simia Inutrs:-

Echinococcus. liyd, hep. 15j. 2.

9. Simia Maimon.

- Spiroptera alata. tur. v. 27. 22.

* Cysticercus tenuicollis. hyd. mes. 19o. 3.

10. Sinzia Midas.

Filaria gracilis. a. 3. 2.

11. Simia Paniscus. .

Filaria gracilis. a. 3...2.

- Ascaris vermicularis. c1. 4f. 31.

12. Simia Rosalia.

* Physaloptera dilatata. r. Is. Apx.

* Echinórhynchus Spirula. cr. 63. 2. Apx. 51:

13. Simia rubra (Patas).

Trichocephalus palacformis, cuec. 16. 2. Cy-sticercus cellulosac. Iyd. mu. 1SO. 4.

14. Simia Sabaea.

Trichocephalizs palaeformis. cr. 16.2.

* Ascaris distans. cr. 38. 2.

Filaria gracilic. a. 3. 2.

15. Simia Sphinx.

* Trichocephalus palaeformis. cr. 16. 2.

16. Simia Sylkanus.

- Trichocephalus palacformis. coec. 16. 2. Cysticercus cellulosae. hyd. mu. 19o. 4 -

17. Simia ursina.

* Trichocephalus palaeformis. cr. 16. 2.

1S. Simiac species incerta.

* Cysticercus tenuicollis, biceps. 545 .

19. Lcmur Mongoz.

Trichocephalus. cr. 1S. 9.

Cysticercus sphaerocephalus. hyd, om, hep, ur, 181. 7.

* - crispus. pI. 1S1. 8 .

\section{Ordo 111. Chiroptera.}

20. Vespertilio auritus.

* Ophiostoma mucronatum. i. 61. 2.

* Distona Linı. i. 117. 117.

Taenia. i. 16g. 9í.

Cysticercus fasciolaris. hyd, hep. 179. 1,

Dub. ? Sírongylus, i. 185, 4. 
21. Vespertilio discolor.

Filaric. a. 7. 21 .

Distoma Lima. i. 117. 117 .

Dub. (Trichosoma). cr. 185. 5.

22. Vespertilio Ferrum equinum;

Disıoma Lima. i. 117. 117.

23. Vespertilio lasioptcrus.

Trichosoma. i. 14. 7 .

Ophiostoma mucronatum. i. 61.2.

Monostoma, i. 87. 26.

Distoma Lima, i. 117.117 .

- Taenia acuta, i, 165. \$2.

24. Vespertilio murinus.

Ophiostoma mucronatum. i. 62, 2.

Distoma Lima. i. 117. 11.7.

* Tdenia obtusata. i. 159. 59 .

25. Vespertilio Noctule.

Distoma, i. 119. 1240

20. Vespertilio Pipistrellus.

Distoma Lima. i. 117. 117.

2\%. Vespertilionis species incertae.

Dub. (Ncmatoidea). hyd. pell. 185. 6.

\section{Ordo 1V. Digitigrada (Ferae).}

\section{Canis familiaris.}

* Trichocephalus depressiusculus. cr. 17. 5 . Strongylus Gigas. ren. 31. 3 .

* - trigonocephalus. tub. art. oe, V. 35.20.

* Ascaris marginata. t. 42. 19.

* Pentastoma taenioides. fr. 123. 1.

- Tacnia serrata. t. 163.72.

* - cucumerina. t. 147. 14.

Cysticercus. hyd.-pi. 1\$1. 10.

$$
\text { 29. Canis Lupus. }
$$

* Trichosoma Plica. iir. 14. 6.

* Spiroptera sanguinolenta. $\iota u b, v .27 .21$.

* Strongylus Gigas. ren. 31. 3 .

i. 36.24 .

* Ascaris microptera. oes. i. 41. 17.

* Distoma alatum, v. 112.96.

* Pentastoma taenioides. fr, 123. 1. 


\section{$-722-$}

* Tzenia opuntioides. t. 1/77. 15.

* - marginatr. t. 163. 70.

Filaria. ren. a. 7. 22. 30. Canis Vulpes.

Trichocephalus depressiusculus. coec, 17. 5.

Strongylus Gigas. ren. 31. 3.

- - tetragonocephílis. t. cr. tub. oes. 35.21.

- gland. mes. 36.25. (185. 7.)

* Ascaris triquetra. t, 41. 19.

- Distoma alatum, i. 112. g6.

* Taenia litterata. t. 148. 16.

* - crassiceps. t. 163. 70.

Ascaris. i. 53. 73 .

$$
\text { 31: Canis Lagopus. }
$$

Taenia. i. 169. 95 .

\section{Canis aureus.}

Ascaris. i. 53.74 .

33. Canis americanus (Vulpes: Agouara-Guazu.) Strongylus Gigas. ren. 31. 3.

34. Felis Catus domesticus.

Strongylus tubaeformis. t. 36. 23.

* Ascaris Mystax. t. vi 41. 21.

* Taenia elliptica. to $158 \cdot 52$.

* - crassicollis. t. 164.75 .

35. Felis Catus ferus.

Ascaris Mystax. i. 41, 21.

Taenia crassicollis. i. 164.75 .

- lineata. t. 169.97.

Dub, (Liorliynchus?) tun. v. 116. Apx.

Filaria, pcll. 7.23.

36. Felis Leo.

- Spiroptera. tub. oe. 27. 25.

* Ascaris leptoptera. v. i. 41. 16.

* Ascaris mystas. j. 41. 21. 37. Felis Lynx.

- Taenia laticollis. i, 164. 74. 38. Felis Pardus.

- Taenia. i. 169.96.

Spirnptera. oe, $\nabla .28,26$.

39. Felis Tigris.

Ascaris. i. 53.75. 


\section{- 723}

40. Viverra Genetia.

* Ascaris brachyoptera. i. 41. 20.

41. Viverrà Narica.

* Echinorhynchus Spirula. i. 51, Apx。

* Taenia crassipora. i. 97. Apx.

42 Viverra Nasua.

* Ascaris alienata. i. 44. Apx.

43. Mustela Lutra.

* Strongylus Gigas, ren. 31. 3.

Fílaria, pell. mu. 3. 24.

44. Mustela Foina.

- tub. pu. 8. 25.

Ascaris, i. 53. 76.

* Distoma trigonocephalum, t. 114. 103.

45. Mustela Martes.

Filaria, pell. mu, 7. 24 .

* - tub. pu. 8. 25.

Strongylus Gigas, ren, 31. 3.

Ascaris (major) i. -53.76 .

- - (parva) t. 48. Apx.

Distoma trigonocephalum. t, 114. 103.

* Taenia intermedia. t. 163. 71.

46. Mustela Putorius.

- Filaria. pell. nu. 7. 24.

- tub. pu. 8. 25.

Trichosoma, du..14. 8.

Echinorhynchus moniliformis (parasit.) $\nabla, 71,33$.

* - ventricosus, t. 74, 42.

* Distoma, squamula. i. 103. 53.

* - trigonocephalum. t. 114, 103.

* Taenia tenuicollis. t. 159. 58.

Cysticercus, liyd. liep. 181. 11.

47. Mustela erminea.

* Taenia brevicollis. i. 159.57 .

48. Mustela vulgaris.

Echinorhynchus. mes, 75, 50 .

* Distoma trigonocephalum. t, 114. 103.

* Tienia tenuicollis. t. 159.53.

\section{Ordo V. Plantigrada:}

49. Ursus Arctos.

* Spiroptera. oes. '(ubb. ren.) 28. 27.

* Ascaris transfuga, i. 40, 15. 
50. Ursus maritinus.

- Ascaris trausfuga. i. 40, 15.

- Taenia. i. 169. 98.

13. Ursus Gulo.

Strongylus ? Gigas. om. 31.3.

Ascaris. i. 53. 77 .

52. Meles Taxus.

* Oxyuris niata, cr, 19:2.

* Sucrigvins criniformis. i. 35. 22.

- Liorhynchus truncatus. t. cr. 62, 1.

* Disioma trigonoceptalum. i. 114. 103.

* Taenia alugustata. i. 148.19.

53. Erinaceus europaeus.

Filaria. pu. S. $20 \hat{.}$

Triclosoma. t. 14. 9.

* Physaloptera clausa, v. 29. 1. Apx. 16́. Strongylus striatus. bro. 34.15.

"Ascaris pusilla. hyd. pi. 46. 42.

- Echinorhynchus napiformis. cr. $6 \% .4$.

$$
\text { - } \quad \text { a. } 76.52 .
$$

Distoma pusillum. hyd, hep. pell. 104. 56.

- - trigonocephalum. i. 114. 103.

* Taenia tripunctata. i. 147.13.

- compacea, i. 16\%. -6 .

* Dizb. (Cestoideum) hyd th, 1S5. S.

54. Talpa europaea.

* Spiroptera strumosa. v. 24 10.

* Ascaris incisa. hyd pi. 40, 42.

* Monostoma ocreatum, i. 34. 11.

- Distoma llexuosmm. v. t. 105.64.

* Taenia bacillaris. i. 154. 38. Cysticercus. hyd, hep. 193. 12.

55. Chrysochlorus capensis (Talpa aurea.).

- Trenia sphueroccphala. i. 9-4. Apx.

56. Nygale moschata (Sorex.).

Dub. Cestoidenm. jell. gla, ax $\nabla, 186,9$.

57. Sorex Araneus.

Echinorhyuchus. i. 6. 5 t.

1 Taenia. ¿. 170. 39.

58. Sorex Eremita.

- Distoma exaspcratum, i, 117, 118.

Taenia. 170. 99. 
59. Sorex fodiens.

Taenia, i, 170. 99.

\section{Ordo V1. Marsupialia.}

6o. Didelphis Cayopollin.

* Trichocephalus minutus, coec, 8. Apx.

- Physaloptera turgida. Y. t, 17. Apx.

- Ascaris tentaculata. coec 41. Apx.

* Echinorhyuchus microcephialus. i. 50. Apx.

61. Didelphis ? virginiana,

* Distoma coronatum, t. 81. Aps.

62. Didelphis murina.

Ascaris. i. 53.78 .

Taenia. i, 170. 100 ,

63. Halmaturus giganteus Kangurls.s.).

Distoma hepaticumu. hep, 92.1.

- Taenia festiva. hep. fell, 16, 7 .

\section{Ordo VII. Glires.}

64. Hystrix cristata.

Spiroptera, tub. oe, 2s. 2s.

6. Casior Fiber.

Tricloocephalus. cpec. 1s. 10.

Așcaris, i. 54.79.

* Amphistuma subtriquetrum. t. crass. 91. 18.

66. Mus amphibius.

Trichomocephalus nodosus. coec, 17. 7. .

Ascaris obveluta. cr. 44. 32 .

* Taenia omphalodes. i. 1,6: 9 .

Cysticercus fnsciolaris, hyd. hep. 179. 1.

Dub. byd. pell ingu. 1 sti. zo.

67. Mus arvalis.

Trichocephalus nociosus. cocc. 17. 7 .

Ascaris oblevata. cä. 44. 2?

* Estinorliynchus monilifornis. i. 71. 33 .

* Taenia omplaalodes. i. 146, 9 .

C) sticercus fasciolaris. hiyd. hep. 179. 1.

* - longicollis. hyd. the 150. 5.

Gs. Mus capensis.

* Triclocephalus contortus, coec, 7. Apx.

Taenia. i, $170,106$. 


\section{$-726$}

69. Mus decumanus.

Taenis. i. 170, 103.

- Cysticercus fasciolaris. hyd, hep. 179. 1.

Taenia. j. 17ò. 105 .

$$
\text { 70. Mus Lemmus. }
$$

Filaria a. א. 29.

$$
\text { 71. Mus Musculus. }
$$

- Trichocephalus nodosus, cocc. 17. 7.

* Spiroptera obtusa. V. 27. 22.

* Ascaris obvelata, cr. 44. 32.

Echinorlyynchus. v. 76.65 .

Distoma. . i. 11.9. 125.

* Taenia pusilla. to 159. 56.

- a. 170.102.

* Cysticercus fasciolaris. hyd. hep. 179. 1. - pisiformis. hyd. hep. 18I. 6.

Dub. lì̀d. pell. ingu. 186.10.

72. Mus Rattus.

Trichocephalus nodosus. cr. 17.7.

* Taenia diuinuta, i. 87. Apx.

- (arı eadem) t. 170.103.

* Cysticercins fasciolaris. hyd. hep. 179. 1.

73. Mus syluaticus.

Tríchoceph halus nodosus. coec. $17 \cdot 7$.

Taenia. i. 170, 104.

Strongylus. i. $36,27$.

74. Myoxus Glis,

Opliostoma cristatum. i. 6o. 1.

* Taenia. i. 170. 101.

Ophiostoma cristatum, i. 60. 1.

75. Myoxus Dryas.

Taenia, i. 170, 101 .

76. Myoxius muscardinus.

Ophiostoma cristatum. i. 6o, 1.

Ascaris, i. 54.80 .

77. Dipus Sagitta.

78. Arcomys Cricetus.

Echinorlyynchus moniliformis, i. 71. 33。

* Taenia straminea. to 16j. 81 .

79. Arctomys Citillus.

* Trichocephalus unguiculatus. coec, 17. 40

- Spiroptera. vo 28.29. 
Ascaris obvelata. cr. 44. 32 .

Eclinorliynclius, i. 76.54 . 80. Arctomys Marmota.

Taenia pectinata. t. 145.3 . 81. Sciurus vulgaris.

Ascaris aculiosima. to 4 '. 33 .

Distoma hepaticum, liyd, hep. 93. 1.

* Taenia dendritica. t. 148. 17.

* Cysticercus tenuicollis. pi. 19o. 3 . 82. Sciurus cinereus.

Cysticercus tenuicóllis hyd. hep. 190. 3.

83. Lepus Cuniculus domesticus.

Oxyuris ambigua, cr, 19. 3 .

Taenva pectinata. to v. a. 145.3 .

Cysticercus pisiformis. lyd. pi. 1\$1. 6.

$S_{\text {t. }}$ Lepus Cinniculus ferus.

* Oxymris ambigua. cr. 19. 3 .

* Trichocephalus unguiculatus. cr. $17 \cdot 4$; Strongylus retortaeformis. i. 34. 17.

Distoma hepaticum, hep. 92. 1.

Taenia pectinata. to 145.3 .

Cysticercus pisiformis. hyd. vi. 181. 6. '

S5. Lepus timidus.

Filaxia. mu. cox. 8. 27.

(? Trichosoma). pu. S. 2S.

* Trichocephalus unguiculatus. cr. $17 . \%$

Oxyutis ambigua, cr. 19. 3.

* Strongylus retortaeformis. t. (cav.) tra. bro. $34,17$.

Distoma hepatićum. hep. 92, 1.

ventastoma serratum, "pu. 124." 4 .

* Taenia pectinata. i. 14.5 .3$.

Cysticercus pisiformis. hyd. hep. pi. 18t: 6.

86. "Lepus variatilis.

Trichocephalus unguiculatus. ${ }^{i}$ cr.! $17: 4$.

- Cystićercus, liyd. mes, 182. 13.

87. Lepus brasiliensis.

- Ascaris veligera. coec. 37. Apx́'.

8s. Cavic Paca.

* Áscaris uncinata, coec. 43 . Apx."

\$9. Cavia Aperea.

- Ascaris uncinata, i. 43. Apx.

9o. Cavia Cobấá.

Pentastoma emarginatumb pu. 124. 3. 


\section{Ordo VIII. Edentata:}

* Spiroptera gracilis. i. 12. Apx.

* Strongylus leptocephalius. cr. 27. Apx.

92. Myrmecophaga didacty'a.

Dab. Nematoidenrn. i. 1S6. 11 .

33: Dasypus novimcinctus,

- Ascaris retusa. cr, - tub. cr. 33. Apx.

Taenia, $\mathrm{cr}_{\circ} 170.10 \%$

\section{Ordo IX. Packydermos:}

9\%. Hyrax capensis,

45. Sus scrofa.

- Trichocephalus crenatus, i. 17.6.

* Spiroptera strongy hina. $\nabla .23 .3^{\circ}$

* Strongylus deutatus, tr. i. 31. 2 .

* - bró. 36.29.

* Ascaris lumbricoîdes. i. 63. 1.

* Distoma hepaticum. 'hep. 1. 92. 1.

* Cysticercus tenuicollis, hyd. pi, pl. 180. 3 .

* - - cellulosae: ' hyd. cer. mir. 1800.4 .

Echinococcus vetérinorum, hÿl. liep. pi, 155.3. 96. Elephas indicus.

Strongy!us, hep. 36.29.

\section{.... .in Crdo X. Bisulca.}

97. Camelus bactrianus.

Trichocephalus, cr, $1 \mathrm{~S} .11$,

Strongylụs. pu. 36. 30.

Distoma hepaticun? hep. 92. 1.

Cysticercus: thyd, pis pl. 18o. 3.

Echingocoscus veterinorum, hyd. hep, 185. 3.

99. Camelus Dromas.

Trichocephalus, cr, 18. 11.

Strongylus. pu. 36. 30.

Echinococcus veterinoruł. hyd. hep, 185. 3:

... 99 Cprous Axis.

$$
\text { 99. Cirvus Axis. }
$$

C)-sticercus tenuicollis, hyd. pi. ISO. 3:

Filasia. a. 8: 30 :

300, Eervus Eilaphus, 


\section{$-7 ? 9=$}

Trichacephalns affinis, coec, 16.3 .

* Strongylus ventricosus. t. 33. 12.

* Amphistoma conicumı. v. $2-3$. 91. 17.

* Disroma hepaticum. hep. to 92. J.

Cysticercus tenuicollis. hyd. pi. 180.3.

101. Cervus Tarandus.

* Cysticercus tenuicollis. hyd. pi. $180,3$.

102. Ceri'us Dama.

Trichocephalus affinis. cr. 16. 3.

Strongylus ventricosus. i, 33. 12.

Amphistoma conicum. v. 91. 17.

Distoma hepatiçum, hep. 92. 1.

193. Cervus Capreolus,

* Trichocephalus affinis, cr. 16. 3 .

* Strongs lus filicollis. t. 32.7 .

- tub. Fen. 36. 31.

Distoma hepaticum.. hep. 92. 1.

* Taenia expansa. i. 144. 1.

- Cysticercus tehnicollis, hyd. pi. 190. 3.

104. Antilupe Corinna.

* Distoma hepaticum. oe. 92: 1 .

105. Antilope Dorcas.

* Trichocephalus affinis. cr. 16.3 .

Strongylus. pus: $3 \% \cdot 32$.

Taenia expansa. i. 144. 1.

Cysticereus tennicollis. hyd. pi. 1go. 3 :

... 306. Antilope Kevclla.

Distoma liepaticum. hep. 0?. J.

Cysticercus tennicollis. hyd. pi. 15o. 3 :

107. Antilope Rupicapra.

* Trichocephalus affinis. coec. 16. 3 .

* Strongy lus hyposiomus. i. 33: 9 .

* Taeria exparsa. - i. 14亿. I.

Cysticercus temuicollis. hyd. pi. 180. 3i

109. Antilope "Saiga.

Cysticcrens tenuicollis. hyd. pi. I\$o. 3.

109. Antilopae africanae species incerta.

Dub. Nematoideum. i. 186.12.

Coenurus cerebraliso lyyd. cer. 181. ?.

110. Capra Hirclis.

* Strongylus venulosus. tr. i. 33. 11 .

* Distoma hepaticuun. hep. i. 92. 3.

* Pentastoma denticulatum. hiep. C._12fo. 2. 


\section{$-750$}

* Taenia. i. 170.108 .

* Cy:icercus tenuicollis. hyd. pi. 180.3.

* Tichocephalus afizis. coer. 16. 1:1. Oris Aries.

* Strongy lus contortis. v. 4. i. 32. 6.

* - $\quad$ filicollis. t. 327.

* - Filaria bro. 32.8 .

Ascáris. i. 51.8 .

* Amphistoma conicum. v.,1.93. 17.

* Distoma h. patictum, hep. y2. 1.

* Taenia expansa. to r.́. I.

* Cysticercus tenuicollisa hyd. pir 180. 3 .

* Comnins.cerebralis bys.cer. 194.

Echinococcus vererinorum. hyd. hep. pu. 185*3.

Dub. Nematoideum. S: 1.3 .

112. Ovis Ammon.

Trichocephalus aftinis. coec. $16 \cdot 3$.

Strongylus Filaria. bro. 328.

Cysticercus tenuicollis. hyd. mes. 180.30

Echinucoccus Veterinorum. Iyd. hicp. pu. 185. 3,

113. Bos Bubalis.

Filaria. a. $8 \cdot 3$,

114. Bes Taurus ferus.

Distoma hepaticun. hep. 92. 1.

Echinococcus Veterinorum. hyd, hep. 195: 3.

115. Bos Taurus domesticus.

* Trichocephalus afinis. çoec. 16. 3.

* Strongylus Gigas 1en. 31.-3.

* - - radiatils, tr. i. 33.10,

- bro. 37.34 .

- Ascrris lumbricoides. t. 3\%. 1.

* Amphistoma conicum v, 1 et 2. 91. 17.

* Dirioma hepuicum. hep. i. 92. 1.

* Taenia denticulata i. $r$ 4. 145. 2.

* Cysticercus re!nicollis. hyd, pi. pl. x80. 3 . Coe urrus curebralis. liyu. ces. 184. 1.

Echinococcus Veterinorur. hyd. hel. 185. 3.

\section{Ordo X\% Solidungula.}

\section{1:6. Equus Caballus.}

* Filaria pajillosa. a, th. oc. 6. 14.

* Oxyuris curvula. coec. 18.1... 
* Spiroptera megastoma. tub. V. 22, 1.

* Strongylus Gigas. ren 31. 3.

* - arnatus. cr. pa. tub. art. 30. 1.

* Ascaris lumbricoides. t. 37. 1.

* Distoma hepaticum. hep. 1, 92,.1. Pentastoma taenioides. fr. 123. 1.

* Taenia plicata, t. v. 145 a 5.

* - perfoliata. col. t. $\nabla .145 .6$.

* Cysticercus fistularis. hyd. pi. 179. 2.

117. Equus Asinus.

Oxyuris curvula coec. 18. 1.

Strongylus armatus. cr. tub. art. 30.10

Ascaris lumbricoides. t. 37. 1.

Distoma hepaticum. hep. 92. 3.

1is. Mulus.

Strongylus armatus. cr. 30. 1.

Pentastoma taenioides. fr. 123. 1.

119. Equus Zebra.

Taenia. i. $17^{1}$. 109 .

$$
\text { 119. Equus Zebra. }
$$

\section{Oräo XII. Palmipedes.}

120. Phoca barbata.

Liorhynchns gracilescens. v. 62. 2 ,

* Distoma tenuicolle. Lep. 93. 5 . Taenia anthocephala, rect. 146.8.

121. Phoca foerida.

Ophiostoma dispar. v. i. 61. 4.

$$
\text { 122. Phoca groenlandica.- }
$$

Ophiostoma dispar. v. i. 61.4 .

* Ascaris osculata. i. 30 . Apx.

123. Phoca vitulina.

* Strongylus Gigas. hep. pu. i. 31. 3.

* Ascaris osculata. จ. 39. 9. Apx. 30.

* Echinurhynchus strumosus. i. 73. $4 \mathrm{r}$.

* Amphiostoma truncatum. hep. v. i. 91, 15.

* Distoma acanthoides. i. 114. 104.

* Ligula. i. 134. 7 .

* Bothriocephalus solidus. rect. 139. 14.

\section{Ordo XIII. Cetaeea.}

12. 1. Balaena rostrata.

* Echinorhynchus porrigens. t. 21. 34. 
125. Df!phinus Phocaenc.

- Strongylus inflexus. ty. bro. 34. 16.

- Ascaris simplex. vo 1. 49. 53.

126. Delphinus Delphis.

Cystiçercus. hygl. ad. vi. \$2. 1任.

Dub. Nematoideum. hyd. pi. - i. 156. 4 :

127. Delphinus gangeticus.

Ascaris. v. $54 . \mathrm{S} 2$.

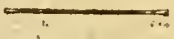

\section{Classis II. Aves.}

Ordo 1. Accipitres.

128. Vuitur cinereus.

Ascaris depressa. . 42.24.

129. Vultur fulvus,

* Ascaris depressa. i. 42. $2 \%$.

130. Falco Albicills.

Ascaris depressa. io $4: 2$.

Echinorhyrachis striatus. i. 74. 43 .

Amphirema macroceptialun. i. 88.3 .

Iigula uniserialis. i. 132.1.

131. Finlco albicollis.

* Echinorhynchus umidu?us. i. 53. Apx;

- Dab. Nemacordeum. 117. Apx.

Ascaris deprassa. i. $43.2 \%$

132. Falco apivorus.

- Amphistoma macrocephalnn. i. SS. 3:

133. Falco ater.

Taenia globifora. i. 155. 5.3 .

\section{Falco Buteo.}

Trichosoma. tun. *. 15. 10.

* Spiroptera leptopicia. tun. v. 26. 19.

Accaris depiessa. i. +3.2 .

* Echiusorhynchus inaequalis. .66 .12$.

$$
\text { - caudalus. 70. } 29 .
$$

Amphistoma macrocuphalum, i. $88 \cdot 3$.

Discoma. to 119 127.

* Taenia globifera. i. 158. 53.

- perlata. i. $1+6.10$.

Dub. Nematoidemin, i. $157 \cdot 17$. 


\section{- 733}

335. Fulco Chrysaëros.

Ascaris depressa. i. 42.24.

Anplistons macrocephalum, i, $8 S .3$.

Disioma. fell. $11 \mathrm{~g} .1: 6$.

Taenia. i. 171.110.

136. Falco cinieraceus.

Spiropiera leptoptera. 5.26 .13$.

Ascuris depressa, i. $42 ; 2 q$,

Echinorlynchus caudatus. i. 70.290

- 1 moniliformis (parasit.) 71.330

Amplistoma mácrocepluilum. i. \$.9. 3 .

13\%. Fuico cyaneus.

Filaria attenuata. aur. uas. 4. 3 .

Ascarišs depressit. i. $4 \underline{2} 2,2\}$.

Echino:hynchus caudatus. i. 70. $2 \dot{3}$.

$$
\text { - i. } 76.56 .
$$

Amphisiona macrocephalum. i. $89.3^{3}$

Tuenia globifera: i. $15 \mathrm{~S}$. 5.7o'

$$
\text { 138. Faico fulius. }
$$

* Lignla nniserialis. i. 13\%. 1.

Dub. Femat. pu. e. 196.15 .

139. Falco gallicus,

* Physaloptera alata. i. 29. 2.

Amplistoma macrocephalun. i. SS. 3 .

Taenia globifera. i. 158 . 153.

140. Falco Eentilis:

Filaria attenuata. a. th. mu. 4. 3 .

141. Falco Haliä̇ios.

Physaloptera tenuicullis. i. 30. 5. Apr. 21,

Ascaris depressa. i. \{r. 2 4.

Echinorlyynchus: i. 76.57 .

* Amphistoma serpens. i. \$.. 2.

nucrocephalum. i. SS. 3.

1'2. Falco lagopus.

* Spiroptera laticeps. oc. 23. 5 .

Ascaris depressa. i. 42, 24.

Echinorhynchus caudatus. i. 70. 29.

Amphistona, macrocephalnm. is 88.3.

* Taenia globifera. i. 1.58. j3.

$$
\text { 14.3. Falco lanarius. }
$$

Filaria attenuata. a. $4 \cdot 3$.

Ascaris depressa. i. 42,34 . 


\section{$-734-$}

Ampinistoma macrocephalum. i. 88.3.

- Taenia globifera. i. 15s. 53 .

144. Falco Leucosoma.

Anphistoma macrocephalum. i. $93 \cdot 3$.

Taenia globifera, i. 158.53 .

145. Fàlco Lithofalco.

Filaria attennata. a. 4. 3 .

Amphistoma macrocephalnm. i. SS. 3.

146. Falco magnirostris,

* Ascaris depressa. i. 35. Apx.

- Echinorhynchus caudatus. i. 54. Apx.

147. Falco Melanaëtos.

Distoma crassiusculum, fell. 112. 97.

Trichosoma. cr. 15. 10.

148. Falco Milvus.

* Ascaris depressa. t. 42. 24.

Echinorhynchus caudatus. i. 70.29.

- Amphistoma macrocephalum. i. $88 \cdot 3$.

- Listoma echinocephalum. rect. 115. 10g.

Taenia flagellum. i. 161.67.

149. Falco naevius.

- Spiroptera sterëura. oc. anr. 23. 2. Ascaris depressa. i. 42. 24 :

Eclinorlyynchus caudatis. 70. 29.

Amphistoma macroceplalum. i. 88.3.

- Dub. (Filaria) oc. nas. 187. 18.

150. Falco Nisus.

- Spiroptera leptoptera. tuu. v. 26. Ig.

- Physaloptera alata. v. 29. 2. Ascaris depressa. i. 42. 24 . Amphistoma macrocephalum. i. $\delta 8 \cdot \hat{3}$. Dub. tub. hep. 187. 16.

151. Falco Palumbarius.

- Ascaris depressa, i. 42. 24. Amphistomá macrocephalum. i. 88.3.

152. Falco pennatus.

Physaloptcra alata. r. 29. 2.

Ascaris depressa. i. 42, 24.

Echinorhynchus candatus. i. 70. 29.

Amphistoma macrocephalum. i. 8S. 3 .

- Distoma albicolle. hep. fell. 98. 3I.

Taenia globifera. i. 158. 53 . 
153. Falio peregrinus.

- Filaria attenuata. a. 4. .3.

Ascai is depressa. i. 42. 2f.

Amphistona macrocephalum. i. SS. 3 .

*

Taenia globifera. i. $15 \% \cdot 53$.

1. 92.19 .

- Spiroptera. pe. 2s. 30.

Anplistoma macrocepluanm. i. S3. 3.

$$
15 \% \text {. Falco rifus. }
$$

Ascaris depressu. i. 4?. $2 \%$

Echinoring ficluns candatus. i. 70. 29.

Aniphistoma macrocephalum. i. 88.3.

Distoma. i. 119. :29.

Taenia globifera. i. $458 \cdot 53$.

156. - Falco Subbuteo.

Filaria attenuata. th. 4.3 .

Amphistona macrocephalum. i. 8s. 3. Taenia glohifera. i. 158. 53 .

157. Falco Tinnunculus.

Spiroptera leptoptera. v. 26.19.

Asraris depressa. i. 42.24.

Echinorhynchus caudatus. i. 70. 29.

Amphistoma macrocephalım. i. $8 \delta \cdot 3$.

- Taenia globifera. i. 158.53.

- Dub. Cestoidenm. pall. 187. 19.

159. Falconis species brasil.

- Spiroptera acutissima. v. 1.\%. $\Lambda$ px.

- Physaloptera alata. v. 19. Apx.

* Echinorhynchus tumidulus. i. 53. Apx. 160. Strix accipitina.

- Dub. Cestoideum. pell. hypoch. 1:7. 20.

$$
\text { 161. Strix Aluco (et Stridula.) }
$$

- Ascaris depressa. i 42. 24.

Echinorhynchus tuba. i. 70.30 .

Amplistoma nacrocephalum. i. 58.3 .

- Distoma apiculatum. i. 116. 111.

- i. 119.129.

th. 119.130.

Taenia candelabraria. i. $160,60$.

16\%. Strix bracilyotus (et Ulula).

Filaria attenuata. pell. aur. 4. 3. 


\section{$-736$}

Amphistoma mactoceplalum. i. 88.3.

* Taenia candelabraria. 160.60. 163. Strix Rubo.

* Trichosoma obtusum. coec. 13. 3..

* Ascaris deprossa. i. 42. 24.

Echinoshyuchus Tuba. i. 7ó. 30 .

* Amplistoma macroeefhalum. i. SS. 3.' Taenia canúclabraria. i. 160.60. . ?6.. Strix dasypus. Trichosona obtisum. coec. 13. 3 . Amphistoma macrocephalum. i. $88 \cdot 3$. 165. Strix flarmea.

- Ascaris depressa: i. 42. $2 \%$.

* Amphistoma macrocophalum. i. SS. 3.

- Echinorliynchns tuba, i. 70. 30. Distema apiculatme cr. 116. 111。 166. Strix Otus,

Trichosoma obtusum. coec. 13. 3 . Ascaris itepressa. i. 42. 24 . Echinorhynchus aequalis。 cr. 700,31 . * Amplistoma macrocephalum. i. 88.3. Taenia candelabraria. i. 160.60. 167: Strix passerina. Trichosona obtusum. coec, 13. 3. Echinorhyuchus globocaudatus, i. 66. 13. Amphistoma macroceplualum. i. SS. 3. 168. Strix Scops.

Spiroptera, tun. v, 28. 31 .

Taenia candelabraria. i. 160.60. 16g. Strigis species brasil,

* Physaloptera saginata. i. 23. Apx.

* Taenia, i. 103. Apx.

* Dub. Nematoiderm. i. 118. Apx. 170. Lanius Collurio:

Filaria. pell. s. 32 .

$$
\text { bro. pl. S. } 33 .
$$

Spiroptera curyoptera, tun. v, 26. 20.

Ascaris. i. 54. 83.

Echinorliyuchus, i. 76.58.

Distuma. i. 119. 131.

Taenia parallclipida, i, $160,61$.

171. Lanius Excubitor.

Spiroptera eturyoptera, tun, $\nabla, 26,20$. 


\section{- 737}

Fílaria. pell. s. 32.

\section{Lanius minor.}

Spiroptera euryoptera. tun. $\nabla .26 .20$.

- Ascaris. i. 54. 83.

* Taenia parallelijipeda. i. 160.61.

Filaria. pell. 8. 32.
Taenid parallelipipeda, 1. 160.61.

Dub. Cestoideum, a. 187. 22。

\section{Ordo II. Levirostres.}

174. Psitrácus aestivus.

- Ascaris truncata. i. 45. 36. Apx. 40.

175. Psitracus dominicensis.

Ascaris truncata. i. 45. $3 \mathrm{G}^{\circ}$

276. Psistacus erithacus.

Taenia filiformis. t. cr. 166. 83.

177. Psittacus leucocephalus,

- Ascaris muncata. i. 45. 36.

1.8. Psitiaci species brasit.

- Ascaris truncata. i. 40. Apx.

\section{Ordo III. Pici.}

Trichosoma. i. 15. 12.

\section{Picus canus.}

Taenia crateriformis. i. 168.91.

1So. Picus lineatus.

* Tilaria obtusocaudara. th. 1. Apx.

* Taenia cruciata. i. 8s. Apx.

Tiichosoma: i. 15. 12.

1S1. Picus major.

Echiroshynchus cylindraceus. i. fig. 2 in

Taenia crenata. t. 146. 11.

- crateriformis. t. 1 (is. 91.

192. Picus martius.

- Taenia craterifornis. i. 168. 91.

Taenia crenata. i. 1.46 .11$.

\$.3. Picus medius.

- crateriformis, i. os. gr.

- Mallens.ii. 171.11.

* Distoma íingens, i, 101. 仵.

15.t. Picus ridacrylis.

A a a 


\section{- 738}

is5. Picus viridis.

Trichosoma. i. 15. 12.

+ Spiroptcra quadilloba. oe. 25. 11.

Echinorhynchins cylindraceus. i. 69. 24.

* Tacnia cralcriformis. i. 105.91.

isu. Yunx Torq́uilia.

* Taenia cratcriformis. i. 168. 91.

157. Suta europaea.

Taenia. i. 171, 112.

18s. Alcedo Ispida.

* Anphistoma denticulatun. i. go. 13.

. 189. 'Alcedinis species brasilo

* Filaria. a. 3. Apx.

Igo. Merops Apiaster.

Filaria. mes. 9. 34 .

* Spiroptera bidens。 tun. v. 24. 8 .

Distoma. i. $120,132$.

Taenia. i. 171. :13。

191. Upupa Epops.

Spiroptera. tum. $\forall .2 g \cdot 32$ 。

* Ascaris pellucida. lep. tun. 39. 10.

* Echinorhynchus ricinoides. mes. 64. 3.

* Taenia crateriformis。 i. $165.91 .(171.114$.

\section{Ordo IV. Coraces.}

192. Crotophaga major.

* Ascaris reclinata. i. 39. Apx.

193. Crotophaga minor.

- Echinorhyrichus. tumidulus. i. 69. 25. Apx. 53.

* Taenia nutabilis. i. i5s. 31.

19.. Corvus Caryocatactes.

Tilaria attenuata. a. 4. 3 .

Trchosoma. i. 15. 11.

Spiroptera Anthuis. tun. v. 25. 13.

* Strougylar papillosus. oe. Lun. 31. 4.

* Amplistoma nicrostónum. i. 8s. 4,

Distoma candale. rect. 103. 54.

Taenia. i. 171. 115.

Filaria attenuata. a. $4 \cdot 3$.

195. Corrus Curax.

Spiroptera Anthuris. tun. v. 25. 13.

Taenia undulata. i. 167. 88 . 


\section{- 739}

196. Corsiss Cornix.

* Filaria attentsata. a. th. 4. 3. Spiroptera Authuis. tum, v. 25. 13. Asç̨ris. v. i. 54. 84.

Echinouliysuchus. i. 76. 59.

* Anplistoma splanernla. i. go. 11.

* Distonia macrumrum. fell. 93. 30.

- ovarnm. bu. 0...?

- Talenia nadulatio '. 17. Ss.

* - serpentinlus. i. 167. \$9.

$$
\text { 197. Corrus Corone, }
$$

Filaria attenuata. a. 4.3 .

Spiroptera Anthuris, thin. v. 25. 13.

Taenia undulata. j. 167.58 :

$$
\text { 19S. Corvus frugilegus, " }
$$

Filaria attennata. a. 4. 3.

* Spiropiera Anthris. tun. v. 25, 13,

Ascaris. i. 5 f. $\$ 6$.

Monostoma pricmatieum, 2. 85, 15 .

* Distuma ovaun. hu. 93.2.

Taenia. 171. 115.

109. Corves giandarius.

Filaria attenuata. a. 4. 3 .

* Spiroptera Anthuris. tuti. r. 25. 13.

* Ascaris. i. 5 q. 85 .

Echinorhynchus compressis. i. 64.5.

Disionıa caudale, rect. 103. 54 .

Taenia stylosa. t. 160.63.

- serpentulus. i. 167.89.

200. Corvus Moncdula.

Filaria attennata. a. $4 \cdot 3$.

Trichosoma. 15. 11.

* Echinorliynclius conipressus, i. 64.5 .

* Distoma cirratum. cr. 100. 40. Taehia。 i. 171. 115.

\section{Corvis Pica.}

Filaria attentata. a. 4.3 .

Tricliosóma. i. 15. 11.

Sprruptera Anthuris, tun. $\nabla .25 .13$.

* Ascaris. i. 54. 87.

Echnoritynchis. i. 76.60 .

Distoma ovatum, bu. 93. 2.

= cirratum. rect, 100. 40. 
- Taenia serpentulus. i. 167.89 .

$$
\text { 202. Corvus Pyrrhocorax. }
$$

Filaria attenuata. a. $4 \cdot 3$.

Spiroptera Anthuris. tun. v. 25. 13.

Distoma caudale. i. 103. 54.

203. Dendrocolaptes (Picucule.)

* Taenia. i. 104. Apx.

204. Coracias Garrula,

* Filaria coronata. pell. coll. 6. 15,

* Spiroptera Anthuris. tun. v. 25. 13.

* Echinorhyuchus gracilis. i. 65. 22.

Taenia. i. 172. 116.

$$
\text { oin. } 77 \cdot 61 \text {. }
$$

\section{Oriolus Galbula.}

- Spiroptera Anthuris. tun. v. 25. 13. Echinorhyncluss. i. 77.62.

* Taenia serpentulus. t. 167. 89. 206. Oriolus cristatus.

* Echinorliynchus. i. 59. Apx.

* Taenia longiceps. i. 89. Apx. 207. Bucco brasil.

* Ascaris forcipatá. i. 42. Apx. 208. Cuculus canorus.

* Taenia difformis. i. 148. 1 s. 209. Cuculus naevius.

* Ascaris forcipata. i. 42. Apx.

210. Cuculus seniculus.

* Physaloptera strongylina. v. i. 20. Apx.

* Ascaris forcipata. i. 42. Apx. 211. Cucislus Tingazu.

* Ascaris forcipata. i, 42. Apx. 212. Cuculi sp. Erasil.

* Echinorhynchus tumidulus. cr. 53. Apx.

\section{Ordo I. Passeres.}

- Filaria unguiculata. a. 4. 5. 213. Alauda ariensis. Trichosonia. cr. 15. 13. Echinorhynchus micracanthus, i. 69. 2\%. Taenia platycephala. i. 154. 10. 214. Alauda cristata. Taenia platycephala, i. 154 . 40 . 


\section{- 741}

215. Alauda nemorosa.

Echinorhyncluss, i. 77.63 .

216. Alauda obscura.

Taenia platycephala. i. 151. 40.

217. Alauda trivialis.

Așcaris. i. 54. 85.

Echinorhynchis. i. 77. 63.:

'racuia platycephala. i. 154. 40.

318. Sturnus Cinclus.

Spiroptera, tun, v. $2 S \cdot 33$.

219. Sturnus vulgaris.

Filuria, th. pu. 9. 35 .

* Ascris crenata. i. 43. 26.

* Echinorhynchus transversus. i. 69 . 36.

* Taenia farcininalis, i. $160.6 \%$.

220. Tur lus arundinaceiss.

Ascaris ensicaudata. i. 42. 25.

221. Turdus cyaneus.

- Trichosoma inflexurn. i. 13. 4 .

* Echiuorhynchus transversus. i. 69. 26. 222. Turdus iliacus.

* Ascaris ensicaudata, $i, 42,25$.

* Discuma mesostomnm. rect. 104. 58.

* Taenia angulata. i. 155. 亿1. 223. Turdus Merulá.

* Ascaris ensicaludata. i 42. 25.

Echinorhyuchus transversus. i. 6g. 26.

Distoma. i. 120.13 .3$.

Taenia angulata. i. 15.5. 41 .

22.;. Turdus musicus

Ascaris ensicaudata. i. 42. 25.

Taenia angulata. i. 155. 4I.

Filaria. a. 9. 36 .

225. Turdus pilarisa

Ascaris ensicaudata, i. 42. 25.

* Taenia angulata to 1j5. 11.

226. Tiurdus saxatilis.

A scaris ensicandața. i. 42.250.

Eclinorlynchns transversus. i. 69. 26.

Taenia angulata. t. 155 . 41 . 
227. Turdus torquatus,

Ascaris ensicandata. i. 42, 25.

Taenia angulata. i. 155 . 41 .

2:8. Turdus viscivorus.

Fil.ıria. a. 9. 36,

Trichosuma.'i. 15.14.

A cerris ensicaudata. i, 42. 25.

Taenia angulata. i. 155: 11.

229. Turli species brasilienses.

* Echinorhynchus transrersus. i. 69. 26.

* Taenia pyranidara. i. 95. Apx.

- - i. 105. Apx.

* Dub. Nematoideum, i. 119. Apx.

$$
\text { 230. Loxia Chloris. }
$$

Distoma. i. 120. 134.

$$
\text { 231. Loxia Coccothraustes. }
$$

* Echinorhynchus micracanthus. i. 69. 27.

Distoma. i. 120.134.

Taenia. i. 172. 11 .

$$
\text { 232. Loxia Curvirostra. }
$$

Taenia. i. 172.117 .

Distoma, i. 120, 134 .

. 233. Loxia Pyrrhula.

234. Emberiza Citrinella.

Taenia. i. 172. 120.

23\%. Emberiza hortuluna.

Ascaris. i. 55. 83.

Taenia. i. 172.120.

236. Emberiza melanocephala.

* Taenia platycephala. i. 15\% 40.

Taenia. i. 172. 120.

$$
\text { 237. Emberiza Queled. }
$$

238. Emberiza brasil.

* Echinorhynchus. i. Go. Apx.

239. Tanagra Tatoa.

- Amphistonia. bu. 63. Apx. ,

$$
\text { 2.jo. Tanagraé sp. brasit. }
$$

- Spiroptera denuilata. i. 13. Apx.

* Echinorhyncluss. i. 61. Apx.

Taenia. i. 172. 119. :

31. Fringilla cannabina.

I ilaria. cox. $9 \cdot 37$.

242. Fringilla Carduelis. 
2ł3. Fringilía cociebs.

Trichosoma. i. 15. 15.

- Echinorliynclus micracinthus. i, 6g. 2\%

Distoma elerrans, i. 100. 39.

Taenia. i. 172. 119.

3if. Fringilla domesticas

- Spiroptera nasuta. 23. 4.

\& Distoma elegans. i. 100. 39.

Taenia. i. 172: 119.

Distoma. i. 120. 135 :

.25. Fringilla Linaria.

Taenia. i. 172. 119.

$$
\text { 2-16. Fringilla montana. }
$$

Distoma elegans. i. 100. 39.

Taenia, i. 172. 119 .

Taenia. i. 172. I19•" Fringilla Spinus.

2.í7. Fringilla montifringilla.

Taenia. i. 172 . 119.

$$
\text { 249. Fringillae sp. hispanষ }
$$

* Filaria affinis. 4. 6. 250, Muscicapa atricapilla.

- Taenia quadrata, i. 164. 7S.

251. Muscicapa audax,

* Taenia ormprnulatio i. 92. Apx.

252. Muscicapa collaris,

Echinorlynchus. i. 77. 64?

* Taenia quadrata. i. 164. 75 .

253. Muscicapa grisolc.

Taenia quadrata. i. 164. 78 .

* Filaria. a. 4. Apx.

254. - Muscicapa brasi?

* Taenia campanulata. i. 92. Apx."

Distoma, i. 120, 135 .

$$
\text { 255. Motacilla albas }
$$

25\%. Motacilla atricapilla.

* Echinorlyynchus arcolatus. i. 69. 23.

Taenia platycephala. i. 154.40.

257. Moracilla cinerea.

Distoma macrost omum. i. 104. 57.

2:js. Motacille Curruca.

Taenia platycephala, i. 154 , 0 . 
* Amphistoma, i. 64. Apx

259. Moiacilla ejanea.

- Taenia. i. 108. Aps.

$$
\text { 260. Motacilla Fitis. }
$$

Echinorliynchus, i. 77. 66 a 261 Moracilla flave.

Distoma macrostomum i. 104. 57.

Taenia platycephala. i 15.4. 40.

$$
\text { 262. Motacilla Auviatilis. }
$$

Distoma macrosiomun. i. 104.57.

263. Motacilla hortensis.

Taenia platycephala. i. 154.40.

\section{Motacilla Luscinia.}

Echinorhynchus, i, 77. 66 。

* Monostoma ventricosum. hep. 86. 19.

- Dietoma macrostomnm。 i. 104. 57.

* Taenia platýcephala. i. 154 f́ $40_{2}$

$$
\text { 265. Motescilla nisoria. }
$$

Echinorhynchus micracautlus. i. 69. 27 .

Distoma macrostomum, i. 104. 57.

Tąenia platycephala. i. 154. 40.

$$
\text { 266. Motacilla Cenanthe. }
$$

Filaria. th. $9 \cdot 38$.

Echinorliynchus micracanthus. i. 69. 27.

Filaria gen. coll. 9.39. 267. Motacilla Philonele

Echiinorhynchus, i, 77. 66 ,

Distoma, i. $120,136$.

$$
\text { 267. Motacilla Phoenicurus. }
$$

Echinorhynchus: i. 77:66,

Monostoma crenulatum. i. 8 i. 9 .

Taesia platyceptiala, i. 15í fo.

168. Motacilla provincialis,

* Taenia. i. 106. 'Apx.

209. Motacilla rubecula:

Filaria. a. th. 9. 38 :

Echinoshynchus, i. 77. 66.

Taenia platycephąla, i. 154. 4a.

Echinoxtyrnchus, in 77. 67.

270. Moracilla Rubetra,

271, Motacilla Rubicola

Echinorhynchus, is 77,66 , 


\section{$-745$}

272. Motacilla stapazina.

* Filaria abbreviata. oc. 4. 7 .

Echinorhynchus transversus. i. $69 \cdot 26$.

Taenia platycephala, i. 15 40.40.

273. Moracilla Sylvià.

Distoma macrostomum. i, 104.57 .

Taenia platycephalia. i. 154 40.

27\%. Motacilla Troglodytes.

Echinorhynchus. i. 7 f5. 66 .

275. Notacillae spec, italica (Ficedula dicta)

* Echinorhynchus micracanthus, i, 69. 27.

276. Motacillae spec, brasilicnsis.

* Distoma deflectens. i. 6S. Apx.

* Taenia. i. 107. Apx

277. Parus ater.

Taenia nasuta. i. 1/77.12.

278 Parus caeruleus.

Distoma. i. 120. 135.

Taenia nissuta. i. 1/7. 12.

279. Parus cristatus,

Taenia nasuta. i., 147. 12.

2So. Parus major.

Echinorkynclu1s, i, 76, 68.

Distoma. i., 120. 135a

* Taenia nasuta, i. 147. 12. 2\$1. Parus palustris.

Distoma. i, 120. 135

Taenia nasuta. i. 147.12.

2\$2. Parus pendulinus.

Distoma, i, 120.135.

Echinorhyuchus. i. 76,69 ,

283. Hirundo, Apus.

Distoma maculosum. réct. 100. 37.

* Tạenia cyathiformis. t. 152. 32.

23.4. Hirundo Melba.

- Taenia cyathuformis. $i_{9} 152.32$.

285. Hirundo riparia.

Filaria obtusa. a. 4. 48

Spiroptera attenuata. tun, v 25, 14.

Distoma maculosinm, i, 100.37.

* Taenia cyathifoımis, i, 152. 32. 256. Hirundo rupestris.

* Taenia cyathiformis, i, $9^{1}, A p x$. 
- Filaria cbtusa, a, 4. 4́.

287. Hirundo rustica.

Trichoson:a: i, 15. 16.

Spiroptera attenuata, tun, v, 25, 14.

Echinorhyuchus, i, 76, 69 .

* Listoma maculosum. i. 100, 37.

* Taenia cyathiformis, i. 152, 32.

285. Hirundo urbica,

Filaria obtusa, a, 4. 4 .

* Spiroptera attennąta, tun, v. 25, 14.

Distoma maculosim, rect, 100, 37.

Taenia cyathiformis. $t, 152.32$.

259. Hirundinis spec, brasil.

- Taenia cyathiformis. i. 91. Apx.

29o. Caprimulgus europaeus

Trichosoma. i. 15. 17.

* Strongylus capitellatus, t, 35. 19.

Ascaris, i. 55. 90.

* Distoma maculusum, i. $100,37$.

* Taenia megacantia. i. 10o. Apx.

191. Caprimulgus ruficollis (hispan.)

* Ascaris subulata, i. 38. 5.

292. Caprimutgus Bacaurau (brasil.)

Ascaris forcipata. i. 42, A x.

293. . Caprimulgus Urutau (Brasil.)

* Ascaris forcipatą. i. 42. Apx.

* Taenia megacantha. i. 10o. Apx.

29.4. Caprimulgi sp. brasii.

Ascaris forcipata. i, 42. A p.y.

- Taenia megacancha. i. 100. Apx.

\section{Ordo VI. Gallinac.}

395. Columba (Ocnas) domestica.

Trichosoma. cr. 15, 18.

* Ascaris maculosa. t. 45. 3j,

* Taenia crassula, i. 101. Apx.

2g6. Columba livia.

* Taenia sphenocephala. i, 15\% 39.

297. Colnmba risoria.

Ascaris maculosa. i. 4j. 3 .

298. Columba turtur.

* Taenia sphenocephal.t. i. 15 i. 39. 


\section{- $747-$}

Ascaris vesicularis. i. 35.3.

Taenia, i, 172. 122.

$$
\text { 300. Titrao Coturnix. }
$$

* Ascaris vesicularis. i. 3S. 3.

- Disioma fuscatum, i. 101. 43.

* Taenia Linea, i, 157. 51.

- Ascaris compar, i, 40. 39.

$$
\text { 301. Tetrao Lagopus. . }
$$

Trichosoma longicolle, i. 1/4. 5.

$$
\text { 302. Tetraó Perdix. }
$$

Ascaris vesicularis. i. $3 \% .3$.

Distonia lineare, tra, 113, 102.

* Taenia linea. i. 157. 51.

$$
\text { 302, Tetrao (Perdix) saxatilis. }
$$

* Taenia linca, th, a. (dub, cest.) 195. 23.

$$
\text { 303. Tetrao Tetrix. }
$$

Trichosoma longicolle. $\mathrm{cr}, 1-\mathfrak{f}_{0} 5$.

$$
\text { 30.. Tetrao Urogallus, }
$$

Triclıosoma longicolle, cóec, 1$\} 5^{\circ}$,

Ascaris vesicularis. i. 38.3.

T'aenia, i, 172. 121.

$$
\text { 305. Tetrao Uru, }
$$

* Ascaris strongylina, i. 29. $A p x$.

$$
\text { 306. Crypturus (Tinamus). }
$$

- Trichosoma. i, 6. Apx.

- Ascaris strongylina, i, 29, Apx.

$$
\text { 307. Numida Meleagris, }
$$

Ascaris vesiculariz, i, 38.3 .

$$
\text { 308. Phasiantus colchiçus. }
$$

Trichosoma longicolle. i. 1f. 5.1

Ascaris vesicularis, cr. 38.3 .

Distoma lineare. trit. 113, 102.

Taenia, i, 172, 183:

\section{3ิ09. Phasianus Gallus,}

* Trichosoma longicoile, i 14. 5 :

* Ascaris vesicularis, cr, 39. 3.

* - inflexa. t. 35. 4 .

$$
\text { - gibbosa, i. } 45.49 \text {. }
$$

Distoma lineare, $1 \mathrm{rin}, \mathrm{cr}, 113,102$.

* Taenia infundibuliformis, i, 152. 33 . 


\section{- 748}

Ascaris resicularis, i. 38. 3 .

310. Phasianus nycthemerus.

* Ascaris vesicularis. cr. 38.3

3i1. Phasianus pictus.

312. Meleagris Gallopavo.

* Ascaris Perspicillum, t. 41, 22.

313. Pavo cristatus.

* Ascaris vesicularis. cr. 38.3.

314. Otis Tarda.

* Strongylus. a. 37. 35.

* Ascaris resicularis. cr. 38. 3.

Echinorhynchus i 76,70 .

* Distoma cuneatum rect. 93. 3.

* Taenia infundibuliformis. $t, 152.33$.

* - villosa t, 153.34.

315 Otis Tetrax.

* Spiroptera laticaudata, tun. v. 2i. 7.

\section{Ordo VII. Struthianes.}

316. Struthio. Camelus.

Taenia. i, 173. 1,24.

\section{Ordo VIII. Grallae.}

\section{Platalea Ajajo.}

* Echinorhynchus murabilis. i. 56. Apx.

* Distoma graude. i. 67. Apx.

- Taenia Capito. i, 102. Apra

$$
\text { 318. Ardea alba. }
$$

Echinorliynclius ? bu, i, 78. 73.

- Amphiscoma longicolle, i. 87. 1.

319. Ardea caerulea.

* Taenia aurita. i. 98. Apx.

" Dub, Cestoidetum, pell, 120. Apx. 320. Ardea Ciconia.

Filaria, pell. a. 9. $39^{\circ}$.

Ascaris. i. 55. 91.

- Distoma excrvatum, i. 10g. 82.

- - ferox, i, 116.114.

* Ligula sparsa, i. $1,334$.

- Taenia unguicula, i.; 173, 125. 


\section{- 749}

321. Ardea cinerea.

* Filaria, mu, pell. g. 41.

* Ascaris serpentulus. i. 53. 72.

- Echinorhynchus striazus. i. 74. 43. Monostoma Cornu. i. 85, 14.

* Amphistona Cornu. i. 89 . 9.

- Distoma complanatum. oe. 98.29.

* Bothriocephalins noilosus, i, 140. 15.

- Taenia unguicula.-j. 173. 125. 322. Arclea cumaia.

- Ascaris microcephala, i. 48. 48.

- Distoma echinatum, i. 115.206. 323. Ardea Egretta.

- Eckinorhynclus mutabilis, i. 56. Apx: Ligula sparsa. i. 133. 4.

- Tatnia unilateralis. 1. 96. Apx. Amphistoma Cornu, i. 89. 9 .

\section{2i. Ardea Gatzetta.}

325. Ardea. Grus,

- Trichosoma obtusiusculum. tun. v, 13, 2. Echinorhynchus. i. 78. 73 .

* Distoma echinatum. i. 115. Jo6. 326. Ardea minuta.

* Fchinorhynchus spiralis, i 70. 28 .

* Distona spatulatum. i. 109. \$4.

Filaria, a. 9. 40 . 327. Ardea nigra.

* Spiroptera alata, tun, v, i. 23. 6. Ascaris, v. 55, 91.

- Dístoma hians, oe, 94. 7 .

- - ferox. i. 116.114. 328. Ardea Nycticorax.

- Ascaris microcephala. i. oe. v. 48. 48-Apx, 47.

* Tchinorhynchus mutabilis. i. 56. Apx.

* Monostuma Cornu, i. 85. 14. Distoma excavatum. i. 109. 82 . Iigula sparsa. i. 1.33. 4 . Taenia unguicula. i. 173. 125. Ascaris. i. 55. $9^{2}$. 3ेg. Ardea pturpurea. Echinorhynchus. i. 79. 72.

* Distonaa heterostomum, oe. li, 102, 50. Taenia unguicula, i, 173, 25. 


\section{- 750}

330. Arclea scapularis,

* Ascaris serpentulus. i. 47. Apx.

Strongylus, v. 37,6 .

331. Ardea stellaris.

* Amphistoma longicolle, i. 87. 1.

Distoma (? ferox). i. 110. 137.

332. Ardea virescens.

* Echinorhynchus mutabilis. 3. 56. Apx.

* Tienia unilircralis, i. 96. Apx.

333. Ardcae species brasilienses.

* Filariar a. 5. Apx.

* Ascaris serpentulus. oe. v. 47. Apx.

* Echinorhyuchus mutabilis, i. 56 Apx;

* Distoma marginatum, fau, 72. Apx,

* Taenia aurita. i. 95. Apx.

334. Tantalus falcinellus. .

Spiroptera, tmn, v. 2S. 34.

* Distoma bilobum. i. 114. 105.

* Taénia microcephala. i. 157: 50.

335. Scolopax aegocephala.

Taenia, i. 173, 128 .

3.36. Scolopax Arquata.

* Distoma militare, rect, 115. 108.

* Taenia sphaerophora, t. 151. 27.

337. Scolopax Calidris.

Distoma, i. 12u. 1.3S

* 'Trenia variabilis, i. 151. 28.

338. Scolopax fusca.

Tacnia, i, 173, $12 \mathrm{~S}$.

339. Scolopax Gallinago

* Monostoma attenuatum. coec. \%4. 10.

* Amplistoma erraticum, i. 89.7.

Disioma militare. i. 115. 108.

Taenia, i 173. 126.

- variabilis, i. 15128.

3+0. Scolopax Gallinula.

* Spiroptera. oc. 28.35.

* Distoma ninirm. cr. 101. 41.

* - militare, cr. 115. 10S.

* Taenia filum, i. 157. 49.

- _ interrupra. i. 161.65.

3i1. Scolopax Glotcis (Totanus chlóropus).

Taenia, i, 173. 129. 


\section{$-751$}

Taenia. i, 173. 128.

342, Scolopar lapponica.

313. Soolopax, Phacopus.

Taenia Nymplaca. i. 158. 54.

34. Scolopax Rusticola.

- Amplistoma creaticun. i. \$9. 7 .

* Taenia filum, t, 157.49.

- paradoxa, i. cr. 161. 64.

345. Scolopax stagratiits (Totantis).

Taenia. i, 173. 2.9.

3i. Sculopar subarquata.

- Taenia variabilis. i. 151, 29.

3i7. Scolopax Totcnus,

Taenia Siliculd, i. 173. 12\%

" Taenia. i. 1ng. Ayr.

34. Scolopacis sp. brasil.

3+9. Tringa alpina.

Filaria. pell. aur 10. \{2.

* Taenia amphitricha. i, 1,j2. 30. 350. Trinza Glareola.

* Taenia variabilis. i. 151. 28

351. Tringa helvetica.

- Spiroptera bicuspis, tun, v. 22.9.

Distoma. i. 120. 139.

Taenia variabilis, i. 151 ? 2 .

* Taenia variabilis. i. 151, 23.

352. Tringa hypoleuca.

* Taenia. i, I73. 130. Apx. 110.

353. Tringa interpres.

354. Tringa ocitropus;

* Taenia Filum, i. 157. 49.

* Taenia Filum。 i, 157. 49.

355. Tringa pugnax.

356. Tringa Vanellus.

Trichosoma, i. 15. 12.

Spiroptera. tun, v, 2y. 36.

* Ascaris semiteres. i. 42.'23. Echinorhynchus, i。73.174.

* Monostoma lineare. i. 83. 8́.

* - pu. e. 87. $2 \%$.

* Distoma cincuum, i. $116,110$.

* Taenia varabilis. i, 151. 28. 
357. Trengae sp. brasil.

* Taenia vaginata. i. 93. Apx.

358. Charadrius Cantianus.

Taenia laevigatab i. 151, 29.

- Taenia, i. 111. Aps.

359. Charadrius collaris.

360: Charadrius Himantopus.

Trichosoma, tun. v. 16. 20.

* Spiroptera revoluta. cun, v, 26. is.

Ascaris, i. 55, 93.

Monostoma, i. 87. 28

* Taenia vaginata, i, 153. $3^{6}$. Apx. 93.

361. Charadrius Hiaticula.

* Taenia laevigata. i. 15i. 29.

Filaria, nas, aur, 10, 43.

362 - Charadrius minor (fluviatilis).

Tricliosoma. tun. vे. 16.20,

Taenia laevigata. iv 151. 20 .

303. Charadrius Morinellus.

Ascaris, i. 55, 93.

Echinorliyuchus, i. 78. 75.-

Ascaris. i. 55, 95,

364. Charadrius Oedicnemus.

Echinorhyuchus, i, 75. 76 .

Ascaris, i. 55. 93.

365. Charadrius pluvialis.

Echinorhyuchus, i. 78. 77.

* Amphistona cornutum. t. 90. 10.

Taenia laevigata. i, 151.29.

366. Redurvirostra Avocetta.

* Taenia polýmórplıa, i. 15\%” 37.

367. Haematopus, Ostralegus.

* Echinorhynchus sphaerocephalus. i. 57. Apx,

- Ascaris. cuec, 55. 95.

365. Glareola austriaca.

- Distoma micrococcum, i, 101. 42.

* Taenia longirostris. i. 168.92.

Spiroptera,otun v. 20.37.

.369. Fulica atra (et fusca).

* Echnorhynchus filicollis. i. 71, 35.

- versicolor, i, 74, 44 。 
- Distoma ovatum. bul. 93. 2.

* Taenia intala, i, 16. 85

370. Fulica Cluloropus.

Echinorleynclus versicolor. i. 74. 4 4.

Monoroma mutibile. a. 85 . IE.

Distoma uncinatum. cr. 115. 107.

Ligula sparsa, i. 133.4 .

371. Rallus aquaticus.

* Distoma holostomum i. 91. 19.

Ascaris. i. 55. 94.

372. Rallus Crex.

'l'aenia. i. 172, 131 .

373. Rallus Porzania.

* Distuma militare. i. 115 log.

374. Ralli spec, brasile

Spiroptera. tun. vo i. 15. Apx

375. Avis riparia incerta (an Ardea?)

"Distoma cucumerinum. tra. 9k. S.

\section{Ordo IX. Anseres.}

376. Sterna cantiaca (stubberica.)

Echinorhynchus. i. $78 \cdot 79$.

- Amphisioma pileatum. i. go. 12.

* Distoma denticulaum. i. 116, 112.

- - cochleariforme. i. 74. Apx.

- Spiroptera. oe. 29. 38 .

377. Sterna Hirundo.

* Amplistorna pileatum. i. go. 12.

- Distoma denticulatum. 1. 116. 112.

- Bothriocephalus nodosus. i. 140, 15 . Taenia. i. 174. 132.

Filaria. i, e. 10. 44 .

378. Sterna Leucopareia,

379. Sterna minuta.

- Echinorhynchus murabilis. i. 56. Apx.

* Distoma cochleariforme. i. 7 i. Apx. 38o. Sterna nigra (fissipess,;

* Spiroptera elongata. tun, v, 26. 17.

* Ascaris. hyd. pi: 55. 96.

Distoma denticulatum. 1. 216, 112.

Ligula sparsa. i. 133. 4.

- Taenia inversa, i. 155.45.

$\mathrm{Bb}$ 


\section{$-754-$}

* Taenia oligotoma. i. 161. 66.

351. Sternae species brasilianae.

- Distoma canaliculatum. i. 66. Apx.

* - cochleariforme. i. 74. Apx.

- Taenia, i. 112. Apx. 382. Colymbus arcticus (et Immer),

Strongylus tubifex. oe. 31.5 .

- Amphistoma erraticum. i. 89. 7. '

Ligula' sparsa. i. 133. 4.

Bothriocephalus nodosus. 140. 15 .

- macrocephalus, 140. 16.

- Taenia capitellata. i. 156. 47.

Ascaris. i. 55.97 .

383. Coljmbus auritus.

* Ligula interrnpta. i. 133. 3.

- Taenia capillariso 't. 156.46.

384. Colymbus cornutus,

Ascaris., i. 55. 97.

Taenia. i. 174.135.

385. Colymbus cristatus.

Filaria. a. 10. 45.

Strongylus dubifex. oe. 31. 5 .

Ascaris. i. 55. 97.

- Distoma gibbosum (parasitice). 107. 710

* - spinulosum. i. 116. 113.

* Ligula sparsa, i. 133. 4 .

* Bothriocephalus nodosus. i. 140.15.

Taeniar i. 174. 134.

386. Colymbus minor.

- Strongylus tubifex. oe. 31. 5 .

Ascaris, i. 55. 97.

Distoma echinatum. i. 115. 136.

* Taenia macrorliyncha. t. 165. 79.

- multistriata. to 166. S4.

- Spiroptera. oe. 29. 39.

387. Colymbus septentrionalis.

* Strongylus iubifex. oe. 31. 5 .

- Ascaris variegata: oe, 49. 52.

- Amphistoma erraticum. tr. i. a. 89. $\%$.

- Distorna spinulosum. i. 116. 113.

Liqula sparsa. -i. 133. 4 .

- Bothriocephalus nodosus. tr. i. 140. 15. 


\section{$-755$}

* Bothriocephalus macrocephalus. tr. i, 140, 16.

* Dub, Cestoid. v. 18S. 2.4. 38s. Colynbus subcristatus.

* Ligula sparsa. i. 133. 4.

* Taenia lanceolata. i. 145. 4.

- - capillaris. i. 156. 46 . 359. Colymbi sp. brasil.

Ascaris. i. 49. Apx.

390. Larus atricilla.

Amphistoma longicolle. i. 87.1 .

*.Bothriocephalus cylindracens. 140. 17. 391. Larus canus.

Lignla alternạus. i. 133. 2. Taenia, i. 174 136 .

\section{9:. Larus cinerarius.}

Ascaris. i. 55.98 .

* Monostoma macrostomum. i. 86.23.

* Distoma spinulosum. i. 116.113.

- Taenia porosa. t. 168. 9\% 39.3. Larus fuscus.

* Amphistoma longiculle. i. 87. 1 .

- Distoma lucipetum. nict. 94. 9 .

- Taenia porosa. i. 16s. jo. Amphistoma. i. 92. 20.

$$
\text { 39\%. Larus glaucus (argentatus.) }
$$

- Distoma spathaceum. i. 10g. 83.

* - lucipetum, nict. 94. 9. Ligula alternans. i, 133. 2.

- Bothriocephalus cylindracens. i. 1.10: 17.

395. Larus minutus.

Filaria, pell. coll. 10. 45 .

* Taenia porosa. i. 168 . go. 396. Larus, naevius.

- Distoma spinulosum, i 116. 11.3. 397. Larus parasiticus.

Ligula alternans. i. 133. 2. 39s. Larus ridibundus.

* Amplistoma longicolle. i. 87. 1. Ligula alternans. i. 133. 2.

* Taenia porosa. i. 165 . 90. 399. Larus tridactylus (Rissa).

- Ligula alternans. i. 133. 2. B b b 2 


\section{- 756}

40n. Lari spec. brasil.

* Echimorhynchus sphaerocephalus. i. 57. Apx. 401. Pelecanus Aquila.

* Ascaris spiculigera. oe. v. 45. Apx.

* Distoma cochleariforme, i, 74. Apx.

* Taenia. i. 113. Apx.

402. Pelecanus trasiliensis (Hálicus).

* Ascaris spiculigera. oe, v. 45. Apx.

Trichosoma, i. 16. 21.

$$
\text { 403. Pelecanus Carbo. }
$$

Strongylus tubifex, oe, 31.5 .

* Ascaris spiculigera, oe. V. 4S. 51.

* Echinorhynchus Hystrix. i. 75, 46.

* Distoma trilobum. i. 1af́ 60.

* - echinatum. i. 115. 106.

* Ligula interrupta. i. 133. 3.

* Taenia scolecina. i. 169. 93. 404. Pelecanus cristatus.

Ascaris spiculigera, oe. v. 48.51 .

405. Felecanus Onocrotalus.

* Ascaris spiculigera. oe. v. 48. 51. 406. Pelecanus pygmaeus.

Strongylus tubifex, oe, 31. 5 .

Ascaris spiculigera. oe. v. 48.51.

Distoma echinatum. i. 115, 106.

Ligula interrupta, i. 133. 3.

407. Pelecanus Sula.

* Distoma planicolle. i. 82, Apx.

Ascaris inflexa, i. 38.4 .

\section{Anas acuta.}

j'aenia sinuosa. i, 166,86 .

- trilineata, i. 166.87 .

4og. Anas Anser.

- Trichosoma brevicolle. coec, 13. 1.

* Spiroptera uncinatá, tüb. oe. 26. 16.

: Strongylus nodularis. oe. $\nabla$ : t. 35.18 .

Ascaris dispar. cóec. 45. 34.

Echinorhyachus versicolor, i. 74. 44.

Monostoma verrucosum, coec, rest, 84. 12.

Distorna lineare. nas. 79. Apx.

- - echinatum. 115. 106.

- Taenia lanceolata. t. 145. 4.

* - sinuosa, i. 99. Apx. 


\section{- 757}

Taenia infundibuliformis. t. 152. 33.

- setigera. $1.115 \mathrm{~S} .35$.

- fasciata, $0.15 \% .48$.

- Malleus. i, 162. 6S."

410. Anas Boschas domestiea

Filaria, co. e. 10, 48.

Ascaris inflexa, cr, t. 38. 4.

Strongylus tubifex. ve, 31.5 .

Echinorhynchus versicolor. i. 74. 440

Distoma (ovatum.) a. 121. 142 .

- lineare. tra. 113.102.

* - echinatum. i. 115. 106.

Ligula sparsa, i. 133. 4.

* Taenia infundioulifornis. t. 152. 33.

- Malleus, t. 162.68.

* - sinuosa. t. $166 . \delta 6$. 411. Anas Boschas fera.

Ascaris inflexa, i, 38, 4.

* Echinorhynchus filicollis. i. 71, 35.

$$
\text { - versicolor. i. 74. 44. }
$$

* Distoma oxycephalunı. i. 9S. 28.

* - echinatum. i. 115.105.

Ligula sparsa, i. 133. 4.

* Taenia sinuosa. i. 166.86 .

- - trilineata. i. 167.87.

- gracilis. i. 158. 55.

- Malleus. t. 162.68.

Ascaris dispar: i. 45. 34 .

$$
\text { 412. Sinas canaderesis. }
$$

Taenia laevis. i. 155. 42 .

$$
\text { 413. Anas Clanguia. }
$$

Echinorhynchus, i. 78. 78 .

Taenia laevis. i. 155.42 . 414. Anas clypeatá

Monostoma attenuatum: coec. $\$ 4.10$.

* Distoma ovatum. bu. 93. 2.

* - oxycephalum. i. 93. 28.

- echinatima. i. 115. 106.

Taenia laevis. i, 155. 42.

- trilineata. i. 167.87 .

$$
\text { 415. Anas Crecca. }
$$

Strongylus tubifex. oe, 31.5. 
Echinorliynchus, - i. 78. 78 :

Taenia laevis., i. 155. 42 .

Taenia laevis. i. $155.4^{2}$

Filaria, a. tr. i: 10" 47 .

$$
\text { 416. Anas cygnoides. }
$$

$$
\text { 417. Anas Cygnus. }
$$

* Taenia áequabilis. t. 155.43.

419. Anas ferind:

Distonja echinatum. i. 115. 106.

Taenia lanceolata, i. 145. 40

Ascaris. i. 71. 35:

419. Anas fuligula.

* Echinorhynahus filicollis. t. 71. 35 .

- - versicolor cr. 74. 44.

* Distoma globulus. i. 109. 81.

$$
\text { 420. Anas fusca. }
$$

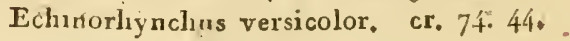

- Distoma. i. 120. 14i.

Taenia sinuosa. i. 165. 86.

421 Anas glacialis:

Spiroptera. tun. v. 29. 40.

$$
422 \text { Anas leucocephala. }
$$

Taenia laevis; i. 155. 42.

Echinorhynclius. i. 78.78 .

$$
\text { 423. Anas Marila. }
$$

Monostoma: i. 87. 29.

$$
\text { 424. Anas mollissima. }
$$

Echinorhynchus filicollis. 1. 71, 35 .

$$
\text { 425. Anas moschata. }
$$

Ascaris inflexa, i. 38.4 .

426. Anas Nyraca (leucophthalma).

Eclinorhynchus. i. 78. 78 .

Distoma echinatun, i. 115. 106.

Taenia lanceolata, i. 145. 4 . 427. Anas Olor.

Echinorlynchus striatus, i. 74. 43.

Taenia aequabilis, i. 155. 43.

$$
\text { 428. Anas Penelope. }
$$

Echinorhynchus, i, 78, 78 .

Distonıa echinatum, i. 115, 106.

Taenia, gracilis. i. $15 \mathrm{~S} .55$.

* - Malleus, i. 162; 6s: 


\section{- 759}

429. Anas Querquedula (Circia).

Trichosoma brevicolle. coec. 13. 1,

Monostoma verrucosum, coec, rect, $8 \%$, 12,

Amphistoma. i. 92. 21 .

Taenia Mallens. i. 162.68 .

- trilineata. i. 167.87.

430. Anas rufina.

Echinorhynchus, i. 78.78 .

431.' Anas segetum.

* Strongylus nodularis. i. 35. 1 s.

* Monostoma verrucosum. i. 84. 12. 432. Anas sponsa.

* Echinorhynchus filicollis, i. 71. 35 .

* Distoma delicatulum. fell. 99. 35 .

433. Anas strepcra.

Distoma echinatum, i. 115. 106. 434. Anas Tadorna.

Amphistoma isostomum! i. 89.5 .

Taenia. i. 174. 137. 435. Anatis sp, brasil.

- Taenia sinuosa, i. 99. Apx. 436. Mergus albellus (et minutus).

Strongylus tubifex, oe, 31.5 .

- Echinorhynchus bacillaris. i. 67, 15.

Amphistoma gracile. i. $\$ 9.6$.

Distoma,' i. 121, 143.

Ligula interrupta. i. 133. 3.

Bothriocephalus nodosus, i, 140, 15.

- Taenia tenuirostris. i. 156. 44.

* Strongylus tubifex, oe, 31. 5. 437. Mergus Merganser (et Castor). Ascaris, i. 56. 100.

* Amplistoma gracile. i. 89. 6. "

* Ligula interrupta. i. 133. 3.

- Bothriocephalus nodosus, i, 140, 15. Taenia Malleus, i, 16z, $6 \mathrm{~s}$.

* - tenuirostris. i. 156. 4f.

Dnb. Cestoideum. oe. 441 .

438. Mergus Serrator.

Strongylus tubifex, oe, 31.5 .

Ascaris. i. 56. 100.

Ligula interrupta, $233,3$. 


\section{$-760-$}

Bothriocephalus nodosus, i. $140,15$.

* Taenia tenuirostris, i. 156. 44.

Taenia, i 174.138.

439., Alca Pica.

- armillaris. i. $175,139$.

Dub, i. 188. 25.

\section{Classis III. Amphibia.}

\section{Ordo 1. Chelonii.}

440. Testudo Mydas (Chelonia).

* Ascaris leptura. i. 48.47.

* - sulcata. i. 48.50 .

* Monostoma trigonocephalum. †. i. 86. 20 r

* Disiona cymbiforme. ur. 9620.

- - gelimnosunı. i. 102. 48.

* - irroratum. v. 105. 63.

* Tetrarhynchus nizerobothrius tun. v. 131, 7 .

* Spiroptcra tortit. v. 25. 32.

441. Testudo orbicularis. (Enrys.)

Cucullanus, i, 21, 10 .

Distoma, i. 121, I4́i

* Polystoma ocellatum, fáu, 125, 2.

* Ascaris dactylura. cr. 40.12.

4.2. Testudo graeca.

* - holoptera. i. 53. 71 .

443. Testud'o indica.

* Ascaris sulcuitz. ti 48.50 .

444. Testudo tabulata.

* Ascaris gulosa. cr. 40, 11.

* $\therefore$ sulcata, t. 48.50 .

\section{Ördo II. Saurii.}

445. Crocodilus Lucius:

* Ascaris tenuicollis. v. tub. $47.45 . \mathrm{b}$.

446: Crocodilus Sclerops.

- Distoma pyxidatum. i. 69: Apx.

- Pentastoma proboscideum. pu. 83. Apx. 447. Lacerta (Monitor) Teguixini

I'hysaloptera retus̀a. v. t. 30. 4. Apx. 21, 


\section{$-T^{6} 1$}

* Strongylus galeatus. i. 25. Apx́.

- Ascaris spinicanda. i. fo. 13. Apx. 31s 448. Lacerta agilis.

Strongylus auricularis. is 33.13 .

* Distoma mentulatum. i. 103. 51.

Dnb. r. i. a. tub. pi. 18S. 29.

$$
\text { 449. Lacerta cacrulescens. }
$$

Distoma. t. 121. 145.

450. Lacerta maculata.

* Distoma mentulatum. i. 103. 51 . . 451. Lacerta margaritacea.

* Physaloptera abbieviata, v. i. 30. 3 .

"Ascaris extenuata. rect. - tub. mu. 47. 46.

\section{Lacerta muralis.}

"Dub. Cestoideum (Ditlyriáinm). hep. 188. 2S.

453. Lacerta siridis.

Strongylus auricularis. i. $33,13 . \ldots$

* Ascaris fallax. v. i. 43. 27.

* Dub. Cestoideum (Dithyridium). tub. hep. 188. 27.

454. Lacertae species hispanica.

* Taenia tuberculata. i. 150. 25.

455. Lacerta (Cordylus) orbicularis,

Taenia, i. 175. 40 .

456. Lacerta Iguana (Ig:ana tuberculata).

* Ascaris megatyphlon. coec. 47. 45.

457. Làreria (Gecko) Stellio Linn.

(Steltio Veterum. Tarentela Ital.)

* Ascaris êclinata. i. 47. 44.

* Taenia dispar. t. 150. 2i.

458. Lacerta. Stellionis sp. Urasil.

* Physaloptera retusa. ¿. 21. Apx.

459. Lacerta. Scinci sp. brasil.

* Ascaris spinicauda, palp. 31. Apx.

460. Laccria Apus. (Seps. Bipes.)

Trichocephalus echinatus. T. 18. \&.

\section{Ordo 11I. Ophidii.}

Strongtlus auricularis. t. 32. 13.

461. Anguis fragilis,

* Ascaris nigrovenosa. put. 43. 25.

* - brevicaudata. cr. 47.43 . 


\section{- $762-$}

Taeniz. i, 175. 141 .

462. Amphisbaena alba:

463. Amplisbaenae spec, brasil。

* Ascaris unguiculata. i. 3.3. Apx.

* Distoma Monas. 'i. Apx. 7r.

464. Boa canina.

* Dubinm, Nematoideum. i. e. 12I.. Apx。 465. Coluber atrosirens.

* Echinorhynchus cinctus. pi. 66. 14.

Filaria. oe. 10. 49 .

466. Coluber austriacus.

Distoma. i. 121. 147 .

46:- Coluber murorum.

Distoma. i. $121,146$.

468. Coluber Natrix.

Ascaris brevicaudata. i. 47.43 .

* Distoma Naja. pu: 99. 32.

* - mentulatum. i. 103.5I.

Dub. tub. hep. 199.30.

Dub. (Nematoidermi) i. 189. 31.

469. Coluber olivaceus.

* Strongylus. v. $1 \$ 9 \cdot 32$.

* Echinorhynchus oligacanthoides. hyd. pi. 64. 7. Apx.52. - f70. - Coluber quadrilineatus.

* Ascaris cephaloptera. i. 46. Apx.

* Echinorhynchus oligacanthus. pi. 64. 6.

47.1. Coluber tesselatus.

'* Strongylus denudatuse pu. 34. 14.

Distoma. i. 121. 147.

472. Colubri species brasilianae.

* Strongylus costatus, i. 24. Apx.

* Ascaris mascula. i. 32. Apx.

* - auriculata. i. 36 . Apx.

- Echitorhynchus oligacanthoides. i. 52. Apx.

- Taenia racemosa. i. go. Apx.

* - i. 114. Apx.

* Dub. (Nematoideum). i. 122. Apx.

Filaria. i. 10. 50.

473. Colubri species Caroliniana.

Distonı. fau. 121. 148.

Ascaris. i. 56.101. 
475. Vipera, Berzs.

* Distoma crystallinum. hyd. co. 100. 36 . 476. Vipera Redii.

Strongylus. i. $37.3 \%$

* Ascaris cephaloptera. i. 52. 70.

* Echinorhynchus cinctus. pi. 66. 14.

Dub. tub. hep. i. 189. 30.

477. Crotalus Durissus.

Trichosoma. i. 16. 22.

Pentastoma proboscideum. a. pu. 124. 5o

478. Hydrus caspius.

Cucullanus. i. 21, 11,

\section{Ordo 1V. Batrachii.}

Ascaris leptocephala. v. 46. 40.

479. Proteus anguinus.

* Dub. Nematoidenm. i. - hyd. pi. I89. 35. 480. Salamandra (Triton) lacustris.

Strongylus auricularis. i. 3.3. 13.

481. Salamandra (Triton) palustris.

- Ascaris leptocephala. v. 96. 40.

* Amphistoma unguiculatum. i. 91. 16.

Strongy hus anicila 482. Salamandra atra.

Ascaris brevicandata. i. 47.43 .

Echinorhynchus falcatus. i. 68. 21.

Distoma crásicolle, rect. 102. 46.

Taenia dispar. i. 150. 2/.

483. Salamandra maculata.

* Ascaris brevicandata. rect. 47.43 .

- Distoma crassicolle. i. 102.46 .

Taenia dispar. t. 150. 24.

484. Bufo cruciatus (R. Calamita).

* Ascaris leptocephala. hyd. spl. -46. 40. 4S5. Bufo fuscus.

Strongylus auricularis. i. 33. 13 . Ascaris nigrovenosa. pu. 43. 2S. Ascaris brevicaudata. rect. 47.43. Taenia dispar. i. 150. 22. 486. Bufo igneus (Rana, Bombina.)

Strongylus aniricularis. i. 33. 13. Ascaris nigrovenosa. $\mathrm{pu}, 4 \mathrm{z}^{2} \cdot 28$. 


\section{$-764-$}

Ascaris Ieptocephala. i. 46. 40.

* - brevicaudata. 47.43.

* Pchinorlivuchus Ixaeruca. i. 67. 18:

* Mnnusioma ellipticum. pu. 8.́. 3.3.

* Anphistome subclavatum, ur. 95. 1/4.

* Discoma cygnoides. ur. o6. 10.

* - crystallinum. hyd. hep. mes. 100. 36. 487. Bufo viridis (R. variabilis.)

Strongylus auricularis. i. 33.13.

Ascaris nigrovenosa. $\mathrm{pu}^{\circ} 4$ 43. 28.

* - brevicaudaia, hyd. oe. 47.43.

* Distoma crystallinum. lygd. hep. mes. 100. 36 .

* - clarigerum. 1. 103. 5?.

Polystoma integerrimura. ur. 125. 1.

- Taenia dispar. i. 150. 24. 488. Bizfo vuigaris (s. cinercus. $R$. Bufo).

- Strongylus auricularis, t. 33. 13.

" Ascaris nigrovenosa. pu. 43. 4S.

* - brericaudara. cr. 47. 43.

Echinorhynchus Haetnca. i. 67. 19.

Monostoma ellipticum. pu. Sf́ 13.

Anphistoma subchavatum. cr. 90. 14,

Distoma clavigerum. i. 103. 52.

* Taenia dispar. i. 150.24 . 489. Rana cornuta.

- Ascaris foecunda. i. 34. Apx. 490. Rana esculenta.

- Filaria rubella. hyd. pi. 5. 10.

- pell. 10. 51 .

Strongylus atricularis. i. 33.13 . Ascaris nigrovenosa. pu. 43. $2 \mathrm{~S}$.

- acuminata. t. 40. 14.

- Echinorhynchus Haeruca. i. 67. 18.

* Amphistoma urnigerum. rect. - tryd. vi. 8g. 8.

- subclavatam. cr. 90. 14.

- Distoma cygnoides. ux. 96. 19.

- - variegatum. pu. 99.33.

* - crystallinum. fell. - Iyd. nies, 100, 36.

Polystoma integeriinum. ur. 125. 1.

* Filaria rudelìa. v. 5. 10.

491. Rana temporaria.

* Strongylus auricularis. t. 33. 12.

* Ascaris nigrovenosa. pu 43. 25 . 


\section{$-765$}

* Ascaris acuminata. t. 40. 14.

* Echinorhynchus Hacruca. i. 67. 19.

- Amphistonia subclavatum. cr. go. 1f.

- Distoma crestallinum. fell. - liyd. hep. 100. 36.

- clarigernm. i. 103. 52.

- - cylindraceum. pu. 106.66.

* Polystoma integerrimum. ur. 125. 1. \{.?2. Rana Pipa.

* Monostoma sulcatum. t. S6. 22.

* Dub. (Nematoid.) hyd. mes. 1\$9. 33. 495. Rana musica brasil.

* Strongylus subaunicularis. i. 26. Apx.

* Distoma Linguatula. i. 7o, Apx.

* Dub. Nemat. i. 123. Apx.

* Distoma Lingnamlá. t. 100. 35. 49\%. Ranate species brasilienses.

- - repandum. i. 73. A px.

- Nemat. Dub. 123. Apr.

40.. H)/a arborea.(Rana L.).

Strongylus auricularis. i. 33.13.

Ascaris acutninata. to $40.3 \%$.

Amphistorna subclavarum. cr. 90. 14.

Distoma clavigerum. i. 103. 5,2." $11 x .121 .149$.

- Taenia dispar. i. 450.24.

- Ascaris foecunda, cr. 34 . Apx. 49. Hylae sp. Grasil.

\section{Classis IV. Pis.ces.}

\section{Ordo 1. Cyclostomi.}

Ligula simplex. a. 134. 6 .

497. Petromyzon branchialis.

\section{Ordo II. Plagiostomí.}

498 Torpedo marmorata. (T. marm. et Galvani Riss.)

- Ascaris, v. 56. 102.

* Distoma appendiculatum. v. 110.85 .

* Scolex polymoriphus. i. 129. 1.

* Bothriocephalus auriculatus, i. 141. Is. 


\section{$-766$}

* Eotfriocephalus coronatus. i. 1/11. 20. 499. Torpedo ocellata. (T. vulgaris et unimaculata Riss.)

* Bothriocephalus punctatus. v. 138.

* - coronatus. i 141. 20.

Dub. i. 190.36 . 500. Torpedo (persica.)

501. Raja Aquila.

Dub. Nematoid. v. i. 19 n. 39 . 502. Rajà Batis.

Bothriocephalus coronatus. cr. 141. 20.

Dub. Nemat. 190. 37 . corollatus. cr. 142. 23 .

503. Raja clavata.

* Ascaris succisa. i. 52. 6g.

* Distoma appendiculatum. corp. 110. 85. 504. Raja fullonica.

Dub. Nemat. $\checkmark .190 .38$.

$$
\text { 505. Raja. Muraletus. }
$$

Ascaris succisa. i. 52. 6ig.

Scolex polymorphus i, $12 \delta$. I.

506. Raja oxyrhynchus.

* Tetrarhynchus scolecinus. pinn. pect. 131. g.

Ascaris. i. 56. 103.

507. Raja Pastinaca.

* Bothriocephalus tumidulus. i. 1ł1. I9.

coronatus. i. 141. 20.

508. Raja Rubus.

Bothriocephalus coronatus. i. I 141, 20.

509. Squalus Acantlias.

Scolex polymorphus. i. 128 . 1 .

Bothriocephalus paleaceus. cr. $\$ 42.24$.

Dub. Nemat. i. 190. 4I.

510. Squalus Catulus.

511. Squalus Centrina.

* Tetrarhynchus scolecinns. carn. 131. g.

- Ascaris rotundata. vo t. 39.8 .

512. Squalus Galeus.

- Distoma niegastomum. v. 102. 49 .

- Bothriocephalus auriculatus. i. 141. 19.

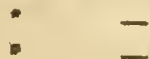

verticillatus. i. 42.22.

uncinatus, i. 1/2. 21.

* Ascaris rotundata. . 39. 3 .

513. Sqíalus glaucus. 


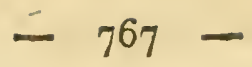

Bothriocephalus, cr. $x .43 \cdot 25$.

Dub. i. 191, 42.

514. Squalus griseus.

Dub. hep. 191. 4\%.

515. Squalus Mustelus.

516. Squalus Spinax.

Bothriocephalus corollatus. cr. 14\% 23. 517. Squalus Squatina.

* Botbriocephalus coronatus. i. 141. 20.

Dub. Nemat. i. 190. 39.

corollatus. cr. 142.23 .

518. Squalus stellari.

- Tetrarliynchus megacephalus. a. 129. 1.

* - scolecinus, carn. 131. 9.

* Bothiocephalus coronatus. i. 141. 20.

Ascaris. i. 56. 10..

519. Squali species incertae.

* Tetrarhyncluus grossus. 129. 2.

$$
\text { - irep. 132. } 11 .
$$

Dub. co. 191. 44.

\section{Ordo III. Schismopnoei.}

* Ascaris rigida. i. 51.61.

520. Lophius piscatorius.

* Echinorhynchus Pumilio. i. 66. I1.

$$
\text { i. } 71.32 \text {. }
$$

* Distona gracilescens. 1. 111. 91.

* Scolex polymorpluns. i. 12S. 1. Tetrarhynchus tenuicollis. pi. 130. 5. Bothriocephalus, i. 143.26.

\section{Ordo IV. Eleutheropiomi.}

521. Accipenser Helops.

Ascaris. rect. 56. 105.

522. Accipenser Huso.

Cucnllanus. i. 22. 12.

Echinorhynchus tereticollis, i. 72.35 .

$$
\text { i. } 78.80 \text {. }
$$

523. Accipenser Ruthenus.

Echinorhynchus. i. 79. 81.

$$
\text { 524. Accipenser Sturio. }
$$

Cucullanug. i. 22, 12. . 


\section{$-768$}

- Opliostona spháerocephalum. cr. 61. 3.

- Echinorhynchus tereticollis. i. 72. 36.

i. 79.82 .

* Monostoma foliaceun?, a. 83, 4.

* Distoma appendiculatum, v. na. I10. 85. :

* - hispidum. i. 11\%. 120.

525. Syngnathus Hippocampus.

* Ascaris. i. 56. 107.

* Distoma tumiuluium, i. 95. 14.

* Triaenophorus nodulosus, i. 135. r. 526. Syngnathus ficus,

Scolex polymorphus. i. 12S. I.

527. Syngnuthus pclagicus.

* Distoma labiatum. hep. 107. 7S.

* Dub. pi. 191. 46.

\section{Ordo T. Teleobranchii.}

528. Orthragoriscus Mola (Diodon. Limn.).

* Ascaris. pi. 56. 106.

* Monestoma filicolle mu. 57.30 .

- Distoma contertum. bra. 118.122.

* - nigrollarum. (Schisturus.) v. i. 118. 123.

* Tristoma coccineum, bra. 113. 1.

- Bothriocephalus microcephalus. v, i. bra. 138.7.

* Anthocephalus elongatus. hyd. mes. hep. 177. 1. 529. Diodon (Nootkensis.)

Tristoma maculatum. corp. 123. 2.

$$
\text { 530. Cyclopterus Lumpus. }
$$

* Echinorhynchus gitbosus. mes. 73. 40.

Distoma. i. 121. 150.

Scolex polymorphus. i. 128. 1 .

531. Centriscus Scolopax.

* Ascaris, pi, 57, 10 .

\section{Ordo VI. Apodes.}

* Cucullauus elegans. i. 19. 1.

532. Muraena Anguilla.

* Ascarrs labiata t. 49. 54.

"Liorhvichus denticulatus. v. 62. 3 . Echunorhynchus globutosis. i. 65. 10.

* Distoma polymorphus. i. (a, ?) 95. 16. 


\section{- $769-$}

* Bothriocephalus claviceps, i. 136. 3. Dub. cestuid. 17)1. 47.

$$
\text { 5.3.3. Mureuna Cassini (myroides). }
$$

- Cucrellanas foverilaras. pi. z'. i).

* Mionostoma Crincibulume i. 83. 6.

* Bothrioceplalus claviceps. i 136.3

53i. Muraena Conger. .

- Ascaris labiata. cr. 49. 5 i.

* Monostoma crucibuluri. i. \$3. 6.

* Distoma mforiride. v. 110.87. Nub, tub. pi. 1y2, 4S.

535. Muraena Helena.

* Cucullanus foreolatus. i. 21. 6 ,

* Distoma grandiporm. v. 110. 88 . Nub, tub. pi. 19249.

\section{Muraena Myrus.}

* Ascaris labiata. pi. 49. 54 .

Dub. tub. pi, 102. 50.

$$
\text { 537. Muraena serpens. }
$$

5.39. Arraenae spec. Brasil.

* Distoma tubulatum i, 67. Apx.

539. Symbrancius laticaudis.

* Filaria cystica. hyd. abd $2 A p x$.

540 Trichiurus leprurus.

* Anthocephalus interruptus. hyd. pi. 178. 5.

- 5 ir. Anarrhichas Lupus.

Distona incisum. v. 94. 11 .

- i. $121.15^{1}$.

542. Ammodytes Cicerelus:

* Tetrarliynchus gracilis i. 132. 10.

* Ascaris. pi. 57. 109.

* Distoma appendiculatum. i. 110. 85 .

* Scolex polymorphus. i, 12g. 1 .

544. Ophidium Vassalli.

- Distoma appendiculatum. i. 110. 85.

545. Ophidium imberbe (Notopterus Riss.)

- Ascaris. pi. 57. 110

* Distoma sinuatum. i. 97.26.

* Ascaris. pi. 192. 51.

5.6. Stromateus Fiatola:

* Distona crisıarrm v. 117. 119.

* Scolex polymorphus. pi. 128: 1 . 
547. Xiphias Gladius.

* Ascaris incurva. br. v. duod.-tub. pi, 51.63.

* Distoma dendriticum. i. 93. 4. Tristoma coccineum. bra 123. 1.

- Tetrishynchus attenuatus. a. bra. 130. 3 .

* Bothriocephalus plicatus. rect. tub. 136. 2.

\section{Ordo VIT. Jugutulares.}

549." Uranoscopus sçaber.

- Filaria globiceps, ov. te. pi. 7. 19.

* Ascaris. pi. 57. 111

- Distoma capitellatum. fell. 99. 34 .

- $\quad$ fallax. v. 117. 115.

Scolex polymorphus. i. 128.1 .

\section{Trachinus Draco:}

* Ascaris constricta. pi. 39. $7^{\circ}$

* Echinorhynchus gibbosus. mes. 73. 40. 550. Trachinus lineatus.

* Ascaris constricta. pi. 397. 551. Gadus Aeglefinus.

* Ascaris clavata. fall. 51.6ł. Echinorhynchus Acus. i. 71. 32 .

Distoma simplex. i. $97 \cdot 22$.

Dut. hyd. ne. cer. $19^{2} \cdot 52$.

\section{Gadus barbatus.}

Filaria piscium. a. 218. 52 .

Ascaris clavata. v. 51.64 .

Echinorhyuchus Acus. 1. 71. 32 .

Distona scabrum. v. 11S. 121.

Bothriocephalus. i. 143 " 28 .

553. Gadus Brosme.

Filaria piscium. hyd. pell. 218. 51 . 554. Gadus Callarias.

Filaria piscinm. 2. 218. 52.

Cucullanus foveolatus. i. 21.6 .

- Echinorhynchus Acus. i. 71. 32 .

tereticollis. i. 72.36 .

Bothriocephalus. i. $143 \cdot 30$.

555. Gadus Chalcogrammus. .

Dub. v. app. hep. 193. 55 .

556. Gadus Lota.

Cucullanus elegans. i. 19. 1. 
Ascaris tenuissima. i. 52.66 .

$$
\text { mucronata. i. } 52 \text {. ii }
$$

- Echinorliynclius tereticollis. i. $72 \cdot 36$.

$$
\begin{aligned}
& \text { - nolulosus. i. } 7237 . \\
& \text { - } \\
& \text { - } \\
& \text { angustatus. i. } 69.19 .
\end{aligned}
$$

* Bothriocephalus rugosus. app. (?-hyd. Jep.) 137. 16. 557. Gadus liescus.

Echinorhyuchus Acus. i. $71 \cdot 3^{2}$

\section{Gadus mediterraneus.}

* Distoma fulvum. i. 9.9. 27.

- Ascaris. a. 51. 64.

$$
\text { 559. Gadus Merlangus. }
$$

- Echinorliynclins Acus i. 71. 32 .

* - terericollis. i 72. 36 . 5 fio. Gadus Merluccius.

* Ascaris. pi. - app. 192. 53.

Echinorhynchus Acus. 71. 32.

* Scolex polymorplus. i. 128 1.

* Bothriocephalus crassiceps. i. 1,39. 13.

* Ascaris. pi. 5 ? 112.

* Bothriocephalus punctatus i 1,8.11. 56. Gadus Molva.

Filaria tub. v. 218. 52 .

Echinorhyuchus Acus. i. $71 \cdot 32$.

- Distoma fulvum. i. 98. 27.

* - furcatum. i. 10772.

- - scabinm, i. 118. 121 . 563. Gadus Morhua.

Cucullanus foveolatus. i. 21. 6 .

Tetrarhynchus. 458 . 14.

Bothioceplalus, i. 143. 29.

Dub. cer. ne. 192. 52.

564. Gadus Mustela.

Bothriocephalus rugosus. app $1,7.6$. $56 ;$. Gadus Pollacitius.

Taenia, an Bothriocephalıs. i 175.142. 566. Gadus virens.

Echinorhynchus Acus. i. 71. 32 .

Dub. cer: 192.52 . 567. Gadus Wachnia.

Echinorbyuchus: i. $79: 83$ : 


\section{- $772-$}

Distoma. i. 122.152.

Dub. vert. 193.53 .

568. Blennius cornutus.

* Distoma divergeas. i. 97.24.

569. Blennius Gattorugize.

* Distoma divergens. i. 97. 24.

570. Elenñius ocellaris.

Scolex polymorphus. i. 128. 1.

571. Blennius Phycis.

* Filaria globiccps. ov. 7. 19.

* Ascaris. pi. 57. 113 .

* Cacullanus forcolatus. pi. 21. 6.

572. Blennius raninus.

Echincrhynchus tereticollis. i. 72. 36 .

573. Blennius tentaculatus.

* Distoma divergens. i. 97. 24.

574. Blennius viviparus.

* Ascaris ancta. i. pi. bra. 50. 57.

* Echinorhynchus tereticollis. i. 72.36 .

Distoma granulum. i. 106. 67.

- divergens. i. 97. 24 .

\section{Orda VIII. Thoracigi.}

575. Cepola rubescens.

* Distoma filiforme. i. 112. 94. Scolex polymorphus. i. 128. 1 . Bothriocephalus. i. 144. 31 .

* Dub. Nemat. pi. 193. 56 .

576. Coryphaena Equiselis.

* Distoma tornatumi. v. 7S. Apx.

* Tetrarhynchus macrobothrius. a. 131. 7 .

577. Coryphaena Hippuris.'

Ophiostoma lepturum. i. 61. 5 .

* Echinorhynchus Pristis. i. 58. Apx.

- Distoma furcatnm. i. 77. Apx.

- - tornatum. 7 . 78. Apx.

- bra. vi. 122.153.

* Tetrarhynchus macrobothrius. mu. fau bra. 86. Apz.

- $\quad$ discophorus. hep. 85. Apx. 578. Coryphaena Novacula (Labrus).

* Ascaris. pi. 58. 125.

579. Gobius Aplya.

* Echinorhynchus globulosus. i. 65. 10. 


\section{- 773}

Ascaris. i. $57 \cdot 314$.

\section{5so. Gobius fozo.}

Echinorhynchus globulosus. i. 65. 10 \$

Scolex polymorphus. i. 128 . i.

Scolcx polymorphus. i. 128.1 .

$$
\text { 5\$2. Gobius nigers }
$$

* Echirorhynchus globulosus. i. 65.10:

* Scolex polymorphus. i. 128. 1.

* Scolex polymorphus. i. $12 S .1$.

583. Lepadogaster Gouani.

$$
\text { 584. Gottus Gobio. }
$$

Echinorhynchus angustatus. i. 68. $19 \mathrm{n}^{\circ}$. tereticollis. i, 72.56

Scolex polymorphus. i. 128. 1 .

* Triaenophorus nodulosis. hep. 135. 1. 585. Cottus Scotpizs.

".Ascaris angulata. mes. 4q 30.

Echinorhyuchus Acus. i. 71. 32.

$$
\text { * }
$$

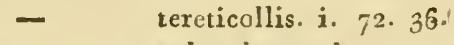

Distoma divergens. i. 97.24.

sphaericus. pi. $73 \cdot 35$.

- granulum. i. 106.67.

* Bothriocephalus punctatus, i. 13s. 11. - solidus. fau. 139.14.

Dub. i. 193.57.

586. Scorpaena massiliensisa

597. Scorpaena Porcus:

- Scolex polymorpirus. i. 128. 1 .

588. Scorpena Scrofei

* Ascaris. i. 57. 115.

Echinorhynchus. 79. 55 .

Distoma. i. 122. 154 .

- Bothriocephalus angustatus. i. 139. 12.

* Distoma appendiculatum. i. 110, 85

5Sg. Zeus Aper.

* Dub. Nematoid. pi. 194. 59.

Filaria. hep, i. 11. 53.

$$
\text { 590. Zcus Faber. }
$$

- Ascaris. V. 57. 116.

Echinorlyynchus. i. 79. 86.

* Distoma caudiporum. i. 96.18. 


\section{- $774-$}

Scolex polymorphus, i. 128, 1 .

* Dub. Nemat. mes. 193. 58 .

591. Lampris guttatus (Chrysotosus, Zeus. Luna.)

* Monostoma tenuicolle. carn. 8517.

592. Pleuronectes Busciia

* Botluriocephalus punctatus. i, 138. 11.

* Dub. Nentatoid. pi. 191.61.

593. Pleuronectes. Flesus.

Ascaris collaris. i. 52,65.

* Cucullanis heterochrors. i. 21,9.

* Echinorliynchus angustatus. i. 69.19.

* - tereticollis. v. 72. 36 .

* Distoma Atomon, v. 95. 15.

59\%. Pleuronectes Linguatula.

* Asciric pi. 5S 11 S.

* Echinorhy nclurs globulosus. i. 65. 10.

* Distonra appeniticulatum. v. 110. 85. Scolex polymorphus, i. 128. 1.

* FiInia fuscr. a, 5. 8.

595. Pléronectes mancus.

* Ascaris collaris. pi. 152. 65 .

- Distoma areolatum. i. 111. 9o.

* Cucullanus alatus, i. 20. 3.

596. Pleuronectes maximus.

* Ascoris collario. i. 52.65.

Echinorhynchus i. 79. 87.

* Distoma appendiculatum. i. 110. 85 .

* Scolex polymorphus. mes. 128 . 1 .

Tetrarhynchus, li. 137. 12.

* Bothriocephalus punçtatus. v. i. 13s. 11. 597. Pleuronectes Passer.

* Cucullanus minutus. i. 21. 8. -

* Distoma appendiculatum. i. 110, \$5. I 59s. Pleuronectes. Pegosa.

* Tetrarhynchus teunicollis. V. 130. 5.

* Bothriocephalus punctatus. i. 138. 11.

599. Pleuronectes Platessa.

* Cucullanus. i. 22. 13.

Echinorhynehus. i 79. SS.

Distoma arcolatum i 111. 9o.

Scolex polymorphus i 128.1 .

6oo. Pleuronectes Platessoides.

Filaria piscium, mu, a. 219. 52. 


\section{- 775}

Echinorhynchus. v. so. $\$ 9$.

601. Pleuronecles Solea.

Cucullanus. i. 22. 14.

Ascaris, i. 5s 117.

* Echinorhynchus angustatus. i. (ig. 19:

* Distoma microstonum. i. 104. 29.

- appendiculatmm, i. 110.85.

- Bothriocephalus punctatus. i. 13S. 11.

Dub. Cest. 194. Go.

602. Pleuronectis $s p$. italica (Sanchetto).

* Bothriocephalus punctatus. i. 138. 11.

603. Chaetodontis sp. Urasil.

* Distoma incomtum, i. 76. Apx.

$$
\text { 6o.. Sparus Alcedo. }
$$

* Anthocephalus Granulum. hyd. pi. I78. 3:

* Dub. Nemat. pi. 194. 63. 605. Sparus Aurata.

Filaria ovar. 10. 54.

* Distoma pallens. i. 111. 89. 6o6. Sparus Boops.

* Ascaris. pi. 5s. 120.

- Distoma Ascidia. i, 109. 77.

* Scolex polymorphus. i. 12S, 1.

$$
\text { 607. Sparus Dentex. }
$$

* Echinorhynclus globulosus. i. 65. 10a

* Distuma carnosum r. 93. 6.

* - fuscescens. i, 113. 99.

Dub. tub. i. 12a. 194. 62:

6os. Sparus erythrinus.

Distoma, i, 122. 155 .

$$
\text { 6og. Sparus Maena, }
$$

Cucullanus. i. 22. 16 .

- Ascaris. i. 58, 119.

610. Sparus Melanurus:

* Dub. pi. 194. 63.

611. Sparus Mormyrus.

* Dub. pi. 194. 63 .

$$
\text { 612. Sparus Pagrus. }
$$

- Distoma Ascidia. i. 108. 77.

$$
\text { 613. Sparus Raji (Brama Raji). }
$$

* Echinorhynchus vasculosus. a, i. $7 \%$. 49 .

* Monostońra filicolle. vert. 85. 18.

- Scolex polymorphus. i, 128.1. 


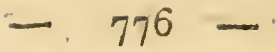

* Gymnorhynchus reptans. carı. 12 1. 1. Apx. 84.

* Tetrarhynchis discophorits. bra. tun. v. 130. 40

* Anthocephalus gracilis. byd. pi. 17s. 2.

614. Sparus Salpe.

- Monostoma orbeculare. i. 83. 5.

* - capitellatum. i. 83. 7.

* Distoma fractum: i, 1n7. 73 .

Ascaris. i. $55 \cdot 122$.

615. Spartis Smaris.

Distonra. i. 122. 15 \%

616 Spari n. sp. Ttalis Schiandra.

Scolex polyznorphus. i. 128. 1.

6\%. Sparin sp. Italis Spiere.

* Ascaris. pi. 58.121 .

6rs. Spari sp. brasil.

* Anthocephalus macromins. hy. pi. 178. 4.

* Ascaric. pi. 58. 1\%3.

-6 9 Labrus Eynaedus.

* Distoma pulchellum. i. 9f. 10.

* Ascaris pi. 58. 12 f.

Ba: Labrus luscus.

* Distoma genu. i. 107.74.

* Scolex polyniorphus. i. pi. 128. 1.

621. Labrus melops.

- Distoma fasciatam. i. 97.25.

622. Labrus niloticus.

Dub. Nematoid. v. 194. 6 f.

623. Labrus rupestris.

Distoma. i. 122. 156 .

624. Labrus Tinca.

* Ascaris crassicanda. i. 50. 56 .

Echinorlynchus. i. 80. 90.

* Distona fasciatum i. 97. 25.

$\therefore$ 625. Sciaena, Aquila.

* Echinorhynchus. pi. So. 91.

* Anthocephalus elongatus. hyd. pi. 177. 1.

Ascaris, pi. 58. 126.

626. Sciaena Umbra.

- Echinorhynchus globulosus. i. 65. 10.

* Distonna inbarium. i. 111. 93.

Uub. tub. i. na. 134. 65.

627. Perca Asper.

Distoma no.?ulosum. i. 113. 100. 


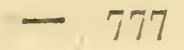

62s. Pirca cernua.

Cucullanus elegans, i. 19. 1.

- Echinorhynclius tereticoliis. i. 72. 36.

Distoma nodulosumi. i. 113. 100.

angustatus. i. $6 S \cdot 19$.

Taenia ocellata. i. 149. 22.

629. Perca cirrosa.

* Cucullanus abbreviatus, i. 21. 7.

* Distoma affine. i. 110.56.

* Dub. pi. 19f. 66.

6,30. Perca fuviatilis.

* Cucullanus elegans. v. app. 19. 1.

- Ascaris truncatnla i mu. - hyd. hep. 49. 55.

- Echinorhynchus angustatus. i. 6s. 19.

* - tereticollis. i. 72, 36.

* Distoma nodulosum i. app. 113.100.

- globiporum. i 96.17 .

Scolex polymorphus. i. 12S. 1 .

Ligula sinplicissima. a 15 f. 6 .

* Triaenophorus nodulosus. i.-hyd. hep. 135. 3*

* Taenia ocellata, i. - hyd. hep. 140. 22.

631. Perca Lucioperca.

- Cucullanus elegans. v. app. a. 19. 1.

* Ascaris truncatula. pi. 49. 55.

Echinorliynchus angustatus, i. $69 \cdot 19$.

Distoma tereticolle, v. 102, 47.

* - nodulosum. i. app. 113. 100.

- truncatunt. v. 122, 159.

Ligula simplicissima. i. 134. 6 .

632. Perca marina (Cabrilla).

* Distorna fasciatum, i. 97. 25.

* - microsoma, i. 1og. so.

* Dub. pi, 195. 67.

Filaria piscium. a. 218.52.

\section{Perce nórvegica.}

Cucullanus i. 22. 15.

Taenia occllata. t, 149. 22.

- octolobata. i. 105. 80.

634. Perca Zingel.

Cucullamus elegans. i, 19.1.

Distoma nodulosum, i. 113. 100.

635. Gastcrostcus aculeation

Ascaris, i, 59. 327. 
* Fichinnrhynchus angustatus. i. 68. 19.

* Ninostomi carrophyllinum. "i. 82. 1.

* iriscoma appenticulacmm, v. 110. 85.

* Tinanuphoris modnfosus. hyd. hep. 135.1.

* Buthriocephalus solidus. a 139. 14.

* Toenia filicollís, i. 1\$8. $20-444$. Lub. v. é.-liyd, v. 19 .69. 636. Centronotus glaucus.

* Monostoma galeatum, i. 8 fo. 2i.

* Authocephalus elongatus. hyd. à. 177. 1.

* Dub. Nemat. pi. 195. 6g. 6.37. Scomber Colias.

* Cucullanus melanocephalus. i. 20.5.

* Echinorhynchas Pristis. i. 75. 47.

* Distoma excisirm. i. 112. 45. .

* Aathocephalus Granulum, hyd. pi. 178. 3.

* Dub. Nemat. pi. 195. 70. 638. I Scomber Pelamis.

*Echinorhyuchus terebra, v. 55. Apx.

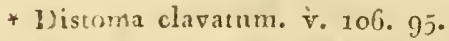

Tetrarhynchus macrobothrius. mu. 131. 7 . - 639. Scomber Rochei:

* Cucullanus melanocephalus. i. 20. 5. * Anthoceplialus gracilis. hyd. pi. 178. 2. 640. Scomber Sarda.

* Cucullanus melanocephalus. i. 20: 5 .

* Tetrarhy uchus inegabothrius, tun. v, i. 130.6.

* Dub. Nemat. pi. I95. 70 .

6\{1. Scomber Scomber.

* Eclinorhynchus Pristis. i. 75. 47.

* Distoma excisum. v. 112. 95 .

- Dub. pi. 195. 70, 6q2. Scomber Thynnus.

- Trichocephalus gibbosus. fell. 10. Apx.

* Cucullanus melanocephalus. i. 11. Apx.

* Distoma clavatum. v. 75. Apx. Polystoma duplicatum. bra. 1.5. 4 .

* Anthocephalus elongatus. hop. hyd. 115. Apx.

6.3. Caranx trachirus (Scomber Lim.).

* Distomia laticolle. i. 117. 116.

* Anthoceplialus Granulum. hyd. pi. 178. 3.

- Dub. Nemat. i. 195. 71. 


\section{$-779-$}

* Dub. Nemat. 195. 71 .

6if. Caranx imperialis.

* Ascaris. i. 59. 128.

* Distoma furcatum, i. 107. 72 .

6i6. Mullus Surmuletus.

- Distoma furcatum. i. 107. 72.

647. Apogon ruber (Afullus inzlerbis).

- Distoma apertum. i. 10879.

* Scolex polymorplins. i. 12S I.

648 Trigla adriatica.

Echinorhynchus simplex. i. 68. 20.

$$
\text { - i. so. } 92 \text {. }
$$

* Distoma appendiculatum. i. 110. 85 . Borhriocephalus puuctatus. i. 238. 11.

* Dub. Nemat. i. p. 195. 73.

$$
\text { 649. Trigla Cuculus. }
$$

Distoma. i. 122. 157.

$$
\text { 6;0. Trigla fasciata. }
$$

* Anthocephalus macrourus, hyd. pi. 543.

$$
\text { 651. Trigla. Gurnardus. }
$$

Echinorhynchus simplex. 68. 20.

Distoma soleatforme. v. Inf. 55.

652. Trigla Hirundo.

- Distoma appendiculatum. v. 110. 55 .

- Dub. Nemat. pi. 195. 72.

* Ascaris. pi. 59. 129.

$$
\text { 653. Trigla Lyra. }
$$

654. Triglae sp. brasil.

* Dub. (Anthocephalus.) a. 124. Apx.

\section{Ordo 1X. Ábdominales.}

Ascaris. i. 59. 130.

\section{Cobitis Barbatula.}

Echinorhynchus cliviceps. i. 65. 9.

Caryophyllaeus mittabilis. i. 12\%. 1.

Bothriocephalus. i. 14i. 32.

656. Cobitis fossilis.

* Distoma transversale. v, 95. 13.

$$
\text { 657. Cubiris, Taenia }
$$

Echinorhyuchus claviceps. i. 65. 9.

Distoma transversalc. v. 95. 13. 


\section{- 780}

Caryoplyyllaens mutabilis. i. 127. 1:

Iignla simplicissimi, a. 134. 6 .

0ij8. Silurus Glanis.

- Cucullanns truncalus. i. 20, 2. Ascuis. i. 59. I31.

- Echinorliynchus aregustatus. i. 68. 19:

$\rightarrow$

* Hiscrima so:ulosuni. i. 111. 92 .

- *Taeria uscula'a. t. 150. 26

Lignia simplicissima. a. 154. 6.

Dub. Nernat. v. 196. 7\%。

6.9. Siluri sp. brasil.

Anthocephalus elongatus, a. tun. i. 115. Apx.

66o. Salmo Albula.

Ascaris.' tub. v.-bra. 59. 1.32.

661. Salmo alpinus.

Distoma seriale. ren. 97. 32.

- lanrcatum. i. 11j. 101.

* Botliriocephalus infundibuliformis. app. 137.5.

Taenia longicollis. hep. hyd. 149. 21.

662. Salmo arcricus.

Filaria. v. c. 218.52.

Bothriocephalus i. 144. 34 .

$$
\text { 663. Salmo Carpio. }
$$

664. Salmo Eperlanus.

Echinorhynchus. tub. a. 80.94 .

Nonostoma gracile, a. 82. 1.

- Taenia longicollis. i. 149. 21.

- a. 175. 144.

Dub. Nemat. tub. a. 196. 75 .

665. Salmo Eriox.

- Distoma liyalinumi, i, 105.61.

Bothriocephalus.' i. 144. 31.

- 666. Salmo Fario.

Cucullanus globosus. i. 20,4.

* Spiroptera Cystidicola. na. 25. 15.

- Ascaris obtusocandata. i. 50. 59.

Echinorliynchus fusiformis. i. 67. 17.

- i. 80.93 .

* Distoma laureatunı, i. 113. 101.

- tereticolle. v. 102. 47 .

Triaenophorus nodulosus app. hep. 135. 1.

Taenia longicollis. hyd. hep. 140. 21. 


\section{6\%. Salmo Hucho.}

Echinorhynchus claviceps. i. 65.5 .

i. 82.93 .

Distoma tereticolle.... 102, 47 .

Tiaenoplorus ao hilosus. hep 149. 21.

* Bothriocephalus proboscideus. i. 137. 4.

6îs. Salmo Lavaretus.

Echinorhynchus. recl, 81. 93.

* Taenia longicollis. t. 149. 21.

Dub. v. e. 196.75 .

66y. Salmo Maraenula.

MTonostoma. hyd. v. $87 \cdot 24$.

* Taenia longicollis. i. 1 \{9. 21. 67o. Salmo Ornul.

Ascaris. $\nabla \cdot 59 \cdot 13,3$.

Taenia, v. app. 175. 14\%。

671. Salmo Salar.

Ascaris Capsularia. pi. 50.60.

Echinorhynchus fusiformis, i. 67. 17.

$$
\text { - i. } 81.9 .3 \text {. }
$$

Distoma varicum. v. 10(1, 59.

$$
\text { appendierlatum. i. 110. } 85 .
$$

Tetrarlyychins appendicalatus. hejp. mu. 31. So

* Bothriocephalus probescidmas. app. 137. 4.

$$
\begin{aligned}
& \text { (parazit.) v. 139. 14. } \\
& \text { 672. Saimo Salvelinus, }
\end{aligned}
$$

Echinorhynchus, i so. 9.3.

Ligula simplicissima. a. 13 i. 6 .

* Botl riocephalus infundibuliformic. t. 137. 5 .

* Ascaris. pi. 59.-13ł.

$$
\text { 673. Salmo Saurus (Osmerus.), }
$$

* Eistoma appendiculatum. จ. 110. 85.

$$
\text { 674. Salmo Spirincinus. }
$$

Dub. na. 196.77 .

$$
\text { 675. Salmo Thymallus. }
$$

Echinorhynchus fusifornis. i. 67. 17.

$$
\text { - i. 80. } 93 .
$$

Distoma varicum. v, Jo6. 69 .

$$
\text { - laureatum, i. } 11 \hat{\jmath} .101 .
$$

Triaenopliorus nodulosus, hep. app. 13\%. 1.

* Taenia longicollis. app. 149, 121.

$$
\text { 676. Salmo Thymallus latus, }
$$

* Spiroptera Cystidicol.s. na. oe. 25. 15. 
677. Salmo Trutta.

Cucullanus globosus. t. app. a. 20. 4 .

Acraris obtusicautata. v. i. 50. 59.

Echinorhynchus fusifornis. i. 67. 17.

$$
\text { - ovatus. pi. } 73 \cdot 38 \text {. }
$$

Distoma laureatum. app. i. 113.101.

- tereticolle v. 102.47 .

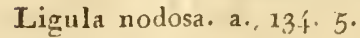

* Triaenophorus nodulosus. pi. app. hyd. hep. 135. 1. Taenia longicollis. i. $149-21$.

\section{Salma Wartmanni.}

Ligula simplicissima. a 134 . 6 .

* Taenia longicollis. i, 149. 21.

* Cucullanus elegans. t. 19. I.

$$
\text { 679. Esox Lucius. }
$$

* Ascaris Acus. i. 43. 29

- adiposa. a. 6o. 135.

* Echinorbyuchus angustatus, i. 6S. 19.

* Distoma folium. ur. 96.21.

$$
\text { ovatus, pi. } 73.79 \text {. }
$$

* - tereticolle. v. 102. 47.

- i. $12 \%$. $15 \mathrm{~S}$.

* Triaenophorus nodulosus. i, - hyd: hep. 135.1. Dub. Cestoid. v. 196. 78 .

* Ascaris Acus a. 4.3. 29.

$$
\text { 6go. Esox Belone. }
$$

* Echinorhynchus angustatus. 43. 29.

* - Pristis. i. 7547.

* Distoma gibbosum. v. 107. 71. Scolex polymorphus i, 12S. 1 . Taenia. i. 175. 143.

$$
\text { 681. Sphyraena Spet. (Esox. Linn.) }
$$

* Echinorhynchus globulosus. i. 65. 10.

* Dub. Nennat, pi. 196 . so.

$$
\text { 6S3: Argentina Sphyraena. }
$$

Accaris a 6o. 136.

Echinorhynchus. a 80. 9̀.

Tetrarhyuchus. 458 13.

Dub Nematoid. a. 19f. 79 .

- Ascaris, pi 6n. 137.

\section{Atherina Hepsetus.}

* Echinorhynchus. i $\$ S .96$.

* Distoma baccigerum. $v, 109,76$. 


\section{$-783$}

65.. Mugil Cephalus.

* Echinorhynchus arilis. 1, 17. 16.

65). Clu. ea. Alosa.

* Ascaris adunca, v. i. 2i), 0.

Echinorhyolins subuiatns, i. 75. 48.

* Distoma ventricosum, i. 105.75.

* - appendiculaum. v. $210 \mathrm{~S}$ S.

* Bothriocephralus fragiliz. app. 1 iS, s.

6,6. Clippea Encrasicolus.

- Ascaris gracilescens. pi. 45. 35.

* Scolex polymorphiss. i. J2\%. 1.

$$
\text { 687. Clupua Harengus. }
$$

* Filaria Capsularia. pi. 6. 13. Asearis. pi. (10. 13s.

* Distoma ocreatum, i. 107. 70. 68.s. Clupea Sprattus.

* Ascaris gracilescerss. i. 4\%. 39.

6s9. Clupeae n. sp. italita (Aliccia, s, Sardone.)

* Scolex polymorphus. i. 12S. 1.

Ggo. Cyprinus A'brurusus.

Echinorliynchus claviceps. i 65.9 . Caryophyllaens mutabilis, i. 127. 1. Ligula simplicissiua. a $1.3 \mathrm{i} .6$

691. Cyprinus amarus.

Caryophyllaeus muahilis i. 127. 1

602. Cyprinus Aspius.

* Cucullanus elegans. i. 19 :.

* Dub. Nicmatoidenm. pi. 197. 81.

693. Cyprimus auratus.

Echinorhynchus claviceps. $165 . \mathrm{C}$.

6o\%. Cyprinus Barbus.

* Ascaris dentata. i. 45. 3\%.

Echinorlyynchus claviceps i. 65.9.

$*$

nodulosus. i. 72.37 .

Monostoma cochleariforme. i. 32.3 .

* Distona punctum. rect. 112. 9S.

Caryophyllaeus nutabilis. i. 127. 1.

- Borluriocephalus fiectangalum. i. 13S. 10. 695. Cyprinus Blicia.

* CaryophyIlaens mutabilis. v. 127. 1.

Ligula simplicissima. a. 13 f. 6 . 696. Cyprirus Brama.

Echinorhynchus claviceps. i. 65. 9. 
* Echinorhynchus nodulosus. i. 72.37 .

* Distoma globipormm. i. 96. 17.

* Caryopliylläeus mutabilis. i.,127. 1.

* Ligula simplicissima, a. 1, \} 6

\section{Echinorhyuclius. i. 82. 9s.}

607. Cyprinus Carassius.

* Caryophyllaeus nutabilis. i. I27. 1.

* Ligula simplicissina. a. 134. 6.

6gs. Cyprinus Carpio.

Echinorliynclius claviceps. i. 95-9.

Distoma globiporum, i. $96,17$.

Caryopliyllaeus mutabilis. i. 127. 1.

Ligula simplicissima, a. 13i 6.

6g9. Cyprinus cultratus.

Ascaris cuneiformis. i. 50. 58.

700. Cyprinus Dubula.

Echinorhyuchus nodulosis i. 72.37 .

* Caryophyllaeus mutabilis. i. 127. 1.

fo1. Cyprinus erythophthalmus.

Ascaris. i. 60139 .

Echinorhynchus claviceps. i. 65.9 .0

$$
\text { - nodulosus, v. i. } 7^{2} 37 .
$$

* Distona globiporum, i. $96 \quad 17$.

Caryophyllaeus mutabilis, i. 127. 1.

Ligula simplicissima. a. 13ł. 6 .

$$
\text { 70:. Cyprinus Gibelio. }
$$

* Filaria sanguinea pinn. cand 5. 9.

* Caryophyllaeus mutabilis. i. 127. 1.

* Ligula simplicissima. a. 134 . 6.

* Filaria ovata. a. 5. 12.

703. Cyprinus Gobio.

Echinorhynchus claviceps. i. 65.9 . nodulosus, i $7.2 \cdot 37$.

Caryoplyyllaeus murabilis. i. 127. 1 .

* Ligula simplicissima, a. 134. 6 .

\section{Cyprinus Idbarus.}

Echinorhynchus. i. $\$ 297$.

\section{Cyprinus Idus}

Ascaris cuneiformis, i. $\therefore 0.58$.

Echinorhyuchus nodulosus, i, 72. 37.

Monostoma, i. 8725.

Taenia. i. 176.146 . 


\section{- 785}

706. Cyprinus Jeses.

* Echinorhyichus nodulosus. i. 72.37.

Distoma inficxum. i. 106. 68 .

Curyoplyyllacus mutabilis. i. 127. 1.

* Tacnia tormiosa. i s.í. 23.

70-.' Cyprinus Leuciscus.

Ciryoplyyllaeus matabilis. i. 127. 1.

Iigula simplicissima. a. 13 i. 6 .

Tacnia turulosa. i. 149. 23. zos. Cyprinus Nasus.

Echinorhruchus clavicens. i. 6j. 9.

Distoma globiporum. i. 96. 17 .

C.irroplyyllaeus mutabilis. i. 127. 1.

Taenia torulosa. i. $\$ 49 \cdot 23$.

$$
\text { 7og. Cyprinus Orfus. }
$$

710. Cyprinus phoxinus.

Filaria orata. a. 5 12.

Echinorhuchus chviceps. i, 6i. 9 .

Cargophyllaens mutabilis. i. 127. 1 .

* Botbriocephalus gramularis. i. 138 - 9.

711. Cyprinus rutilus.

Ichinorliynchus tuberosis. mes. 65. S.

$$
\text { - claviceps. i. } 65 \cdot 9 \text {. }
$$

Caryophyllaeus murabilis. i. $12 \%$. 1.

Ligula simplicissima. a. '134.' 6 .

Dub. Nemat. a. 107. \$2.

Cuculląnus. i. 22. 1\%

\section{Cyprinus Tincs:}

Echinorhyuclus claviceps. i. 659 .

$$
\text { nodulosus. i. } 72 \cdot 37 \text {. }
$$

Distoma globiporum. i. g6. 17 .

Caryophyllaeus mutabilis, i. 127, 1.

Ligula simplicissima. a. 134. 6.

7:3. Cyprinus Vimba.

* Echinorhynchus nodulosus. i. 72. 37 .

714. Cyprini sp. Barbo affinis, Italis Lasca dreta.

* Ligula simplicissima. a. 134 . 6

715. Pisces hand determinati.

Distoma binote. i. 123. 160 .

- distichum. j. 123. 161. 


\section{$-786$}

Classis V. Insecta.

Ordo 1. 'Crustacea.

Dub. app. 197. 83 .

716. Cancer depressus.

- 717. Cancer Ruviatilis.

* Distoma isostomum. hep. (ne?) 105. 62. 718. Cancer Locusta.

Dub. ves. ov. v. $197 \cdot 85$.

Dub. ves. ov. $197 \cdot 84$.

719. Cancer Pagurus.

Filaria. a. 12. 64.

Ordo 11. Arachnides.

721. Phalangium cornutum (et Opilio).

* Filaria truncatula. a. 6. 17 \%

722. Araneae species incerta.

Filaria. a. 12. 63 .

\section{Ordo III. Coleoptera.}

Ascaris. cr. 6o. 140 .

$$
\text { 7.23. Lucanus Capreolus. }
$$

724. Geotrupes Nasicornis. Larva.

* Ascaris cuspidata. cr. 52.68 .

725. Cltrysomela Alni.

Filaria. a. 11. 58 .

726. Chrysomela Tanaceti.

Filaria. a. 11. 57.

Filaria. a. ì. 56 .

727. Silpha obscura.

728. Buprestis. (Species non dicta.)

Filaria. a. 1i. 59

Filaria. a. 11. 55 .

729. Carabus blapoides,

Dub. v. 197. 86.

730. Blaps Mrortisaga. 


\section{- 787}

\section{Ordo IV. Orthoptera.}

* Filaria. a. 11. 60

731. Forficuia Auricularia.

Filaria. a. 11. 61.

732. Locusta hemitogict.

Filaria. a. 11, 61.

733. Locissta verrucipora.

Filaria. a. 11, 61.

734. Locusta siridissima.

Ordo $V$. Neuroptera.

735. Phryganea. Species non dicts.

Filaria. a. 12. 66.

\section{$\therefore$ Ordo II. Hymenoptera.}

Filaria. a. 12.67.

736. Tenthredinis Lar'á.

Filaria. ส. 12. 62 .

\section{Ordo VII. Hemiptera.}

737. Cercopis spumaria.

\section{Ordo VIIT. Lepidoptera}

Filaria. a. 12. 65;

735. Papilionis Antiopés Larya.'

739. Papilionis Betulae larva.

Filaria. a. 12. 65 .

Filaria. a. 12. 65.

740. Papilionis Polychlori larya.

741. Papilionis Quercus larya.

Filaria. a. 12. 65 .

742. Papilionis Urticae larya.

Filaria. a. 12. 65 .

743. Sphingis Euphorbii larva.

Filária. a: 12. 65 .

744. Bombycis Alni larva.

Filaria. a. 12. 65 .

745. Bombycis Cajae larva.

Filaria. a. 12. 65.

746. Bombycis Quercus Larwa.

Filaria. a. 12. 65.

Ddd 2 


\section{- 788 -}

747. Bombycis Salicis larva.

* Filaria plicata. a. 7. 18.

7.48. Bombycis Trifolii larva. Filaria. a. 12. 65.

749. Bombycis Ziczac laria.

Tilaria. a. 12. 65 .

7.jo. Noctuae nuptae laria.

Filaria acuminata. a. 6. 16 .

751. Pyralidis pomanae larva.

* Filaria a. 12. 65.

752. Tineae Padellae larva:

* Filaria truncata. a. 5. 11.

\section{Classis V1. Vermes.}

\section{Ordo 1. Cephalopoda.}

753. Sepia officinalis.

- Tetrarhynchus megabothrius. tuin. v. 130. 6 .

754. Loligo ulgaris (Sepia Loligo).

Dub. i. $198 \cdot 89$.

755. Polypus vulgaris (Sepia octopodia).

- Scolex polymorphus. i. 128. 1.

Dub. tub. v. 199. 88 .

\section{Ordo 11. Gasteropoda:}

756. Helix putris.

Dub, tent. a. 198. 91. 


\section{Index alphabeticus} genera Animalium, in quibus Entozoa hactenus reperta sunt, indicans.

Numeri iilis Animalium Vermibus obnoxiorum Catalogo praemisso enumeratorum respondent.
A ccipenser 521-524a
$30 \mathrm{mbyx} 74 \hat{\imath}-749$ :
Bos 11.3-115.
Alca 439.
Rradypus 91.
Alcedo $18 S$, 189.
Ammodytes 542.
Amphisbaena 462, 463 .
Anarrhichas 541.
Anas 408 - 435 .
Anguis 46 .
Antilope 104-109,
Apogon 647.
Aranea 722.
Arctomys $78-80$.
Ardea 318-333.
Argentina 68\%。
Birccu 207.
Bufo $484-488$.
Buprescis 728 .
Camelus $97,98 a$
Canis 28 - 3 .
Cancer $716-719$ a
Caraux 643, 644o
Capra 110.
Capsimulgus 290-294.
Carabus $7299_{2}$
Castor 65.
Astaciss 77.
Atherina 683.
Çavia $88-90$.
Centriscus 531.
Balaena 124,
Centronosus 636 .
Bipes $\{60$.
Cepula 575.
Blaps 7.30 .
Cercopis 737.
Blemius $568-5740$
Cervizs 99-103.
Boa 464 .
Cliaetodon tioz. Charadrius $358-36 j$. 
Chelnnia 44 ก

Chry cochlorus 550-

Clirysomela 725,726 .

Clirysorosus 591.

Clupea 68; 6.89 .

Cohitis $6 ; ;-557$.

Colnber 465-473.

Columba 295298 .

Colymbus $3 x^{2}-389$.

Coracias 204.

Cordylus 4i5.

Corrous 19í-202.

Coryphaena 576-578.

- Coltus 584, 585.

Cricetus 7.9.

Crosulilus 445,446 .

Crotalus 477.

Crotophaga 192, 193.

Ci) purus 306.

Cifulus 208-212.

Cyclopterus 530 .

Cypinus 69o-714.

Dasypus 93.

Delphinus 125-127.

Dendrocolaptes 203.

Didel Lhis 6u-62.

Diorton 529 .

Dipus 77。.

Elephas 96. .

Enuberiza 234-238.

Emys 4i1.

Equus $116-119$.

Eriuaceus 53.

Esox 679-681.

Falco 130-159.

Felis $31-39$.

Forficula 731 .

Fringilla $241-2$. 9 .

Fulica 369,370 . -
Gadus $.551-567$.

- Gasterostens 635 .

Gecko 457.

Georhychus, 66. 68 .

Geotrupes 724.

Glareola 368 .

Gobius 579-582.

Gulo 5:.

Haematopus $36 \%$.

Halieus 402.

Halmaturus 63 .

Helix 756 .

Hirundo 283-289。

Hy'drus 478 .

Hyla 495,496 .

Hyrax 9\%.

Hystrix 64.

Iguana 456 .

Kangurus 63 .

Labrus 619-624。

Lacerta $4+7-460$.

Lampris 591.

Lanius $170-173$.

Larus $390-400$.

Lemar 19.

Lepadogaster 583.

Lepus $83-87$.

Locusta $732-734$.

Luligo 754 .

Lophins 520.

Loxia $230-233$.

Lucanus 723 .

Lutra 43.

Meleagris 312.

Meles 52.

Mergus 436-438.

Merops 190. 


\section{$-791$}

Monitor $44 \%$.

Monoculus 720 .

Motacilla 255-276.

Mngil 684.

Mullus $645-647$.

Muraena $532-538$.

Mus $66-73$.

Muscicapa 250-254.

Mirstela 43-48.

M) gale 56 .

Myoxus 74-76.

Myrmecophaga 92.

Nasua 42.

Noctua 750.

Notopterus 545.

Novacula 578 .

Numida 307.

Ophidium 543-545.

Oriolus 205, 206.

Orthragoriscus 528.

Otis 314,315 .

Ovis 111, 112 .

Papilio $738-742$.

Parus 277-282.

Pavo 313.

Pelecanus 401 - $\$ 07$.

Perca 627-63\%.

Petromyzon . 97 .

Phalaena $744-750$.

Phalangium 721 .

Phasianus $308-311$.

Phoca $120-123$.

Phryganea $735^{\circ}$.

Picucule 203.

Picus 179-295.

Platalea 317.

Pleuronectes 592-602.

Polypus 755.

Proleus 4.9.
Psittacus 174 × 178 .

Pyralis 751 .

Raja 501-508:

Rallus $371-374$.

Rana 489-494.

Recurvirostra 366:

Salamandra $480-483$.

Salmo 66o-67s.

Sciaena 625,626 .

Scincus 459 .

Scinrus 81, 82 .

Scolopax 33j-348.

Scomber $637-644$.

Scorpaena 586-588.

Sepia $753-755$.

Seps 460 .

Silpha 727.

Silurus 658, 659:

Simia 2,- 18 .

Sitta 187 8 \%

Sorex $55-59$.

Sparus 6of-618.

Splinx 743 .

Sphyraena 681 .

Squálus $509-519$.

Stellio 458 .

Sterna $376-381$.

Strix $160-169$.

Stromateus 546 .

Struthio 316.

Sturuus 218, 219.

Sus $95^{\circ}$

Sylvia conf. Motacilla.

Syubranclius 539 .

Syngnathus $525-527$.

Talpa 54, 55.

Tanagra 239, 240 .

Tantalus 334 .

Tenthredo 736 . 
Testudo $410-$ 你 Tetrao $299-30^{\circ} 5$.

Tinamus 306 .

Tinea 752.

Torpedo $498-500$.

Totanus $341.345 \cdot 347$.

Trachinus 549,550 .

Trichiurus 540 .

Trigh 6is -654 .

Tringa $349-357$.

Triton 48\%, 481 .

Turdus $220-229$.
Upıpa IgI.

Uranoscopus 548.

Ursus $49-51$.

Vespertilio $20-27$.

Vipera $474-476$.

Viverra $40-42$.

Vultur 128-129。

Xiphias 547.

Yunx 186.

Zeus 589, 590* 


\section{Index Entozoorum alphabeticus.}

Synonyma litteris inclinatis impressa sunt, Nusmeri paginam indicant,

Amphistoma Anatis 92. conicum 91. 360.

Cornu $89 \cdot 357$.

cornu. 85 .

cornntum go.

denticulatum go. 355 . erraticum 89.356 .

Falconis palumbarii. 89 .

Falconis peregrini. 92.362。 gracile. $\$ 9 \cdot 355$.

isostomum. $89 \cdot 355$.

Lari glauci $9 \%$.

longicclle. $87 \cdot 332$.

macroceplialum SS. 354 .

microstomim $S \delta$.

pileatum. 90, 358 .

serpens $85 \cdot 353$.

Sphaerula. 90. 358.

striatum. SS.

subclavatum go. 358 .

subtriquetrum 91. 360 .

Sylviae 675 .

Tadornae SO.

Tanagrae 67千.

truncatum 91. 359.

unciforme 674 .

ungniculatum 360 .

urnigerum $S 9.356$.
Anthocephalus

elongatus $177.537 \cdot 709$.

gracilis 178.540 .

granulum. 178. 541.

interrujtus. 178. 543 .

macrourus. $178 \cdot 542.714$.

Ascaris

acuminata 40 .

acus 43.

acuta 51.

acutissima 44.

adiposa. 6o. adunca 39.270 ,

Alaudae 54.

Albulae 59 .

alienata 661 .

Ammodyris 56 .

angulata. 44. Ardearum 55.

Argentinae 60. 303:

aucta 50 .

anriculata 655 .

Barbatulae 59 .

Boopis 5S. 301.

brachyoptera 41. 275 .

brevicaudata. 47. 283,

Canis aurei 53.

Canis Lagopodis 53. 
Ascaris

Caprimulgi. 55.

Capsularia 50.

Casturis 54 .

Centrisci 57. 299.

cephaloptera 52. 295.

Cliaradriorum 55.

Ciconiae 55. 29S.

clavata 51.293.

Clupearum 6o. 303.

collaris 52, 294.

Colymborun , 55. 664.

compar 46. 282.

constricta $39 \cdot 270$.

Coruicis 54 .

Corvi frugilegi 54 .

Corvi glandarii. 5f. 297.

crassicauda 50. 291.

crenata, 43.

cuneiformis. 50 .

cisspida:a 52. 294.

Cynaedi. 5S. 301.

Cyprini, 6 .

dactyluris $10,272$. .

Delphini 54. 296.

dentata 45. 291.

depressa 42.276 .655 .

Didel phidis 53.

Dipodis 54 .

dispax 45.

distans 38 .

echinata 47. 28 4.

elongata. 650 .

Emberizae. 55 .

ensicaudata. $\{2$. 275 .

extesulata 47.287 .

Fabri 57.300.

fallax, 4.3. 279 .

foecunda $: 654$.

forcipata 659 .

Fuligulae 56 .

Gadi minuti 57.300 .

Gasterostci 59 .
Ascaris

gibbosa. 48.

Glareolae 55. 235 .

Gobii. 57. 300.

gracilescens $45.2 \$ 2$.

Gulonis. 53 . gullosa 40. 271.

Helopis 56 .

IIippocampi 56. 299.

holoptera 53. 295.

incisa 46.

incurva 51. 292.

inflexa. 3S. 268 .

labiata 49. 290.

Labri lusci. 58. 301.

lancea. 42.

Laniorum. 54. 296 .

Lari 55.

leptocephala 46. $2 \$ 2$.

leptoptern. 41. 274 .

leptura. 48. 288.

lineata. 56.

Linguatulae 58. 300.

Lilcani 6o. 304 .

lumbricoides $37.26 \%$.

Lyrae 59. 302.

maculosa. 45.

Maenae 58. 301.

marginata. 41 .

Marlis G6.í.

Martis 53 a

mascula. 653 .

megatyphlon 47. 285.

Mergorum. 56.

microcephala. 4S. 2 SS.

microptera 41.275.

mucronata 52.

Mulli 59. 302.

Mustelaruni 53 .

mystax 42. 276 .

nigrovenosa. $43 \cdot 2 \pi 9$.

Novaculae 58. 302.

obtusa 27. 
Ascaris

obtusncaudata 50. 291. obvelata 4 \% 2 SO.

Ophidii barbati 57. 299 .

Ophidii imherbis. 47.299.

Orthragorisci. 56. 209.

osculata. 39.651 .

Ovis. 54 .

Pastinacae 56.

pellucida 39. 270 .

Perspicillum 42.

Phycidis 57. 300 .

Picae 54. 297.

pusilla. 46 .

Ralli 5 .

reclinata 657 .

retusa 656 .

rigida 51. 292.

rotundata 39.270 .

sagitlata 24 .

Salamandrae 47.

Salmonis Omul 59.

Sauri 59. 302.

Sciaenae 58. $3 q^{2}$.

Scorpaenae 57. 300 .

semitercs. 42. 276 .

serpentulus 53.296 .

Siluri 59.

simplex 49 .

Smaris 58.

Soleac 58.

Spari Spicre 58. 301.

sphaerocephala 61 .

spiculigera 48.290 .662$.

spinicauda 40. 272.652 .

spiralis 42.

Squali 56 .

Sternae 55. 298.

strongylina 651 .

strumosa 24 .

subulata $38 \cdot 269$.

succisa 52.

sulcata 48 . 289 .
Ascaris

tentaculata 658 .

tenuicollis 47.286 .

tenuissima 52 .

testudinis 25 .

Tigridis. 53. 296.

Torpedinis 56 . 29 .

transfuga. 40. 273 .

triquetra, 41 .

truncata $45.281,657$.

truncatula 40 .

truttae 50.

uncinata 661 .

unguiculata 6.53 .

Uranoscopi 57. 299.

Urogalli 38 .

variegata 49 .

veligera 656 .

vermicularis 44.279 .

vesicularis, 38,268 .

Bothriocephalus

angistatus 139.476 .

auriculatus, 141, 479 .

Barbatulae 14f.

Carpionis 1.44.

Cepolae ráf.

claviceps 136. 472.

corollatus 142.485 .

coronatus. 141. 481 .

crassiceps 139. 476 .

cylindraceus 140.478 .

Eriucis 1.4\%.

fragilis 135 .

Gadi barbati 143 .

Gadi Callariae 143.

Gadi Morhuae 1/43.

Gadi Merluccii 143.

Gadi Redianus 143.

granularis 138. 474.

infundibuliformis. $137.4733^{\circ}$

latus 130.469 .

Lophii : 4.

macrocephalus 1.fo. 478 . 


\section{$-796$}

Bothriocephalns

micrncephalns' 138. 473。 .

noticins. 140.597.

paleacens 142.

plicatus 136.470.

proboscidens 137. 472 .

punciatus 138.475.

rectangulum 135. 474a

rugosus 137 .

solidus 139. 477. 597.

Squali glauci 1.43. tunidulns. 141. 4SO.

uncinatus 142. 483 .

ventricosus 685 .

verticillatus, $142.4 \$ 4$.

Capsala. 430.

Caryophyllaeus mutabilis. 127. 441.

Coenuruz cerebralis. 192 .

Cuculianus

abbreviatus 2r. 234.

Accipenseris 22。

alaetus 20 .

arntatus 19.

ascaroides 567.-

coronatus 20.

eleginis 19. 230 .

foreolatus 21. 233.

globosus.' 20.

heterochrous $2 \mathrm{I}$.

IIydri 2 I.

melanocephalus 20. 232. 640.

minntas. 21.235 .

papillosus 19.

Percae 22.

Platessae 22.

Soleale 22.

Testudinis 21.

Tincae 22.

truncalus. 20. $23 \mathrm{I}$.

Cysticercus

Canis 381.
Cysicercus

cellulosae 1SO. 546 .

crispus 1\$1. 549 .

Délphini 182. 5.51。

fasciolaris 179. 554 .

fistularis 179. 544.

Gadi Lotae 1.35 .

Leporis variabilis 182.550 .

longicollis 180. 547 .

Lucıi 135.

Percae 135.

pisiformis. 181. 54 S.

Pntorii 1.81.

Salmonum 149.

Simiae visceralis 180. sphaerocephalus, 181. 5ís. Talpae 181.

tenuicollis. 180: 545 .

Diacanthus. 184.

Dibothrius. 469 .

Diceras 184 .

Dipodiun 197.

Distoma

acanthoides 114.415.

affine. 110. 406 .

alatum 112. 412.

albicolle 98. 376 .

Aluconis intestinale. 119.

Alnconis thoracicum. 119.

amphistomoides 361 .

Anarrhichae 121.

Anatis domesticae 121.

Aultis fuscae 120.

Anguillae 359.

apertum 108. 400.

apiculatum. 136 .

appendicalatum. 110. 40.4.

Ardene stellaris 120.

areolatum. 111. 40 S.

Ascudia 10S. 399.

Alomon 95.

baccigerum. 10,8. 398.

bilobum 114.416 . 


\section{$-797$.}

Distoma

binode 123.

Buteonis 119.

Calidris 120.

causiliculatum. 696 .

capitellatum. n? 3790

carnosum. 93. 366.

callitile roz.

cantiporrm. 96.398 .

Chrysaéti. 119.

cinctum 116 .

cirratum. 100.

clavatum 106. 394. $6 \$ 2$.

clavigerum 103. 399. .

Clupeae. 110.

cochleariforme 681.

Collurionis. 119.

Culnbri americani 121.

Colubri murorum. 121.

Colubri Natricis intest. 103.

Colubri Natrecis pulm. 99.

Colubri tesselati 121.

complanatum 98. 376 .

contorzum. 118. 424.

coronatnm 686 .

Coryjphaemae. 12\%. 685.

crassicolle 102.

crassiusculum 112.

crenatum. 110.

crisiatum $11 \%$. $\{22$.

Crorali Durissi 124.

crystallinum 100. 380.

cucumerinum. 94.

cuneatum 93.

C) clopieri 121.

cyynoides 96.370 .

cylindıaceum. 106.

cyuriforme 96.371 . :

deflectens 677 .

delicatulum 99 .

dendriticum 93. 364 .

deniculatum 116. 419 .

distichun1 123.
Distoma

divergens 97.372.

echiratum $115 \quad 416.685$.

echinocephalum 115. 41 .

elegans 100.

erraticum $\mathbf{3} 20$.

exa"peratum 117. 421 .

excaralum 10\%. 402.

ext:sirm. 11\%. 11 .

Fạlconis rufi 119 .

fallax 117. 420.

facciatum $97 \cdot 37 \hat{\jmath}$.

ferox 110.419.

filiforme 112, 411.

flexuosum 105.

folium $y 6.37$.

fractrm $10 \% 397$.

fulvum 99. 374.

furcatum 107. 396. 683.

fuscrtum 101. 384 .

fuscescens $113.41 \hat{\jmath}$.

gelatinosum 10\% 386 .

genu 307397

gibbocum 107.345 .

glohiporim 96 .

globulus 109.401.

gracilescens 11?. 409. ,

grande 676 .

grandiporam 110. 407 .

granulam 106.

hepaticum 92. 363 .

heterostomum. 102. 385.

hiank. 94. 366.

hispidum, 118. 423 .

holostomum. 9\%. 36 s.

hyalinum 105.

Hylae 121.

incissum 94.

incomtum. 683.

inerme 375.

inflexum 106.

involutum 101.

irroratum, 105. 393. 
Distoma

isoitamum 105392.

lainiarum 108. 400.

Labri 122.

Lacertae 121.

laticolle 117.421.

laureatum 113. 413 .

lima 117 .

lineare 113. 414. 605.

Linguatula 100.383.679.

longicauda 9S.

Loxiae 120.

Lucii 123.

Incipetunı 94. 367 .

macrostomum 104 .

macrourum 9S.

maculosum 1100. 383.

marginatum. 680 .

megastomum 102. 387.

mentnlatum 103. 388 .

Mergi 121.

Meropis 120.

mesostomium 104 .

micrococcum 101. 383.

microsoma 109. 401.

micristomum 104.

militare $115 \cdot 41$.

Milvi 115.

N!onas 679 .

Musculi 119.

Naja 99. 377.

nanum 101.

nigruflavum 118. 425.

Noculate 119.

noilulosinm 113.

Ocreallum.107.

ovirum 93.

oxycephalum 9S. 375 .

pallens 111. 408.

Pailomelae 120.

planiculle $6 \times 6$.

polymor phum 95. 369.

pulchellum 9.367.
Disroma.

punctum 112.

pusillum 104.

pyxidatnm 67s.

repandum 681 .

ringens 101.385 .

rufoviride 110.406 .

scabrum 118.424.

Scorpaenae 122.

simplex 97.

sinnalum. 97. 374 .

seriale 97 .

solea eforme 10\%.

Spari 122.

spathaceum 109. 403.

spatulatum 109. 403.

spinulosum 116.419.

squamula 103. 391.

sturionis $11 \mathrm{~g}$.

tenuicolle 93 .

teretícolle 102. 386 .

Testudinis 121.

tornatum 68 ;

torulosum 111. \%10.

trachea 11.f. 605 .

transversale $05 \cdot 36 \mathrm{~S}$.

Triglae 122

trigunocephalum 114.

trilubum 10\% $39 \%$.

Tringae helveticae 120.

truncatum 122.

mbarium 111. 410 .

unbulantum 675 .

tumidulum 95. 369 .

Turdi 120.

uncinatum 115. 417\%, varicum 106.

vaцiegatum 99. 378.

ventricosilm 108. 398 .

Wachniąe 122. 427.

Dithyidium. 559 .

Ditrachyceras, 18 fo 


\section{$-799$}

Dub.

Accipenseris Sturionis 191. Alcae Prae 1:35.

Anilop: 156 .

Apis mellificae 197.

Ardeac caemleae 711.

Argentinae 106 .

Blaptis Mortisagae 107.

Boae caninae 712.

Cancri depressi 19\%.

Cancri Locustae 137.

Cancri Pagmi 197.

Carancis rrachuri 195.566 .

Cati feri 710.

Centronoti glauci 195.565.

Cepolae rubescentis 193.

Colubri 712.

563.

Colubri Natricis 199.

Colnbri olivacei 199.559.

Colymbi septentrionalis. 158 .

Cyprini Aspii 197. 507.

Cyprini rutili 197 .

Delphini 186.

Erinacei 185. 554.

Esocis Lucii 19j.

Falconis' 187.556.

Falconis albicollis 711 .

Falconis Buttunis 187 .

Falconis naevii $18 \% .555$.

Falconis Nisi $3^{n} .187$.

Fiatolae 1 $32.56 \%$.

Fringillae ưonest, 157.557.

Gadi Aeglefini 192.

Gadi Chalcogrammi 193.

Gadi Merluccil 192. 562.

Gadi Morhuse 192.

Gadi virentis 19:.

Gadi Wachniae 193.562 .

Gasterostei 195.

Helicis putris 198. 567.

Labri nilotici 194.
Dub.

Lucertae muralis 195.559.

Lacertae viridis $18355 \mathrm{~s}$.

Licrrtarum 1SS.

Lanii pomerani 187.557.

Loliginis vnlguris 103 .

Lumbruci tenestias 30 .

- Muraenae Angurilat 191.

- Muraenae Congri. 192.

- Muraenae Helenae I92.

Mnraenae serpentis 192.

Mnris amphibii $\mathbf{1} 96$.

Muris. Musculi $18 \sigma$.

Musizlarum, s.

Mygales moschatae. 186.

Myrmecophagae 186.

$O$ ris 186.

Percae cirrosae 194. 565.

Percae marinae 195.565 .

Perdicis saxatiliz 18ร. 559. - Plenronectis Plemronectis Soleae 194.564 .

Polypi rulgaris 19S.

Prutei anguimi 199. 559.

Pterotrucitace ing.

Rajate Aquilae 19o.

- Rajae Batis 190. 560.

Rajae Fnllonicae 190.

Pajae P.sstinacae 190.

Rauae brasil. 713.

Rauae Pipae 159.

Ranarum $\perp S g$.

Salmonis Eperlani 196.

Salm. Lavaresi 195.

Salnu. Spirinchi 196 .

Sciaenae Umbrae 19' 565 .

Scombri Colide 195. 566.

Scombri Saldie 195. 566 .

Scombri Scombri 395.566.

Scorpaenae massil. 193.

Sepiae. $19 \delta$.

Sepiae Loliginis 130.

Sepiae officinalis 130. 
Dub. =

Serpentum 1S9.

Siluri Glanidis 196.567.

Soricis moschati 186.

Spari_Alcedinis 194. 564.

Spari Boopis 194. 565 .

Spari Denticis 194.

Spari Melanuri 19'. 565.

Spari Mormyri 194. 565.

Spliyraenae Spet 196. 567.

Squali 191.

Squali Catuli 190.

Squali grisei 191 .

Squali Musteli $19 z$.

Squatinae 190.

Strigis 711.

Strigis accipitrinae $\mathrm{I} 87.556$. Sturionis 191.

Syngnathi pelngici 191: 561.

Testudinis marizae $\mathbf{1} \delta 8$.

Testud. terrestris. 40.

Torpedinis, 190.

Triglae adriaticae 195.566 . Triglae brasil. 713 .

Triglae Hirundinis 195.

Turdi 711 . 566.

Vespertilionis $185^{\circ}$

Vespertil. allriti 155.553

Vespert, discoloris is 5.554 .

Viperae marinae 192.

Vulpis 155 .

Xiphiae. 51.

Zenis Apri 10\% 564.

Zenis Fabri 193. 563.

Dyacanthus polycephalus.

Echinococcus 1 S4.

Honinis 183.551.

Simiae 183.

Vetcrinorum 183. 551.

Echinorlynchus

Accipenseris Futheni 79 .
Echinorliynchus

Acus 71. 32.4.

aequalis 70 .

affinis Gs.

afilis $67 \cdot 316$.

Alaudae 77 .

Alcae 188.

Anatis mollissimae. 72 .

Anatum 78.

angustatus 68. 318.

Ardeae albae 78 .

Ardeae purpureae 78 .

areolatus 69. 319 .

Argentinae so.

Atherinae so. 336 .

bacillaris $67 \cdot 316$.

Balaenae 71.

Barbi 72.-

Bramie 72.

Cirassii 81.

caudatus 70.323 - 66\%.

Charadrii pluvialis 78 .

finctus $66.31 \mathrm{~d}$.

Citilli. 76 .

claviceps 65 .

collaris 74 .

Collurionis 76 .

compressus 6.t.

constrictus 74 .

Coraciae 77 .

Cornicis 76 .

cylindraceus 69 .

Emberizae 673.

Eperlani so.

Erinacei abdominalis 76 .

Erinacei subcutaneus 76 .

Fabri 79.

falcatus 6 s.

Falconis cyanei 76 .

filicollis 71.327 .

fusiformis 67.317 .

gibbosuls 73 .

Gigas 63. 310 . 


\section{$-801$}

Echinorhynchus

globocaudatus 66. 314.

globulosus 65.313.

Gobii ;9.

gracilis. 68. 319.

Gruis 78 .

Haeruca 67.317 .

Haliaèti 76 .

Hirundinum 77 .

Husonis 75 .

Hystrix 75.332.

Idbari $\delta 1$.

inaequalis 66 .

inflarus 67.

Labri so.

Lavareti so.

lineolatus $7 \mathbf{1 .}$

Lophii 71 .

micracanthus $6 \mathrm{~g} .322$.

microcephalus 665 .

minutus 74 .

moniliformis 71.324 .

Morinelli 78 .

Motacillae atricapillae $7 \%$.

Muris 76 .

Muscicapae 77 .

Mustelae 75. 335 .

mutabilis. 669 .

rapaeformis 64 .

nodulosus. 72.328 .

Oedicnemi 78 .

oligacanthoides $64 \cdot 311.666$.

oligacanthus 64. 311.

Orioli 77.

Orioli cristati 673 .

ovatus 73.

Pari 77 .

Picae 76 .

Platessae 79 .

Platessoidae 80.

Pletronect. maximi 79 .

polymorphus 672.

porrigens 71.325 .
Echinorleynchus

Pristis $75.33 \% 672$.

pumilio $\dot{ } 6.31 .4$.

pyriformis 74.331.

quadrirostris 131.

ricinoides 64 .

Rubetrae 77 .

rutili 65.

Salmonum So.

Sciaenae 80. 335:

Scombri 75 .

Scorpaenae 79.

simplex 6s.

Soricis -6 .

sphaericus 73 .

sphierocephalus $670^{\circ}$

Sphyraenae 8o.

spiralis 70, 323.

Spirula 63. 310. 665 .

Stermae $780^{\circ}$

striatus 74.329.

strumosus 73 .

Sturionis 79 .

sublobatus go.

subulatus 75 .

Sylviarum 77 .

Tanagrae 673.

Tardae 77 .

tcrebra 668 .

tereticollis 72.328 .

transversus 69.321 .

Triglae so.

tuba 70.324 .

tuberosins 65.312 .

tumidulus 6. 320. 667.

Vanelli 78 .

vasculosus 75.334 .

ventricosus 74 .

versicolor 74.330 .

Wachniae 79. 335.

Xiphiae $1, j 6$.

Fasciolu appendiculard, 375.

barbata 130.

I e a 


\section{- 802}

Fasciola longicollis 377 . $r$ anae $3 \mathrm{~S}$.

Festucarià Oti 354 .

Filaria

abbreviata 4. 210.

acuminata 6 .

affinis. 4. 209 .

alata 27. 219.

Alaudae 4.

Alcedinis 635 .

Anatis 10.

Aquilae 4.,

Araneae 12.

Ardeae cinereae 9 .

Ardeae nigrae 9. 21 s.

Ardearum 636 .

attennata $4 \cdot 20 \mathrm{~S}$.

Bubali 8 .

Buprestis 11.

capsularia 6.

Carduelis 9 .

Cercopidis 12.

Cervi S.

Charadrii io.

Chrysomelae Alni 11.

Clirysom. Tanaceti 11.

Ciconiae 9.

Coleopterorum 1 .

Collurionis pulm. S. 217 .

Collurionis intest. 8.217.

Colubri americani 10.

Colubri austriaci 10.

Colynibi 10.

coronata 6 .

Cygni. 10.

c) stica $63 \%$

Erinacei 8 .

Erucarum 12.

Fulconum 4 .

Forficulae 11. 219.

fusca $5.21 \mathrm{x}$.

globiceps 7.215 .

gracilis 3.20 s.
Filaria

Hominis bronchialis. $7 \cdot 215$.

Lari 10.

Leonis 7 .

Leporis pulm. 8.216.

Leporis subcut, $S$.

Locustae. 11.

medinensis $3 \cdot 205$.

Meropis $q$.

Mbnoculi 12 .

Motacillae brasil. $C_{35}$.

MIotacillaruni 9 .

Musculi S.

Mustelarnm pulm. 8. 216.

Mustelaruin subcut. 7.216 . obtusa 4 .

obinsocaudata 63f.

ovata 5.213.

papillosa 6.213 .

Phalangii 6 .

Pliryganeae 12.

piscium 10. 218 .

plicata $7 \cdot 324$.

Ranae, esculentae 10 .

rubella 5. 212.

sanguinea 5. 211.

Silphae 11.

Spari Aniatae 11.

Sternae 10.

Strigis 4 .

Stunini 9 .

Tenthredinis 12.

Tringae 10.

truncata 5 .

truncatula 6.214.

Turdorum 9 .

unguiculata 4 . 209.

Vespertilionis 7 .

Vulpis 7.

Zenis Fabri 11.

Fimbriaria Malleus 521. Mitra 521.

Floriceps 178. 710. 
Gymnobothrius 469 .

Gymnorhynchus reptans 129. 444. 688. Hacruca 76.

Hamuluria lymphatica 7. nodulosa 14 .

subcompressa 7 .

Hepatoxylon 132. 456.

Hexathyridium conf. Polystoma.

Hypostoma 81. 87.

Ligula

acuminata 134 .

alternans 133.460.

cingulum 134.

Cobitidis 134 .

Co'ymbi cristati 133.

Culymbi Immeris 133.

corstringens 134 .

contortrix. 134 .

crispa 134. $4670^{\circ}$

Cyprini Alburni 13.4.

Cypr. Carpionis 13f.

Cypr. Gobionis 13 f.

Cypr. Leucisci 134.

Cypr. Tincae 13\%.

Cypr. Wartmanni 134.

interrupta 133. 460 .

Mergorum 133.

nodosa 134. 464 .

Salvelini 134.

simplicissima 134.465.

Soricis moschati 156.

sparsa 133. 462 .

uniserialis 132. 459 .

Liorhynchus

dericulatus 63. 307 .

gracilescens 62 .

truncatus 62 .

Nonostoma

attenuatum 8f.

capitellatum 83.343 .

caryophyllinum 82 .
Monostoma

cochleariforme $\mathbf{8 2}$.

cornu $\$ 5.345$.

crenulatum $S$ f́.

crucibulum $\$ 3.342$.

ellipticum 84 . 344 .

filicolle 85.347 .

foliaceum 83 . 3.to.

galeatum. $86 \cdot 349$.

gracile 82.

Ilimantopodis 87.

Idi $\$ 7$.

lineare 83.3 iz.

macrostomum $\delta 6$.

Maraenulae 87.

Marilae 87.

Molae 87.350 .

ocreatum 84 .

orbiculare $83.34 \%$

pileatum go.

prismaticun 85 .

sulcatum $\delta 6$.

tenuicolle $\$ 5.346$.

trigonocephalum 85,349 .

Vanelli 87.350.

ventricusum $8 G$.

verrucosum $8 \% \cdot 34$.

Vespertiliouis $87:$

Onchobothrius 469 .

Ophiostoma

cristatum 6o. 30\%.

Cystidicola 26.

dispar 61.

lepturum 61.

mucronatim 61. 305.

sphaerocephalum 61.305.

Oxyuris

alata 19.229.

anıbigua 19.229.

curvula 18.229.

vermicularis 4 .

Pentastoma

denticulatum 12\%.

Ee 2 


\section{Pentastoma}

emarginatum 124.433.

proboscideum I24. 434.687 .

serratum 124.

raenioides $12,3 \cdot 432$.

Phylline Diodontis 430.

Physaluptera

abbreviala $30 \quad 257$.

alata 29,$2 ; 6 \quad 645$.

clansa $29 \cdot 2556643$.

dilatata 644 .

retusa 30. 258.646 .

saginata 6 f́n.

strongylina 6 47.

tenuicollis 30. 258. 6. 7 . turgida 644 .

Polystoma

denticulatum 123.

- duplicatum 125. 438 . integerianum 125. ocellatun 125. 436 .

Pinguicola 125. 437.

serratum 124.

tạenioides 123.

Thynni 438.

Venarım 126.

Porocephalus 124. 434.

Prionoderma ascaroides 196. taenioides 123.

Pseudoechinorhynchus 76 .

Rliynchobotirius 469 .

Rhytlelminthus 444.

Riciularia 61.

Schisturus 118.

Scolex

Cyclopteri 128.

Gigas 129. 444.

Lopliii 128.

Percae $12 \delta$.

polymorphus 125. 442 .

quadrilobus 128.

zetrastomus 149.
Spiroptera

acutissima 642 .

alata 23. 239.

Anatis 29 .

Anthuris 25.243.

atteunata 25. 244 .

bicuspis 24. 240.

bidens 24. 2 亿0.

Citilli 29. 254.

Colymbi 29.

contorta $25.2 / 2$.

cystidicola 25.245.

denudata 6.4 .

elongata 26.246.

entycoptera 26. 2.48 .

Falciuelli 28 .

Falconis $25 \cdot 254$.

Fulicae 29.

Gallinulae 28.

gracilis $64 \%$.

Hominis 27. 250 .

Hystricis 28 .

laticaudata 24. 239.

laticeps $23.23 \delta$.

Leonis 27.

leptoptera 26. 247.

megastoma 22. 236 .

nasuta 23. 238.

obtus. 27. 249.

quadriloba 25. 2 1.

Ralli 6 亿 2 .

revolnta 26.247 .

sanguinolenta $27 \cdot 249$.

Simiae $27.25 j$.

sterëura 23. 237.

Sternae 29.

Strigis 28 .

strongylina $23.23 \%$.

strumosa 24 . 241.

Sturni 28 .

Tigridis 28 .

uncinata 26.246.

- Upupae 28. 


\section{- 805}

Spiroptera

Ursi 2S. 253.

Vanelli 29.

Strigea candida 355.

Strongylus

ambiguus 29.

Ammonis 37 .

Anatis 32.

Ardeac stellaris 37.

armatus 30. 259.

auricularis 33 .

Cameli 36 .

capitellatus $35,265$.

Capreoli 36 .

Colubri 37.

contortus 32.

costatus 647 .

crenulatus 29.

criniformis 35 .

dentatus 31:

denudatus 34. $26 \mathbf{3}$.

Dorcadis 37.

Elephanti 36 , ।

Filan ia 32.

filicollis $32,26 \mathrm{j}$.

galeatus 648 .

Gigas 31. 260.

horridus 28.

lyypostomus 33.263 .

Hystricis 28.

inflexus 34 .

Leonis 27:

leptocephalus 649 .

Lupi 36.

Lupi 27.

Mergorum 32.

Myoxi 36.

nodularis 35.264 .

papillosus 31. 261.

radiatus 33 .

retortaeformis 5 f. 264 .

striat us 3 ;

subauricularis 649 .
Strongylus

Suis $36,266$.

Tardae $3 \%$.

tetrag̨onocephalus $35,265$.

tubaeformis 36 .

tubifex 33, 262.

Ursi $2 S$.

Vanelli 29.

ventricosus 33.

venulosus 33 .

Viperae 37.

Vitulorum 37.

Vulpis glandularis 36.266 :

Vulpis oesophagens $3^{\text {f. }}$. 266.

\section{Taenia}

acuta 165.525 .

aequabilis 155 .

Alaudae 155.

Alcae Picae 174.

Amphisbacnae 175 .

amphitricha 152.501.

angulata 155. 509 .

angustata 148. 494 .

anthocephala 146.

armillaris 175.

aurita $€ g 8$.

bacillaris 154. 506.

Belones 175.

Bonasiae 172.

brevicollis $159 \cdot 516$.

brevicollis 494.

calycina 151.

campanulata 693.

Canis lagopodis 169 .

candelabraria 160.518.

capillaris 156.511 .

capitellata 156. 511.

capito 704.

Caprae 170.

Capreali 14 i.

Charadrii 708 . 
Taenia

Charadrii Hiaticulae $15 \mathrm{~T}$.

Cirarcirii Hmantop. 153.

Chiysaëti 171"

Coccoiliraustis 172.

Colubri brasil. 709 .

Coly'mbi cornuti 174 .

Colyumbi cristati 174 .

Colymbi Troiles 174 .

compacta 164 .

Coraciae 172.

Cornicis 167.

Corvi frugilegi $16 \%$.

Corvorum 171.

crasșiceps 163. crasicollis 164.524 .

crassipora 697 .

crassula jo2.

crateriformis 168.531.

cienata 145. $4 y^{2}$.

cruciata 696 .

cucumerina 147.

cunciceps $15 \mathrm{~S}$.

Curvirostrae 172.

cyathiformis $152.502,692$.

Cyprini Idi 175.

dondritica 349. 493.

Dentroculajtis 705 .

denticulata 145 .

Didel phidis 170 .

difformis 1.45 . 43 .

diminuta 689 .

dispar 150. 495.

elliptica 158.514.

Enberizarum 172 .

Eperlani 175.'

expansa 1.44. 497.

farcininalis 160.519.

fasciata $157,700$.

liclis Pardi 100.

festiva 145.490

filamentosa 154 .

flicollis 145 .
Taenia

filiformis 166 .

filum $157 \cdot 512$.

flagellum 161. 520.

Fringillarum 172 .

Fulicae 166.

furcigera $5: 8$.

Gallinaginis 175 .

globifera 153.514 .

globulosa 515 .

Igracilis 3 .

IIyracis $170^{\circ}$.

Idi 175 .

inflata 166. 527 .

infundibuliformis 152.503 .

intermedia 163. 701 .

interrupta 161.

inversa 156.510.

Lacertae $175^{\circ}$

laevigata 151.500.

laevis. 155. 700 .

lanceolata 145. 4S8.

lanceslata. 43\%.

Lari cani 174 .

lata 136.

laticollis 164524 .

Lemmi 170.

Linea 157. 513 .

lineata 169 .

litterata 148 .

longiceps $69 \mathrm{i}$.

longicollis 149. 495 .

Jongirostris 168.532 .

longirostris 528 .

macrorliyncha 165 .

maculaia 155.

Malleus 262.521.

mirginata $16,3.523$.

Marmorae. 4SS.

megacantha 701 .

Merupis 171.

microcephala $157 \cdot 513$. 


\section{$\div 807 \div$}

Taenia

Motacillae brasil. 706 .

Motacil. cyaneae. jo6.

Motacil. prorincialis 705 . multistriata 165.526.

Muris capensis 170 .

Muis sylvatici 170 .

Musculi 170.

Mustelce vulgaris 159.

mutabilis $15 \% 502$.

Myoxi 170. 53千.

nasuta 147.

Nymṕliaea 158.

obtusata $359 \cdot 517$.

ocellata 149 .

octolobata 165 .

oligotoma $\mathrm{I} 6 \mathrm{t} \cdot 520$.

omphalodes I 46 . 401 .

opuntioides 147.493.

Orivli 16s.

osculata 150. 497.

paradoxa 1 fí.

parallelipipeda 160.519.

pectinata 145.458.

Pelecani Aquilae 7og.

perfoliata- 145 .

perlata 146 .

Phasiani colchici 172 :

Pici 171.

platycephala 154.508 .

plicata 145.490.

Pollachii 175 .

polymorpha $154 \cdot 505$.

porosa 168.529 .

pusilla 159.

Puzorii 159.

pyramidata 696 .

quadrata $164 .-525$.

raçemosa, 692.

Rajae Butis 14I.

Ralli 173.

Ratti 170.
Trenia

Rentine 495 .

Salmonis Omul 175.

sculeciua 169. 532.

Sculopacis 173

Scolopacis brasil. 706 .

Serpentulus $167 \cdot 529$.

ser rata 163 .

setigera 153 .

silicula 173.

sinuosa 360.527 .699 .

Sitrat 171 .

Solinm I62. 523 .

Soricis 170 .

spluacrocephala' 695 .

splacroptora I51. 499.

splienocephala 154: 505.

stentorea 495.

Sternae 174 .

Sternac brasil. 70\%.

straminea 165 .

Strigis 704 .

Struthionis 173.

stylosa 160.520.

Tulornae 17 f. 534 .

tenuicollis 159.517 .

tenuirostris $156.50 \mathrm{~g}$.

Tetrodontis Molae 138.

iornlosa 149.

Totani 173 .

trilineata $16 \%, 529$.

Tringae 173.

Tringae brasil. 707 .

tripunctata 147 .

tuberculata, 150.406 .

Turdi brásil. 705.

Turdorum 155 .

undnlata 167.528 .

Unguicula 173.53 f.

unilateralis 696 .

Upupae 171.

Urogalli $17 \%$

Ursi maritimi $16 \mathrm{~g}$. 


\section{$-808$}

Taenia

vaginata $153.503 \cdot 694$.

variabilis 151. 498 .

Vespertilionis 159 .

Vospertil. auriti 169 .

villosa 153.

Zebrae 171 .

Tentacularia vid. Tetrarhynchus.

Tetrabothrius 469 .

Tetragulus 124. 433:

Tetrarhynchus

appendiculatus 131. 454 .

Argentinae 458 .

attenuatus 130 . 449 .

disoophorus 130.450 .688 .

elongatus $45 \mathrm{~S}$.

gracilis 131.456 .

grossus 129.448 .

lingualis 132 .

macrobothrius 131. 453.

6.89.

megabothrins 130.451.

megacephalus 129. 447 .

Morhuae 458 .

papillosus -131. 453 .

Pleuronectis maximi 132.

\subsection{7 .}

scolecinus 131. 454.

Squali $132.45^{6}$.

tenuicollis 130. 451 .

Tetrathyridium, 514.

Triaenophorus

nodulosus 135. 467 .

Trichocephalus

affinis 16. 225.

Cameli 1s. 228.

capillaris 13.

Castoris $1 \mathrm{~S} .22 \mathrm{~S}$.

contortius 637 .
Trichocephalus

crenatus 17.226.

dispar 16.

echinatus 18.

gibbosus 638 .

gracilis 638 .

Lemuris 18. 228.

minutus 638 .

nodosus 17.227.

palaeformis 16.223.

tenuissimus 13.

unguiculatus 17.225 .

Trichosoma

Alaudae 15.

brevicolle 13.

Caprimulgi 15 .

Carbonis 16.

Charadrii 16.

Columbae 15 .

Corvorum 15. $223^{\circ}$

Crotali 16.

Crypturi 636.

Erinacei 1.4.

Falconum 15. 222:

Fringillae 15.

Hirundinis 15.

inflexum 13. 221,

obtusiusculum 13. 220 .

obtusım 13. 220.

Picorum 15.

plica 14. 222.

Putorii 1.f.

Turdi 15 .

Vauelli 15.

Vespertilionis 14.1

Tricuspidaria. 135.

Tristoma

coccinenm 123. $42 S$.

maculatum 123. 430 . 


\title{
Explicatio 'Tabularum.
}

\author{
T a b. I.
}

Fig. 1. Filaria sanguinea.

2. Physaloptera clausa. Mas magnit. naturali.

3. Ejusdem apex caudae magnitudine aucta.

4. Echinorlynchus porrigens, adulzus, magnit, nat.

5. Ejusdem apex receptáculi.

6. Idem Echinorlynchus pusillus.

7. Tristoma coccineum a latere infferiore, magnit. uat.

S. Idem a latere superiore.

9. Tristoma maculatum a latere inferiore, magnit. naturali.

10. Idem a datere superiore。

\section{T a b. I r.}

Fig. 1. Monostoma tenuicolle a parte dextra et dorsali, magnitudine naturali.

2. Ilen a parte sinistra et inferiore.

3. Idcu magnitudine ancta.

4. Ejusdem particula corporis magis aucta, vasis majoribus, minoribus et minimis ope fitterarum a, b. c. distinctis.

5. Distona tereticolle Iucii duabus excrescentis no. iatum, de quibus 1. 600. conferenda. Magnit. naturali.

(j. Polystoma duplicatum. a. MInsura maguitudinis naturalis. b. Ninguitudive auctum. c. Porusdu. plicatus nugis auctus.

7. Tcurarhynchus megaceplualus a parte superioré, magnitudine natutali.

S. Iilem a latére. 
Fig. 9. Tetrarhynchus grossus, proboscidibus retractis, magn, nat.

10. Idem apertus, proboscidum receptaculis sub oculos cadentibus.

11. Tetrarhynchus macrobothrius a latere planiusculo, dorsali vel abdominali, magnitudine naturali.

12. Idem a latere.

13. Idem papilla caudali biloba bene exhibita.

14. Tetrarhyuchus megabothrius, linea apposita magnitudinem naturalem indicante.

\section{T a b. III.}

Fig. 1, Particula Ligulae sparsae ovariis" lenıniscatis in. signis, conf. P. 462: Magnitudine parum aucta. 2. Bochriocephalus plicatus parte corporis maxime contracta. Magnitudine naturali.

3. 4. Ova Taeniae variabilis magnitudine aucta.

5. 6. Eadem magis ancta.

7. S. Ova Taeniae porosae pariter aucta.

9. Ovum Taeniac crateriformis.

10. 11. Ova Taeniae polymorphae, magnitudine diverso modo aucta?

12. Anthocephali elongati mesenterici vesica externa, magnit, naturali?

13. 14, Ejusdem vesicae internae, verme protracto in. timae adhaereute.

15. Idem situ naturali.

I6. Idem aperiıs, quo proboscidum receptacula pateant. 17. Ejusdem proboscis magnitudine valdopere aucta.

18. Cysticercus Simiąe biceps, magnitudine naturali, conf: p. 545 :

19. Ova Taeniaje crassulac, magnitudine aucta.

20. Ova T'aeniae sphenocephalae, pari modo aucta ac praecedentia:

21. Ovum Taeniae longicipitis. 


\section{Corrigend a.}

P. 12. pone lin. 12. adde: Ent. 1. p. 79. n. 40.

- 31. lin. 9. Mant. n. 2. deleatur.

- 53. - 7. Mydae l. graecae.

- 59. pone lin. 31. adde: Mant. 13 \%.

- 65. lin. 14. loco 99. 1. 98 .

- 73. - 2. pone Aprili adde: Tincae ib. Majo, Brauv.

- 75. - 6. Junio 1. Martio.

- 93. - 1. tanri 1. Tanri feri.

- 97. - 4. et lin. 13. signum crucis addatur.

- 95. - 31. 370.1.372。

- 99. - 10. n. $7 \cdot 1$. n. 71 .

- 106. - 3. signum crucis deleatm.

- 112. - 30. Hilitëtorum 1. Nielanaëtorum.

- 114. - 6. Pdronis deleatur.

- 115. - S. pone Anatis adde: Anseris.

- 120: - 28, 140. Distoma Ralli deleatme, idem enim est ac D. holostomum p. 94. n. I2.

- 124. pone lin. 5. inseratur: Ent. I. F. 447 . n. 2. Polystoma denticulatum.

- 131: lin. 12, forsan Coryphaente Hippuridis, 1. Coryphaenae Equiselis.

- 155. - 3. Luscini !. Lnsciniae Majo.

- 23. Addatur crucis signum.

- I'4. - 20. pone 11. 61, adde: Mant. 75 .

- IS4. pone lin. 1. add. Mant. I3.

- - 4. signum crucis deleatur, addatur: Mant. I4, 
Berolini.

Litteris b. 7. W. SCHMIDT VIDE.AE et FiLYT. 



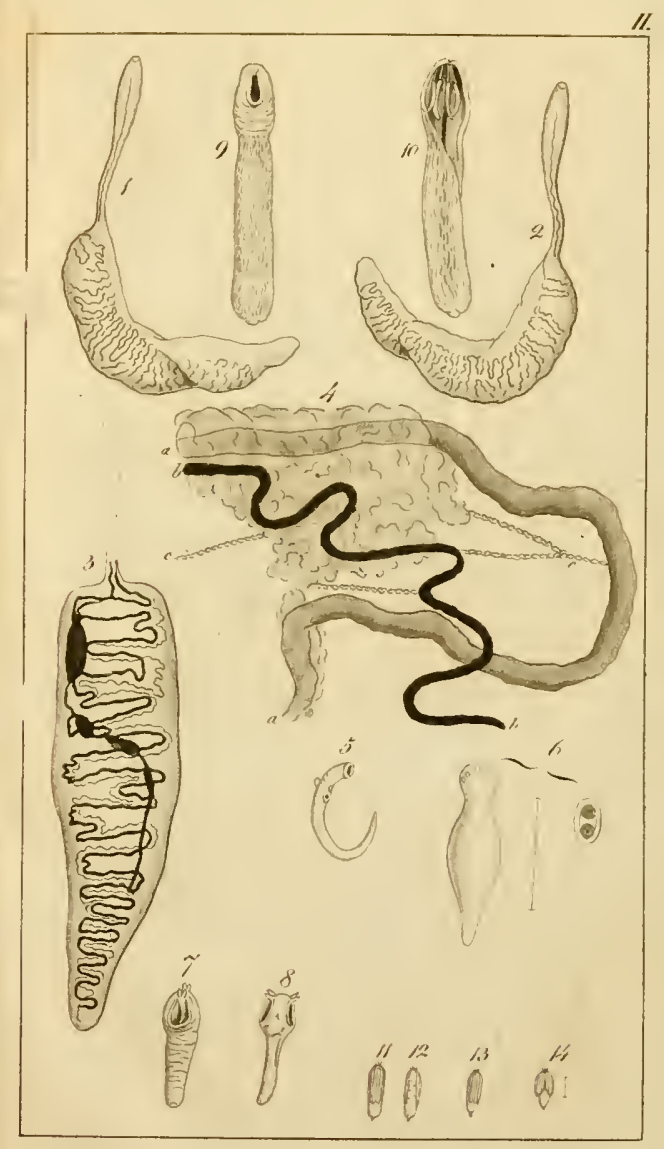





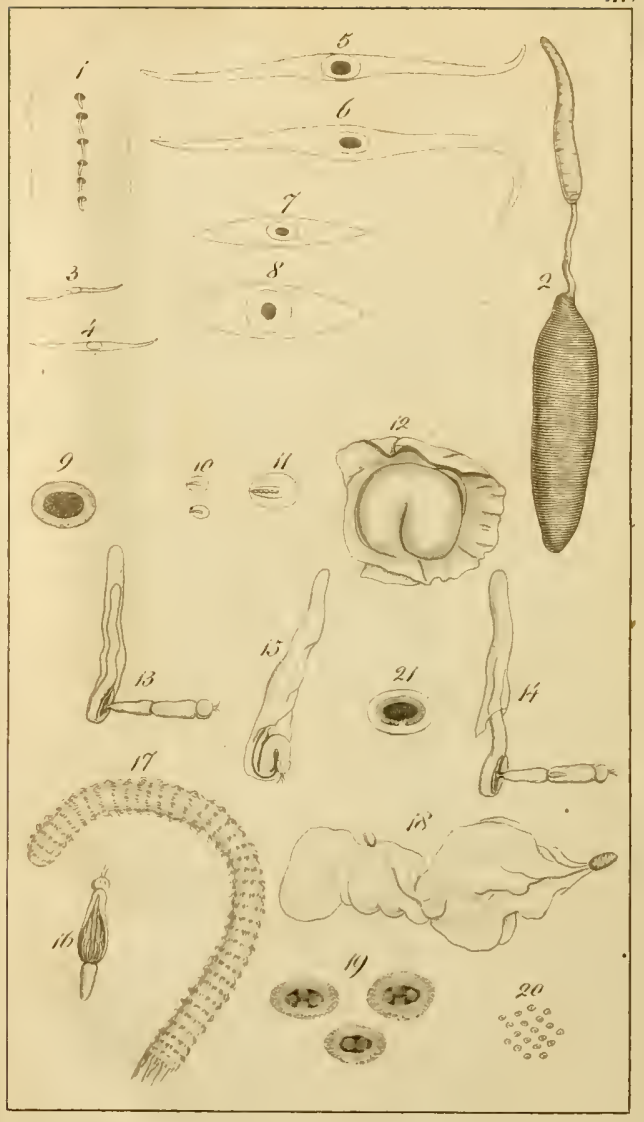









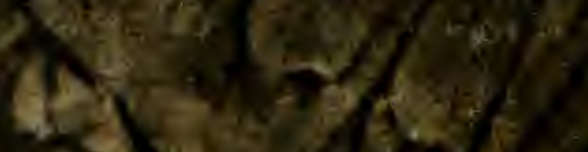